\title{
Synthesis of modified nucleoside oligophosphates simplified: Fast, pure, and protecting group free
}

Jyoti Singh ${ }^{\mathrm{a}}$, Alexander Ripp ${ }^{\mathrm{a}}$, Thomas M. Haas ${ }^{\mathrm{a}}$, Danye Qiu ${ }^{\mathrm{a}}$, Manfred Keller ${ }^{\mathrm{a}}$, Paul A. Wender ${ }^{\mathrm{b}}$, Jay S. Siegel $^{\mathrm{c}, \mathrm{d} *}$, Kim K. Baldridge ${ }^{\mathrm{c} *}$, Henning J. Jessen ${ }^{\mathrm{a}, \mathrm{d}, \mathrm{e} *}$

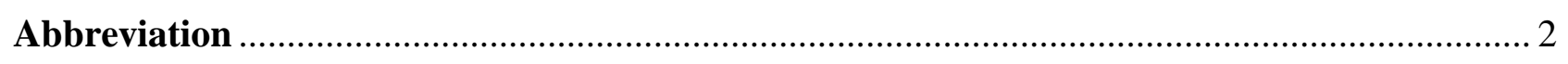

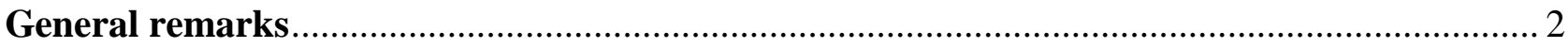

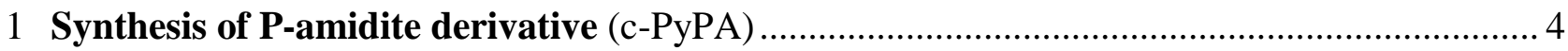

1.1 General procedure for preparation of c-PyPA .............................................................. 4

1.1.1 Cyclic pyrophosphoryl-P-amidite (c-PyPA, -O-, $\left.\mathrm{A}_{1}\right)$................................................... 4

1.1.2 Cyclic methylenediphosphoryl-P-amidite (c-PyсH2 $\left.\mathrm{PA}, \mathrm{A}_{2}\right)$........................................... 5

1.1.3 Cyclic difluoromethylenediphosphoryl-P-amidite (c-PyCF2 $\left.\mathrm{PA}, \mathrm{A}_{3}\right)$................................5

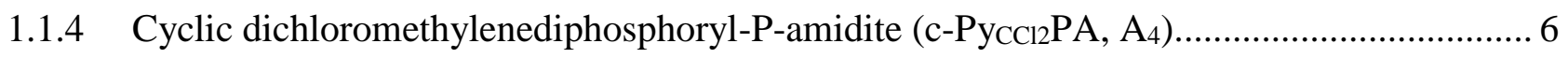

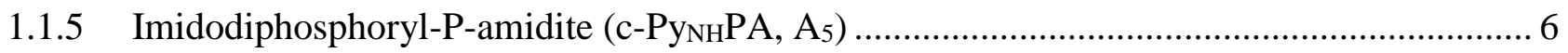

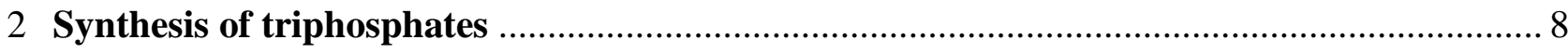

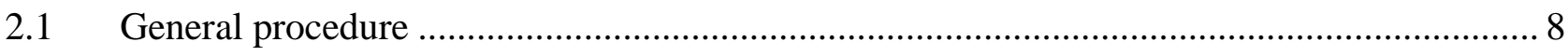

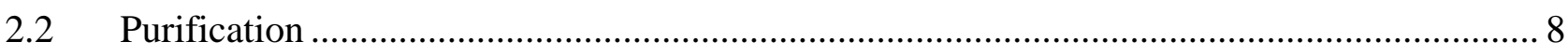

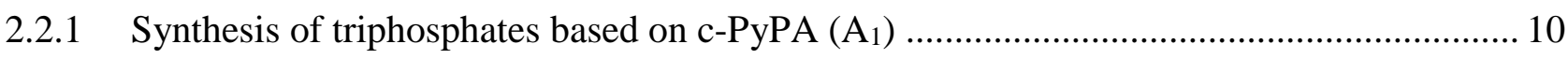

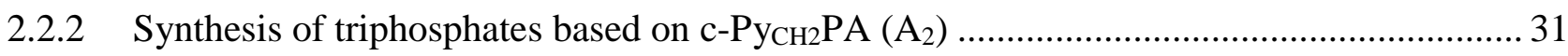

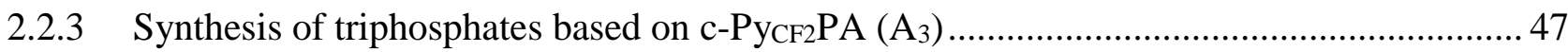

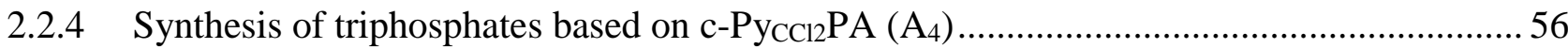

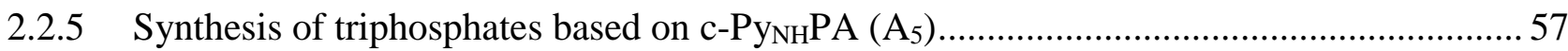

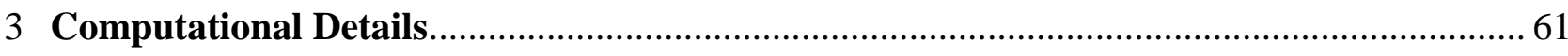

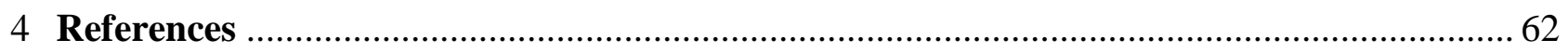




$\begin{array}{lll}\text { Abbreviation } & & \\ \text { TBA } & : & \text { Tetrabutylammonium } \\ \text { ETT } & : & \text { 5-(Ethylthio)-1H-tetrazole } \\ \text { DCI } & : & \text { Dicyanoimidazole } \\ m \text { CPBA } & : & \text { meta-Chloroperbenzoic acid } \\ \text { EtOAc } & : & \text { Ethyl acetate } \\ \text { Et } 2 \mathrm{O} & : & \text { Diethyl ether } \\ \mathrm{Et}_{3} \mathrm{~N} & : & \text { Triethylamine } \\ \mathrm{DIPEA} & : & \text { Diisopropylethylamine } \\ \mathrm{MeCN} & : & \text { Acetonitrile } \\ \mathrm{DMF} & : & \text { Dimethylformamide } \\ \mathrm{PP}_{\mathrm{i}} & : & \text { Pyrophosphate } \\ \mathrm{PCP} & : & \text { Methylene diphosphate } \\ \mathrm{d} 4 \mathrm{~T} & : & \text { Stavudine } \\ \mathrm{AZT} & : & \text { Azidothymidine } \\ \mathrm{NH}_{4} \mathrm{HCO}_{3} & : & \text { Ammonium bicarbonate } \\ \mathrm{NaClO}_{4} & : & \text { Sodium perchlorate } \\ \mathrm{TEAA} & : & \text { Triethylammonium Acetate }\end{array}$

\section{General remarks}

Reagents were purchased from commercial suppliers (Acros, Sigma Aldrich, Fluka, TCI, Chem Genes Corp.) and used without further purification, unless noted otherwise.

Starting precursors were obtained from Sigma Aldrich, Fluka, or TCI as sodium salts and converted into their tetrabutylammonium (TBA) salts by ion exchange on Dowex 50WX8 $\left(\mathrm{H}^{+}\right)$followed by neutralization with TBA hydroxide and subsequent lyophilization. The accurate amounts of TBA counterions in the obtained salts were determined by ${ }^{1} \mathrm{H}-\mathrm{NMR}$ and ${ }^{31} \mathrm{P}-\mathrm{NMR}$ by the use of tetramethylphosphonium bromide as internal standard. Furthermore, all the polyPs for e.g. pyrophosphate, methylenediphosphate, difluoromethylenediphosphate, dichloromethylenediphosphate and imidodiphosphate were stored over molecular sieves $(3 \AA)$ in anhydrous DMF or MeCN.

Adenosine triphosphate and fluoromonophosphate was obtained from Sigma Aldrich stored over molecular sieves $(4 \AA)$ in DMF.

Solvents were obtained in analytical grade and used as received.

Dry solvents were purchased in analytical grade and used without further purification.

Oxidation and hydrolysis sensitive reactions were performed with dry solvents which were stored under an argon (Ar) atmosphere. Diethyl ether $\left(\mathrm{Et}_{2} \mathrm{O}\right)$, dichloromethane (DCM) and tetrahydrofuran (THF) were purified using Braun Solvent Purification System 800 and stored under Ar atmosphere.

Acetonitrile (MeCN) was purchased from Acros (Acetonitril, 99.9\%, Extra dry over Molecular Sieve, AcroSeal $^{T M}$ ) and stored over molecular sieves ( $3 \AA$ ) under Ar atmosphere. 
$N, N$-Dimethylformamide (DMF) was purchased from Sigma-Aldrich (,$N$-Dimethylformamide,

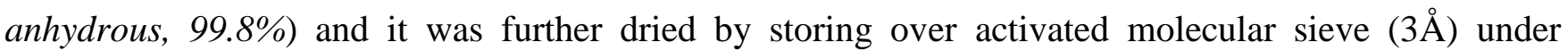
Ar atmosphere.

$\mathrm{N}, \mathrm{N}$-Diisopropylethylamine (DIPEA) and triethylamine $\left(\mathrm{Et}_{3} \mathrm{~N}\right)$ was distilled under $\mathrm{Ar}$ atmosphere and was stored over activated molecular sieve $(3 \AA)$ and Ar atmosphere.

Deuterated solvents for NMR and reactions were obtained from Aldrich and deutero Germany, in the indicated purity grade and used as received for NMR spectroscopy.

Reactions were carried out under $\mathrm{Ar}$ or $\mathrm{N}_{2}$ atmosphere, the gasses were pass through drying column $\left(\mathrm{P}_{2} \mathrm{O}_{5}\right)$ and NMR tubes were preheated prior to use and samples were prepared under inert atmosphere.

Anion exchange chromatography was performed using Q Sepharose ${ }^{\circledR}$ Fast Flow or DEAE Sepharose ${ }^{\circledR}$ Fast Flow (GE Healthcare). Crude products were loaded with water and were eluted using ammonium bicarbonate buffer by stepwise increase of the buffer concentration. Fractions were lyophilised and analyzed by ${ }^{31} \mathrm{P}$ NMR.

Medium pressure liquid chromatography (MPLC) was performed using PuriFlash $®$ from interchim ${ }^{\circledR}$. Lyophilizations were done with Alpha 1-4 LD plus Freeze Dryer from Christ.

${ }^{1}$ H-NMR spectra were recorded on Bruker $300 \mathrm{MHz}$, Bruker $400 \mathrm{MHz}$ and Bruker $500 \mathrm{MHz}$ spectrometers in the indicated deuterated solvent. Data are reported as follows: chemical shift $(\delta, \mathrm{ppm})$, multiplicity (s, singlet; d, doublet; t, triplet; q, quartet; m, multiplet; $\mathrm{m}_{\mathrm{c}}$, centered multiplet; br, broad signal), coupling constant(s) (J, Hz), integration. All signals were referenced to the internal solvent signal as standard $\left(\mathrm{CDCl}_{3}, \delta 7.26 ; \mathrm{D}_{2} \mathrm{O}, \delta 4.79 ; \mathrm{CD}_{3} \mathrm{OD}, \delta 3.31 ; \mathrm{DMSO}_{-} \mathrm{d}_{6}, \delta 2.50\right)$.

${ }^{13} \mathbf{C}\left\{{ }^{1} \mathbf{H}\right\}$-NMR spectra were recorded on Bruker $101 \mathrm{MHz}$ (with cryoprobe) and Bruker $126 \mathrm{MHz}$ (without cryoprobe) spectrometers at $298 \mathrm{~K}$ in the indicated deuterated solvent.

${ }^{31} \mathbf{P}\left\{{ }^{1} \mathbf{H}\right\}$-NMR spectra and ${ }^{31} \mathbf{P}$-NMR spectra were recorded on Bruker $162 \mathrm{MHz}$ (equipped with a cryo platform) or $202 \mathrm{MHz}$ spectrometers at $298 \mathrm{~K}$ in the indicated deuterated solvent. All signals were referenced to an internal standard phosphate (PPP).

${ }^{19}$ F-NMR spectra were recorded on Bruker $377 \mathrm{MHz}$ (equipped with a cryo platform) at 298K in the indicated deuterated solvent.

Mass Spectrometer were recorded by Analytical department of the institute of Organic Chemistry at ALU-Freiburg using a Thermo LCQ Advantage [spray voltage: $2.5-4.0 \mathrm{kV}$, spray current: $5 \mu \mathrm{A}$; ion transfer tube: $250(150)^{\circ} \mathrm{C}$, Vapourizer temperature: $\left.50-400{ }^{\circ} \mathrm{C}\right]$ and Exactive with Orbitrap-Analysator, Thermo Scientific used for HRMS. 


\section{Synthesis of P-amidite derivative ( $c$-PyPA)}

\subsection{General procedure for preparation of $c$-PyPA}

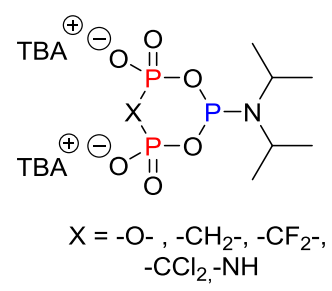

(A)

$\mathrm{PxP}_{\mathrm{i}} \times 1.6$ - 2.2 TBA (1.0 eq.) was coevaporated with dry $\mathrm{MeCN}(3 \times 4 \mathrm{ml})$, then dissolved in appropriate amount of dry MeCN or in dry DMF under an Ar atmosphere. Distilled Et ${ }_{3} \mathrm{~N}$ or DIPEA (2.0 - 3.0 eq.) stored over molecular sieves $(3 \AA)$ was added and the mixture was cooled to $-4^{\circ} \mathrm{C}$. Afterwards, distilled (iPr) ${ }_{2} \mathrm{~N}-\mathrm{PCl}_{2}$ (1.0 eq.) was added dropwise into the reaction mixture, which was then stirred at $-4^{\circ} \mathrm{C}$ to $-10^{\circ} \mathrm{C}$ for $5-10$ mins and the reaction was followed by ${ }^{31} \mathrm{P}-\mathrm{NMR}$. The reaction mixture was stored at $-20^{\circ} \mathrm{C}$ under Ar atmosphere for up to four weeks and added directly for coupling reactions without further manipulations.

Note-: Additionally, activated molecular sieves $(3 \AA)$ could be added after the coevaporation step in order to further prevent hydrolysis within the formation of c-PyPA.

\subsubsection{Cyclic pyrophosphoryl-P-amidite ( $c$-PyPA, -O-, $\left.\mathrm{A}_{1}\right)$}

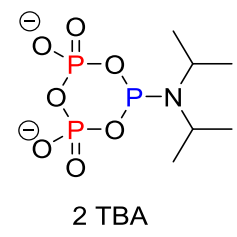

$\left(A_{1}\right)$

$\mathrm{PP}_{\mathrm{i}} \times 2.0 \mathrm{TBA}(2.09 \mathrm{~g}, 3.02 \mathrm{mmol}, 1.0$ eq. $)$ was coevaporated with dry MeCN $(2 \times 20 \mathrm{ml})$ and then dissolved in dry $\mathrm{MeCN}$ (40 ml) under an Ar atmosphere. Additionally, activated molecular sieves $(3 \AA)$ was added in the above flask. $\mathrm{Et}_{3} \mathrm{~N}(1.50 \mathrm{ml}, 10.5 \mathrm{mmol}, 3.5$ eq. $)$ was added and the mixture was cooled to $-10^{\circ} \mathrm{C}$. Previously distilled $(i \mathrm{Pr})_{2} \mathrm{~N}-\mathrm{PCl}_{2}(520 \mu \mathrm{l}, 3.02 \mathrm{mmol}, 1.0$ eq. $)$ was then slowly added into the mixture and it was stirred at $-10^{\circ} \mathrm{C}$. After 5 mins of stirring, ${ }^{31} \mathrm{P}-\mathrm{NMR}$ of an aliquot of the reaction mixture in dry $\mathrm{CDCl}_{3}$ confirmed full conversion. The reaction mixture was stored at $-20^{\circ} \mathrm{C}$ under $\mathrm{Ar}$ atmosphere for up to four weeks and added directly without further manipulations for coupling reactions. The analytical data is consistent with the literature. ${ }^{1}$

${ }^{31} \mathbf{P}\left\{{ }^{1} \mathbf{H}\right\}$ NMR $\left(162 \mathrm{MHz}\right.$, aliquot of the reaction mixture in dry $\left.\mathrm{CDCl}_{3}, \delta / \mathrm{ppm}\right): 130.9(\mathrm{t}, \mathrm{J}=22.8 \mathrm{~Hz}$, 1P), $-17.4(\mathrm{~d}, \mathrm{~J}=22.8 \mathrm{~Hz}, 2 \mathrm{P}) .{ }^{31} \mathbf{P}$ NMR $\left(162 \mathrm{MHz}\right.$, aliquot of the reaction mixture in dry $\left.\mathrm{CDCl}_{3}, \delta / \mathrm{ppm}\right)$ : 130.9 (tt, J = 21.9, $11.6 \mathrm{~Hz}, 1 \mathrm{P}),-17.4$ (d, J = 22.7 Hz, 2P). 


\subsubsection{Cyclic methylenediphosphoryl-P-amidite $\left(c-\mathrm{Py}_{\mathrm{CH} 2} \mathbf{P A}, \mathbf{A}_{2}\right)$}

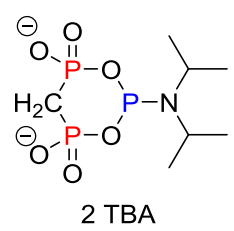

$\left(A_{2}\right)$

Methylenediphosphonic acid $\times 1.9$ TBA (1.00 g, 1.57 mmol, 1.0 eq. $)$ was coevaporated with dry MeCN $(4 \times 2 \mathrm{ml})$, then dissolved in dry $\mathrm{MeCN}(20 \mathrm{ml})$ under an Ar atmosphere. Additionally, activated molecular sieves ( $3 \AA ̊)$ was added in the above flask. $\mathrm{Et}_{3} \mathrm{~N}(670 \mu 1,4.71 \mathrm{mmol}, 3.0$ eq. $)$ was added and the mixture was cooled to $-4^{\circ} \mathrm{C}$. Previously distilled ( $\left.i \mathrm{Pr}\right)_{2} \mathrm{~N}-\mathrm{PCl}_{2}(250 \mu \mathrm{l}, 1.57 \mathrm{mmol}, 1.0$ eq. $)$ was then slowly added into the mixture and it was stirred at $-4{ }^{\circ} \mathrm{C}$. After 5 mins of stirring, ${ }^{31} \mathrm{P}-\mathrm{NMR}$ of an aliquot of the reaction mixture in dry $\mathrm{CDCl}_{3}$ confirmed full conversion. The reaction mixture was stored at $-20^{\circ} \mathrm{C}$ under an Ar atmosphere for up to two weeks and added directly without further manipulations for coupling reactions.

${ }^{31} \mathbf{P}\left\{{ }^{1} \mathbf{H}\right\}$ NMR $\left(122 \mathrm{MHz}\right.$, aliquot of the reaction mixture in dry $\left.\mathrm{CDCl}_{3}, \delta / \mathrm{ppm}\right): 125.7(\mathrm{t}, \mathrm{J}=9.0 \mathrm{~Hz}, 1 \mathrm{P})$, $6.25(\mathrm{~d}, \mathrm{~J}=9.0 \mathrm{~Hz}, 2 \mathrm{P}) \cdot{ }^{31} \mathbf{P} \mathrm{NMR}\left(162 \mathrm{MHz}\right.$, aliquot of the reaction mixture in dry $\left.\mathrm{CDCl}_{3}, \delta / \mathrm{ppm}\right): 125.7$ $(\mathrm{p}, \mathrm{J}=10.1 \mathrm{~Hz}, 1 \mathrm{P}), 6.25(\mathrm{td}, \mathrm{J}=18.6,8.7 \mathrm{~Hz}, 2 \mathrm{P}) .{ }^{1} \mathbf{H}$ NMR $(400 \mathrm{MHz}$, aliquot of the reaction mixture in dry $\mathrm{CDCl}_{3}, \delta / \mathrm{ppm}$ contains1.9 TBA salt): $3.25-3.18(\mathrm{~m}, 2 \mathrm{H}), 2.92-2.40(\mathrm{~m}, 16 \mathrm{H}), 1.16(\mathrm{tt}, \mathrm{J}=8.3$, $5.9 \mathrm{~Hz}, 16 \mathrm{H}), 0.93(\mathrm{~h}, \mathrm{~J}=7.3 \mathrm{~Hz}, 16 \mathrm{H}), 0.79-0.65(\mathrm{~m}, 14 \mathrm{H}), 0.56(\mathrm{t}, \mathrm{J}=7.3 \mathrm{~Hz}, 24 \mathrm{H})$.

HRMS (ESI) $\mathrm{m} / \mathrm{z}$ for $\left[\mathrm{C}_{7} \mathrm{H}_{17} \mathrm{NO}_{6} \mathrm{P}_{3}\right]^{-}$: calcd 304.0274, found 304.0273.

\subsubsection{Cyclic difluoromethylenediphosphoryl-P-amidite ( $c$-Pyсr2 $\left.\mathbf{P A}, \mathbf{A}_{3}\right)$}

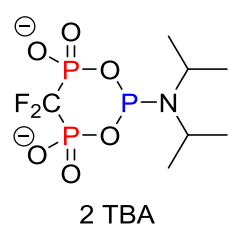

$\left(A_{3}\right)$

Difluoromethylenediphosphonic acid $\times 1.95 \mathrm{TBA}(500 \mathrm{mg}, 0.73 \mathrm{mmol}, 1.0$ eq.) was coevaporated with dry MeCN $(2 \times 2 \mathrm{~mL})$, then dissolved in dry MeCN $(10 \mathrm{ml})$ under an Ar atmosphere. Additionally, activated molecular sieves $(3 \AA)$ was added in the flask. $\mathrm{Et}_{3} \mathrm{~N}(310 \mu 1,2.20 \mathrm{mmol}, 3.0$ eq. $)$ was added and the mixture was cooled to $-4^{\circ} \mathrm{C}$. Previously, distilled $(i \mathrm{Pr})_{2} \mathrm{~N}-\mathrm{PCl}_{2}(135 \mu 1,0.730 \mathrm{mmol}, 1.0$ eq. $)$ was then slowly added into the mixture and it was stirred at $-4^{\circ} \mathrm{C}$. After 5 mins of stirring, ${ }^{31} \mathrm{P}-\mathrm{NMR}$ of an aliquot of the reaction mixture in dry $\mathrm{CD}_{3} \mathrm{CN}$ confirmed the reaction was completed by total consumption of starting material, but due to the instability of reagent, NMR was variable. The reaction mixture was stored 
at $-20^{\circ} \mathrm{C}$ under $\mathrm{Ar}$ atmosphere for up to three weeks and added directly without further manipulations for coupling reactions.

${ }^{31} \mathbf{P}\left\{{ }^{1} \mathbf{H}\right\}$ NMR $\left(162 \mathrm{MHz}\right.$, aliquot of the reaction mixture in dry $\left.\mathrm{CD}_{3} \mathrm{CN}, \delta / \mathrm{ppm}\right): 128.8-126.8(\mathrm{~m}, 1 \mathrm{P})$, -5.48 (ddd, $\mathbf{J}=82.3,77.6,13.0 \mathrm{~Hz}, 2 \mathrm{P}) .{ }^{31} \mathbf{P}$ NMR (162 MHz, aliquot of the reaction mixture in dry $\left.\mathrm{CD}_{3} \mathrm{CN}, \delta / \mathrm{ppm}\right): 130.1-125.7(\mathrm{~m}, 1 \mathrm{P}),-5.49(\mathrm{ddd}, \mathrm{J}=82.2,77.4,13.0 \mathrm{~Hz}, 2 \mathrm{P}) .{ }^{31} \mathbf{P}\left\{{ }^{19} \mathbf{F}\right\} \mathbf{N M R}(162$ $\mathrm{MHz}$, aliquot of the reaction mixture in dry $\left.\mathrm{CD}_{3} \mathrm{CN}, \delta / \mathrm{ppm}\right): 127.7(\mathrm{~m}, 1 \mathrm{P}),-5.50(\mathrm{~d}, \mathrm{~J}=13.0 \mathrm{~Hz}, 2 \mathrm{P})$.

HRMS (ESI) $\mathrm{m} / \mathrm{z}$ for $\left[\mathrm{C}_{7} \mathrm{H}_{15} \mathrm{~F}_{2} \mathrm{NO}_{6} \mathrm{P}_{3}\right]^{-}$: calcd 340.0086 , found 340.0082 .

\subsubsection{Cyclic dichloromethylenediphosphoryl-P-amidite $\left(c\right.$-Py $\left.\mathbf{y C C l 2}_{2} \mathbf{P A}, \mathbf{A}_{4}\right)$}

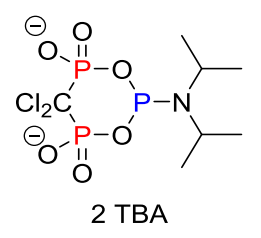

$\left(\mathbf{A}_{4}\right)$

Dichloromethylenediphosphonic acid $\times 2.0$ TBA ( $1.00 \mathrm{~g}, 1.38 \mathrm{mmol}, 1.0$ eq. $)$ was coevaporated with dry $\mathrm{MeCN}(5 \times 2 \mathrm{ml})$, then dissolved in dry $\mathrm{MeCN}(20 \mathrm{ml})$ under Ar atmosphere. Additionally, activated molecular sieves ( $3 \AA$ ) was added in the flask. $\mathrm{Et}_{3} \mathrm{~N}(580 \mu \mathrm{l}, 4.13 \mathrm{mmol}, 3.0$ eq.) was added and the mixture was cooled to $-4^{\circ} \mathrm{C}$. Previously distilled $(i \mathrm{Pr})_{2} \mathrm{~N}-\mathrm{PCl}_{2}(250 \mu \mathrm{l}, 1.38 \mathrm{mmol}, 1.0$ eq. $)$ was then slowly added into the mixture and it was stirred at $-4^{\circ} \mathrm{C}$. After 5 mins of stirring, ${ }^{31} \mathrm{P}-\mathrm{NMR}$ of an aliquot of the reaction mixture in dry $\mathrm{CDCl}_{3}$ confirmed full conversion. The reaction mixture was stored at $-20^{\circ} \mathrm{C}$ under $\mathrm{Ar}$ atmosphere for up to three weeks and added directly without further manipulations for coupling reactions. ${ }^{31} \mathbf{P}\left\{{ }^{1} \mathbf{H}\right\}$ NMR $\left(122 \mathrm{MHz}\right.$, aliquot of the reaction mixture in dry $\left.\mathrm{CD}_{3} \mathrm{CN}, \delta / \mathrm{ppm}\right): 129.0(\mathrm{t}, \mathrm{J}=9.0 \mathrm{~Hz}$, 1P), $-1.33(\mathrm{~d}, \mathrm{~J}=9.5 \mathrm{~Hz}, 2 \mathrm{P}) .{ }^{31} \mathbf{P}$ NMR $\left(162 \mathrm{MHz}\right.$, aliquot of the reaction mixture in dry $\left.\mathrm{CD}_{3} \mathrm{CN}, \delta / \mathrm{ppm}\right)$ : $131.2-126.6(\mathrm{~m}, 1 \mathrm{P}),-1.33(\mathrm{~d}, \mathrm{~J}=9.2 \mathrm{~Hz}, 2 \mathrm{P})$.

HRMS (ESI) $\mathrm{m} / \mathrm{z}$ for $\left[\mathrm{C}_{7} \mathrm{H}_{15} \mathrm{Cl}_{2} \mathrm{NO}_{6} \mathrm{P}_{3}\right]^{-}$: calcd 371.9495 , found 371.9491 .

\subsubsection{Imidodiphosphoryl-P-amidite ( $c$-Py $\left.\mathbf{y}_{\mathrm{NH}} \mathbf{P A}, \mathbf{A}_{5}\right)$}

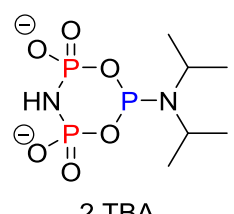

2 TBA

$\left(A_{5}\right)$

Imidodiphosphonic acid $\times 2.0$ TBA (1.00 g, $1.52 \mathrm{mmol}, 1.0$ eq.) was coevaporated with dry MeCN $(5 \times 2 \mathrm{~mL})$, then dissolved in dry $\mathrm{MeCN}(20 \mathrm{ml})$ under an Ar atmosphere. Additionally, activated molecular sieves $(3 \AA)$ was added in the flask. $\mathrm{Et}_{3} \mathrm{~N}(650 \mu 1,1.52 \mathrm{mmol}, 3.0$ eq. $)$ was added and the mixture 
was cooled to $-4^{\circ} \mathrm{C}$. Previously distilled $(i \mathrm{Pr})_{2} \mathrm{~N}-\mathrm{PCl}_{2}(270 \mu \mathrm{l}, 1.52 \mathrm{mmol}, 1.0$ eq. $)$ was then slowly added into the mixture and it was stirred at $-4^{\circ} \mathrm{C}$. After 5 mins of stirring, ${ }^{31} \mathrm{P}-\mathrm{NMR}$ of an aliquot of the reaction mixture in dry $\mathrm{CDCl}_{3}$ confirmed total consumption of the starting material, but due to the instability of the product, ${ }^{31} \mathrm{P}-\mathrm{NMR}$ was variable. Nevertheless, a triplet at $+128 \mathrm{ppm}$ confirmed the formation of Pamidite (A5). The reaction mixture was stored at $-20^{\circ} \mathrm{C}$ under Ar atmosphere and added directly without further manipulations for coupling reactions.

${ }^{31} \mathbf{P}\left\{{ }^{1} \mathbf{H}\right\}$ NMR $\left(122 \mathrm{MHz}\right.$, aliquot of the reaction mixture in dry $\left.\mathrm{CD}_{3} \mathrm{CN}, \delta / \mathrm{ppm}\right): 128.7(\mathrm{t}, \mathrm{J}=9.0 \mathrm{~Hz}$, 1P).

HRMS (ESI) $\mathrm{m} / \mathrm{z}$ for $\left[\mathrm{C}_{6} \mathrm{H}_{16} \mathrm{~N}_{2} \mathrm{O}_{6} \mathrm{P}_{3}\right]^{-}$: calcd 305.0227, found 305.0224.

Note -: The preparation method of the imidodiphosphonic acid was another reason for the lower purity of this P-amidite $\left(\mathrm{A}_{5}\right)$ since the imidodiphosphosphate is acid labile. It was partially decomposed while passing through Dowex 50WX8 $\left(\mathrm{H}^{+}\right)$. The column was performed according to the reported literature. ${ }^{2}$ However, it was employed without any further manipulation proceeded for coupling reaction. 


\section{Synthesis of triphosphates}

\subsection{General procedure}

\section{Procedure 1}

Note -: The coevaporation worked better in Oven-dried pear shaped flask.

Alcohol (1.2 - 1.5 eq.) and ETT (3.0 - 5.0 eq.) were coevaporated together in an oven-dried pear shaped flask with dry $\mathrm{MeCN}(2 \times 2 \mathrm{ml})$. Under an Ar atmosphere, a reaction mixture containing $c$-PyPA (A) $(0.075 \mathrm{M}, 1.0$ eq. in $\mathrm{MeCN})$ was added to the dried solids. The mixture was stirred at r.t. for 5-10 mins and the reaction was followed by ${ }^{31} \mathrm{P}$ NMR (complete conversion of the reaction was monitored by ${ }^{31} \mathrm{P}$ NMR, shifting a triplet from $+130 \mathrm{ppm}$ to $+100 \mathrm{ppm})$. Afterwards; the reaction mixture was cooled to $0^{\circ} \mathrm{C}$ or $-4^{\circ} \mathrm{C}, m \mathrm{CPBA}\left(1.5\right.$ - 2.0 eq.) was added and the mixture was stirred for 5 mins until ${ }^{31} \mathrm{P}$ NMR confirmed complete oxidation (a formation of triplet at $-22 \mathrm{ppm}$ which is diagnostic signal for the cyclotriphosphate B). The cyclic-intermediates were ring-opened by adding the reaction mixture to nucleophiles stirred well for 5 mins at r.t. (in solution) and then linearized products were isolated and purified by methods as given in purification section respectively.

\section{Procedure 2}

Nucleoside (1.2 - 1.5 eq.) and DCI (3.0 - 5.0 eq.) were coevaporated together in an oven-dried pear shaped flask with dry MeCN $(2 \times 2 \mathrm{ml})$. Under an Ar atmosphere, both solids were dissolved in dry DMF $(1 \mathrm{~mL})$ and a reaction mixture containing $c$-PyPA (A) $(0.075 \mathrm{M}$ or $0.5 \mathrm{M}, 1.0$ eq. in $\mathrm{MeCN}$ or DMF) was added. The mixture was stirred at r.t. for 5-10 mins and the reaction was followed by ${ }^{31} \mathrm{P}$ NMR (complete conversion of the reaction was monitored by ${ }^{31} \mathrm{P}-\mathrm{NMR}$, shifting a triplet from $+130 \mathrm{ppm}$ to $\left.+100 \mathrm{ppm}\right)$. Upon cooling to $0^{\circ} \mathrm{C}$ or $-4^{\circ} \mathrm{C}, m \mathrm{CPBA}(1.5$ - 2.0 eq.) was added and the mixture was stirred for 5 mins until ${ }^{31}$ P NMR confirmed complete oxidation (a formation of triplet at $-22 \mathrm{ppm}$ which is diagnostic signal for the cyclotriphosphate $\mathbf{B}$ ). The cyclic-intermediates were ring-opened by adding the reaction mixture to nucleophiles stirred well for 5 mins at r.t. (in solution) and the linearized products were isolated and purified.

\subsection{Purification}

Method A -: The product was precipitated from the reaction mixture by $\mathrm{NaClO}_{4}$ sol. $\left(-20^{\circ} \mathrm{C}, 0.5 \mathrm{M}\right.$ in acetone), it was stand at $-4^{\circ} \mathrm{C}$ for $10-20$ mins and the resulting precipitate was collected via centrifugation. The organic layer was discarded, the precipitate was thoroughly washed twice with acetone and dried under vacuum. The organic layer was stored at r.t. and the residue was again precipitated by the addition of cold $\mathrm{NaClO}_{4}$ sol. $\left(-20^{\circ} \mathrm{C}, 0.5 \mathrm{M}\right.$ in acetone $)$ at the next day. 
Note -: In some cases crude sample could be contaminated by remaining benzoic acid, which would be completely removed by washing the precipitate with the minimal amount of $\mathrm{MeOH}$ and acetone mixture $(1: 5, \mathrm{v} / \mathrm{v})$.

Method B -: Reaction mixture was precipitated by Method A and the crude product was further purified by Strong anion-exchange chromatography (SAX) (Q Sepharose ${ }^{\circ}$ Fast Flow, increasing concentrations of aqueous $\mathrm{NH}_{4} \mathrm{HCO}_{3}$ ) and freeze drying of the fractions eluted with specific concentrations of buffer.

Method C -: Reaction mixture was precipitated by Method A and the crude product was analyzed by analytical HPLC:

Column: Hypersil GOLD ${ }^{\mathrm{TM}}$ aq.,

Dimension: 150 × $3 \mathrm{~mm}$,

Particle size: $3 \mu \mathrm{m}$,

Flow rate: $1 \mathrm{ml} / \mathrm{min}$,

Mobile phase: Phase A: Water, Phase B: $100 \mathrm{mM}$ of TEAA (pH adjusted at 8.5), Phase C: Acetonitrile. Gradient:

1. 0 min $\quad$ : $\quad 60 \%$ mobile phase A $40 \%$ mobile phase $\mathrm{B}$

2. $5 \mathrm{~min} \quad$ : $60 \%$ mobile phase $\mathrm{A}$ $40 \%$ mobile phase $\mathrm{B}$

3. $16 \mathrm{~min} \quad$ : $\quad 60 \%$ mobile phase B $40 \%$ mobile phase $\mathrm{C}$

4. $21 \mathrm{~min} \quad$ : $\quad 60 \%$ mobile phase $\mathrm{A}$ $40 \%$ mobile phase B

The crude product was purified on a preparative scale with the above mentioned method by MPLC. Therefore, the precipitate was dissolved in deionized water and loaded on puriFlash C18 aqueous column: Column size: F0012 (20 g), Particle size: $30 \mu \mathrm{m}$, Flow rate: 10ml/min,

Mobile Phase: Phase A: Water, Phase B: $100 \mathrm{mM}$ of TEAA (pH adjusted at 8.5), Phase C: Acetonitrile. Gradient:

1. 0 min $\quad$ : $\quad 60 \%$ mobile phase A $40 \%$ mobile phase B

2. $15 \mathrm{~min} \quad: \quad 60 \%$ mobile phase $\mathrm{A}$ $40 \%$ mobile phase $\mathrm{B}$

3. $45 \mathrm{~min} \quad: \quad 60 \%$ mobile phase $\mathrm{B}$ 


\section{$40 \%$ mobile phase $\mathrm{C}$}

The fractions containing product were concentrated under reduced pressure to the minimal amount of the solvent and then product was precipitated by $\mathrm{NaClO}_{4}$ sol. $(0.5 \mathrm{M}$ in acetone). The precipitate was washed twice with acetone.

Note-: It was found that purification of adenosine triphosphates by Strong anion-exchange chromatography (Method B) is straightforward as they give 2'-3'cyclophosphates as byproducts, which can be easily removed from the product.

In the case of (deoxy)nucleoside such as thymidine, cPyPA is also phosphitylating the $3^{\prime} \mathrm{OH}$. The resulting 3'-triphosphate can be removed by RP-LC.

\subsubsection{Synthesis of triphosphates based on $c$-PyPA $\left(\mathrm{A}_{1}\right)$}

3'-Azido-3'-deoxythymidine 5'-cyclotriphosphate (AZT cyclotriphosphate B1)

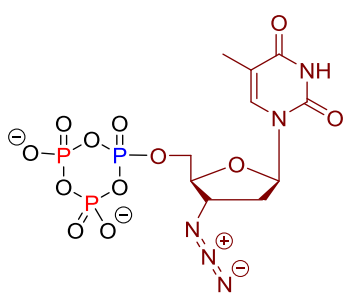

$\left(B_{1}\right)$

AZT (50 mg, $0.18 \mathrm{mmol}, 1.2$ eq.) and ETT (78 mg, $0.60 \mathrm{mmol}, 4.0$ eq.) were coevaporated with dry $\mathrm{MeCN}(2 \times 1 \mathrm{ml})$. Under an $\mathrm{Ar}$ atmosphere, a reaction mixture containing the $c$-PyPA $\mathbf{A}_{1}(0.075 \mathrm{M}$ in $\mathrm{MeCN}, 2 \mathrm{ml}, 0.15 \mathrm{mmol}, 1.0$ eq.) was added to the dried solids and it was stirred at r.t. for $10 \mathrm{mins}$. Upon cooling to $0^{\circ} \mathrm{C}, m \mathrm{CPBA}(\leq 77 \%, 39 \mathrm{mg}, 0.22 \mathrm{mmol}, 1.5 \mathrm{eq}$.) was added and the mixture was stirred for 5 mins until ${ }^{31} \mathrm{P}-\mathrm{NMR}$ confirmed complete oxidation (formation of triplet at $-23 \mathrm{ppm}$ which is diagnostic signal for the cyclotriphosphate $\left.\mathbf{B}_{1}\right)^{3}$

Aliquots of this reaction mixture were used for the ring opening with various nucleophiles. The AZT cyclotriphosphate $\left(\mathbf{B}_{1}\right)$ is stable in reaction mixture at $-20^{\circ} \mathrm{C}$ up to 2 weeks.

${ }^{31} \mathbf{P}\left\{{ }^{1} \mathbf{H}\right\}$ NMR $\left(122 \mathrm{MHz}, \mathrm{CDCl}_{3}, \delta / \mathrm{ppm}\right):-23.2--25.2(\mathrm{~m}, 1 \mathrm{P}),-25.8(\mathrm{~d}, \mathrm{~J}=21.1 \mathrm{~Hz}, 2 \mathrm{P})$. 
Ring opening by using propargylamine: 3'-Azido-3'-deoxythymidine 5'- $\gamma$-P-propargylamino triphosphate (1)

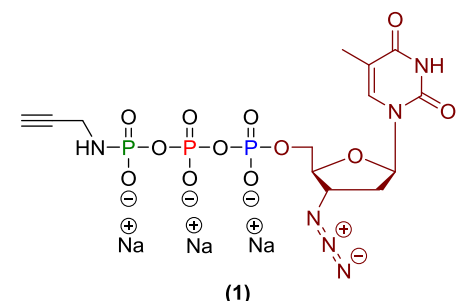

(1)

The reaction mixture of $\mathbf{B}_{1}(250 \mu \mathrm{L}, 18.7 \mu \mathrm{mol}, 1.0$ eq.) was added to propargylamine ( $250 \mu \mathrm{L})$ and then $\mathrm{D}_{2} \mathrm{O}(100 \mu \mathrm{l})$ was added (stirred it well for 5 mins). The product $\mathbf{1}$ was isolated by Method A, affording a colourless solid $(8.20 \mathrm{mg}, 13.0 \mu \mathrm{mol}, 72 \%)$.

\section{$\underline{\text { Reaction on large scale (1) }}$}

AZT (344 mg, $1.28 \mathrm{mmol}, 1.0$ eq.) and ETT (540 mg, $4.15 \mathrm{mmol}, 3.2$ eq.) were coevaporated with dry $\mathrm{MeCN}(2 \times 4 \mathrm{ml})$ in oven dried pear shape $25 \mathrm{ml}$ flask. Under an atmosphere of dry Ar, a mixture of c-PyPA (A1) (0.075M, $17.0 \mathrm{ml}, 1.27 \mathrm{mmol}, 1.0$ eq.) in $\mathrm{MeCN}$ was added to the dried solids. The mixture was stirred at r.t. for 10 mins. Upon cooling to $-4{ }^{\circ} \mathrm{C}, m \mathrm{CPBA}(\leq 77 \%, 439 \mathrm{mg}, 2.55 \mathrm{mmol}, 2.0 \mathrm{eq}$.) was added and the mixture was stirred for 10 mins until ${ }^{31} \mathrm{P}-\mathrm{NMR}$ confirmed complete oxidation (formation of triplet at $-23 \mathrm{ppm}$ which is diagnostic signal for the cyclotriphosphate $\mathbf{B}_{1}$ ). Then propargylamine ( $520 \mu \mathrm{l}, 8.28 \mathrm{mmol}, 6.5$ eq.) was added to the reaction mixture at $-4^{\circ} \mathrm{C}$ and the mixture was stirred at r.t. for 15 mins.

The product 1 was isolated by Method A, affording a colourless solid (712 mg, $1.16 \mathrm{mmol}, 91 \%$ ).

${ }^{1}$ H NMR (300 MHz, D $\left.2 \mathrm{O}, \delta / \mathrm{ppm}\right): 7.70(\mathrm{~s}, 1 \mathrm{H}), 6.24$ (t, J = 6.9 Hz, 1H), 4.53 (s, 1H), 4.17 (m, 3H), 3.64 $(\mathrm{dd}, \mathrm{J}=9.8,2.2 \mathrm{~Hz}, 2 \mathrm{H}), 2.51(\mathrm{t}, \mathrm{J}=2.5 \mathrm{~Hz}, 1 \mathrm{H}), 2.48-2.36(\mathrm{~m}, 2 \mathrm{H}), 1.88(\mathrm{~s}, 3 \mathrm{H}) .{ }^{31} \mathbf{P}\left\{{ }^{1} \mathbf{H}\right\} \mathbf{N M R}$ (122 MHz, D $2 \mathrm{O}, \delta / \mathrm{ppm}):-1.99$ (d, J = 20.7 Hz, 1P), -10.5 (d, J = 19.0 Hz, 1P), -20.7 (t, J = 19.9 Hz, 1P). 31P NMR (162 MHz, D $2 \mathrm{O}, \delta / \mathrm{ppm}):-1.99$ (dt, J = 19.4, 9.2 Hz, 1P), -10.5 (dt, J = 19.1, 5.6 Hz, 1P), -20.7 $(\mathrm{t}, \mathrm{J}=19.8 \mathrm{~Hz}, 1 \mathrm{P}) .{ }^{13} \mathrm{C}$ NMR $\left(101 \mathrm{MHz}, \mathrm{D}_{2} \mathrm{O}, \delta / \mathrm{ppm}\right): 166.8,151.8,137.2,111.8,84.8,82.9$ (d, J = 9.3 $\mathrm{Hz}), 71.3,65.6(\mathrm{~d}, \mathrm{~J}=5.6 \mathrm{~Hz}), 60.8,36.3,30.9$ (d, J = 3.7 Hz), 11.7.

HRMS (ESI) m/z for $\left[\mathrm{C}_{13} \mathrm{H}_{18} \mathrm{~N}_{6} \mathrm{NaO}_{12} \mathrm{P}_{3}\right]^{-}$: calcd. 566.0099, found 566.0088.

AZT triphosphate 2 can also be obtained from 1 by dissolving in water and adjusting the $\mathrm{pH}$ with $\mathrm{HCl}$ at 2.5 resulted in completed hydrolysis of amidate $(\mathrm{P}-\mathrm{N})$ bond. Afterwards, the product was isolated by Method A. 
Ring opening by using aq. sodium hydroxide: 3'-Azido-3'-deoxythymidine 5'-triphosphate (2)

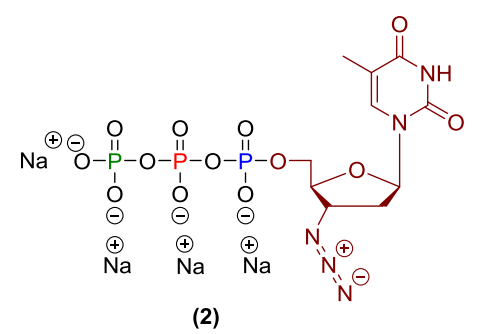

The reaction mixture of $\mathbf{B}_{1}(250 \mu \mathrm{L}, 18.7 \mu \mathrm{mol}, 1.0$ eq. $)$ was added to aq. $\mathrm{NaOH}(1 \mathrm{M}, 250 \mu \mathrm{L})$ and then $\mathrm{D}_{2} \mathrm{O}(100 \mu \mathrm{l})$ was added (stirred it well for 5 mins). The product 2 was isolated by Method A, affording a colourless solid (7.60 mg, $13.3 \mu \mathrm{mol}, 71 \%)$.

The analytical data are consistent with literature. ${ }^{3}$

Note -: It was found that use of aq. $\mathrm{NaOH}$ as a nucleophile, was a superior way to open the cyclotriphosphate as compared to $\mathrm{H}_{2} \mathrm{O}$.

${ }^{1}$ H NMR (300 MHz, D $\left.2 \mathrm{O}, \delta / \mathrm{ppm}\right): 7.63$ (s, 1H), $6.36(\mathrm{t}, \mathrm{J}=7.0 \mathrm{~Hz}, 1 \mathrm{H}), 4.69-4.52(\mathrm{~m}, 1 \mathrm{H}), 4.29-4.11$ (m, 3H), $2.46(\mathrm{dd}, \mathrm{J}=7.0,5.1 \mathrm{~Hz}, 2 \mathrm{H}), 1.91$ (s, 3H). ${ }^{31} \mathbf{P}\left\{{ }^{1} \mathbf{H}\right\}$ NMR $\left(122 \mathrm{MHz}, \mathrm{D}_{2} \mathrm{O}, \delta / \mathrm{ppm}\right):-7.02$ (d, J $=20.3 \mathrm{~Hz}, 1 \mathrm{P}),-11.5(\mathrm{~d}, \mathrm{~J}=19.8 \mathrm{~Hz}, 1 \mathrm{P}),-22.4(\mathrm{t}, \mathrm{J}=20.1 \mathrm{~Hz}, 1 \mathrm{P}) .{ }^{31} \mathbf{P}$ NMR $\left(162 \mathrm{MHz}, \mathrm{D}_{2} \mathrm{O}, \delta / \mathrm{ppm}\right)$ : $-7.01(\mathrm{~d}, \mathrm{~J}=20.8 \mathrm{~Hz}, 1 \mathrm{P}),-11.5(\mathrm{dt}, \mathrm{J}=21.7,4.7 \mathrm{~Hz}, 1 \mathrm{P}),-22.4(\mathrm{t}, \mathrm{J}=20.1 \mathrm{~Hz}, 1 \mathrm{P}) .{ }^{13} \mathrm{C}$ NMR $(101 \mathrm{MHz}$, $\left.\mathrm{D}_{2} \mathrm{O}, \delta / \mathrm{ppm}\right): 166.6,151.7,137.2,111.8,84.8,83.0$ (d, J = 9.3 Hz), 65.7 (d, J = 5.6 Hz), 60.9, 36.3, 11.6. HRMS (ESI): calculated for $\left[\mathrm{C}_{10} \mathrm{H}_{14} \mathrm{~N}_{5} \mathrm{NaO}_{13} \mathrm{P}_{3}\right]^{-}$: calcd. 527.9704 found: 527.9706.

Ring opening by using water: 3'-Azido-3'-deoxythymidine 5'-triphosphate (2)

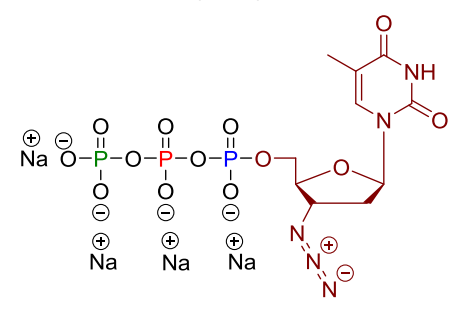

(2)

The reaction mixture of $\mathbf{B}_{1}(250 \mu \mathrm{L}, 18.7 \mu \mathrm{mol}, 1.0$ eq. $)$ was added to $\mathrm{D}_{2} \mathrm{O}(250 \mu \mathrm{l})$ and it was stirred at r.t. for $2.5 \mathrm{hr}$. The product 2 was isolated by Method A, affording a colourless solid (6.4 mg, $10.7 \mu \mathrm{mol}$, $57 \%)$.

${ }^{1}$ H NMR (300 MHz, D $\left.2 \mathrm{O}, \delta / \mathrm{ppm}\right): 7.63$ (s, 1H), 6.36 (t, J = 7.0 Hz, 1H), $4.69-4.52(\mathrm{~m}, 1 \mathrm{H}), 4.29-4.11$ (m, 3H), $2.46(\mathrm{dd}, \mathrm{J}=7.0,5.1 \mathrm{~Hz}, 2 \mathrm{H}), 1.91(\mathrm{~s}, 3 \mathrm{H}) .{ }^{31} \mathbf{P}\left\{{ }^{1} \mathbf{H}\right\} \mathbf{N M R}\left(162 \mathrm{MHz}, \mathrm{D}_{2} \mathrm{O}, \delta / \mathrm{ppm}\right):-7.02(\mathrm{~d}, \mathrm{~J}$ $=20.3 \mathrm{~Hz}, 1 \mathrm{P}),-11.5(\mathrm{~d}, \mathrm{~J}=19.8 \mathrm{~Hz}, 1 \mathrm{P}),-22.4$ (t, J = 20.1 Hz, 1P). ${ }^{31} \mathbf{P}$ NMR $\left(162 \mathrm{MHz}, \mathrm{D}_{2} \mathrm{O}, \delta / \mathrm{ppm}\right)$ : $-7.01(\mathrm{~d}, \mathrm{~J}=20.8 \mathrm{~Hz}, 1 \mathrm{P}),-11.5(\mathrm{dt}, \mathrm{J}=21.7,4.7 \mathrm{~Hz}, 1 \mathrm{P}),-22.4(\mathrm{t}, \mathrm{J}=20.1 \mathrm{~Hz}, 1 \mathrm{P}) .{ }^{13} \mathbf{C ~ N M R}(101 \mathrm{MHz}$, $\left.\mathrm{D}_{2} \mathrm{O}, \delta / \mathrm{ppm}\right):$ 166.6, 151.7, 137.2, 111.8, 84.8, 83.0 (d, J = 9.3 Hz), 65.7 (d, J = 5.6 Hz), 60.9, 36.3, 11.6. HRMS (ESI): calculated for $\left[\mathrm{C}_{10} \mathrm{H}_{14} \mathrm{~N}_{5} \mathrm{NaO}_{13} \mathrm{P}_{3}\right]^{-}$: calcd. 527.9704 found: 527.9706. 
Ring opening by using aq. ammonia: 3 '-Azido-3'-deoxythymidine 5'- $\gamma$-P-amino triphosphate (3)

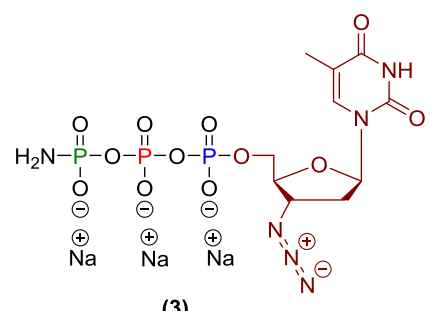

(3)

The reaction mixture of $\mathbf{B}_{1}\left(250 \mu \mathrm{L}, 18.7 \mu\right.$ mol, 1.0 eq.) was added to $25 \%$ aq. $\mathrm{NH}_{4} \mathrm{OH}(250 \mu \mathrm{L})$ and then $\mathrm{D}_{2} \mathrm{O}(100 \mu \mathrm{l})$ was added afterwards it was stirred for 5 mins. The product $\mathbf{3}$ was isolated by Method A, affording a colourless solid (7.90 mg, $13.8 \mu \mathrm{mol}, 73 \%)$.

${ }^{1}$ H NMR (300 MHz, D $\left.2 \mathrm{O}, \delta / \mathrm{ppm}\right): 7.67(\mathrm{~d}, \mathrm{~J}=1.4 \mathrm{~Hz}, 1 \mathrm{H}), 6.21(\mathrm{t}, \mathrm{J}=6.9 \mathrm{~Hz}, 1 \mathrm{H}), 4.52(\mathrm{dt}, \mathrm{J}=6.3,3.8$ $\mathrm{Hz}, 1 \mathrm{H}), 4.22-4.01(\mathrm{~m}, 3 \mathrm{H}), 2.84-2.25(\mathrm{~m}, 2 \mathrm{H}), 1.85(\mathrm{~s}, 3 \mathrm{H}) .{ }^{31} \mathbf{P}\left\{{ }^{1} \mathbf{H}\right\} \mathbf{N M R}\left(122 \mathrm{MHz}, \mathrm{D}_{2} \mathrm{O}, \delta / \mathrm{ppm}\right)$ : $-2.71(\mathrm{~d}, \mathrm{~J}=20.4 \mathrm{~Hz}, 1 \mathrm{P}),-11.6(\mathrm{~d}, \mathrm{~J}=19.2 \mathrm{~Hz}, 1 \mathrm{P}),-22.6$ (t, J = 19.9 Hz, 1P). ${ }^{31} \mathbf{P}$ NMR $\left(162 \mathrm{MHz}, \mathrm{D}_{2} \mathrm{O}\right.$,

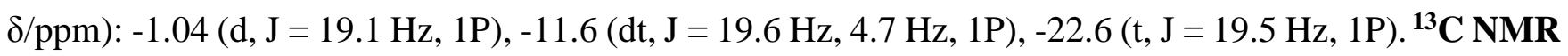
(101 MHz, $\left.\mathrm{D}_{2} \mathrm{O}, \delta / \mathrm{ppm}\right): 166.6,151.7,137.2,111.8,84.8,83.02(\mathrm{~d}, \mathrm{~J}=9.3 \mathrm{~Hz}), 65.7$ (d, J = 5.6 Hz), $60.9,36.3,11.6$.

HRMS (ESI): for $\left[\mathrm{C}_{10} \mathrm{H}_{15} \mathrm{~N}_{6} \mathrm{O}_{12} \mathrm{P}_{3}\right]^{2-}$ : calcd. 251.9986 , found: 251.9986.

Ring opening by using diethylamine: 3'-Azido-3'-deoxythymidine 5'- $\gamma$-P-diethylamino triphosphate (4)

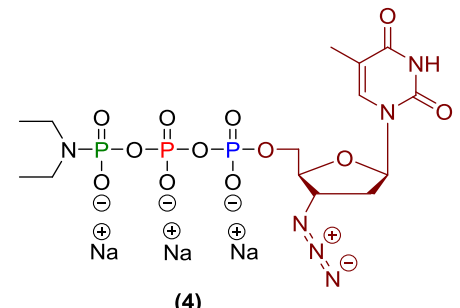

(4)

The reaction mixture of $\mathbf{B}_{1}(250 \mu \mathrm{L}, 18.7 \mu \mathrm{mol}, 1.0$ eq.) was added to diethylamine $(250 \mu \mathrm{L})$ and then $\mathrm{D}_{2} \mathrm{O}(100 \mu \mathrm{l})$ was added afterwards it was stirred for 5 mins. The product 4 was isolated by Method A, affording a colourless solid $(8.80 \mathrm{mg}, 14.0 \mu \mathrm{mol}, 76 \%)$.

${ }^{1}$ H NMR (300 MHz, D $\left.2 \mathrm{O}, \delta / \mathrm{ppm}\right): 7.68(\mathrm{~s}, 1 \mathrm{H}), 6.22(\mathrm{t}, \mathrm{J}=6.9 \mathrm{~Hz}, 1 \mathrm{H}), 4.51(\mathrm{dt}, \mathrm{J}=6.4,3.6 \mathrm{~Hz}, 1 \mathrm{H})$, $4.25-3.94$ (m, 3H), 2.96 (dq, J = 11.3, 7.2 Hz, 4H), $2.52-2.36(\mathrm{~m}, 2 \mathrm{H}), 1.85$ (d, J = 1.1 Hz, 3H), 0.99 $(\mathrm{t}, \mathrm{J}=7.1 \mathrm{~Hz}, 6 \mathrm{H}) .{ }^{31} \mathbf{P}\left\{{ }^{1} \mathbf{H}\right\}$ NMR $\left(122 \mathrm{MHz}, \mathrm{D}_{2} \mathrm{O}, \delta / \mathrm{ppm}\right):-0.55(\mathrm{~d}, \mathrm{~J}=24.2 \mathrm{~Hz}, 1 \mathrm{P}),-11.8(\mathrm{~d}, \mathrm{~J}=$ $19.1 \mathrm{~Hz}, 1 \mathrm{P}),-23.0(\mathrm{dd}, \mathrm{J}=24.2,19.0 \mathrm{~Hz}, 1 \mathrm{P}) .{ }^{31} \mathbf{P}$ NMR $\left(162 \mathrm{MHz}, \mathrm{D}_{2} \mathrm{O}, \delta / \mathrm{ppm}\right):-0.55$ (dp, J = 23.9, 11.5 Hz, 1P), -11.8 (dt, J = 18.6, 4.5 Hz, 1P), -23.0 (dd, J = 24.4, $18.8 \mathrm{~Hz}, 1 \mathrm{P}) .{ }^{13} \mathbf{C}$ NMR $\left(101 \mathrm{MHz}, \mathrm{D}_{2} \mathrm{O}\right.$,

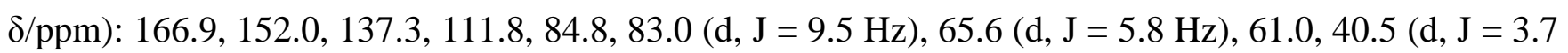
$\mathrm{Hz}), 36.2,13.8$ (d, J = 3.7 Hz), 11.7.

HRMS (ESI) $\mathrm{m} / \mathrm{z}$ for $\left[\mathrm{C}_{14} \mathrm{H}_{23} \mathrm{~N}_{6} \mathrm{O}_{12} \mathrm{P}_{3}\right]^{2-}$ : calcd. 280.0299, found 280.0298 . 
Ring opening by using morpholine: 3'-Azido-3'-deoxythymidine 5'- $\gamma$-P-morpholino triphosphate (5)

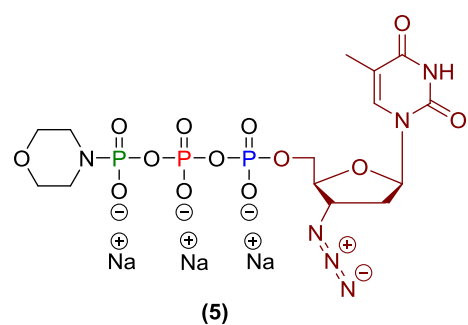

The reaction mixture of $\mathbf{B}_{1}(250 \mu \mathrm{L}, 18.7 \mu \mathrm{mol}, 1.0$ eq.) was added to morpholine ( $250 \mu \mathrm{L})$ and then $\mathrm{D}_{2} \mathrm{O}$ $(100 \mu \mathrm{l})$ was added afterwards it was stirred for 5 mins. The product 5 was isolated by Method A, affording a colourless solid $(9.60 \mathrm{mg}, 14.9 \mu \mathrm{mol}, 80 \%)$.

${ }^{1}$ H NMR (300 MHz, D $\left.2 \mathrm{O}, \delta / \mathrm{ppm}\right): 7.71(\mathrm{~s}, 1 \mathrm{H}), 6.23(\mathrm{t}, \mathrm{J}=6.9 \mathrm{~Hz}, 1 \mathrm{H}), 4.61-4.52(\mathrm{~m}, 1 \mathrm{H}), 4.17(\mathrm{~d}, \mathrm{~J}=$ $4.5 \mathrm{~Hz}, 3 \mathrm{H}), 3.91-3.75(\mathrm{~m}, 2 \mathrm{H}), 3.66-3.56(\mathrm{~m}, 2 \mathrm{H}), 3.09-2.91(\mathrm{~m}, 4 \mathrm{H}), 2.70-2.37(\mathrm{~m}, 2 \mathrm{H}), 1.88$ (s, 3H). ${ }^{31} \mathbf{P}\left\{{ }^{1} \mathbf{H}\right\}$ NMR $\left(122 \mathrm{MHz}, \mathrm{D}_{2} \mathrm{O}, \delta / \mathrm{ppm}\right):-3.36(\mathrm{~d}, \mathrm{~J}=23.9 \mathrm{~Hz}, 1 \mathrm{P}),-11.7$ (d, J = 19.3 Hz, 1P), -22.7 $(\mathrm{dd}, \mathrm{J}=23.8,18.9 \mathrm{~Hz}, 1 \mathrm{P}) .{ }^{31} \mathbf{P}$ NMR $\left(162 \mathrm{MHz}, \mathrm{D}_{2} \mathrm{O}, \delta / \mathrm{ppm}\right):-2.98--3.77$ (m, 1P), -11.7 (dt, J = 18.6, $4.5 \mathrm{~Hz}, 1 \mathrm{P}),-22.7$ (dd, J = 23.9, $19.3 \mathrm{~Hz}, 1 \mathrm{P}) .{ }^{13} \mathbf{C}$ NMR (101 MHz, $\left.\mathrm{D}_{2} \mathrm{O}, \delta / \mathrm{ppm}\right): 166.8,151.9,137.3$, 111.8, 84.9, 83.0 (d, J = 9.1 Hz), 66.9 (d, J = 8.2 Hz), 65.6 (d, J = 5.6 Hz), 64.9, 44.9, 36.9, 11.7.

HRMS (ESI) m/z for $\left[\mathrm{C}_{14} \mathrm{H}_{21} \mathrm{~N}_{6} \mathrm{NaO}_{13} \mathrm{P}_{3}\right]^{-}$: calcd.: 597.0283, found 597.0291.

Ring opening by using aniline: 3'-Azido-3'-deoxythymidine 5'- $\gamma$-P-anilino triphosphate (6)

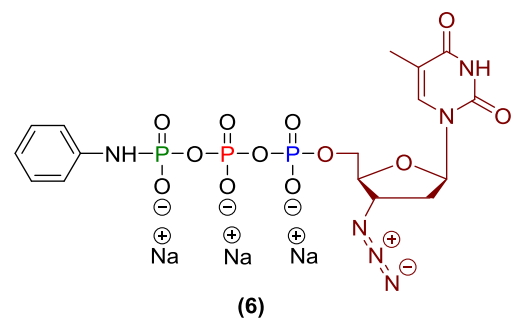

The reaction mixture of $\mathbf{B}_{1}(250 \mu \mathrm{L}, 18.7 \mu \mathrm{mol}, 1.0$ eq.) was added to aniline (250 $\mu \mathrm{L})$ and then $\mathrm{D}_{2} \mathrm{O}$ $(100 \mu \mathrm{l})$ was added afterwards it was stirred for 5 mins. The product 6 was isolated by Method A, affording a colourless solid $(9.45 \mathrm{mg}, 14.5 \mu \mathrm{mol}, 77 \%)$.

${ }^{1} \mathbf{H}$ NMR $\left(300 \mathrm{MHz}, \mathrm{D}_{2} \mathrm{O}, \delta / \mathrm{ppm}\right): 7.57(\mathrm{~s}, 1 \mathrm{H}), 7.16\left(\mathrm{~m}_{\mathrm{c}}, 2 \mathrm{H}\right), 6.98\left(\mathrm{~m}_{\mathrm{c}}, 2 \mathrm{H}\right), 6.80\left(\mathrm{~m}_{\mathrm{c}}, 1 \mathrm{H}\right), 6.12(\mathrm{t}, \mathrm{J}=$ $6.9 \mathrm{~Hz}, 1 \mathrm{H}), 4.51-4.31(\mathrm{~m}, 1 \mathrm{H}), 4.02-3.94(\mathrm{~m}, 2 \mathrm{H}), 3.94-3.81(\mathrm{~m}, 1 \mathrm{H}), 2.42(\mathrm{t}, \mathrm{J}=6.5 \mathrm{~Hz}, 1 \mathrm{H}), 2.37$ $-2.25(\mathrm{~m}, 1 \mathrm{H}), 1.81(\mathrm{~s}, 3 \mathrm{H}) .{ }^{31} \mathbf{P}\left\{{ }^{1} \mathbf{H}\right\}$ NMR $\left(122 \mathrm{MHz}, \mathrm{D}_{2} \mathrm{O}, \delta / \mathrm{ppm}\right):-10.1$ (d, J = 19.1 Hz, $\left.1 \mathrm{P}\right),-11.6$ $(\mathrm{d}, \mathrm{J}=18.5,1 \mathrm{P}),-21.4--24.0(\mathrm{~m}, 1 \mathrm{P}) .{ }^{31} \mathbf{P}$ NMR $\left(162 \mathrm{MHz}, \mathrm{D}_{2} \mathrm{O}, \delta / \mathrm{ppm}\right):-10.1$ (d, J = 19.1 Hz, 1P), $11.9(\mathrm{dt}, \mathrm{J}=18.5,4.5 \mathrm{~Hz}, 1 \mathrm{P}),-21.4--24.0(\mathrm{~m}, 1 \mathrm{P}) .{ }^{13} \mathrm{C} \mathbf{N M R}\left(101 \mathrm{MHz}, \mathrm{D}_{2} \mathrm{O}, \delta / \mathrm{ppm}\right): 166.5,151.6$, 141.9, 137.1, 129.1, 120.1, $117.3(\mathrm{~d}, \mathrm{~J}=7.3 \mathrm{~Hz}), 111.8,84.7,82.9$ (d, J = 9.5 Hz), 65.5 (d, J = 5.4 Hz), $60.9,36.4,11.7$.

HRMS (ESI) m/z for $\left[\mathrm{C}_{16} \mathrm{H}_{18} \mathrm{~N}_{6} \mathrm{Na}_{2} \mathrm{O}_{12} \mathrm{P}_{3}\right]^{-}$: calcd. 624.9996, found 624.9999. 
Ring opening by using NaOMe solution: 3'-Azido-3'-deoxythymidine 5'- $\gamma$-P-methoxy triphosphate (7)

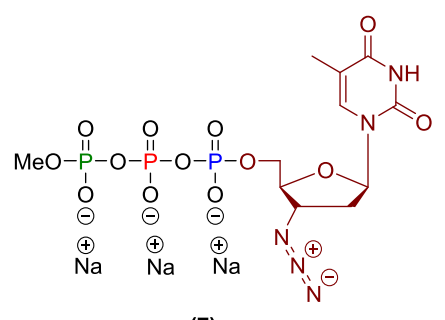

(7)

The reaction mixture of $\mathbf{B}_{1}(250 \mu \mathrm{L}, 18.7 \mu \mathrm{mol}, 1.0$ eq.) was added to NaOMe Solution ( $0.5 \mathrm{M}$ in methanol, $300 \mu \mathrm{l})$ and then $\mathrm{D}_{2} \mathrm{O}(100 \mu \mathrm{l})$ was added afterwards it was stirred for 5 mins. The product 7 was isolated by Method A, affording a colourless solid (9.00 mg, $15.3 \mu \mathrm{mol}, 81 \%)$.

${ }^{1} \mathbf{H}$ NMR (300 MHz, D $\left.2 \mathrm{O}, \delta / \mathrm{ppm}\right): 7.63(\mathrm{~s}, 1 \mathrm{H}), 6.36(\mathrm{t}, \mathrm{J}=7.0 \mathrm{~Hz}, 1 \mathrm{H}), 4.69-4.52(\mathrm{~m}, 1 \mathrm{H}), 4.29$ $4.11(\mathrm{~m}, 3 \mathrm{H}), 3.48-3.45(\mathrm{~m}, 3 \mathrm{H}) 2.46(\mathrm{dd}, \mathrm{J}=7.0,5.1 \mathrm{~Hz}, 2 \mathrm{H}), 1.91(\mathrm{~s}, 3 \mathrm{H}) .{ }^{31} \mathbf{P}\left\{{ }^{1} \mathbf{H}\right\} \mathbf{N M R}(162 \mathrm{MHz}$, $\left.\mathrm{D}_{2} \mathrm{O}, \delta / \mathrm{ppm}\right):-9.63(\mathrm{~d}, \mathrm{~J}=19.1 \mathrm{~Hz}, 1 \mathrm{P}),-11.6(\mathrm{~d}, \mathrm{~J}=19.4 \mathrm{~Hz}, 1 \mathrm{P}),-22.9$ (t, J = 19.1 Hz, 1P). ${ }^{31} \mathbf{P}$ NMR (162 MHz, D $2 \mathrm{O}, \delta / \mathrm{ppm}):-9.64(\mathrm{dq}, \mathrm{J}=19.3,11.8 \mathrm{~Hz}, 1 \mathrm{P}),-11.6(\mathrm{dt}, \mathrm{J}=19.1,5.2 \mathrm{~Hz}, 1 \mathrm{P}),-22.9(\mathrm{t}, \mathrm{J}=$ $19.1 \mathrm{~Hz}, 1 \mathrm{P}) .{ }^{13} \mathrm{C}$ NMR (101 MHz, $\left.\mathrm{D}_{2} \mathrm{O}, \delta / \mathrm{ppm}\right): 166.6,151.7,137.2,111.8,95.7,84.8,83.0(\mathrm{~d}, \mathrm{~J}=$ $9.3 \mathrm{~Hz}), 65.7$ (d, J = 5.6 Hz), 60.9, 36.3, 11.6.

HRMS (ESI): calculated for $\left[\mathrm{C}_{11} \mathrm{H}_{17} \mathrm{~N}_{5} \mathrm{O}_{13} \mathrm{P}_{3}\right]^{-}$: calcd. 520.0041 found: 520.0045 .

Ring opening by using monofluorophosphate: 3 ''Azido-3'-deoxythymidine 5 ' $-\delta$ - $P$-fluoro tetraphosphate $(8)$

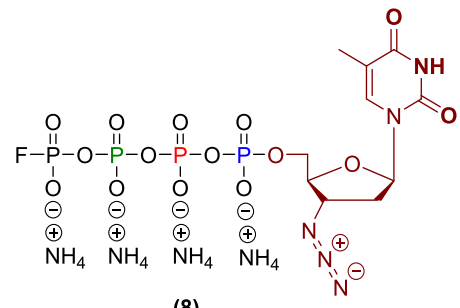

(8)

The reaction mixture of $\mathbf{B}_{1}$ (2.00 ml, $0.15 \mathrm{mmol}, 1.0$ eq.) was added to monofluorophosphate $\times 1.2$ TBA (0.12 g, $0.30 \mathrm{mmol}, 2.0$ eq.) and subsequently addition of anhydrous $\mathrm{MgCl}_{2}(0.08 \mathrm{~g}, 0.84 \mathrm{mmol}, 5.6$ eq.) and further addition of dry DMF $(1 \mathrm{~mL})$. The reaction mixture was stirred for $1 \mathrm{hr}$ and the crude product was then purified by Method B (the product was eluted at $300-500 \mathrm{mM} \mathrm{NH} \mathrm{NCO}_{3}$ buffer). The title compound 8 (51.9 mg, $0.07 \mathrm{mmol}, 52 \%$ ) was obtained as a colourless solid.

${ }^{1}$ H NMR (400 MHz, D $\left.2 \mathrm{O}, \delta / \mathrm{ppm}\right): 7.53(\mathrm{~s}, 1 \mathrm{H}), 6.08(\mathrm{t}, \mathrm{J}=6.9 \mathrm{~Hz}, 1 \mathrm{H}), 4.37(\mathrm{~m}, 1 \mathrm{H}), 4.06-3.98(\mathrm{~m}$, 3H), 2.39 - 2.13 (m, 2H), 1.73 (s, 3H). ${ }^{31} \mathbf{P}\left\{{ }^{1} \mathbf{H}\right\}$ NMR (162 MHz, D $\left.{ }_{2} \mathrm{O}, \delta / p p m\right):-11.1$ - -12.1 (m, 1P), $18.2(\mathrm{dd}, \mathrm{J}=934.0,16.2 \mathrm{~Hz}, 1 \mathrm{P}),-22.9--23.9$ (m, 2P). ${ }^{31} \mathbf{P}$ NMR $\left(162 \mathrm{MHz}, \mathrm{D}_{2} \mathrm{O}, \delta / \mathrm{ppm}\right):-11.7(\mathrm{~m}$, 1P), -17.7 - -18.4 (m, 1P), -23.0 - -23.9 (m, 2P). ${ }^{13}$ C NMR (126 MHz, $\left.\mathrm{D}_{2} \mathrm{O}, \delta / \mathrm{ppm}\right): 166.7,165.6,151.8$, 
137.1, 111.7, $82.8(\mathrm{~d}, \mathrm{~J}=9.1 \mathrm{~Hz}), 65.7(\mathrm{~d}, \mathrm{~J}=5.5 \mathrm{~Hz}), 60.8,36.1,11.6 .{ }^{19} \mathbf{F}$ NMR $\left(377 \mathrm{MHz}, \mathrm{D}_{2} \mathrm{O}, \delta / \mathrm{ppm}\right)$ : $-73.1(\mathrm{~d}, \mathrm{~J}=935.1 \mathrm{~Hz}, 1 \mathrm{P})$.

HRMS (ESI) $\mathrm{m} / \mathrm{z}$ for $\left[\mathrm{C}_{10} \mathrm{H}_{16} \mathrm{FN}_{5} \mathrm{O}_{15} \mathrm{P}_{4}\right]^{-}$: calcd. 588.9577, found 588.9583.

Ring opening by using imidazole: 3'-Azido-3'-deoxythymidine 5'- $\gamma$-P-imidazole triphosphate (9)

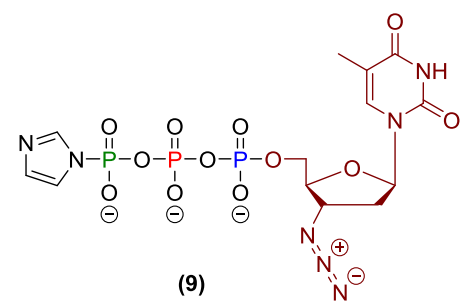

AZT (50 mg, $0.18 \mathrm{mmol}, 1.2$ eq.) and ETT (78 mg, $0.60 \mathrm{mmol}, 4.0$ eq.) were coevaporated together with dry $\mathrm{MeCN}(2 \times 1 \mathrm{~mL})$. Under an Ar atmosphere, a freshly prepared reaction mixture containing the $c$ - PyPA A1 (0.075M in MeCN, $2 \mathrm{~mL}, 0.15 \mathrm{mmol}, 1.0$ eq.) was added to the dried solids. The mixture was stirred at r.t. for 10 mins. Upon cooling to $0{ }^{\circ} \mathrm{C}, m \mathrm{CPBA}(\leq 77 \%, 39 \mathrm{mg}, 0.22 \mathrm{mmol}, 1.5 \mathrm{eq}$.) was added and the mixture was stirred for 5 mins.

A solution of imidazole in dry DMF $(1.47 \mathrm{M}, 4.00 \mathrm{ml}, 5.88 \mathrm{mmol}, 39.2 \mathrm{eq}$.) was added to the cyclotriphosphate $\left(\mathbf{B}_{1}\right)$ and reaction was stirred for $15 \mathrm{mins}$ (the reaction progress was followed by ${ }^{31} \mathrm{P}$ NMR) which results in quantitative conversion to 9.

Note -: Imidazole was dried before using by coevaporation with $\mathrm{MeCN}$ and it was further stored of molecular sieve $(3 \AA)$.

${ }^{31}$ P NMR (162 MHz, D $\left.2 \mathrm{O}, \delta / \mathrm{ppm}\right):-11.0(\mathrm{~d}, \mathrm{~J}=19.5 \mathrm{~Hz}, 1 \mathrm{P}),-20.5(\mathrm{~d}, \mathrm{~J}=19.1 \mathrm{~Hz}, 1 \mathrm{P}),-23.4(\mathrm{t}, \mathrm{J}=$ $19.3 \mathrm{~Hz}, 1 \mathrm{P}) .{ }^{31} \mathbf{P}$ NMR $\left(162 \mathrm{MHz}, \mathrm{D}_{2} \mathrm{O}, \delta / \mathrm{ppm}\right):-11.0$ (dt, J = 20.4, $\left.10.1 \mathrm{~Hz}, 1 \mathrm{P}\right),-20.5$ (d, J = $19.0 \mathrm{~Hz}$, 1P), -23.4 (t, J = 19.4 Hz, 1P).

HRMS (ESI) m/z for $\left[\mathrm{C}_{13} \mathrm{H}_{17} \mathrm{~N}_{7} \mathrm{O}_{12} \mathrm{P}_{3}\right]^{-}$: calcd. 556.0154, found 556.0156.

\section{5'-Adenosyl 3'-azido-3'-deoxy-5'-thymidinyl hexaphosphate (Ap6AZT, P1)}

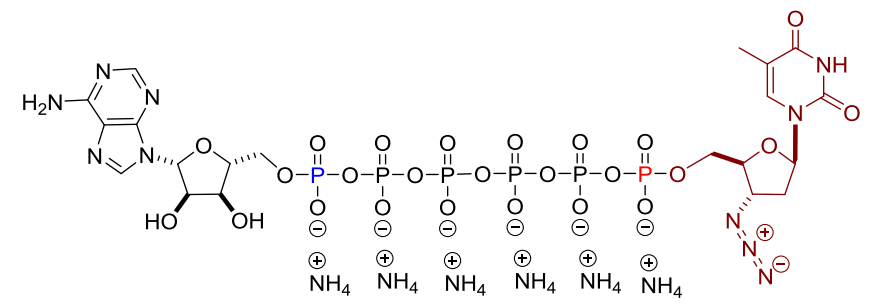

(P1)

Anhydr. $\mathrm{ZnCl}_{2}(0.25$ g, $1.8 \mathrm{mmol}, 20$ eq. $)$ and ATP x 2.1 TBA (0.25 g, 0.24 mmol, 2.7 eq.) were added to a freshly prepared solution of $9(0.09 \mathrm{mmol})$. The reaction mixture was then diluted with dry DMF: DMSO (v:v, 1:1, $8 \mathrm{ml})$ in order to increase the solubility and it was stirred at r.t. for $6 \mathrm{hrs}$. The crude 
product was purified by Method B and target product was eluted with 400-600 mM conc. of $\mathrm{NH}_{4} \mathrm{HCO}_{3}$ buffer. Purification gave P1 as a colorless solid (0.03 g, $0.03 \mathrm{mmol}, 35 \%)$.

Note -: The reactions for capped hexapolyPs were not optimized and therefore the yield could still be improved.

${ }^{1}$ H NMR (300 MHz, D $\left.2 \mathrm{O}, \delta / \mathrm{ppm}\right): 8.45$ (s, 1H), $8.16(\mathrm{~s}, 1 \mathrm{H}), 7.61(\mathrm{~s}, 1 \mathrm{H}), 6.13(\mathrm{t}, \mathrm{J}=6.9 \mathrm{~Hz}, 1 \mathrm{H})$, $6.04(\mathrm{~d}, \mathrm{~J}=6.2 \mathrm{~Hz}, 1 \mathrm{H}), 4.66$ - $4.44(\mathrm{~m}, 2 \mathrm{H}), 4.44-4.32(\mathrm{~m}, 1 \mathrm{H}), 4.26$ - $4.08(\mathrm{~m}, 5 \mathrm{H}), 2.37$ (dd, J = 6.9, $5.2 \mathrm{~Hz}, 2 \mathrm{H}), 1.81(\mathrm{~s}, 3 \mathrm{H})$. The DHO peak overlaps with the signal $4.66(1 \mathrm{H}) .{ }^{31} \mathbf{P}\left\{{ }^{1} \mathbf{H}\right\} \mathbf{~ N M R}(122 \mathrm{MHz}$, $\left.\mathrm{D}_{2} \mathrm{O}, \delta / \mathrm{ppm}\right):-11.3(\mathrm{~d}, \mathrm{~J}=19.8 \mathrm{~Hz}, 1 \mathrm{P}),-11.6(\mathrm{~d}, \mathrm{~J}=19.8 \mathrm{~Hz}, 1 \mathrm{P}),-21.4--23.4(\mathrm{~m}, 4 \mathrm{P}) .{ }^{31} \mathbf{P}$ NMR (162 MHz, $\left.\mathrm{D}_{2} \mathrm{O}, \delta / \mathrm{ppm}\right):-11.2--11.5$ (br, 1P), -11.5--11.7 (br, 1P), -22.1 - -23.6 (m, 4P). ${ }^{13} \mathbf{C}$ NMR (101 MHz, $\left.\mathrm{D}_{2} \mathrm{O}, \delta / \mathrm{ppm}\right): 166.4,155.6,152.8,151.5,149.1,139.8,137.1,118.5,111.6,86.5,84.8,84.1$ (d, J = 9.3 Hz), 82.9 (d, J = 9.3 Hz), 74.2, 70.4, 65.7 (d, J = 5.7 Hz), 65.4 (d, J = 5.7 Hz), 60.9, 36.3, 11.6. HRMS (ESI) $\mathrm{m} / \mathrm{z}$ for $\left[\mathrm{C}_{20} \mathrm{H}_{28} \mathrm{~N}_{10} \mathrm{O}_{25} \mathrm{P}_{6}\right]^{2-}$ : calcd. 496.9832, found 496.9831.

\section{3'-Azido-3'-deoxythymidine 5'- $\alpha$-thiocyclotriphosphate (AZT $\alpha$-(S)-cyclotriphosphate: B2)}

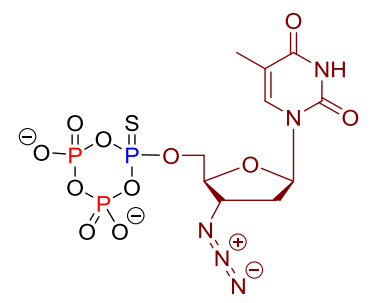

$\left(B_{2}\right)$

AZT (45 mg, $0.17 \mathrm{mmol}, 1.5$ eq.) and ETT (44 mg, $0.34 \mathrm{mmol}, 3.0$ eq.) were coevaporated with dry $\mathrm{MeCN}(2 \times 2 \mathrm{~mL})$. Under an Ar atmosphere, a freshly prepared reaction mixture containing the $c$-PyPA A 1 $(0.075 \mathrm{M}$ in $\mathrm{MeCN}, 1.5 \mathrm{ml}, 0.11 \mathrm{mmol}, 1.0$ eq.) was added to the dried solids and it was stirred at r.t. for 10 mins. Upon cooling to $0^{\circ} \mathrm{C}$, Becauge's reagent ( $45 \mathrm{mg}, 0.22 \mathrm{mmol}, 2.0$ eq.) was added and the mixture was stirred for 5 mins until ${ }^{31} \mathrm{P}-\mathrm{NMR}$ confirmed complete oxidation (the oxidation product shows a triplet at +43 ppm which is diagnostic signal for the $\alpha-(S)$-cyclotriphosphate $\left.\mathbf{B}_{2}\right){ }^{4,5}$

${ }^{31} \mathbf{P}\left\{{ }^{1} \mathbf{H}\right\}$ NMR $\left(122 \mathrm{MHz}, \mathrm{CDCl}_{3}, \delta / \mathrm{ppm}\right): 42.6(\mathrm{t}, \mathrm{J}=35.0 \mathrm{~Hz}, 1 \mathrm{P}),-24.7$ (d, J = 35.3 Hz, 2P).

Ring opening by using propargylamine: 3'-Azido-3'-deoxythymidine 5'- $\gamma$-P-propargylamino $\alpha$ thiotriphosphate (10)

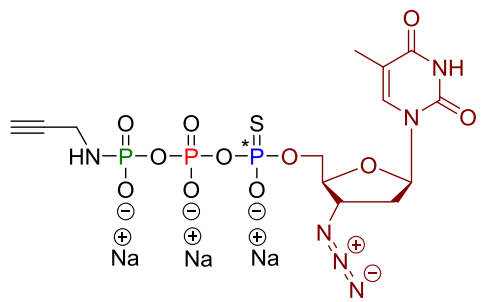

(10) 
The reaction mixture of $\mathbf{B}_{2}(250 \mu \mathrm{L}, 18.0 \mu \mathrm{mol}, 1.0$ eq. $)$ was added to propargylamine $(250 \mu \mathrm{l})$ and then $\mathrm{D}_{2} \mathrm{O}(100 \mu \mathrm{l})$ was added afterwards it was stirred for 5 mins. The product $\mathbf{1 0}$ was isolated by Method A, affording a colourless solid, whereas products were obtained as mixtures of diastereoisomers (10, $7.01 \mathrm{mg}, 11.2 \mu \mathrm{mol}, 59 \%)$.

${ }^{1}$ H NMR (300 MHz, D $\left.2 \mathrm{O}, \delta / \mathrm{ppm}\right): 7.70(\mathrm{~s}, 1 \mathrm{H}), 6.20(\mathrm{t}, \mathrm{J}=6.8 \mathrm{~Hz}, 1 \mathrm{H}), 4.50(\mathrm{dt}, \mathrm{J}=6.6,4.0 \mathrm{~Hz}, 1 \mathrm{H})$, $4.23-4.09(\mathrm{~m}, 3 \mathrm{H}), 3.67-3.51(\mathrm{~m}, 2 \mathrm{H}), 2.56(\mathrm{~m}, 1 \mathrm{H}), 2.52-2.43(\mathrm{~m}, 2 \mathrm{H}), 1.87(\mathrm{~s}, 3 \mathrm{H}) .{ }^{31} \mathbf{P}\left\{{ }^{1} \mathbf{H}\right\} \mathbf{N M R}$ (122 MHz, $\left.\mathrm{D}_{2} \mathrm{O}, \delta / \mathrm{ppm}\right): 42.7$ (m,1P), -2.93 (dd, J = 21.2, $\left.4.1 \mathrm{~Hz}, 1 \mathrm{P}\right),-23.0--24.8$ (m,1P). ${ }^{31} \mathbf{P}$ NMR (162 MHz, $\left.\mathrm{D}_{2} \mathrm{O}, \delta / \mathrm{ppm}\right): 42.8$ (m, 1P), -1.73 - -6.95 (m,1P), -24.0 (t, J = $\left.25.3 \mathrm{~Hz}, 1 \mathrm{P}\right) .{ }^{13} \mathrm{C}$ NMR $(101$ MHz, $\left.\mathrm{D}_{2} \mathrm{O}, \delta / \mathrm{ppm}\right): 166.8,151.9,137.3,111.9,84.8,83.0(\mathrm{~d}, \mathrm{~J}=9.0 \mathrm{~Hz}), 71.3,65.6(\mathrm{~d}, \mathrm{~J}=5.5 \mathrm{~Hz}), 60.9$, $36.3,31.0,11.7$.

HRMS (ESI): calculated for $\left[\mathrm{C}_{13} \mathrm{H}_{18} \mathrm{~N}_{6} \mathrm{O}_{11} \mathrm{P}_{3} \mathrm{~S}\right]^{-}$: calcd. 558.9973 found: 558.9978.

\section{AZT $\alpha$-(Se)-cyclotriphosphate: 3'-Azido-3'-deoxythymidine 5'- $\alpha$-selenocyclotriphosphate (B3)}

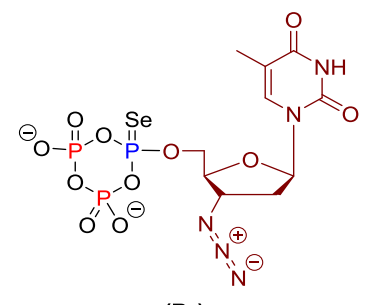

$\left(\mathrm{B}_{3}\right)$

AZT (115 mg, $0.43 \mathrm{mmol}, 1.2$ eq.) and ETT (150 mg, $1.15 \mathrm{mmol}, 3.2$ eq.) were coevaporated with dry $\mathrm{MeCN}(2 \times 4 \mathrm{~mL})$. Under an Ar atmosphere, a freshly prepared reaction mixture containing the $c$-PyPA $\mathbf{A}_{1}$ $(0.075 \mathrm{M}$ in $\mathrm{MeCN}, 5.00 \mathrm{~mL}, 0.36 \mathrm{mmol}, 1.0$ eq. $)$ was added to the dried solids and it was stirred at r.t. for 10 mins. Upon cooling to $0{ }^{\circ} \mathrm{C}$, potassium selenocyanate $(650 \mathrm{mg}, 4.51 \mathrm{mmol}, 12.5 \mathrm{eq}$.) was added and the mixture was stirred for 15 mins. A colourless precipitate was observed, which was further enhanced by addition of acetone and it was collected via centrifugation. The colourless residue was further checked by ${ }^{31} \mathrm{P}-\mathrm{NMR}$ confirmed complete oxidation (the oxidation product shows triplet at $+33 \mathrm{ppm}$ for the $\alpha$ - (Se)-cyclotriphosphate $\mathbf{B} 3$ ).

${ }^{31} \mathbf{P}\left\{{ }^{1} \mathbf{H}\right\}$ NMR (122 MHz, $\left.\mathrm{D}_{2} \mathrm{O}, \delta / \mathrm{ppm}\right): 35.8-30.6$ (m, 1P), -20.8 - -23.5 (m, 1P), -23.7 - -26.1 (m, 1P) ${ }^{31}$ P NMR (162 MHz, D $\left.2 \mathrm{O}, \delta / \mathrm{ppm}\right): 34.6$ - 32.5 (m, 1P), -20.2 - -23.2 (m, 1P), -23.8 - -25.1 (m, 1P). HRMS (ESI) m/z for $\left[\mathrm{C}_{10} \mathrm{H}_{15} \mathrm{~N}_{5} \mathrm{O}_{12} \mathrm{P}_{3} \mathrm{Se}\right]^{-}$: calcd.: 569.9101 , found 569.9103 . 
Ring opening by using propargylamine: 3'-Azido-3'-deoxythymidine 5'- $\gamma$-P-propargylamino $\alpha$ selenotriphosphate (11)

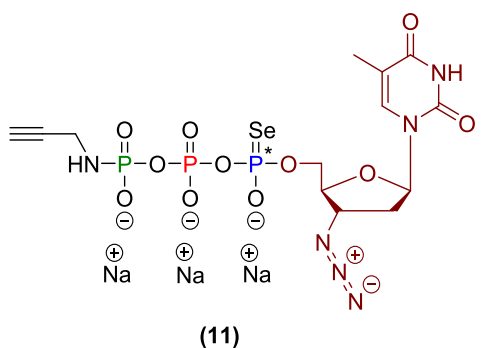

The precipitated product $\mathbf{B}_{3}$ was further dissolved in $\mathrm{D}_{2} \mathrm{O}(1 \mathrm{ml})$ and treated with propargylamine $(1 \mathrm{ml})$ for 15 mins at r.t. The product $\mathbf{1 1}$ was obtained by Method B as a colourless solid (the product was eluted at 300-500 $\mathrm{mM} \mathrm{NH}_{4} \mathrm{HCO}_{3}$ buffer) (98 mg, $\left.0.14 \mathrm{mmol}, 40 \%\right)$.

${ }^{1}$ H NMR (300 MHz, D $\left.2 \mathrm{O}, \delta / \mathrm{ppm}\right): 7.73(\mathrm{~s}, 1 \mathrm{H}), 6.23(\mathrm{t}, \mathrm{J}=6.9 \mathrm{~Hz}, 1 \mathrm{H}), 4.61-4.49(\mathrm{~m}, 1 \mathrm{H}), 4.28-$ 4.09 (m, 3H), $3.65(\mathrm{dt}, \mathrm{J}=9.8,2.9 \mathrm{~Hz}, 2 \mathrm{H}), 2.50(\mathrm{t}, \mathrm{J}=2.7 \mathrm{~Hz}, 1 \mathrm{H}), 2.48-2.34(\mathrm{~m}, 2 \mathrm{H}), 1.91(\mathrm{~s}, 3 \mathrm{H})$. ${ }^{31} \mathbf{P}\left\{{ }^{1} \mathbf{H}\right\}$ NMR (122 MHz, D $\left.2 \mathrm{O}, \delta / \mathrm{ppm}\right): 33.2$ (dd, J =32.4, $\left.19.9 \mathrm{~Hz}\right),-2.89$ (dd, J = 21.5, 3.0 Hz), -24.5 (ddd, J = 33.1, 21.4, 3.0 Hz). ${ }^{31} \mathbf{P}$ NMR (122 MHz, $\left.\mathrm{D}_{2} \mathrm{O}, \delta / \mathrm{ppm}\right): 33.2-32.2(\mathrm{~m}, 1 \mathrm{P}),-2.89--3.40(\mathrm{~m}$, 1P), -24.5 - -25.6 (m, 1P). ${ }^{13} \mathrm{C}$ NMR (101 MHz, $\left.\mathrm{D}_{2} \mathrm{O}, \delta / \mathrm{ppm}\right): 166.8,151.8,137.2,111.8,84.8,82.9$ (d, $\mathrm{J}=9.3 \mathrm{~Hz}), 82.4,71.3,65.6(\mathrm{~d}, \mathrm{~J}=5.6 \mathrm{~Hz}), 60.8,30.9,11.7$.

HRMS (ESI) m/z for $\left[\mathrm{C}_{13} \mathrm{H}_{18} \mathrm{~N}_{6} \mathrm{O}_{11} \mathrm{P}_{3} \mathrm{Se}\right]^{-}$: calcd. 606.9417, found 606.9415 .

\section{2',3'-didehydro-2',3'-dideoxythymidine 5'-cyclotriphosphate (d4T cyclotriphosphate $B_{4}$ )}

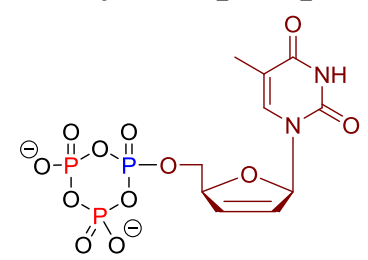

$\left(B_{4}\right)$

d4T (70 mg, $0.31 \mathrm{mmol}, 1.4$ eq.) and ETT (113 mg, $0.87 \mathrm{mmol}, 4.0$ eq.) were coevaporated with dry $\mathrm{MeCN}(2 \times 1 \mathrm{~mL})$. Under an Ar atmosphere, a reaction mixture containing the $c$-PyPA $\mathbf{A}_{1}(0.075 \mathrm{M}$ in MeCN, $2.9 \mathrm{ml}, 0.22 \mathrm{mmol}, 1.0$ eq.) was added to the dried solids and it was stirred at r.t. for 10 mins. Upon cooling to $0^{\circ} \mathrm{C}, m \mathrm{CPBA}(\leq 77 \%, 56 \mathrm{mg}, 0.32 \mathrm{mmol}, 1.5 \mathrm{eq}$.) was added and the mixture was stirred for 5 mins until ${ }^{31} \mathrm{P}-\mathrm{NMR}$ confirmed complete oxidation (the oxidation product shows triplet at $-23 \mathrm{ppm}$ which is diagnostic signal for the cyclotriphosphate $\mathbf{B} 4)$. Aliquot of this reaction mixture were used for the ring opening with various nucleophiles. The $\mathrm{d} 4 \mathrm{~T}$ cyclotriphosphate $\mathbf{B}_{\mathbf{4}}$ is stable in reaction mixture at $-20^{\circ} \mathrm{C}$ upto 2 weeks.

Ring opening was monitored by ${ }^{31} \mathrm{P}-\mathrm{NMR}$ of the resulting reaction mixtures.

${ }^{31} \mathbf{P}\left\{{ }^{1} \mathbf{H}\right\}$ NMR $\left(122 \mathrm{MHz}, \mathrm{CDCl}_{3}, \delta / \mathrm{ppm}\right):-22.1--23.9(\mathrm{t}, 1 \mathrm{P}),-26.5(\mathrm{~d}, \mathrm{~J}=21.1 \mathrm{~Hz}, 2 \mathrm{P})$. 
Ring opening by using propargylamine: 2',3'-didehydro-2',3'-dideoxythymidine 5'- $\gamma-P$ propargylamino triphosphate (12)

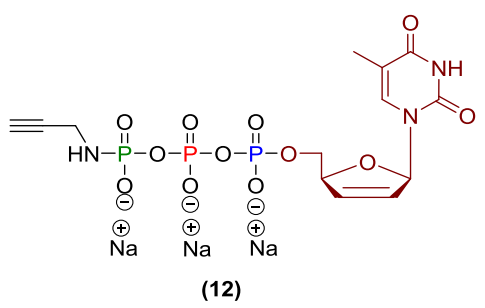

The reaction mixture of $\mathbf{B}_{4}(250 \mu \mathrm{L}, 18.7 \mu \mathrm{mol}, 1.0 \mathrm{eq})$ was added to propargylamine $(250 \mu \mathrm{L})$ and then $(100 \mu \mathrm{l})$ was added afterwards it was stirred for 5 mins. The product 12 was obtained by Method A as a colourless solid (7.70 mg, $13.8 \mu \mathrm{mol}, 74 \%)$.

${ }^{1}$ H NMR (300 MHz, D $\left.2 \mathrm{O}, \delta / \mathrm{ppm}\right): 7.58(\mathrm{~s}, 1 \mathrm{H}), 6.91(\mathrm{dt}, \mathrm{J}=3.3,1.7 \mathrm{~Hz}, 1 \mathrm{H}), 6.48(\mathrm{dt}, \mathrm{J}=6.2,1.8 \mathrm{~Hz}$, $1 \mathrm{H}), 5.92-5.86(\mathrm{~m}, 1 \mathrm{H}), 5.06(\mathrm{~s}, 1 \mathrm{H}), 4.13(\mathrm{dt}, \mathrm{J}=6.1,3.1 \mathrm{~Hz}, 2 \mathrm{H}), 3.61(\mathrm{dd}, \mathrm{J}=9.9,2.4 \mathrm{~Hz}, 2 \mathrm{H}), 2.49$ $(\mathrm{t}, \mathrm{J}=2.5 \mathrm{~Hz}, 1 \mathrm{H}), 1.85(\mathrm{~s}, 3 \mathrm{H}) .{ }^{31} \mathbf{P}\left\{{ }^{1} \mathbf{H}\right\} \mathbf{N M R}\left(122 \mathrm{MHz}, \mathrm{D}_{2} \mathrm{O}, \delta / \mathrm{ppm}\right):-2.81(\mathrm{~d}, \mathrm{~J}=20.9 \mathrm{~Hz}, 1 \mathrm{P}),-11.5$ (d, J = 19.4 Hz, 1P), -22.5 - -23.4 (m, 1P). ${ }^{31} \mathbf{P}$ NMR (162 MHz, D $\left.\mathrm{O}, \delta / \mathrm{ppm}\right):-1.97$ (dt, J = 19.1, 9.4 Hz, 1P), -10.3 (dt, J = 19.4, $6.0 \mathrm{~Hz}, 1 \mathrm{P}),-20.6$ (t, J = 19.9). ${ }^{13} \mathbf{C}$ NMR (101 MHz, $\left.\mathrm{D}_{2} \mathrm{O}, \delta / \mathrm{ppm}\right): 167.0,152.5$, 138.2, 134.3, 125.3, 111.6, 89.9, 85.9 (d, J = 8.8 Hz), 83.1, 66.5 (d, J = 6.0 Hz), 30.9, 11.5.

HRMS (ESI) m/z for $\left[\mathrm{C}_{13} \mathrm{H}_{17} \mathrm{~N}_{3} \mathrm{NaO}_{12} \mathrm{P}_{3}\right]^{-}$: calcd. 522.9928, found 522.9927.

Ring opening by using diethylamine: 2',3'-didehydro-2',3'-dideoxythymidine 5'- $\gamma$-P-diethylamino triphosphate (13)

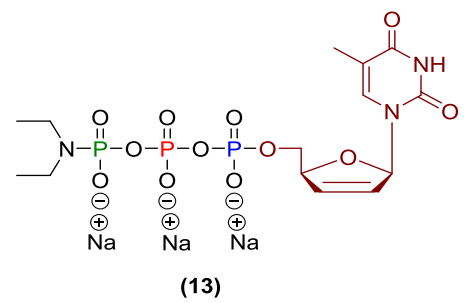

The reaction mixture of $\mathbf{B}_{4}(250 \mu \mathrm{L}, 18.7 \mu \mathrm{mol}, 1.0$ eq.) was added to diethylamine $(250 \mu \mathrm{L})$ and then $\mathrm{D}_{2} \mathrm{O}(100 \mu \mathrm{l})$ was added afterwards it was stirred for 5 mins. The Product 13 was obtained by Method A as a colourless solid ( $8.80 \mathrm{mg}, 15.0 \mu \mathrm{mol}, 80 \%)$.

${ }^{1}$ H NMR (300 MHz, D $\left.2 \mathrm{O}, \delta / \mathrm{ppm}\right): 7.50(\mathrm{~s}, 1 \mathrm{H}), 6.90(\mathrm{dt}, \mathrm{J}=3.4,1.7 \mathrm{~Hz}, 1 \mathrm{H}), 6.44(\mathrm{dt}, \mathrm{J}=6.2,1.8 \mathrm{~Hz}$, $1 \mathrm{H}), 5.86(\mathrm{dt}, \mathrm{J}=5.9,1.9 \mathrm{~Hz}, 1 \mathrm{H}), 5.20-4.94(\mathrm{~m}, 1 \mathrm{H}), 4.20-3.98(\mathrm{~m}, 2 \mathrm{H}), 2.93(\mathrm{dq}, \mathrm{J}=11.3,7.2 \mathrm{~Hz}$, 4H), 1.81 (s, 3H), 0.97 (t, J = 7.1 Hz, 6H). ${ }^{31} \mathbf{P}\left\{{ }^{1} \mathbf{H}\right\}$ NMR $\left(122 \mathrm{MHz}, \mathrm{D}_{2} \mathrm{O}, \delta / \mathrm{ppm}\right):-0.51(\mathrm{~d}, \mathrm{~J}=24.7 \mathrm{~Hz}$, 1P), $-11.5(\mathrm{~d}, \mathrm{~J}=19.0 \mathrm{~Hz}, 1 \mathrm{P}),-22.9(\mathrm{dd}, \mathrm{J}=24.6,18.6 \mathrm{~Hz}, 1 \mathrm{P}) .{ }^{31} \mathbf{P}$ NMR $\left(162 \mathrm{MHz}, \mathrm{D}_{2} \mathrm{O}, \delta / \mathrm{ppm}\right)$ : - $0.51(\mathrm{dp}, \mathrm{J}=22.9,11.4 \mathrm{~Hz}),-11.5(\mathrm{dt}, \mathrm{J}=19.1,6.5 \mathrm{~Hz}),-22.9(\mathrm{dd}, \mathrm{J}=24.3,18.9 \mathrm{~Hz}) .{ }^{13} \mathbf{C}$ NMR $(101$ MHz, $\left.\mathrm{D}_{2} \mathrm{O}, \delta / \mathrm{ppm}\right): 166.0,152.5,137.8,133.9,125.5,111.6,90.0,85.8(\mathrm{~d}, \mathrm{~J}=8.5 \mathrm{~Hz}), 66.6(\mathrm{~d}, \mathrm{~J}=$ $5.7 \mathrm{~Hz}), 40.5(\mathrm{~d}, \mathrm{~J}=3.7 \mathrm{~Hz}), 13.8(\mathrm{~d}, \mathrm{~J}=3.7 \mathrm{~Hz}), 11.9$.

HRMS (ESI) $\mathrm{m} / \mathrm{z}$ for $\left[\mathrm{C}_{14} \mathrm{H}_{23} \mathrm{~N}_{3} \mathrm{O}_{12} \mathrm{P}_{3}\right]^{-}$: calcd. 518.0500, found 518.0501. 
Ring opening by using aq. ammonia: 2',3'-didehydro-2',3'-dideoxythymidine 5'- $\gamma$ - $P$-amino triphosphate (14)

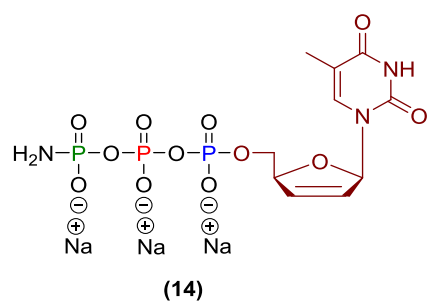

The reaction mixture of $\mathbf{B}_{4}\left(250 \mu \mathrm{L}, 18.7 \mu\right.$ mol, 1.0 eq.) was added to $25 \%$ aq. $\mathrm{NH}_{4} \mathrm{OH}(250 \mu \mathrm{L})$ and then $\mathrm{D}_{2} \mathrm{O}(100 \mu \mathrm{l})$ was added afterwards it was stirred for 5 mins. The product 14 was obtained by Method A as a colourless solid (7.73 mg, $14.6 \mu \mathrm{mol}, 79 \%)$.

${ }^{1}$ H NMR (300 MHz, D $\left.2 \mathrm{O}, \delta / \mathrm{ppm}\right): 7.55(\mathrm{~s}, 1 \mathrm{H}), 6.91(\mathrm{dt}, \mathrm{J}=3.3,1.7 \mathrm{~Hz}, 1 \mathrm{H}), 6.48$ (dt, J = 6.2, $1.8 \mathrm{~Hz}$, $1 \mathrm{H}), 5.89(\mathrm{~d}, \mathrm{~J}=6.3 \mathrm{~Hz}, 1 \mathrm{H}), 5.06(\mathrm{~s}, 1 \mathrm{H}), 4.11(\mathrm{dt}, \mathrm{J}=6.3,3.3 \mathrm{~Hz}, 2 \mathrm{H}), 1.84(\mathrm{~s}, 3 \mathrm{H}) .{ }^{31} \mathbf{P}\left\{{ }^{1} \mathbf{H}\right\} \mathbf{N M R}(122$ MHz, $\left.\mathrm{D}_{2} \mathrm{O}, \delta / \mathrm{ppm}\right):-1.08(\mathrm{~d}, \mathrm{~J}=19.3 \mathrm{~Hz}, 1 \mathrm{P}),-11.5(\mathrm{~d}, \mathrm{~J}=20.1 \mathrm{~Hz}, 1 \mathrm{P}),-22.7(\mathrm{t}, \mathrm{J}=19.4 \mathrm{~Hz}, 1 \mathrm{P})$. ${ }^{31} \mathbf{P}$ NMR $\left(162 \mathrm{MHz}, \mathrm{D}_{2} \mathrm{O}, \delta / \mathrm{ppm}\right):-0.33(\mathrm{~d}, \mathrm{~J}=19.1 \mathrm{~Hz}, 1 \mathrm{P}),-10.9$ (dt, J = 19.4, $\left.6.5 \mathrm{~Hz}, 1 \mathrm{P}\right),-21.6(\mathrm{t}, \mathrm{J}$ $=19.3 \mathrm{~Hz}, 1 \mathrm{P}) .{ }^{13} \mathrm{C}$ NMR (101 MHz, $\left.\mathrm{D}_{2} \mathrm{O}, \delta / \mathrm{ppm}\right): 166.8,152.3,138.2,134.4,125.0,111.5,89.9,86.0$ $(\mathrm{d}, \mathrm{J}=8.5 \mathrm{~Hz}), 66.4(\mathrm{~d}, \mathrm{~J}=5.8 \mathrm{~Hz}), 11.4$.

HRMS (ESI) $\mathrm{m} / \mathrm{z}$ for $\left[\mathrm{C}_{10} \mathrm{H}_{15} \mathrm{~N}_{3} \mathrm{Na}_{2} \mathrm{O}_{12} \mathrm{P}_{3}\right]^{+}$: calcd. 507.9658, found 507.9660.

Ring opening by using aq. sodium hydroxide: 2',3'-didehydro-2',3'-dideoxythymidine 5'triphosphate (15)

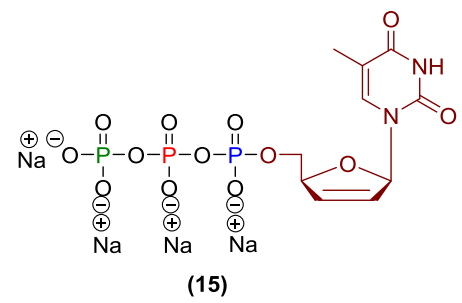

The reaction mixture of $\mathbf{B}_{4}(250 \mu \mathrm{L}, 18.7 \mu$ mol, 1.0 eq.) was added to $1 \mathrm{M}$ aq. $\mathrm{NaOH}(250 \mu \mathrm{L})$ and then $\mathrm{D}_{2} \mathrm{O}(100 \mu \mathrm{l})$ was added afterwards it was stirred for 5 mins. The product 15 was obtained by Method A as a colourless solid (7.95 mg, $14.9 \mu \mathrm{mol}, 80 \%)$.The analytical data are consistent with literature. ${ }^{6}$

${ }^{1}$ H NMR (300 MHz, D $\left.2 \mathrm{O}, \delta / \mathrm{ppm}\right): 7.55$ (s, 1H), $6.90(\mathrm{dt}, \mathrm{J}=3.4,1.7 \mathrm{~Hz}, 1 \mathrm{H}), 6.49$ (dt, J = 6.2, $1.8 \mathrm{~Hz}$, $1 \mathrm{H}), 5.87(\mathrm{dt}, \mathrm{J}=6.2,1.9 \mathrm{~Hz}, 1 \mathrm{H}), 5.05(\mathrm{td}, \mathrm{J}=3.6,1.9 \mathrm{~Hz}, 1 \mathrm{H}), 4.32-3.98(\mathrm{~m}, 2 \mathrm{H}), 1.83(\mathrm{~s}, 3 \mathrm{H}) .{ }^{31} \mathbf{P}\left\{{ }^{1} \mathbf{H}\right\}$ NMR (122 MHz, D $2 \mathrm{O}, \delta / \mathrm{ppm}):-10.9$ (d, J = 19.6 Hz, 1P), -11.6 (d, J = 20.1 Hz, 1P), -23.4 (t, J = 19.8Hz, 1P). ${ }^{31}$ P NMR (162 MHz, D $\left.2 \mathrm{O}, \delta / \mathrm{ppm}\right):-9.68$ (d, J = $\left.18.3 \mathrm{~Hz}, 1 \mathrm{P}\right),-10.7$ (dt, J = 19.4, $6.5 \mathrm{~Hz}, 1 \mathrm{P}$ ), -21.6 (m,1P). ${ }^{13} \mathrm{C}$ NMR (101 MHz, $\left.\mathrm{D}_{2} \mathrm{O}, \delta / \mathrm{ppm}\right): 166.8,152.3,138.2,134.4,125.0,111.5,89.9,86.0$ (d, $\mathrm{J}=8.5 \mathrm{~Hz}), 66.4(\mathrm{~d}, \mathrm{~J}=5.8 \mathrm{~Hz}), 11.5$.

HRMS (ESI) m/z for $\left[\mathrm{C}_{10} \mathrm{H}_{14} \mathrm{~N}_{2} \mathrm{O}_{13} \mathrm{P}_{3}\right]^{-}$: calcd. 462.9714, found 462.9712 . 
Ring opening by caesium fluoride: 2',3'-didehydro-2',3'-dideoxythymidine 5'- $\gamma$ - $P$ fluorotriphosphate (16)

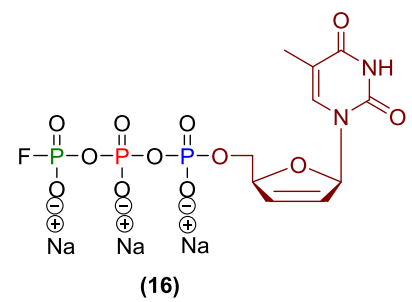

The reaction mixture of $\mathbf{B}_{4}(300 \mu \mathrm{L}, 13.5 \mu \mathrm{mol}, 1.0 \mathrm{eq}$.) was added to caesium fluoride (100 mg, $661 \mu \mathrm{mol}$, 50 eq) and then immediately $\mathrm{D}_{2} \mathrm{O}(200 \mu \mathrm{l})$ was added afterwards it was stirred for 5 mins. The crude product was isolated by Method A and the impurity $\mathrm{CsClO}_{4}$ was co-precipitated which was further removed by dissolving the crude product in water $(2 \mathrm{~mL})$. The insoluble residue was removed by filtration (syringe filter, pore size $0.45 \mu \mathrm{m}$ ). Afterwards, the product $\mathbf{1 6}$ was isolated from the aq. solution again by Method A and a colourless solid was obtained (5.9 mg, $11.0 \mu \mathrm{mol}, 82 \%)$.

${ }^{1}$ H NMR (300 MHz, D $\left.2 \mathrm{O}, \delta / \mathrm{ppm}\right): 7.53(\mathrm{~s}, 1 \mathrm{H}), 6.97-6.81(\mathrm{~m}, 1 \mathrm{H}), 6.57-6.40(\mathrm{~m}, 1 \mathrm{H}), 5.95-5.81$ (m, 1H), $5.04(\mathrm{~s}, 1 \mathrm{H}), 4.08(\mathrm{dd}, \mathrm{J}=6.4,3.4 \mathrm{~Hz}, 2 \mathrm{H}), 1.82(\mathrm{~s}, 3 \mathrm{H}) .{ }^{31} \mathbf{P}\left\{{ }^{1} \mathbf{H}\right\} \mathbf{~ N M R}\left(122 \mathrm{MHz}, \mathrm{D}_{2} \mathrm{O}, \delta / \mathrm{ppm}\right)$ : $-11.5(\mathrm{~d}, \mathrm{~J}=19.2 \mathrm{~Hz}, 1 \mathrm{P}),-17.9(\mathrm{dd}, \mathrm{J}=932.9,17.3 \mathrm{~Hz}, 1 \mathrm{P}),-22.3--23.5(\mathrm{~m}, 1 \mathrm{P}) .{ }^{31} \mathbf{P}$ NMR $(162 \mathrm{MHz}$ $\left.\mathrm{D}_{2} \mathrm{O}, \delta / \mathrm{ppm}\right):-11.5(\mathrm{dt}, \mathrm{J}=19.3,6.4 \mathrm{~Hz}, 1 \mathrm{P}),-17.9(\mathrm{dd}, \mathrm{J}=932.9,17.3 \mathrm{~Hz}, 1 \mathrm{P}),-22.4--24.2(\mathrm{~m}, 1 \mathrm{P})$ ${ }^{31} \mathbf{P}\left\{{ }^{19} \mathbf{F}\right\}$ NMR $\left(162 \mathrm{MHz}, \mathrm{D}_{2} \mathrm{O}, \delta / \mathrm{ppm}\right):-11.5(\mathrm{dt}, \mathrm{J}=19.5,6.4 \mathrm{~Hz}, 1 \mathrm{P}),-17.9(\mathrm{~d}, \mathrm{~J}=17.2 \mathrm{~Hz}, 1 \mathrm{P}),-21.6$ - -23.5 (m,1P). ${ }^{13}$ C NMR (101 MHz, $\left.\mathrm{D}_{2} \mathrm{O}, \delta / \mathrm{ppm}\right): 166.8,152.3,138.2,134.4,125.0,111.5,89.9,86.0$ $(\mathrm{d}, \mathrm{J}=8.5 \mathrm{~Hz}), 66.4(\mathrm{~d}, \mathrm{~J}=5.8 \mathrm{~Hz}), 11.5 .{ }^{19} \mathrm{~F}$ NMR $\left(377 \mathrm{MHz}, \mathrm{D}_{2} \mathrm{O}, \delta / \mathrm{ppm}\right):-73.1$ (d, J = 933.5 Hz, 1F). HRMS (ESI) m/z for $\left[\mathrm{C}_{10} \mathrm{H}_{13} \mathrm{FN}_{2} \mathrm{O}_{12} \mathrm{P}_{3}\right]^{-}$: calcd. 464.9671, found 464.9678 .

Ring opening by using lithium azide: 2',3'-didehydro-2',3'-dideoxythymidine 5'- $\gamma$-Pazidotriphosphate (17)

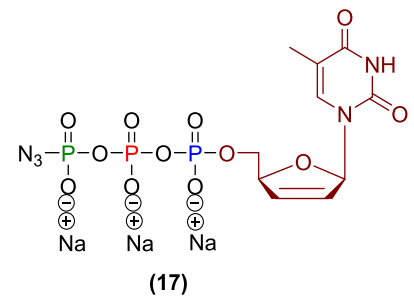

The reaction mixture of $\mathbf{B}_{4}\left(250 \mu \mathrm{L}, 18.7 \mu \mathrm{mol}, 1.0\right.$ eq.) was added to the lithium azide $(500 \mu \mathrm{L})$ and $\mathrm{D}_{2} \mathrm{O}$ $100 \mu 1$ was added afterwards it was stirred for 5 mins. The product was purified by Method A, affording a colourless solid 17 (9.89 mg, $17.8 \mu \mathrm{mol}, 95 \%)$.

Note -: As the commercial availability of $\mathrm{LiN}_{3}$ solution is $20 \%$ wt. in water which results in $\mathrm{H}_{2} \mathrm{O}$ is also acting as a nucleophile, so the product contains $30 \%$ of hydrolysed $\mathrm{d} 4 \mathrm{~T}$ triphosphate $\mathbf{1 5}$, indicated yield does not refer to the pure product. 
${ }^{31} \mathbf{P}\left\{{ }^{1} \mathbf{H}\right\}$ NMR $\left(122 \mathrm{MHz}, \mathrm{D}_{2} \mathrm{O}, \delta / \mathrm{ppm}\right):-10.8(\mathrm{~d}, \mathrm{~J}=17.2 \mathrm{~Hz}, 1 \mathrm{P}),-12.9(\mathrm{~d}, \mathrm{~J}=19.5 \mathrm{~Hz}, 1 \mathrm{P}),-21.5-$ -22.2 (m, 1P). ${ }^{31} \mathbf{P}$ NMR (162 MHz, $\left.\mathrm{D}_{2} \mathrm{O}, \delta / \mathrm{ppm}\right):-10.9$ (dt, J = 17.5 Hz, 1P), -12.9 (d, J = $\left.19.4 \mathrm{~Hz}, 1 \mathrm{P}\right)$, $-21.8(\mathrm{t}, \mathrm{J}=18.4 \mathrm{~Hz}, 1 \mathrm{P})$.

HRMS (ESI) $\mathrm{m} / \mathrm{z}$ for $\left[\mathrm{C}_{10} \mathrm{H}_{13} \mathrm{~N}_{5} \mathrm{O}_{12} \mathrm{P}_{3}\right]^{-}$: calcd. 487.9779, found 487.9785 .

\section{2',3'-O-Isopropylideneadenosine 5'-cyclotriphosphate (B)}

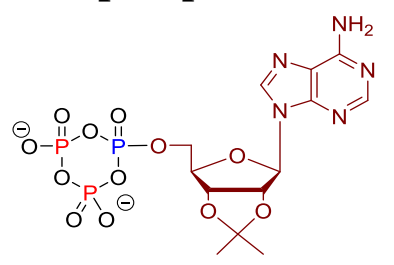

$\left(B_{5}\right)$

2',3'-O-Isopropylideneadenosine (70 mg, $0.22 \mathrm{mmol}, 1.5$ eq.) and ETT ( $80 \mathrm{mg}, 0.61 \mathrm{mmol}, 4.0$ eq.) were coevaporated with dry $\mathrm{MeCN}(2 \times 1 \mathrm{~mL})$. Under an Ar atmosphere, both solids were dissolved in dry $\operatorname{DMF}(2 \mathrm{~mL})$, a reaction mixture containing the $c$-PyPA $\mathbf{A}_{1}(0.075 \mathrm{M}$ in MeCN, $2.0 \mathrm{~mL}, 0.15 \mathrm{mmol}, 1.0$ eq.) was added and it was stirred at r.t. for $10 \mathrm{mins}$. Upon cooling to $0^{\circ} \mathrm{C}, \mathrm{mCPBA}(\leq 77 \%, 40 \mathrm{mg}, 0.23 \mathrm{mmol}$, 1.5 eq.) was added and the mixture was stirred for 5 mins until ${ }^{31} \mathrm{P}-\mathrm{NMR}$ confirmed complete oxidation (formation of triplet at $-22 \mathrm{ppm}$ which is diagnostic signal for the cyclotriphosphate $\mathbf{B}_{5}$ ). The final concentration of cyclotriphosphate $\mathbf{B}_{5}$ is $0.037 \mathrm{M}$.

${ }^{31} \mathbf{P}\left\{{ }^{1} \mathbf{H}\right\}$ NMR $\left(122 \mathrm{MHz}, \mathrm{CDCl}_{3}, \delta / \mathrm{ppm}\right):-23.2--25.2(\mathrm{~m}, 1 \mathrm{P}),-25.8(\mathrm{~d}, \mathrm{~J}=21.1 \mathrm{~Hz}, 2 \mathrm{P})$.

Ring opening by using aq. ammonia: 2',3'-O-Isopropylideneadenosine 5'- $\gamma$ - $P$-amino triphosphate (S-1)

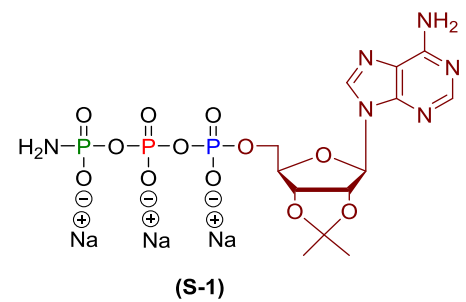

The reaction mixture of $\mathbf{B}_{5}\left(500 \mu \mathrm{L}, 18.7 \mu\right.$ mol, 1.0 eq.) was added to $25 \%$ aq. $\mathrm{NH}_{4} \mathrm{OH}(500 \mu \mathrm{L})$ and then $\mathrm{D}_{2} \mathrm{O}(200 \mu \mathrm{l})$ was added afterwards it was stirred for 5 mins. The product $\mathbf{S - 1}$ was isolated by Method A, affording a colourless solid (7.50 mg, $12.5 \mu \mathrm{mol}, 66 \%)$.

${ }^{1}$ H NMR (400 MHz, D $\left.2 \mathrm{O}, \delta / \mathrm{ppm}\right): 8.40(\mathrm{~s}, 1 \mathrm{H}), 8.20(\mathrm{~s}, 1 \mathrm{H}), 6.24(\mathrm{~d}, \mathrm{~J}=3.5 \mathrm{~Hz}, 1 \mathrm{H}), 5.36$ (dd, J = 6.2, $3.5 \mathrm{~Hz}, 1 \mathrm{H}), 5.20(\mathrm{dd}, \mathrm{J}=6.2,2.2 \mathrm{~Hz}, 1 \mathrm{H}), 4.33-4.04(\mathrm{~m}, 2 \mathrm{H}), 1.64(\mathrm{~s}, 3 \mathrm{H}), 1.41$ (s, 3H). The DHO peak overlaps with the signal $4.50(1 \mathrm{H}) .{ }^{31} \mathbf{P}\left\{{ }^{1} \mathbf{H}\right\}$ NMR $\left(122 \mathrm{MHz}, \mathrm{D}_{2} \mathrm{O}, \delta / \mathrm{ppm}\right):-1.04(\mathrm{~d}, \mathrm{~J}=19.4 \mathrm{~Hz}$, 1P), -11.6 (d, J = 19.7 Hz, 1P), -22.6 (t, J = 19.4 Hz, 1P). ${ }^{31}$ P NMR (162 MHz, $\left.\mathrm{D}_{2} \mathrm{O}, \delta / \mathrm{ppm}\right):-0.98$ (d, J $=19.1 \mathrm{~Hz}, 1 \mathrm{P}),-11.6(\mathrm{dt}, \mathrm{J}=19.7,5.7 \mathrm{~Hz}, 1 \mathrm{P}),-22.5(\mathrm{t}, \mathrm{J}=19.2 \mathrm{~Hz}, 1 \mathrm{P}) .{ }^{13} \mathbf{C} \mathbf{N M R}\left(101 \mathrm{MHz}, \mathrm{D}_{2} \mathrm{O}\right.$, 


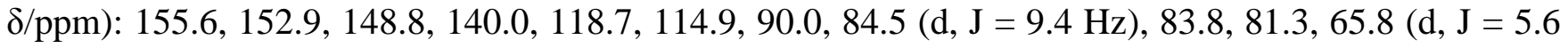
Hz), 26.1, 24.4.

HRMS (ESI) $\mathrm{m} / \mathrm{z}$ for $\left[\mathrm{C}_{13} \mathrm{H}_{20} \mathrm{~N}_{6} \mathrm{O}_{12} \mathrm{P}_{3}\right]^{-}$: calcd. 545.0358, found 545.0359.

Ring opening by using propargylamine: 2 ', 3'-O-Isopropylideneadenosine 5'- $\gamma$ - $P$-propargylamino triphosphate (S-2)

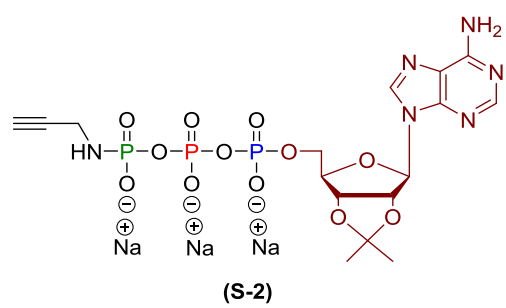

The reaction mixture of $\mathbf{B} \mathbf{5}$ ( $500 \mu \mathrm{L}, 18.7 \mu \mathrm{mol}, 1.0$ eq.) was added to propargylamine (500 $\mu \mathrm{L}$ ) and then $\mathrm{D}_{2} \mathrm{O}(200 \mu \mathrm{l})$ was added afterwards it was stirred for 5 mins. The product $\mathbf{S - 2}$ was isolated by Method A, affording a colourless solid (9.56 mg, $14.6 \mu \mathrm{mol}, 79 \%)$.

${ }^{1} \mathbf{H}$ NMR $\left(400 \mathrm{MHz}, \mathrm{D}_{2} \mathrm{O}, \delta / \mathrm{ppm}\right): 8.41(\mathrm{~s}, 1 \mathrm{H}), 8.20(\mathrm{~s}, 1 \mathrm{H}), 6.23(\mathrm{~d}, \mathrm{~J}=3.6 \mathrm{~Hz}, 1 \mathrm{H}), 5.36(\mathrm{dd}, \mathrm{J}=6.2$, $3.5 \mathrm{~Hz}, 1 \mathrm{H}), 5.20(\mathrm{dd}, \mathrm{J}=6.2,2.2 \mathrm{~Hz}, 1 \mathrm{H}), 4.27-4.03(\mathrm{~m}, 2 \mathrm{H}), 3.56(\mathrm{~d}, \mathrm{~J}=9.9 \mathrm{~Hz}, 2 \mathrm{H}), 2.51(\mathrm{t}, \mathrm{J}=2.5$ $\mathrm{Hz}, 1 \mathrm{H}) 1.64(\mathrm{~s}, 3 \mathrm{H}), 1.41(\mathrm{~s}, 3 \mathrm{H})$. The DHO peak overlaps with the signal $4.55(1 \mathrm{H}) .{ }^{31} \mathbf{P}\left\{{ }^{1} \mathbf{H}\right\} \mathbf{N M R}(122$ MHz, $\left.\mathrm{D}_{2} \mathrm{O}, \delta / \mathrm{ppm}\right):-2.76(\mathrm{~d}, \mathrm{~J}=20.9 \mathrm{~Hz}, 1 \mathrm{P}),-11.6(\mathrm{~d}, \mathrm{~J}=19.2 \mathrm{~Hz}, 1 \mathrm{P}),-21.2--23.5$ (m, 1P). ${ }^{31} \mathbf{P}$ NMR (162 MHz, $\left.\mathrm{D}_{2} \mathrm{O}, \delta / \mathrm{ppm}\right):-2.76(\mathrm{dt}, \mathrm{J}=20.4,10.1 \mathrm{~Hz}, 1 \mathrm{P}),-11.6(\mathrm{~d}, \mathrm{~J}=18.3 \mathrm{~Hz}, 1 \mathrm{P}),-22.0--23.1(\mathrm{~m}$, 1P). ${ }^{13} \mathrm{C}$ NMR (101 MHz, $\left.\mathrm{D}_{2} \mathrm{O}, \delta / \mathrm{ppm}\right): 155.7,152.9,148.9,140.0,118.7,114.9,89.9,84.5$ (d, J = 9.7 Hz), 83.8, 81.3, 65.8 (d, J = 5.5 Hz), 42.4, 30.9, 26.2, 24.4.

HRMS (ESI) m/z for $\left[\mathrm{C}_{16} \mathrm{H}_{21} \mathrm{~N}_{6} \mathrm{O}_{12} \mathrm{P}_{3}\right]^{2-}$ : calcd. 291.5260, found 291.5251 .

Ring opening by using aq. sodium hydroxide: 2',3'-O-Isopropylideneadenosine 5'-triphosphate (S- 3)

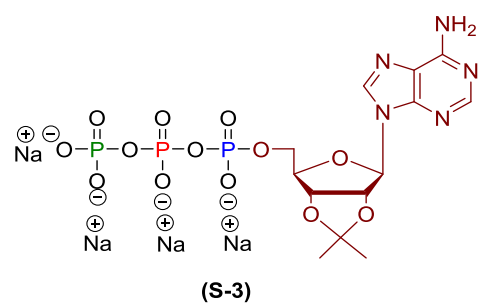

The reaction mixture of $\mathbf{B} 5(500 \mu \mathrm{L}, 18.7 \mu \mathrm{mol}, 1.0$ eq.) was added to $1 \mathrm{M}$ aq. $\mathrm{NaOH}(500 \mu \mathrm{L})$ and then $\mathrm{D}_{2} \mathrm{O}(200 \mu \mathrm{l})$ was added. The product $\mathbf{S - 3}$ was isolated by Method A, affording a colourless solid (9.10 mg, $14.7 \mu \mathrm{mol}, 78 \%)$.

The analytical data is consistent with literature. ${ }^{7}$

${ }^{1}$ H NMR (400 MHz, D $\left.2 \mathrm{O}, \delta / \mathrm{ppm}\right): 8.41(\mathrm{~s}, 1 \mathrm{H}), 8.20(\mathrm{~s}, 1 \mathrm{H}), 6.23(\mathrm{~d}, \mathrm{~J}=3.5 \mathrm{~Hz}, 1 \mathrm{H}), 5.35$ (dd, J = 6.1, $3.6 \mathrm{~Hz}, 1 \mathrm{H}), 5.21(\mathrm{dd}, \mathrm{J}=6.1,2.2,1 \mathrm{H}), 4.43-4.05(\mathrm{~m}, 2 \mathrm{H}), 1.63(\mathrm{~s}, 3 \mathrm{H}), 1.41(\mathrm{~s}, 3 \mathrm{H})$. The DHO peak 
overlaps with the signal $4.55(1 \mathrm{H}) .{ }^{31} \mathbf{P}\left\{{ }^{1} \mathbf{H}\right\}$ NMR $\left(122 \mathrm{MHz}, \mathrm{D}_{2} \mathrm{O}, \delta / \mathrm{ppm}\right):-5.55(\mathrm{~d}, \mathrm{~J}=20.1 \mathrm{~Hz}, 1 \mathrm{P})$, $11.2(\mathrm{~d}, \mathrm{~J}=18.6 \mathrm{~Hz}, 1 \mathrm{P}),-21.5(\mathrm{t}, \mathrm{J}=19.2 \mathrm{~Hz}, 1 \mathrm{P}) .{ }^{31} \mathbf{P}$ NMR $\left(162 \mathrm{MHz}, \mathrm{D}_{2} \mathrm{O}, \delta / \mathrm{ppm}\right):-5.56(\mathrm{~d}, \mathrm{~J}=20.0$ $\mathrm{Hz}, 1 \mathrm{P}),-11.2$ (d, J = 18.7 Hz, 1P), -21.1 - -21.8 (m, 1P). ${ }^{13} \mathrm{C}$ NMR (101 MHz, D $\left.2 \mathrm{O}, \delta / \mathrm{ppm}\right): 155.6$, 152.9, 148.9, 140.0, 118.7, 114.9, 90.0, 84.5 (d, J = 9.4 Hz), 83.8, 81.3, 65.8 (d, J = 5.6 Hz), 26.1, 24.4. HRMS (ESI) m/z for $\left[\mathrm{C}_{13} \mathrm{H}_{17} \mathrm{~N}_{5} \mathrm{Na}_{4} \mathrm{O}_{13} \mathrm{P}_{3}\right]^{+}$: calcd. 635.9621, found 635.9623.

\section{4-Pentyne-1-cyclotriphosphate $\left(\mathbf{B}_{6}\right)$}

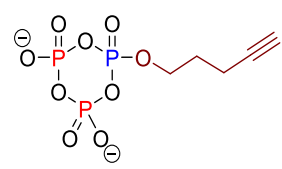

$\left(B_{6}\right)$

4-Pentyn-1-ol (50 mg, $0.59 \mathrm{mmol}, 1.5$ eq.) and ETT (118 mg, $0.90 \mathrm{mmol}, 2.3$ eq.) were coevaporated with dry MeCN $(2 \times 1 \mathrm{~mL})$. Under an atmosphere of dry Ar, a reaction mixture containing the $c$-PyPA A1 (0.079 M in MeCN, $5.0 \mathrm{~mL}, 0.39 \mathrm{mmol}, 1.0$ eq.) was added and it was stirred at r.t. for $10 \mathrm{mins}$. Upon cooling to $0^{\circ} \mathrm{C}, m \mathrm{CPBA}(\leq 77 \%, 75 \mathrm{mg}, 0.43 \mathrm{mmol}, 1.1 \mathrm{eq}$.) was added and the mixture was stirred for 5 mins until ${ }^{31} \mathrm{P}-\mathrm{NMR}$ confirmed complete oxidation (formation of triplet at $-21 \mathrm{ppm}$ which is diagnostic signal for the cyclotriphosphate $\mathbf{B}_{\mathbf{6}}$ ).

${ }^{31} \mathbf{P}\left\{{ }^{1} \mathbf{H}\right\}$ NMR (122 MHz, $\left.\mathrm{CDCl}_{3}, \delta / \mathrm{ppm}\right):-21.2--23.1(\mathrm{~m}, 1 \mathrm{P}),-24.3$ (d, J = 20.7 Hz, 2P).

Ring opening by using aq. ammonia: 4-Pentyne $\gamma$-P -amino triphosphate (S-4)

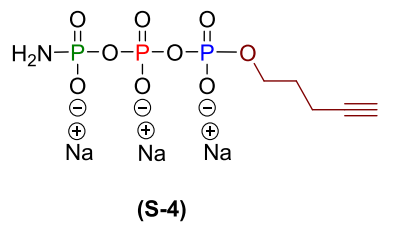

The reaction mixture of $\mathbf{B}_{6}(250 \mu \mathrm{L}, 19.7 \mu \mathrm{mol}, 1.0$ eq. $)$ was added to $25 \%$ aq. $\mathrm{NH}_{4} \mathrm{OH}(250 \mu \mathrm{L})$ and then $\mathrm{D}_{2} \mathrm{O}(100 \mu \mathrm{l})$ was added. The product $\mathbf{S}-\mathbf{4}$ was isolated by Method A, affording a colourless solid (7.55 mg, $17.9 \mu \mathrm{mol}, 91 \%$ ).

${ }^{1} \mathbf{H}$ NMR (400 MHz, D $\left.2 \mathrm{O}, \delta / p p m\right): 3.98(\mathrm{q}, \mathrm{J}=6.5 \mathrm{~Hz}, 2 \mathrm{H}), 2.26(\mathrm{t}, \mathrm{J}=7.2 \mathrm{~Hz}, 1 \mathrm{H}), 2.15$ (m, 2H), 1.79

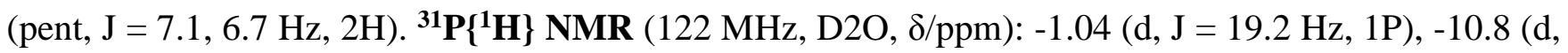
$\mathrm{J}=20.1 \mathrm{~Hz}, 1 \mathrm{P}),-22.6$ (t, J = 19.4 Hz, 1P). ${ }^{31} \mathbf{P}$ NMR (162 MHz, D $\left.2 \mathrm{O}, \delta / \mathrm{ppm}\right):-0.70$ (d, J = 23.5 Hz, 1P), $-10.3(\mathrm{dt}, \mathrm{J}=9.2 \mathrm{~Hz}, 1 \mathrm{P}),-18.9--22.1$ (m, 1P). ${ }^{13} \mathrm{C}$ NMR (101 MHz, $\left.\mathrm{D}_{2} \mathrm{O}, \delta / \mathrm{ppm}\right):$ 85.2, 69.4, 65.3 (d, $\mathrm{J}=5.9 \mathrm{~Hz}), 28.8(\mathrm{~d}, \mathrm{~J}=7.2 \mathrm{~Hz}), 14.2$.

HRMS (ESI) $\mathrm{m} / \mathrm{z}$ for $\left[\mathrm{C}_{5} \mathrm{H}_{11} \mathrm{NO}_{9} \mathrm{P}_{3}\right]^{-}$: calcd. 321.9652, found 321.9652. 


\section{Ring opening by using aq. sodium hydroxide: 4-Pentyne-1-triphosphate (S-5)}

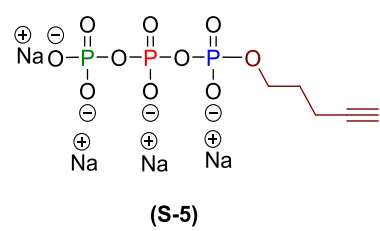

The reaction mixture of $\mathbf{B}_{6}(250 \mu \mathrm{L}, 19.7 \mu$ mol, 1.0 eq. $)$ was added to aq. $\mathrm{NaOH}(1 \mathrm{M}, 250 \mu \mathrm{L})$ and then $\mathrm{D}_{2} \mathrm{O}(100 \mu \mathrm{l})$ was added. The crude product was purified by Method A, affording a colourless solid S-5 (7.80 mg, $18.9 \mu \mathrm{mol}, 95 \%)$.

${ }^{1} \mathbf{H}$ NMR (400 MHz, D $\left.2 \mathrm{O}, \delta / \mathrm{ppm}\right): 3.99$ (dd, J = 7.2, $\left.6.3 \mathrm{~Hz}, 2 \mathrm{H}\right), 2.29$ (t, J = 7.2, 1H), 2.19 (m, 2H), 1.82 $(\mathrm{p}, \mathrm{J}=6.7 \mathrm{~Hz}, 2 \mathrm{H}) .{ }^{31} \mathbf{P}\left\{{ }^{1} \mathbf{H}\right\}$ NMR $(122 \mathrm{MHz}, \mathrm{D} 2 \mathrm{O}, \delta / \mathrm{ppm}):-4.33$ (d, J = 18.0 Hz, 1P), -9.74 (d, J = 17.8 $\mathrm{Hz}, 1 \mathrm{P}),-19.7$ (t, J = 18.0 Hz, 1P). ${ }^{31} \mathbf{P}$ NMR (162 MHz, $\left.\mathrm{D}_{2} \mathrm{O}, \delta / \mathrm{ppm}\right):-4.35$ (d, J = 18.0 Hz, 1P), -9.75 $(\mathrm{dt}, \mathrm{J}=17.6,6.9 \mathrm{~Hz}, 1 \mathrm{P}),-19.7$ (t, J = 17.9 Hz, 1P). ${ }^{13} \mathrm{C}$ NMR (101 MHz, $\left.\mathrm{D}_{2} \mathrm{O}, \delta / \mathrm{ppm}\right): 85.1,69.3,65.3$ $(\mathrm{d}, \mathrm{J}=5.9 \mathrm{~Hz}), 28.8(\mathrm{~d}, \mathrm{~J}=7.1 \mathrm{~Hz}), 14.2$.

HRMS (ESI) $\mathrm{m} / \mathrm{z}$ for $\left[\mathrm{C}_{5} \mathrm{H}_{10} \mathrm{O}_{10} \mathrm{P}_{3}\right]^{-}$: calcd. 322.9492, found 322.9468 .

\section{Ring opening by using propargylamine: 2'-deoxythymidine 5'- $\gamma$-P-triphosphate (18)}

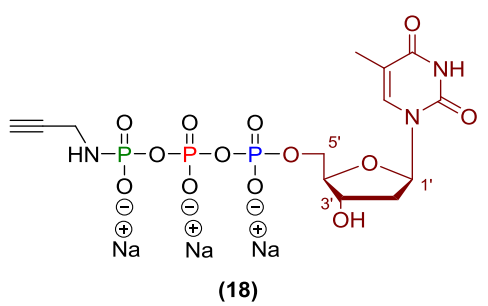

Thymidine (470 mg, $1.95 \mathrm{mmol}, 1.3 \mathrm{eq}$.$) and DCI (700 mg, 6.00 \mathrm{mmol}, 4.0$ eq.) were coevaporated together with dry MeCN $(2 \times 5 \mathrm{~mL})$. Under an Ar atmosphere, both solids were dissolved in dry DMF $(5 \mathrm{~mL})$ and a reaction mixture containing the $c$-PyPA A1 (0.075M in MeCN, $20.0 \mathrm{ml}, 1.50 \mathrm{mmol}, 1.0$ eq.) was added. The mixture was stirred for 10 mins at r.t. and the reaction was followed by ${ }^{31} \mathrm{P}-\mathrm{NMR}$ (of an aliquot of the reaction mixture in dry $\mathrm{CDCl}_{3}$ ) which shows characteristic peak triplet at $-100 \mathrm{ppm}$ confirms complete coupling. Upon cooling to $-4^{\circ} \mathrm{C}, \mathrm{mCPBA}$ (460 mg, $2.70 \mathrm{mmol}, 1.8 \mathrm{eq}$.) was added in portions. The mixture was stirred mixture was stirred for 5 mins until ${ }^{31} \mathrm{P}-\mathrm{NMR}$ confirmed complete oxidation (a triplet at - $22 \mathrm{ppm}$ revealed formation of cyclotriphosphate). Then propargylamine $(1.52 \mathrm{ml}, 24.0 \mathrm{mmol}$, 16.0 eq.) was added to the reaction mixture at $-4^{\circ} \mathrm{C}$ and the mixture was stirred another for 15 mins at r.t. The product was further purified by Method $\mathrm{C}$ and obtained as a colourless solid, details of the MPLC purification can be found in Supporting figure S3. (18, $520 \mathrm{mg}, 0.89 \mathrm{mmol}, 64 \%)$.

Fraction 1: 2'-deoxythymidine 5'- $\gamma$ - $P$-propargylamino triphosphate

${ }^{1}$ H NMR (400 MHz, D $\left.2 \mathrm{O}, \delta / \mathrm{ppm}\right): 7.69(\mathrm{~s}, 1 \mathrm{H}), 6.28(\mathrm{t}, \mathrm{J}=6.9 \mathrm{~Hz}, 1 \mathrm{H}), 4.58(\mathrm{dt}, \mathrm{J}=6.3,3.4 \mathrm{~Hz}, 1 \mathrm{H})$, $4.13(\mathrm{q}, \mathrm{J}=4.8,4.4 \mathrm{~Hz}, 3 \mathrm{H}), 3.62(\mathrm{dd}, \mathrm{J}=9.9,2.5 \mathrm{~Hz}, 2 \mathrm{H}), 2.49(\mathrm{dt}, \mathrm{J}=3.5,1.8 \mathrm{~Hz}, 1 \mathrm{H}), 2.37-2.23(\mathrm{~m}$, 2H), 1.86 (s, 3H). ${ }^{31} \mathbf{P}\left\{{ }^{1} \mathbf{H}\right\}$ NMR $\left(162 \mathrm{MHz}, \mathrm{D}_{2} \mathrm{O}, \delta / \mathrm{ppm}\right):-2.75(\mathrm{~d}, \mathrm{~J}=20.7 \mathrm{~Hz}, 1 \mathrm{P}),-11.5$ (d, J = 19.4 
$\mathrm{Hz}, 1 \mathrm{P}),-22.8(\mathrm{t}, \mathrm{J}=20.1 \mathrm{~Hz}, 1 \mathrm{P}) .{ }^{31} \mathbf{P}$ NMR $\left(162 \mathrm{MHz}, \mathrm{D}_{2} \mathrm{O}, \delta / \mathrm{ppm}\right):-2.75$ (dt, J = 20.2, $\left.9.8 \mathrm{~Hz}, 1 \mathrm{P}\right)$, - 11.5 (dt, J = 19.4, $5.2 \mathrm{~Hz}, 1 \mathrm{P}),-22.8$ (t, J = $19.9 \mathrm{~Hz}, 1 \mathrm{P}) .{ }^{13} \mathrm{C}$ NMR (126 MHz, D $\left.2 \mathrm{O}, \delta / \mathrm{ppm}\right): 166.6$, 151.7, 137.9, 111.8, $85.4(\mathrm{~d}, \mathrm{~J}=9.2 \mathrm{~Hz}), 84.9,82.8(\mathrm{~d}, \mathrm{~J}=11.5 \mathrm{~Hz}), 71.3,70.8,65.3(\mathrm{~d}, \mathrm{~J}=5.7 \mathrm{~Hz}), 46.6$, $23.0(\mathrm{~d}, \mathrm{~J}=1.3 \mathrm{~Hz}), 11.6$.

HRMS (ESI) m/z for $\left[\mathrm{C}_{13} \mathrm{H}_{19} \mathrm{~N}_{3} \mathrm{O}_{13} \mathrm{P}_{3}\right]^{-}$: calcd. 518.0136, found 518.0139.

Fraction 2: 2'-deoxythymidine 3'- $\gamma$ - $P$-propargylamino triphosphate

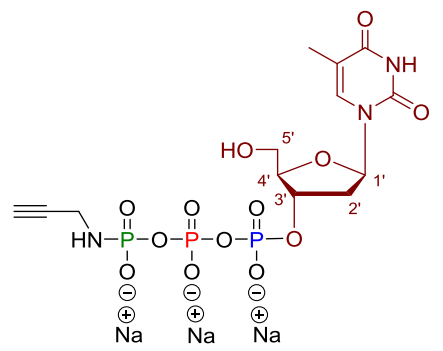

${ }^{1} \mathbf{H}$ NMR (400 MHz, D $\left.2 \mathrm{O}, \delta / \mathrm{ppm}\right): 7.61(\mathrm{~s}, 1 \mathrm{H}), 6.27(\mathrm{t}, \mathrm{J}=7.0,1 \mathrm{H}), 4.85(\mathrm{~m}, 1 \mathrm{H}), 4.26-4.08(\mathrm{~m}, 1 \mathrm{H})$, $3.78(\mathrm{~m}, 2 \mathrm{H}), 3.62(\mathrm{~d}, \mathrm{~J}=7.2 \mathrm{~Hz}, 2 \mathrm{H}), 2.74-2.47(\mathrm{~m}, 1 \mathrm{H}), 2.45-2.36(\mathrm{~m}, 2 \mathrm{H}), 1.67(\mathrm{~s}, 3 \mathrm{H}) .{ }^{31} \mathbf{P}\left\{{ }^{1} \mathbf{H}\right\}$ NMR (162 MHz, D $2 \mathrm{O}, \delta / \mathrm{ppm}):-2.61$ (d, J = 20.3 Hz, 1P), -12.1 (d, J = 19.0 Hz, 1P), -22.7 (t, J = 19.9 $\mathrm{Hz}, 1 \mathrm{P}) .{ }^{31} \mathbf{P}$ NMR $\left(162 \mathrm{MHz}, \mathrm{D}_{2} \mathrm{O}, \delta / \mathrm{ppm}\right):-2.61(\mathrm{dt}, \mathrm{J}=20.4,9.9 \mathrm{~Hz}, 1 \mathrm{P}),-12.1$ (dd, J = 19.0, $7.9 \mathrm{~Hz}$, 1P), -22.7 ( $\mathrm{t}, \mathrm{J}=20.0 \mathrm{~Hz}, 1 \mathrm{P})$.

Ring opening by using aq. ammonia: 2'-deoxythymidine 5'- $\gamma$-P-amino triphosphate (19)

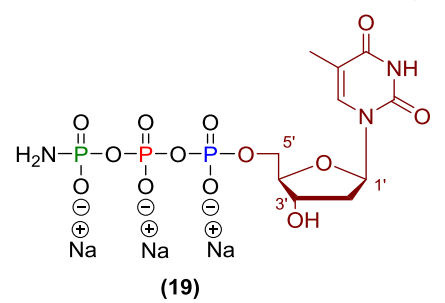

Thymidine (110 mg, $0.46 \mathrm{mmol}, 1.5$ eq.) and DCI (120 mg, $1.05 \mathrm{mmol}, 3.5 \mathrm{eq})$ were coevaporated together with dry MeCN $(2 \times 5 \mathrm{~mL})$. Under an Ar atmosphere, both solids were dissolved in dry DMF $(2 \mathrm{~mL})$ and a reaction mixture containing the $c$-PyPA A1 (0.075M in MeCN, $4.0 \mathrm{ml}, 0.30 \mathrm{mmol}, 1.0$ eq.) was added. The mixture was stirred for 5 mins at r.t. and the reaction was followed by ${ }^{31} \mathrm{P}-\mathrm{NMR}$ (of an aliquot of the reaction mixture in dry $\mathrm{CDCl}_{3}$ ) which shows characteristic peak triplet at -100 ppm confirms complete coupling. Upon cooling to $-4^{\circ} \mathrm{C}$ to $0^{\circ} \mathrm{C}, m \mathrm{CPBA}(80 \mathrm{mg}, 0.45 \mathrm{mmol}, 1.5 \mathrm{eq})$ was added in portion wise and the mixture was stirred for 5 mins until ${ }^{31} \mathrm{P}-\mathrm{NMR}$ confirmed complete oxidation (reaction progress was followed by ${ }^{31} \mathrm{P}$ NMR, and formation of triplet at $-22 \mathrm{ppm}$ which is diagnostic signal for the cyclotriphosphate). Then $25 \%$ aq. $\mathrm{NH}_{4} \mathrm{OH}(0.5 \mathrm{ml})$ was added to the reaction mixture at $-4^{\circ} \mathrm{C}$ and the mixture was stirred another for 15 mins at r.t. 
The product was further purified by Method $\mathrm{C}$ and obtained as a colourless solid. (19, $98 \mathrm{mg}, 0.18 \mathrm{mmol}$, $59 \%)$.

Fraction 1: 2'-deoxythymidine 5'- $\gamma$ - $P$-amino triphosphate

${ }^{1} \mathbf{H}$ NMR (400 MHz, D $\left.2 \mathrm{O}, \delta / \mathrm{ppm}\right): 7.67(\mathrm{~s}, 1 \mathrm{H}), 6.28(\mathrm{t}, \mathrm{J}=7.0 \mathrm{~Hz}, 1 \mathrm{H}), 4.62-4.51(\mathrm{~m}, 1 \mathrm{H}), 4.27-3.94$ (m, 3H), $2.62-2.19(\mathrm{~m}, 2 \mathrm{H}), 1.86(\mathrm{~s}, 3 \mathrm{H}) .{ }^{31} \mathbf{P}\left\{{ }^{1} \mathbf{H}\right\}$ NMR $\left(162 \mathrm{MHz}, \mathrm{D}_{2} \mathrm{O}, \delta / \mathrm{ppm}\right):-0.97$ (d, J = $19.0 \mathrm{~Hz}$,

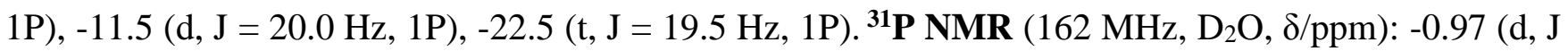
$=19.0 \mathrm{~Hz}, 1 \mathrm{P}),-11.5(\mathrm{dt}, \mathrm{J}=19.4,5.2 \mathrm{~Hz}, 1 \mathrm{P}),-22.5$ (t, J = $19.5 \mathrm{~Hz}, 1 \mathrm{P}) .{ }^{13} \mathbf{C} \mathbf{N M R}\left(101 \mathrm{MHz}, \mathrm{D}_{2} \mathrm{O}\right.$,

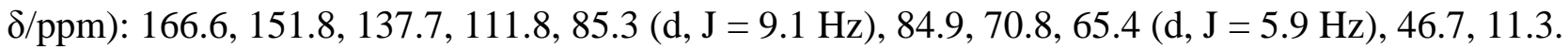

HRMS (ESI) $\mathrm{m} / \mathrm{z}$ for $\left[\mathrm{C}_{10} \mathrm{H}_{17} \mathrm{~N}_{3} \mathrm{Na}_{2} \mathrm{O}_{13} \mathrm{P}_{3}\right]^{+}$: calcd. 525.9764, found 525.9765.

Fraction 2: 2'-deoxythymidine 3'- $\gamma$ - $P$-amino triphosphate ${ }^{31} \mathbf{P}\left\{{ }^{1} \mathbf{H}\right\}$ NMR $\left(162 \mathrm{MHz}, \mathrm{D}_{2} \mathrm{O}, \delta / \mathrm{ppm}\right):-0.87$ (d, J = 19.1 Hz, 1P), -12.0 (d, J = 19.3 Hz, 1P), -22.4 (t, J $=19.3 \mathrm{~Hz}, 1 \mathrm{P}) .{ }^{31} \mathbf{P}$ NMR $\left(162 \mathrm{MHz}, \mathrm{D}_{2} \mathrm{O}, \delta / \mathrm{ppm}\right):-0.88$ (d, J = 19.0 Hz, 1P), -12.0 (dd, J = 19.5, 8.0 Hz, 1P), $-22.4(\mathrm{t}, \mathrm{J}=19.2 \mathrm{~Hz}, 1 \mathrm{P})$.

\section{Adenosine-5' -cyclotriphosphate $\left(B_{7}\right)$}

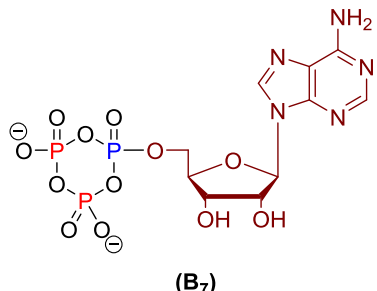

Adenosine (120 mg, $0.45 \mathrm{mmol}, 1.5$ eq.) and DCI (94 mg, $0.80 \mathrm{mmol}, 2.7$ eq.) were coevaporated together with dry $\mathrm{MeCN}(2 \times 5 \mathrm{~mL})$. Under an atmosphere of dry Ar, both solids were dissolved in dry DMF $(5.5 \mathrm{ml})$. A reaction mixture containing the $c$-PyPA A 1 (0.5M in DMF, $0.60 \mathrm{ml}, 0.30 \mathrm{mmol}, 1.0$ eq.) was added to the above flask. The mixture was stirred for 5 mins at r.t. and the reaction was followed by ${ }^{31} \mathrm{P}$ NMR (of an aliquot of the reaction mixture in dry $\mathrm{CDCl}_{3}$ ) which shows characteristic peak triplet at $100 \mathrm{ppm}$ confirms complete coupling. Upon cooling to $-4^{\circ} \mathrm{C}, \mathrm{mCPBA}(100 \mathrm{mg}, 0.60 \mathrm{mmol}, 2.0 \mathrm{eq}$.) was added in the mixture and stirred for 5 mins until ${ }^{31} \mathrm{P}-\mathrm{NMR}$ confirmed complete oxidation (reaction progress was followed by ${ }^{31} \mathrm{P} \mathrm{NMR}$, and formation of triplet at $-22 \mathrm{ppm}$ which is diagnostic signal for the cyclotriphosphate $\mathbf{B}_{7}$ ).

${ }^{31} \mathbf{P}\left\{{ }^{1} \mathbf{H}\right\}$ NMR $\left(122 \mathrm{MHz}, \mathrm{CDCl}_{3}, \delta / \mathrm{ppm}\right):-23.0(\mathrm{t}, \mathrm{J}=24.5 \mathrm{~Hz}, 1 \mathrm{P}),-24.0--25.9(\mathrm{~m}, 2 \mathrm{P})$. 
Ring opening by using aq. ammonia: Adenosine 5'- $\gamma$-P -amino triphosphate (20)

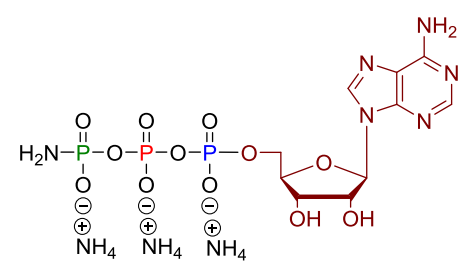

(20)

The reaction mixture of $\mathbf{B}_{7}\left(500 \mu \mathrm{l}, 25.0 \mu \mathrm{mol}, 1.0\right.$ eq.) was added to $25 \%$ aq. $\mathrm{NH}_{4} \mathrm{OH}(100 \mu \mathrm{l})$ and $\mathrm{D}_{2} \mathrm{O}$ $(100 \mu \mathrm{l})$ was added. The crude product was purified by Method B and freeze drying of the fractions $(2 \times)$ eluted at $6.4-9.2 \%$ of aq. $1 \mathrm{M} \mathrm{NH}_{4} \mathrm{HCO}_{3}$ buffer afforded the title compound $(\mathbf{2 0}, 6.50 \mathrm{mg}, 12.0 \mu \mathrm{mol}$, $47 \%$ ) as colourless solid.

Analytical data are consistent with those reported in the literature (reported in DMSO-d 6 ). ${ }^{8}$

1H-NMR: (300 MHz, D $2 \mathrm{O}, \delta / \mathrm{ppm}): 8.54$ (s, 1H), 8.29 (s, 1H), 6.17 (d, J = 6.0 Hz, 1H), 4.60 (dd, J = 5.2, $3.6 \mathrm{~Hz}, 1 \mathrm{H}), 4.48-4.40(\mathrm{~m}, 1 \mathrm{H}), 4.35-4.22(\mathrm{~m}, 2 \mathrm{H})$. The DHO peak overlaps with the signal $4.88(1 \mathrm{H})$ ${ }^{31} \mathbf{P}\left\{{ }^{1} \mathbf{H}\right\}$-NMR: $\left(122 \mathrm{MHz}, \mathrm{D}_{2} \mathrm{O}, \delta / \mathrm{ppm}\right):-0.99(\mathrm{~d}, \mathrm{~J}=19.2 \mathrm{~Hz}, 1 \mathrm{P}),-11.4(\mathrm{~d}, \mathrm{~J}=20.3 \mathrm{~Hz}, 1 \mathrm{P}),-$ $22.6(\mathrm{t}, \mathrm{J}=19.5 \mathrm{~Hz}, 1 \mathrm{P})$.

HRMS (ESI) $\mathrm{m} / \mathrm{z}$ for $\left[\mathrm{C}_{10} \mathrm{H}_{16} \mathrm{~N}_{6} \mathrm{O}_{12} \mathrm{P}_{3}\right]^{-}$: calcd. 505.0045, found 505.0046.

Ring opening by using propargylamine: Adenosine 5'- $\gamma$-P-propargylamino triphosphate (21)

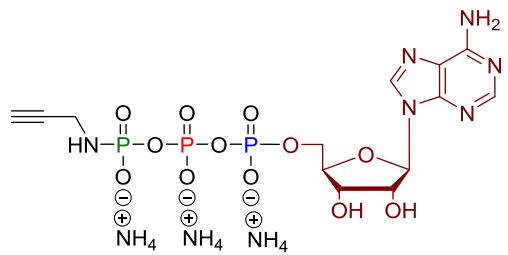

(21)

The reaction mixture of $\mathbf{B}_{7}(400 \mu 1,20.0 \mu$ mol, 1.0 eq.) was added to propargylamine (100 $\mu 1)$ and $\mathrm{D}_{2} \mathrm{O}$ $(100 \mu \mathrm{l})$ was added. The crude product was purified by Method B and freeze drying of the fractions $(2 \times)$ eluted at $8.7-13 \%$ of aq. $1 \mathrm{M} \mathrm{NH}_{4} \mathrm{HCO}_{3}$ buffer afforded the title compound (21, $\left.4.30 \mathrm{mg}, 7.20 \mu \mathrm{mol}, 37 \%\right)$ as colourless solid.

Analytical data are consistent with those reported in the literature. ${ }^{9}$

${ }^{1}$ H-NMR: (300 MHz, D $\left.2 \mathrm{O}, \delta / \mathrm{ppm}\right): 8.56$ (s, 1H), 8.29 (s, 1H), 6.17 (d, J = 5.8 Hz, 1H), 4.63 - 4.56 (m, $1 \mathrm{H}), 4.46-4.40(\mathrm{~m}, 1 \mathrm{H}), 4.35-4.23(\mathrm{~m}, 2 \mathrm{H}), 3.67(\mathrm{~d}, \mathrm{~J}=9.9 \mathrm{~Hz}, 2 \mathrm{H}), 2.50\left(\mathrm{~m}_{\mathrm{c}}, 1 \mathrm{H}\right)$. The DHO peak overlaps with the signal $4.88(1 \mathrm{H}) .{ }^{31} \mathbf{P}\left\{{ }^{1} \mathbf{H}\right\}$-NMR: $\left(122 \mathrm{MHz}, \mathrm{D}_{2} \mathrm{O}, \delta / \mathrm{ppm}\right):-2.72(\mathrm{~d}, \mathrm{~J}=20.7 \mathrm{~Hz}, 1 \mathrm{P})$, -11.4 (d, J = 19.5 Hz, 1P), -22.8 (t, J = 19.5 Hz, 1P).

HRMS (ESI) $\mathrm{m} / \mathrm{z}$ for $\left[\mathrm{C}_{13} \mathrm{H}_{18} \mathrm{~N}_{6} \mathrm{O}_{12} \mathrm{P}_{3}\right]^{-}$: calcd. 543.0201, found 543.0202. 


\section{Ring opening by using imidazole: 4-Pentyne $\gamma-P$-imidazole triphosphate (S-6)}

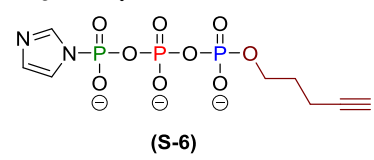

4-Pentyn-1-ol (51 mg, $0.60 \mathrm{mmol}, 2.0$ eq.) and ETT (156 mg, $1.20 \mathrm{mmol}, 4.0$ eq.) were coevaporated with dry $\mathrm{MeCN}(2 \times 1 \mathrm{~mL})$. Under an atmosphere of dry Ar, a freshly prepared reaction mixture containing the $c$-PyPA A 1 (0.075M, $4.0 \mathrm{~mL}, 0.30 \mathrm{mmol}, 1.0$ eq.) in $\mathrm{MeCN}$ was added in the above reaction mixture. The mixture was stirred at r.t. for 10 mins. Upon cooling to $0{ }^{\circ} \mathrm{C}, \mathrm{mCPBA}(\leq 77 \%, 80 \mathrm{mg}$, $0.47 \mathrm{mmol}, 1.5$ eq.) was added and the mixture was stirred for $5 \mathrm{mins}$ until ${ }^{31} \mathrm{P}-\mathrm{NMR}$ confirmed complete oxidation (reaction progress was followed by ${ }^{31} \mathrm{P} \mathrm{NMR}$, and formation of triplet at $-23 \mathrm{ppm}$ which is diagnostic signal for the cyclotriphosphate $\mathbf{B}$ ).

A solution of imidazole in dry DMF $(1.47 \mathrm{M}, 9.00 \mathrm{ml}, 13.2 \mathrm{mmol}, 45 \mathrm{eq}$.$) was added to the$ cyclotriphosphate $\left(\mathbf{B}_{6}\right)$ and reaction was stirred for another 20 mins (the reaction progress was followed by ${ }^{31} \mathrm{P}$ NMR) which results in the quantative conversion to $\mathbf{S - 6}$.

Note -: Imidazole was dried before using by coevaporation with $\mathrm{MeCN}$ and it was further stored of molecular sieve $(3 \AA$ ).

${ }^{31} \mathbf{P}\left\{{ }^{1} \mathbf{H}\right\}$ NMR (122 MHz, D $\left.2 \mathrm{O}, \delta / \mathrm{ppm}\right):-11.1(\mathrm{~d}, \mathrm{~J}=19.9 \mathrm{~Hz}, 1 \mathrm{P}),-20.2(\mathrm{~d}, \mathrm{~J}=19.3 \mathrm{~Hz}, 1 \mathrm{P}),-23.8$ (t, J $=19.6 \mathrm{~Hz}, 1 \mathrm{P}) .{ }^{31} \mathbf{P}$ NMR $\left(122 \mathrm{MHz}, \mathrm{D}_{2} \mathrm{O}, \delta / \mathrm{ppm}\right):-11.1(\mathrm{dt}, \mathrm{J}=19.9,5.5 \mathrm{~Hz}, 1 \mathrm{P}),-20.2(\mathrm{~d}, \mathrm{~J}=19.3 \mathrm{~Hz}$, 1P), $-23.9(\mathrm{t}, \mathrm{J}=19.6 \mathrm{~Hz}, 1 \mathrm{P})$.

\section{5'-Adenosyl 4-Pentyne hexaphosphate (P2)}

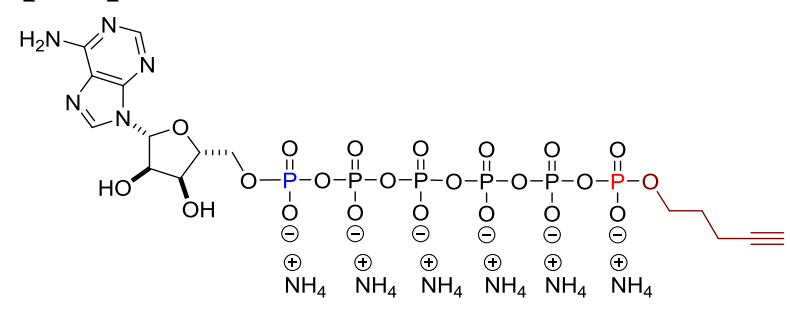

(P2)

Anhydr. $\mathrm{ZnCl}_{2}(389 \mathrm{mg}, 2.84 \mathrm{mmol}, 9.5$ eq.) and ATP x 2.1 TBA (390 mg, 0.28 mmol, 1.3 eq.) were added to a freshly prepared solution of $\mathbf{S - 6}(0.30 \mathrm{mmol})$. The reaction mixture was then diluted with dry DMF: DMSO (v:v, 1:1, $16 \mathrm{ml}$ ) in order to increase the solubility and it was stirred at r.t. for $48 \mathrm{hrs}$. The crude product was purified by Method B and target product was eluted with 400-600 mM conc. of $\mathrm{NH}_{4} \mathrm{HCO}_{3}$ buffer. Purification gave $\mathbf{P 2}$ as a colorless solid (0.03 g, $\left.0.03 \mathrm{mmol}, 13 \%\right)$.

Note-: The reactions for capped hexapolyPs were not optimized and therefore the yield could still be improved.

${ }^{1}$ H NMR (300 MHz, D $\left.2 \mathrm{O}, \delta / p p m\right): 8.29$ (s, 1H), 8.05 (s, 1H), 5.93 (d, J = 5.8 Hz, 1H), 4.56 (s, 2H), 4.43 $-4.28(\mathrm{~m}, 1 \mathrm{H}), 4.19(\mathrm{~s}, 1 \mathrm{H}), 4.04(\mathrm{~s}, 1 \mathrm{H}), 3.84(\mathrm{q}, \mathrm{J}=6.4 \mathrm{~Hz}, 2 \mathrm{H}), 2.25-2.08(\mathrm{~m}, 3 \mathrm{H}), 1.64(\mathrm{p}, \mathrm{J}=6.7$, 
2H). ${ }^{31} \mathbf{P}\left\{{ }^{1} \mathbf{H}\right\}$ NMR (122 MHz, D $\left.2 \mathrm{O}, \delta / \mathrm{ppm}\right):-10.6--11.2(\mathrm{~m}, 1 \mathrm{P}),-11.2--11.6(\mathrm{~m}, 1 \mathrm{P}),-21.7--23.4$ (m, 4P). ${ }^{31} \mathbf{P}$ NMR (162 MHz, $\left.\mathrm{D}_{2} \mathrm{O}, \delta / \mathrm{ppm}\right):-10.2--11.1$ (m, 1P), -11.1 - -11.7 (m, 1P), -20.8 - -23.9

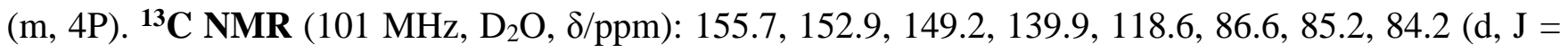
$9.2 \mathrm{~Hz}), 74.2,70.4,69.5,65.4(\mathrm{~d}, \mathrm{~J}=5.6 \mathrm{~Hz}), 65.04(\mathrm{~d}, \mathrm{~J}=5.9 \mathrm{~Hz}), 28.8(\mathrm{~d}, \mathrm{~J}=7.6 \mathrm{~Hz}), 14.2(\mathrm{~d}, \mathrm{~J}=3.9$ $\mathrm{Hz})$.

HRMS (ESI) m/z for $\left[\mathrm{C}_{15} \mathrm{H}_{25}{ }^{2} \mathrm{HN}_{5} \mathrm{O}_{22} \mathrm{P}_{6}\right]^{+}$: calcd. 814.9552, found 814.9548.

\subsubsection{Synthesis of triphosphates based on $c$-Pyсн2 $\mathrm{PA}\left(\mathrm{A}_{2}\right)$}

3'-Azido-3'-deoxythymidine 5'- $\beta, \gamma$-methylene cyclotriphosphate (B8)

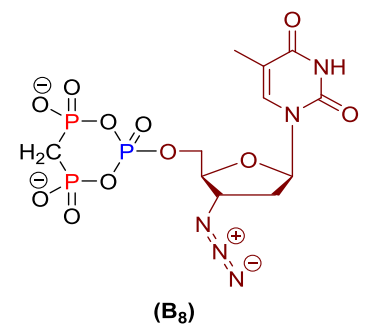

AZT (50 mg, $0.18 \mathrm{mmol}, 1.2$ eq.) and ETT ( $81 \mathrm{mg}, 0.62 \mathrm{mmol}, 4.0$ eq.) were coevaporated with dry $\mathrm{MeCN}(2 \times 1 \mathrm{~mL})$. Under an Ar atmosphere, a reaction mixture containing the $c$-Pусн $2 \mathrm{PA} \mathbf{A}_{2}(0.078 \mathrm{M}$ in $\mathrm{MeCN}, 2.0 \mathrm{~mL}, 0.15 \mathrm{mmol}, 1.0$ eq.) was added to the dried solids and it was stirred at r.t. for 10 mins. Upon cooling to $0^{\circ} \mathrm{C}, \mathrm{mCPBA}(\leq 77 \%, 40 \mathrm{mg}, 0.23 \mathrm{mmol}, 1.5$ eq.) was added and the mixture was stirred for 5 mins until ${ }^{31} \mathrm{P}-\mathrm{NMR}$ confirmed complete oxidation (a formation of triplet at $-24 \mathrm{ppm}$ which is diagnostic signal for $\mathbf{B} 8$ ).

${ }^{31} \mathbf{P}\left\{{ }^{1} \mathbf{H}\right\}$ NMR $\left(122 \mathrm{MHz}, \mathrm{CDCl}_{3}, \delta / \mathrm{ppm}\right): 5.06(\mathrm{~d}, \mathrm{~J}=18.5 \mathrm{~Hz}, 2 \mathrm{P}),-24.1(\mathrm{t}, \mathrm{J}=18.6 \mathrm{~Hz}, 1 \mathrm{P}) .{ }^{31} \mathbf{P}$ NMR (162 MHz, $\left.\mathrm{CDCl}_{3}, \delta / \mathrm{ppm}\right): 5.06$ (q, J = 19.4 Hz, 2P), -23.2 - -24.6 (m, 1P).

Ring opening by using propargylamine: 3'-Azido-3'-deoxythymidine 5'- $\gamma$-P-propargylamino $\beta, \gamma$ methylenetriphosphate (22)

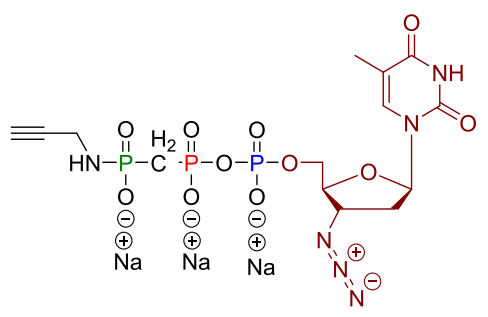

(22)

The reaction mixture of $\mathbf{B}_{8}\left(250 \mu \mathrm{L}, 19.5 \mu \mathrm{mol}, 1.0\right.$ eq) was added to propargylamine $(250 \mu \mathrm{L})$ and $\mathrm{D}_{2} \mathrm{O}$ $(100 \mu \mathrm{l})$ was added. The crude product was purified by Method A, affording a pale yellow solid 22 (9.10 mg, $15.0 \mu \mathrm{mol}, 79 \%)$. 


\section{Reaction on large scale (22)}

AZT (220 mg, $0.820 \mathrm{mmol}, 1.0$ eq.) and ETT (325 mg, $2.49 \mathrm{mmol}, 3.2$ eq.) were coevaporated with dry $\mathrm{MeCN}(2 \times 4 \mathrm{~mL})$. Under Ar atmosphere, a reaction mixture containing the $c$ - $\mathrm{PyCH}_{2} \mathrm{PA} \mathbf{A}_{2}(0.078 \mathrm{M}$ in $\mathrm{MeCN}, 10 \mathrm{ml}, 0.78 \mathrm{mmol}, 1.0$ eq.) was added to the dried solids and it was stirred at r.t. for 10 mins. Upon cooling to $-4 \mathrm{C}, m \mathrm{CPBA}(\leq 77 \%, 300 \mathrm{mg}, 1.74 \mathrm{mmol}, 2.2 \mathrm{eq}$.) was added and the mixture was stirred for 5 mins until ${ }^{31} \mathrm{P}-\mathrm{NMR}$ confirmed complete oxidation (formation of triplet at $-24 \mathrm{ppm}$ which is diagnostic signal for the cyclotriphosphate B8). Then propargylamine (1.00 ml, $15.6 \mathrm{mmol}, 20 \mathrm{eq})$ was added to the reaction mixture at $-4^{\circ} \mathrm{C}$ and it was stirred at r.t. for 15 mins.

The product 22 was isolated by Method A, affording a colourless solid (411 mg, $0.67 \mathrm{mmol}, 85 \%$ ).

${ }^{1} \mathbf{H}$ NMR (400 MHz, D $\left.2 \mathrm{O}, \delta / \mathrm{ppm}\right): 7.70(\mathrm{~s}, 1 \mathrm{H}), 6.23(\mathrm{t}, \mathrm{J}=6.9 \mathrm{~Hz}, 1 \mathrm{H}), 4.52(\mathrm{q}, \mathrm{J}=4.8,1 \mathrm{H})$, 4.25 - $4.11(\mathrm{~m}, 3 \mathrm{H}), 3.61(\mathrm{dd}, \mathrm{J}=10.1,2.4 \mathrm{~Hz}, 2 \mathrm{H}), 2.54(\mathrm{t}, \mathrm{J}=2.5 \mathrm{~Hz}, 1 \mathrm{H}), 2.44(\mathrm{dd}, \mathrm{J}=6.6,4.6 \mathrm{~Hz}$, 2H), 2.30 (dd, J = 20.6, $18.8 \mathrm{~Hz}, 2 \mathrm{H}), 1.89$ (d, J = 1.1 Hz, 3H). ${ }^{31} \mathbf{P}\left\{{ }^{1} \mathbf{H}\right\} \mathbf{N M R}\left(122 \mathrm{MHz}, \mathrm{D}_{2} \mathrm{O}, \delta / \mathrm{ppm}\right)$ : $17.3(\mathrm{~d}, \mathrm{~J}=8.6 \mathrm{~Hz}, 1 \mathrm{P}), 10.2(\mathrm{dd}, \mathrm{J}=25.0,8.6 \mathrm{~Hz}, 1 \mathrm{P}),-9.95$ (d, J = 25.0 Hz, 1P). ${ }^{31} \mathbf{P}$ NMR $(162 \mathrm{MHz}$, $\left.\mathrm{D}_{2} \mathrm{O}, \delta / \mathrm{ppm}\right): 17.3(\mathrm{dq}, \mathrm{J}=18.2,9.0 \mathrm{~Hz}, 1 \mathrm{P}), 10.2(\mathrm{dtd}, \mathrm{J}=26.9,18.2,6.68 \mathrm{~Hz}, 1 \mathrm{P}),-9.96$ (dt, $\mathrm{J}=26.2,5.2 \mathrm{~Hz}, 1 \mathrm{P}) .{ }^{13} \mathbf{C} \mathbf{N M R}\left(101 \mathrm{MHz}, \mathrm{D}_{2} \mathrm{O}, \delta / \mathrm{ppm}\right): 166.8,151.9,137.3,111.8,84.9,82.9(\mathrm{~d}, \mathrm{~J}=$ $9.2 \mathrm{~Hz}), 76.1,69.9,65.4$ (d, J = 5.5 Hz), 60.9, 36.2, 31.7 - 27.6 (dd, J = 129.2, 107.1 Hz), 11.7.

HRMS (ESI) $\mathrm{m} / \mathrm{z}$ for $\left[\mathrm{C}_{14} \mathrm{H}_{20} \mathrm{~N}_{6} \mathrm{O}_{11} \mathrm{P}_{3}\right]^{-}$: calcd. 541.0408, found 541.0409.

Ring opening by using aq. ammonia: 3'-Azido-3'-deoxythymidine 5'- $\gamma$ - $P$-amino $\beta$, $\gamma$-methylene triphosphate (23)

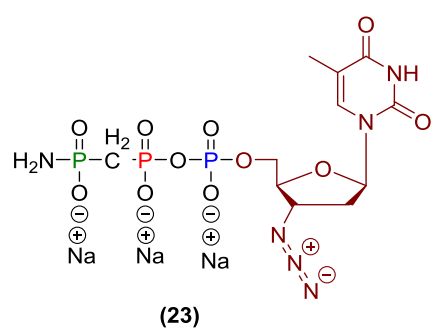

The reaction mixture of $\mathbf{B}_{8}\left(250 \mu \mathrm{L}, 19.5 \mu \mathrm{mol}, 1.0\right.$ eq.) was added to $25 \%$ aq. $\mathrm{NH}_{4} \mathrm{OH}(250 \mu \mathrm{L})$ and then addition of $\mathrm{D}_{2} \mathrm{O}(100 \mu \mathrm{l})$. The product 23 was isolated by Method A, affording a colourless solid (9.54 $\mathrm{mg}$, $16.7 \mu \mathrm{mol}, 86 \%)$.

${ }^{1} \mathbf{H}$ NMR (400 MHz, D $\left.2 \mathrm{O}, \delta / \mathrm{ppm}\right): 7.55(\mathrm{~s}, 1 \mathrm{H}), 6.14(\mathrm{t}, \mathrm{J}=6.9 \mathrm{~Hz}, 1 \mathrm{H}), 4.41(\mathrm{td}, \mathrm{J}=5.7,5.2,3.6 \mathrm{~Hz}$, $1 \mathrm{H}), 4.10-3.85(\mathrm{~m}, 3 \mathrm{H}), 2.38-2.30(\mathrm{~m}, 2 \mathrm{H}), 2.19(\mathrm{dd}, \mathrm{J}=20.6,19.1 \mathrm{~Hz}, 2 \mathrm{H}), 1.77(\mathrm{~s}, 3 \mathrm{H}) .{ }^{31} \mathbf{P}\left\{{ }^{1} \mathbf{H}\right\}$ NMR (122 MHz, D $2 \mathrm{O}, \delta / \mathrm{ppm}): 18.2(\mathrm{~d}, \mathrm{~J}=6.7 \mathrm{~Hz}, 1 \mathrm{P}), 9.70(\mathrm{dd}, \mathrm{J}=25.5,6.7 \mathrm{~Hz}, 1 \mathrm{P}),-10.6$ (d, J = 25.5 $\mathrm{Hz}, 1 \mathrm{P}) .{ }^{31} \mathbf{P}$ NMR $\left(162 \mathrm{MHz}, \mathrm{D}_{2} \mathrm{O}, \delta / \mathrm{ppm}\right): 18.2$ (td, J = 18.9, 6.7 Hz, 1P), 9.70 (dtd, J = 26.9, 20.4, 6.7 $\mathrm{Hz}, 1 \mathrm{P}),-9.52--11.4$ (m, 1P). ${ }^{13} \mathrm{C}$ NMR (101 MHz, D $\left.2 \mathrm{O}, \delta / \mathrm{ppm}\right): 169.3,153.8,136.9,111.9,84.9,82.8$ $(\mathrm{d}, \mathrm{J}=9.3 \mathrm{~Hz}), 65.4$ (d, J = 5.5 Hz), 60.9, 36.2, 31.9 (dd, J = 129.2, 107.1 Hz), 11.9.

HRMS (ESI) m/z for $\left[\mathrm{C}_{11} \mathrm{H}_{18} \mathrm{~N}_{6} \mathrm{O}_{11} \mathrm{P}_{3}\right]^{-}$: calcd. 503.0252, found 503.0253. 
Ring opening by using diethylamine: $3^{\prime}$-Azido-3'-deoxythymidine $5^{\prime}$ - $\gamma$-P-diethylamino $\beta, \gamma$ methylene triphosphate (24)

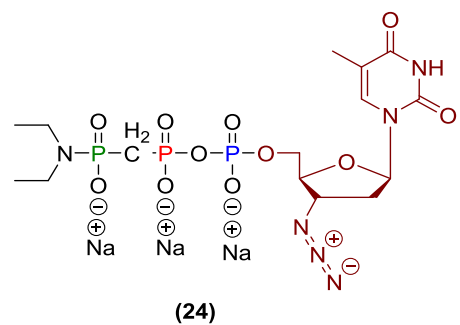

The reaction mixture of $\mathbf{B} 8(250 \mu \mathrm{L}, 19.5 \mu \mathrm{mol}, 1.0$ eq.) was added to diethylamine $(250 \mu \mathrm{L})$ and then (100 $\mu \mathrm{l})$ was added. The product $\mathbf{2 4}$ was isolated by Method A, affording a colourless solid (7.10 $\mathrm{mg}$, $11.0 \mu \mathrm{mol}, 59 \%)$.

${ }^{1}$ H NMR (400 MHz, D $\left.2 \mathrm{O}, \delta / \mathrm{ppm}\right): 7.63(\mathrm{~s}, 1 \mathrm{H}), 6.24(\mathrm{t}, \mathrm{J}=6.9 \mathrm{~Hz}, 1 \mathrm{H}), 4.66-4.40(\mathrm{~m}, 1 \mathrm{H}), 4.24-4.03$ $(\mathrm{m}, 3 \mathrm{H}), 2.90(\mathrm{dq}, \mathrm{J}=9.9,7.1 \mathrm{~Hz}, 4 \mathrm{H}), 2.52-2.32(\mathrm{~m}, 2 \mathrm{H}), 2.16(\mathrm{dd}, \mathrm{J}=20.6,19.1 \mathrm{~Hz}, 2 \mathrm{H}), 1.85(\mathrm{~d}, \mathrm{~J}=$ $1.2 \mathrm{~Hz}, 3 \mathrm{H}), 0.98(\mathrm{t}, \mathrm{J}=7.1 \mathrm{~Hz}, 6 \mathrm{H}) .{ }^{31} \mathbf{P}\left\{{ }^{1} \mathbf{H}\right\} \mathbf{N M R}\left(122 \mathrm{MHz}, \mathrm{D}_{2} \mathrm{O}, \delta / \mathrm{ppm}\right): 16.7$ (d, J = 9.9 Hz, 1P), $10.2(\mathrm{dd}, \mathrm{J}=26.1,10.0 \mathrm{~Hz}, 1 \mathrm{P}),-10.6$ (d, J = 26.2 Hz, 1P). ${ }^{31} \mathbf{P}$ NMR $\left(162 \mathrm{MHz}, \mathrm{D}_{2} \mathrm{O}, \delta / \mathrm{ppm}\right): 17.3-$ $16.3(\mathrm{~m}, 1 \mathrm{P}), 10.1(\mathrm{dtd}, \mathrm{J}=36.0,20.9,10.4 \mathrm{~Hz}, 1 \mathrm{P}),-10.6(\mathrm{dt}, \mathrm{J}=26.2,5.2 \mathrm{~Hz}, 1 \mathrm{P}) .{ }^{13} \mathrm{C}$ NMR $(101 \mathrm{MHz}$, $\left.\mathrm{D}_{2} \mathrm{O}, \delta / \mathrm{ppm}\right): 166.5,152.9,137.8,111.9,84.7,83.0(\mathrm{~d}, \mathrm{~J}=9.5 \mathrm{~Hz}), 65.6(\mathrm{~d}, \mathrm{~J}=5.8 \mathrm{~Hz}), 61.0,40.5(\mathrm{~d}, \mathrm{~J}$ = 3.7 Hz), 36.2, $30.9(\mathrm{dd}=129.4,107.1 \mathrm{~Hz}), 13.8(\mathrm{~d}, \mathrm{~J}=3.7 \mathrm{~Hz}), 11.7$.

HRMS (ESI) m/z for $\left[\mathrm{C}_{15} \mathrm{H}_{25} \mathrm{~N}_{6} \mathrm{O}_{11} \mathrm{P}_{3}\right]^{2-}$ : calcd. 279.0403, found 279.0400.

Ring opening by using aq. sodium hydroxide: 3'-Azido-3'-deoxythymidine 5'- $\beta, \gamma$ methylenetriphosphate (25)

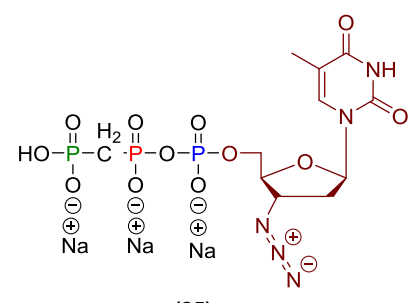

(25)

The reaction mixture of $\mathbf{B} 8(250 \mu \mathrm{L}, 19.7 \mu \mathrm{mol}, 1.0$ eq. $)$ was added to $1 \mathrm{M}$ aq. $\mathrm{NaOH}(250 \mu \mathrm{L})$ and then $\mathrm{D}_{2} \mathrm{O}(100 \mu \mathrm{l})$ was added. The crude product was purified by Method A, affording a colourless solid 25 (7.25 mg, $12.0 \mu \mathrm{mol}, 66 \%)$. The analytical data are consistent with literature. ${ }^{10}$

${ }^{1} \mathrm{H}$ NMR (400 MHz, D $\left.2 \mathrm{O}, \delta / \mathrm{ppm}\right): 7.58(\mathrm{~s}, 1 \mathrm{H}), 6.24(\mathrm{t}, \mathrm{J}=7.0 \mathrm{~Hz}, 1 \mathrm{H}), 4.48(\mathrm{td}, \mathrm{J}=5.2,3.5 \mathrm{~Hz}, 1 \mathrm{H})$, $4.26-4.01(\mathrm{~m}, 3 \mathrm{H}), 2.48-2.28(\mathrm{~m}, 2 \mathrm{H}), 2.12-1.99(\mathrm{~m}, 2 \mathrm{H}), 1.83(\mathrm{~s}, 3 \mathrm{H}) .{ }^{31} \mathbf{P}\left\{{ }^{1} \mathbf{H}\right\} \mathbf{N M R}(122 \mathrm{MHz}$, D2O, $\delta / \mathrm{ppm}): 13.5$ (dd, J = 27.0, 7.0 Hz, 1P), $11.6(\mathrm{~d}, \mathrm{~J}=6.9 \mathrm{~Hz}, 1 \mathrm{P}),-11.1$ (d, J = 26.4 Hz). ${ }^{31} \mathbf{P}$ NMR (162 MHz, D $2 \mathrm{O}, \delta / p p m): 13.5(\mathrm{dtd}, \mathrm{J}=27.9,21.1,7.0 \mathrm{~Hz}, 1 \mathrm{P}), 11.6(\mathrm{td}, \mathrm{J}=18.7,7.0 \mathrm{~Hz}, 1 \mathrm{P}),-11.2(\mathrm{dt}, \mathrm{J}$ = 27.0, $5.7 \mathrm{~Hz}, 1 \mathrm{P}) .{ }^{13} \mathrm{C}$ NMR $\left(101 \mathrm{MHz}, \mathrm{D}_{2} \mathrm{O}, \delta / \mathrm{ppm}\right): 164.6,156.5,136.5,111.9,84.8,82.7(\mathrm{~d}, \mathrm{~J}=8.9$ $\mathrm{Hz}), 65.4$ (d, J = 5.6 Hz), 61.06, 48.8, 30.7 (dd, J = 129.9, 117.4 Hz), 12.4.

HRMS (ESI) $\mathrm{m} / \mathrm{z}$ for $\left[\mathrm{C}_{11} \mathrm{H}_{17} \mathrm{~N}_{5} \mathrm{O}_{12} \mathrm{P}_{3}\right]^{-}$: calcd. 504.0092, found 504.0098. 
Ring opening by using morpholine: 3'-Azido-3'-deoxythymidine 5'- $\gamma$ - $P$-morpholino $\beta, \gamma$-methylenetriphosphate (26)

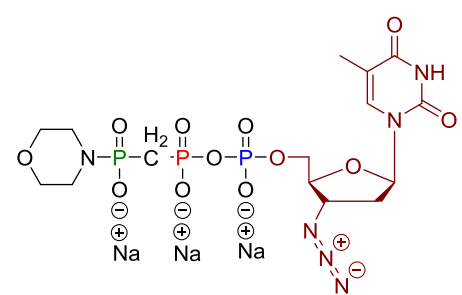

(26)

The reaction mixture of $\mathbf{B}_{8}(500 \mu \mathrm{L}, 37.5 \mu$ mol, 1.0 eq.) was added to morpholine (500 $\mu \mathrm{L})$ and then $\mathrm{D}_{2} \mathrm{O}$ $(200 \mu \mathrm{l})$ was added. The product $\mathbf{2 6}$ was isolated by Method A, affording a colourless solid (18.2 $\mathrm{mg}$, $28.5 \mu \mathrm{mol}, 76 \%)$.

${ }^{1}$ H NMR (400 MHz, D $\left.2 \mathrm{O}, \delta / \mathrm{ppm}\right): 7.69$ (s, 1H), $6.21(\mathrm{t}, \mathrm{J}=6.9 \mathrm{~Hz}, 1 \mathrm{H}), 4.56-4.42(\mathrm{~m}, 1 \mathrm{H}), 4.23$ - 4.03 $(\mathrm{m}, 3 \mathrm{H}), 3.60(\mathrm{~m}, 4 \mathrm{H}), 2.98(\mathrm{~m}, 4 \mathrm{H}), 2.50-2.34(\mathrm{~m}, 2 \mathrm{H}), 2.24-2.00(\mathrm{~m}, 2 \mathrm{H}), 1.86(\mathrm{~s}, 3 \mathrm{H}) .{ }^{31} \mathbf{P}\left\{{ }^{1} \mathbf{H}\right\}$

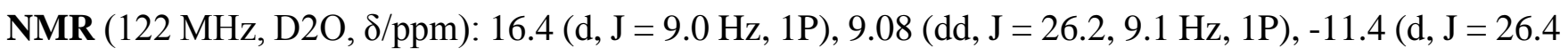
$\mathrm{Hz}, 1 \mathrm{P}) .{ }^{31} \mathbf{P}$ NMR $\left(162 \mathrm{MHz}, \mathrm{D}_{2} \mathrm{O}, \delta / \mathrm{ppm}\right): 16.4$ (tdt, $\left.\mathrm{J}=17.8,8.7,4.4 \mathrm{~Hz}, 1 \mathrm{P}\right), 9.07$ (dtd, J = 26.5, 21.2, $9.0 \mathrm{~Hz}, 1 \mathrm{P}),-11.4(\mathrm{dt}, \mathrm{J}=27.0,5.7 \mathrm{~Hz}, 1 \mathrm{P}) .{ }^{13} \mathbf{C}$ NMR (101 MHz, $\left.\mathrm{D}_{2} \mathrm{O}, \delta / \mathrm{ppm}\right): 166.8,151.9,137.9$, 111.8, 84.8, $82.9(\mathrm{~d}, \mathrm{~J}=9.2 \mathrm{~Hz}), 67.3(\mathrm{~d}, \mathrm{~J}=6.7 \mathrm{~Hz}), 65.4(\mathrm{~d}, \mathrm{~J}=5.6 \mathrm{~Hz}), 64.6,44.4(\mathrm{~d}, \mathrm{~J}=1.2 \mathrm{~Hz}), 36.3$, $27.9(\mathrm{dd}, \mathrm{J}=130.9,110.1 \mathrm{~Hz}), 11.7$.

HRMS (ESI) m/z for $\left[\mathrm{C}_{15} \mathrm{H}_{24} \mathrm{~N}_{6} \mathrm{O}_{12} \mathrm{P}_{3}\right]^{-}$: calcd. 573.0671, found 573.0670.

Ring opening by using aniline: 3'-Azido-3'-deoxythymidine 5'- $\gamma$-P-anilino $\beta, \gamma$-methylene triphosphate (27)

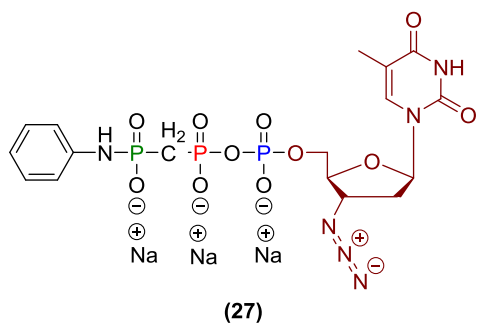

The reaction mixture of $\mathbf{B} 8(250 \mu \mathrm{L}, 18.7 \mu \mathrm{mol}, 1.0$ eq.) was added to aniline (300 $\mu \mathrm{L})$ and $\mathrm{D}_{2} \mathrm{O}(100 \mu \mathrm{l})$ was added. The product 27 was isolated by Method A, affording a colourless solid $(8.50 \mathrm{mg}, 13.1 \mu \mathrm{mol}$, $70 \%)$.

${ }^{1} \mathbf{H}$ NMR $\left(400 \mathrm{MHz}, \mathrm{D}_{2} \mathrm{O}, \delta / \mathrm{ppm}\right): 7.68(\mathrm{~s}, 1 \mathrm{H}), 7.21\left(\mathrm{~m}_{\mathrm{c}}, 2 \mathrm{H}\right), 6.92-6.76\left(\mathrm{~m}_{\mathrm{c}}, 3 \mathrm{H}\right), 6.22(\mathrm{t}, \mathrm{J}=6.9 \mathrm{~Hz}$, 1H), $4.57-4.45(\mathrm{~m}, 1 \mathrm{H}), 4.24-4.06(\mathrm{~m}, 3 \mathrm{H}), 2.48-2.39(\mathrm{~m}, 2 \mathrm{H}), 2.27(\mathrm{t}, \mathrm{J}=20.4 \mathrm{~Hz}, 2 \mathrm{H}), 1.87(\mathrm{~s}$,

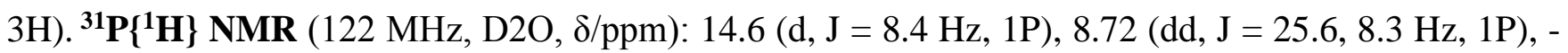
$11.3(\mathrm{~d}, \mathrm{~J}=25.4 \mathrm{~Hz}, 1 \mathrm{P}) .{ }^{31} \mathbf{P}$ NMR $\left(162 \mathrm{MHz}, \mathrm{D}_{2} \mathrm{O}, \delta / \mathrm{ppm}\right): 14.6$ (td, J = 19.9, 8.4 Hz, 1P), 8.72 (dtd, J $=29.2,21.0,8.5 \mathrm{~Hz}, 1 \mathrm{P}),-11.3(\mathrm{dt}, \mathrm{J}=24.8,5.5 \mathrm{~Hz}, 1 \mathrm{P}) .{ }^{13} \mathbf{C}$ NMR $\left(101 \mathrm{MHz}, \mathrm{D}_{2} \mathrm{O}, \delta / \mathrm{ppm}\right): 166.5$, 151.7, 145.1, 137.3, 129.5, 120.1, 116.8, 111.8, 84.9, 83.0 (d, J = 9.2 Hz), 65.5 (d, J = 5.7 Hz), 60.9, 36.3, $29.6(\mathrm{dd}, \mathrm{J}=130.9,110.1 \mathrm{~Hz}), 11.7$. 
HRMS (ESI) m/z for $\left[\mathrm{C}_{17} \mathrm{H}_{22} \mathrm{~N}_{6} \mathrm{O}_{11} \mathrm{P}_{3}\right]^{-}$: calcd. 579.0565, found 579.0569.

Ring opening by using imidazole: 3'-Azido-3'-deoxythymidine 5'- $\gamma$-P-imidazole $\beta, \gamma$-methylene triphosphate (28)

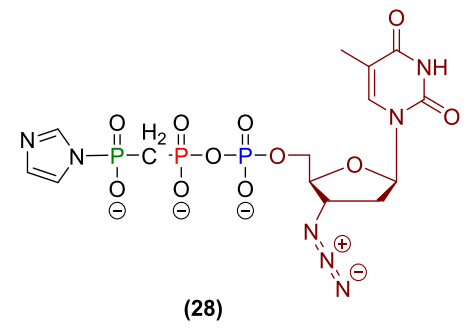

AZT (0.12 g, $0.46 \mathrm{mmol}, 1.2$ eq.) and ETT (0.15 g, $1.2 \mathrm{mmol}, 3.0$ eq. $)$ were coevaporated with dry MeCN $(2 \times 1 \mathrm{~mL})$. Under an Ar atmosphere, a reaction mixture containing the $\mathrm{Py}_{\mathrm{CH}} \mathrm{PA} \mathrm{A}_{2}(0.078 \mathrm{M}$ in $\mathrm{MeCN}$, $5.0 \mathrm{~mL}, 0.39 \mathrm{mmol}, 1.0$ eq.) was added to the dried solids and the mixture was stirred at r.t. for 10 mins. Upon cooling to $-10^{\circ} \mathrm{C}, \mathrm{mCPBA}(\leq 77 \%, 0.13 \mathrm{~g}, 0.78 \mathrm{mmol}, 2.0 \mathrm{eq}$.) was added and the mixture was stirred for 5 mins until ${ }^{31} \mathrm{P}-\mathrm{NMR}$ confirmed complete oxidation (reaction progress was followed by ${ }^{31} \mathrm{P}$ NMR, and formation of triplet at -24 ppm which is diagnostic signal for the $\mathbf{B} 8$ ).

A solution of imidazole in dry DMF (1.5M, $10 \mathrm{ml}, 15 \mathrm{mmol}, 37.0$ eq.) was added to the cyclotriphosphate B8 and reaction was stirred for another 20 mins (the reaction progress was followed by ${ }^{31} \mathrm{P} N \mathrm{NR}$ ) which results in quantative conversion to $\mathbf{2 8}$.

Note -: Imidazole was dried before using by coevaporation with $\mathrm{MeCN}$ and it was further stored of molecular sieve $(3 \AA)$.

${ }^{31} \mathbf{P}\left\{{ }^{1} \mathbf{H}\right\}$ NMR $(122 \mathrm{MHz}, \mathrm{D} 2 \mathrm{O}, \delta / \mathrm{ppm}): 8.33(\mathrm{~d}, \mathrm{~J}=8.1 \mathrm{~Hz}, 1 \mathrm{P}), 4.65(\mathrm{dd}, \mathrm{J}=25.7,8.0 \mathrm{~Hz}, 1 \mathrm{P}),-11.6$ $(\mathrm{d}, \mathrm{J}=25.7 \mathrm{~Hz}, 1 \mathrm{P}) .{ }^{31} \mathbf{P}$ NMR $\left(162 \mathrm{MHz}, \mathrm{D}_{2} \mathrm{O}, \delta / \mathrm{ppm}\right): 8.76-7.98$ (m, 1P), 4.58 (dtd, J = 26.5, 21.2, 9.0 $\mathrm{Hz}, 1 \mathrm{P}),-11.6(\mathrm{~d}, \mathrm{~J}=26.3,1 \mathrm{P})$. 


\section{5'-Adenosyl 3'-azido-3'-deoxythymidinyl $\delta, \varepsilon$-methylene hexaphosphate (P3)}

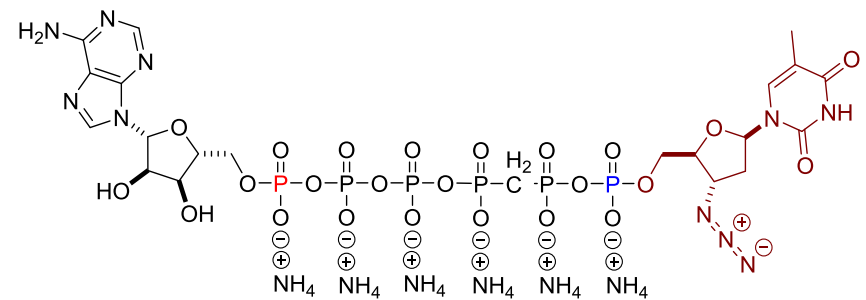

(P3)

Anhydr. $\mathrm{ZnCl}_{2}(0.25 \mathrm{~g}, 1.81 \mathrm{mmol}, 9.3$ eq. $)$ and ATP x 2.1 TBA (0.48 g, 0.39 mmol, 2.0 eq. $)$ were added to a freshly prepared solution of $\mathbf{2 8}(0.19 \mathrm{mmol})$. The reaction mixture was then diluted with dry DMF: DMSO (v:v, 1:1, $16 \mathrm{ml}$ ) in order to increase the solubility and it was stirred at r.t. for $48 \mathrm{hrs}$. The crude product was purified by Method B and the target product was eluted with 500-600 mM conc. of $\mathrm{NH}_{4} \mathrm{HCO}_{3}$ buffer. Purification gave $\mathbf{P 3}$ as a colorless solid (0.027 g, $0.02 \mathrm{mmol}, 13 \%)$.

Note -: The reactions for capped hexapolyPs were not optimized and therefore the yield could still be improved.

${ }^{1} \mathrm{H}$ NMR (400 MHz, D $\left.{ }_{2} \mathrm{O}, \delta / \mathrm{ppm}\right): 8.47(\mathrm{~s}, 1 \mathrm{H}), 8.16(\mathrm{~s}, 1 \mathrm{H}), 7.61(\mathrm{~s}, 1 \mathrm{H}), 6.14(\mathrm{t}, \mathrm{J}=6.9 \mathrm{~Hz}, 1 \mathrm{H})$, $6.04(\mathrm{~d}, \mathrm{~J}=6.2 \mathrm{~Hz}, 1 \mathrm{H}), 4.57-4.41(\mathrm{~m}, 2 \mathrm{H}), 4.33(\mathrm{t}, \mathrm{J}=2.9 \mathrm{~Hz}, 1 \mathrm{H}), 4.25-4.04(\mathrm{~m}, 5 \mathrm{H}), 2.66-2.26$ $(\mathrm{m}, 5 \mathrm{H}), 1.80(\mathrm{~s}, 3 \mathrm{H})$. The DHO peak overlaps with the signal $4.88(1 \mathrm{H}) . .{ }^{31} \mathbf{P}\left\{{ }^{1} \mathbf{H}\right\} \mathbf{N M R}(122 \mathrm{MHz}, \mathrm{D} 2 \mathrm{O}$,

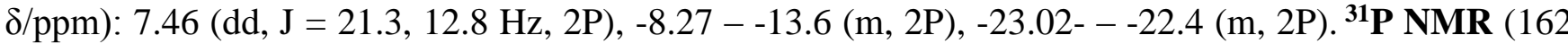
MHz, $\left.\mathrm{D}_{2} \mathrm{O}, \delta / \mathrm{ppm}\right): 8.78-6.84(\mathrm{~m}, 2 \mathrm{P}),-9.62--12.9(\mathrm{~m}, 2 \mathrm{P}),-19.5--24.9(\mathrm{~m}, 2 \mathrm{P}) .{ }^{13} \mathrm{C}$ NMR $(101 \mathrm{MHz}$, $\left.\mathrm{D}_{2} \mathrm{O}, \delta / \mathrm{ppm}\right): 166.4,155.6,152.8,151.6,149.1,139.9,137.1,118.5,111.7,86.6,84.8,84.2(\mathrm{~d}, \mathrm{~J}=9.3$ $\mathrm{Hz}), 82.9(\mathrm{~d}, \mathrm{~J}=9.3 \mathrm{~Hz}), 74.3,70.4,65.4(\mathrm{~d}, \mathrm{~J}=5.6 \mathrm{~Hz}), 65.4(\mathrm{~d}, \mathrm{~J}=5.6 \mathrm{~Hz}), 60.9,36.2,27.9(\mathrm{dd}, \mathrm{J}=$ 130.9, $110.1 \mathrm{~Hz}), 11.6$.

HRMS (ESI) $\mathrm{m} / \mathrm{z}$ for $\left[\mathrm{C}_{21} \mathrm{H}_{31} \mathrm{~N}_{10} \mathrm{O}_{24} \mathrm{P}_{6}\right]^{-}$: calcd. 992.9944, found 992.9942.

AZT 5'- $\alpha$-P-Seleno- $\beta, \gamma$-methylenecyclotriphosphate: 3'-Azido-3'-deoxythymidine 5'- $\alpha$-P-seleno$\beta, \gamma$-methylene 5'-cyclotriphosphate (B9)

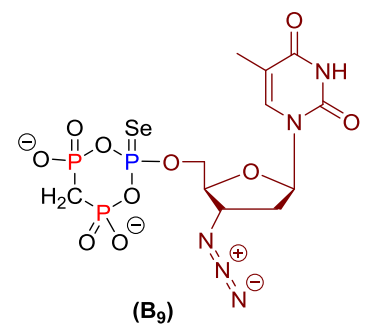

AZT (120 mg, $0.45 \mathrm{mmol}, 1.2$ eq.) and ETT (147 mg, $1.12 \mathrm{mmol}, 3.0$ eq.) were coevaporated with dry $\mathrm{MeCN}(2 \times 4 \mathrm{~mL})$. Under an $\mathrm{Ar}$ atmosphere, a reaction mixture containing the $c-\mathrm{Py}_{\mathrm{CH} 2} \mathrm{PA} \mathbf{A}_{2}(0.075 \mathrm{M}$ in $\mathrm{MeCN}, 5.00 \mathrm{~mL}, 0.37 \mathrm{mmol}, 1.0$ eq.) was added and the mixture was stirred at r.t. for 10 mins. Upon cooling to $0^{\circ} \mathrm{C}$, potassium selenocyanate $(550 \mathrm{mg}, 3.82 \mathrm{mmol}, 10.5 \mathrm{eq}$.) was added and the mixture was stirred for 10 mins. A colourless precipitate was observed and acetone was added to complete the 
precipitation. The precipitate was then collected via centrifugation and a ${ }^{31} \mathrm{P}-\mathrm{NMR}$ confirmed the formation of B9. Afterwards, the crude product was purified by Method B, affording the pure product eluted at $150 \mathrm{mM}-250 \mathrm{mM}$ conc. of $\mathrm{NH}_{4} \mathrm{HCO}_{3}$ buffer as a colourless solid $\mathbf{B} \mathbf{9}(0.09 \mathrm{~g}, 0.16 \mathrm{mmol}, 42 \%)$.

Note -: The isolated product B9 was very unstable.

${ }^{1}$ H NMR (400 MHz, D $\left.2 \mathrm{O}, \delta / \mathrm{ppm}\right): 7.73(\mathrm{~s}, 1 \mathrm{H}), 6.22(\mathrm{t}, \mathrm{J}=6.8,1 \mathrm{H}), 4.57-4.50(\mathrm{~m}, 1 \mathrm{H}), 4.32-4.14$

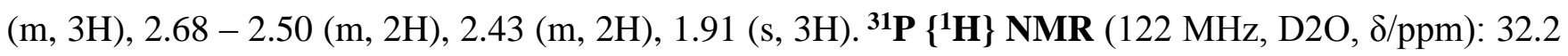
$(\mathrm{dd}, \mathrm{J}=35.7,27.0 \mathrm{~Hz}, 1 \mathrm{P}), 7.91-7.24$ (m, 2P). ${ }^{31} \mathbf{P}$ NMR (162 MHz, $\left.\mathrm{D}_{2} \mathrm{O}, \delta / \mathrm{ppm}\right): 32.2$ (dd, J = 35.2, $27.7 \mathrm{~Hz}, 1 \mathrm{P}), 9.42-5.79$ (m, 2P). ${ }^{13} \mathrm{C}$ NMR (126 MHz, D $\left.2 \mathrm{O}, \delta / \mathrm{ppm}\right): 166.6,151.7,137.3,111.9$ (d, J = $2.6 \mathrm{~Hz}), 84.9$ (d, J = 4.4 Hz), $82.8(\mathrm{t}, \mathrm{J}=9.3 \mathrm{~Hz}), 65.8$ (dd, J = 61.3, 6.2), 61.0 (d, J = 44.6 Hz), 36.3 (d, J $=12.4 \mathrm{~Hz}), 32.4,11.9$.

Ring opening by using propargylamine: 3'-Azido-3'-deoxythymidine 5'- $\gamma$-P-propargylamino $\alpha$ - $P$ seleno- $\beta, \gamma$-methylene-triphosphate (29)

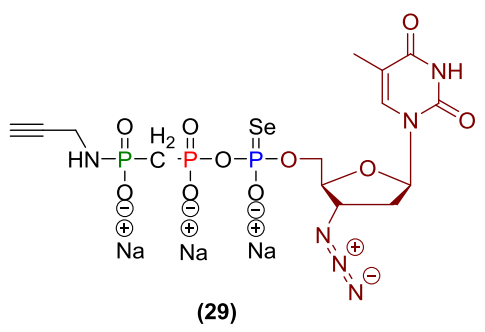

Freshly prepared B9 was dissolved, after precipitation but without any purification, in $\mathrm{D}_{2} \mathrm{O}(1 \mathrm{~mL})$. propargylamine $(1 \mathrm{~mL})$ was added and the mixture was stirred at r.t. for 15 mins. The product 29 was obtained after purification with Method B, eluted with $300-450 \mathrm{mM}$ conc. of $\mathrm{NH}_{4} \mathrm{HCO}_{3}$ buffer, as colourless solid (111 mg, $0.16 \mathrm{mmol}, 45 \%)$.

${ }^{1}$ H NMR (400 MHz, D $\left.2 \mathrm{O}, \delta / \mathrm{ppm}\right): 7.72(\mathrm{~s}, 1 \mathrm{H}), 6.22(\mathrm{t}, \mathrm{J}=6.8,1 \mathrm{H}), 4.58-4.49(\mathrm{~m}, 1 \mathrm{H}), 4.34-4.11$ $(\mathrm{m}, 3 \mathrm{H}), 3.61(\mathrm{dd}, \mathrm{J}=10.1,2.5 \mathrm{~Hz}, 2 \mathrm{H}), 2.56-2.51(\mathrm{~m}, 1 \mathrm{H}), 2.48-2.39(\mathrm{~m}, 2 \mathrm{H}), 2.34-2.23(\mathrm{~m}, 2 \mathrm{H})$,

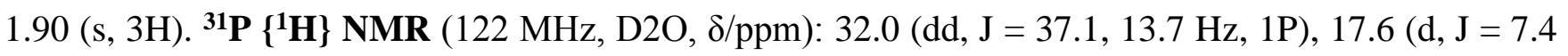
$\mathrm{Hz}, 1 \mathrm{P}), 8.4$ (ddd, J = 36.7, 7.3, 4.9 Hz, 1P). ${ }^{31} \mathbf{P}$ NMR (162 MHz, D $\left.2 \mathrm{O}, \delta / \mathrm{ppm}\right): 33.1$ - 31.5 (m, 1P), 18.8

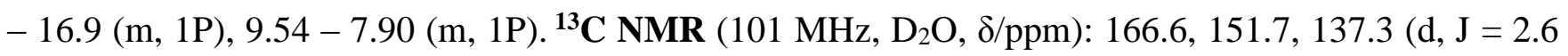
Hz), 111.8, 84.8 (d, J = 15.8 Hz), 82.7, 71.5, 65.7 (dd, J = 47.6, 6.0 Hz), 60.9 (d, J = 45.2 Hz), 36.2 (d, J $=8.4 \mathrm{~Hz}), 30.9,31.0-27.6(\mathrm{dd}, \mathrm{J}=129.9,117.4), 11.8$.

HRMS (ESI) m/z for $\left[\mathrm{C}_{14} \mathrm{H}_{22} \mathrm{~N}_{6} \mathrm{O}_{10} \mathrm{P}_{3} \mathrm{Se}\right]^{+}$: calcd. 606.9770, found 606.9769. 
3'-Azido-3'-deoxythymidine 5'- $\alpha$-P-thio $\beta, \gamma$-methylene cyclotriphosphate (B10)

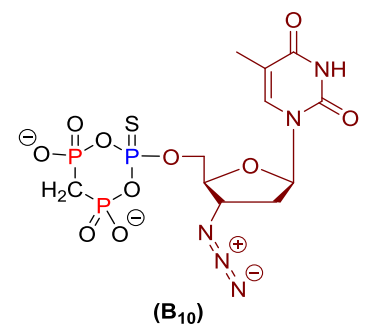

AZT (50 mg, $0.18 \mathrm{mmol}, 1.2$ eq.) and ETT (78 mg, $0.60 \mathrm{mmol}, 4.0$ eq.) were coevaporated with dry $\mathrm{MeCN}(2 \times 1 \mathrm{~mL})$. Under an Ar atmosphere, a reaction mixture containing the $\mathrm{Pу}_{\mathrm{CH}} \mathrm{PA} \mathbf{A 2}_{2}(0.075 \mathrm{M}$ in $\mathrm{MeCN}, 2 \mathrm{~mL}, 0.15 \mathrm{mmol}, 1.0$ eq.) was added to the dried solids and it was stirred at r.t. for $10 \mathrm{mins}$. Upon cooling to $0^{\circ} \mathrm{C}$, Becauge's reagent $(50 \mathrm{mg}, 0.25 \mathrm{mmol}, 1.7$ eq.) was added and the mixture was stirred for 10 mins until ${ }^{31} \mathrm{P}-\mathrm{NMR}$ confirmed complete oxidation (formation of triplet at $+40 \mathrm{ppm}$ which is diagnostic signal for the $\alpha-(\mathrm{S})$-cyclotriphosphate $\left.\mathbf{B}_{10}\right)$.

${ }^{31} \mathbf{P}\left\{{ }^{1} \mathbf{H}\right\}$ NMR (122 MHz, $\left.\mathrm{CDCl}_{3}, \delta / \mathrm{ppm}\right): 39.3(\mathrm{t}, \mathrm{J}=30.6 \mathrm{~Hz}, 1 \mathrm{P}), 2.38(\mathrm{~d}, \mathrm{~J}=30.5 \mathrm{~Hz}, 2 \mathrm{P}) .{ }^{31} \mathrm{P}$ NMR (122 MHz, $\left.\mathrm{CDCl}_{3}, \delta / \mathrm{ppm}\right): 40.3$ (m, 1P), 2.38 (dt, J = 30.6, $19.5 \mathrm{~Hz}, 2 \mathrm{P}$ ).

Ring opening by using propargylamine: 3'-Azido-3'-deoxythymidine 5'- $\gamma$-P-propargylamino $\alpha-P$ thio $\beta, \gamma$-methylene triphosphate (30)

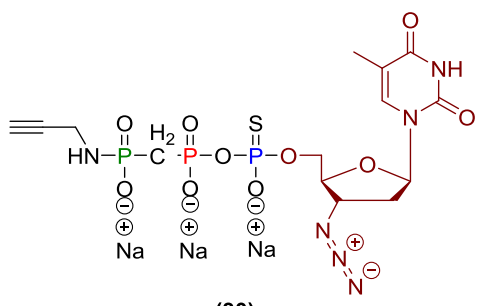

(30)

The reaction mixture of $\mathbf{B}_{10}\left(250 \mu \mathrm{L}, 18.7 \mu \mathrm{mol}, 1.0\right.$ eq.) was added to propargylamine $(250 \mu \mathrm{L})$ and $\mathrm{D}_{2} \mathrm{O}$ $(100 \mu \mathrm{l})$ was added. The product 30 was isolated by Method A, affording a colourless solid whereas products were obtained as mixtures of diastereoisomers ( $8.70 \mathrm{mg}, 14.0 \mu \mathrm{mol}, 75 \%$ ).

${ }^{1} \mathbf{H}$ NMR $\left(400 \mathrm{MHz}, \mathrm{D}_{2} \mathrm{O}, \delta / \mathrm{ppm}\right): 7.74(\mathrm{~s}, 1 \mathrm{H}), 6.23(\mathrm{t}, \mathrm{J}=6.9 \mathrm{~Hz}, 1 \mathrm{H}), 4.57-4.46(\mathrm{~m}, 1 \mathrm{H}), 4.27$ - 4.12 $(\mathrm{m}, 3 \mathrm{H}), 3.62(\mathrm{dd}, \mathrm{J}=9.9,2.3 \mathrm{~Hz}, 2 \mathrm{H}), 2.60-2.38(\mathrm{~m}, 5 \mathrm{H}), 1.91(\mathrm{~s}, 3 \mathrm{H}) .{ }^{31} \mathbf{P}\left\{{ }^{1} \mathbf{H}\right\} \mathbf{N M R}(122 \mathrm{MHz}, \mathrm{D} 2 \mathrm{O}$, $\delta / \mathrm{ppm}): 42.1(\mathrm{dd}, \mathrm{J}=33.3,23 ., 1 \mathrm{P}), 19.0$ - $16.6(\mathrm{~m}, 1 \mathrm{P}), 9.28$ - $8.47(\mathrm{~m}, 1 \mathrm{P}) .{ }^{31} \mathbf{P}$ NMR $\left(162 \mathrm{MHz}, \mathrm{D}_{2} \mathrm{O}\right.$,

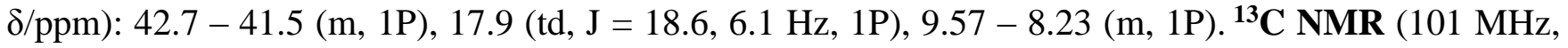
$\left.\mathrm{D}_{2} \mathrm{O}, \delta / \mathrm{ppm}\right): 166.8,151.9,137.3,111.8,84.9,82.9$ (d, J = 9.2 Hz), 76.1, 69.9, 65.4 (d, J = 5.5 Hz), 60.9, $36.2,31.7-27.6(\mathrm{dd}, \mathrm{J}=129.2,107.1 \mathrm{~Hz}), 11.7$. 
Ring opening by using aq. ammonia: 3'-Azido-3'-deoxythymidine 5'- $\gamma$-P-amino $\alpha$-P-thio $\beta, \gamma$ methylene triphosphate (31)

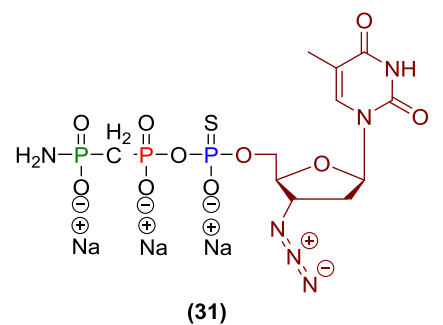

The reaction mixture of $\mathbf{B}_{10}(250 \mu \mathrm{L}, 18.7 \mu \mathrm{mol}, 1.0$ eq. $)$ was added to $25 \%$ aq. $\mathrm{NH}_{4} \mathrm{OH}(250 \mu \mathrm{L})$ and of $\mathrm{D}_{2} \mathrm{O}(100 \mu \mathrm{l})$ was added. The product 31 was isolated by Method A, affording a colourless solid whereas products were obtained as mixtures of diastereoisomers $(8.50 \mathrm{mg}, 14.5 \mu \mathrm{mol}, 80 \%)$.

${ }^{1} \mathbf{H}$ NMR (400 MHz, D $\left.2 \mathrm{O}, \delta / \mathrm{ppm}\right): 7.73$ (s, 1H), $6.23(\mathrm{t}, \mathrm{J}=6.9 \mathrm{~Hz}, 1 \mathrm{H}), 4.65$ - 4.47 (m, 1H), $4.46-4.20$ (m, 3H), $2.57-2.41(\mathrm{~m}, 2 \mathrm{H}), 2.33(\mathrm{~m}, 2 \mathrm{H}), 1.90$ (s, 3H). ${ }^{31} \mathbf{P}\left\{{ }^{1} \mathbf{H}\right\}$ NMR (122 MHz, D2O, $\left.\delta / \mathrm{ppm}\right): 45.8-$ 43.2 (m, 1P), 17.2 (d, J = 8.1, 1P), 9.80 (d, J = 31.4, 1P). ${ }^{31}$ P NMR (162 MHz, D $\left.2 \mathrm{O}, \delta / p p m\right): ~ 45.3-43.6$ (m, 1P), 18.1 - 16.4 (m, 1P), 10.7 - 9.10 (m, 1P). ${ }^{13}$ C NMR (101 MHz, $\left.\mathrm{D}_{2} \mathrm{O}, \delta / \mathrm{ppm}\right): 169.3,153.8,136.9$, 111.9, 84.9, $82.8(\mathrm{~d}, \mathrm{~J}=9.3 \mathrm{~Hz}), 65.4(\mathrm{~d}, \mathrm{~J}=5.5 \mathrm{~Hz}), 60.9,36.2,31.9(\mathrm{dd}, \mathrm{J}=129.2,107.1 \mathrm{~Hz}), 11.9$.

\section{2',3'-didehydro-2',3'-dideoxythymidine 5'- $\beta, \gamma$-methylene cyclotriphosphate (B11)}

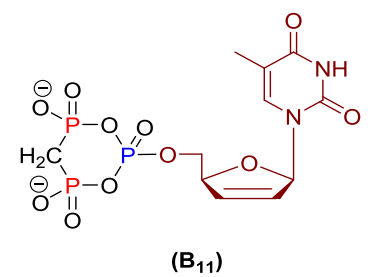

d4T (50 mg, $0.22 \mathrm{mmol}, 1.5$ eq.) and ETT (69 mg, $0.52 \mathrm{mmol}, 3.5$ eq.) were coevaporated with dry MeCN $(2 \times 1 \mathrm{~mL})$ and the dried solids were dissolved in dry DMF $(1 \mathrm{~mL})$. Under an Ar atmosphere, a reaction mixture containing the $\mathrm{Py}_{\mathrm{CH}} \mathrm{PA} \mathrm{A}_{2}(0.075 \mathrm{M}$ in $\mathrm{MeCN}, 2.0 \mathrm{~mL}, 0.15 \mathrm{mmol}, 1.0$ eq.) was added and it was stirred at r.t. for 10 mins. Upon cooling to $0^{\circ} \mathrm{C}, \mathrm{mCPBA}(\leq 77 \%, 39 \mathrm{mg}, 0.22 \mathrm{mmol}, 1.5 \mathrm{eq}$.) was added and the mixture was stirred for 5 mins until ${ }^{31} \mathrm{P}-\mathrm{NMR}$ confirmed complete oxidation (formation of triplet at $-25 \mathrm{ppm}$ which is diagnostic signal for the $\mathrm{d} 4 \mathrm{~T}$ cyclotriphosphate $\mathbf{B}_{11}$ ).

${ }^{31} \mathbf{P}\left\{{ }^{1} \mathbf{H}\right\}$ NMR (122 MHz, $\left.\mathrm{CDCl}_{3}, \delta / \mathrm{ppm}\right): 5.16(\mathrm{~d}, \mathrm{~J}=18.5 \mathrm{~Hz}, 2 \mathrm{P}),-24.9$ (t, J = $\left.18.6 \mathrm{~Hz}, 1 \mathrm{P}\right)$. 
Ring opening by using aq. ammonia: 2',3'-didehydro-2',3'-dideoxythymidine 5'- $\gamma$ - $P$-amino $\beta, \gamma$ methylene triphosphate (32)

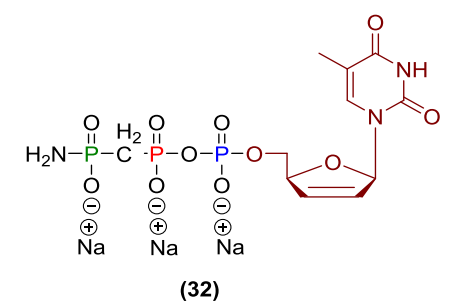

The reaction mixture of $\mathbf{B}_{11}\left(250 \mu \mathrm{L}, 12.5 \mu \mathrm{mol}, 1.0\right.$ eq.) was added to $25 \%$ aq. $\mathrm{NH}_{4} \mathrm{OH}(250 \mu \mathrm{L})$ and then $\mathrm{D}_{2} \mathrm{O}(100 \mu \mathrm{l})$ was added. The product 32 was isolated by Method A, affording a colourless solid (5.31 mg, $10.7 \mu \mathrm{mol}, 80 \%)$.

${ }^{1}$ H NMR (400 MHz, D $\left.2 \mathrm{O}, \delta / \mathrm{ppm}\right): 7.53$ (s, 1H), 6.89 (m, 1H), 6.45 (m, 1H), 5.87 (m, 1H), 5.03 (d, J $=4.2 \mathrm{~Hz}, 1 \mathrm{H}), 4.07(\mathrm{dt}, \mathrm{J}=6.1,3.1 \mathrm{~Hz}, 2 \mathrm{H}), 2.25(\mathrm{dd}, \mathrm{J}=20.6,19.1 \mathrm{~Hz}, 2 \mathrm{H}), 1.82(\mathrm{~s}, 3 \mathrm{H}) .{ }^{31} \mathbf{P}\left\{{ }^{1} \mathbf{H}\right\} \mathbf{N M R}$ (122 MHz, D2O, ס/ppm): 17.9 (d, J = 6.1 Hz, 1P), 9.14 (dd, J = 26.1, 6.1 Hz, 1P), -11.5 (d, J = 26.1 Hz, 1P). ${ }^{31} \mathbf{P}$ NMR (162 MHz, D $\left.2 \mathrm{O}, \delta / \mathrm{ppm}\right): 17.9$ (td, J = 19.1, $\left.6.1 \mathrm{~Hz}, 1 \mathrm{P}\right), 8.91$ (dtd, J = 26.4, 20.3, 6.1 Hz, 1P), -11.4 (dt, J = 26.1, 6.6 Hz, 1P). ${ }^{13} \mathrm{C}$ NMR (101 MHz, $\left.\mathrm{D}_{2} \mathrm{O}, \delta / \mathrm{ppm}\right): 167.3,152.6,138.1,134.2,125.2$, 111.5, 89.9, 85.9 (d, J = 8.7 Hz), 66.3 (d, J = 5.7 Hz), 31.9 (dd, J =129.8, 106.3 Hz), 11.5.

HRMS (ESI) m/z for $\left[\mathrm{C}_{11} \mathrm{H}_{16} \mathrm{~N}_{3} \mathrm{NaO}_{11} \mathrm{P}_{3}\right]^{-}$: calcd. 481.9901, found 481.9901.

Ring opening by using propargylamine: 2',3'-didehydro-2',3'-dideoxythymidine 5'- $\gamma-P$ propargylamino $\beta, \gamma$-methylene triphosphate (33)

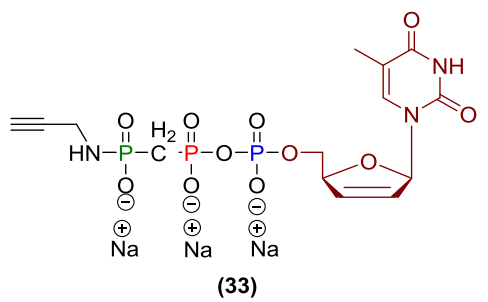

The reaction mixture of $\mathbf{B}_{11}(250 \mu \mathrm{L}, 12.5 \mu \mathrm{mol}, 1.0$ eq.) was added to propargylamine $(250 \mu \mathrm{L})$ and then $\mathrm{D}_{2} \mathrm{O}(100 \mu \mathrm{l})$ was added. The product 33 was isolated by Method A, affording a colourless solid (5.45 mg, $9.64 \mu \mathrm{mol}, 75 \%)$.

${ }^{1}$ H NMR (400 MHz, D $\left.2 \mathrm{O}, \delta / \mathrm{ppm}\right): 7.54(\mathrm{dd}, \mathrm{J}=5.3,1.4 \mathrm{~Hz}, 1 \mathrm{H}), 6.89(\mathrm{~m}, 1 \mathrm{H}), 6.45(\mathrm{~m}, 1 \mathrm{H}), 5.87(\mathrm{~m}$, 1H), $5.03(\mathrm{dq}, \mathrm{J}=3.7,1.9 \mathrm{~Hz}, 1 \mathrm{H}), 4.07$ (dt, J = 5.7, $2.7 \mathrm{~Hz}, 2 \mathrm{H}), 3.56(\mathrm{~d}, \mathrm{~J}=10.1 \mathrm{~Hz}, 1 \mathrm{H}), 2.34-2.17$ (m, 2H), 1.83 (s, 3H). ${ }^{31} \mathbf{P}\left\{{ }^{1} \mathbf{H}\right\} \mathbf{N M R}(122 \mathrm{MHz}, \mathrm{D} 2 \mathrm{O}, \delta / \mathrm{ppm}): 17.8(\mathrm{~d}, \mathrm{~J}=7.8 \mathrm{~Hz}, 1 \mathrm{P}), 8.53$ (dd, J = 26.2, $7.1 \mathrm{~Hz}, 1 \mathrm{P}),-11.4$ (d, J = 27.1, 1P). ${ }^{31} \mathbf{P}$ NMR (162 MHz, D $\left.2 \mathrm{O}, \delta / \mathrm{ppm}\right): 18.6-17.4$ (m, 1P), 8.5 (dtd, J = 27.5, 20.9, 7.3 Hz, 1P), -11.4 (dt, J = 26.3, 6.3 Hz, 1P). ${ }^{13} \mathbf{C}$ NMR (101 MHz, D $\left.2 \mathrm{O}, \delta / \mathrm{ppm}\right): 166.8,152.3$, $138.1(\mathrm{~d}, \mathrm{~J}=4.4 \mathrm{~Hz}), 134.3,125.2,111.5,89.9,85.9(\mathrm{dd}, \mathrm{J}=8.7,3.0 \mathrm{~Hz}), 74.7,66.3(\mathrm{~d}, \mathrm{~J}=5.6 \mathrm{~Hz}), 30.4$ $(\mathrm{d}, \mathrm{J}=37.9 \mathrm{~Hz}), 29.0$ (dd, J =129.8, 106.3 Hz), 11.5.

HRMS (ESI) $\mathrm{m} / \mathrm{z}$ for $\left[\mathrm{C}_{14} \mathrm{H}_{19} \mathrm{~N}_{3} \mathrm{O}_{11} \mathrm{P}_{3}\right]^{-}$:calcd. 498.0238, found 498.0238. 
Ring opening by using aq. sodium hydroxide: 2',3'-didehydro-2',3'-dideoxythymidine 5'- $\beta, \gamma$ methylene triphosphate (34)

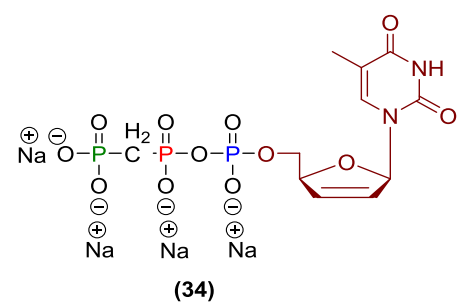

The reaction mixture of $\mathbf{B}_{11}(250 \mu \mathrm{L}, 12.5 \mu \mathrm{mol}, 1.0$ eq.) was added to aq. $\mathrm{NaOH}(1 \mathrm{M}, 250 \mu \mathrm{L})$ and then $\mathrm{D}_{2} \mathrm{O}(100 \mu \mathrm{l})$ was added. The product 34 was isolated by Method A, affording a colourless solid (4.92 mg, $8.94 \mu \mathrm{mol}, 71 \%)$. The analytical data are consistent with literature. ${ }^{11}$

${ }^{1}$ H NMR (400 MHz, D $\left.2 \mathrm{O}, \delta / \mathrm{ppm}\right): 7.51(\mathrm{~s}, 1 \mathrm{H}), 6.89(\mathrm{dt}, \mathrm{J}=3.4,1.7 \mathrm{~Hz}, 1 \mathrm{H}), 6.44(\mathrm{dt}, \mathrm{J}=6.1,1.8 \mathrm{~Hz}$, $1 \mathrm{H}), 5.86(\mathrm{dt}, \mathrm{J}=6.2,1.9 \mathrm{~Hz}, 1 \mathrm{H}), 5.19-4.95(\mathrm{~m}, 1 \mathrm{H}), 4.06(\mathrm{dd}, \mathrm{J}=6.6,3.7 \mathrm{~Hz}, 1 \mathrm{H}), 2.09(\mathrm{dd}, \mathrm{J}=21.3$, $18.9 \mathrm{~Hz}, 1 \mathrm{H}), 1.81$ (s, 2H). ${ }^{31} \mathbf{P}\left\{{ }^{1} \mathbf{H}\right\}$ NMR (122 MHz, D2O, $\left.\delta / \mathrm{ppm}\right): 13.07$ (dd, J = 25.4, 6.6 Hz, 1P), 11.7 $(\mathrm{d}, \mathrm{J}=6.8 \mathrm{~Hz}, 1 \mathrm{P}),-11.1(\mathrm{~d}, \mathrm{~J}=26.5 \mathrm{~Hz}, 1 \mathrm{P}) .{ }^{31} \mathbf{P}$ NMR $\left(162 \mathrm{MHz}, \mathrm{D}_{2} \mathrm{O}, \delta / \mathrm{ppm}\right): 14.1-12.4(\mathrm{~m}, 1 \mathrm{P})$, $12.3-11.4(\mathrm{~m}, 1 \mathrm{P}),-11.1(\mathrm{dt}, \mathrm{J}=26.7,6.7 \mathrm{~Hz}, 1 \mathrm{P}) .{ }^{13} \mathbf{C}$ NMR $\left(101 \mathrm{MHz}, \mathrm{D}_{2} \mathrm{O}, \delta / \mathrm{ppm}\right): 168.7,153.7$, 137.9, 134.1, 125.3, 111.6, 90.0, 85.9 (d, J = 8.5 Hz), $66.3(\mathrm{~d}, \mathrm{~J}=5.5 \mathrm{~Hz}), 30.6(\mathrm{dd}, \mathrm{J}=129.5,117.8 \mathrm{~Hz})$, 11.7.

HRMS (ESI) m/z for $\left[\mathrm{C}_{11} \mathrm{H}_{16} \mathrm{~N}_{2} \mathrm{O}_{12} \mathrm{P}_{3}\right]^{-}$: calcd. 460.9922, found, 460.9928.

Ring opening by tetramethyl guanidinium azide: 2',3'-didehydro-2',3'-dideoxythymidine 5'- $\gamma$ - $P$ azido $\beta, \gamma$-methylene triphosphate (35)

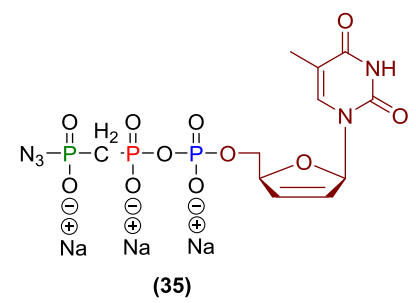

The reaction mixture of $\mathbf{B}_{11}(250 \mu \mathrm{L}, 12.5 \mu \mathrm{mol}, 1.0$ eq.) was added to tetramethyl guanidinium azide (400 mg, $2.52 \mathrm{mmol}, 200$ eq.) and then $\mathrm{D}_{2} \mathrm{O}(200 \mu \mathrm{l})$ were added. The crude product was isolated by Method A and the product was precipitated as guanidinium salt which was again by dissolved in water (2 $\mathrm{mL}$ ).Afterwards, the product $\mathbf{3 5}$ was isolated from the aq. solution again by Method A and a colourless solid was obtained (6.40 mg, $11.2 \mu \mathrm{mol}, 92 \%)$.

Note -: Alternative, lithium azide can also be used as a nucleophile for ring-opening. But, commercially available $\mathrm{LiN}_{3}$ solution is $20 \%$ wt. in water which results in $\mathrm{H}_{2} \mathrm{O}$ acts as a competitive nucleophile giving rise to lower yield. In this case, simultaneously, $\mathrm{H}_{2} \mathrm{O}$ is also acting as a nucleophile the product was obtained as a mixture of $\mathbf{3 4 : 3 5}$ in 20:80 ratio. 
Additionally, it was found that triphosphoazidate $\mathbf{3 5}$ is decomposed into the $\mathbf{3 4}$ in two weeks which, was further confirmed by spiking experiments (see the NMR supporting).

${ }^{1}$ H NMR (400 MHz, D $\left.2 \mathrm{O}, \delta / \mathrm{ppm}\right): 7.45(\mathrm{~s}, 1 \mathrm{H}), 6.90(\mathrm{~m}, 1 \mathrm{H}), 6.44(\mathrm{~m}, 1 \mathrm{H}), 5.86(\mathrm{~m}, 1 \mathrm{H}), 5.19-4.95$ $(\mathrm{m}, 1 \mathrm{H}), 4.06(\mathrm{dd}, \mathrm{J}=6.6,3.7 \mathrm{~Hz}, 2 \mathrm{H}), 2.09(\mathrm{dd}, \mathrm{J}=21.3,18.9 \mathrm{~Hz}, 2 \mathrm{H}), 1.81(\mathrm{~s}, 3 \mathrm{H}) .{ }^{31} \mathbf{P}\left\{{ }^{1} \mathbf{H}\right\} \mathbf{N M R}(162$ MHz, D $2 \mathrm{O}, \delta / p p m) 16.4(\mathrm{~d}, \mathrm{~J}=7.8 \mathrm{~Hz}, 1 \mathrm{P}), 6.94(\mathrm{dd}, \mathrm{J}=24.1,8.0 \mathrm{~Hz}, 1 \mathrm{P}),-10.5$ (d, J = 23.9 Hz, 1P). ${ }^{31} \mathbf{P}$ NMR (162 MHz, D $2 \mathrm{O}, \delta / \mathrm{ppm}): 16.4$ (td, J = 19.7, $7.8 \mathrm{~Hz}, 1 \mathrm{P}), 8.07$ - 5.95 (m, 1P), -10.5 (td, J = 24.4, $6.2 \mathrm{~Hz}, 1 \mathrm{P}) .{ }^{13} \mathrm{C}$ NMR (101 MHz, $\left.\mathrm{D}_{2} \mathrm{O}, \delta / \mathrm{ppm}\right): 167.3,152.6,138.1,134.2,125.2,111.5,89.9,85.9$ (d, J $=8.7 \mathrm{~Hz}), 66.3(\mathrm{~d}, \mathrm{~J}=5.7 \mathrm{~Hz}), 31.9(\mathrm{dd}, \mathrm{J}=129.8,106.3 \mathrm{~Hz}), 11.5$.

HRMS (ESI) $\mathrm{m} / \mathrm{z}$ for $\left[\mathrm{C}_{11} \mathrm{H}_{15} \mathrm{~N}_{5} \mathrm{O}_{11} \mathrm{P}_{3}\right]^{-}$: calcd. 485.9986, found, 485.9987.

Ring opening by caesium fluoride: 2',3'-didehydro-2',3'-dideoxythymidine 5'- $\gamma$-P-fluoro $\beta, \gamma$ methylene triphosphate (36)

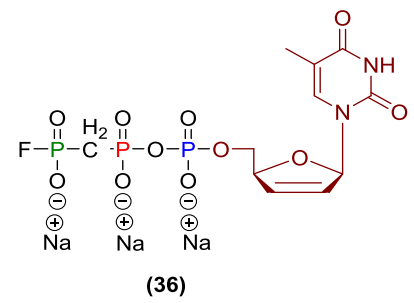

The reaction mixture of $\mathbf{B}_{11}(250 \mu \mathrm{L}, 11.2 \mu \mathrm{mol}, 1.0$ eq.) was added to caesium fluoride (105 mg, $691 \mu \mathrm{mol}, 58$ eq.) and then immediately $\mathrm{D}_{2} \mathrm{O}(250 \mu \mathrm{l})$ was added. The crude product was isolated by Method A and the impurity $\mathrm{CsClO}_{4}$ was co-precipitated which was further removed by dissolving the crude product in water $(2 \mathrm{~mL})$. The insoluble residue was removed by filtration (syringe filter, pore size $0.45 \mu \mathrm{m})$. Afterwards, the product 36 was isolated from the aq. solution again by Method A and a colourless solid was obtained (5.8 mg, $10.9 \mu \mathrm{mol}, 97 \%)$.

${ }^{1} \mathbf{H}$ NMR (400 MHz, D $\left.2 \mathrm{O}, \delta / \mathrm{ppm}\right): 7.51(\mathrm{~s}, 1 \mathrm{H}), 6.89(\mathrm{dt}, \mathrm{J}=3.4,1.7 \mathrm{~Hz}, 1 \mathrm{H}), 6.44(\mathrm{dt}, \mathrm{J}=6.1,1.8 \mathrm{~Hz}$, 1H), $5.86(\mathrm{dt}, \mathrm{J}=6.2,1.9 \mathrm{~Hz}, 1 \mathrm{H}), 5.19-4.95(\mathrm{~m}, 1 \mathrm{H}), 4.06(\mathrm{dd}, \mathrm{J}=6.6,3.7 \mathrm{~Hz}, 2 \mathrm{H}), 2.09$ (dd, J = 21.3, $18.9 \mathrm{~Hz}, 2 \mathrm{H}), 1.81(\mathrm{~s}, 3 \mathrm{H}) .{ }^{31} \mathbf{P}\left\{{ }^{1} \mathbf{H}\right\} \mathbf{N M R}\left(122 \mathrm{MHz}, \mathrm{D}_{2} \mathrm{O}, \delta / \mathrm{ppm}\right): 17.3(\mathrm{dd}, \mathrm{J}=962.3,7.9 \mathrm{~Hz} ; 1 \mathrm{P}), 6.06$ (m, 1P), -11.1 (d, J = 24.3 Hz, 1P). ${ }^{31} \mathbf{P}$ NMR (162 MHz, D $\left.2 \mathrm{O}, \delta / \mathrm{ppm}\right): 17.8$ (dtd, J = 971.1, 21.2, 9.4 Hz, 1P), 5.98 (qd, J = 21.9, 21.3, 9.2 Hz, 1P), -11.3 (dt, J = 24.9, 6.2 Hz, 1P). ${ }^{31} \mathbf{P}\left\{{ }^{19} \mathbf{F}\right\}$ NMR $\left(162 \mathrm{MHz}, \mathrm{D}_{2} \mathrm{O}\right.$,

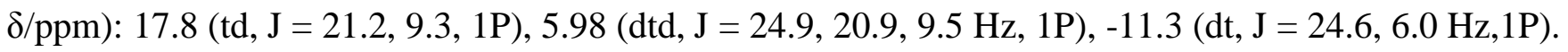
${ }^{13} \mathrm{C}$ NMR (101 MHz, D $\left.2 \mathrm{O}, \delta / \mathrm{ppm}\right): 168.7,153.7,137.9,134.1,125.3,111.6,90.0,85.9$ (d, J = 8.5 Hz), $66.3(\mathrm{~d}, \mathrm{~J}=5.5 \mathrm{~Hz}), 30.6(\mathrm{dd}, \mathrm{J}=129.5,117.8 \mathrm{~Hz}), 11.7 .{ }^{19} \mathbf{F} \mathbf{N M R}\left(377 \mathrm{MHz}, \mathrm{D}_{2} \mathrm{O}, \delta / \mathrm{ppm}\right):-54.5(\mathrm{~d}$, $\mathrm{J}=971.5 \mathrm{~Hz}, 1 \mathrm{~F})$.

HRMS (ESI) m/z for $\left[\mathrm{C}_{11} \mathrm{H}_{14} \mathrm{FN}_{2} \mathrm{NaO}_{11} \mathrm{P}_{3}\right]^{-}$: calcd. 484.9490, found, 484.9479. 
Ring opening by using methanolic sodium methoxide: 2',3'-didehydro-2',3'-dideoxythymidine 5'$\gamma$-P-methoxy $\beta, \gamma$-methylene triphosphate (37)

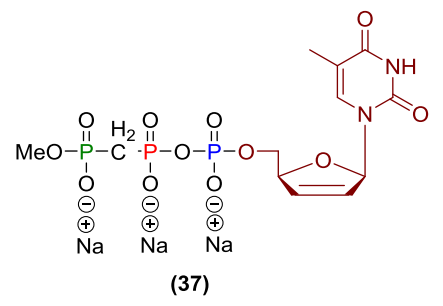

The reaction mixture of $\mathbf{B}_{11}(250 \mu \mathrm{L}, 11.2 \mu \mathrm{mol}, 1.0$ eq.) was added to $\mathrm{NaOMe}(0.5 \mathrm{M}$ in methanol, $1.00 \mathrm{~mL}, 50.0 \mu \mathrm{mol}, 4.5$ eq. $)$ and then $\mathrm{D}_{2} \mathrm{O}(200 \mu \mathrm{l})$ was added. The product 37 was isolated by Method A, affording a colourless solid $(4.90 \mathrm{mg}, 9.03 \mu \mathrm{mol}, 81 \%)$.

${ }^{1}$ H NMR (400 MHz, D $\left.2 \mathrm{O}, \delta / \mathrm{ppm}\right): 7.52(\mathrm{~s}, 1 \mathrm{H}), 6.88(\mathrm{~s}, 1 \mathrm{H}), 6.44(\mathrm{~d}, \mathrm{~J}=6.0 \mathrm{~Hz}, 1 \mathrm{H}), 5.88(\mathrm{~d}, \mathrm{~J}=6.2$ Hz, 1H), $5.04(\mathrm{~s}, 1 \mathrm{H}), 4.05(\mathrm{dd}, \mathrm{J}=6.4,3.6 \mathrm{~Hz}, 2 \mathrm{H}), 3.50(\mathrm{~d}, \mathrm{~J}=10.8 \mathrm{~Hz}, 2 \mathrm{H}), 3.12(\mathrm{~d}, \mathrm{~J}=7.6 \mathrm{~Hz}, 1 \mathrm{H})$, 2.15 (s, 5H). ${ }^{31} \mathbf{P}\left\{{ }^{1} \mathbf{H}\right\}$ NMR (122 MHz, D $\left.2 \mathrm{O}, \delta / \mathrm{ppm}\right): 18.7$ (d, J = 8.0 Hz, 1P), 7.97 (dd, J = 25.4, 7.8 Hz,

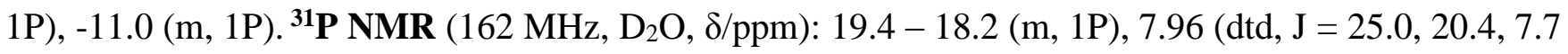
$\mathrm{Hz}, 1 \mathrm{P}),-11.2$ (dt, J = 25.3, $6.5 \mathrm{~Hz}, 1 \mathrm{P}) .{ }^{13} \mathrm{C}$ NMR (101 MHz, $\left.\mathrm{D}_{2} \mathrm{O}, \delta / \mathrm{ppm}\right): 168.7,153.7,137.9,134.1$, 125.3, 111.6, 90.0, 85.9 (d, J = 8.5 Hz), 66.3 (d, J = 5.5 Hz), 51.7 (d, J = 5.6 Hz), 30.6 (dd, J = 129.5, $117.8 \mathrm{~Hz}), 11.5$.

HRMS (ESI) $\mathrm{m} / \mathrm{z}$ for $\left[\mathrm{C}_{12} \mathrm{H}_{18} \mathrm{~N}_{2} \mathrm{O}_{12} \mathrm{P}_{3}\right]^{-}$: calcd. 475.0078, found, 475.0075 .

2',3'-O-Isopropylideneadenosine $\beta, \gamma$-methylene cyclotriphosphate $\left(\mathbf{B}_{12}\right)$

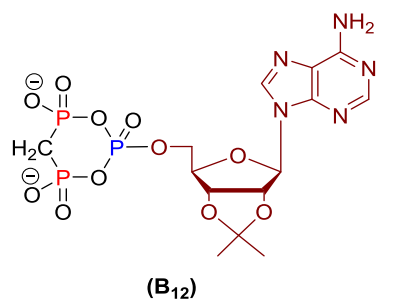

2', 3'-O-Isopropylideneadenosine (59 mg, $0.19 \mathrm{mmol}, 1.2$ eq.) and ETT (60 mg, $0.49 \mathrm{mmol}, 3.0$ eq.) were coevaporated with dry $\mathrm{MeCN}(2 \times 1 \mathrm{~mL})$. Under an Ar atmosphere, both solids were dissolved in dry DMF (1 mL), a reaction mixture containing the Русн2 $\mathrm{PA} \mathbf{A}_{2}(0.078 \mathrm{M}, 2.0 \mathrm{~mL}, 0.15 \mathrm{mmol}, 1.0$ eq.) in $\mathrm{MeCN}$ was added and it was stirred at r.t. for 10 mins. Upon cooling to $0^{\circ} \mathrm{C}, \mathrm{mCPBA}(\leq 77 \%, 47 \mathrm{mg}$, $0.27 \mathrm{mmol}, 1.8 \mathrm{eq}$.) was added and the mixture was stirred for 5 mins until ${ }^{31} \mathrm{P}-\mathrm{NMR}$ confirmed complete oxidation (formation of triplet at $-23 \mathrm{ppm}$ which is diagnostic signal for the cyclotriphosphate $\mathbf{B}_{12}$ ).

${ }^{31} \mathbf{P}\left\{{ }^{1} \mathbf{H}\right\}$ NMR $\left(122 \mathrm{MHz}, \mathrm{CDCl}_{3}, \delta / \mathrm{ppm}\right): 5.79(\mathrm{~d}, \mathrm{~J}=18.5 \mathrm{~Hz}, 2 \mathrm{P}),-25.0(\mathrm{t}, \mathrm{J}=18.6 \mathrm{~Hz}, 1 \mathrm{P})$. 
Ring opening by using aq. ammonia: 2',3'-O-Isopropylideneadenosine 5'- $\gamma$ - $P$-amino $\beta, \gamma$ methylenetriphosphate (38)

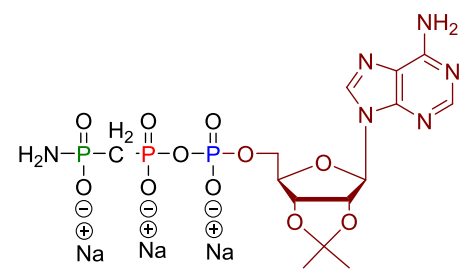

(38)

The reaction mixture of $\mathbf{B}_{12}\left(250 \mu \mathrm{L}, 13.0 \mu \mathrm{mol}, 1.0\right.$ eq.) was added to $25 \%$ aq. $\mathrm{NH}_{4} \mathrm{OH}(250 \mu \mathrm{L})$ and then $\mathrm{D}_{2} \mathrm{O}(100 \mu \mathrm{l})$ was added. The product $\mathbf{3 8}$ was isolated by Method A, affording a colourless solid (6.80 mg, $11.1 \mu \mathrm{mol}, 85 \%)$.

${ }^{1}$ H NMR (400 MHz, D $\left.2 \mathrm{O}, \delta / \mathrm{ppm}\right): 8.37$ (s, 1H), $8.18(\mathrm{~s}, 1 \mathrm{H}), 6.21(\mathrm{~d}, \mathrm{~J}=3.5 \mathrm{~Hz}, 1 \mathrm{H}), 5.34$ (dd, J = 6.2, $3.5 \mathrm{~Hz}, 1 \mathrm{H}), 5.17(\mathrm{dd}, \mathrm{J}=6.2,2.2 \mathrm{~Hz}, 1 \mathrm{H}), 4.61$ (The DHO peak overlaps with the signal $(1 \mathrm{H})), 4.09(\mathrm{~m}$, 2H), $2.25(\mathrm{dd}, \mathrm{J}=20.7,19.1 \mathrm{~Hz}, 2 \mathrm{H}), 1.61(\mathrm{~s}, 3 \mathrm{H}), 1.38(\mathrm{~s}, 3 \mathrm{H}) .{ }^{31} \mathbf{P}\left\{{ }^{1} \mathbf{H}\right\} \mathbf{N M R}(122 \mathrm{MHz}, \mathrm{D} 2 \mathrm{O}, \delta / \mathrm{ppm})$ : $18.1(\mathrm{~d}, \mathrm{~J}=7.1 \mathrm{~Hz}, 1 \mathrm{P}), 9.88(\mathrm{dd}, \mathrm{J}=25.0,7.0 \mathrm{~Hz}, 1 \mathrm{P}),-10.5(\mathrm{~d}, \mathrm{~J}=24.9 \mathrm{~Hz}, 1 \mathrm{P}) .{ }^{31} \mathbf{P}$ NMR $(162 \mathrm{MHz}$, $\left.\mathrm{D}_{2} \mathrm{O}, \delta / \mathrm{ppm}\right): 18.1(\mathrm{td}, \mathrm{J}=18.8,6.8 \mathrm{~Hz}, 1 \mathrm{P}), 10.4-9.39(\mathrm{~m}, 1 \mathrm{P}),-10.5(\mathrm{dt}, \mathrm{J}=24.9,6.5 \mathrm{~Hz}, 1 \mathrm{P}) .{ }^{\mathbf{1 3}} \mathrm{C}$ NMR (101 MHz, $\left.\mathrm{D}_{2} \mathrm{O}, \delta / \mathrm{ppm}\right):$ 155.6, 152.9, 148.8, 140.0, 118.7, 114.9, 90.0, 84.5 (d, J = 9.4 Hz), 83.1, 81.3, 65.5 (d, J = 5.4 Hz), 31.9 (dd, J = 129.4, 106.3 Hz), 26.1, 24.3.

HRMS (ESI) m/z for $\left[\mathrm{C}_{14} \mathrm{H}_{22} \mathrm{~N}_{6} \mathrm{O}_{11} \mathrm{P}_{3}\right]^{-}$: calcd. 543.0565, found, 543.0566.

Ring opening by using aq. sodium hydroxide: 2',3'-O-Isopropylideneadenosine 5'- $\beta, \gamma$-methylene triphosphate (39)

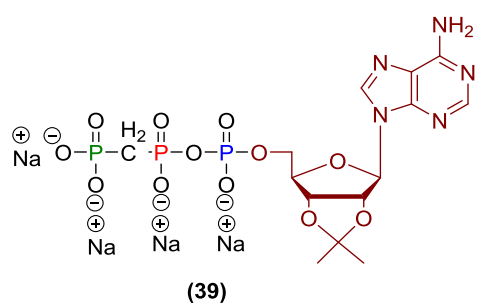

The reaction mixture of $\mathbf{B}_{12}(250 \mu \mathrm{L}, 13.0 \mu \mathrm{mol}, 1.0$ eq.) was added to $1 \mathrm{M}$ aq. $\mathrm{NaOH}(250 \mu \mathrm{L})$ and then $\mathrm{D}_{2} \mathrm{O}(100 \mu \mathrm{l})$ was added. The product 39 was isolated by Method A, affording a colourless solid (5.98 mg, $9.44 \mu \mathrm{mol}, 72 \%)$.

${ }^{1}$ H NMR (400 MHz, D $\left.2 \mathrm{O}, \delta / \mathrm{ppm}\right): 8.39$ (s, 1H), 8.18 (s, 1H), $6.21(\mathrm{~d}, \mathrm{~J}=3.5 \mathrm{~Hz}, 1 \mathrm{H}), 5.33$ (dd, J = 6.1, $3.6 \mathrm{~Hz}, 1 \mathrm{H}), 5.17(\mathrm{dd}, \mathrm{J}=6.2,2.2 \mathrm{~Hz}, 1 \mathrm{H}), 4.61$ (The DHO peak overlaps with the signal $(1 \mathrm{H})$ ), 4.28 $4.02(\mathrm{~m}, 2 \mathrm{H}), 2.20-1.99(\mathrm{~m}, 2 \mathrm{H}), 1.61(\mathrm{~s}, 3 \mathrm{H}), 1.38(\mathrm{~s}, 3 \mathrm{H}) .{ }^{31} \mathbf{P}\left\{{ }^{1} \mathbf{H}\right\} \mathbf{N M R}(122 \mathrm{MHz}, \mathrm{D} 2 \mathrm{O}, \delta / \mathrm{ppm})$ : $15.1(\mathrm{dd}, \mathrm{J}=23.9,6.2 \mathrm{~Hz}, 1 \mathrm{P}), 12.9(\mathrm{~d}, \mathrm{~J}=6.6 \mathrm{~Hz}, 1 \mathrm{P}),-10.0(\mathrm{~d}, \mathrm{~J}=23.9 \mathrm{~Hz}, 1 \mathrm{P}) .{ }^{31} \mathbf{P}$ NMR $(162 \mathrm{MHz}$, $\left.\mathrm{D}_{2} \mathrm{O}, \delta / \mathrm{ppm}\right): 15.5$ - $14.7(\mathrm{~m}, 1 \mathrm{P}), 12.9(\mathrm{td}, \mathrm{J}=18.4,6.6 \mathrm{~Hz}, 1 \mathrm{P}),-10.0(\mathrm{dt}, \mathrm{J}=24.2,6.5 \mathrm{~Hz}, 1 \mathrm{P}) .{ }^{13} \mathrm{C}$ NMR (101 MHz, D $\mathrm{O}, \delta / \mathrm{ppm}):$ 155.6, 152.9, 148.8, 140.0, 118.7, 114.9, 90.0, 84.5 (d, J = 9.4 Hz), 83.1, 81.3, 65.5 (d, J = 5.3 Hz), 32.0 (dd, J = 128.9, 106.5 Hz), 26.1, 24.3. 
HRMS (ESI) $\mathrm{m} / \mathrm{z}$ for $\left[\mathrm{C}_{14} \mathrm{H}_{20} \mathrm{~N}_{5} \mathrm{NaO}_{12} \mathrm{P}_{3}\right]^{-}$: calcd. 566.0224, found, 566.022

Ring opening by propargylamine: 2',3'- $O$-Isopropylideneadenosine 5 ' $-\gamma$ - $P$-propargylamino $\beta, \gamma-$ methylenetriphosphate (40)

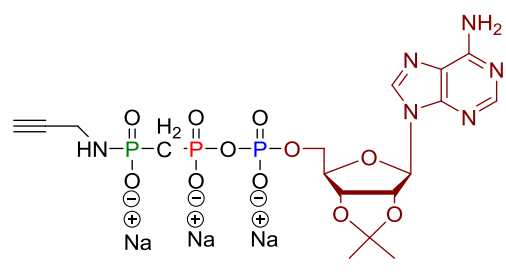

(40)

The reaction mixture of $\mathbf{B}_{12}(250 \mu \mathrm{L}, 13.0 \mu \mathrm{mol}, 1.0$ eq.) was added to propargylamine $(250 \mu \mathrm{L})$ and then $\mathrm{D}_{2} \mathrm{O}(100 \mu \mathrm{l})$ was added. The product 40 was isolated by Method A, affording a colourless solid (5.54 mg, $8.54 \mu \mathrm{mol}, 65 \%)$.

${ }^{1}$ H NMR (400 MHz, D $\left.2 \mathrm{O}, \delta / \mathrm{ppm}\right): 8.39$ (s, 1H), 8.18 (s, 1H), $6.21(\mathrm{~d}, \mathrm{~J}=3.5,1 \mathrm{H}), 5.34$ (dd, J=6.1, 3.5, $1 \mathrm{H}), 5.17(\mathrm{dd}, \mathrm{J}=6.1,2.2 \mathrm{~Hz}, 1 \mathrm{H}), 4.61$ (The DHO peak overlaps with the signal $(1 \mathrm{H})$ ), $4.17-4.03(\mathrm{~m}$, 2H), $3.53(\mathrm{dd}, \mathrm{J}=10.1,2.4 \mathrm{~Hz}, 2 \mathrm{H}), 2.42(\mathrm{t}, \mathrm{J}=2.5 \mathrm{~Hz}, 1 \mathrm{H}), 2.24(\mathrm{dd}, \mathrm{J}=20.6,18.9 \mathrm{~Hz}, 2 \mathrm{H}), 1.61(\mathrm{~s}$, $3 \mathrm{H}), 1.38$ (s, 3H). ${ }^{31} \mathbf{P}\left\{{ }^{1} \mathbf{H}\right\}$ NMR $\left(122 \mathrm{MHz}, \mathrm{D}_{2} \mathrm{O}, \delta / \mathrm{ppm}\right): 17.7$ (d, J = 7.1 Hz, 1P), 8.70 (dd, J = 26.3, 7.1 $\mathrm{Hz}, 1 \mathrm{P}),-11.5$ (d, J = 26.2 Hz, 1P). ${ }^{31}$ P NMR (162 MHz, D $\left.2 \mathrm{O}, \delta / \mathrm{ppm}\right): 18.9-16.9$ (m, 1P), 8.70 (dtd, J $=27.5,20.8,7.2,1 \mathrm{P}),-11.5(\mathrm{dt}, \mathrm{J}=27.5,5.2 \mathrm{~Hz}, 1 \mathrm{P}) .{ }^{13} \mathbf{C} \mathbf{N M R}\left(101 \mathrm{MHz}, \mathrm{D}_{2} \mathrm{O}, \delta / \mathrm{ppm}\right): 166.6,155.6$, 152.9, 148.8, 140.0, 118.7, 114.9, 90.0, $84.6(\mathrm{~d}, \mathrm{~J}=9.4 \mathrm{~Hz}), 83.9,81.3,65.6(\mathrm{~d}, \mathrm{~J}=4.9 \mathrm{~Hz}), 30.6(\mathrm{dd}, \mathrm{J}=$ 128.9, 106.5 Hz), 29.3, 26.1, 24.3.

HRMS (ESI) $\mathrm{m} / \mathrm{z}$ for $\left[\mathrm{C}_{17} \mathrm{H}_{22} \mathrm{~N}_{6} \mathrm{Na}_{3} \mathrm{O}_{11} \mathrm{P}_{3}\right]^{-}$: calcd: 648.0252, found, 648.0259.

Ring opening by using imidazole: 4'-Pentyne $\gamma$-imidazole $\beta, \gamma$-methylene triphosphate (S-7)

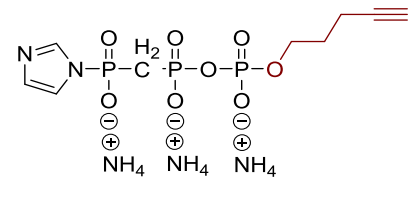

(S-7)

4-Pentyn-1-ol (40 mg, $0.45 \mathrm{mmol}, 1.5$ eq.) and ETT (98 mg, $0.75 \mathrm{mmol}, 2.5$ eq.) were coevaporated with dry MeCN $(2 \times 1 \mathrm{~mL})$. Under an atmosphere of dry Ar, a Pусн $2 \mathrm{PA} \mathrm{A2}(0.075 \mathrm{M}$ in $\mathrm{MeCN}, 4.0 \mathrm{~mL}$, $0.30 \mathrm{mmol}, 1.0$ eq.) was added in the above reaction mixture. The mixture was stirred at r.t. for 5 mins. Upon cooling to $0^{\circ} \mathrm{C}, \mathrm{mCPBA}(\leq 77 \%, 79 \mathrm{mg}, 0.45 \mathrm{mmol}, 1.5$ eq.) was added and the mixture was stirred for 5 mins until ${ }^{31} \mathrm{P}-\mathrm{NMR}$ confirmed complete oxidation.

A solution of imidazole in dry $\operatorname{DMF}(1.47 \mathrm{M}, 10.0 \mathrm{ml}, 14.7 \mathrm{mmol}, 49.0$ eq.) was added to the cyclotriphosphate and the reaction was stirred for another 15 mins (the reaction progress was followed by ${ }^{31} \mathrm{P}$ NMR) which results in quantative conversion to $\mathbf{S - 7}$. 
Note -: Imidazole was dried before using by coevaporation with $\mathrm{MeCN}$ and it was further stored of molecular sieve $(3 \AA)$.

${ }^{31} \mathbf{P}\left\{{ }^{1} \mathbf{H}\right\}$ NMR (122 MHz, D2O, $\left.\delta / p p m\right): 8.80(\mathrm{~d}, \mathrm{~J}=7.8 \mathrm{~Hz}, 1 \mathrm{P}), 4.30(\mathrm{dd}, \mathrm{J}=25.8,8.1 \mathrm{~Hz}, 1 \mathrm{P}),-10.9$ $(\mathrm{d}, \mathrm{J}=25.5 \mathrm{~Hz}, 1 \mathrm{P})$.

\section{5'-Adenosyl 4-Pentyne $\delta, \varepsilon$-methylene hexaphosphate (P4)}

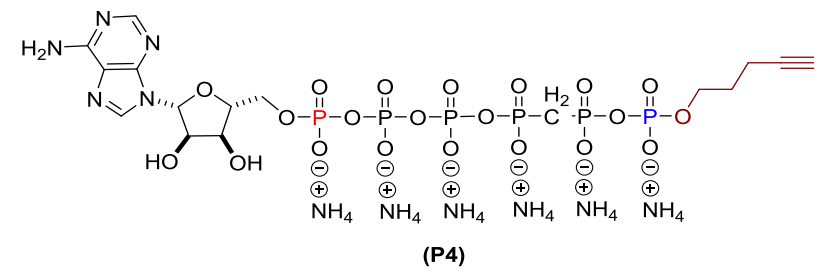

Anhydr. $\mathrm{ZnCl}_{2}(0.33$ g, $2.4 \mathrm{mmol}, 11$ eq. $)$ and ATP x 2.1 TBA (0.40 g, 0.39 mmol, 1.7 eq. $)$ were added to a freshly prepared solution of $\mathbf{S - 7}(0.22 \mathrm{mmol})$. The reaction mixture was then diluted with dry DMF: DMSO (v:v, 1:1, $16 \mathrm{ml}$ ) in order to increase the solubility and it was stirred at r.t. for $20 \mathrm{hrs}$. The crude product was purified by Method $\mathrm{B}$ and the target product was eluted with 550-700 mM conc. of $\mathrm{NH}_{4} \mathrm{HCO}_{3}$ buffer. Purification gave P4 as a colorless solid (0.03 g, $0.04 \mathrm{mmol}, 22 \%)$.

Note -: The reaction for capped hexapolyPs were not optimized.

${ }^{1}$ H NMR (500 MHz, D $\left.\mathrm{O}, \delta / \mathrm{ppm}\right): 8.47$ (s, 1H), 8.19 (s, 1H), 6.07 (d, J = 6.2 Hz, 1H), 4.74 (t, J = 5.7 Hz, 1H), 4.53 (dd, J = 5.2, $3.3 \mathrm{~Hz}, 1 \mathrm{H}), 4.35(\mathrm{~m}, \mathrm{~J}=2.9 \mathrm{~Hz}, 1 \mathrm{H}), 4.22$ (dt, J = 6.1, $2.9 \mathrm{~Hz}, 1 \mathrm{H}), 4.15$ (ddd, $\mathrm{J}=11.7,4.6,3.0 \mathrm{~Hz}, 1 \mathrm{H}), 3.96(\mathrm{q}, \mathrm{J}=6.5 \mathrm{~Hz}, 2 \mathrm{H}), 2.44(\mathrm{t}, \mathrm{J}=21.1 \mathrm{~Hz}, 2 \mathrm{H}), 2.36-2.24(\mathrm{~m}, 3 \mathrm{H}), 1.79$ $(\mathrm{m}, 2 \mathrm{H}) .{ }^{31} \mathbf{P}\left\{{ }^{1} \mathbf{H}\right\}$ NMR $\left(202 \mathrm{MHz}, \mathrm{D}_{2} \mathrm{O}, \delta / \mathrm{ppm}\right): 8.61-7.57(\mathrm{~m}, 1 \mathrm{P}), 7.31-6.77(\mathrm{~m}, 1 \mathrm{P}),-10.6(\mathrm{~d}, \mathrm{~J}=$ $26.1 \mathrm{~Hz}, 1 \mathrm{P}),-11.3$ (d, J = 22.1 Hz, 1P), -22.5 - -23.8 (m, 2P). ${ }^{31} \mathbf{P}$ NMR (162 MHz, $\left.\mathrm{D}_{2} \mathrm{O}, \delta / \mathrm{ppm}\right): 8.37-$ 7.59 (m, 1P), $7.59-6.29$ (m, 1P), -10.6 (dt, J = 26.0, 7.1, 1P), -11.4 (d, J = 16.6, 5.1 Hz, 1P), -21.6 -23.2 (m, 2P). ${ }^{13} \mathrm{C}$ NMR (126 MHz, $\left.\mathrm{D}_{2} \mathrm{O}, \delta / \mathrm{ppm}\right): 155.7,152.9,149.2,139.9,118.6,86.6,85.2,84.2$ (d, $\mathrm{J}=9.2 \mathrm{~Hz}), 74.2,70.4,69.5,65.4(\mathrm{~d}, \mathrm{~J}=5.6 \mathrm{~Hz}), 65.0(\mathrm{~d}, \mathrm{~J}=5.9 \mathrm{~Hz}), 29.9(\mathrm{t}, \mathrm{J}=131.5 \mathrm{~Hz}), 28.8(\mathrm{~d}, \mathrm{~J}=$ $7.6 \mathrm{~Hz}), 14.2(\mathrm{~d}, \mathrm{~J}=3.9 \mathrm{~Hz})$.

HRMS (ESI) $\mathrm{m} / \mathrm{z}$ for $\left[\mathrm{C}_{16} \mathrm{H}_{27} \mathrm{DN}_{5} \mathrm{O}_{21} \mathrm{P}_{6}\right]^{+}$calcd 812.9760, found 812.9750. 


\subsubsection{Synthesis of triphosphates based on $c$-Pyсr2 $\mathrm{PA}\left(\mathrm{A}_{3}\right)$}

3'-Azido-3'-deoxythymidine $\beta, \gamma$-difluoromethylene cyclotriphosphate (B13)

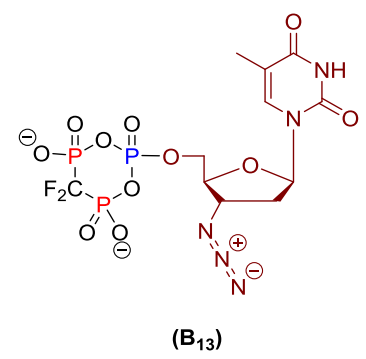

AZT (44 mg, $0.16 \mathrm{mmol}, 1.5$ eq.) and ETT (50 mg, $0.38 \mathrm{mmol}, 3.5$ eq.) were coevaporated with dry $\mathrm{MeCN}(2 \times 1 \mathrm{~mL})$. Under an $\mathrm{Ar}$ atmosphere, a reaction mixture containing the $c$ - $\mathrm{Py}_{\mathrm{CF} 2} \mathrm{PA} \mathbf{A}_{3}(0.073 \mathrm{M}$ in $\mathrm{MeCN}, 1.5 \mathrm{~mL}, 0.11 \mathrm{mmol}, 1.0$ eq.) was added to the dried solids and it was stirred at r.t. for 10 mins. Upon cooling to $0^{\circ} \mathrm{C}, \mathrm{mCPBA}(\leq 77 \%, 24 \mathrm{mg}, 0.16 \mathrm{mmol}, 1.5$ eq. $)$ was added and the mixture was stirred for 5 mins until ${ }^{31} \mathrm{P}-\mathrm{NMR}$ confirmed complete oxidation (a formation of triplet at $-25 \mathrm{ppm}$ which is diagnostic signal for $\left.\mathbf{B}_{13}\right)$.

${ }^{31} \mathbf{P}\left\{{ }^{1} \mathbf{H}\right\}$ NMR (122 MHz, CD $\left.3 \mathrm{CN}, \delta / p p m\right):-9.21(\mathrm{dt}, \mathrm{J}=87.1,23.8 \mathrm{~Hz}, 2 \mathrm{P}),-25.4(\mathrm{t}, \mathrm{J}=23.6 \mathrm{~Hz}, 1 \mathrm{P})$. ${ }^{31} \mathbf{P}$ NMR (162 MHz, $\left.\mathrm{CD}_{3} \mathrm{CN}, \delta / \mathrm{ppm}\right):-9.22(\mathrm{dt}, \mathrm{J}=86.8,23.6 \mathrm{~Hz}, 2 \mathrm{P}),-24.5--25.9$ (m, 1P).

Ring opening by using propargylamine: 3'-Azido-3'-deoxythymidine 5'- $\gamma$-P-propargylamine $\beta, \gamma$ difluoromethylene triphosphate (41)

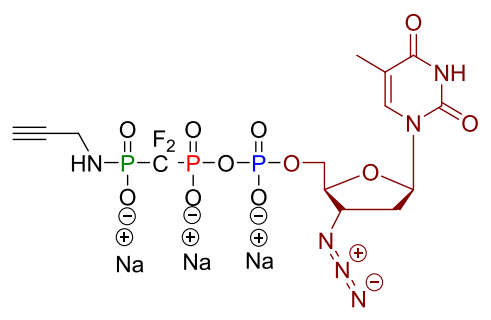

(41)

The reaction mixture of $\mathbf{B}_{13}(500 \mu \mathrm{L}, 36.5 \mu \mathrm{mol}, 1.0$ eq.) was added to propargylamine (500 $\mu \mathrm{L})$ and then $\mathrm{D}_{2} \mathrm{O}(100 \mu \mathrm{l})$ was added. The product 41 was isolated by Method A, affording a colourless solid (18.4 mg, $30.8 \mu \mathrm{mol}, 85 \%)$.

${ }^{1}$ H NMR (400 MHz, D $\left.2 \mathrm{O}, \delta / \mathrm{ppm}\right): 7.69(\mathrm{~s}, 1 \mathrm{H}), 6.21(\mathrm{t}, \mathrm{J}=6.9 \mathrm{~Hz}, 1 \mathrm{H}), 4.55-4.46(\mathrm{~m}, 1 \mathrm{H}), 4.33-4.03$ $(\mathrm{m}, 3 \mathrm{H}), 3.66(\mathrm{dd}, \mathrm{J}=8.7,2.2 \mathrm{~Hz}, 2 \mathrm{H}), 2.47(\mathrm{t}, \mathrm{J}=2.5 \mathrm{~Hz}, 1 \mathrm{H}), 2.46-2.33(\mathrm{~m}, 2 \mathrm{H}), 1.86(\mathrm{~s}, 3 \mathrm{H}) .{ }^{31} \mathbf{P}\left\{{ }^{1} \mathbf{H}\right\}$ NMR (122 MHz, $\left.\mathrm{D}_{2} \mathrm{O}, \delta / \mathrm{ppm}\right): 7.56-5.23$ (m, 1P), -5.38 (tdd, J = 87.9, 59.4, 31.4 Hz, 1P), -11.6 (d, J = $31.8 \mathrm{~Hz}, 1 \mathrm{P}) .{ }^{31} \mathbf{P}$ NMR $\left(162 \mathrm{MHz}, \mathrm{D}_{2} \mathrm{O}, \delta / \mathrm{ppm}\right): 7.44-5.29(\mathrm{~m}, 1 \mathrm{P}),-5.41$ (tdd, J = 87.6, 59.3, $31.3 \mathrm{~Hz}$, 1P), -11.6 (dt, J = 27.5, 5.2 Hz, 1P). ${ }^{13}$ C NMR (101 MHz, D $\left.2 \mathrm{O}, \delta / p p m\right): 166.7,151.8,137.2,111.9,84.8$, $83.0(\mathrm{~d}, \mathrm{~J}=9.4 \mathrm{~Hz}), 70.5,65.6(\mathrm{~d}, \mathrm{~J}=5.8 \mathrm{~Hz}), 60.9,36.3,30.9,11.7 .{ }^{19} \mathbf{F}$ NMR $\left(377 \mathrm{MHz}, \mathrm{D}_{2} \mathrm{O}, \delta / \mathrm{ppm}\right)$ : $-119.2(\mathrm{dd}, \mathrm{J}=87.8,78.0 \mathrm{~Hz}, 2 \mathrm{~F})$.

HRMS (ESI) $\mathrm{m} / \mathrm{z}$ for $\left[\mathrm{C}_{14} \mathrm{H}_{18} \mathrm{~F}_{2} \mathrm{~N}_{6} \mathrm{NaO}_{11} \mathrm{P}_{3}\right]$ : calcd. 600.0112 found 600.0108 . 
Ring opening by using aq. ammonia: 3'-Azido-3'-deoxythymidine 5 '- $\gamma$ - $P$-amino $\beta, \gamma$ difluoromethylene triphosphate (42)

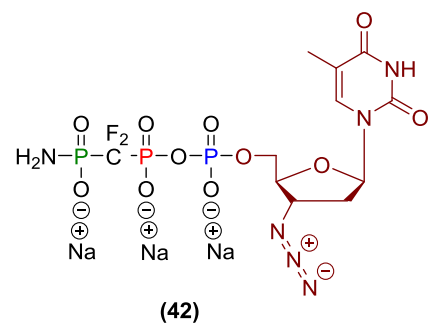

The reaction mixture of $\mathbf{B}_{13}\left(500 \mu \mathrm{L}, 36.5 \mu\right.$ mol, 1.0 eq.) was added to $25 \%$ aq. $\mathrm{NH}_{4} \mathrm{OH}(500 \mu \mathrm{L})$ and $\mathrm{D}_{2} \mathrm{O}(100 \mu \mathrm{l})$ was added. The product 42 was isolated by Method A, affording a colourless solid (14.1 mg, $26.7 \mu \mathrm{mol}, 73 \%)$.

The analytical data are consistent with literature. ${ }^{12}$

${ }^{1}$ H NMR (400 MHz, D $\left.2 \mathrm{O}, \delta / \mathrm{ppm}\right): 7.66(\mathrm{~s}, 1 \mathrm{H}), 6.21(\mathrm{t}, \mathrm{J}=6.9 \mathrm{~Hz}, 1 \mathrm{H}), 4.65-4.46(\mathrm{~m}, 1 \mathrm{H}), 4.23-3.97$ (m, 3H), $2.59-2.33(\mathrm{~m}, 2 \mathrm{H}), 1.85(\mathrm{~s}, 3 \mathrm{H}) .{ }^{31} \mathbf{P}\left\{{ }^{1} \mathbf{H}\right\}$ NMR (122 MHz, $\left.\mathrm{D}_{2} \mathrm{O}, \delta / \mathrm{ppm}\right): 8.36$ (td, J = 78.7, $59.5 \mathrm{~Hz}, 1 \mathrm{P}),-5.18$ (tdd, J = 87.9, 59.4, 31.4 Hz, 1P), -11.56 (d, J = 31.2, 1P). ${ }^{31} \mathbf{P}$ NMR $\left(162 \mathrm{MHz}, \mathrm{D}_{2} \mathrm{O}\right.$,

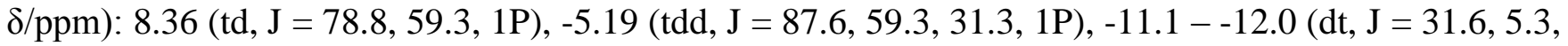
1P). ${ }^{13} \mathrm{C}$ NMR (101 MHz, $\left.\mathrm{D}_{2} \mathrm{O}, \delta / \mathrm{ppm}\right): 168.7,153.3,137.0,111.9,84.9,82.9$ (d, J = 9.4 Hz), 65.7 (d, J $=5.8 \mathrm{~Hz}), 61.0,36.2,11.9 .{ }^{19} \mathbf{F}$ NMR $\left(377 \mathrm{MHz}, \mathrm{D}_{2} \mathrm{O}, \delta / \mathrm{ppm}\right):-121.1(\mathrm{dd}, \mathrm{J}=87.9,78.1 \mathrm{~Hz}, 2 \mathrm{~F})$.

HRMS (ESI) $\mathrm{m} / \mathrm{z}$ for $\left[\mathrm{C}_{11} \mathrm{H}_{15} \mathrm{~F}_{2} \mathrm{~N}_{6} \mathrm{NaO}_{11} \mathrm{P}_{3}\right]^{-}$: calcd 560.9883 found 560.9886.

Ring opening by using aq. sodium hydroxide: 3'-Azido-3'-deoxythymidine 5'- $\beta, \gamma$ difluoromethylene triphosphate (43)

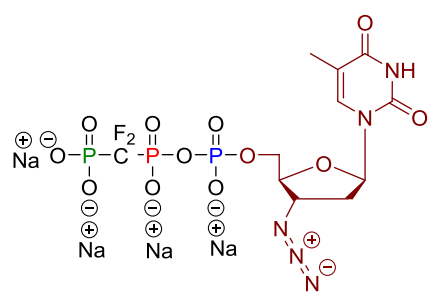

(43)

The reaction mixture of $\mathbf{B}_{13}(500 \mu \mathrm{L}, 36.5 \mu \mathrm{mol}, 1.0$ eq.) was added to aq. $\mathrm{NaOH}(1 \mathrm{M}, 500 \mu \mathrm{L})$ and then $\mathrm{D}_{2} \mathrm{O}(100 \mu \mathrm{l})$ was added. The product 43 was isolated by Method A, affording a colourless solid (15.1 mg, $23.8 \mu \mathrm{mol}, 65 \%)$.

The analytical data are consistent with literature. ${ }^{12}$

${ }^{1}$ H NMR (400 MHz, D $\left.2 \mathrm{O}, \delta / \mathrm{ppm}\right): 7.65$ (s, 1H), $6.22(\mathrm{t}, \mathrm{J}=7.0 \mathrm{~Hz}, 1 \mathrm{H}), 4.58$ - 4.44 (m, 1H), 4.28 - 4.05

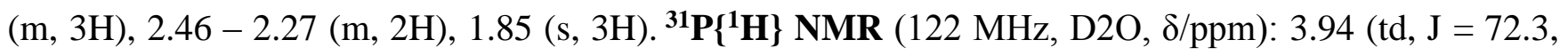
$56.6 \mathrm{~Hz}, 1 \mathrm{P}),-2.70$ (tdd, J = 89.5, 56.7, $32.2 \mathrm{~Hz}, 1 \mathrm{P}),-11.2$ (d, J = 32.2, 1P). ${ }^{31} \mathbf{P}$ NMR $\left(162 \mathrm{MHz}, \mathrm{D}_{2} \mathrm{O}\right.$,

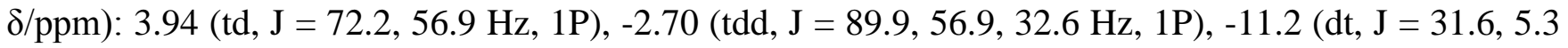
$\mathrm{Hz}, 1 \mathrm{P}) .{ }^{13} \mathrm{C}$ NMR $\left(101 \mathrm{MHz}, \mathrm{D}_{2} \mathrm{O}, \delta / \mathrm{ppm}\right): 168.7,153.3,137.0,111.9,84.8,82.9$ (d, J = 9.4 Hz), 65.6 $(\mathrm{d}, \mathrm{J}=5.8 \mathrm{~Hz}), 61.0,36.2,11.9 .{ }^{19} \mathbf{F}$ NMR $\left(377 \mathrm{MHz}, \mathrm{D}_{2} \mathrm{O}, \delta / \mathrm{ppm}\right):-118.1(\mathrm{dd}, \mathrm{J}=89.9,72.8 \mathrm{~Hz}, 2 \mathrm{~F})$. 
HRMS (ESI) $\mathrm{m} / \mathrm{z}$ for $\left[\mathrm{C}_{11} \mathrm{H}_{14} \mathrm{~F}_{2} \mathrm{~N}_{5} \mathrm{O}_{12} \mathrm{P}_{3}\right]^{2-}$ : calcd 269.4915, found 269.4916.

Ring opening by using morpholine: 3'-Azido-3'-deoxythymidine 5'- $\gamma$ - $P$-morpholino $\beta, \gamma$ difluoromethylene triphosphate (44)

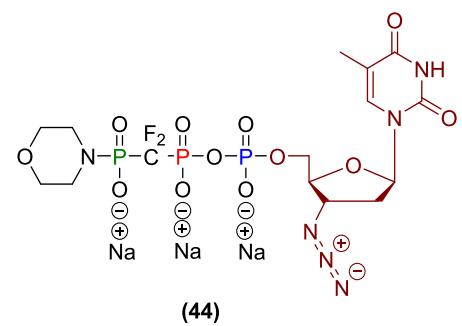

The reaction mixture of $\mathbf{B}_{13}(250 \mu \mathrm{L}, 20.2 \mu \mathrm{mol}, 1.0$ eq.) was added to morpholine (250 $\mu \mathrm{L})$ and then $\mathrm{D}_{2} \mathrm{O}$ $(100 \mu \mathrm{l})$ was added. The product 44 was isolated by Method A, affording a colourless solid (10.2 mg, $15.1 \mu \mathrm{mol}, 74 \%)$.

${ }^{1}$ H NMR (400 MHz, D $\left.2 \mathrm{O}, \delta / \mathrm{ppm}\right): 7.70(\mathrm{~s}, 1 \mathrm{H}), 6.23(\mathrm{t}, \mathrm{J}=6.9 \mathrm{~Hz}, 1 \mathrm{H}), 4.55-4.49$ (m, 1H), 4.16 (d, J $=4.6 \mathrm{~Hz}, 3 \mathrm{H}), 3.61(\mathrm{~m}, 4 \mathrm{H}), 3.07(\mathrm{~s}, 4 \mathrm{H}), 2.75-2.25(\mathrm{~m}, 2 \mathrm{H}), 1.87(\mathrm{~s}, 3 \mathrm{H}) .{ }^{31} \mathbf{P}\left\{{ }^{1} \mathbf{H}\right\} \mathbf{~ N M R}(122 \mathrm{MHz}$, $\left.\mathrm{D}_{2} \mathrm{O}, \delta / \mathrm{ppm}\right): 5.08(\mathrm{td}, \mathrm{J}=77.4,58.0 \mathrm{~Hz}, 1 \mathrm{P}),-5.51(\mathrm{tdd}, \mathrm{J}=86.9,58.1,31.7 \mathrm{~Hz}, 1 \mathrm{P}),-11.5(\mathrm{~d}, \mathrm{~J}=31.6$ $\mathrm{Hz}, 1 \mathrm{P}) .{ }^{31} \mathbf{P}$ NMR $\left(162 \mathrm{MHz}, \mathrm{D}_{2} \mathrm{O}, \delta / \mathrm{ppm}\right): 7.96-3.69(\mathrm{~m}, 1 \mathrm{P}),-3.35$ - -7.45 (m, 1P), -11.5 (dt, J = 32.3, $5.3 \mathrm{~Hz}, 1 \mathrm{P}) .{ }^{13} \mathrm{C}$ NMR (101 MHz, $\left.\mathrm{D}_{2} \mathrm{O}, \delta / \mathrm{ppm}\right): 166.8,151.9,137.3,111.9,84.9,83.0$ (d, J = 9.5 Hz), $67.7(\mathrm{~d}, \mathrm{~J}=3.9 \mathrm{~Hz}), 65.6(\mathrm{~d}, \mathrm{~J}=5.5 \mathrm{~Hz}), 64.9,44.8(\mathrm{~d}, \mathrm{~J}=2.2 \mathrm{~Hz}), 36.3,11.7 .{ }^{19} \mathbf{F}$ NMR $\left(471 \mathrm{MHz}, \mathrm{D}_{2} \mathrm{O}\right.$, $\delta / \mathrm{ppm}):-118.1$ (ddd, $\mathrm{J}=87.6,77.3,3.5 \mathrm{~Hz}, 2 \mathrm{~F})$.

HRMS (ESI) $\mathrm{m} / \mathrm{z}$ for $\left[\mathrm{C}_{15} \mathrm{H}_{22} \mathrm{~F}_{2} \mathrm{~N}_{6} \mathrm{NaO}_{12} \mathrm{P}_{3}\right]^{-}$: calcd 632.0374 , found 632.0371 .

Ring opening by using aniline: 3'-Azido-3'-deoxythymidine 5 '- $\gamma$-P-anilino $\beta$, $\gamma$-difluoromethylene triphosphate (45)

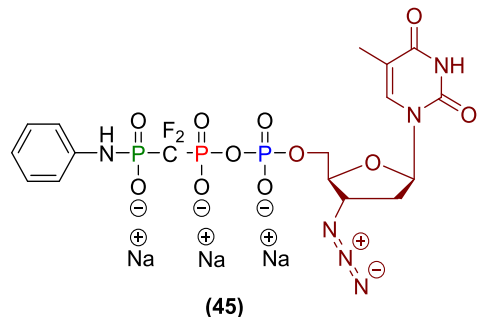

The reaction mixture of $\mathbf{B}_{13}\left(250 \mu \mathrm{L}, 20.2 \mu \mathrm{mol}, 1.0\right.$ eq.) was added to aniline $(250 \mu \mathrm{L})$ and then $\mathrm{D}_{2} \mathrm{O}$ $(100 \mu \mathrm{l})$ was added. The product $\mathbf{4 5}$ was isolated by Method A, affording a colourless solid (11.0 mg, $16.1 \mu \mathrm{mol}, 79 \%)$.

${ }^{1}$ H NMR (400 MHz, D $\left.2 \mathrm{O}, \delta / \mathrm{ppm}\right): 7.55$ (s, 1H), $7.30-7.00$ (m, 4H), 6.83 (tt, J = 7.0, $\left.1.4 \mathrm{~Hz}, 1 \mathrm{H}\right), 6.13$ $(\mathrm{t}, \mathrm{J}=6.9 \mathrm{~Hz}, 1 \mathrm{H}), 4.34(\mathrm{dt}, \mathrm{J}=6.9,3.6 \mathrm{~Hz}, 1 \mathrm{H}), 4.11-3.97(\mathrm{~m}, 3 \mathrm{H}), 2.36-2.18(\mathrm{~m}, 2 \mathrm{H}), 1.80$ (s, 3H). ${ }^{31} \mathbf{P}\left\{{ }^{1} \mathbf{H}\right\}$ NMR $\left(122 \mathrm{MHz}, \mathrm{D}_{2} \mathrm{O}, \delta / \mathrm{ppm}\right): 1.44(\mathrm{td}, \mathrm{J}=80.8,59.1 \mathrm{~Hz}, 1 \mathrm{P}),-5.44$ (tdd, J = 87.0, 59.2, $30.8 \mathrm{~Hz}, 1 \mathrm{P}),-11.4$ (d, J = $30.8 \mathrm{~Hz}, 1 \mathrm{P}) .{ }^{31} \mathbf{P}$ NMR (162 MHz, $\left.\mathrm{D}_{2} \mathrm{O}, \delta / \mathrm{ppm}\right): 1.44$ (td, J = 80.8, $59.4 \mathrm{~Hz}$, 1P), -3.62 - -6.92 (m, 1P), -11.4 (dt, J = 30.6, $5.3 \mathrm{~Hz}, 1 \mathrm{P}) .{ }^{13} \mathrm{C}$ NMR (101 MHz, $\left.\mathrm{D}_{2} \mathrm{O}, \delta / \mathrm{ppm}\right): 166.5$, 
151.6, 141.8, 137.1, 128.8, 120.9, 118.4 (d, J = 5.4 Hz), 111.8, 84.7, 82.9 (d, J = 9.7 Hz), 65.5 (d, J = 5.5 $\mathrm{Hz}), 60.8,36.2,11.6 .{ }^{19} \mathbf{F}$ NMR (471 MHz, $\left.\mathrm{D}_{2} \mathrm{O}, \delta / \mathrm{ppm}\right):-119.3$ (ddd, $J=88.5,77.7,11.2 \mathrm{~Hz}, 2 \mathrm{~F}$ ).

HRMS (ESI) $\mathrm{m} / \mathrm{z}$ for $\left[\mathrm{C}_{17} \mathrm{H}_{21} \mathrm{~F}_{2} \mathrm{~N}_{6} \mathrm{NaO}_{11} \mathrm{P}_{3}\right]^{+}$: calcd 639.0341, found 639.0337 .

Ring opening by using diethylamine: 3'-Azido-3'-deoxythymidine 5'- $\gamma$-P-diethylamino $\beta, \gamma$ difluoromethylene triphosphate (46)

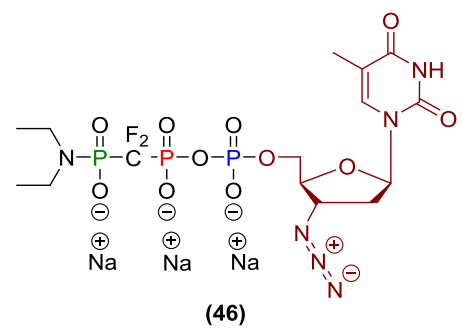

The reaction mixture of $\mathbf{B}_{13}(250 \mu \mathrm{L}, 20.2 \mu \mathrm{mol}, 1.0$ eq.) was added to diethylamine (250 $\mu \mathrm{L})$ and then $(100 \mu \mathrm{l})$ was added. The product 46 was isolated by Method A, affording a colourless solid (7.40 mg, $11.2 \mu \mathrm{mol}, 55 \%)$.

${ }^{1}$ H NMR (400 MHz, D $\left.2 \mathrm{O}, \delta / \mathrm{ppm}\right): 7.68(\mathrm{~s}, 1 \mathrm{H}), 6.24(\mathrm{t}, \mathrm{J}=6.9 \mathrm{~Hz} 1 \mathrm{H}), 4.63-4.46(\mathrm{~m}, 1 \mathrm{H}), 4.36-4.27$ (m, 1H), $4.32-4.04(\mathrm{~m}, 2 \mathrm{H}), 3.09-2.95(\mathrm{~m}, 4 \mathrm{H}), 2.70-2.33(\mathrm{~m}, 2 \mathrm{H}), 1.87(\mathrm{~s}, 3 \mathrm{H}), 1.21(\mathrm{t}, \mathrm{J}=7.3 \mathrm{~Hz}$, $3 \mathrm{H}), 1.01(\mathrm{t}, \mathrm{J}=7.0 \mathrm{~Hz}, 3 \mathrm{H}) .{ }^{31} \mathbf{P}\left\{{ }^{1} \mathbf{H}\right\} \mathbf{N M R}\left(122 \mathrm{MHz}, \mathrm{D}_{2} \mathrm{O}, \delta / \mathrm{ppm}\right): 8.32(\mathrm{td}, \mathrm{J}=75.4,57.6 \mathrm{~Hz}, 1 \mathrm{P})$, 4.77 (tdd, J = 88.8, 57.6, $32.1 \mathrm{~Hz}, 1 \mathrm{P}),-11.4$ (d, J = 32.2 Hz, 1P). ${ }^{31} \mathbf{P}$ NMR $\left(162 \mathrm{MHz}, \mathrm{D}_{2} \mathrm{O}, \delta / \mathrm{ppm}\right)$ : 8.31 (tdt, J = 76.1, 57.4, 9.9 Hz, 1P), -4.78 (tdd, J = 88.8, 57.5, 31.9 Hz, 1P), -11.4 (dd, J = 31.5, 5.7 Hz, 1P). ${ }^{13} \mathrm{C}$ NMR (101 MHz, D $\left.2 \mathrm{O}, \delta / \mathrm{ppm}\right): 166.7,152.0,137.3,111.8,84.8,83.0$ (d, J = 9.5 Hz), 65.6 (d, J $=5.8 \mathrm{~Hz}), 61.0,40.5(\mathrm{~d}, \mathrm{~J}=3.7 \mathrm{~Hz}), 36.2,13.8(\mathrm{~d}, \mathrm{~J}=3.7 \mathrm{~Hz}), 11.7 .{ }^{19} \mathbf{F}$ NMR $\left(471 \mathrm{MHz}, \mathrm{D}_{2} \mathrm{O}, \delta / \mathrm{ppm}\right)$ : -117.7 (dd, $\mathrm{J}=88.5,74.7 \mathrm{~Hz}, 2 \mathrm{~F})$.

HRMS (ESI) $\mathrm{m} / \mathrm{z}$ for $\left[\mathrm{C}_{15} \mathrm{H}_{24} \mathrm{~F}_{2} \mathrm{~N}_{6} \mathrm{O}_{11} \mathrm{P}_{3}\right]^{-:}$calcd 595.0689, found 595.0683.

\section{3'-Azido-3'-deoxythymidine 5'- $\beta, \gamma$-difluoromethylene $\alpha$-P-thiocyclotriphosphate $\left(B_{14}\right)$}

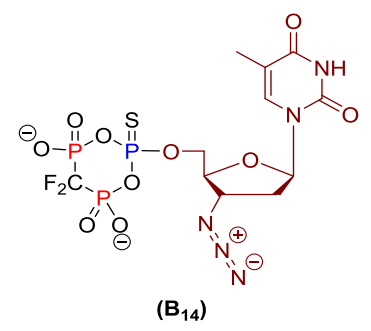

AZT (52 mg, $0.19 \mathrm{mmol}, 1.2$ eq.) and ETT ( $84 \mathrm{mg}, 0.65 \mathrm{mmol}, 4.0$ eq.) were coevaporated with dry $\mathrm{MeCN}(2 \times 2 \mathrm{~mL})$. Under an Ar atmosphere, a reaction mixture containing the $c$-PyCF2 $\mathrm{PA} \mathrm{A3}(0.081 \mathrm{M}$ in $\mathrm{MeCN}, 2.0 \mathrm{~mL}, 0.16 \mathrm{mmol}, 1.0$ eq.) was added to the dried solids and it was stirred at r.t. for $10 \mathrm{mins}$. Upon cooling to $0^{\circ} \mathrm{C}$, Becauge's reagent $(55 \mathrm{mg}, 0.27 \mathrm{mmol}, 1.7 \mathrm{eq}$.$) was added and the mixture was$ stirred for 5 mins until ${ }^{31} \mathrm{P}-\mathrm{NMR}$ confirmed complete oxidation (formation of triplet at $+40.0 \mathrm{ppm}$ which is diagnostic signal for the $\alpha-(\mathrm{S})$-cyclotriphosphate $\left.\mathbf{B}_{14}\right)$. 
${ }^{31} \mathbf{P}\left\{{ }^{1} \mathbf{H}\right\}$ NMR $\left(122 \mathrm{MHz}, \mathrm{CDCl}_{3}, \delta / \mathrm{ppm}\right): 39.4(\mathrm{t}, \mathrm{J}=35.8 \mathrm{~Hz}, 1 \mathrm{P}),-8.72(\mathrm{td}, \mathrm{J}=88.0,35.9 \mathrm{~Hz}, 2 \mathrm{P})$.

Ring opening by using aq. ammonia: 3'-Azido-3'-deoxythymidine $\quad 5^{\prime}-\gamma$ - $P$-amino $\beta, \gamma$ difluoromethylene- $\alpha$-P-thiotriphosphate (47)

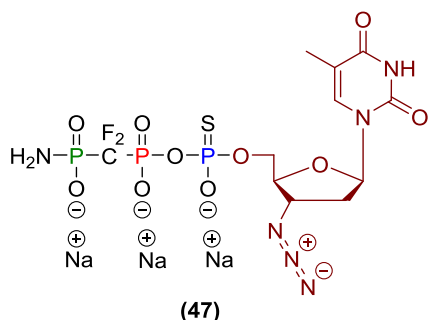

The reaction mixture of $\mathbf{B}_{14}\left(250 \mu \mathrm{L}, 20.2 \mu \mathrm{mol}, 1.0\right.$ eq.) was added to $25 \%$ aq. $\mathrm{NH}_{4} \mathrm{OH}(250 \mu \mathrm{L})$ and then $\mathrm{D}_{2} \mathrm{O}(100 \mu \mathrm{l})$ was added. The product 47 was isolated by Method A, affording a colourless solid, whereas products were obtained as mixtures of diastereoisomers $(8.70 \mathrm{mg}, 13.9 \mu \mathrm{mol}, 69 \%)$.

${ }^{1}$ H NMR (300 MHz, D $\left.2 \mathrm{O}, \delta / \mathrm{ppm}\right): 7.78$ (s, 1H), $6.24(\mathrm{t}, \mathrm{J}=6.9,1 \mathrm{H}), 4.54(\mathrm{~m}, 1 \mathrm{H}), 4.22(\mathrm{~m}, 3 \mathrm{H}), 2.64$ $2.31(\mathrm{~m}, 2 \mathrm{H}), 1.90$ (s, 3H). ${ }^{31} \mathbf{P}\left\{{ }^{1} \mathbf{H}\right\}$ NMR (122 MHz, D $\left.2 \mathrm{O}, \delta / \mathrm{ppm}\right): 42.9$ (dd, J = 38.2, 29.2, 1P), 12.0 $5.88(\mathrm{~m}, 1 \mathrm{P}),-5.60(\mathrm{tdd}, \mathrm{J}=87.7,58.9,38.0 \mathrm{~Hz}, 1 \mathrm{P}) .{ }^{31} \mathbf{P}$ NMR $\left(162 \mathrm{MHz}, \mathrm{D}_{2} \mathrm{O}, \delta / \mathrm{ppm}\right): 44.2-41.7(\mathrm{~m}$, 1P), 12.5 - 6.70 (m, 1P), -3.77 - -7.02 (m, 1P). ${ }^{13}$ C NMR (101 MHz, $\left.\mathrm{D}_{2} \mathrm{O}, \delta / \mathrm{ppm}\right): 168.7,153.3,137.0$, 111.9, 84.9, $82.9(\mathrm{~d}, \mathrm{~J}=9.4 \mathrm{~Hz}), 65.7(\mathrm{~d}, \mathrm{~J}=5.8 \mathrm{~Hz}), 61.0,36.2,11.9 .{ }^{19} \mathbf{F}$ NMR $\left(377 \mathrm{MHz}, \mathrm{D}_{2} \mathrm{O}\right.$, $\delta / \mathrm{ppm}):-121.0(\mathrm{ddd}, \mathrm{J}=87.7,78.7,6.2 \mathrm{~Hz}, 2 \mathrm{~F})$.

HRMS (ESI) $\mathrm{m} / \mathrm{z}$ for $\left[\mathrm{C}_{11} \mathrm{H}_{16} \mathrm{~F}_{2} \mathrm{~N}_{6} \mathrm{O}_{10} \mathrm{P}_{3} \mathrm{~S}\right]^{-}$: calcd 554.9835, found 554.9839.

2',3'-didehydro-2',3'-dideoxythymidine 5'- $\beta, \gamma$-difluoromethylene cyclotriphosphate (B15)

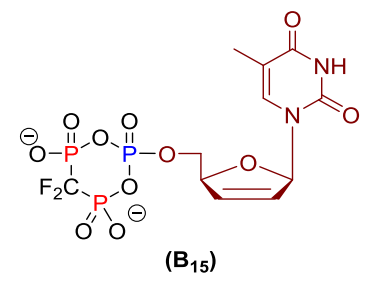

d4T (40 mg, $0.17 \mathrm{mmol}, 1.2$ eq.) and ETT (67 mg, $0.5 \mathrm{mmol}, 3.5$ eq.) were coevaporated with dry MeCN $(2 \times 1 \mathrm{~mL})$ and the dried solids were dissolved in dry DMF $(1 \mathrm{~mL})$. Under an Ar atmosphere, a reaction mixture containing the $c$-PyCF2 $\mathrm{PA} \mathbf{A}_{3}(0.073 \mathrm{M}$ in $\mathrm{MeCN}, 2.0 \mathrm{~mL}, 0.15 \mathrm{mmol}, 1.0$ eq.) was added and it was stirred at r.t. for 10 mins. Upon cooling to $0^{\circ} \mathrm{C}, \mathrm{mCPBA}(\leq 77 \%, 45 \mathrm{mg}, 0.26 \mathrm{mmol}, 1.7 \mathrm{eq}$.) was added and the mixture was stirred for 5 mins until ${ }^{31} \mathrm{P}-\mathrm{NMR}$ confirmed complete oxidation (reaction progress was followed by ${ }^{31} \mathrm{P}$ NMR, and formation of triplet at $-23 \mathrm{ppm}$ which is diagnostic signal for the d4T cyclotriphosphate $\mathbf{B} 15)$.

${ }^{31} \mathbf{P}\left\{{ }^{1} \mathbf{H}\right\}$ NMR $\left(162 \mathrm{MHz}, \mathrm{CD}_{3} \mathrm{CN}, \delta / \mathrm{ppm}\right):-9.21(\mathrm{dt}, \mathrm{J}=87.1,23.8 \mathrm{~Hz}, 2 \mathrm{P}),-25.4(\mathrm{t}, \mathrm{J}=23.6 \mathrm{~Hz}, 1 \mathrm{P})$. 
Ring opening by using propargylamine: 2',3'-didehydro-2',3'-dideoxythymidine 5'- $\gamma$ propargylamine $\beta, \gamma$-(difluoromethylene)triphosphate (48)

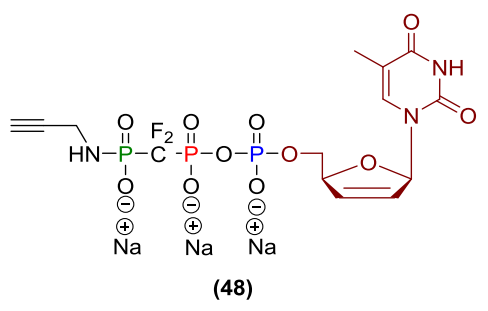

The reaction mixture of $\mathbf{B}_{15}(250 \mu \mathrm{L}, 12.2 \mu \mathrm{mol}, 1.0$ eq.) was added to propargylamine $(250 \mu \mathrm{L})$ and then $\mathrm{D}_{2} \mathrm{O}(100 \mu \mathrm{l})$ was added afterwards it was stirred for 5 mins. The product $\mathbf{4 8}$ was isolated by Method A, affording a colourless solid. The obtained colourless precipitate was washed with methanol twice and isolated by centrifugation and dried under reduced pressure $(6.60 \mathrm{mg}, 10.8 \mu \mathrm{mol}, 89 \%)$.

${ }^{1} \mathbf{H}$ NMR (400 MHz, D $\left.2 \mathrm{O}, \delta / \mathrm{ppm}\right): 7.55(\mathrm{~s}, 1 \mathrm{H}), 6.90(\mathrm{dt}, \mathrm{J}=3.4,1.7 \mathrm{~Hz}, 1 \mathrm{H}), 6.46(\mathrm{dt}, \mathrm{J}=6.2,1.8 \mathrm{~Hz}$, 1H), $5.96-5.75(\mathrm{~m}, 1 \mathrm{H}), 5.14-4.97(\mathrm{~m}, 1 \mathrm{H}), 4.23-3.83(\mathrm{~m}, 2 \mathrm{H}), 3.65(\mathrm{dd}, \mathrm{J}=8.8,2.5 \mathrm{~Hz}, 2 \mathrm{H}), 2.48$ $(\mathrm{t}, \mathrm{J}=2.5 \mathrm{~Hz}, 1 \mathrm{H}), 1.83(\mathrm{~s}, 3 \mathrm{H}) .{ }^{31} \mathbf{P}\left\{{ }^{1} \mathbf{H}\right\} \mathbf{N M R}\left(122 \mathrm{MHz}, \mathrm{D}_{2} \mathrm{O}, \delta / \mathrm{ppm}\right): 6.36(\mathrm{td}, \mathrm{J}=78.1,58.1 \mathrm{~Hz}, 1 \mathrm{P})$, -5.24 (tdd, $\mathrm{J}=87.6,58.2,30.7 \mathrm{~Hz}, 1 \mathrm{P}),-11.3$ (d, J = 31.0, 1P). ${ }^{31} \mathbf{P}$ NMR $\left(162 \mathrm{MHz}, \mathrm{D}_{2} \mathrm{O}, \delta / \mathrm{ppm}\right)$ : $8.26-5.58(\mathrm{~m}, 1 \mathrm{P}),-5.25(\mathrm{tdd}, \mathrm{J}=88.1,58.3,30.7,1 \mathrm{P}),-11.3(\mathrm{dt}, \mathrm{J}=30.8,6.1 \mathrm{~Hz}, 1 \mathrm{P}) .{ }^{13} \mathrm{C}$ NMR $(101$ MHz, $\left.\mathrm{D}_{2} \mathrm{O}, \delta / \mathrm{ppm}\right): 166.9,152.3,138.2,134.3,125.2,111.6,89.9,87.5,85.9$ (d, J = 9.1 Hz), 66.5 (d, J $=5.9 \mathrm{~Hz}), 27.0(\mathrm{~d}, \mathrm{~J}=18.6 \mathrm{~Hz}), 11.5 .{ }^{19} \mathbf{F}$ NMR $\left(377 \mathrm{MHz}, \mathrm{D}_{2} \mathrm{O}, \delta / \mathrm{ppm}\right):-119.7(\mathrm{dd}, \mathrm{J}=87.7,78.0 \mathrm{~Hz}$, 2F).

HRMS (ESI) $\mathrm{m} / \mathrm{z}$ for $\left[\mathrm{C}_{14} \mathrm{H}_{17} \mathrm{~F}_{2} \mathrm{~N}_{3} \mathrm{Na}_{2} \mathrm{O}_{11} \mathrm{P}_{3}\right]^{+}$: calcd 579.9834 , found 579.9833 .

Ring opening by using aq. sodium hydroxide: 2',3'-didehydro-2',3'-dideoxythymidine 5'- $\beta, \gamma$ difluoromethylene triphosphate (49)

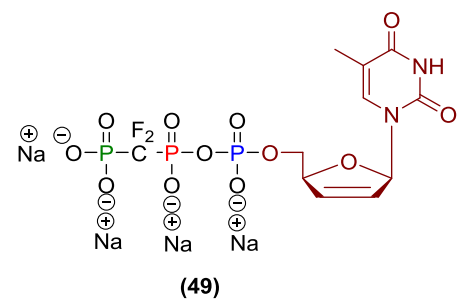

The reaction mixture of $\mathbf{B}_{15}$ ( $250 \mu \mathrm{L}, 12.2 \mu \mathrm{mol}, 1.0$ eq.) was added to aq. $\mathrm{NaOH}(1 \mathrm{M}, 250 \mu \mathrm{L})$ and then $\mathrm{D}_{2} \mathrm{O}(100 \mu \mathrm{l})$ was added afterwards it was stirred for 5 mins. The product 49 was isolated by Method A, affording a colourless solid (5.30 mg, $9.04 \mu \mathrm{mol}, 74 \%)$.

${ }^{1} \mathbf{H}$ NMR (400 MHz, D $\left.2 \mathrm{O}, \delta / p p m\right): 7.54(\mathrm{~s}, 1 \mathrm{H}), 6.89(\mathrm{~d}, \mathrm{~J}=3.4 \mathrm{~Hz}, 1 \mathrm{H}), 6.47(\mathrm{~d}, \mathrm{~J}=6.2 \mathrm{~Hz}, 1 \mathrm{H}), 5.86$ $(\mathrm{dt}, \mathrm{J}=6.2 \mathrm{~Hz}, 1 \mathrm{H}), 5.11-4.90(\mathrm{~m}, 1 \mathrm{H}), 4.23-4.04(\mathrm{~m}, 2 \mathrm{H}), 1.82(\mathrm{~s}, 3 \mathrm{H}) .{ }^{31} \mathbf{P}\left\{{ }^{1} \mathbf{H}\right\} \mathbf{~ N M R}(122 \mathrm{MHz}$, $\left.\mathrm{D}_{2} \mathrm{O}, \delta / \mathrm{ppm}\right): 3.93(\mathrm{td}, \mathrm{J}=72.5,56.9 \mathrm{~Hz}, 1 \mathrm{P}),-2.87$ (tdd, J = 89.5, 57.0, $\left.32.2 \mathrm{~Hz}, 1 \mathrm{P}\right),-11.0(\mathrm{~d}, \mathrm{~J}=32.2$ $\mathrm{Hz}, 1 \mathrm{P}) .{ }^{31} \mathbf{P}$ NMR $\left(162 \mathrm{MHz}, \mathrm{D}_{2} \mathrm{O}, \delta / \mathrm{ppm}\right): 3.93$ (td, J = 72.8, 57.0 Hz, 1P), -2.87 (tdd, J = 89.3, 57.0, $32.3 \mathrm{~Hz}, 1 \mathrm{P}),-11.0$ (dt, J = 32.1, $6.6 \mathrm{~Hz}, 1 \mathrm{P}) .{ }^{13} \mathrm{C}$ NMR (101 MHz, $\left.\mathrm{D}_{2} \mathrm{O}, \delta / \mathrm{ppm}\right): 166.8,152.3,138.2$, 
134.4, 125.4, 111.5, 89.9, $85.9(\mathrm{~d}, \mathrm{~J}=8.7 \mathrm{~Hz}), 66.4(\mathrm{~d}, \mathrm{~J}=5.9 \mathrm{~Hz}), 11.5 .{ }^{19} \mathbf{F}$ NMR $\left(377 \mathrm{MHz}, \mathrm{D}_{2} \mathrm{O}\right.$, $\delta / \mathrm{ppm}):-118.0(\mathrm{dd}, \mathrm{J}=89.9,72.8 \mathrm{~Hz}, 2 \mathrm{~F})$.

HRMS (ESI) $\mathrm{m} / \mathrm{z}$ for $\left[\mathrm{C}_{11} \mathrm{H}_{14} \mathrm{~F}_{2} \mathrm{~N}_{2} \mathrm{O}_{12} \mathrm{P}_{3}\right]^{-}$: calcd 496.9733 , found 496.9733 .

Ring opening by using aq. ammonia: 2',3'-didehydro-2',3'-dideoxythymidine 5'- $\gamma$ - $P$-amino $\beta, \gamma$ difluoromethylene triphosphate $(50)$

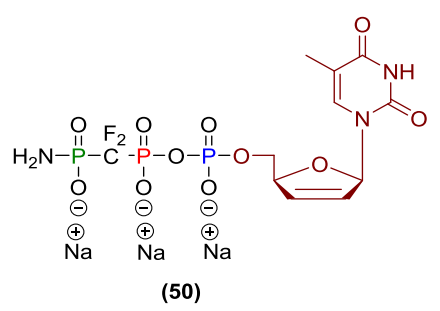

The reaction mixture of $\mathbf{B}_{15}\left(250 \mu \mathrm{L}, 12.2 \mu \mathrm{mol}, 1.0\right.$ eq.) was added to $25 \%$ aq. $\mathrm{NH}_{4} \mathrm{OH}(250 \mu \mathrm{L})$ and then $\mathrm{D}_{2} \mathrm{O}(100 \mu \mathrm{l})$ was added afterwards it was stirred for 5 mins. The product $\mathbf{5 0}$ was isolated by Method A, affording a colourless solid (5.60 mg, $10.1 \mu \mathrm{mol}, 87 \%)$.

${ }^{1} \mathbf{H}$ NMR (400 MHz, D $\left.2 \mathrm{O}, \delta / \mathrm{ppm}\right): 7.53(\mathrm{~s}, 1 \mathrm{H}), 6.89$ (dt, J = $\left.3.4 \mathrm{~Hz}, 1 \mathrm{H}\right), 6.45$ (dt, J = $\left.6.1 \mathrm{~Hz}, 1 \mathrm{H}\right)$, $5.86(\mathrm{~d}, \mathrm{~J}=6.2 \mathrm{~Hz}, 1 \mathrm{H}), 5.18-4.97(\mathrm{~m}, 1 \mathrm{H}), 4.09(\mathrm{~m}, 2 \mathrm{H}), 1.82(\mathrm{~s}, 3 \mathrm{H}) .{ }^{31} \mathbf{P}\left\{{ }^{1} \mathbf{H}\right\} \mathbf{N M R}\left(122 \mathrm{MHz}, \mathrm{D}_{2} \mathrm{O}\right.$, $\delta / \mathrm{ppm}): 8.39$ (td, $\mathrm{J}=78.7,59.3 \mathrm{~Hz}, 1 \mathrm{P}),-5.29$ (tdd, $\mathrm{J}=87.6,59.4,31.2 \mathrm{~Hz}, 1 \mathrm{P}),-11.4(\mathrm{~d}, \mathrm{~J}=31.1 \mathrm{~Hz}$, 1P). ${ }^{31}$ P NMR (162 MHz, D $\left.2 \mathrm{O}, \delta / \mathrm{ppm}\right): 8.38$ (td, J = 78.7, $\left.59.3 \mathrm{~Hz}, 1 \mathrm{P}\right),-5.29$ (tdd, J = 87.5, 59.3, 31.1 Hz, 1P), -11.4 (dt, J = 31.4, 6.4 Hz, 1P). ${ }^{13}$ C NMR (101 MHz, D $\left.2 \mathrm{O}, \delta / \mathrm{ppm}\right): 167.2,152.6,138.1,134.3,125.1$, 111.5, 89.9, $85.9(\mathrm{~d}, \mathrm{~J}=8.6 \mathrm{~Hz}), 66.5(\mathrm{~d}, \mathrm{~J}=6.0 \mathrm{~Hz}), 11.5 .{ }^{19} \mathbf{F}$ NMR $\left(377 \mathrm{MHz}, \mathrm{D}_{2} \mathrm{O}, \delta / \mathrm{ppm}\right)$ : - 121.10 (dd, $\mathrm{J}=87.4,78.7 \mathrm{~Hz}, 2 \mathrm{~F})$.

HRMS (ESI) $\mathrm{m} / \mathrm{z}$ for $\left[\mathrm{C}_{11} \mathrm{H}_{17} \mathrm{~N}_{3} \mathrm{O}_{11} \mathrm{P}_{3}\right]^{-}$: calcd 460.0081, found 460.0095 .

Ring opening by using caesium fluoride: 2',3'-didehydro-2',3'-dideoxythymidine 5'- $\gamma$-P-fluoro $\beta, \gamma$ difluoromethylene triphosphate (51)

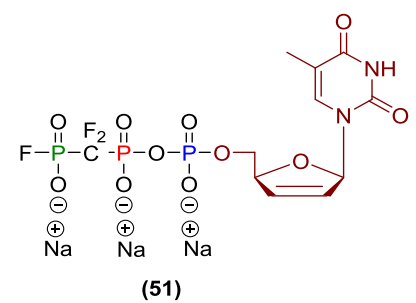

The reaction mixture of $\mathbf{B}_{15}(250 \mu \mathrm{L}, 12.2 \mu \mathrm{mol}, 1.0$ eq.) was added to caesium fluoride (105 mg, $691 \mu \mathrm{mol}, 58$ eq.) and then immediately addition of $\mathrm{D}_{2} \mathrm{O}(250 \mu \mathrm{l})$ afterwards it was stirred for 5 mins. The crude product was isolated by Method A and the impurity $\mathrm{CsClO}_{4}$ was co-precipitated which was further removed by dissolving the crude product in water $(2 \mathrm{~mL})$. The insoluble residue was removed by filtration (syringe filter, pore size $0.45 \mu \mathrm{m}$ ). Afterwards, the product $\mathbf{5 1}$ was isolated from the aq. solution again by Method A and a colourless solid was obtained (6.0 mg, $11.3 \mu \mathrm{mol}, 93 \%)$. 
${ }^{1}$ H NMR (400 MHz, D $\left.2 \mathrm{O}, \delta / \mathrm{ppm}\right): 7.52$ (s, 1H), 6.89 (dt, J = 3.3, 1.7 Hz, 1H), 6.45 (dt, J = 6.2, $1.8 \mathrm{~Hz}$, $1 \mathrm{H}), 5.88(\mathrm{dt}, \mathrm{J}=6.1,2.0 \mathrm{~Hz}, 1 \mathrm{H}), 5.04(\mathrm{~d}, \mathrm{~J}=4.1 \mathrm{~Hz}, 1 \mathrm{H}), 4.28-4.03(\mathrm{~m}, 2 \mathrm{H}), 1.82(\mathrm{~s}, 3 \mathrm{H}) .{ }^{31} \mathbf{P}\left\{{ }^{1} \mathbf{H}\right\}$ NMR (122 MHz, D $2 \mathrm{O}, \delta / \mathrm{ppm}): 5.82$ - -2.25 (m, 1P), -7.52 (tdd, J = 82.2, 64.2, $29.3 \mathrm{~Hz}, 1 \mathrm{P}),-11.4$ (d, $\mathrm{J}=29.1 \mathrm{~Hz}, 1 \mathrm{P}) .{ }^{31} \mathbf{P}$ NMR (162 MHz, $\left.\mathrm{D}_{2} \mathrm{O}, \delta / \mathrm{ppm}\right): 1.76$ (dtd, J = 1027.9, 90.9, 64.2 Hz, 1P), -7.53 (tdd, $\mathrm{J}=82.3,64.2,29.3 \mathrm{~Hz}, 1 \mathrm{P}),-11.4(\mathrm{dt}, \mathrm{J}=29.3,6.2 \mathrm{~Hz}, 1 \mathrm{P}) .{ }^{31} \mathbf{P}\left\{{ }^{19} \mathbf{F}\right\} \mathbf{N M R}\left(162 \mathrm{MHz}, \mathrm{D}_{2} \mathrm{O}, \delta / \mathrm{ppm}\right): 1.77$ $(\mathrm{d}, \mathrm{J}=64.1 \mathrm{~Hz}, 1 \mathrm{P}),-7.52(\mathrm{dd}, \mathrm{J}=64.2,29.1 \mathrm{~Hz}, 1 \mathrm{P}),-11.4(\mathrm{dt}, \mathrm{J}=29.3,6.2 \mathrm{~Hz}, 1 \mathrm{P}) .{ }^{13} \mathbf{C}$ NMR $(101$ $\left.\mathrm{MHz}, \mathrm{D}_{2} \mathrm{O}, \delta / \mathrm{ppm}\right): 166.8,152.3,138.1,134.3,125.1,111.5,89.9,85.9(\mathrm{~d}, \mathrm{~J}=8.6 \mathrm{~Hz}), 66.5(\mathrm{~d}, \mathrm{~J}=$ 6.0 Hz), 11.5. ${ }^{19} \mathrm{~F}$ NMR (377 MHz, $\left.\mathrm{D}_{2} \mathrm{O}, \delta / \mathrm{ppm}\right)$ : -68.7 (d, J = $\left.1027.9 \mathrm{~Hz}, 1 \mathrm{~F}\right),-121.4$ (ddd, J = 90.9, $82.3,3.3 \mathrm{~Hz}, 2 \mathrm{~F})$.

HRMS (ESI) $\mathrm{m} / \mathrm{z}$ for $\left[\mathrm{C}_{11} \mathrm{H}_{13} \mathrm{~F}_{3} \mathrm{~N}_{2} \mathrm{O}_{11} \mathrm{P}_{3}\right]^{-}$: calcd 498.9690, found 498.9689.

Ring opening by using tetra methylgaunidinium azide: 2',3'-didehydro-2',3'-dideoxythymidine 5'$\gamma$-P-azido $\beta, \gamma$-difluoromethylene triphosphate (52)

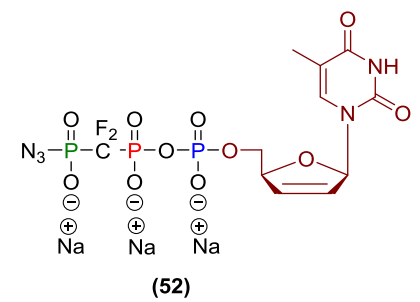

The reaction mixture of $\mathbf{B}_{15}(250 \mu \mathrm{L}, 12.2 \mu \mathrm{mol}, 1.0$ eq.) was added to a solution of tetramethyl guanidinium azide (420 mg $2.62 \mathrm{mmol}, 220$ eq.) in $\mathrm{MeCN}(1 \mathrm{~mL})$ and $\mathrm{D}_{2} \mathrm{O}(100 \mu \mathrm{l})$ was added subsequently. The crude product was isolated by Method A and the product was precipitated as gaunidinium salt which was again by dissolved in water $(2 \mathrm{~mL})$.Afterwards, the product $\mathbf{5 2}$ was isolated from the aq. solution again by Method A and a colourless solid was obtained (6.20 mg, $11.2 \mu \mathrm{mol}, 94 \%$ ). ${ }^{1}$ H NMR (400 MHz, D $\left.2 \mathrm{O}, \delta / \mathrm{ppm}\right): 7.53(\mathrm{~s}, 1 \mathrm{H}), 7.03-6.76(\mathrm{~m}, 1 \mathrm{H}), 6.45(\mathrm{~d}, \mathrm{~J}=6.1,1.8,1 \mathrm{H}), 5.86$ (d, $\mathrm{J}=6.2,1.9,1 \mathrm{H}), 5.03(\mathrm{~s}, 1 \mathrm{H}), 4.37-3.98(\mathrm{~m}, 2 \mathrm{H}), 1.82(\mathrm{~s}, 3 \mathrm{H}) .{ }^{31} \mathbf{P}\left\{{ }^{1} \mathbf{H}\right\} \mathbf{N M R}(162 \mathrm{MHz}, \mathrm{D} 2 \mathrm{O}, \delta / \mathrm{ppm})$ : $3.11(\mathrm{td}, \mathrm{J}=85.4,63.9 \mathrm{~Hz}, 1 \mathrm{P}),-6.35--8.09$ (m, 1P), -11.2 (d, J = 29.7 Hz, 1P). ${ }^{31} \mathbf{P}$ NMR $(162 \mathrm{MHz}$, $\left.\mathrm{D}_{2} \mathrm{O}, \delta / \mathrm{ppm}\right): 3.11(\mathrm{td}, \mathrm{J}=85.8,64.1 \mathrm{~Hz}, 1 \mathrm{P}),-7.18(\mathrm{tdd}, \mathrm{J}=82.5,63.9,29.5 \mathrm{~Hz}, 1 \mathrm{P}),-11.2(\mathrm{dt}, \mathrm{J}=29.5$, $6.1 \mathrm{~Hz}, 1 \mathrm{P}).) .{ }^{13} \mathrm{C}$ NMR $\left(101 \mathrm{MHz}, \mathrm{D}_{2} \mathrm{O}, \delta / \mathrm{ppm}\right): 167.2,152.6,138.1,134.3,125.1,111.5,89.9,85.9$ (d, $\mathrm{J}=8.6 \mathrm{~Hz}), 66.5(\mathrm{~d}, \mathrm{~J}=6.0 \mathrm{~Hz}), 11.5 .{ }^{19} \mathbf{F}$ NMR $\left(377 \mathrm{MHz}, \mathrm{D}_{2} \mathrm{O}, \delta / \mathrm{ppm}\right):-119.2(\mathrm{dd}, \mathrm{J}=87.8,78.0 \mathrm{~Hz}$, $2 \mathrm{~F})$.

HRMS (ESI) $\mathrm{m} / \mathrm{z}$ for $\left[\mathrm{C}_{11} \mathrm{H}_{13} \mathrm{~F}_{2} \mathrm{~N}_{5} \mathrm{O}_{11} \mathrm{P}_{3}\right]^{-}$: calcd 521.9798, found 521.9801 . 
Ring opening by using Imidazole: 4'-Pentyne $\gamma$-P-imidazole $\beta, \gamma$-difluoromethylene triphosphate (S- 8)

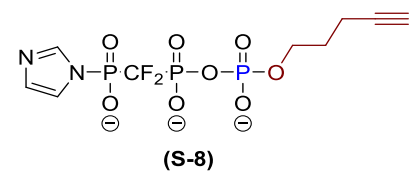

4-Pentyn-1-ol (24 mg, $0.27 \mathrm{mmol}, 1.2$ eq.) and ETT (102 mg, $0.78 \mathrm{mmol}, 3.5$ eq.) were coevaporated with dry $\mathrm{MeCN}(2 \times 1 \mathrm{~mL})$. Under an Ar atmosphere, a reaction mixture containing the $c$-PyCF2 $\mathrm{PA} \mathrm{A} \mathbf{3}$ $(0.075 \mathrm{M}$ in $\mathrm{MeCN}, 3.0 \mathrm{ml}, 0.22 \mathrm{mmol}, 1.0$ eq.) was added to the dried solids and it was stirred at r.t. for 10 mins. Upon cooling to $0^{\circ} \mathrm{C}, \mathrm{mCPBA}(\leq 77 \%, 24 \mathrm{mg}, 0.16 \mathrm{mmol}, 1.5$ eq.) was added and the mixture was stirred for 5 mins until ${ }^{31} \mathrm{P}-\mathrm{NMR}$ confirmed complete oxidation (reaction progress was followed by ${ }^{31} \mathrm{P}$ NMR, and formation of triplet at $-25 \mathrm{ppm}$ which is diagnostic signal for the cyclotriphosphate).

A solution of imidazole in dry DMF $(1.47 \mathrm{M}, 4.00 \mathrm{ml}, 5.88 \mathrm{mmol}, 26.0$ eq.) was added to the above cyclotriphosphate and reaction was stirred for another 30 mins (the reaction progress was followed by ${ }^{31} \mathrm{P}$ NMR) which results in quantative conversion to $\mathbf{S - 8}$.

Note -: Imidazole was dried before using by coevaporation with $\mathrm{MeCN}$ and it was further stored of molecular sieve $(3 \AA)$.

${ }^{31} \mathbf{P}\left\{{ }^{1} \mathbf{H}\right\}$ NMR $\left(122 \mathrm{MHz}, \mathrm{D}_{2} \mathrm{O}, \delta / \mathrm{ppm}\right):-4.94(\mathrm{td}, \mathrm{J}=80.5,61.7 \mathrm{~Hz}, 1 \mathrm{P}),-6.64--8.64$ (m, 1P), -12.3 (d, $\mathrm{J}=27 \mathrm{~Hz}, 1 \mathrm{P})$.

\section{5'-Adenosine 4'-Pentyne $\beta, \gamma$-difluoromethylene hexaphosphate (P5)}

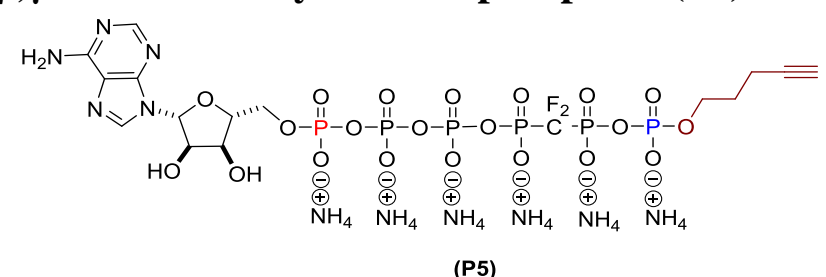

Anhydr. $\mathrm{ZnCl}_{2}$ (0.26 g, 1.9 mmol, 8.4 eq.) and ATP x 2.1 TBA (0.36 g, 0.35 mmol, 1.6 eq.) were added to a freshly prepared solution of $\mathbf{S - 8}(0.22 \mathrm{mmol})$. The reaction mixture was then diluted with dry DMF: DMSO (v:v, 1:1, $16 \mathrm{ml})$ in order to increase the solubility and it was stirred at r.t. for $24 \mathrm{hrs}$. The crude product was purified by Method B and the target product was eluted with 500-600 mM conc. of $\mathrm{NH}_{4} \mathrm{HCO}_{3}$ buffer. Compound P5 was obtained as a colorless solid. (0.02 g, $0.03 \mathrm{mmol}, 12 \%)$.

Note -: The reaction for capped hexapolyPs were not optimized.

${ }^{1}$ H NMR (400 MHz, D $\left.2 \mathrm{O}, \delta / \mathrm{ppm}\right): 8.42(\mathrm{~s}, 1 \mathrm{H}), 8.16(\mathrm{~s}, 1 \mathrm{H}), 6.05(\mathrm{~d}, \mathrm{~J}=6.0 \mathrm{~Hz}, 1 \mathrm{H}), 4.65(\mathrm{~s}, 1 \mathrm{H})$, $4.57-4.45(\mathrm{~m}, 1 \mathrm{H}), 4.43-4.22(\mathrm{~m}, 1 \mathrm{H}), 4.25-4.09(\mathrm{~m}, 2 \mathrm{H}), 4.04-3.88(\mathrm{~m}, 2 \mathrm{H}), 2.55-2.00(\mathrm{~m}, 3 \mathrm{H})$, $1.91-1.65(\mathrm{~m}, 2 \mathrm{H}) .{ }^{31} \mathbf{P}\left\{{ }^{1} \mathbf{H}\right\} \mathbf{N M R}\left(122 \mathrm{MHz}, \mathrm{D}_{2} \mathrm{O}, \delta / \mathrm{ppm}\right):-5.63--7.57$ (m, 2P), -10.6 (d, J = 26.4 Hz, 1P), $-11.4(\mathrm{~d}, \mathrm{~J}=15.8 \mathrm{~Hz}, 1 \mathrm{P}),-22.0--23.9$ (m, 2P). ${ }^{31} \mathbf{P}$ NMR $\left(202 \mathrm{MHz}, \mathrm{D}_{2} \mathrm{O}, \delta / \mathrm{ppm}\right):-5.69--7.97$ $(\mathrm{m}, 2 \mathrm{P}),-10.3--10.9(\mathrm{~m}, 1 \mathrm{P}),-11.1--11.8(\mathrm{~m}, 1 \mathrm{P}),-22.1--23.8(\mathrm{~m}, 2 \mathrm{P}) .{ }^{13} \mathrm{C}$ NMR $\left(126 \mathrm{MHz}, \mathrm{D}_{2} \mathrm{O}\right.$, 


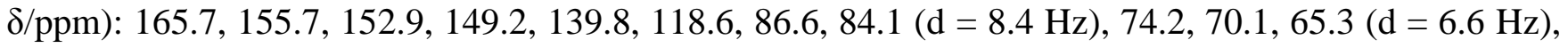
65.4, 28.7 (d, J = 7.3 Hz), 14.1. ${ }^{19}$ F NMR (471 MHz, $\left.\mathrm{D}_{2} \mathrm{O}, \delta / \mathrm{ppm}\right):-120.5$ (t, J = 83.7, 2F).

HRMS (ESI) $\mathrm{m} / \mathrm{z}$ for $\left[\mathrm{C}_{16} \mathrm{H}_{25} \mathrm{DF}_{2} \mathrm{~N}_{5} \mathrm{O}_{21} \mathrm{P}_{6}\right]^{+}$: calcd 848.9571, found 848.9587.

\subsubsection{Synthesis of triphosphates based on $c$-Py $\mathrm{CyCl}_{2} \mathrm{PA}\left(\mathrm{A}_{4}\right)$}

3'-Azido-3'-deoxythymidine $\beta, \gamma$-dichloromethylene cyclotriphosphate $\left(B_{16}\right)$

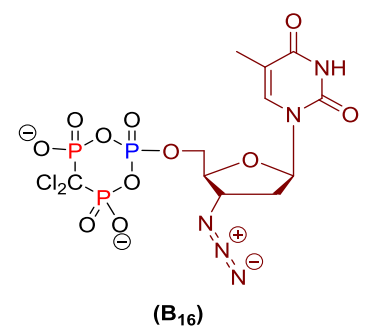

AZT (68 mg, $0.25 \mathrm{mmol}, 1.2$ eq.) and ETT (0.13 g, $0.99 \mathrm{mmol}, 4.8$ eq.) were coevaporated with dry $\mathrm{MeCN}(2 \times 1 \mathrm{~mL})$. Under an Ar atmosphere, a reaction mixture containing the $c$ - $\mathrm{PyCC1}_{2} \mathrm{PA} \mathrm{A}_{4}(0.069 \mathrm{M}$ in $\mathrm{MeCN}, 3.0 \mathrm{~mL}, 0.20 \mathrm{mmol}, 1.0$ eq.) was added to the dried solids and it was stirred at r.t. for 45 mins. Upon cooling to $0^{\circ} \mathrm{C}, m \mathrm{CPBA}(\leq 77 \%, 54 \mathrm{mg}, 0.31 \mathrm{mmol}, 1.5$ eq.) was added and the mixture was stirred for 10 mins until ${ }^{31} \mathrm{P}-\mathrm{NMR}$ confirmed complete oxidation (reaction progress was followed by ${ }^{31} \mathrm{P}$ NMR, and formation of triplet at $-26 \mathrm{ppm}$ which is diagnostic signal for $\left.\mathbf{B}_{16}\right)$.

${ }^{31} \mathbf{P}\left\{{ }^{1} \mathbf{H}\right\}$ NMR $\left(122 \mathrm{MHz}, \mathrm{CDCl}_{3}, \delta / \mathrm{ppm}\right):-4.54(\mathrm{~d}, \mathrm{~J}=21.8 \mathrm{~Hz}, 2 \mathrm{P}),-26.3(\mathrm{t}, \mathrm{J}=21.7 \mathrm{~Hz}, 1 \mathrm{P})$.

Ring opening by using propargylamine: 3'-Azido-3'-deoxythymidine 5'- $\gamma$-P-propargylamino $\beta, \gamma$ dichloromethylene triphosphate (53)

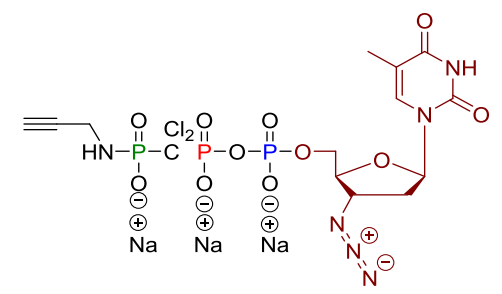

(53)

The reaction mixture of $\mathbf{B}_{16}(250 \mu \mathrm{L}, 17.2 \mu \mathrm{mol}, 1.0$ eq.) was added to propargylamine $(250 \mu \mathrm{L})$ and then $\mathrm{D}_{2} \mathrm{O}(100 \mu \mathrm{l})$ was added afterwards it was stirred for 5 mins. The product $\mathbf{5 3}$ was isolated by Method A, affording a colourless solid $(5.8 \mathrm{mg}, 8.58 \mu \mathrm{mol}, 50 \%)$.

${ }^{1} \mathbf{H}$ NMR (400 MHz, D $\left.2 \mathrm{O}, \delta / \mathrm{ppm}\right): 7.68(\mathrm{~s}, 1 \mathrm{H}), 6.22(\mathrm{t}, \mathrm{J}=6.9 \mathrm{~Hz}, 1 \mathrm{H}), 4.53(\mathrm{dt}, \mathrm{J}=6.5,3.7 \mathrm{~Hz}, 1 \mathrm{H})$, $4.18(\mathrm{~m}, 3 \mathrm{H}), 3.76-3.51(\mathrm{~m}, 2 \mathrm{H}), 2.53-2.36(\mathrm{~m}, 3 \mathrm{H}), 1.86(\mathrm{~s}, 3 \mathrm{H}) \cdot{ }^{31} \mathbf{P}\left\{{ }^{1} \mathbf{H}\right\} \mathbf{N M R}\left(122 \mathrm{MHz}, \mathrm{D}_{2} \mathrm{O}\right.$,

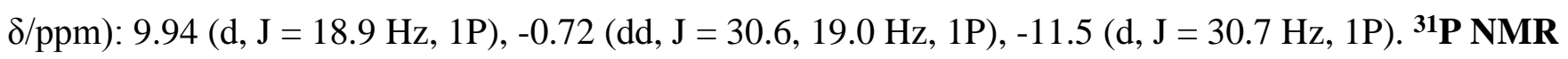
$\left(162 \mathrm{MHz}, \mathrm{D}_{2} \mathrm{O}, \delta / \mathrm{ppm}\right): 9.92(\mathrm{dt}, \mathrm{J}=19.4,8.1 \mathrm{~Hz}, 1 \mathrm{P}),-0.73(\mathrm{dd}, \mathrm{J}=30.5,19.3 \mathrm{~Hz}, 1 \mathrm{P}),-11.6(\mathrm{dt}, \mathrm{J}=$ 31.4, $6.4 \mathrm{~Hz}, 1 \mathrm{P}) .{ }^{13} \mathrm{C}$ NMR (101 MHz, $\left.\mathrm{D}_{2} \mathrm{O}, \delta / \mathrm{ppm}\right): 166.8,151.8,137.2,111.8,84.8,82.9$ (d, J = 9.3 $\mathrm{Hz}), 71.3,65.6(\mathrm{~d}, \mathrm{~J}=5.6 \mathrm{~Hz}), 60.8,36.3,30.9(\mathrm{~d}, \mathrm{~J}=3.7 \mathrm{~Hz}), 11.7$. 
HRMS (ESI) m/z for $\left[\mathrm{C}_{14} \mathrm{H}_{19} \mathrm{Cl}_{2} \mathrm{~N}_{6} \mathrm{O}_{11} \mathrm{P}_{3}\right]^{-}$: calcd 609.9707, found 609.9695.

\subsubsection{Synthesis of triphosphates based on $c$-PyNHPA (A5)}

3'-Azido-3'-deoxythymidine $\beta, \gamma$-imido cyclotriphosphate (B17)

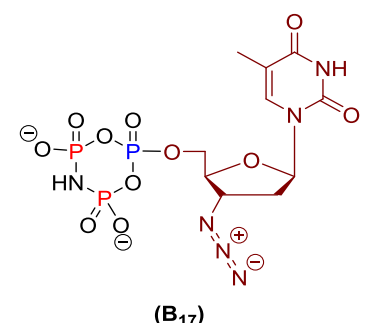

AZT (52 mg, $0.19 \mathrm{mmol}, 1.3$ eq.) and ETT (69 mg, $0.52 \mathrm{mmol}, 3.5$ eq.) were coevaporated with dry $\mathrm{MeCN}(2 \times 1 \mathrm{~mL})$. Under an Ar atmosphere, a reaction mixture containing the $c$ - $\mathrm{Py}_{\mathrm{NH}} \mathrm{PA} \mathbf{A}_{5}(0.075 \mathrm{M}$ in $\mathrm{MeCN}, 2.0 \mathrm{~mL}, 0.15 \mathrm{mmol}, 1.0$ eq.) was added to the dried solids and it was stirred at r.t. for 5 mins. Upon cooling to $-4^{\circ} \mathrm{C}, m \mathrm{CPBA}(\leq 77 \%, 39 \mathrm{mg}, 0.22 \mathrm{mmol}, 1.5 \mathrm{eq}$.) was added and the mixture was stirred for 5 mins until ${ }^{31} \mathrm{P}-\mathrm{NMR}$ confirmed complete oxidation (a formation of triplet at $-23 \mathrm{ppm}$ which is diagnostic signal for $\mathbf{B}_{17}$ ).

${ }^{31} \mathbf{P}\left\{{ }^{1} \mathbf{H}\right\}$ NMR $\left(122 \mathrm{MHz}, \mathrm{CDCl}_{3}, \delta / \mathrm{ppm}\right):-13.7(\mathrm{~d}, \mathrm{~J}=17.9 \mathrm{~Hz}, 2 \mathrm{P}),-22.5(\mathrm{t}, \mathrm{J}=19.2 \mathrm{~Hz}, 1 \mathrm{P})$.

Ring opening by using aq. ammonia: 3'-Azido-3'-deoxythymidine 5 '- $\gamma$ - $P$-amino $\beta, \gamma$ imidotriphosphate (54)

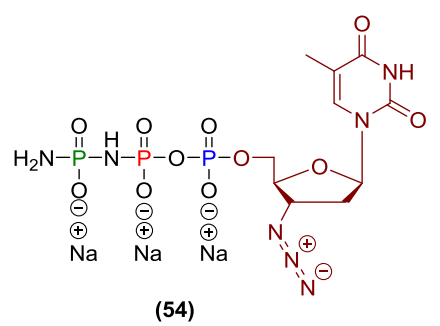

The reaction mixture of $\mathbf{B}_{17}\left(500 \mu \mathrm{L}, 37.5 \mu \mathrm{mol}, 1.0\right.$ eq.) was added to $25 \%$ aq. $\mathrm{NH}_{4} \mathrm{OH}(500 \mu \mathrm{L})$ and then $\mathrm{D}_{2} \mathrm{O}(200 \mu \mathrm{l})$ afterwards it was stirred for 5 mins. The product 54 was isolated by Method C, affording a colourless solid (11.9 mg, $20.8 \mu \mathrm{mol}, 55 \%)$.

${ }^{1}$ H NMR (300 MHz, D $\left.2 \mathrm{O}, \delta / \mathrm{ppm}\right): 7.67$ (s, 1H), 6.21 (t, J = 6.9 Hz, 1H), 4.50 (dt, J = 6.1, 4.1 Hz, 1H),

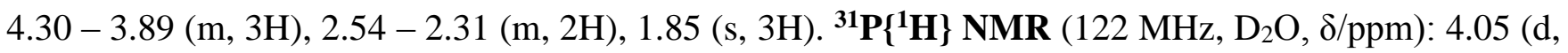
$\mathrm{J}=5.3 \mathrm{~Hz}, 1 \mathrm{P}),-10.5(\mathrm{dd}, \mathrm{J}=20.2,5.4 \mathrm{~Hz}, 1 \mathrm{P}),-11.1(\mathrm{~d}, \mathrm{~J}=20.2 \mathrm{~Hz}, 1 \mathrm{P}) .{ }^{31} \mathbf{P}$ NMR $\left(122 \mathrm{MHz}, \mathrm{D}_{2} \mathrm{O}\right.$, $\delta / \mathrm{ppm}): 4.67(\mathrm{~d}, \mathrm{~J}=6.8 \mathrm{~Hz}, 1 \mathrm{P}),-10.6(\mathrm{dd}, \mathrm{J}=21.2,6.2 \mathrm{~Hz}, 1 \mathrm{P}),-11.4(\mathrm{dt}, \mathrm{J}=21.8,5.8 \mathrm{~Hz}, 1 \mathrm{P}) .{ }^{13} \mathrm{C}$ NMR (101 MHz, D $2 \mathrm{O}, \delta / \mathrm{ppm}):$ 167.1, 152.1, 137.2, 111.8, 84.8, 82.9 (d, J = 9.0 Hz), 65.4 (d, J =5.5 Hz), $60.8,36.2,11.7$.

HRMS (ESI) $\mathrm{m} / \mathrm{z}$ for $\left[\mathrm{C}_{10} \mathrm{H}_{17} \mathrm{~N}_{7} \mathrm{O}_{11} \mathrm{P}_{3}\right]^{-}$: calcd 504.0204, found 504.0204. 
Ring opening by using propargylamine: 3'-Azido-3'-deoxythymidineb 5'- $\gamma$ - $P$-propargylamino $\beta, \gamma$ imidotriphosphate (55)

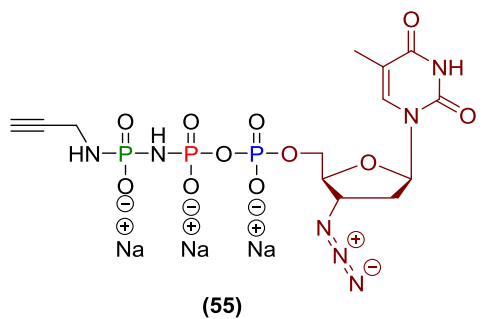

The reaction mixture of $\mathbf{B}_{17}$ (2.0 mL, $0.15 \mathrm{mmol}, 1.0$ eq.) was added to propargylamine ( $3.0 \mathrm{ml}, 4.7 \mathrm{mmol}$, $31 \mathrm{eq}$ ) and stirred at r.t. for 15 mins. The product $\mathbf{5 5}$ was isolated by Method C (details of the MPLC purification can be found in Supporting figure S1), affording a colourless solid (59.8 $\mathrm{mg}, 0.09 \mathrm{mmol}$, $65 \%)$.

${ }^{1} \mathbf{H}$ NMR $\left(300 \mathrm{MHz}, \mathrm{D}_{2} \mathrm{O}, \delta / \mathrm{ppm}\right): 7.68(\mathrm{~s}, 1 \mathrm{H}), 6.21(\mathrm{t}, \mathrm{J}=6.8 \mathrm{~Hz}, 1 \mathrm{H}), 4.63-4.42(\mathrm{~m}, 1 \mathrm{H}), 4.32-4.03$ $(\mathrm{m}, 3 \mathrm{H}), 3.56(\mathrm{dd}, \mathrm{J}=9.7,2.5,2 \mathrm{H}), 2.49(\mathrm{t}, \mathrm{J}=2.5,1 \mathrm{H}), 2.42(\mathrm{td}, \mathrm{J}=6.2,4.8,2.7,2 \mathrm{H}), 1.86(\mathrm{~s}, 3 \mathrm{H})$. ${ }^{31} \mathbf{P}\left\{{ }^{1} \mathbf{H}\right\}$ NMR (122 MHz, D $\left.2 \mathrm{O}, \delta / \mathrm{ppm}\right): 4.05$ (d, J = 5.3 Hz, 1P), -10.5 (dd, J = 20.2, $\left.5.4 \mathrm{~Hz}, 1 \mathrm{P}\right),-11.1$ $(\mathrm{d}, \mathrm{J}=20.2,1 \mathrm{P}) .{ }^{31} \mathbf{P}$ NMR $\left(122 \mathrm{MHz}, \mathrm{D}_{2} \mathrm{O}, \delta / \mathrm{ppm}\right): 4.00(\mathrm{td}, \mathrm{J}=9.7,5.5 \mathrm{~Hz}, 1 \mathrm{P}),-10.7$ (dd, J = 20.6, $5.6 \mathrm{~Hz}, 1 \mathrm{P}),-11.03--11.5$ (m, 1P). ${ }^{13} \mathrm{C}$ NMR (101 MHz, $\left.\mathrm{D}_{2} \mathrm{O}, \delta / \mathrm{ppm}\right): 166.8,160.2,151.7,137.2,111.8$, 84.8, 83.02 (d, J = 9.3 Hz), 71.3, 65.7 (d, J = 5.6 Hz), 60.9, 36.3, 11.6.

HRMS (ESI) $\mathrm{m} / \mathrm{z}$ for $\left[\mathrm{C}_{13} \mathrm{H}_{18} \mathrm{~N}_{7} \mathrm{NaO}_{11} \mathrm{P}_{3}\right]^{-}$: calcd 564.0180, found 564.0182. 


\section{Analytical Data (Chromatogram and IR)}
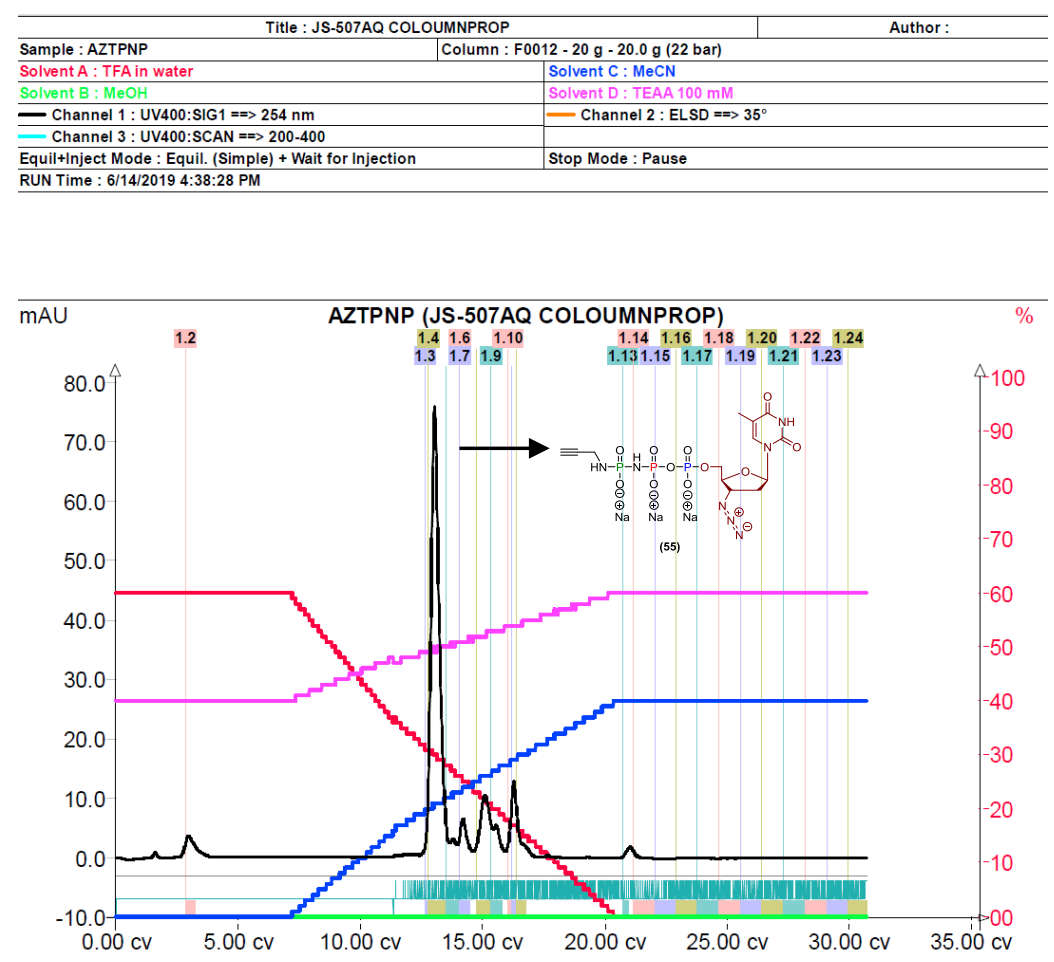

Supporting Figure S1: MPLC Chromatogram of crude $\mathbf{5 5 . 1 0 0 ~ m M ~ o f ~ T E A A ~ b u f f e r ~ w a s ~ a d j u s t e d ~ a t ~ p H ~}$ $=8.55$. The crude product was dissolved in deionized water and loaded on puriFlash C18 aqueous column, Column size: F0012 (20 g), Particle size: $30 \mu \mathrm{m}$, Flow rate: $10 \mathrm{ml} / \mathrm{mins}$, Mobile Phase: $100 \mathrm{mM}$ of TEAA (pH adjusted at 8.5) and MeCN, Gradient: $40 \mathrm{mM}$ of TEAA for 15 mins then $60 \mathrm{mM}$ of TEAA: $40 \mathrm{mM}$ of $\mathrm{MeCN}$ for 45 mins. The target fractions were pooled and concentrated to the minimal amount of the solvent and then product was precipitated by $\mathrm{NaClO}_{4}(0.5 \mathrm{~m}$ in acetone). The precipitate was washed two times with acetone $(10-20 \mathrm{ml})$. 


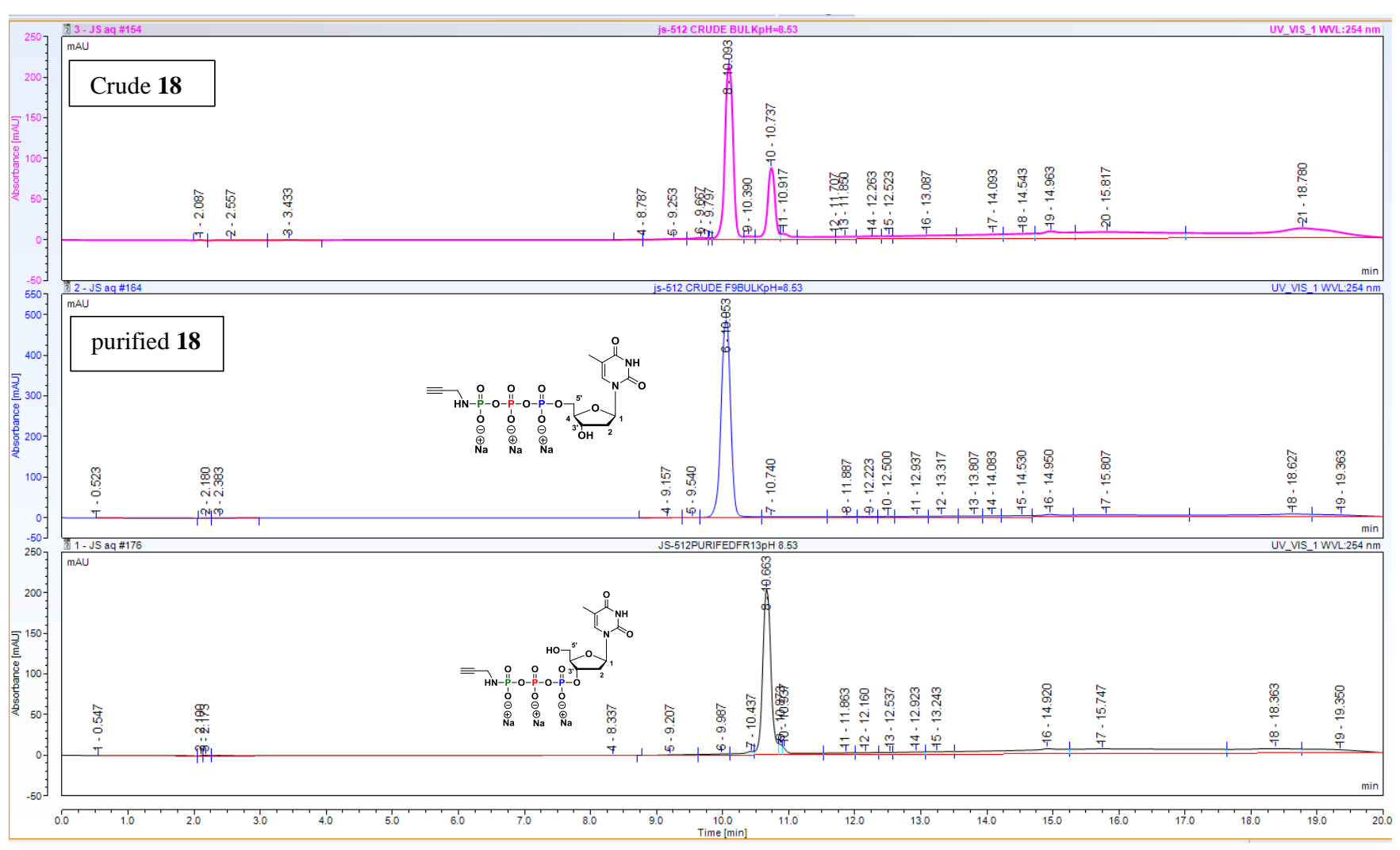

Supporting Figure S2: Analytical RP-HPLC Profile of crude product 18.

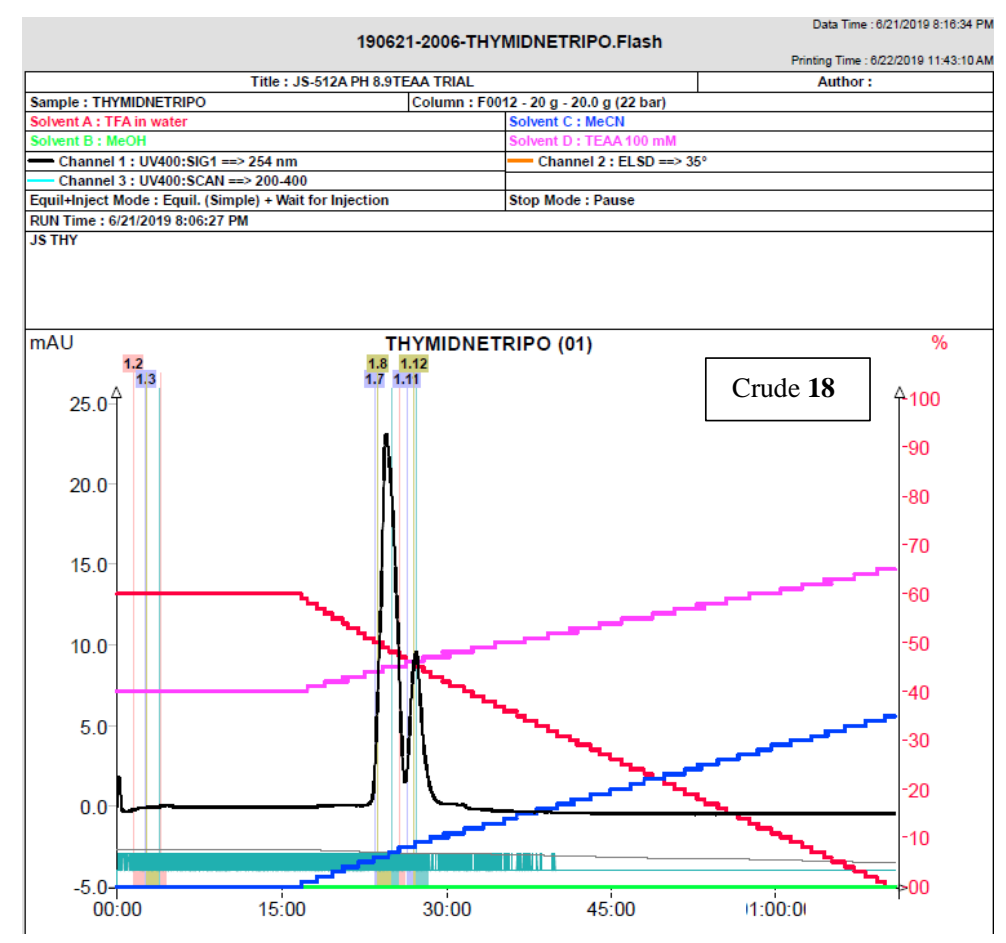

Supporting Figure S3: MPLC Chromatogram of crude 18. $100 \mathrm{mM}$ of TEAA buffer was adjusted at pH $=8.5$. The crude product was dissolved in deionized water and loaded on puriFlash C18 aqueous column, Column size: F0012 (20 g), Particle size: $30 \mu \mathrm{m}$, Flow rate: $10 \mathrm{ml} / \mathrm{mins}$, Mobile Phase: $100 \mathrm{mM}$ of TEAA ( $\mathrm{pH}$ adjusted at 8.5) and MeCN, Gradient: $40 \mathrm{mM}$ of TEAA for 15 mins then $60 \mathrm{mM}$ of TEAA: $40 \mathrm{mM}$ of $\mathrm{MeCN}$ for 45 mins. The target fractions were pooled and concentrated reduced pressure to the minimal 
amount of the solvent and then product was precipitated by $\mathrm{NaClO}_{4}(0.5 \mathrm{~m}$ in acetone $)$. The precipitate was washed two times with acetone $(10-20 \mathrm{ml})$.

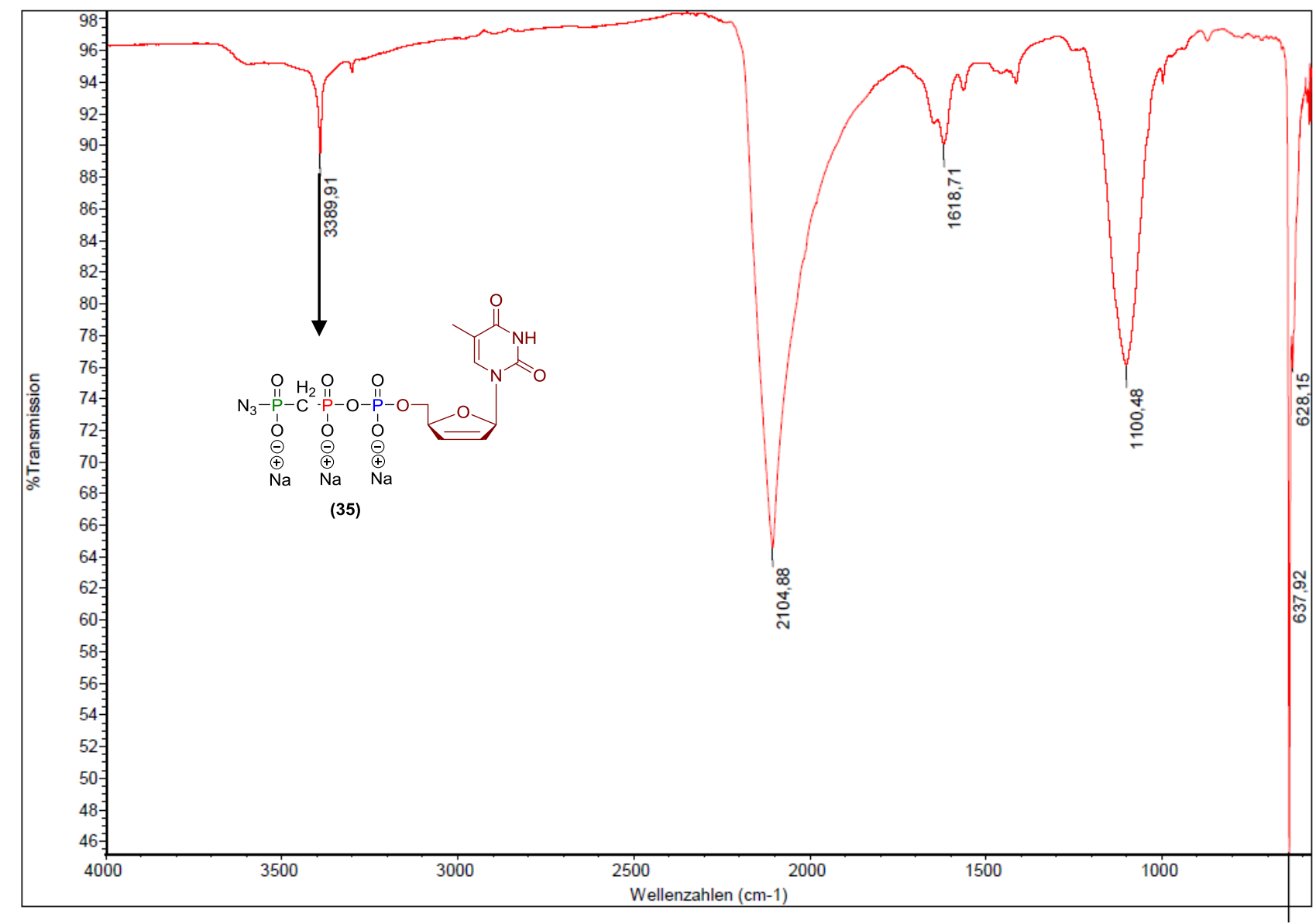

Supporting Figure S4: IR Spectrum of crude 35 indicating presence of an azide group.

\section{Computational Details}

Computed structure and property results were carried out using the GAMESS ${ }^{13}$ and Gaussian09 ${ }^{21}$ software packages. The B97- ${ }^{14}$ density functional was used in combination with the Def2-TZVPPD basis set ${ }^{15}$ for geometry optimizations, Hessian evaluations, and property calculations. Full geometry optimizations were performed and uniquely characterized via second derivatives (Hessian) analysis to determine the number of imaginary frequencies $(0=$ minima; $1=$ transition state $)$, and effects of zero point energy. Effects of solvation were taken into account using the COSab method, ${ }^{16,17}$ using a dielectric in accord with experiment and solvent radii from Klamt. ${ }^{18}$ Visualization and analysis of structural and property results were obtained using Avogadro ${ }^{19}$ and WEBMO. ${ }^{20}$ 


\section{References}

(1) Singh, J.; Steck, N.; De, D.; Hofer, A.; Ripp, A.; Captain, I.; Keller, M.; Wender, P. A.; Bhandari, R.; Jessen, H. J. A Phosphoramidite Analogue of Cyclotriphosphate Enables Iterative Polyphosphorylations. Angewandte Chemie (International ed. in English) 2019, 58, 3928-3933, DOI: 10.1002/anie.201814366.

(2) Yount, R. G.; Babcock, D.; Ballantyne, W.; Ojala, D. Adenylyl imidodiphosphate, an adenosine triphosphate analog containing a P--N--P linkage. Biochemistry 1971, 10, 2484-2489, DOI: 10.1021/bi00789a009.

(3) Mohamady, S.; Taylor, S. D. Synthesis of nucleoside 5'-tetraphosphates containing terminal fluorescent labels via activated cyclic trimetaphosphate. The Journal of organic chemistry 2014, 79, 2308-2313, DOI: 10.1021/jo500051y.

(4) Janos Ludwig and Fritz Eckstein. Rapid and efficient synthesis of nucleoside 5'-0-(1thiotriphosphates), 5'-triphosphates and 2',3'-cyclophosphorothioates using 2-chloro-4H-1,3,2benzodioxaphosphorin-4-one.

(5) Janos Ludwig and Fritz Eckstein. Synthesis of nucleoside 5'-O-(1,3-dithiotriphosphates) and 5'-O(1,1-dithiotriphosphates).

(6) Sun, Q.; Liu, S.; Sun, J.; Gong, S.-S. An H-phosphonate strategy for the synthesis of 2',3'dideoxynucleoside triphosphates and homodinucleotides. Chinese Chemical Letters 2014, 25, 427-430, DOI: 10.1016/j.cclet.2013.11.029.

(7) Dal Ben, D.; Buccioni, M.; Lambertucci, C.; Marucci, G.; Spinaci, A.; Marchenkova, A.; Abdelrahman, A.; Nistri, A.; Müller, C. E.; Volpini, R. Investigation on 2',3'-O-Substituted ATP Derivatives and Analogs as Novel P2X3 Receptor Antagonists. ACS medicinal chemistry letters 2019, 10, 493-498, DOI: 10.1021/acsmedchemlett.8b00524.

(8) Ahmadibeni, Y.; Tiwari, R. K.; Sun, G.; Parang, K. Synthesis of nucleoside mono-, di-, and triphosphoramidates from solid-phase cyclosaligenyl phosphitylating reagents. Organic letters 2009, 11, 2157-2160, DOI: 10.1021/o1900320r.

(9) Lee, S. E.; Elphick, L. M.; Anderson, A. A.; Bonnac, L.; Child, E. S.; Mann, D. J.; Gouverneur, V. Synthesis and reactivity of novel gamma-phosphate modified ATP analogues. Bioorganic \& medicinal chemistry letters 2009, 19, 3804-3807, DOI: 10.1016/j.bmcl.2009.04.028.

(10) Ahmadibeni, Y.; Dash, C.; Le Grice, S. F. J.; Parang, K. Solid-Phase Synthesis of 5'-O- $\beta, \gamma-$ Methylenetriphosphate Derivatives of Nucleosides and Evaluation of Their Inhibitory Activity Against HIV-1 Reverse Transcriptase. Tetrahedron Letters 2010, 51, 3010-3013, DOI: 10.1016/j.tetlet.2010.04.005.

(11) You, Y. H.; Gong, S. S.; Sun, Q. Practical Synthesis of $\beta, \gamma$-Bridging Oxygen-Modified Stavudine 5'-Triphosphates. AMR 2014, 908, 207-210, DOI: 10.4028/www.scientific.net/AMR.908.207. 
(12) Wang, G.; Boyle, N.; Chen, F.; Rajappan, V.; Fagan, P.; Brooks, J. L.; Hurd, T.; Leeds, J. M.; Rajwanshi, V. K.; Jin, Y. et al. Synthesis of AZT 5'-triphosphate mimics and their inhibitory effects on HIV-1 reverse transcriptase. Journal of medicinal chemistry 2004, 47, 6902-6913, DOI: 10.1021/jm040116w.

(13) Schmidt, M. W.; Baldridge, K. K.; Boatz, J. A.; Elbert, S. T.; Gordon, M. S.; Jensen, J. H.; Koseki, S.; Matsunaga, N.; Nguyen, K. A.; Su, S. et al. General atomic and molecular electronic structure system. J. Comput. Chem. 1993, 14, 1347-1363, DOI: 10.1002/jcc.540141112.

(14) Grimme, S. Semiempirical GGA-type density functional constructed with a long-range dispersion correction. Journal of computational chemistry 2006, 27, 1787-1799, DOI: 10.1002/jcc.20495.

(15) Rappoport, D.; Furche, F. Property-optimized gaussian basis sets for molecular response calculations. The Journal of chemical physics 2010, 133, 134105, DOI: 10.1063/1.3484283.

(16) Klamt, A.; Schüürmann, G. COSMO: A new approach to dielectric screening in solvents with explicit expressions for the screening energy and its gradient. J. Chem. Soc., Perkin Trans. 2 1993, 799805, DOI: 10.1039/P29930000799.

(17) Baldridge, K.; Klamt, A. First principles implementation of solvent effects without outlying charge error. The Journal of chemical physics 1997, 106, 6622-6633, DOI: 10.1063/1.473662.

(18) Klamt, A.; Jonas, V.; Bürger, T.; Lohrenz, J. C. W. Refinement and Parametrization of COSMORS. J. Phys. Chem. A 1998, 102, 5074-5085, DOI: 10.1021/jp980017s.

(19) Hanwell, M. D.; Curtis, D. E.; Lonie, D. C.; Vandermeersch, T.; Zurek, E.; Hutchison, G. R. Avogadro: an advanced semantic chemical editor, visualization, and analysis platform. Journal of cheminformatics 2012, 4, 17, DOI: 10.1186/1758-2946-4-17.

(20) http://www.webmo.net/index.html.

(21) Frisch, M. J.; Trucks, G. W.; Schlegel, H. B.; Scuseria, G. E.; Robb, M. A.; Cheeseman, J. R.; Scalmani, G.; Barone, V.; Mennucci, B.; Petersson, G. A.; Nakatsuji, H.; Caricato, M.; Li, X.; Hratchian, H. P.; Izmaylov, A. F.; Bloino, J.; Zheng, G.; Sonnenberg, J. L.; Hada, M.; Ehara, M.; Toyota, K.; Fukuda, R.; Hasegawa, J.; Ishida, M.; Nakajima, T.; Honda, Y.; Kitao, O.; Nakai, H.; Vreven, T.; Montgomery, J. A., Jr., Peralta, J. E.; Ogliaro, F.; Bearpark, M.; Heyd, J. J.; Brothers, E.; Kudin, K. N.; Staroverov, V. N.; Keith, T.; Kobayashi, R.; Normand, J.; Raghavachari, K.; Rendell, A.; Burant, J. C.; Iyengar, S. S.; Tomasi, J.; Cossi, M.; Rega, N.; Millam, J. M.; Klene, M.; Knox, J. E.; Cross, J. B.; Bakken, V.; Adamo, C.; Jaramillo, J.; Gomperts, R.; Stratmann, R. E.; Yazyev, O.; Austin, A. J.; Cammi, R.; Pomelli, C.; Ochterski, J. W.; Martin, R. L.; Morokuma, K.; Zakrzewski, V. G.; Voth, G. A.; Salvador, P.; Dannenberg, J. J.; Dapprich, S.; Daniels, A. D.; Farkas, O.; Foresman, J. B.; Ortiz, J. V.; Cioslowski, J.; Fox, D. J. Gaussian 09, Revision E.01, Gaussian, Inc., Wallingford CT, 2013. 


\section{Triphosphates based on c-PyPA}

(A)

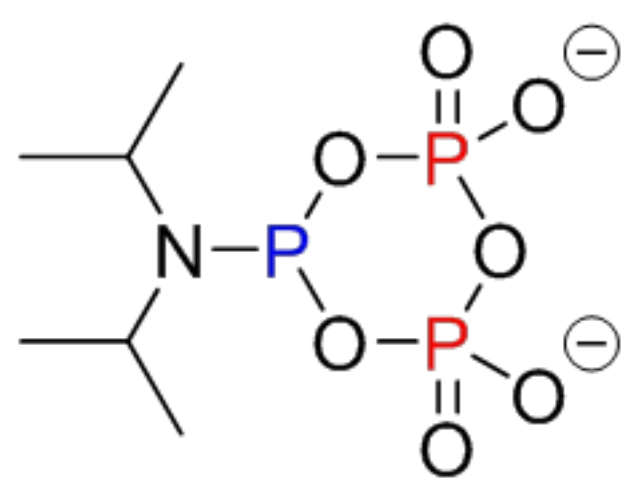




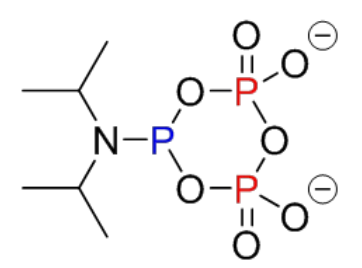

(in dry acetonitrile)

\begin{tabular}{|c|}
\hline$A(t)$ \\
130.68 \\
$J(22.55)$ \\
\hline
\end{tabular}

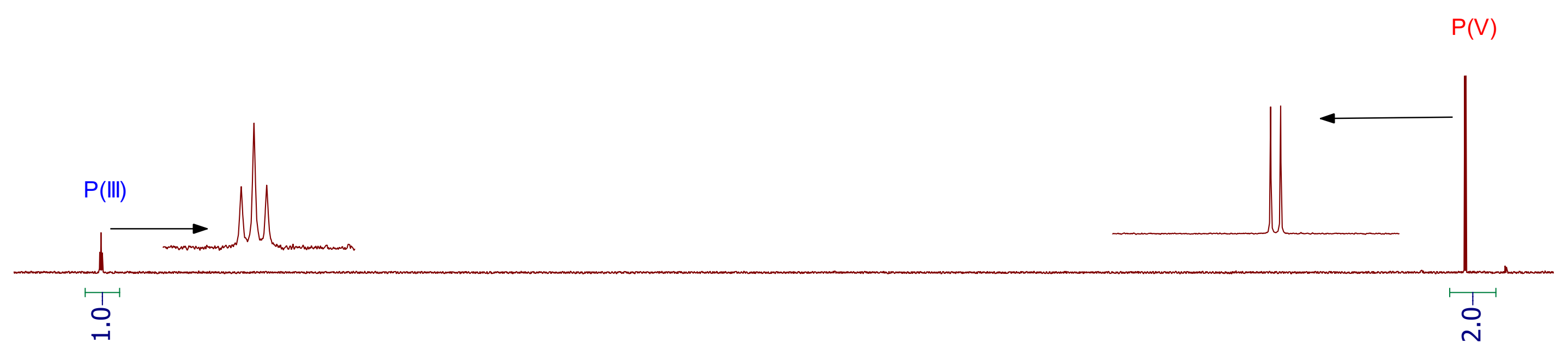

$\begin{array}{lllllllllllllllll} & 10 & 130 & 120 & 110 & 100 & 90 & 80 & 70 & \begin{array}{c}60 \\ \mathrm{f} 1(\mathrm{ppm})\end{array} & 50 & 30 & 20 & 10 & 0 & -10 & -20\end{array}$




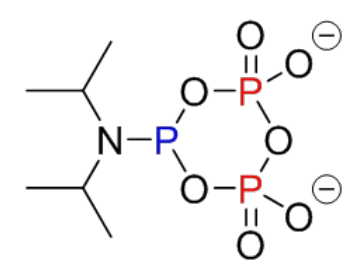

(in dry acetonitrile)

P(III) 


\section{${ }^{31} \mathrm{P}\left\{{ }^{1} \mathrm{H}\right\} \mathrm{NMR}$}

\section{$\underline{\text { P-Amidite }}$}

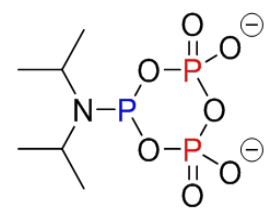

P

In

1) Coupling
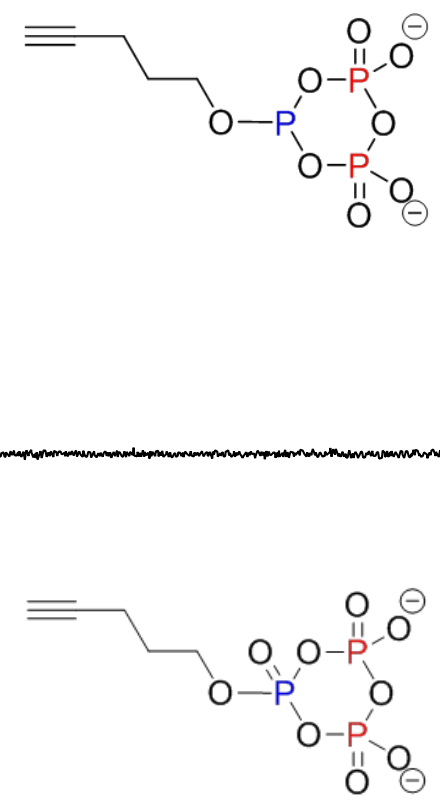


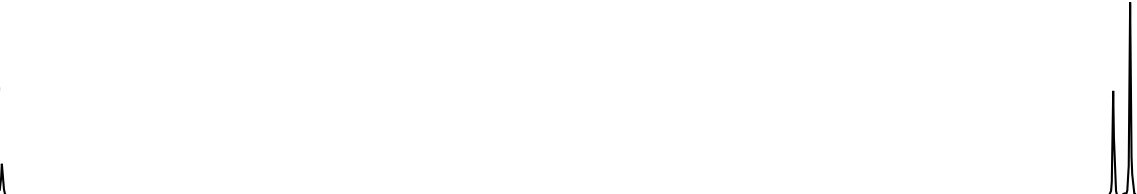

Ring opening with Sodium hydroxide ${ }^{31} \mathrm{P}\left\{{ }^{1} \mathrm{H}\right\}$ NMR

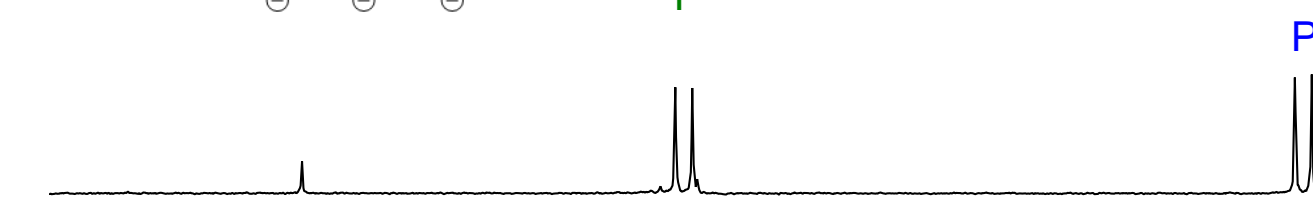

${ }^{31} P\left\{{ }^{1} H\right\} N M R$
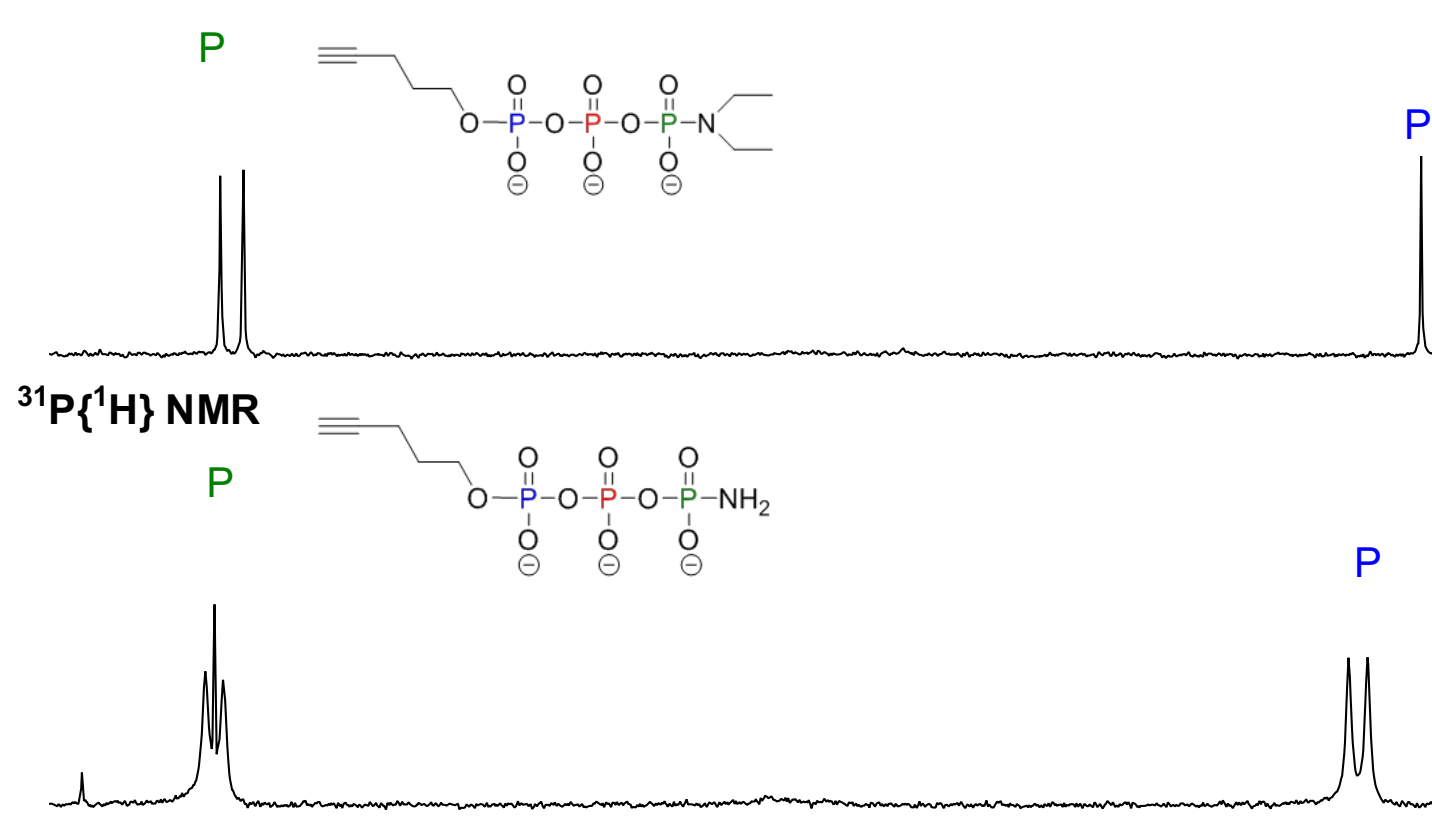

$\mathrm{P}$

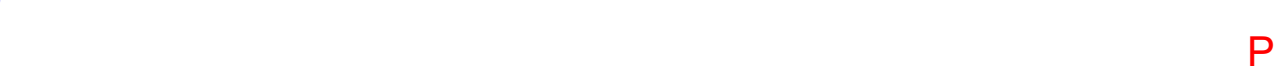

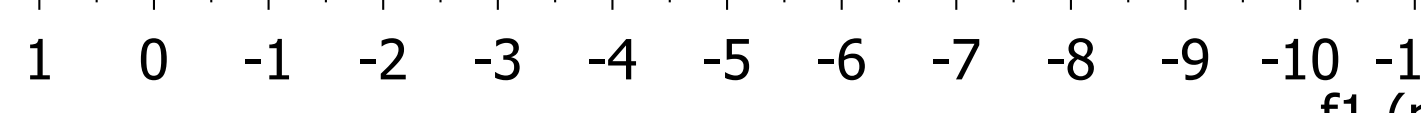

Ring opening with diethylamine

$\mathrm{P}$

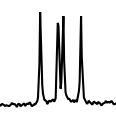

Ring opening with Ammonium hydroxide

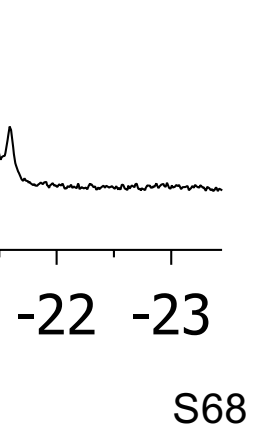



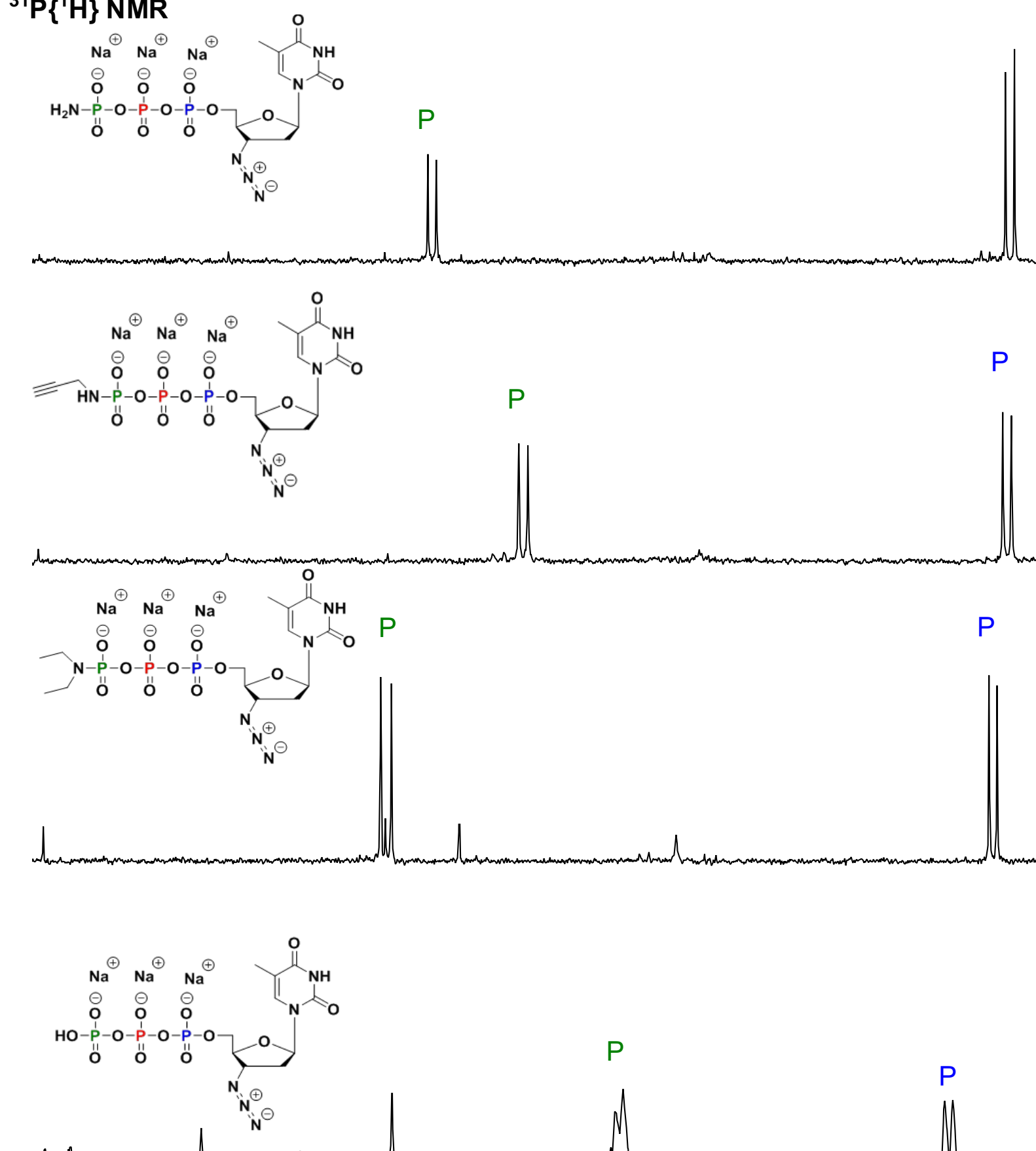

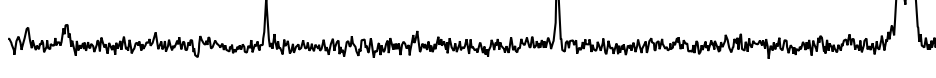

Ring opening with Ammonium hydroxide

$\mathrm{P}$

Ring opening with Propargylamine

$P$

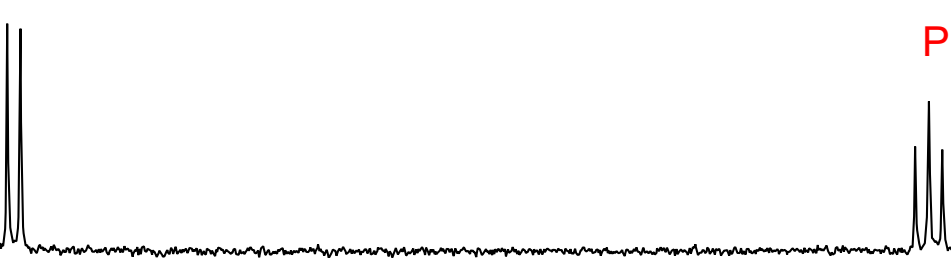

Ring opening with diethylamine

$\mathrm{P}$

$P$

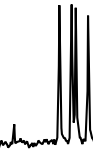

Ring opening with Sodium hydroxide 

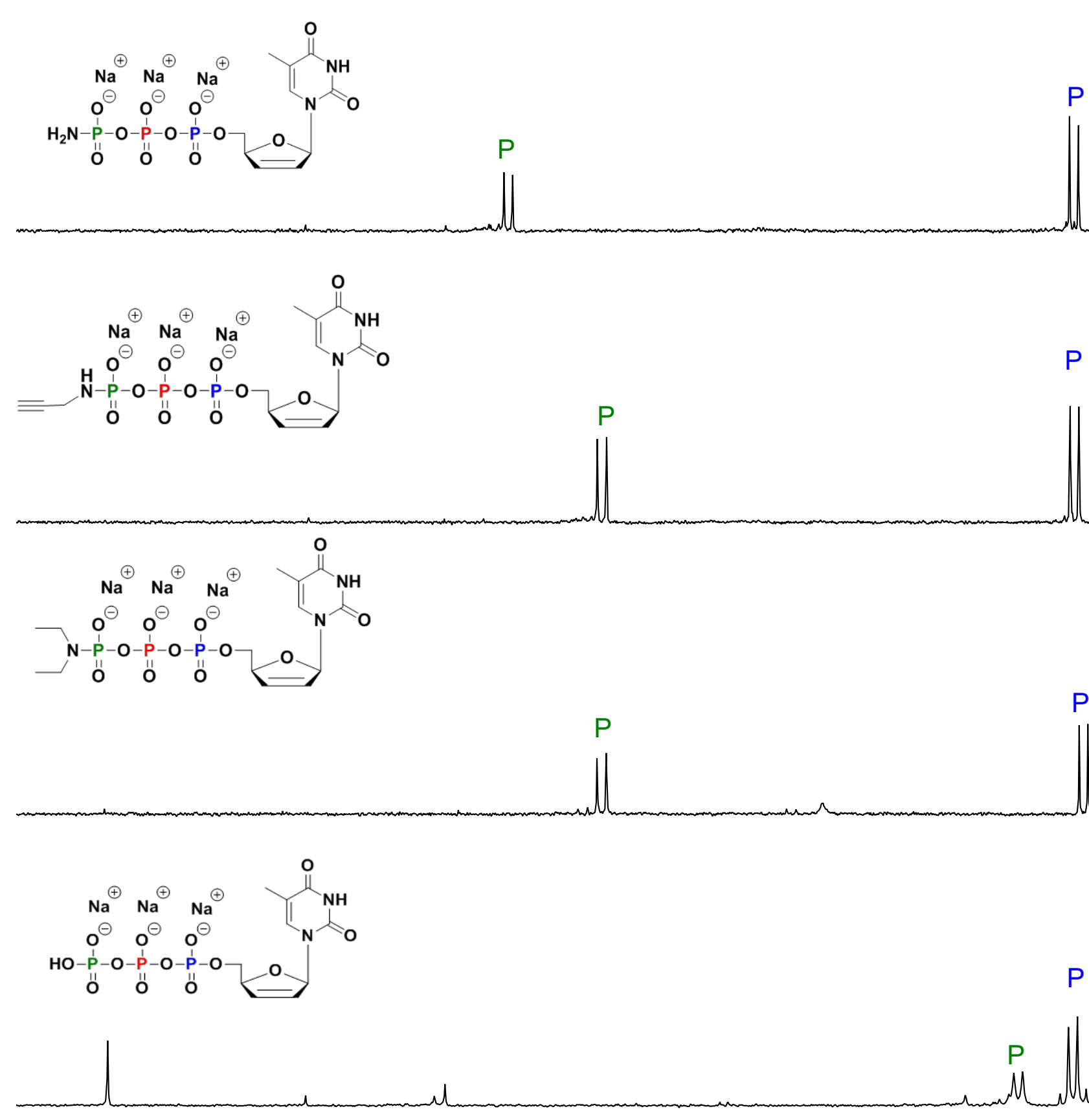

$\begin{array}{lllllllll}7 & 5 & 3 & 1 & -1 & -3 & -5 & -7 & -9 \\ & & & & & & & & \mathrm{f} 1(\mathrm{ppm})\end{array}$

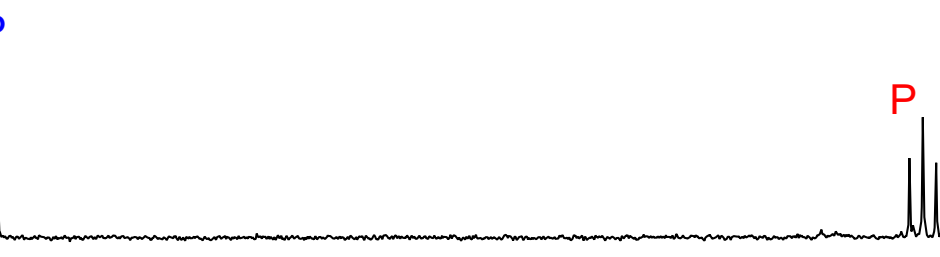

Ring opening with Propargylamine

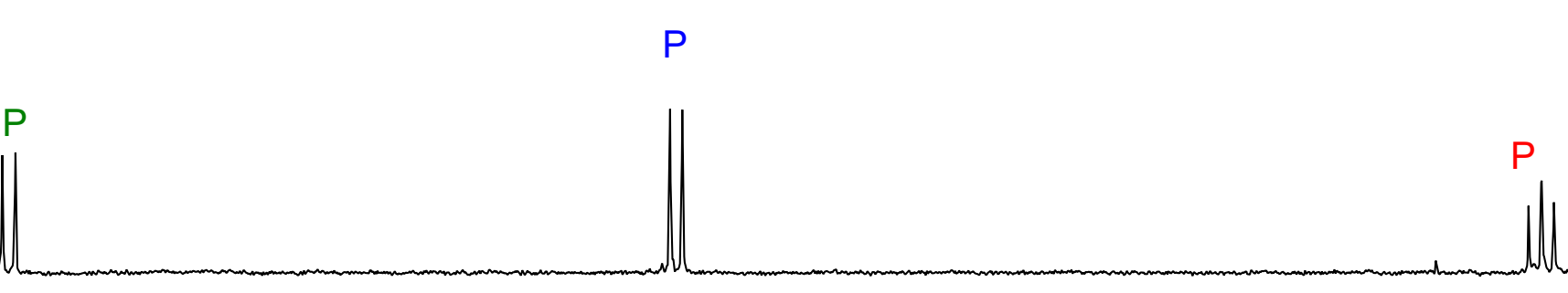

Ring opening with diethylamine

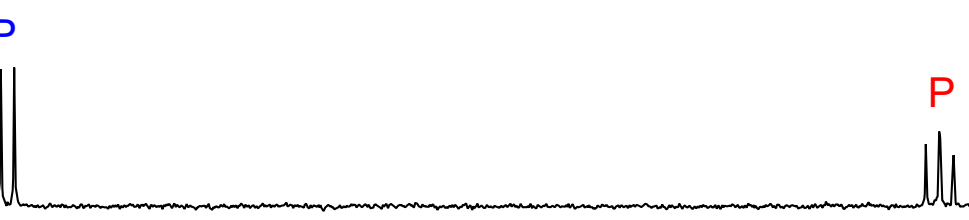

Ring opening with Sodium hydroxide 


\section{${ }^{31} \mathrm{P}\left\{{ }^{1} \mathrm{H}\right\}$ NMR}
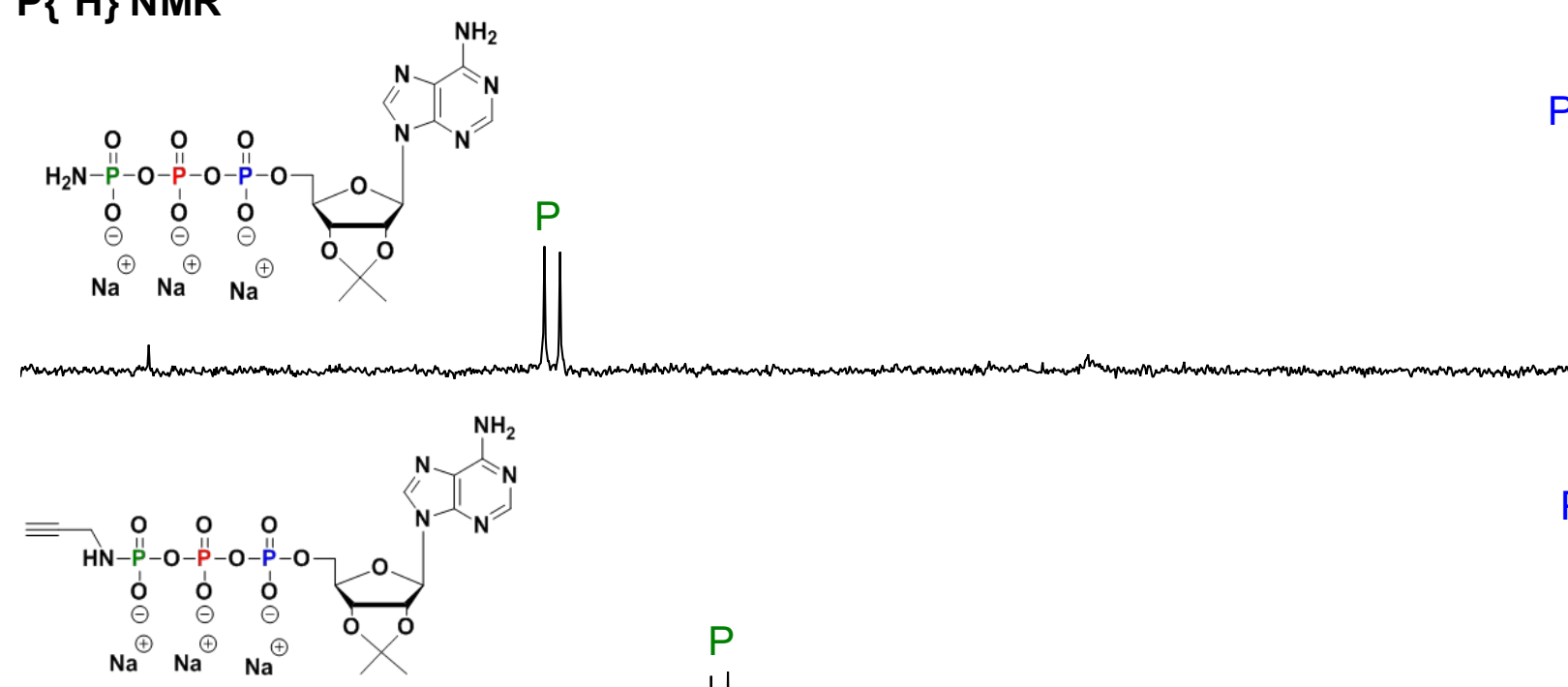

\section{.}

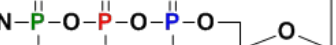

$\mathrm{Na}^{\oplus} \mathrm{Na}^{\oplus} \mathrm{Na}^{\oplus} \stackrel{\ominus}{\mathrm{O}}$

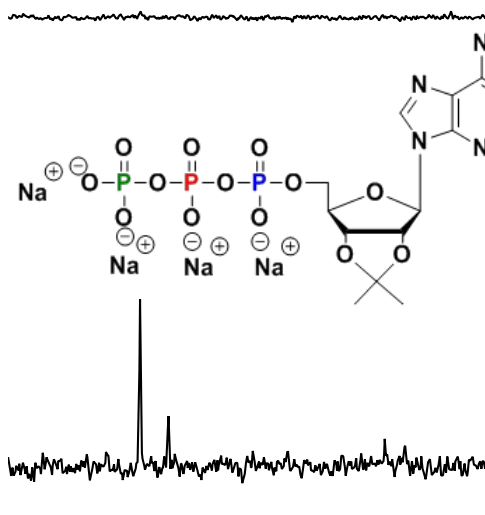

N
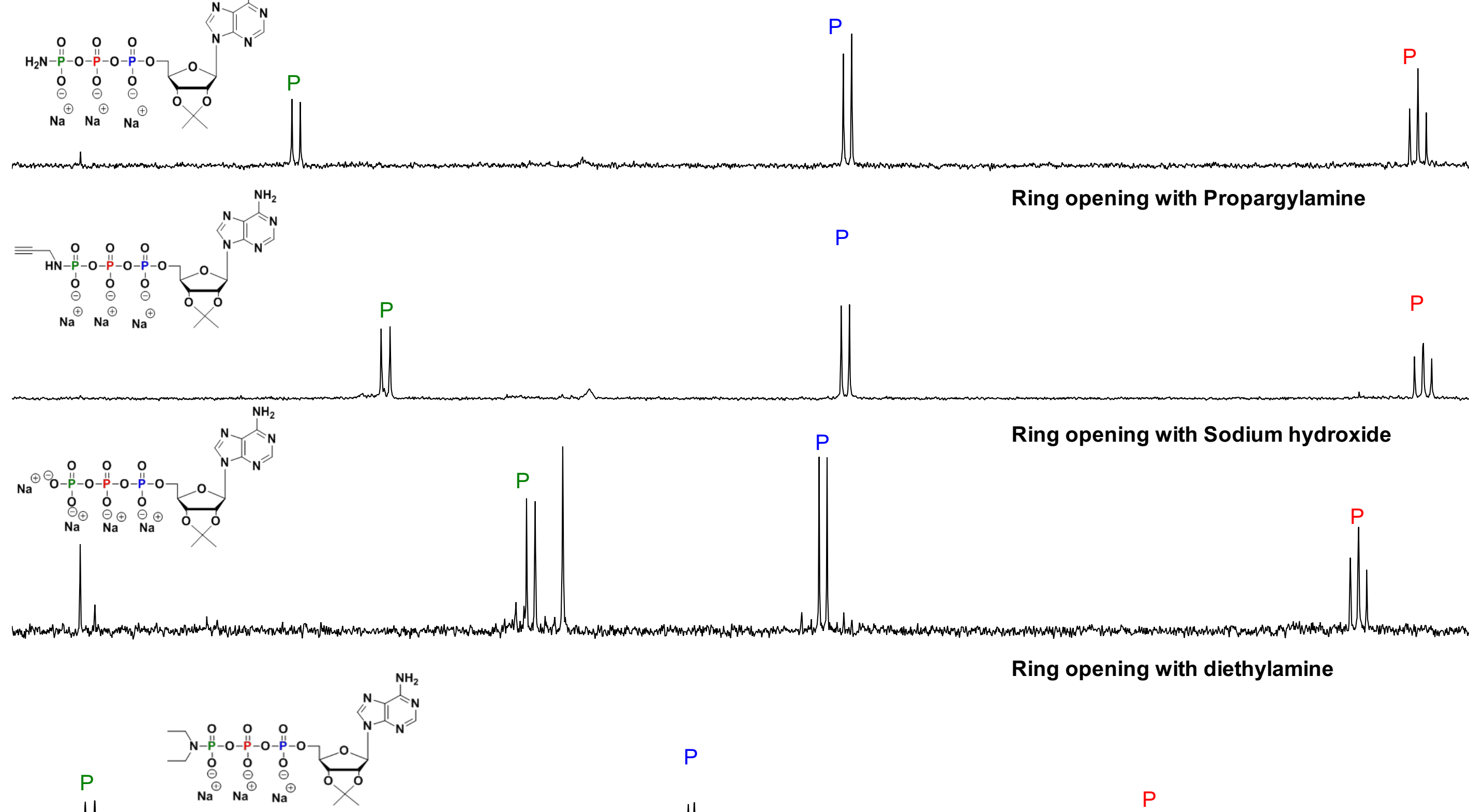

Ring opening with Propargylamine

$\mathrm{P}$

$\mathrm{P}$

Ring opening with Sodium hydroxide

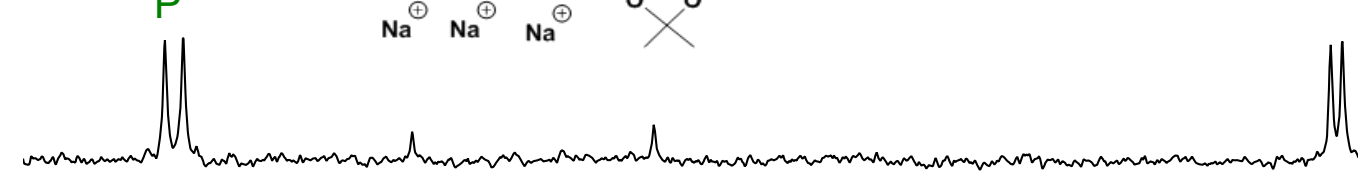

Ring opening with diethylamine

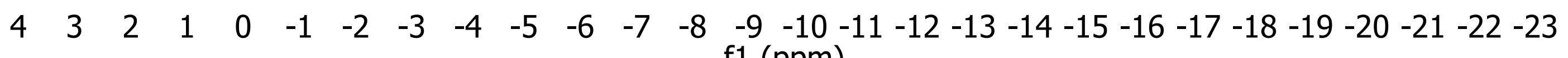
f1 (ppm) 


\section{${ }^{31} \mathrm{P}\left\{{ }^{1} \mathrm{H}\right\} \mathrm{NMR}$}

\section{1) Coupling}

OH

5'-P

|| 3'-P

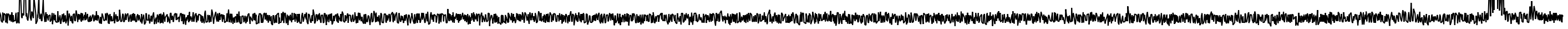

\section{2) Oxidation}

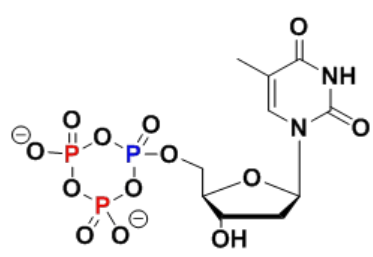

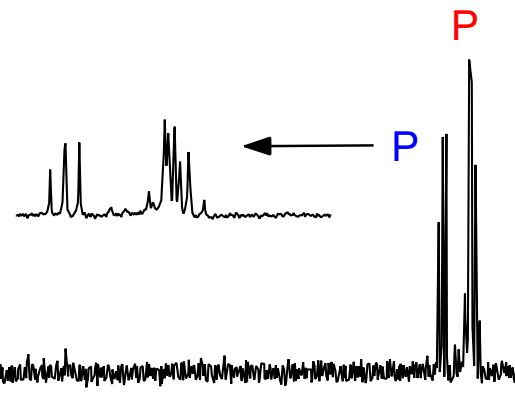




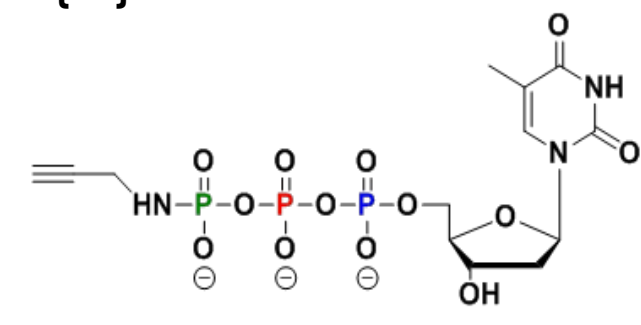

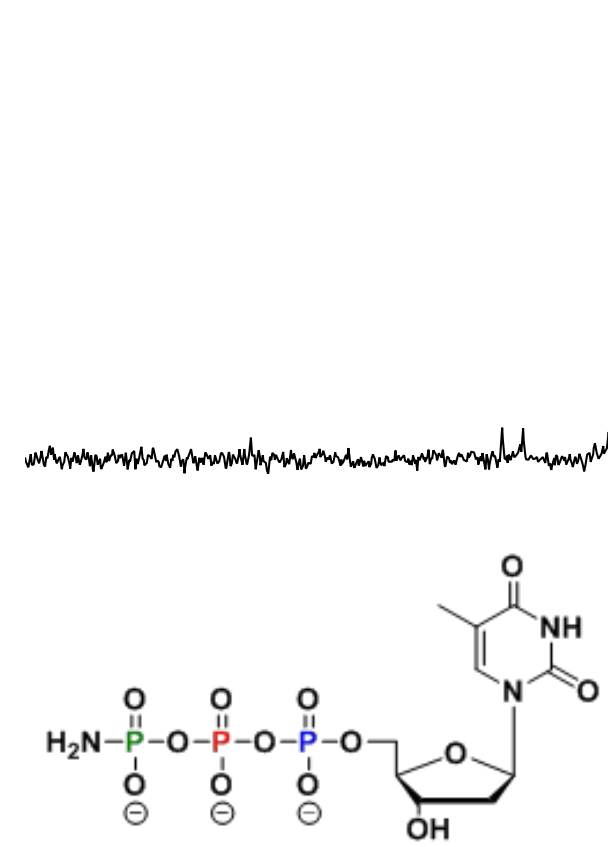

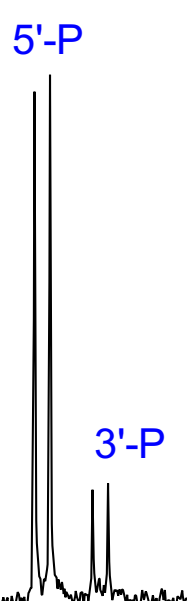

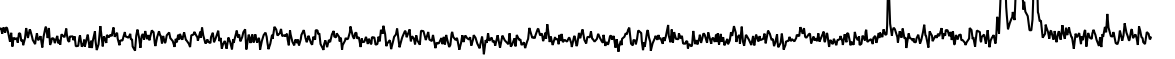

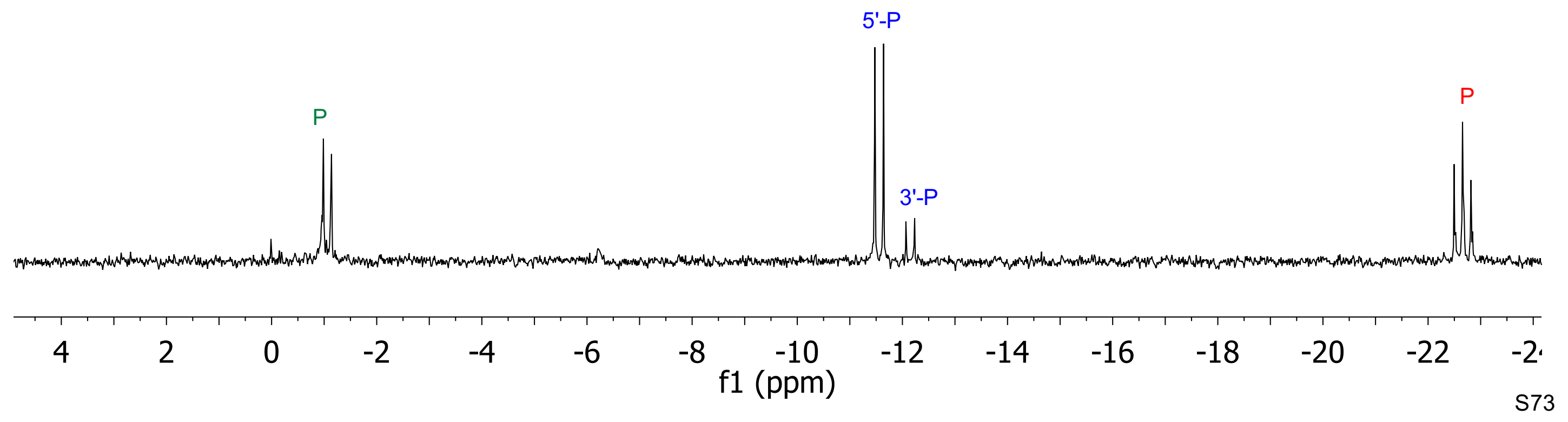




\section{${ }^{31} \mathrm{P}\left\{{ }^{1} \mathrm{H}\right\}$ NMR}

(1)

\section{Oxidation}
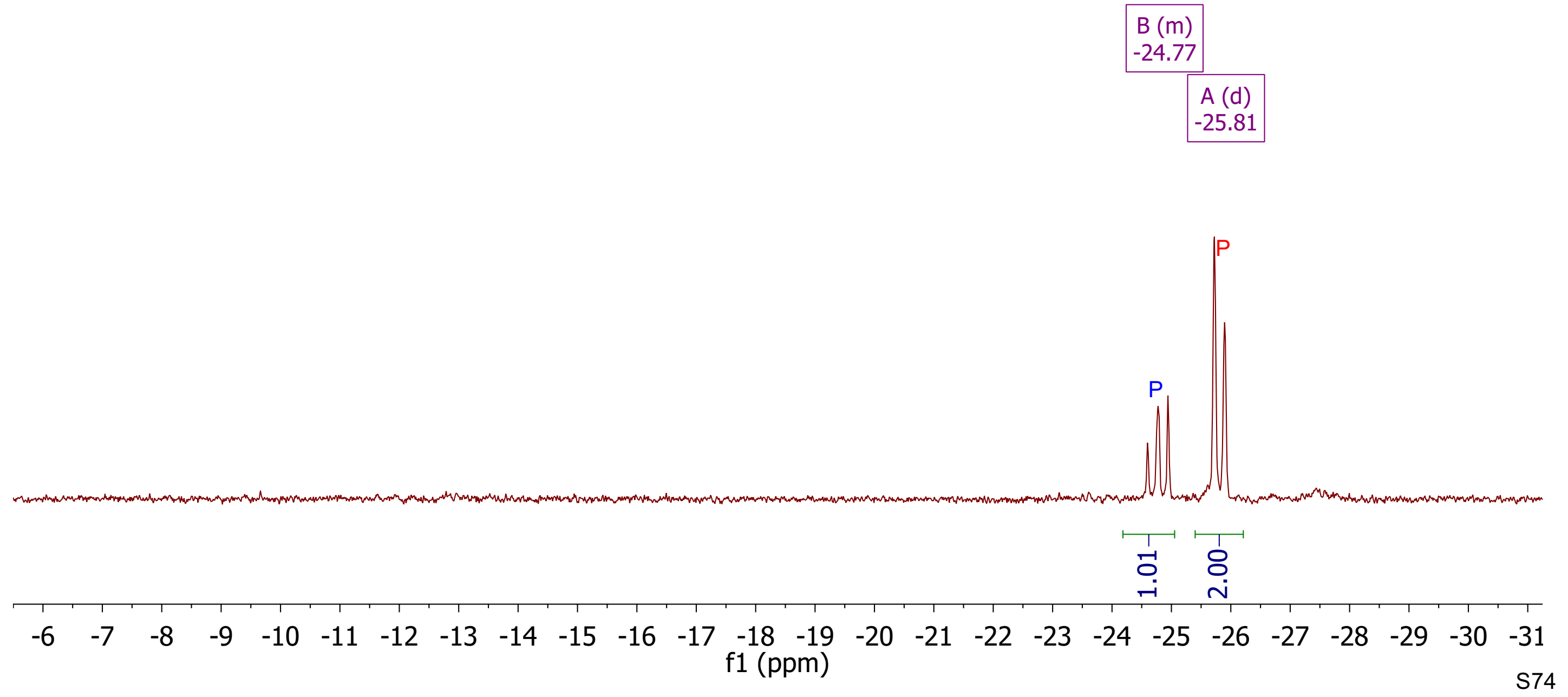


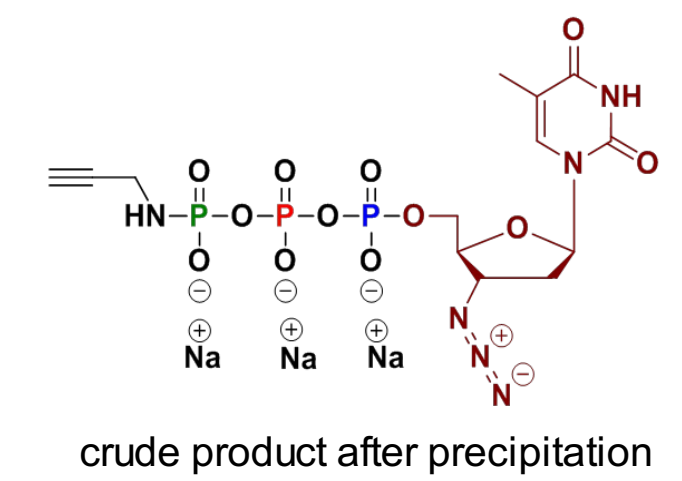

crude product after precipitation
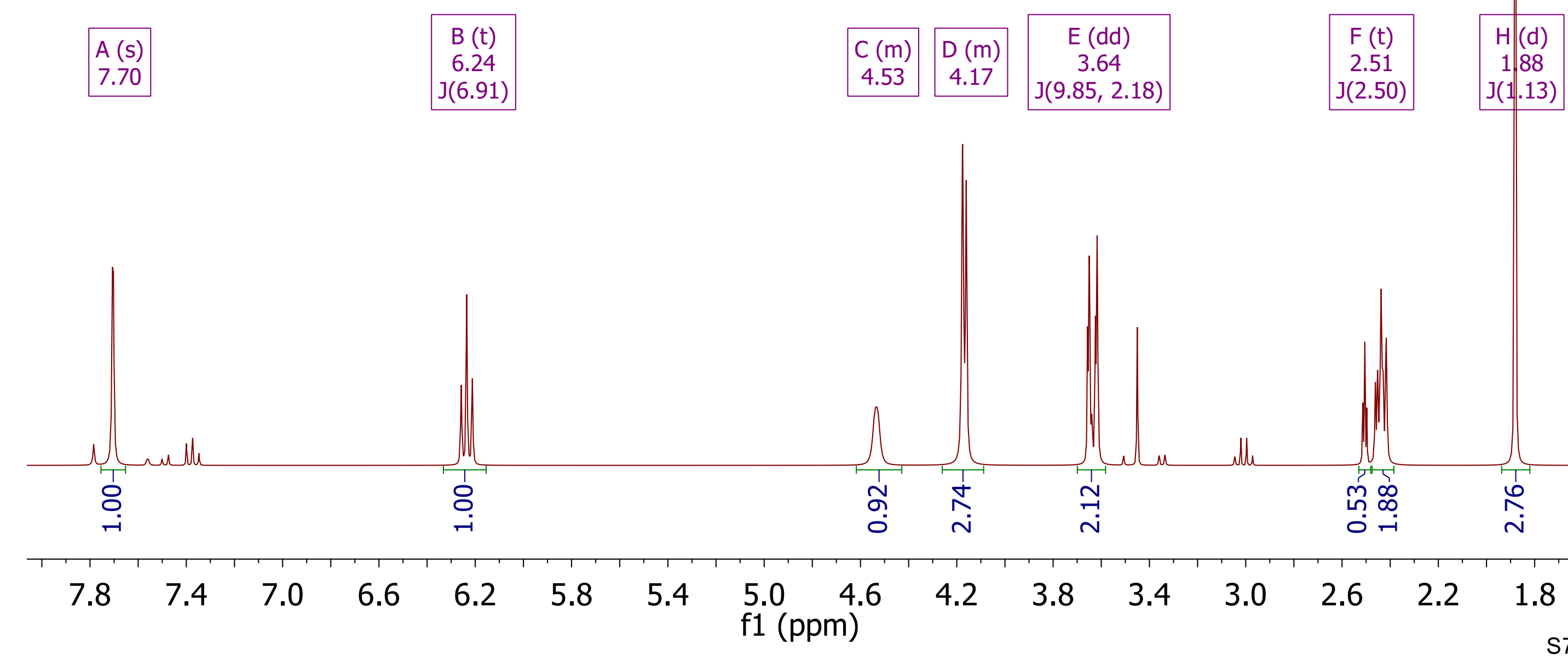

$\mathrm{G}(\mathrm{m})$

2.44 


\section{${ }^{31} \mathrm{P}\left\{{ }^{1} \mathrm{H}\right\}$ NMR}

ตุ

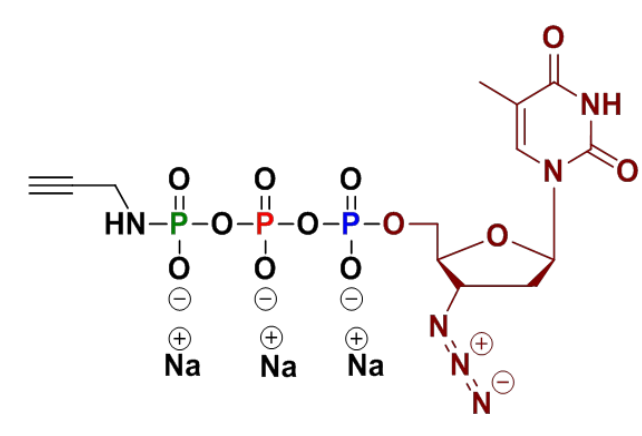

crude product after precipitation

$$
\begin{aligned}
& A(d) \\
& -1.99
\end{aligned}
$$

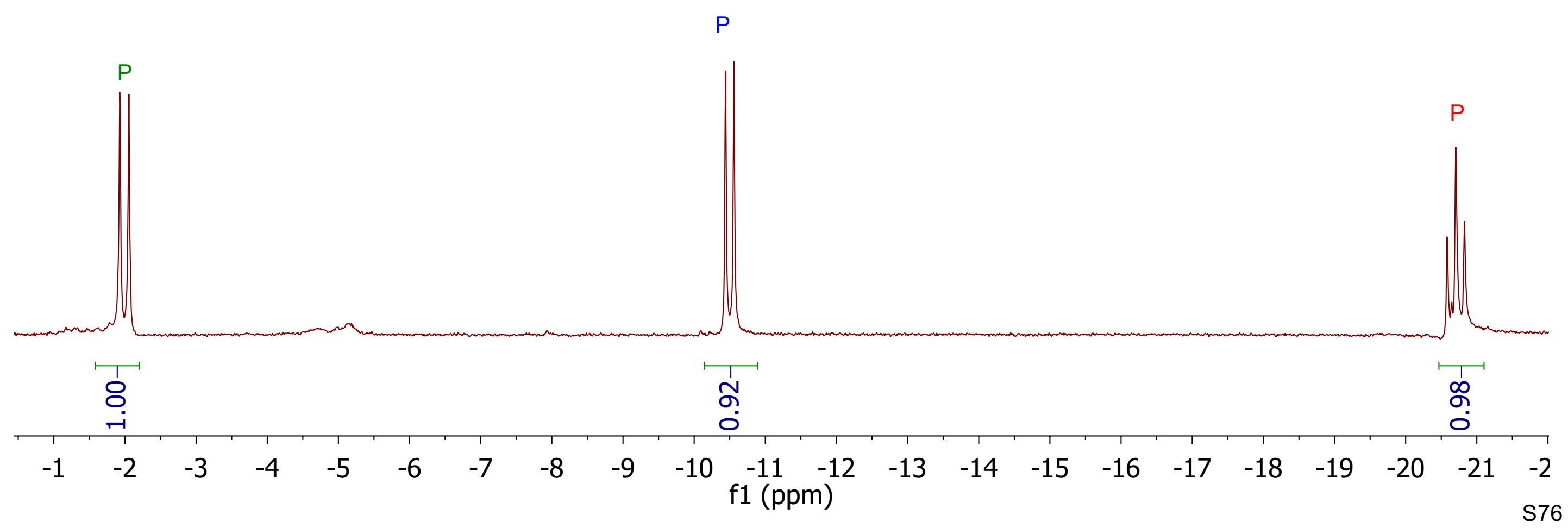




\section{${ }^{31} \mathrm{P}$ NMR}

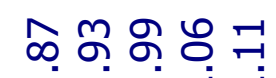

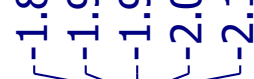

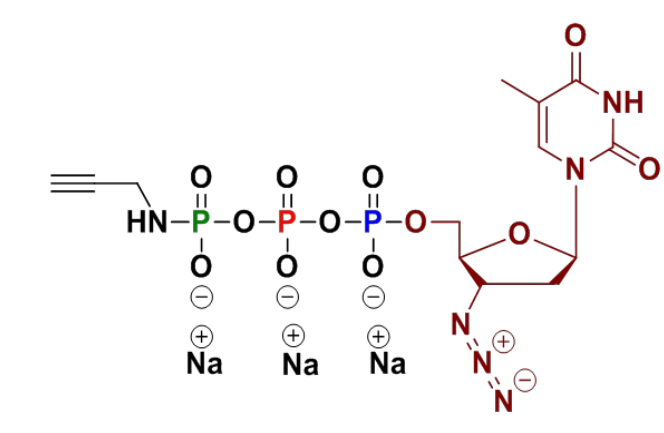

crude product after precipitation

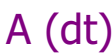

$-1.99$

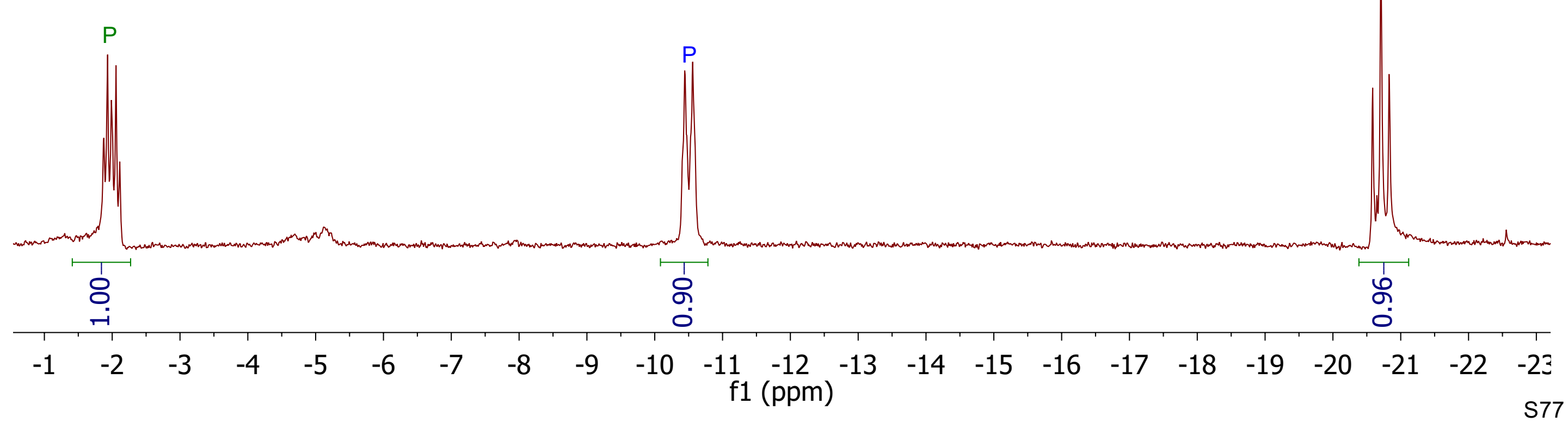



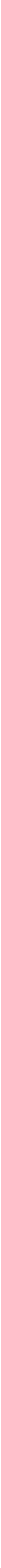


\section{${ }^{31} \mathrm{P}\left\{{ }^{1} \mathrm{H}\right\} \mathrm{NMR}$}
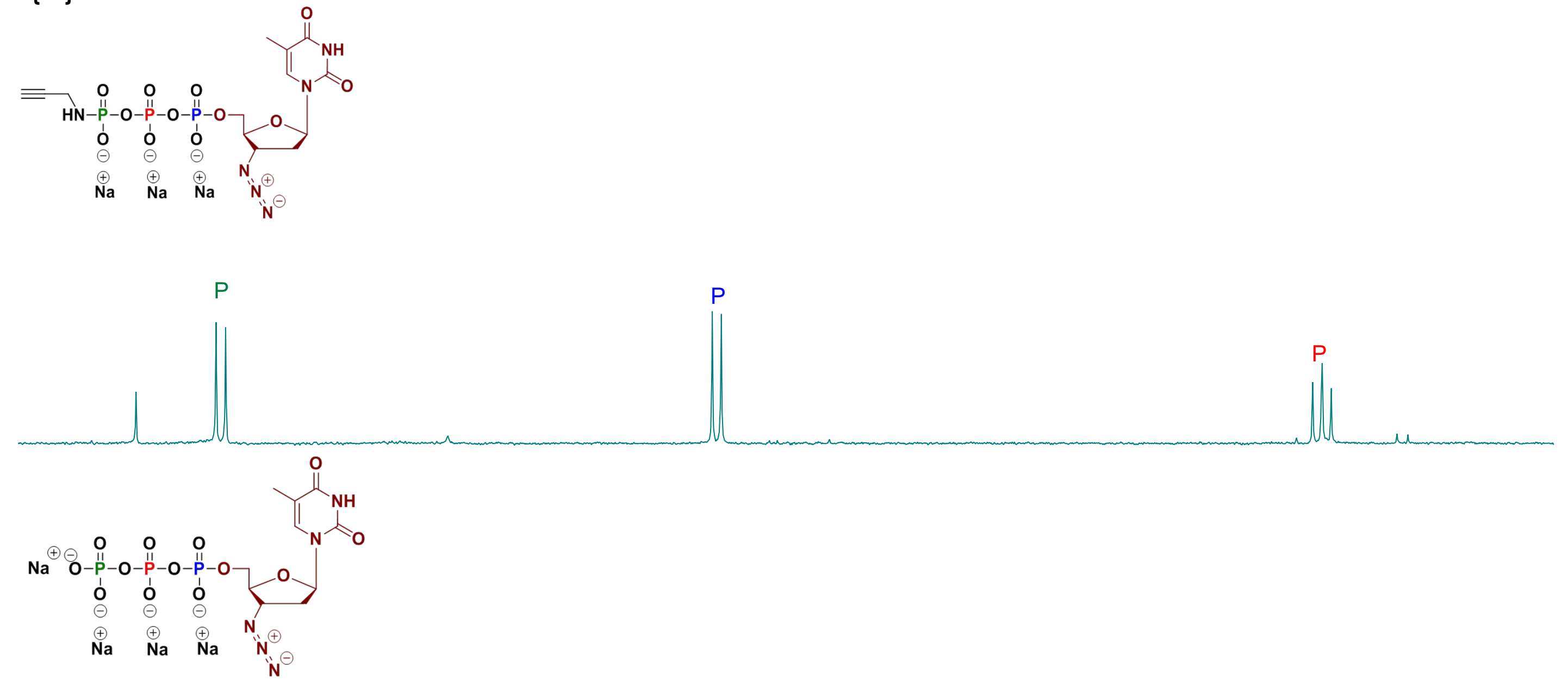

Acidic cleavage of propargyl amine at $\mathrm{pH}=4.5$

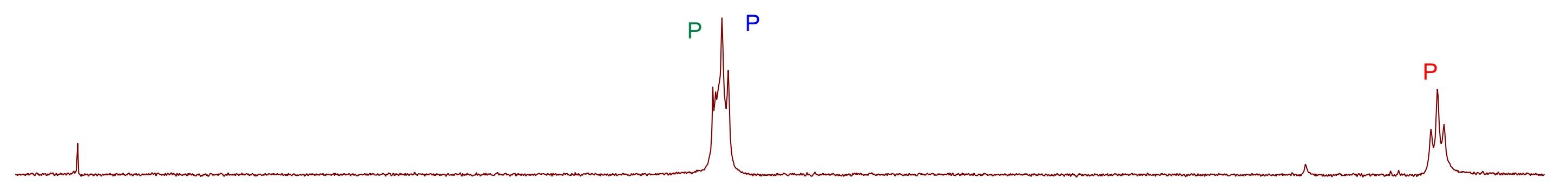




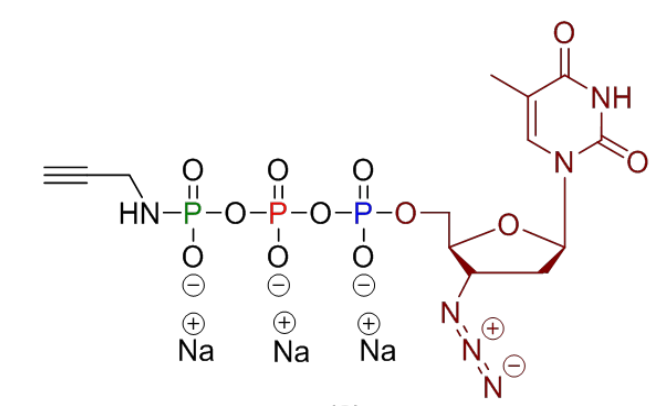

(2)

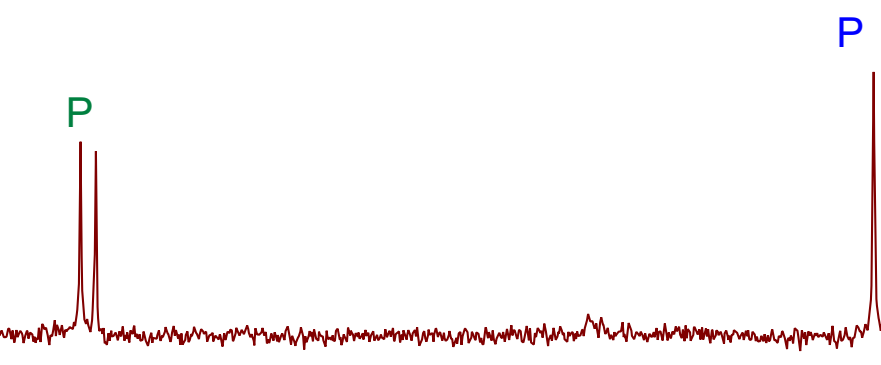

P

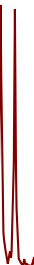

Acidic cleavage of propargylamidate triphosphate 2

(400 $\mathrm{mg}$ of 2 reacted with $\mathrm{HCl}$ resulted in 3 at $\mathrm{pH}=2.5$ )

crude product after precipitation
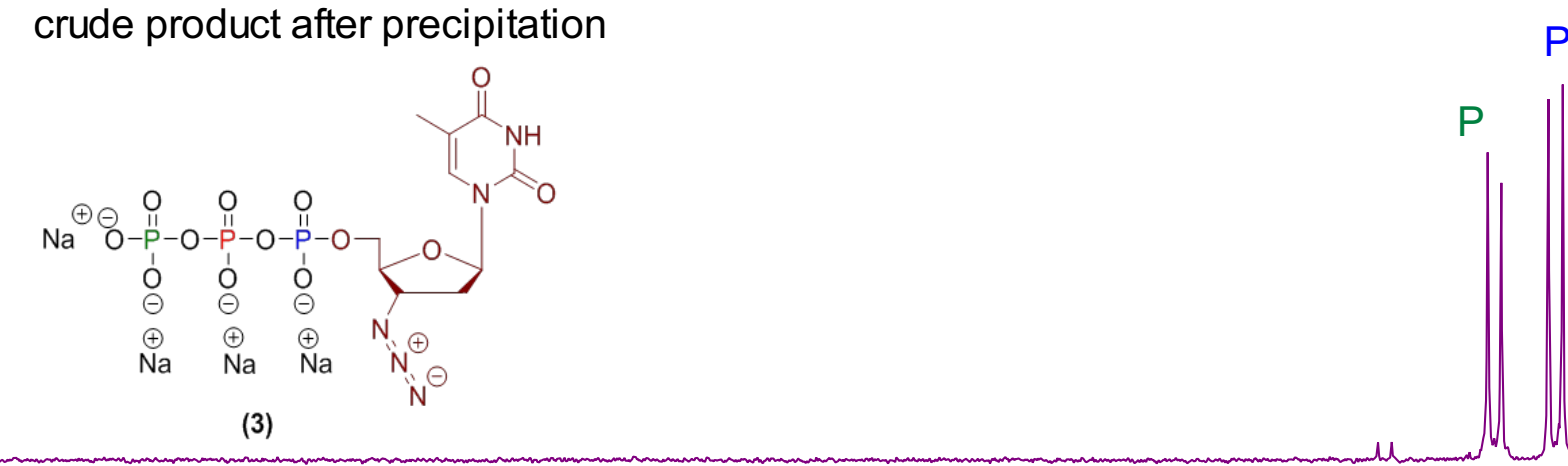

\section{${ }^{31}$ P NMR}
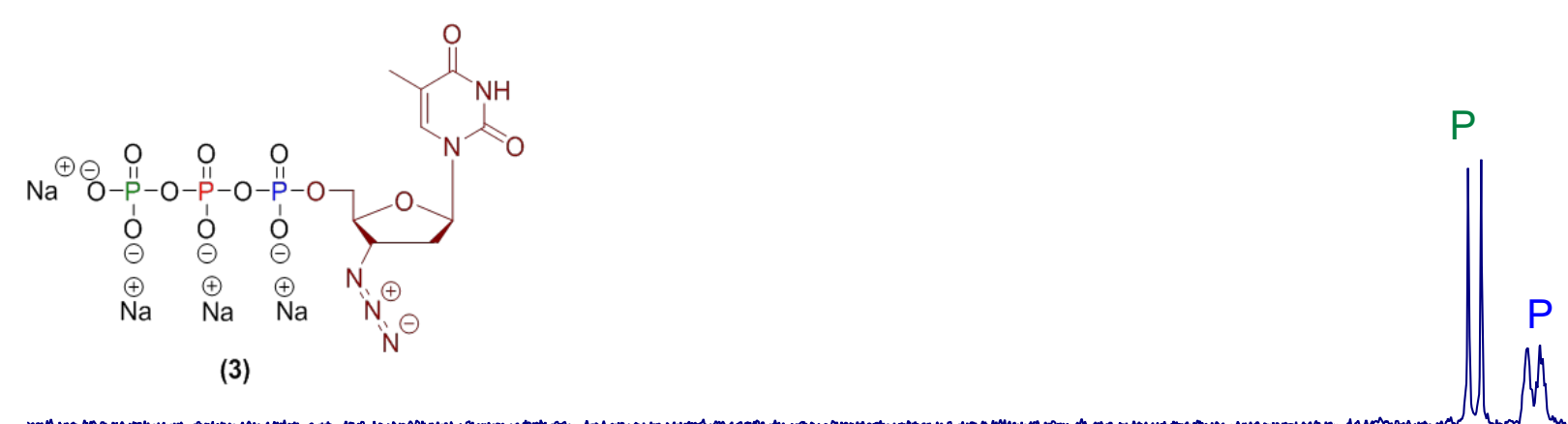

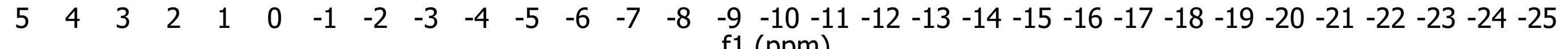
f1 (ppm) 

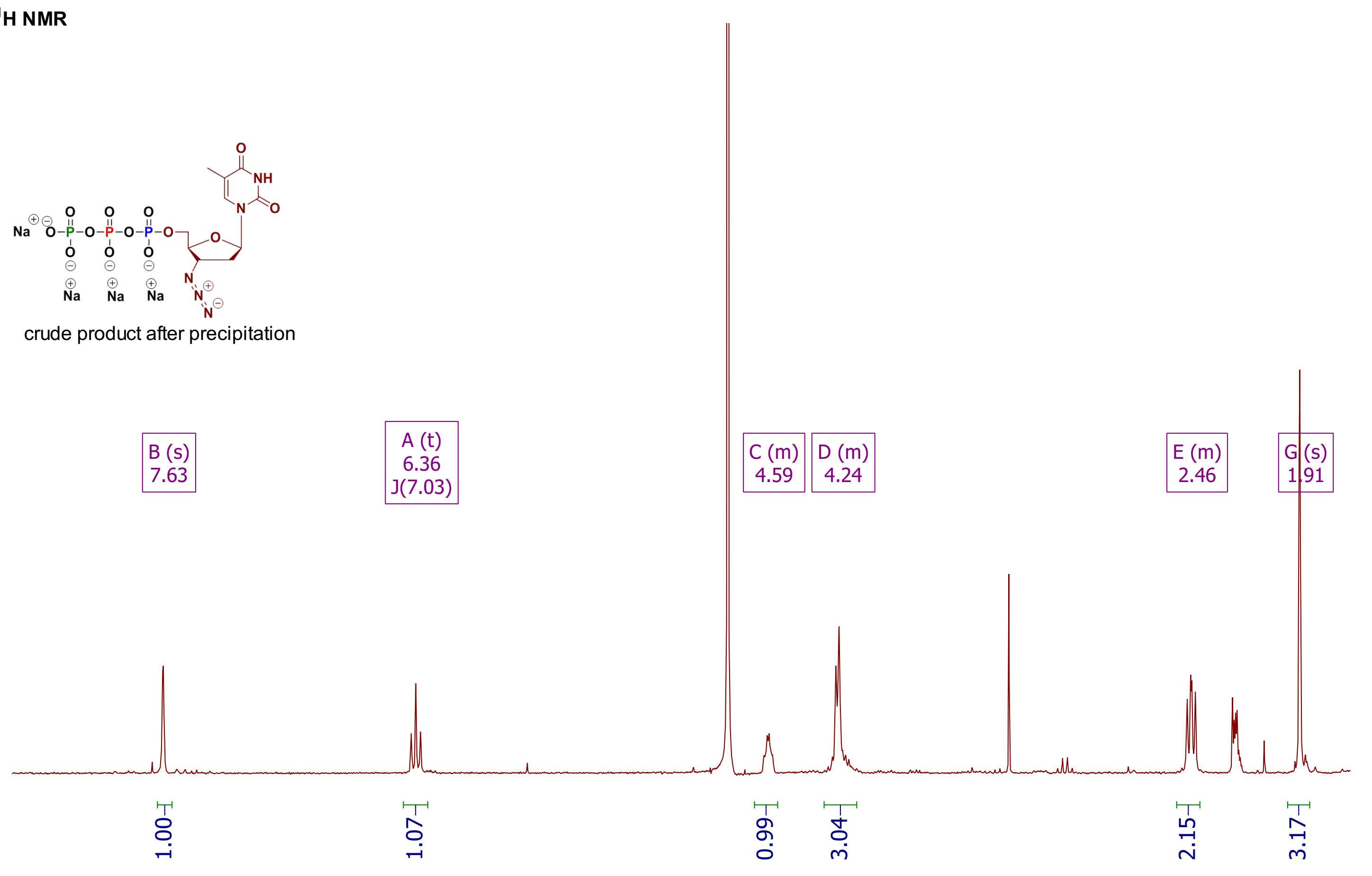

\begin{tabular}{|c|c|c|c|c|c|c|c|c|c|c|c|c|c|c|c|c|}
\hline 8.2 & 7.8 & 7.4 & 7.0 & 6.6 & 6.2 & 5.8 & 5.4 & $\begin{array}{l}5.0 \\
(\mathrm{ppm})\end{array}$ & 4.6 & 4.2 & 3.8 & 3.4 & 3.0 & 2.6 & 2.2 & $\begin{array}{r}1.8 \\
58\end{array}$ \\
\hline
\end{tabular}




\section{${ }^{31} \mathrm{P}\left\{{ }^{1} \mathrm{H}\right\}$ NMR}

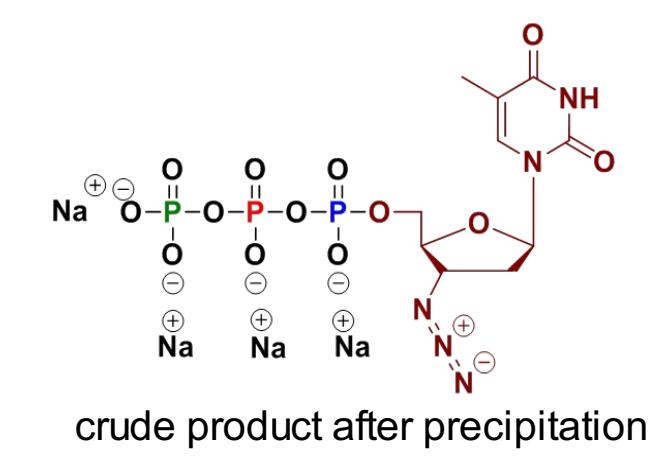

\begin{tabular}{|c|c|}
\hline$A(d)$ & $B(d)$ \\
\hline-7.02 & -11.53 \\
\hline
\end{tabular}

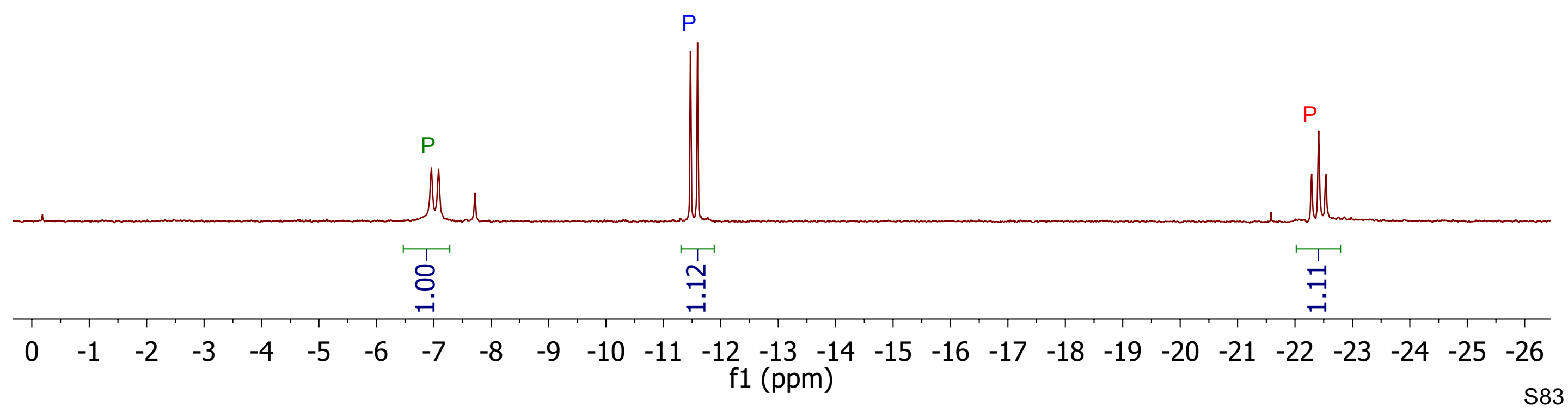




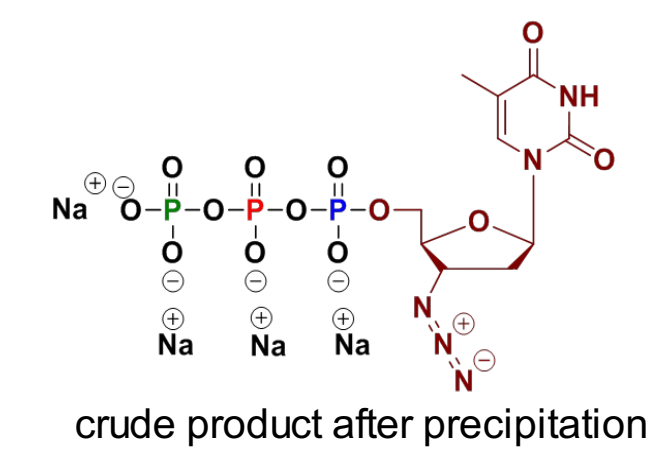

\begin{tabular}{|c|c|}
\hline $\begin{array}{l}B(d) \\
-7.01\end{array}$ & $\begin{array}{l}A(d t) \\
-11.54\end{array}$ \\
\hline
\end{tabular}

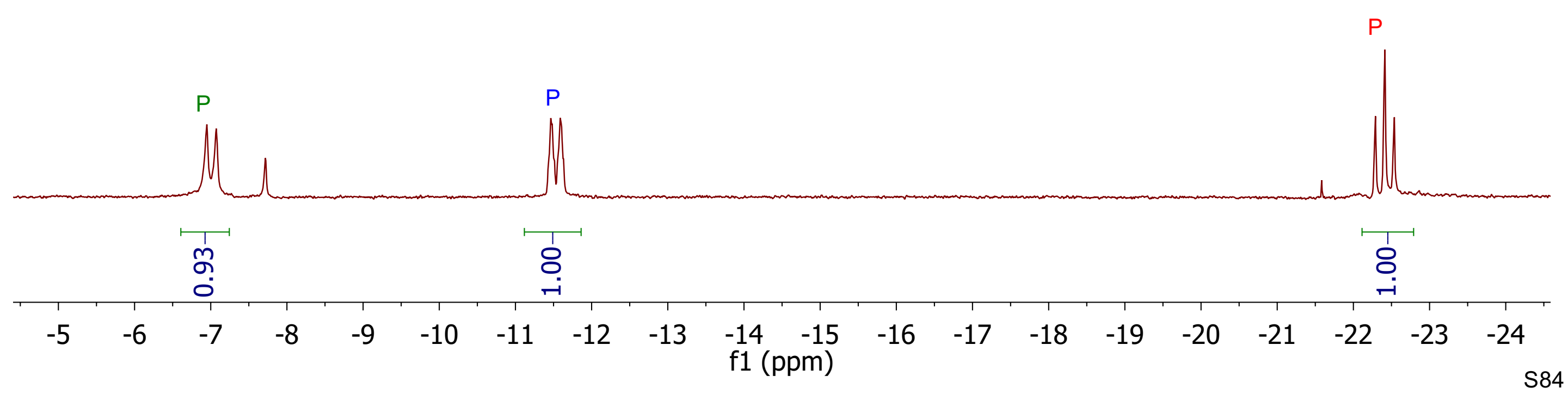




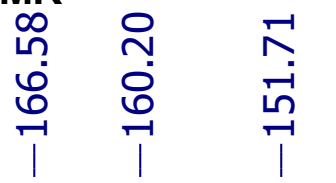

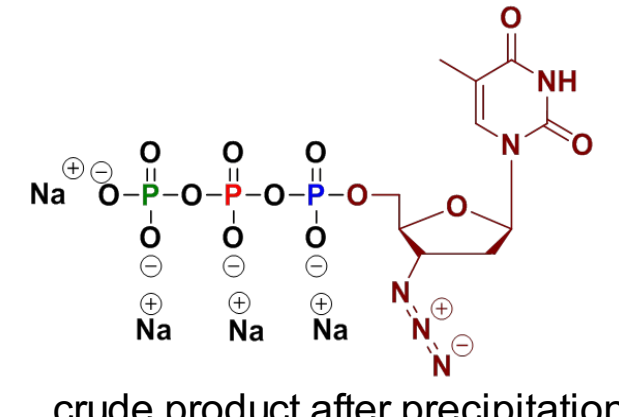

crude product after precipitation

\begin{tabular}{|c|c|c|}
\hline$A(s)$ & $C(s)$ & $D(s)$ \\
166.58 & 151.71 & 137.25 \\
\hline
\end{tabular}

$E(s)$
111.81

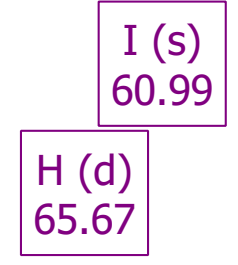

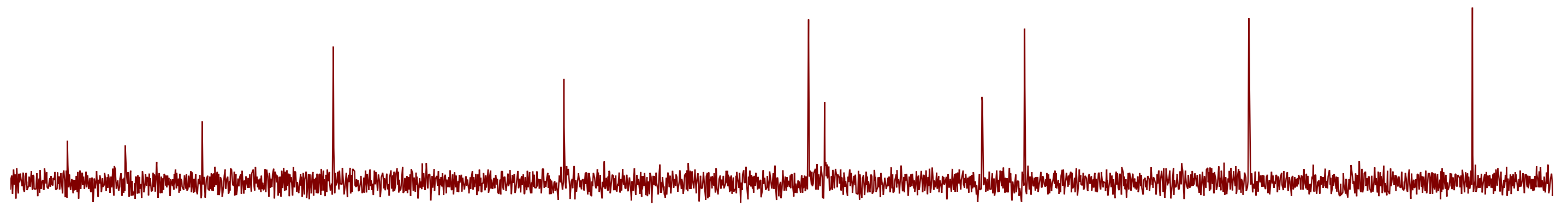

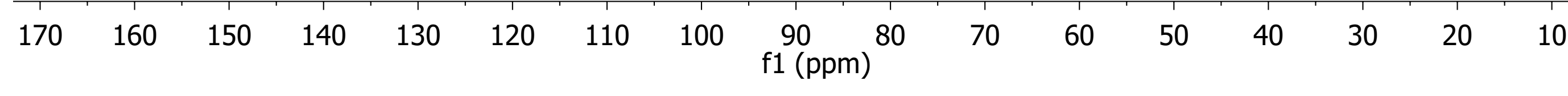




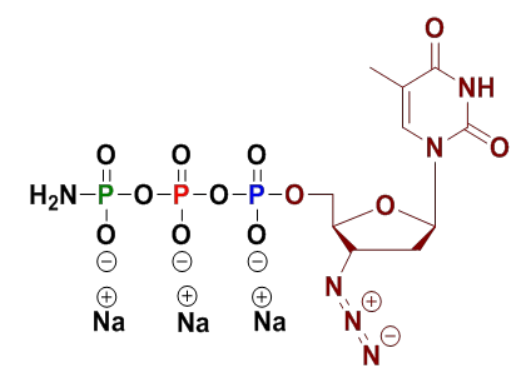

crude product after precipitation

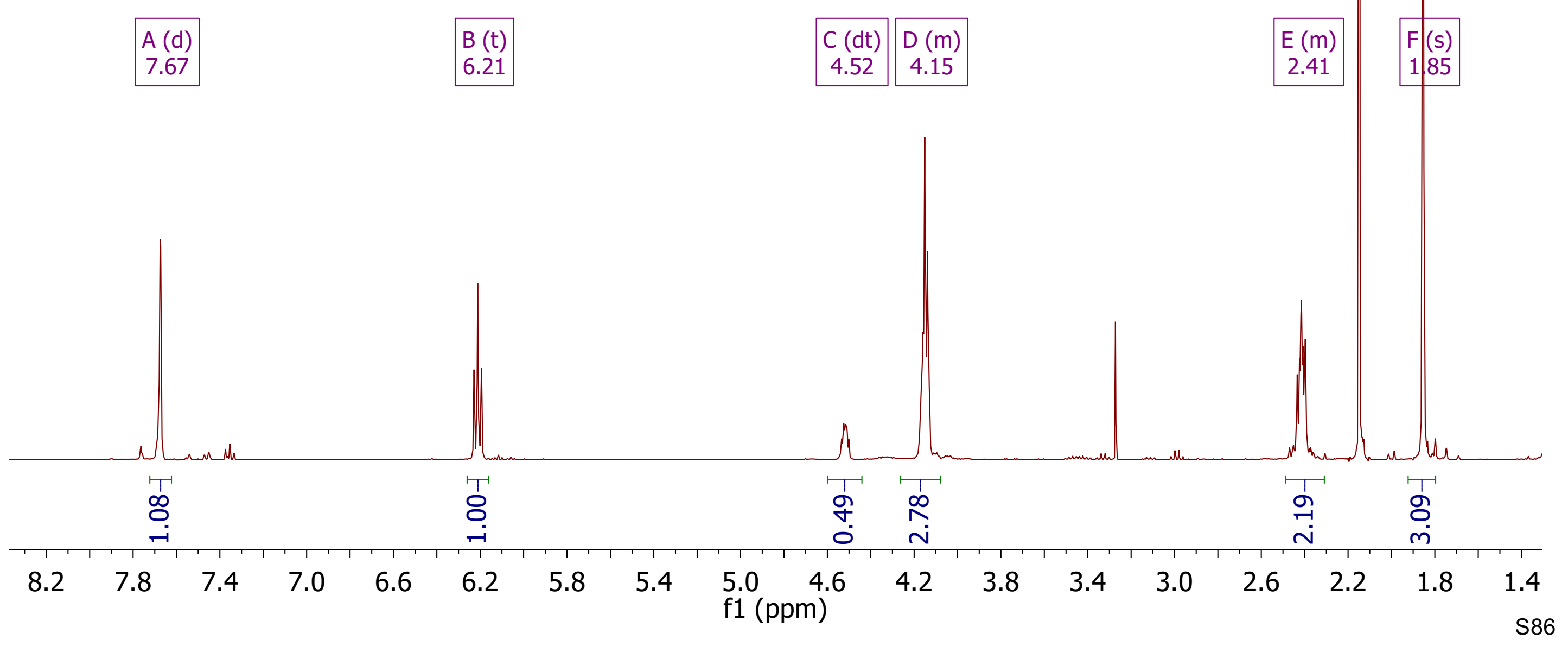


crude product after precipitation

$$
\begin{array}{l|}
C(d) \\
-1.04
\end{array}
$$
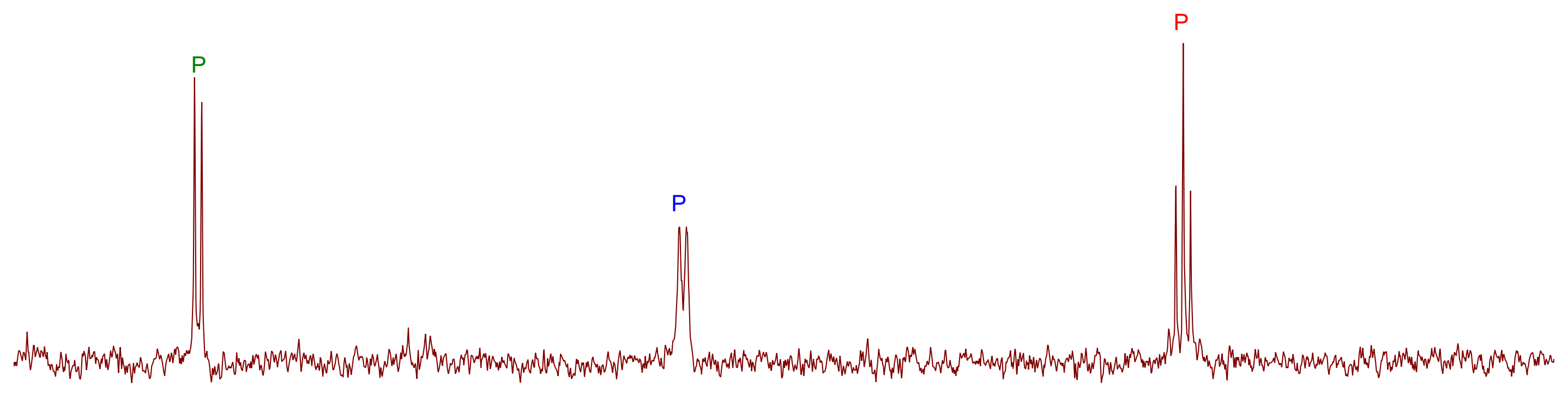

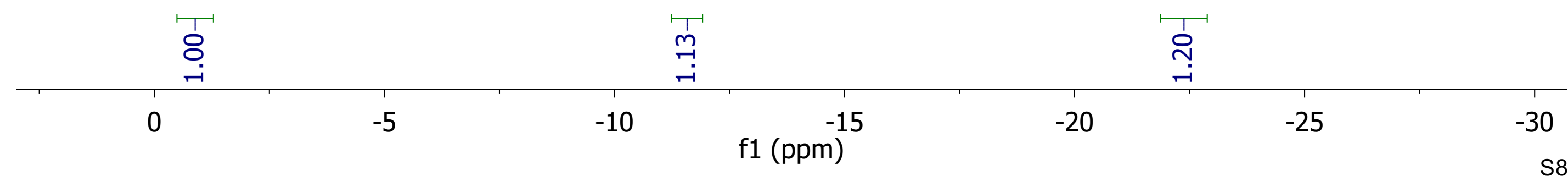




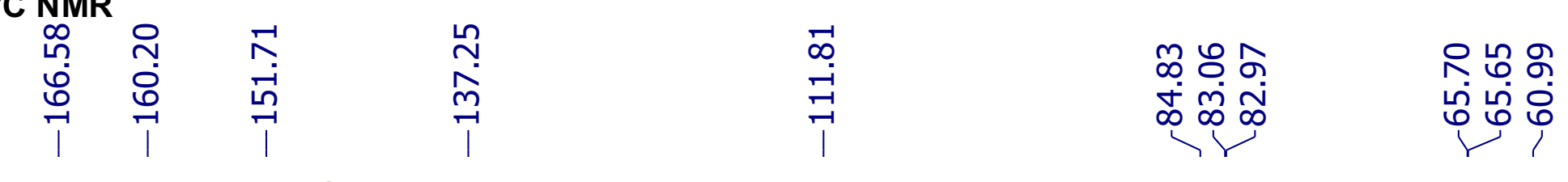

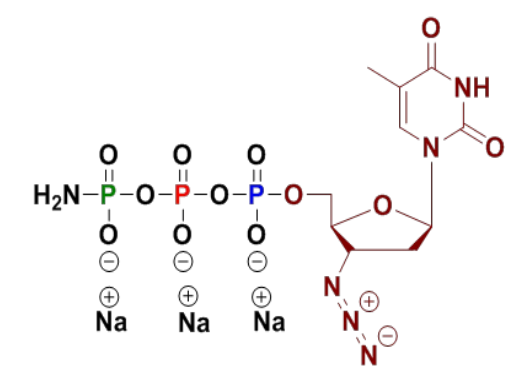

crude product after precipitation

\begin{tabular}{|c|}
\hline$C(s)$ \\
151.71
\end{tabular}

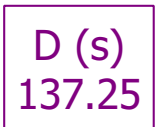

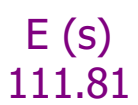

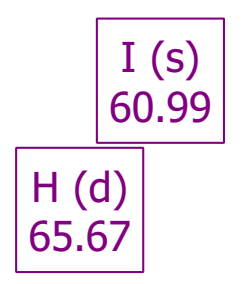

$\mathrm{J}(\mathrm{s})$

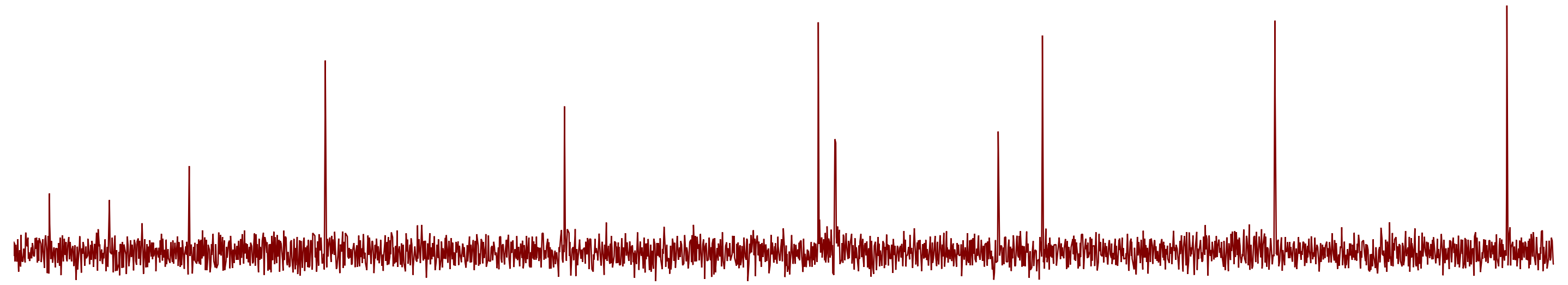

\begin{tabular}{|c|c|c|c|c|c|c|c|c|c|c|c|c|c|c|c|}
\hline 70 & 160 & 150 & 140 & 130 & 120 & 110 & 100 & $\begin{array}{c}90 \\
\mathrm{f} 1(\mathrm{ppm})\end{array}$ & 80 & 70 & 60 & 50 & 40 & 30 & 20 \\
\hline
\end{tabular}




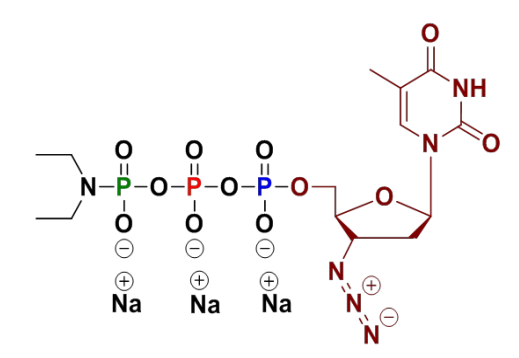

crude product after precipitation

\begin{tabular}{|l|l|}
\hline$A(s)$ & $B(t)$ \\
7.68 & 6.22 \\
\hline
\end{tabular}

$$
\begin{array}{|c|c|}
\hline C(d t) & D(m) \\
4.51 & 4.14 \\
\hline
\end{array}
$$

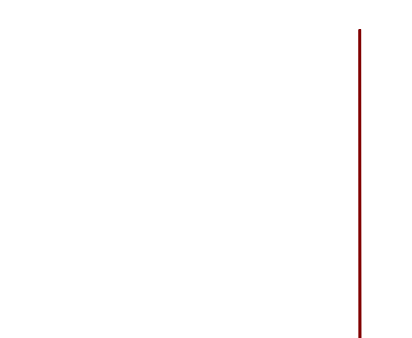

$$
\begin{array}{|c|c|}
\hline E(m) & F(m) \\
2.96 & 2.41 \\
\hline
\end{array} \quad \begin{array}{c|c|}
G & (d) \\
1 & 85 \\
\hline
\end{array}
$$
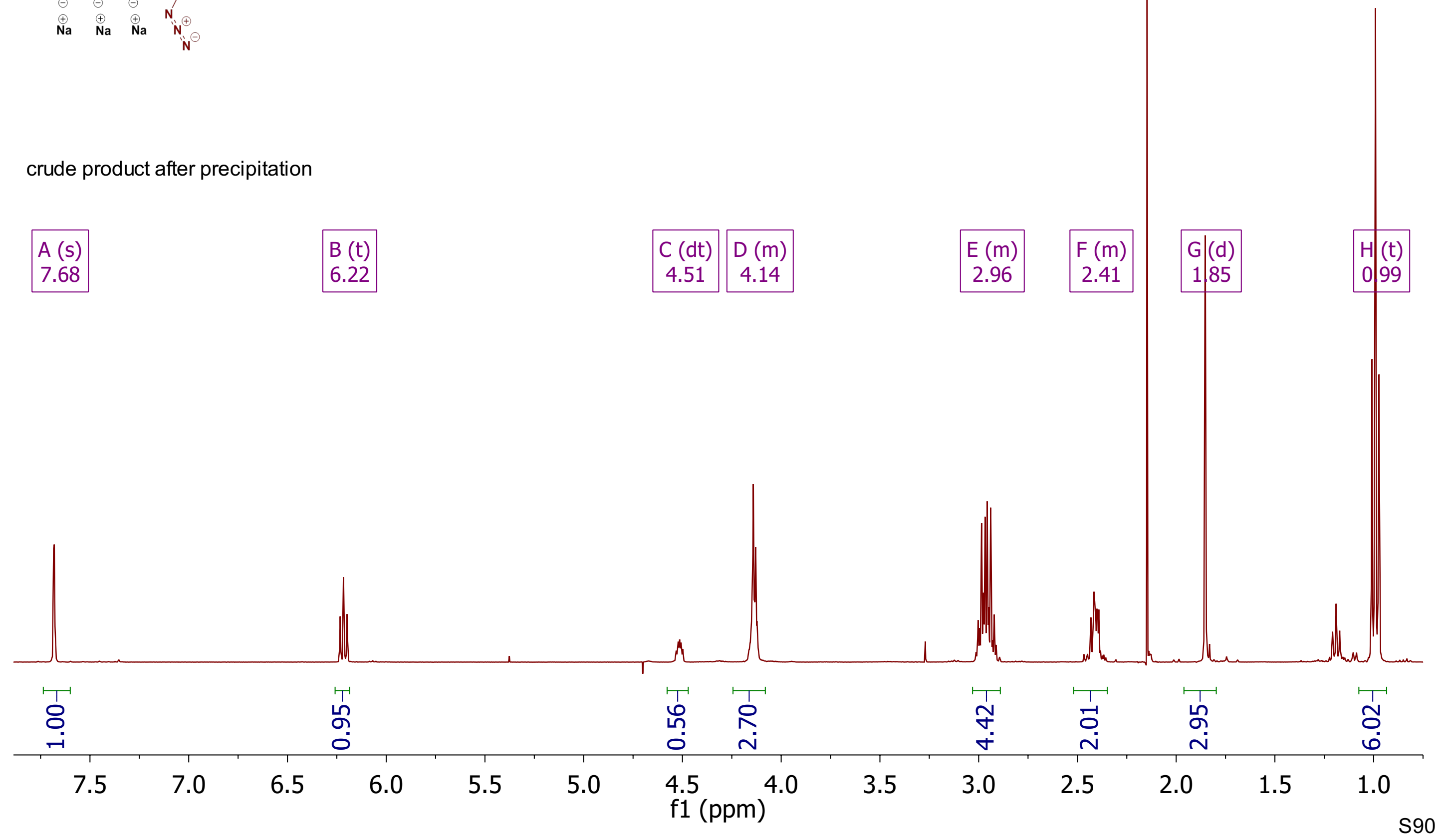
the

crude product after precipitation

\begin{tabular}{|c|}
\hline$A(d p)$ \\
-0.55 \\
\hline
\end{tabular}

B (dt)
-11.78

C (dd)

$-23.05$

P

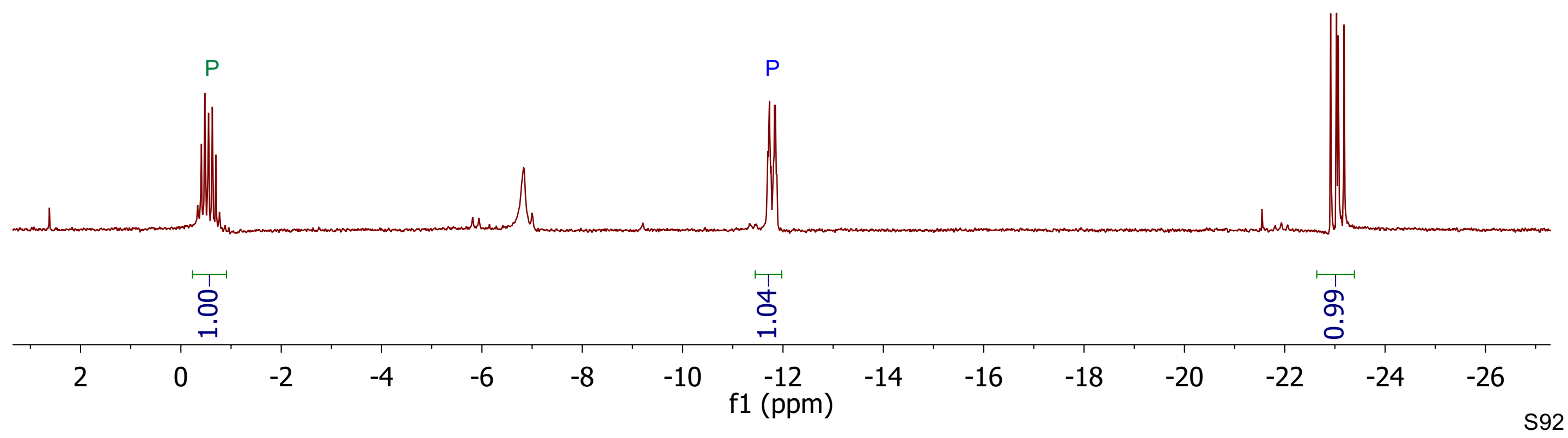




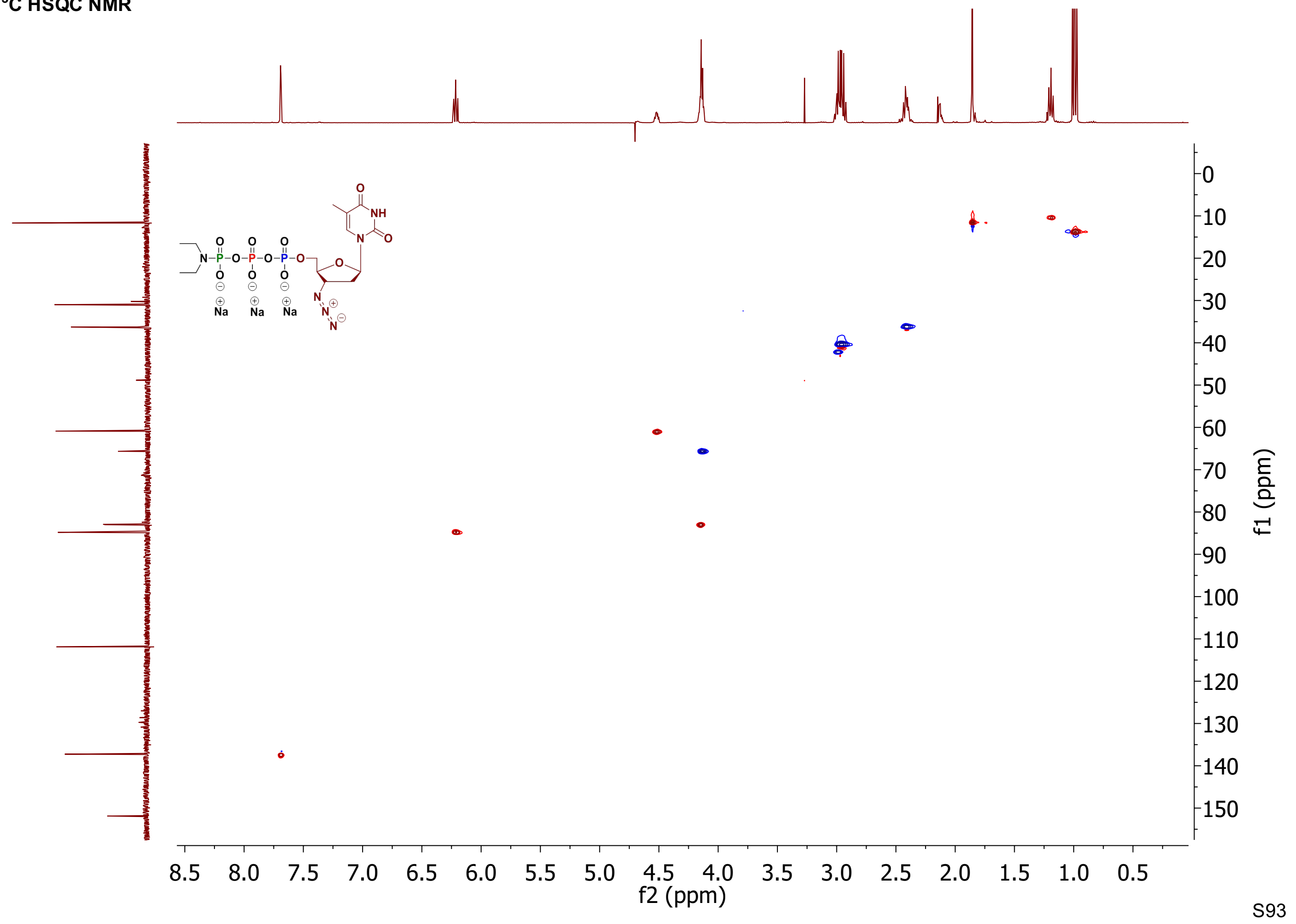




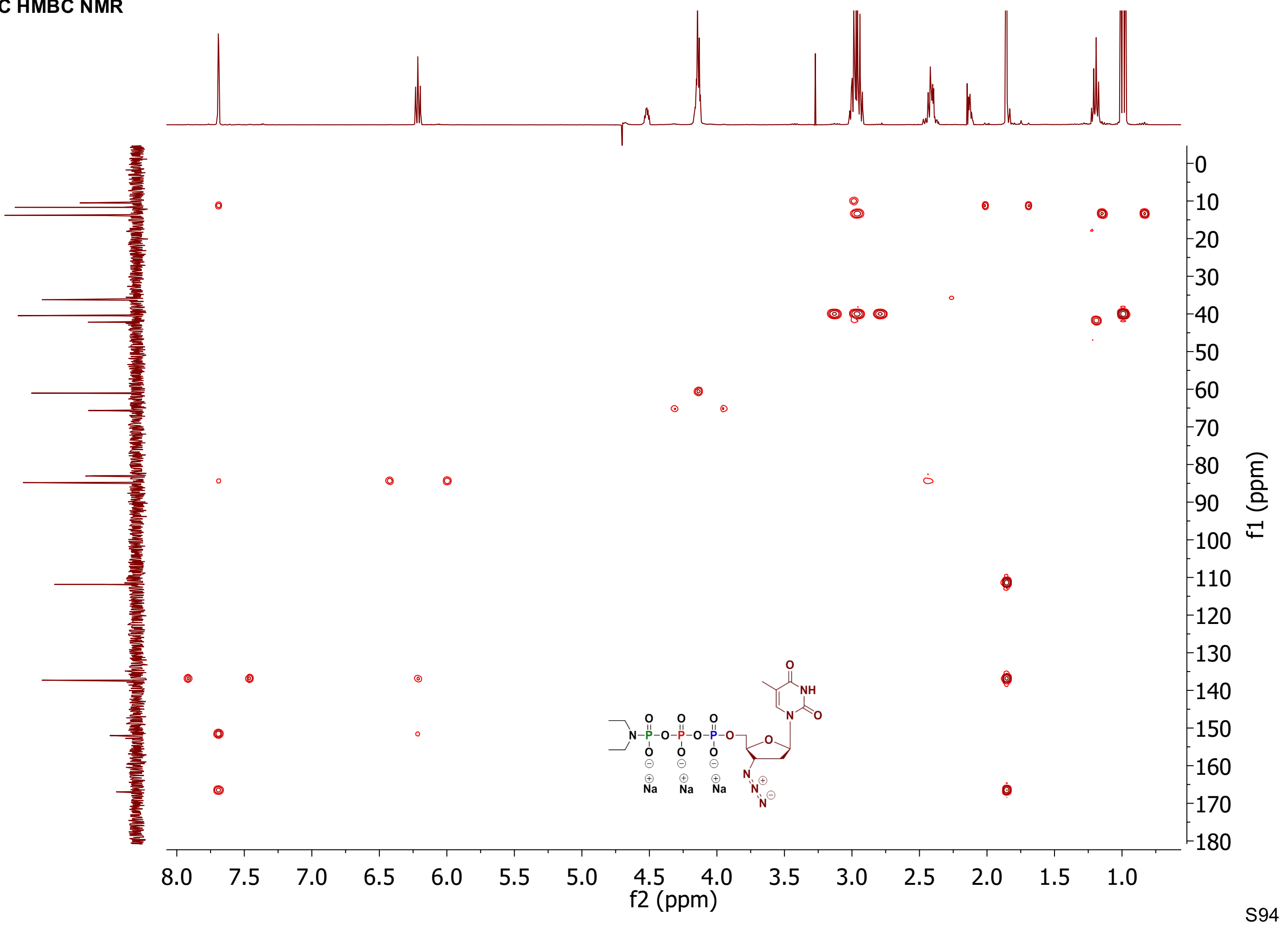




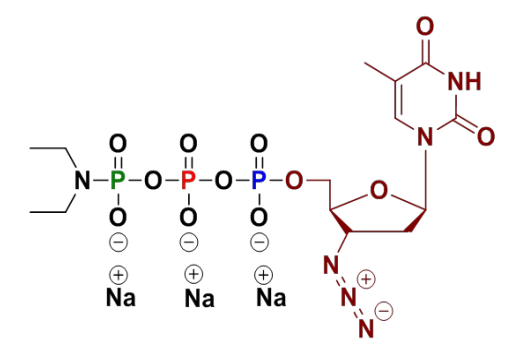

crude product after precipitation

\begin{tabular}{ccc}
\hline$N(s)$ & $A(s)$ & $B(s)$ \\
166.96 & 152.00 & 137.29 \\
\hline
\end{tabular}

\begin{tabular}{|c|}
\hline$E(d)$ \\
83.02 \\
\hline$D(s)$ \\
84.81 \\
\hline
\end{tabular}

\begin{tabular}{|l|}
\hline G (s) \\
61.04 \\
\hline$F(d)$ \\
65.63 \\
\hline
\end{tabular}

\begin{tabular}{|c|c|}
\hline $\begin{array}{l}\text { I (d) } \\
40.4\end{array}$ & \\
\hline $\begin{array}{l}\mathrm{H}(\mathrm{s}) \\
42.20\end{array}$ & \\
\hline & $\begin{array}{c}\text { J (s) } \\
36.23\end{array}$ \\
\hline
\end{tabular}

\begin{tabular}{|c|}
\hline$L(s)$ \\
11.68 \\
\hline$K(d)$ \\
13.83 \\
\hline
\end{tabular}

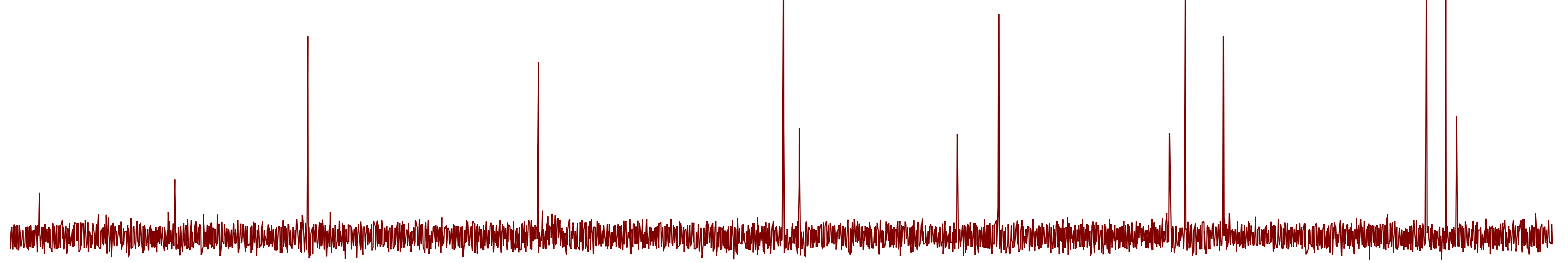

$\begin{array}{rrrrrrrrrrrrrrrrrr}70 & 160 & 150 & 140 & 130 & 120 & 110 & 100 & 90 & 80 & 70 & 60 & 50 & 40 & 30 & 20 & 10 & (\mathrm{f} 1 \text { (ppm) }\end{array}$




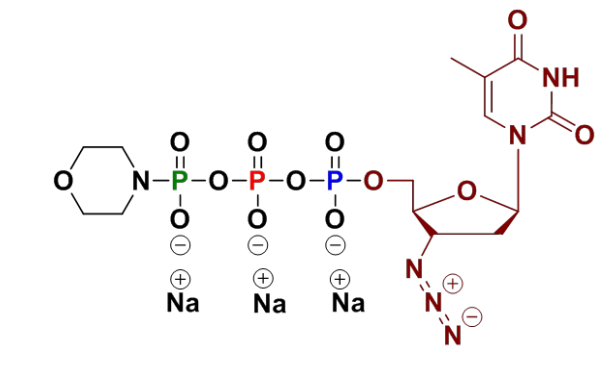

crude product after precipitation

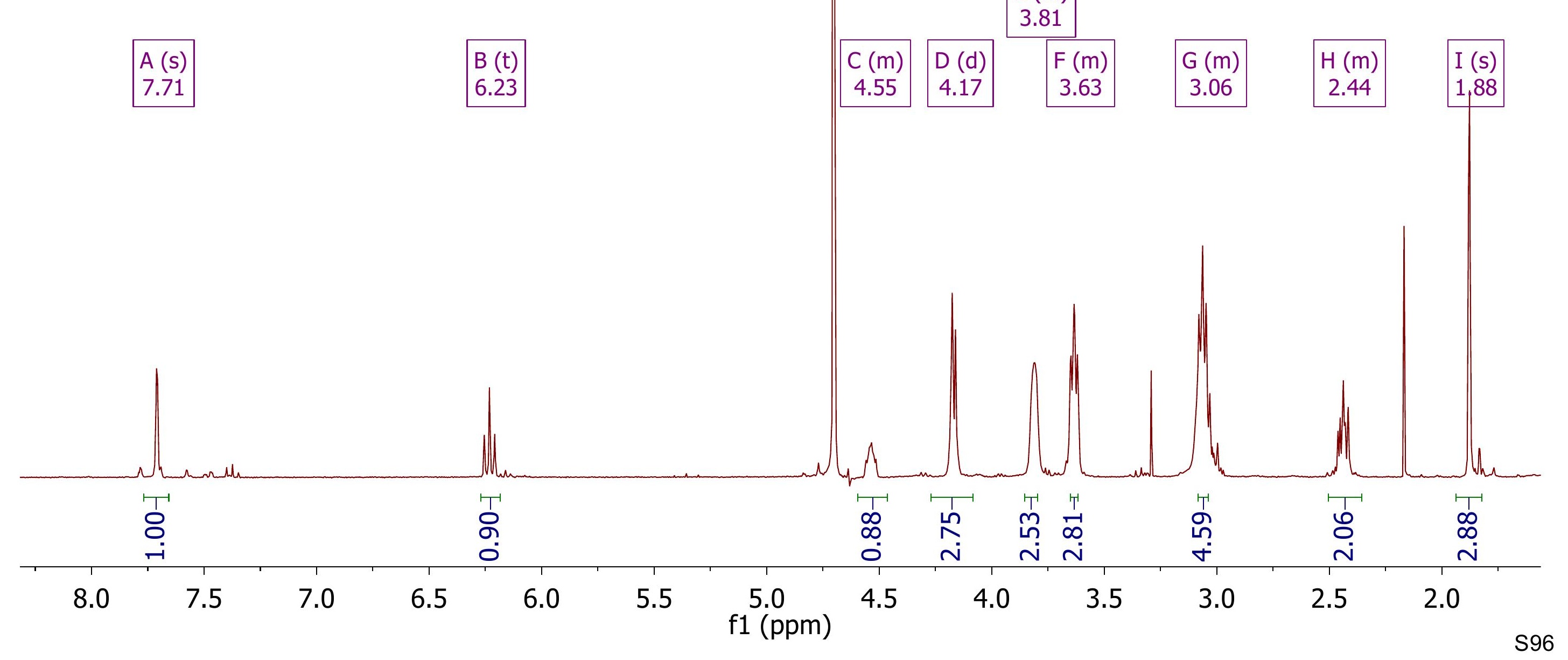




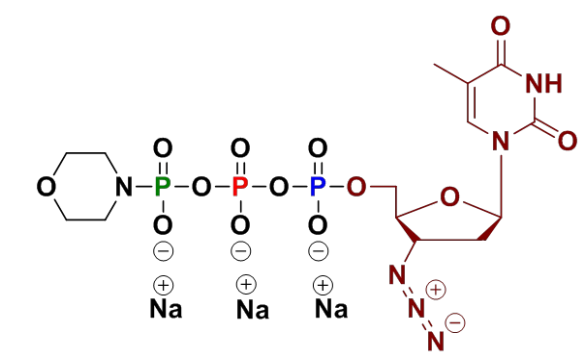

crude product after precipitation
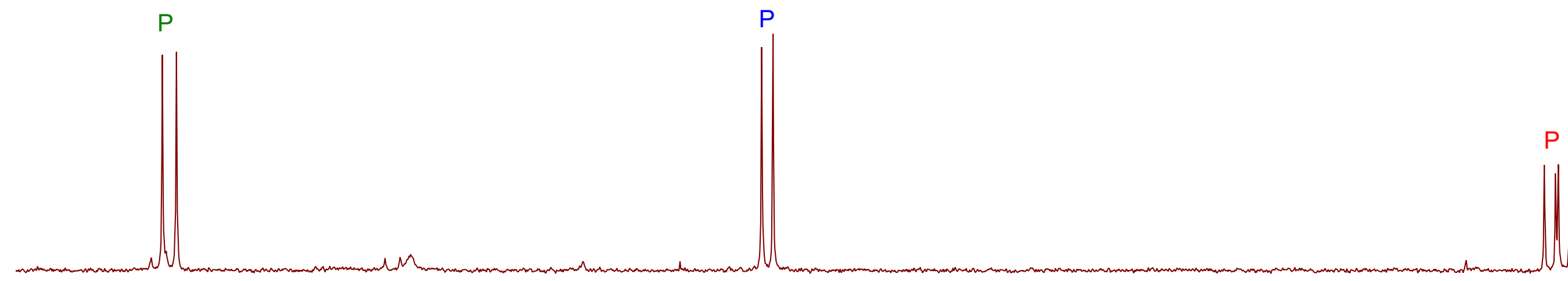


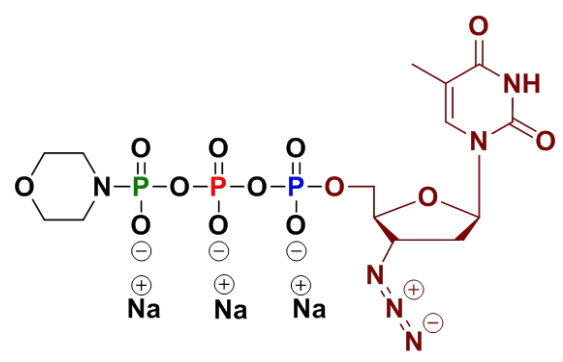

crude product after precipitation

$$
\begin{aligned}
& C(m) \\
& -3.30
\end{aligned}
$$

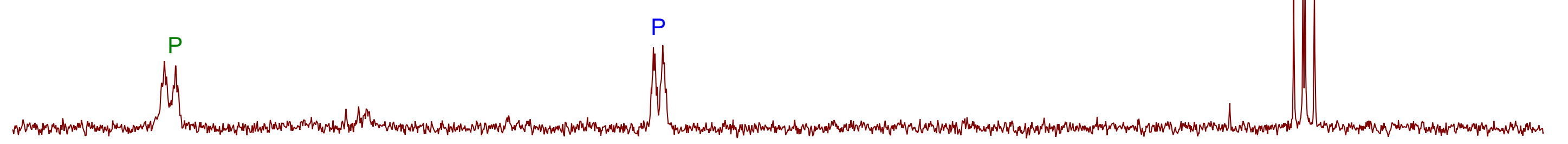

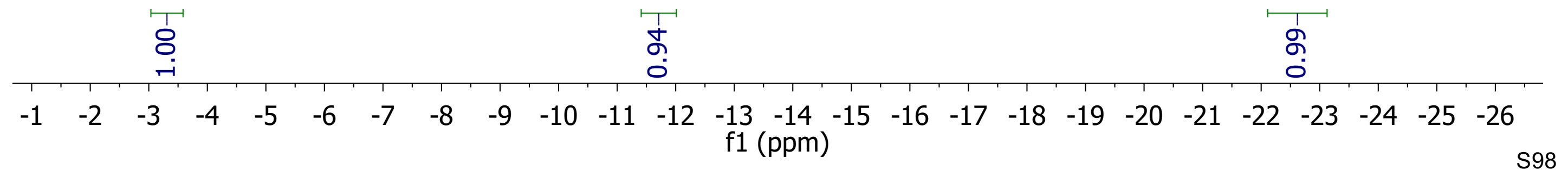




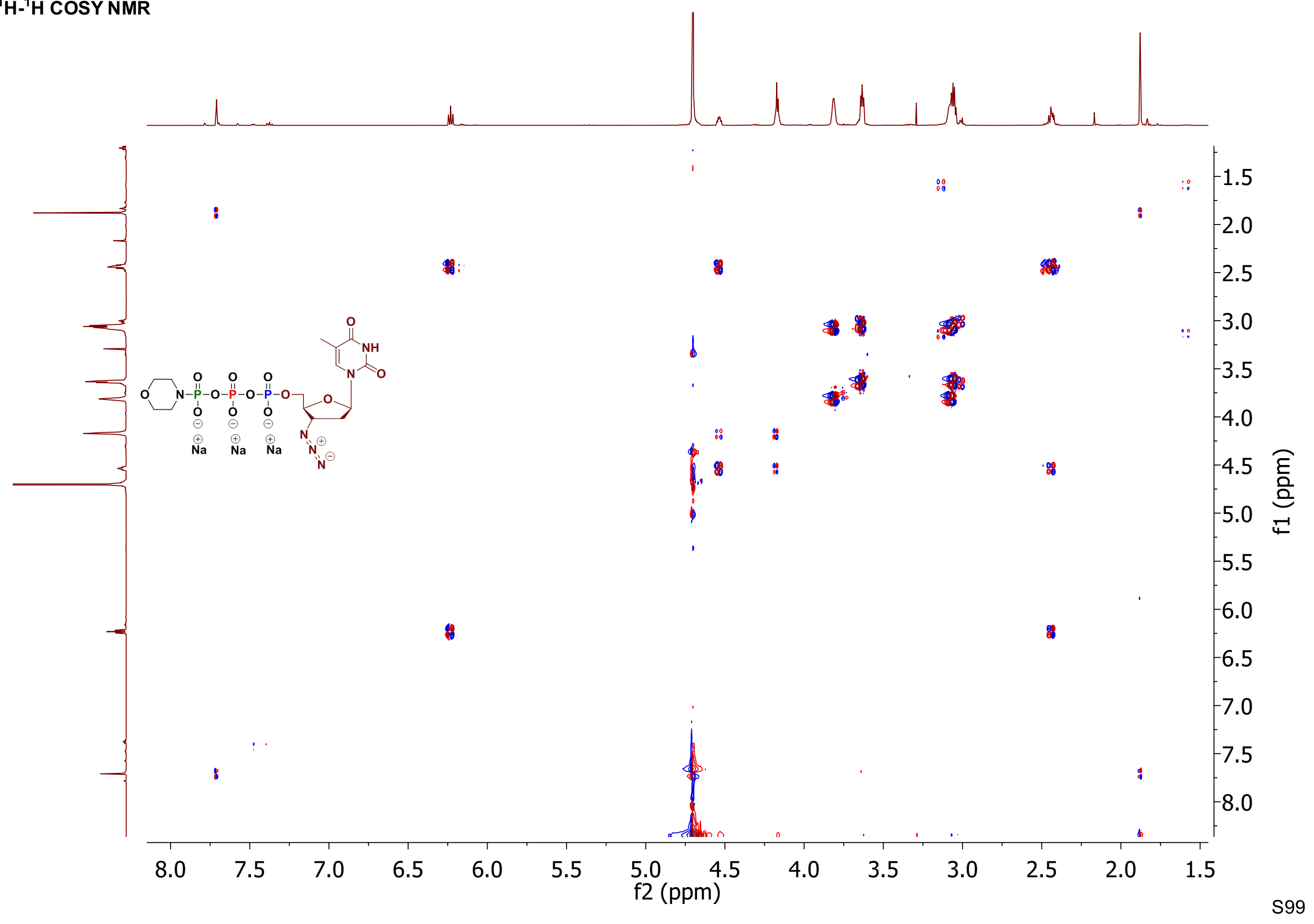




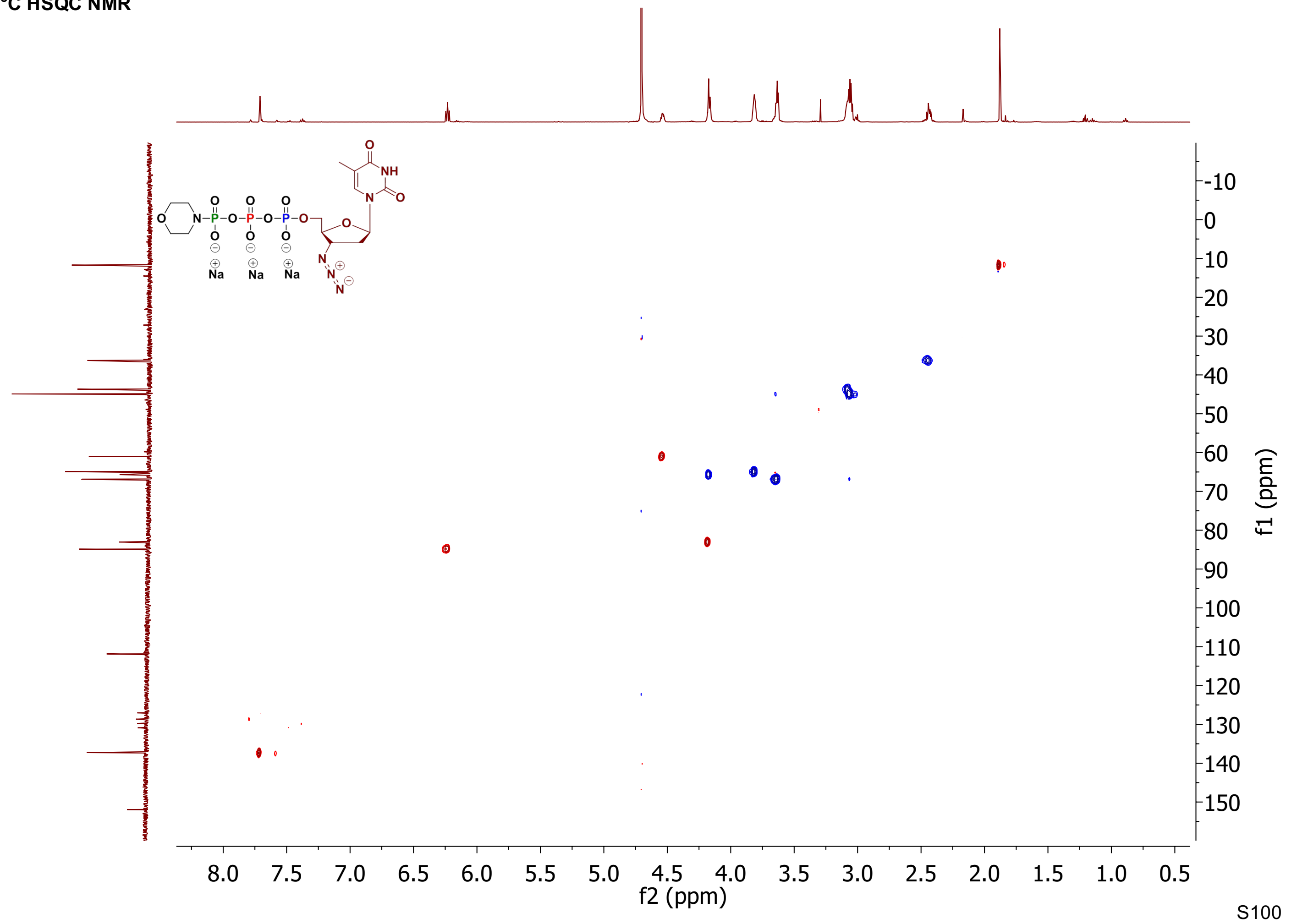




\section{${ }^{13} \mathrm{C}$ NMR}

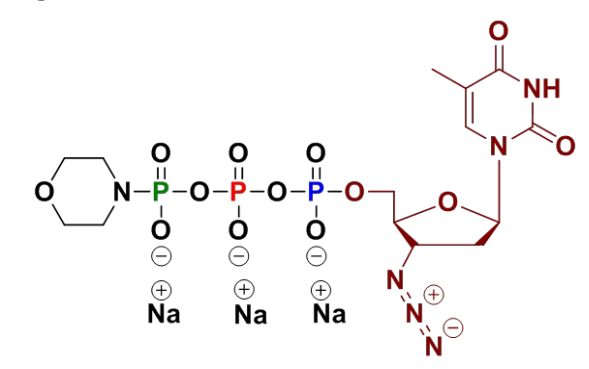

crude product after precipitation

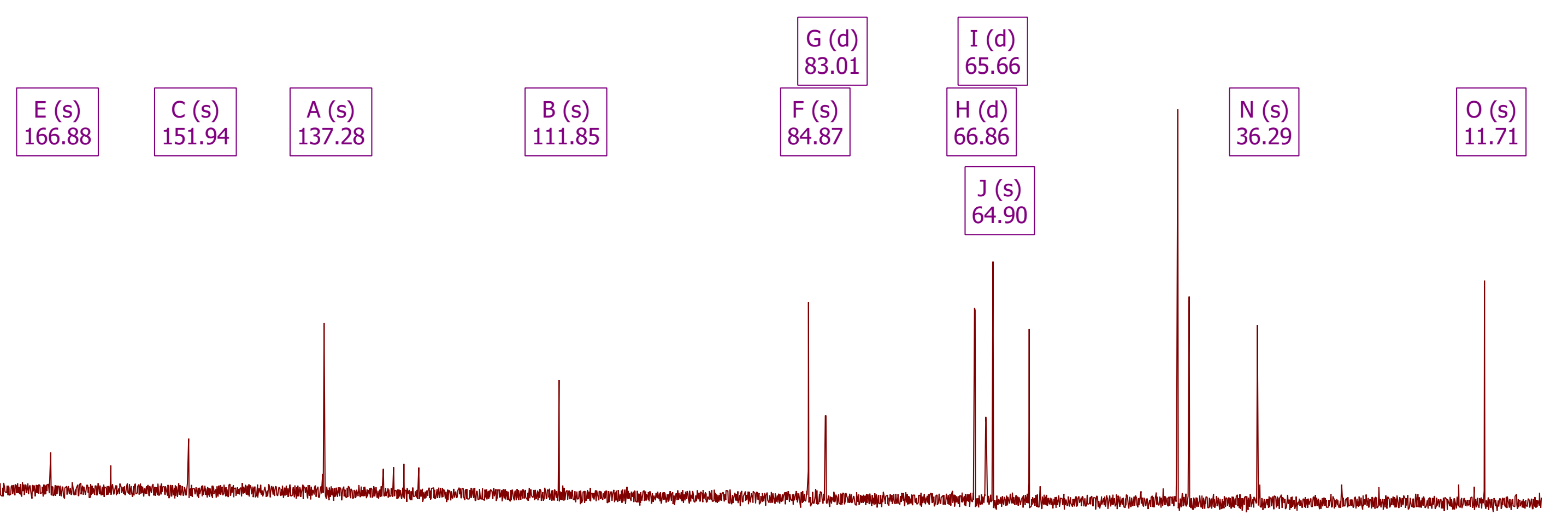

\begin{tabular}{|c|c|c|c|c|c|c|c|c|c|c|c|c|c|c|c|}
\hline & 160 & 150 & 140 & 130 & & & & $\begin{array}{c}90 \\
\mathrm{f} 1(\mathrm{ppm})\end{array}$ & 80 & 70 & 60 & 50 & 40 & 30 & 20 \\
\hline
\end{tabular}




\section{${ }^{1}$ H NMR}

$$
\text { the }
$$

crude product after precipitation

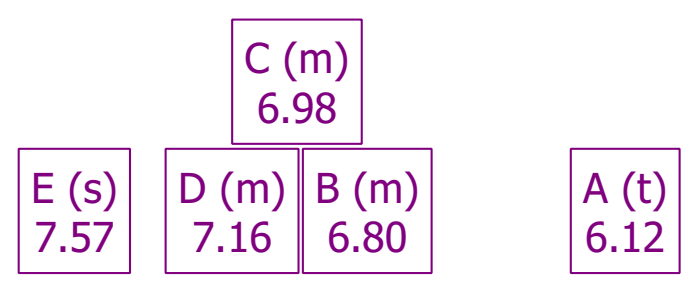

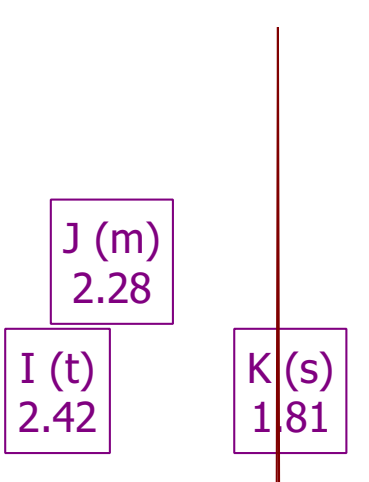

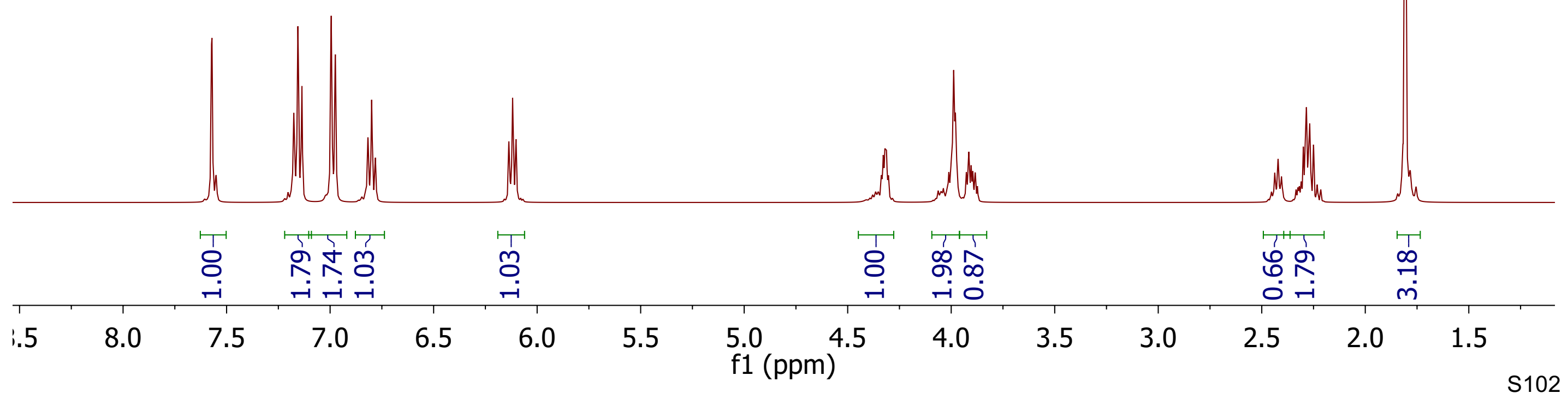




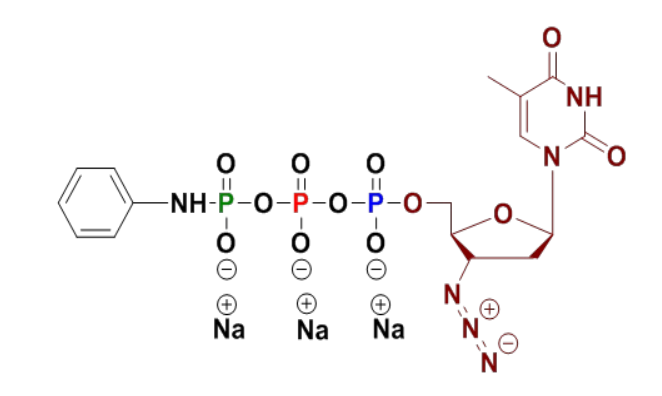

crude product after precipitation
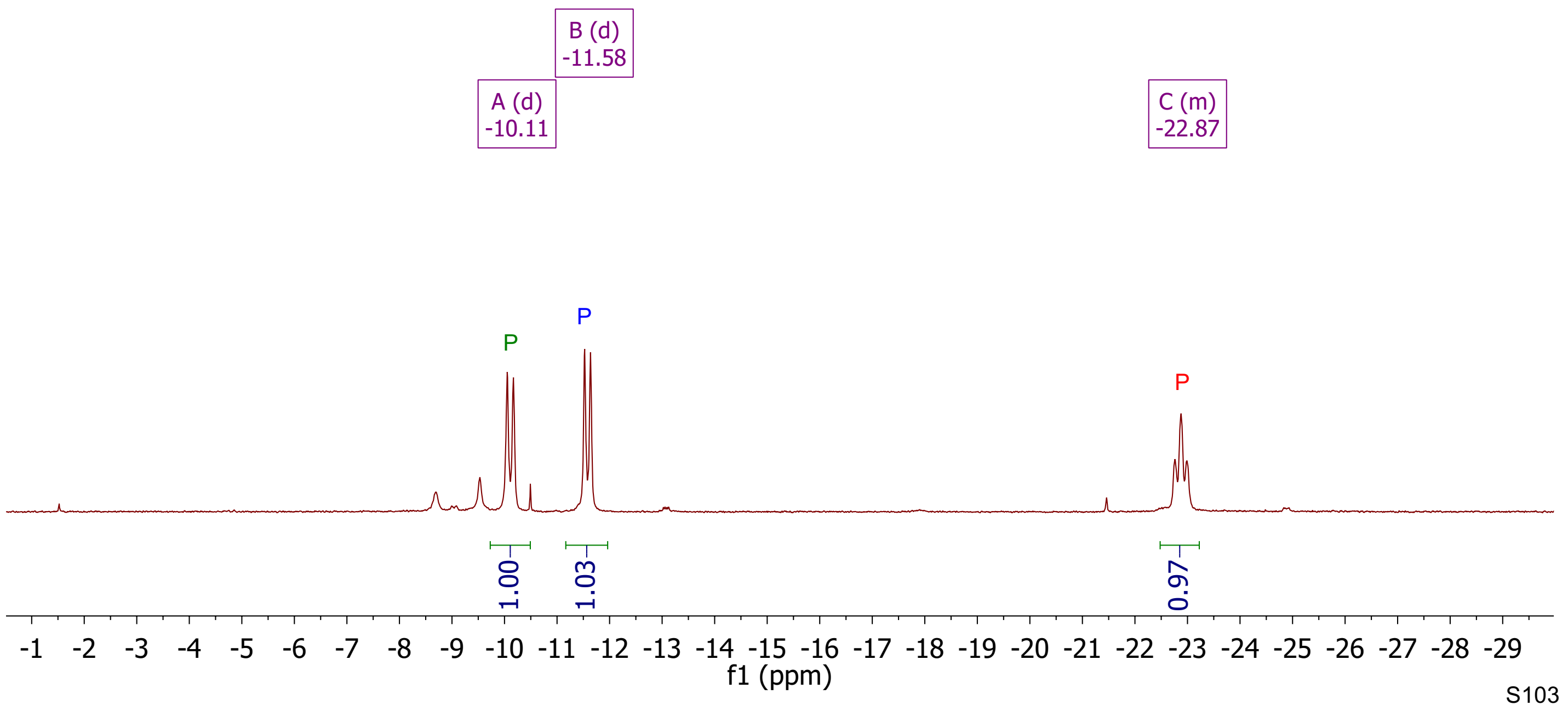


\section{${ }^{31} \mathrm{P}$ NMR}

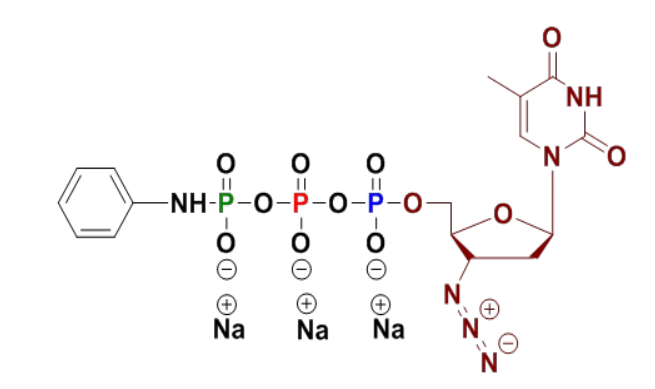

crude product after precipitation

$$
\begin{array}{|c||c|}
\hline A(d) & B(d t) \\
-10.11 & -11.58 \\
\hline
\end{array}
$$

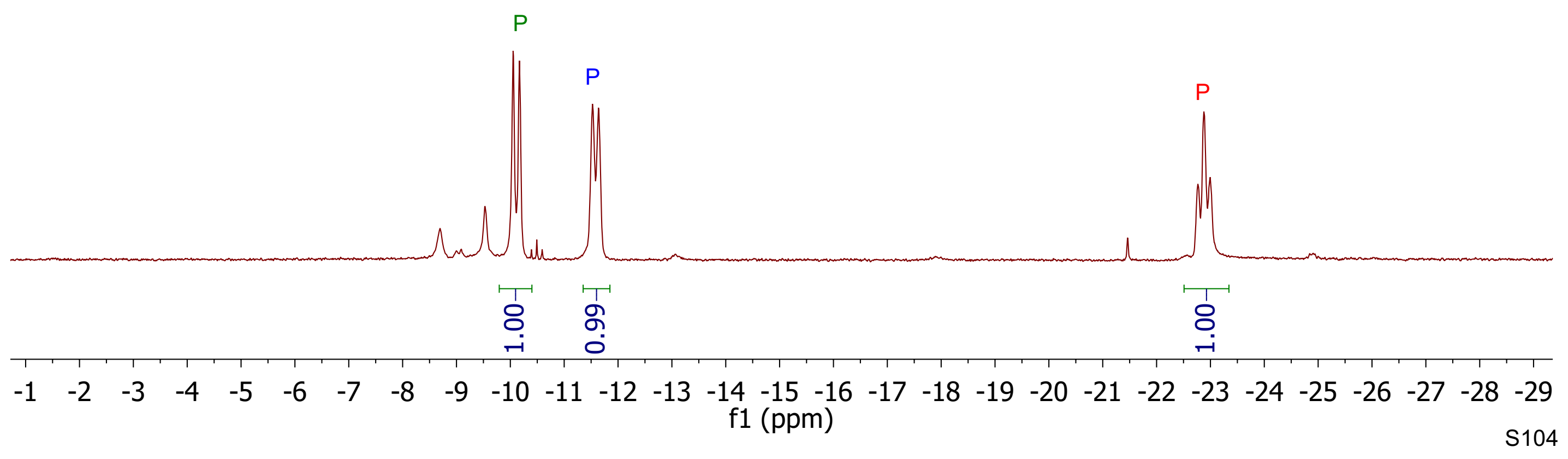




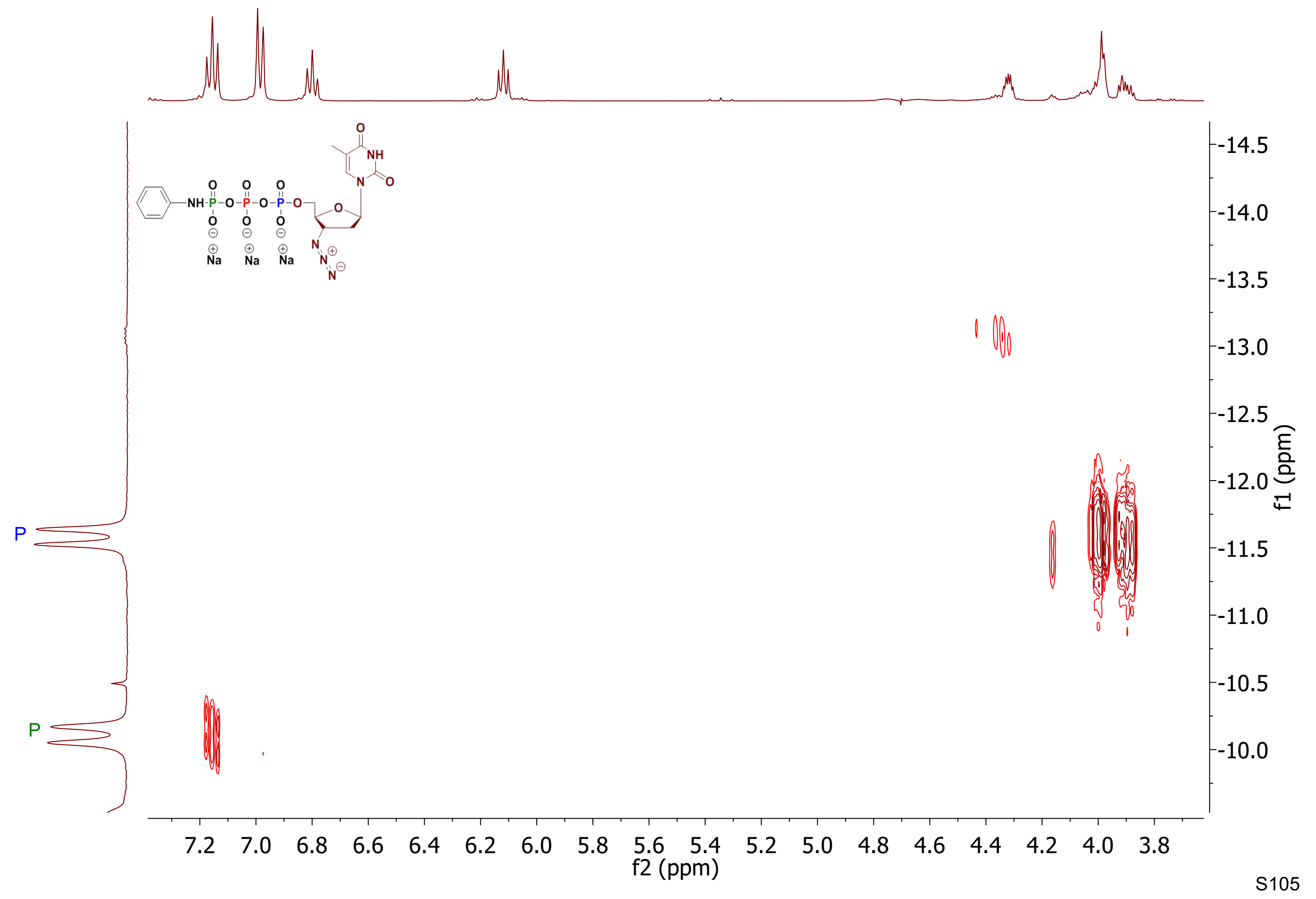




$$
\begin{aligned}
& \begin{array}{llll}
0 & 0 & 0
\end{array}
\end{aligned}
$$

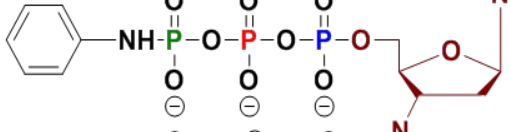

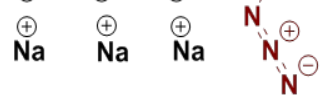

crude product after precipitation

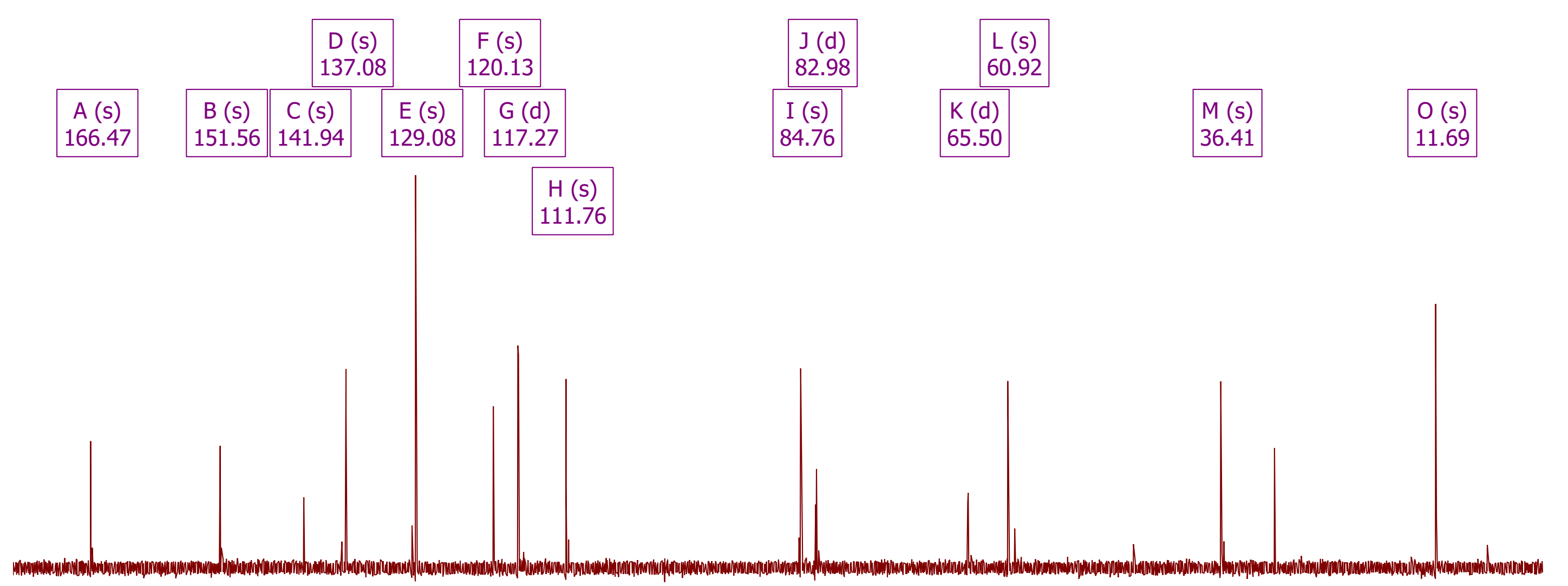

$\begin{array}{llllllllllllllllll}170 & 160 & 150 & 140 & 130 & 120 & 110 & 100 & 90 & 80 & 70 & 60 & 50 & 40 & 30 & 20 & 10 & 0\end{array}$




\section{${ }^{31} \mathrm{P}\left\{{ }^{1} \mathrm{H}\right\}-\mathrm{NMR}$}

$$
\begin{aligned}
& \cos ^{-12}
\end{aligned}
$$

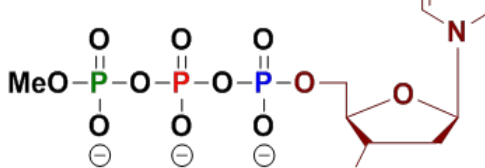

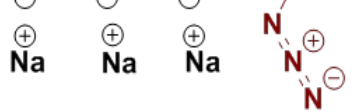

crude product after precipitation

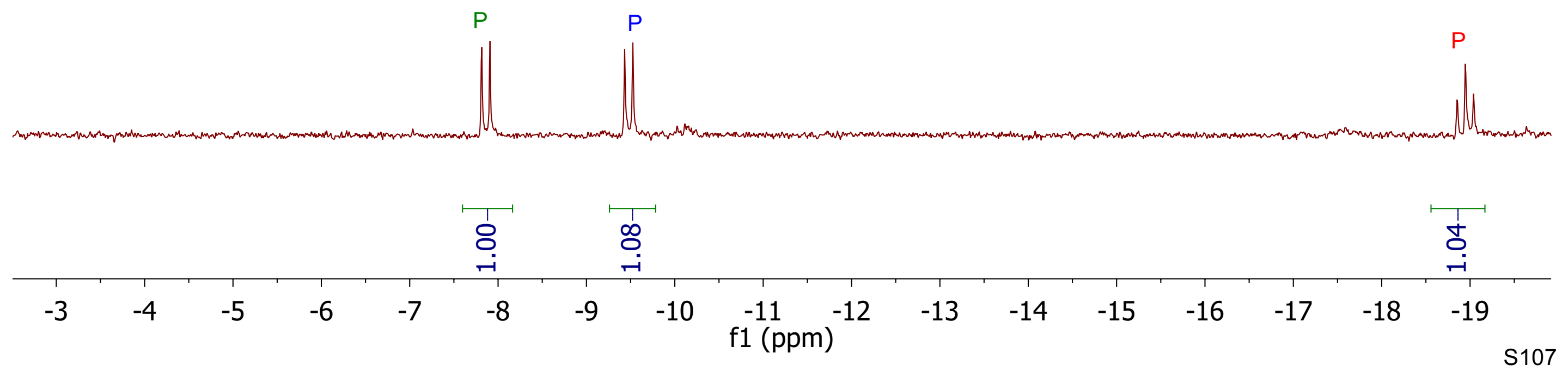




\section{${ }^{31} \mathrm{P}\left\{{ }^{1} \mathrm{H}\right\}-\mathrm{NMR}$}

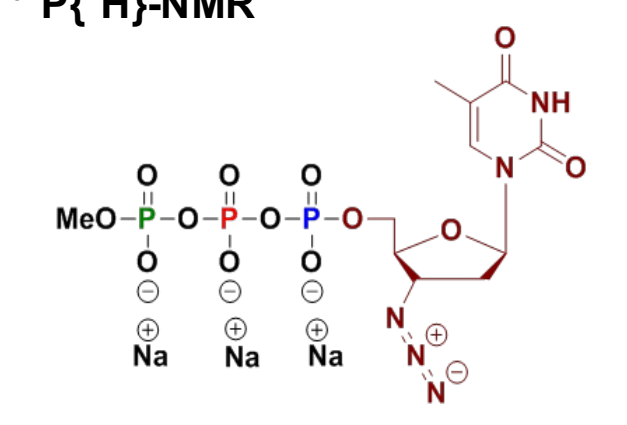

crude product after precipitation

\begin{tabular}{|c|c|}
\hline$A(d q)$ & $B(d t)$ \\
-7.87 & -9.49 \\
\hline
\end{tabular}

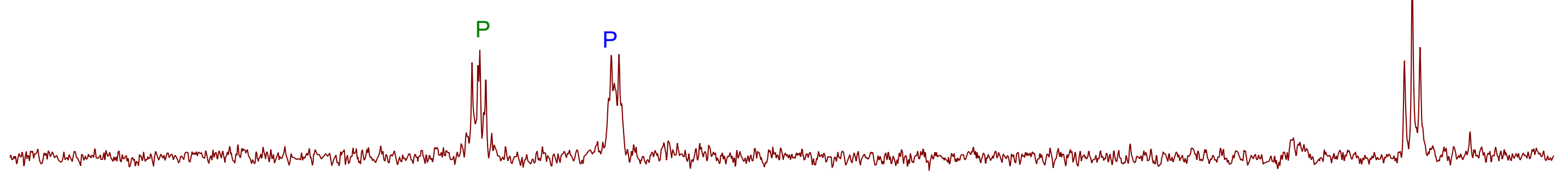

\begin{tabular}{|c|c|c|c|c|c|c|c|c|c|c|c|c|c|c|c|}
\hline & & & & & $\begin{array}{l}1 \\
\text { g } \\
0\end{array}$ & & & & & & & & & & 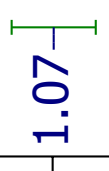 \\
\hline-3 & -4 & -5 & -6 & -7 & -8 & -9 & -10 & $\begin{array}{l}-11 \\
\mathrm{f1}(\mathrm{ppm})\end{array}$ & -13 & -14 & -15 & -16 & -17 & -18 & -19 \\
\hline
\end{tabular}




\section{${ }^{31} \mathrm{P}\left\{{ }^{1} \mathrm{H}\right\}-N M R$}

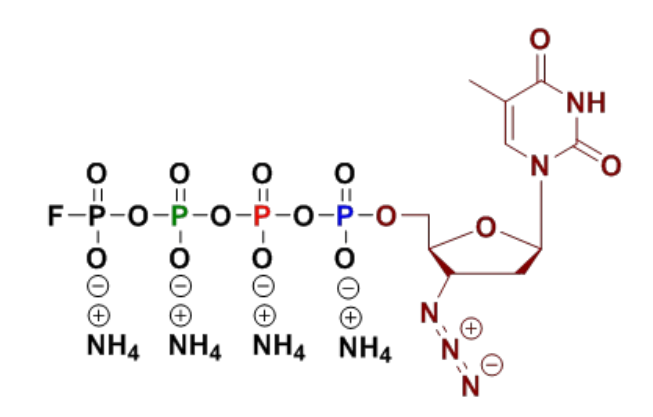

Product after purification by SAX
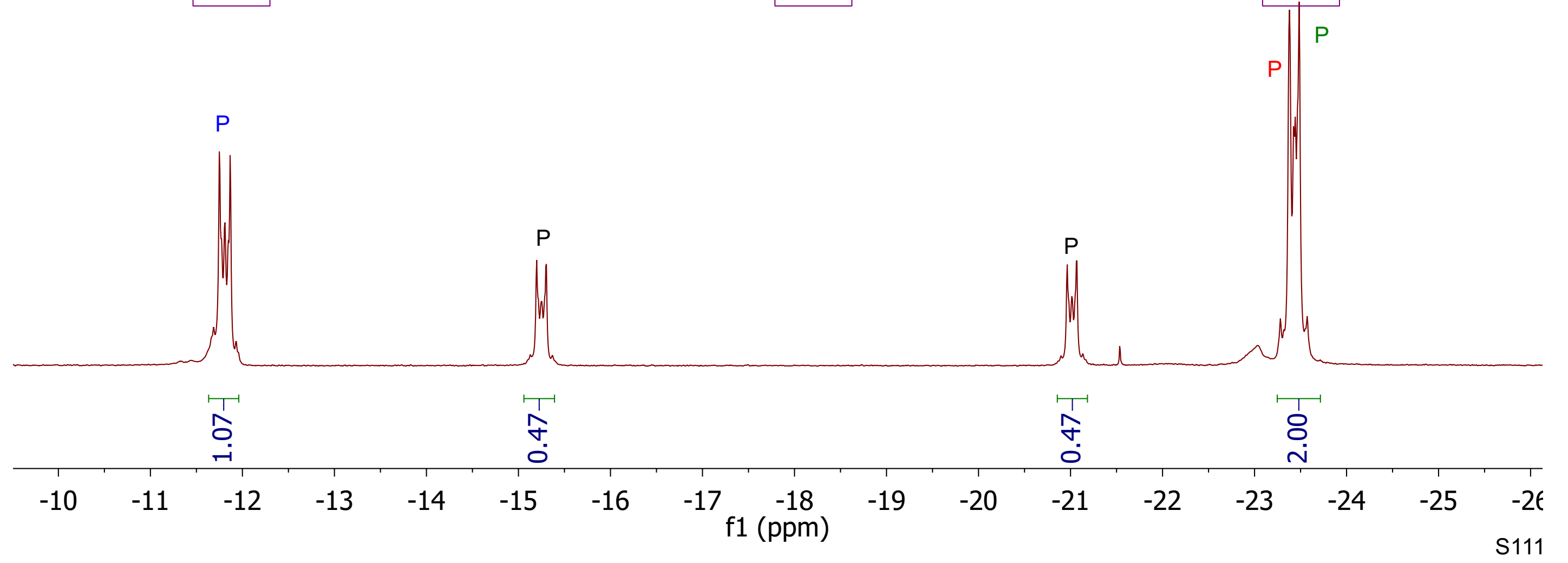


\section{${ }^{31} P\left\{{ }^{19} F\right\} N M R$}

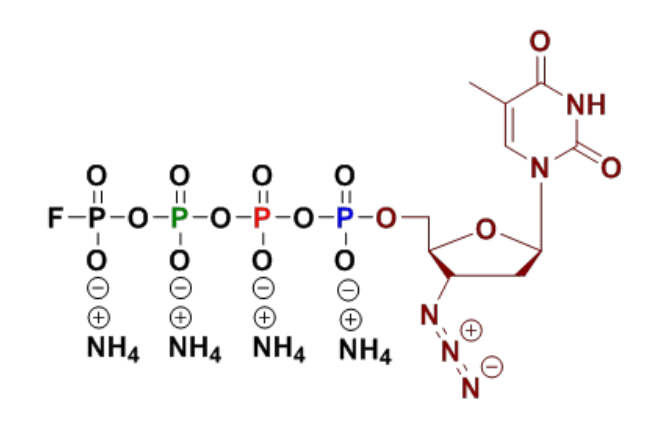

Product after purification by SAX

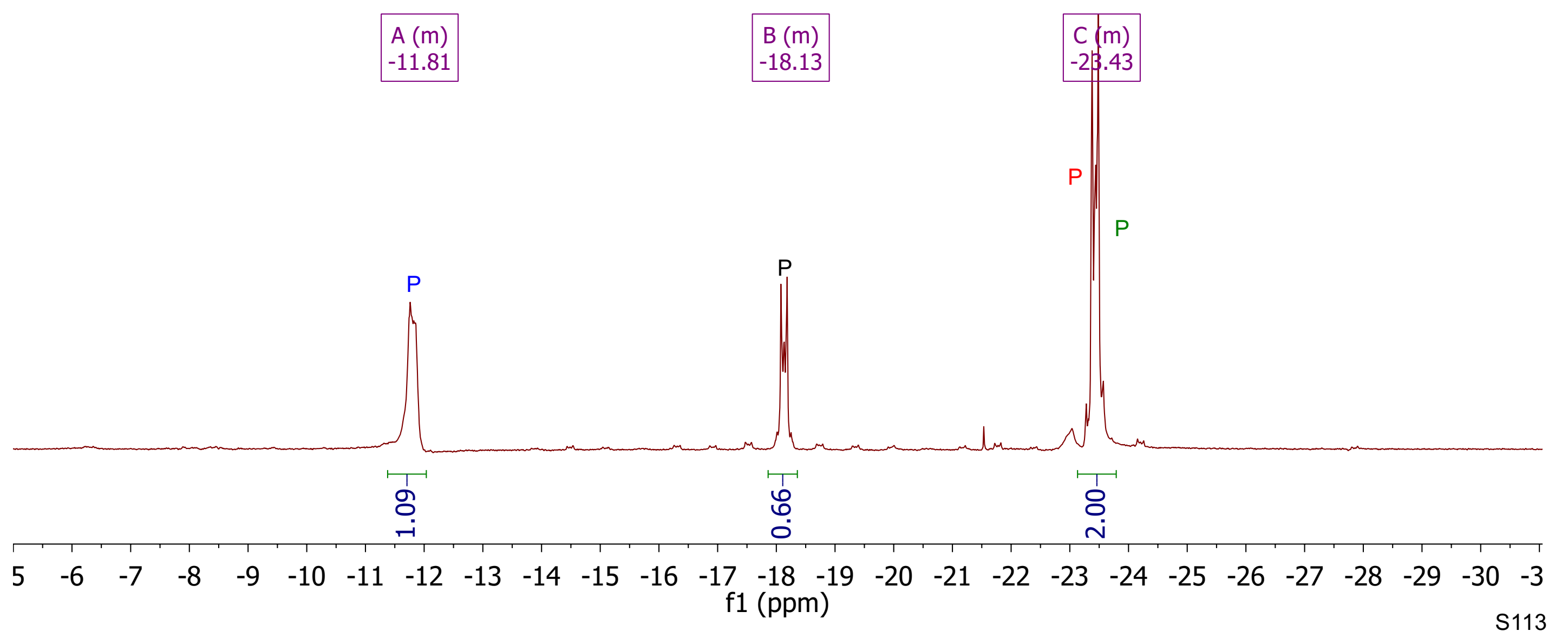



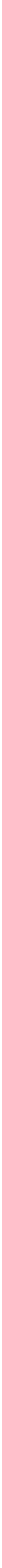


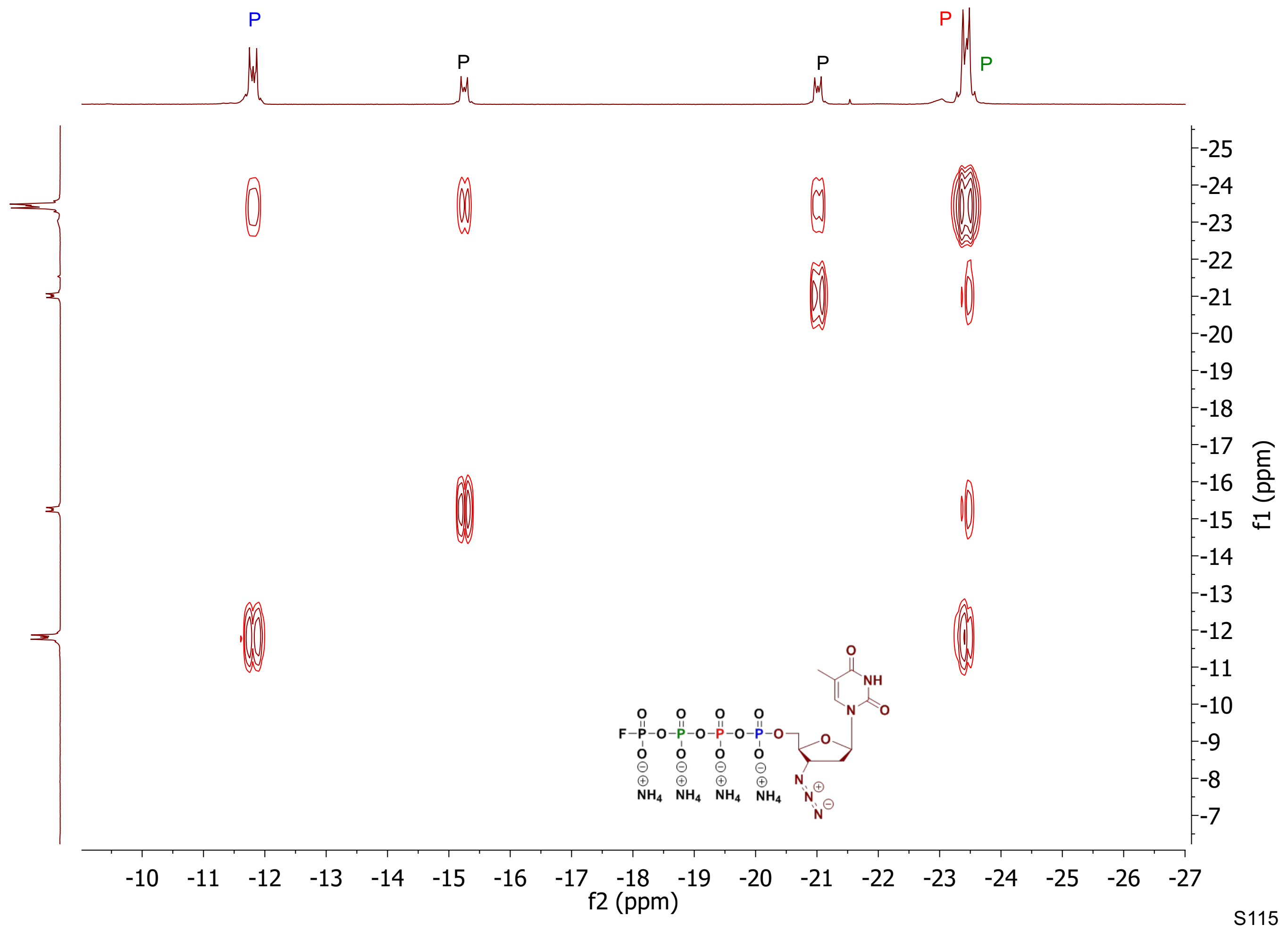




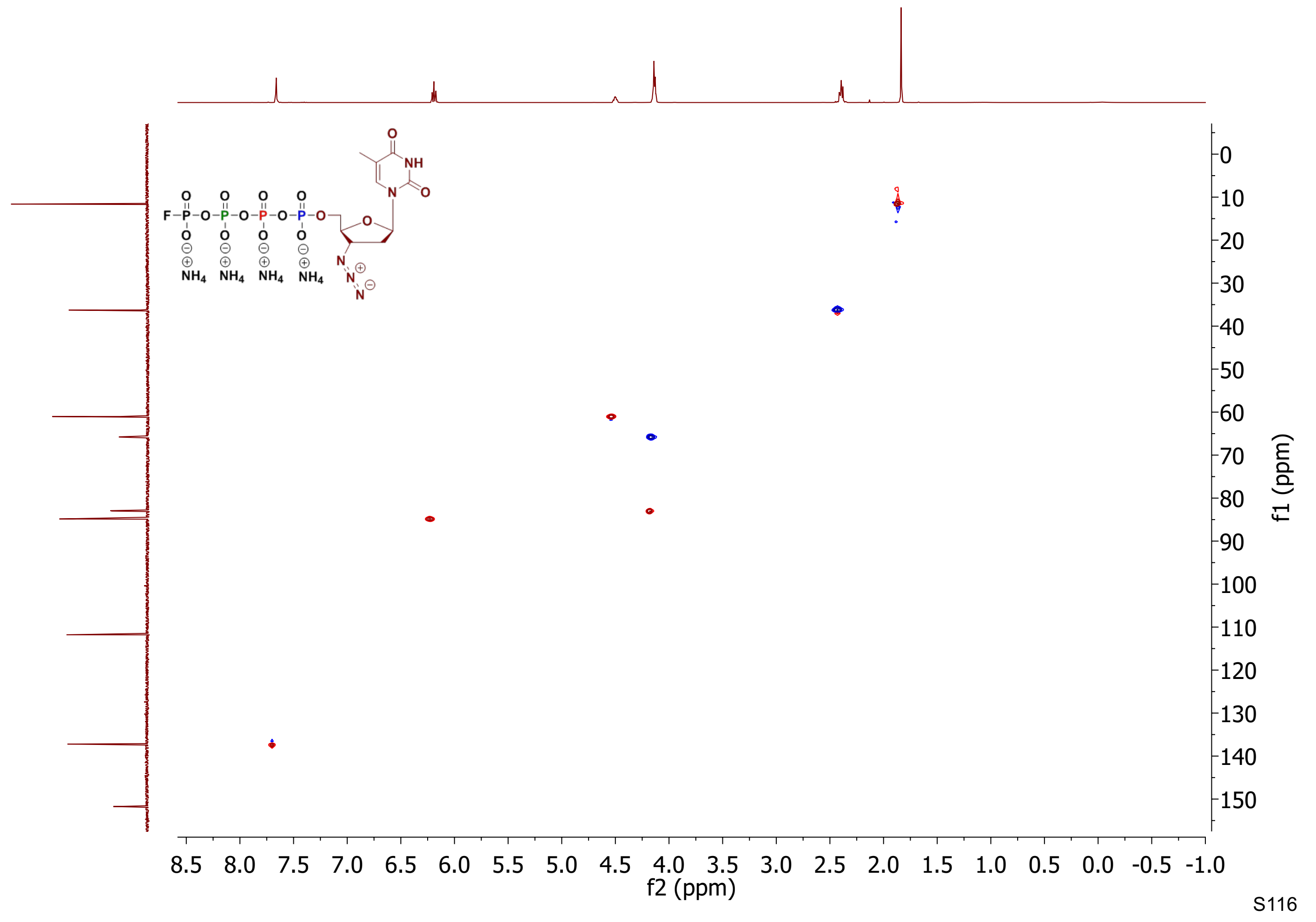




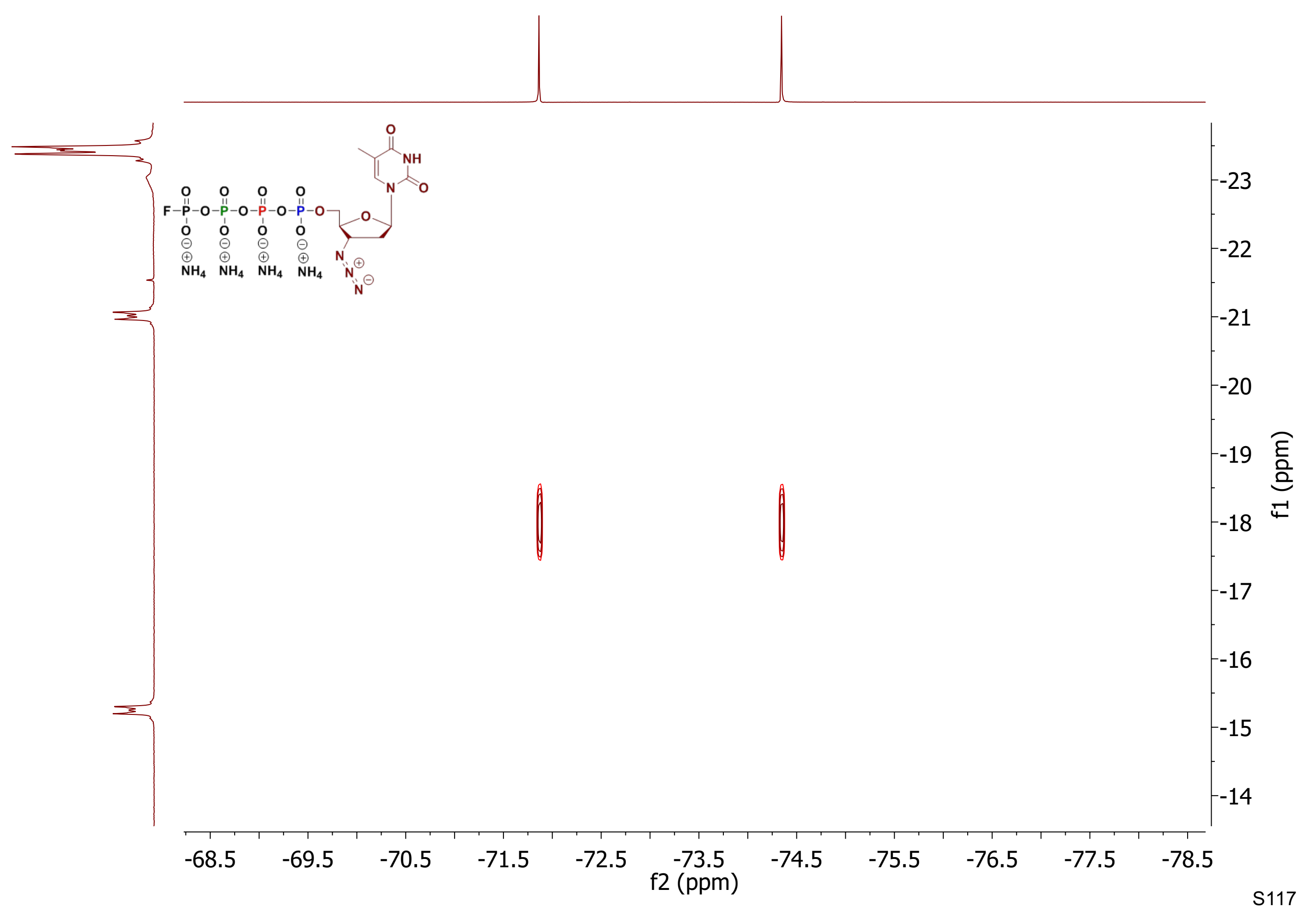




\section{${ }^{31} P\left\{{ }^{1} H\right\} N M R$}
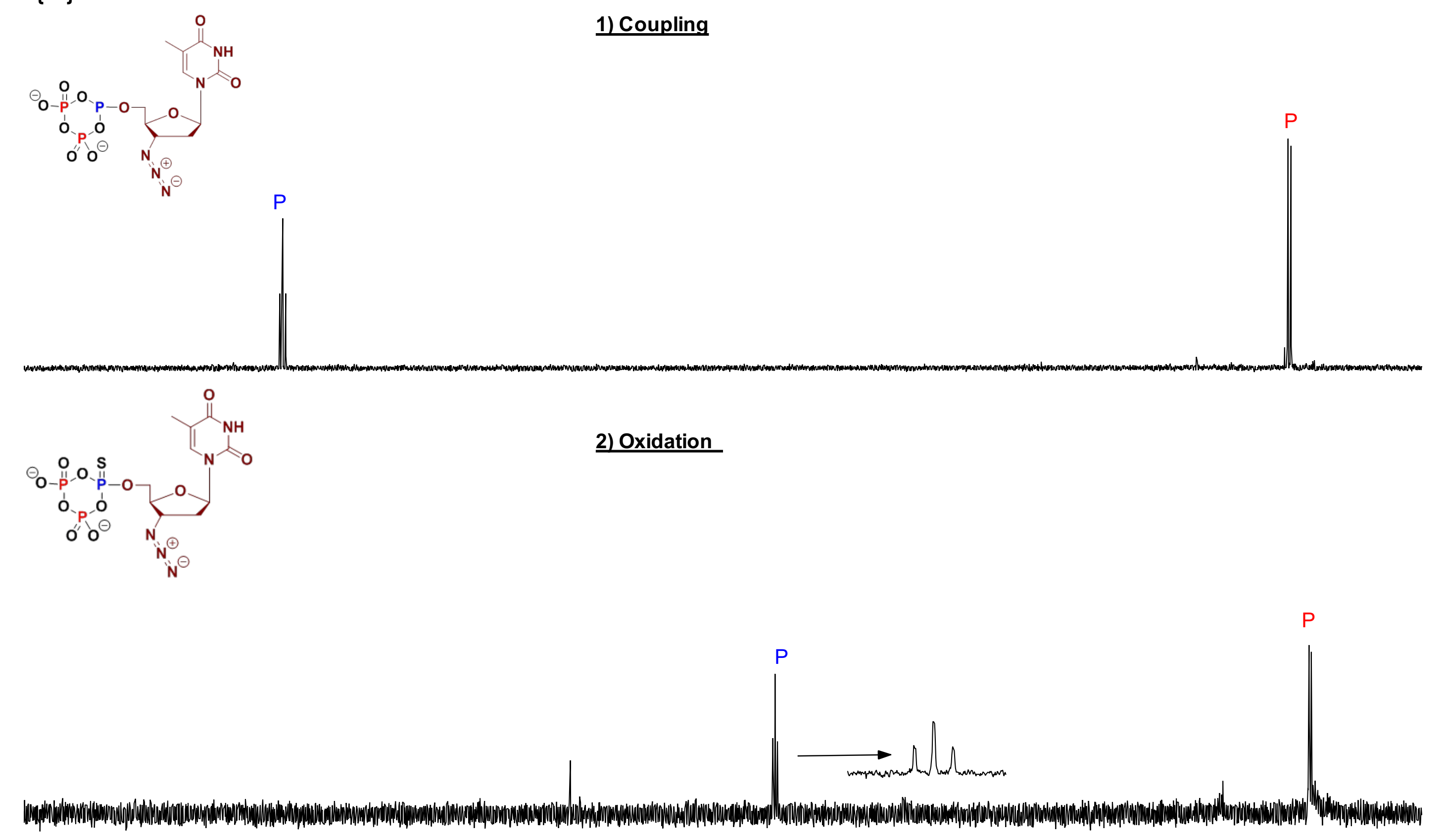


\section{${ }^{1} \mathrm{H}$ NMR}

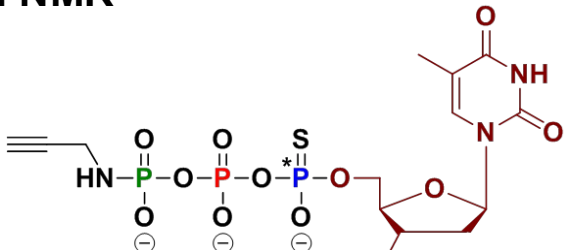

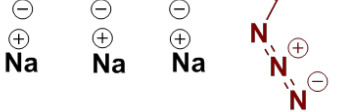

crude product after precipitation

$$
\begin{aligned}
& \text { A (s) } \\
& 7.70
\end{aligned}
$$$$
\mathrm{B}(\mathrm{t})
$$$$
\text { C (dt) D (m) }
$$

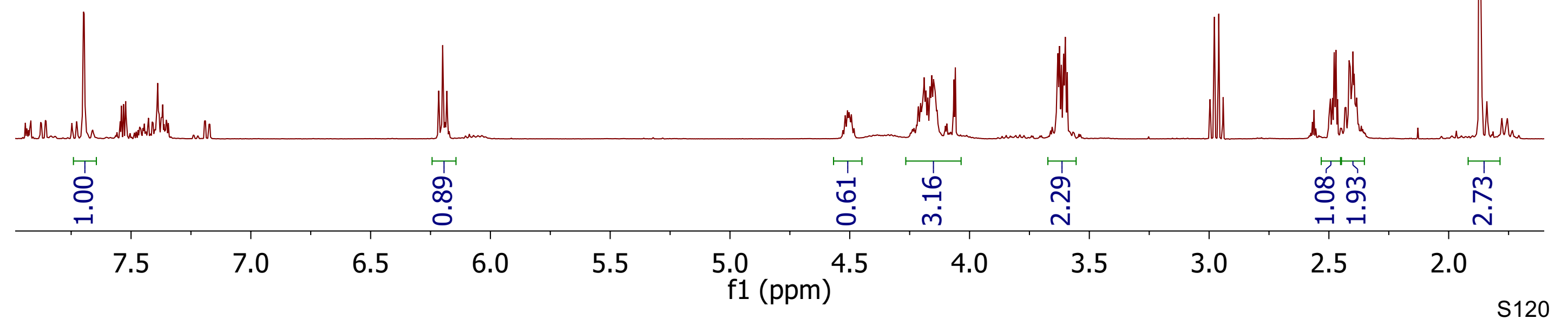




\section{${ }^{31} \mathrm{P}\left\{{ }^{1} \mathrm{H}\right\} \mathrm{NMR}$}

$$
{ }^{2} \mathbf{P}\{\mathrm{H}\} \text { NMR }
$$

crude product after precipitation

$$
\begin{array}{|l|}
\hline B(d d) \\
42.89 \\
\hline
\end{array}
$$

$$
\begin{gathered}
A(d d) \\
-2.85 \\
\hline
\end{gathered}
$$

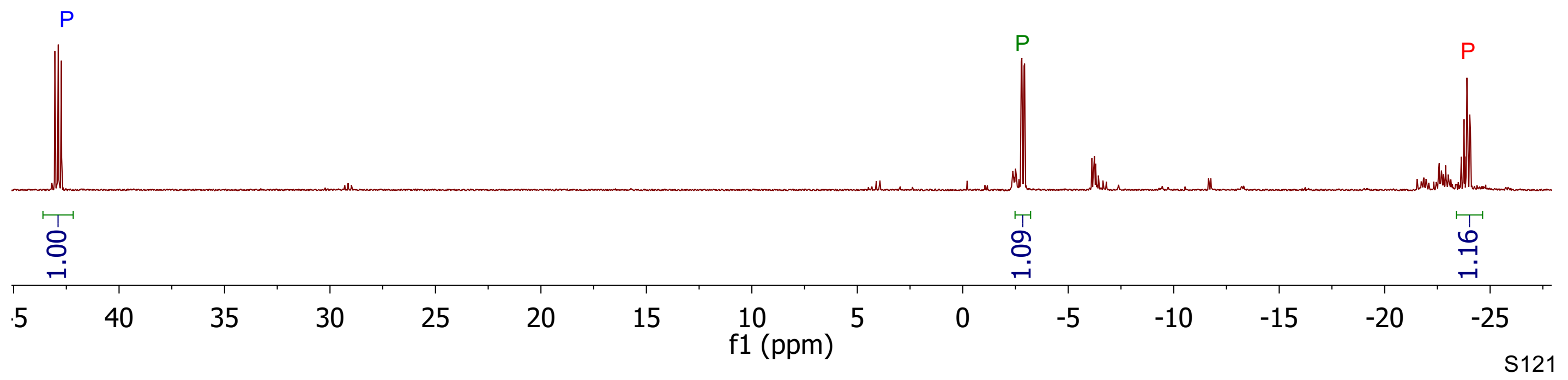


${ }^{31} \mathbf{P}$ NMR

crude product after precipitation
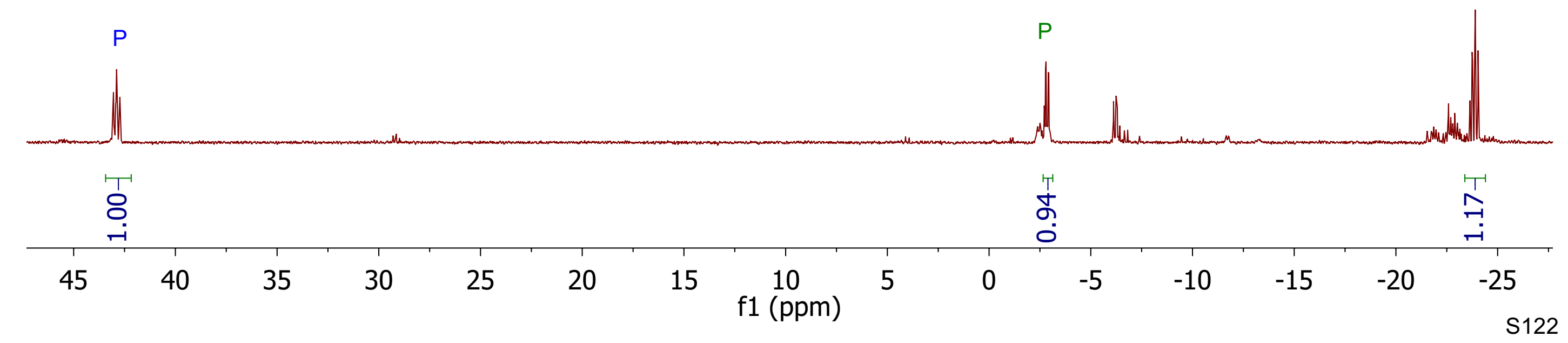


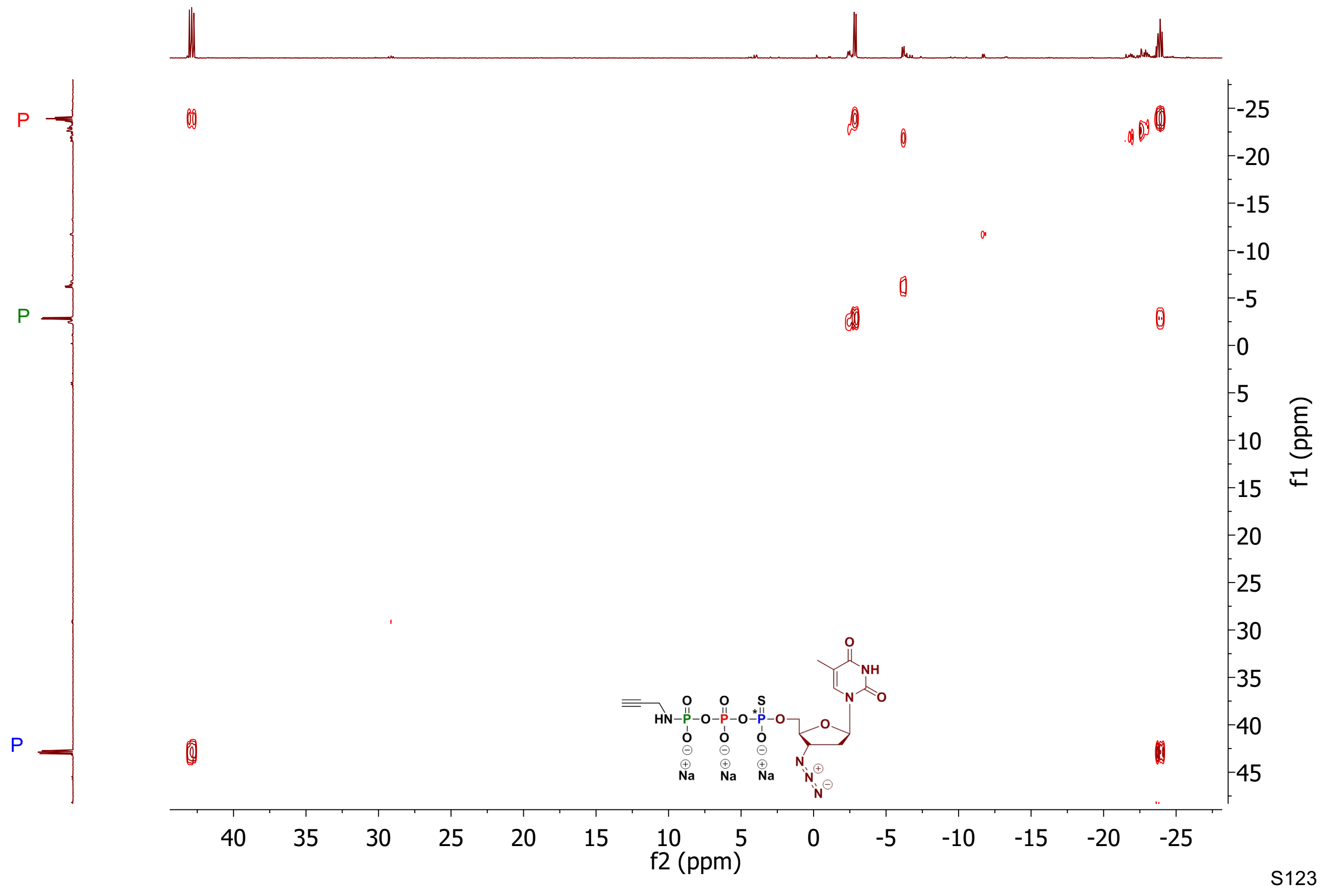




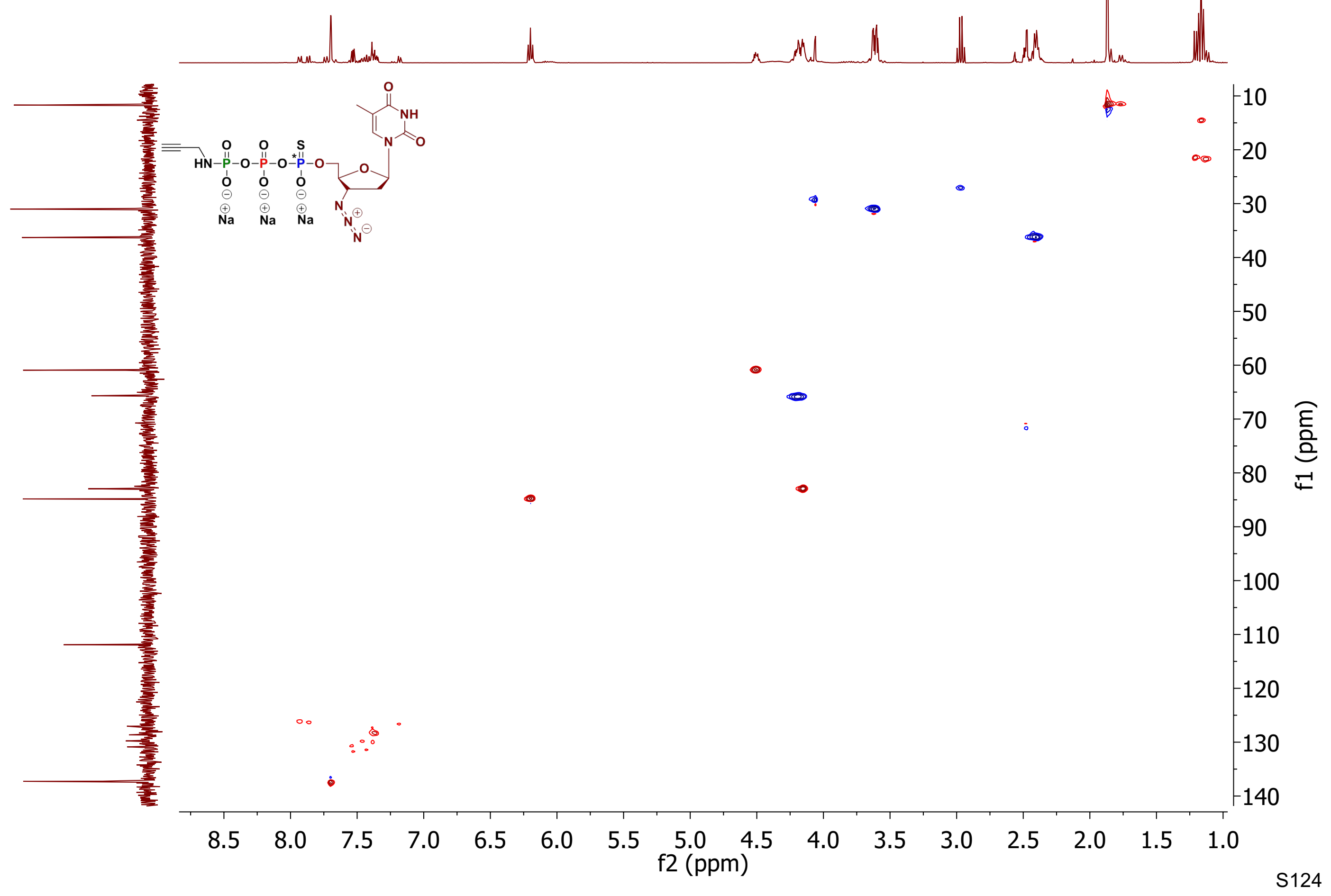


(1)

Product after purification by SAX

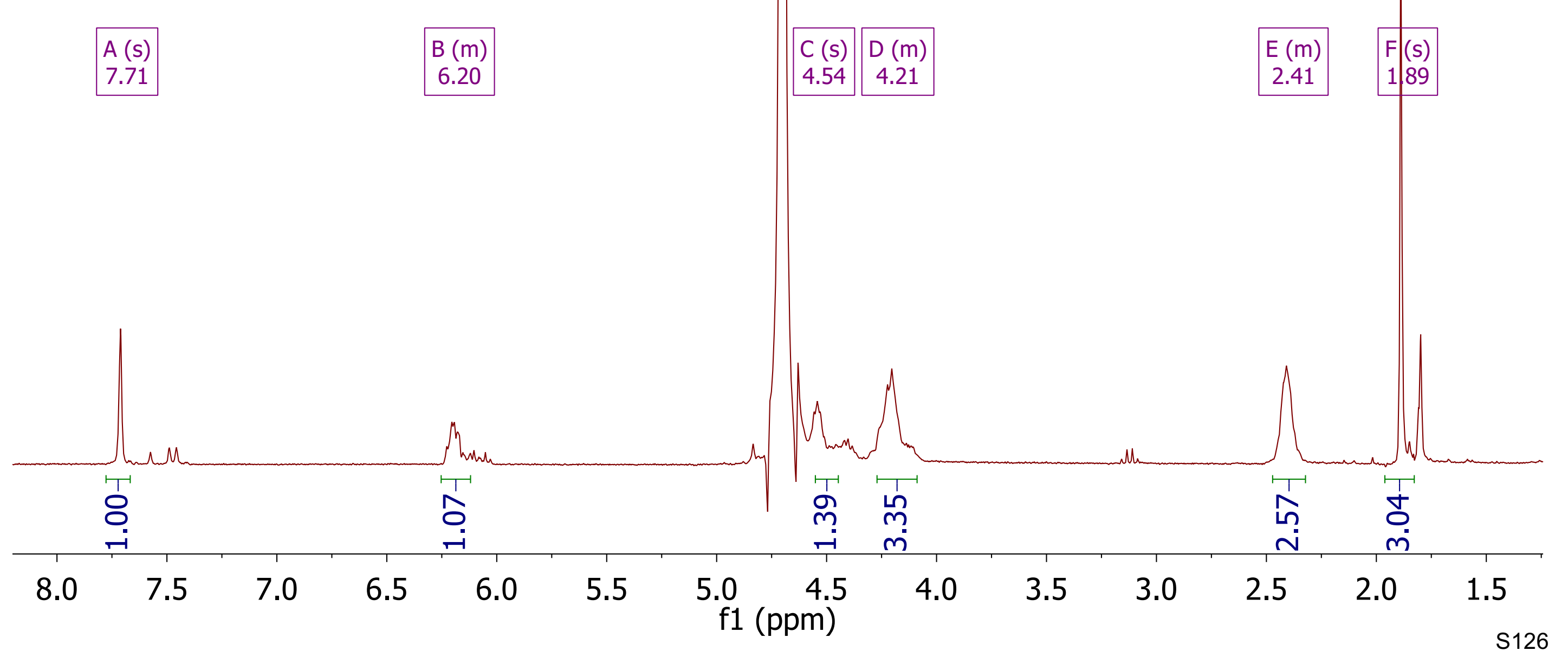




\section{${ }^{31} \mathrm{P}\left\{{ }^{1} \mathrm{H}\right\} \mathrm{NMR}$}

每)

Product after purification by SAX
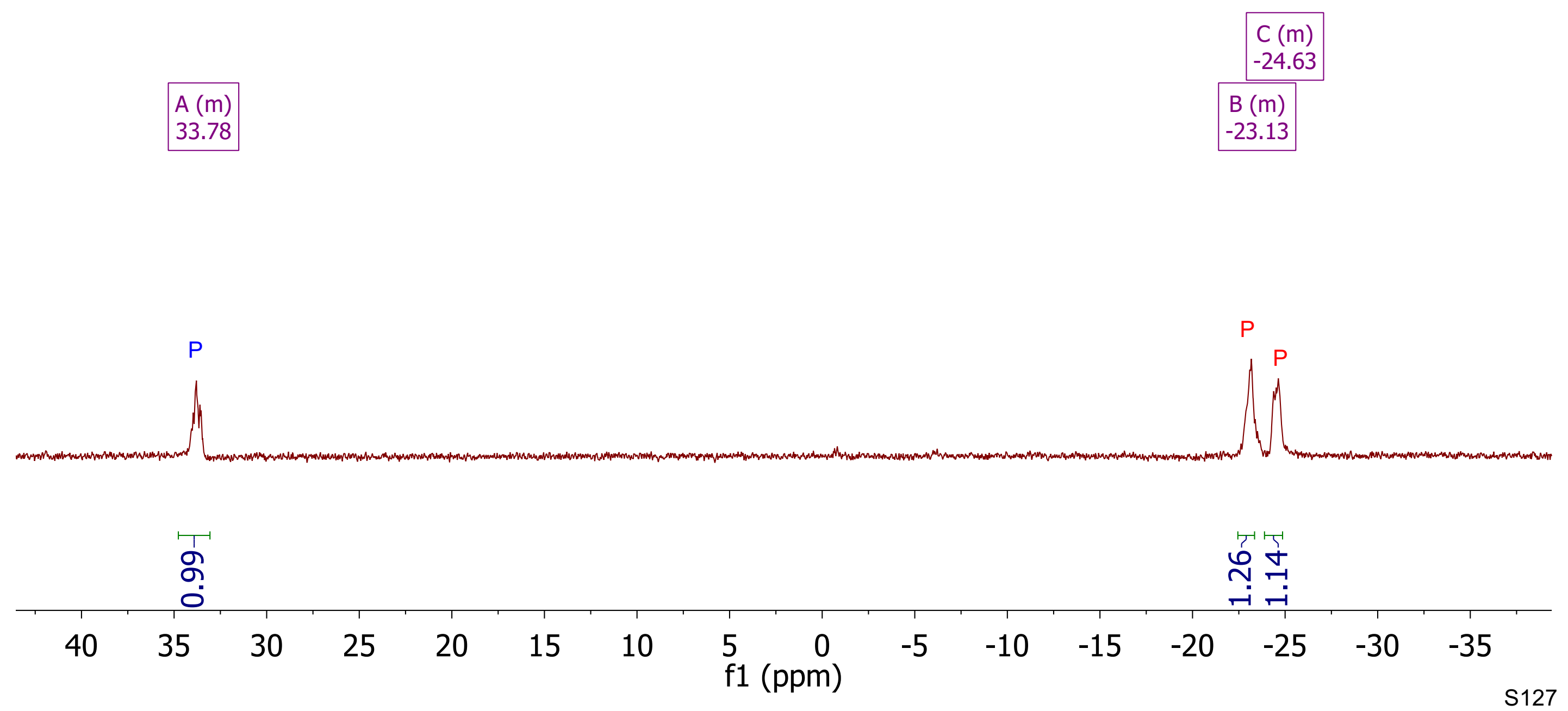
(1)

Product after purification by SAX

$$
\begin{aligned}
& A(\mathrm{~m}) \\
& 33.78 \\
& \hline
\end{aligned}
$$
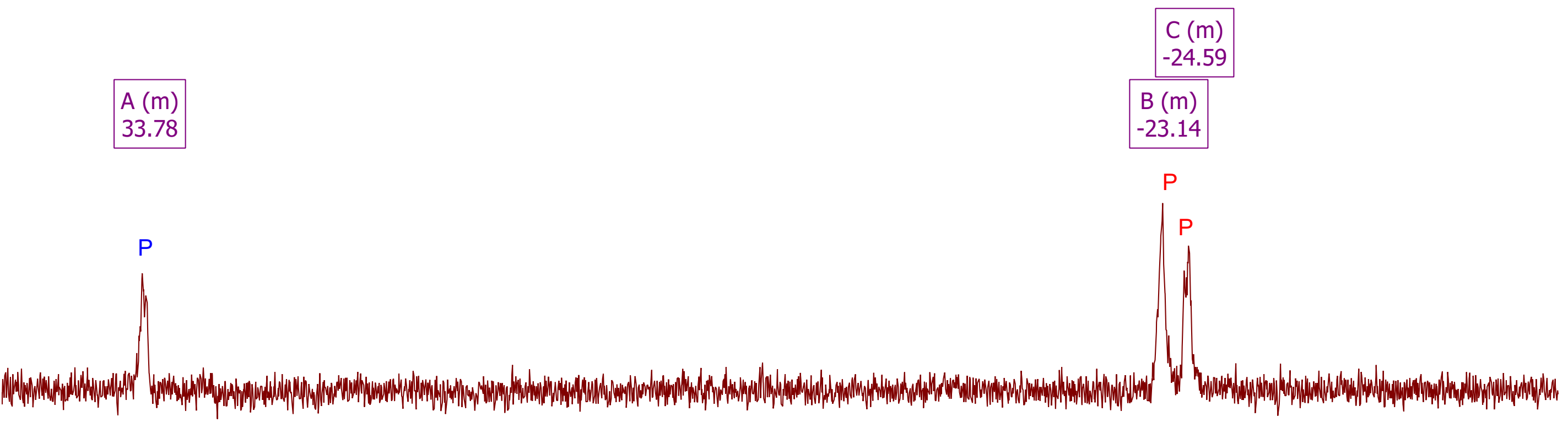

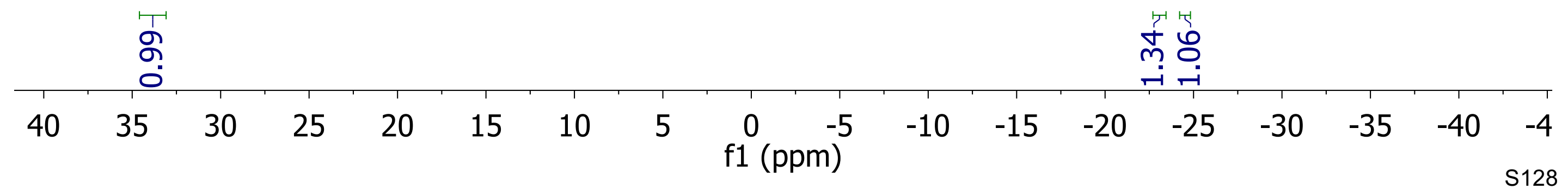




\section{${ }^{1} \mathrm{H}$ NMR}

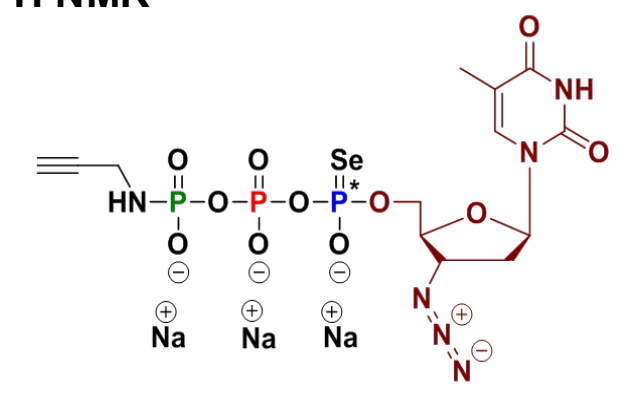

Product after purification by SAX
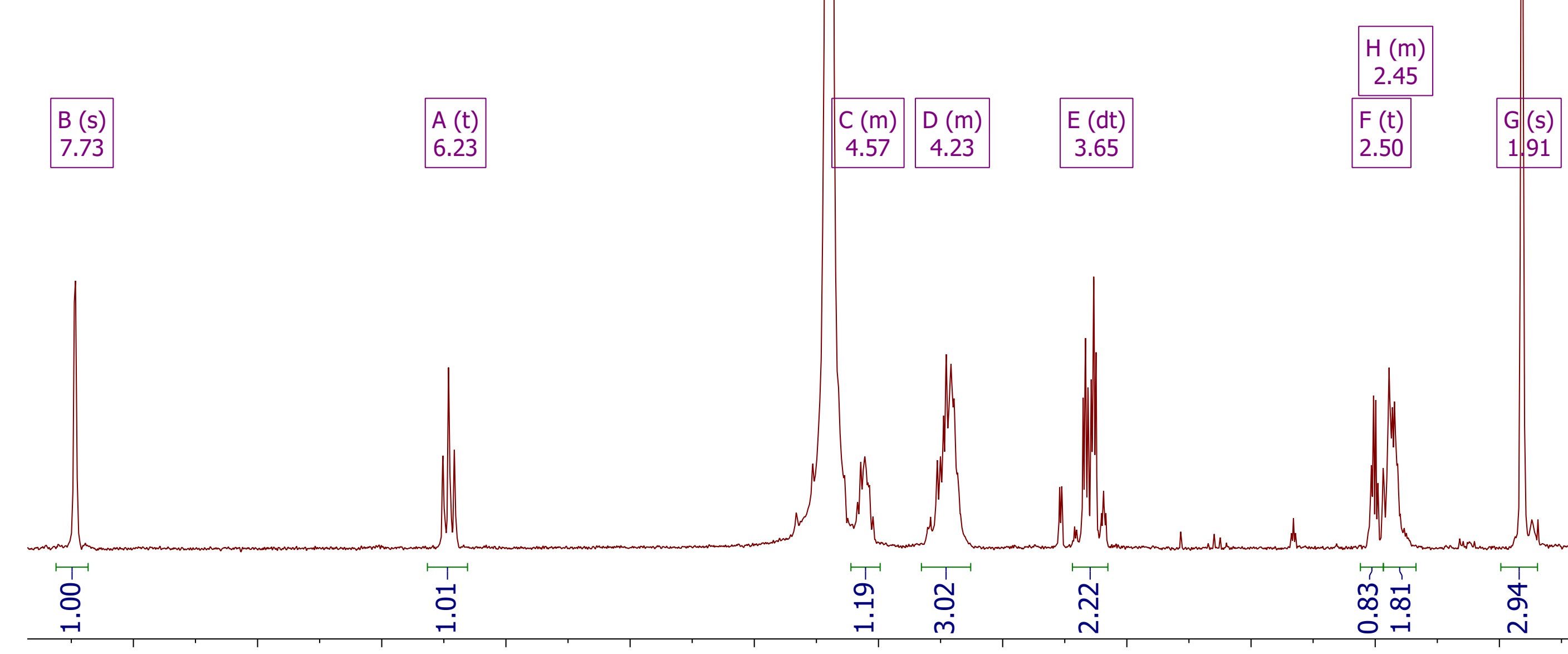

$\begin{array}{llllll}7.5 & 7.0 & 6.5 & 6.0 & 5.5 & \begin{array}{l}5.0 \\ \mathrm{f} 1(\mathrm{ppm})\end{array}\end{array}$

$\begin{array}{ll}4.0 & 3.5\end{array}$

$3.5 \quad 3.0$

$3.0-2.5$

2.5 


\section{${ }^{31} \mathrm{P}\left\{{ }^{1} \mathrm{H}\right\}$ NMR}

ธิต ำ ㄲำ

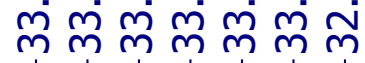

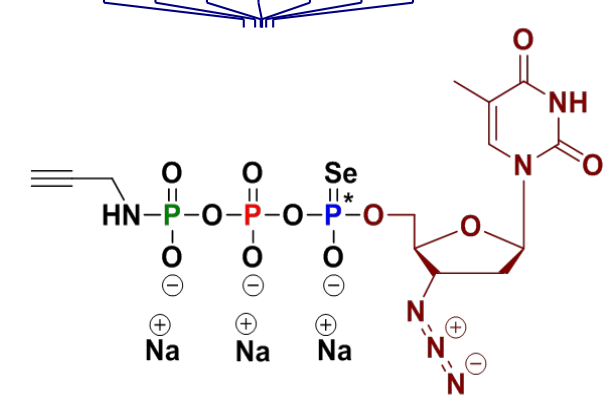

Product after purification by SAX

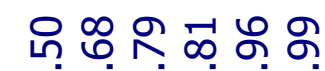

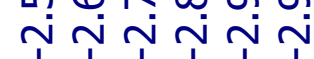
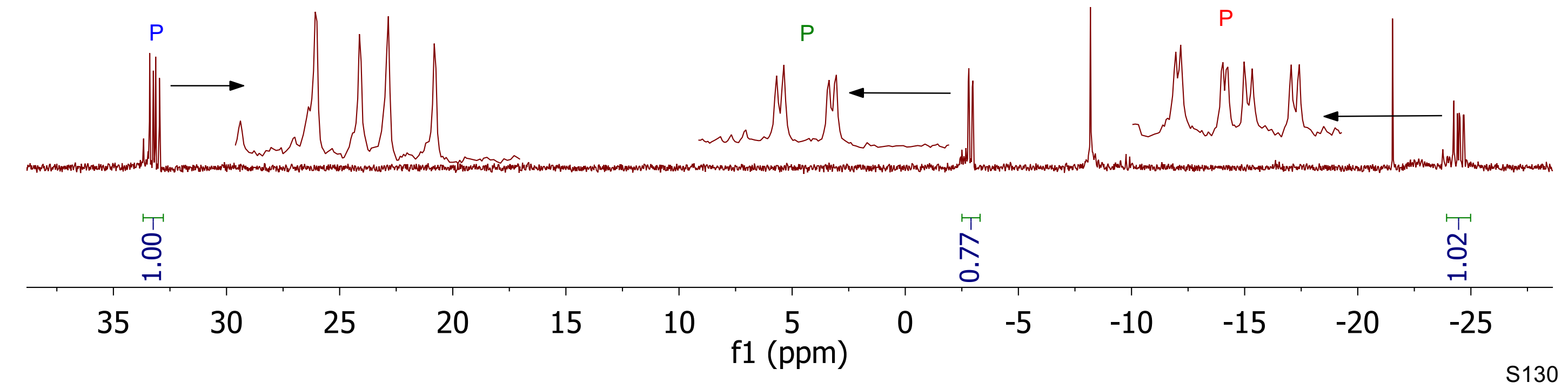
능 ดั

mं

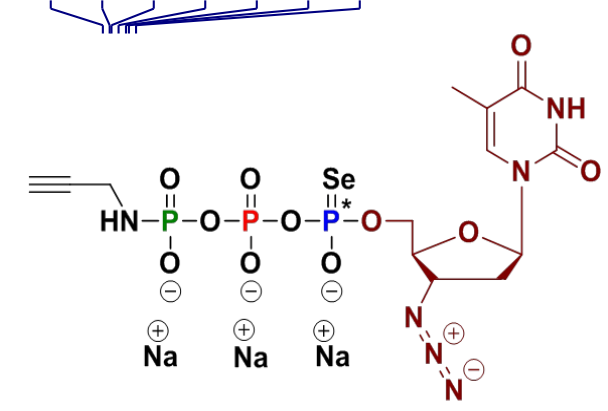

\begin{tabular}{l}
$A(m)$ \\
33.61 \\
\hline
\end{tabular}

\section{Product after purification by SAX}

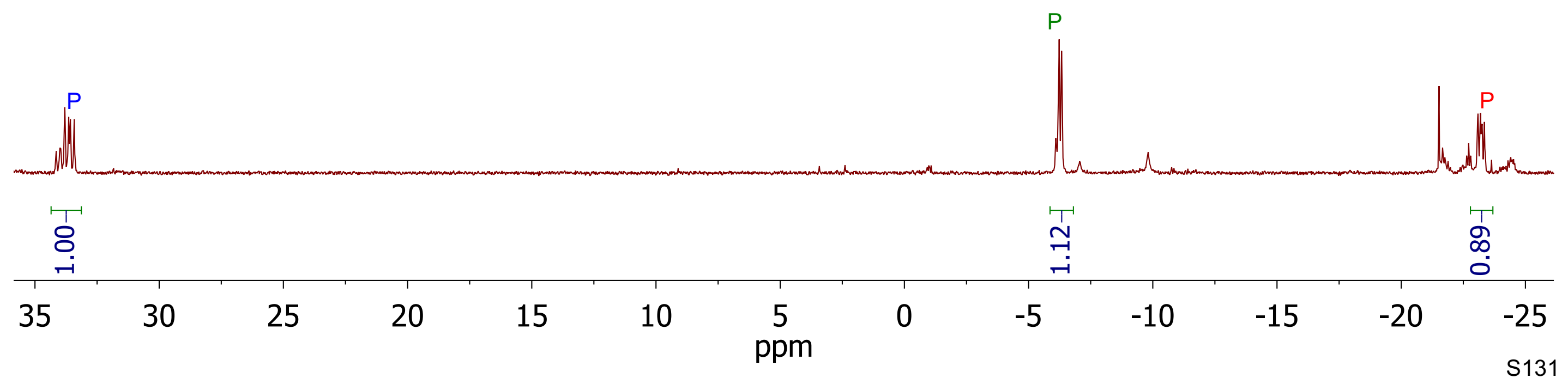




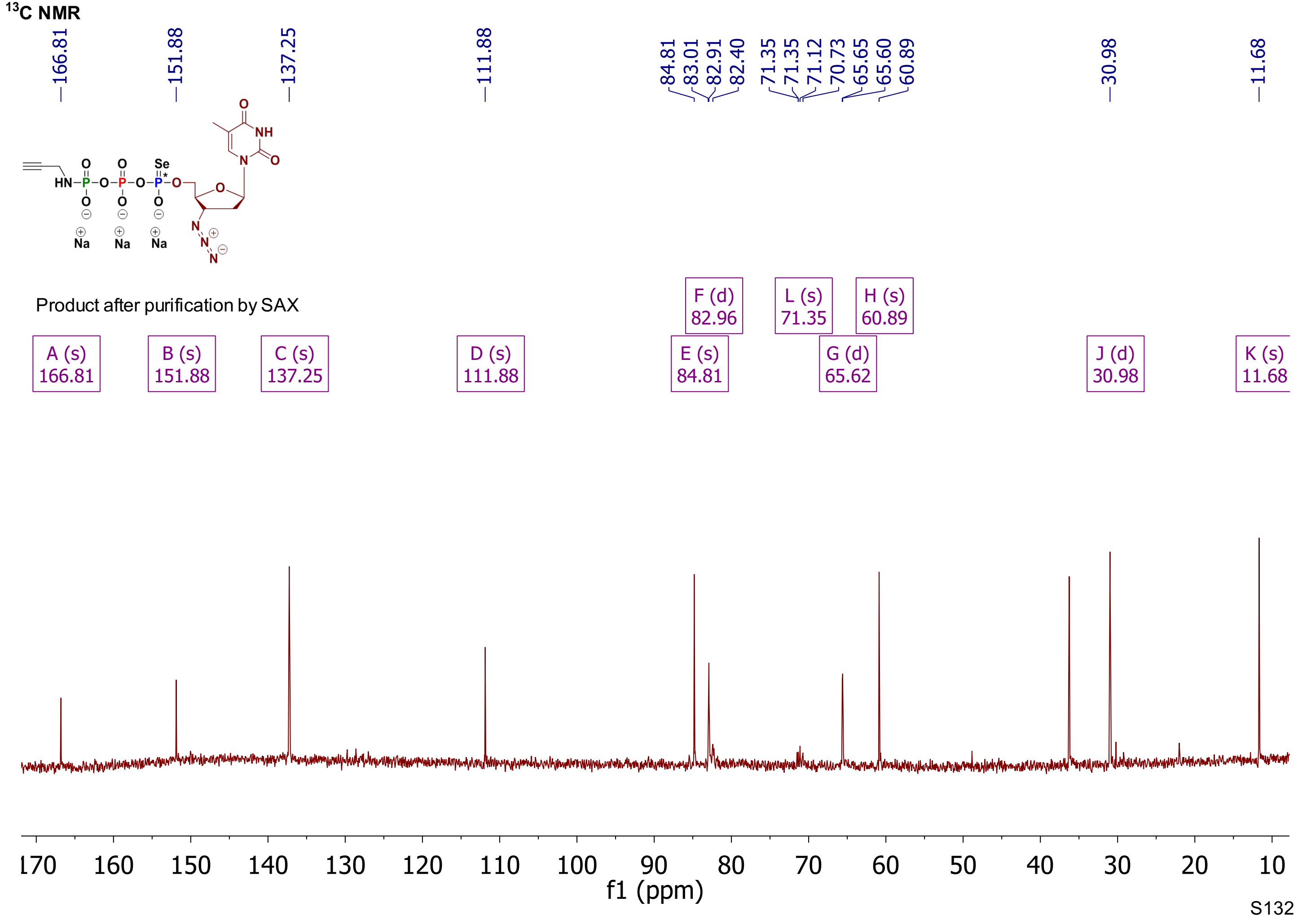




\section{${ }^{1}$ H NMR}

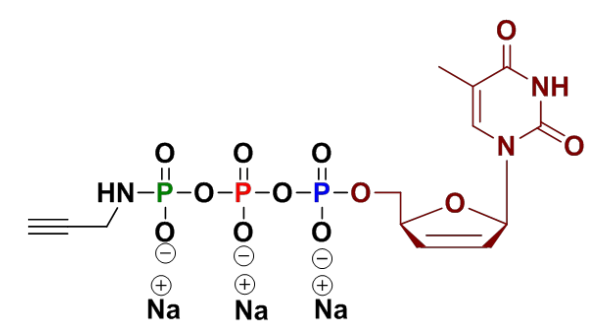

Crude product after precipitation
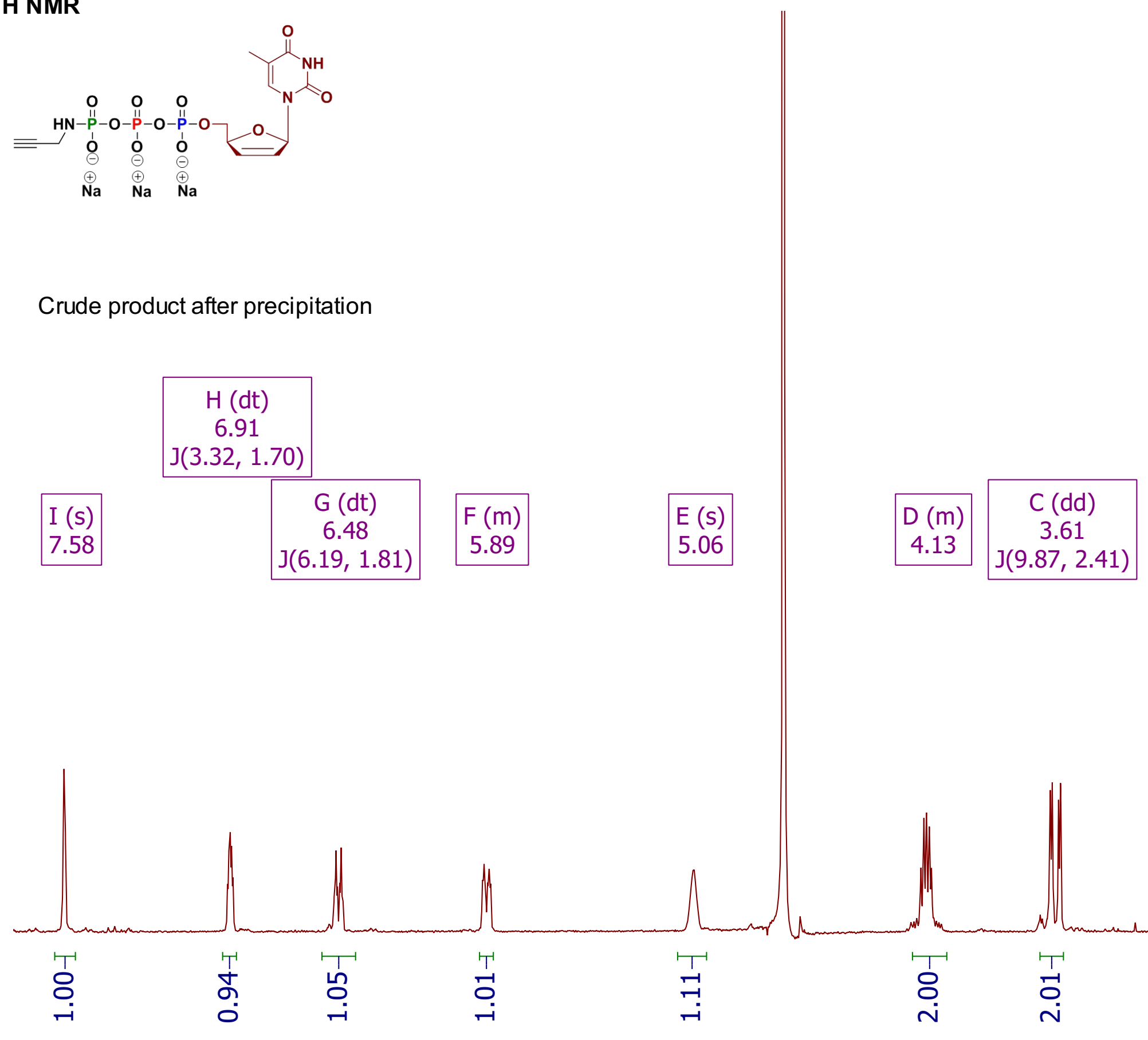

\begin{tabular}{|c|c|c|c|c|c|c|c|c|c|c|c|}
\hline 7.5 & 7.0 & 6.5 & 6.0 & 5.5 & 5.0 & $\begin{array}{c}4.5 \\
\mathrm{f} 1(\mathrm{ppm})\end{array}$ & 4.0 & 3.5 & 3.0 & 2.5 & 2.0 \\
\hline
\end{tabular}


${ }^{31} \mathrm{P}\left\{{ }^{1} \mathrm{H}\right\} \mathrm{NMR}$

Crude product after precipitation

\begin{tabular}{|c|}
\hline$A(d)$ \\
-2.81 \\
$J(20.88)$ \\
\hline
\end{tabular}

\begin{tabular}{|c|}
\hline$B(d)$ \\
-11.54 \\
$J(19.42)$ \\
\hline
\end{tabular}

$\mathrm{P}$

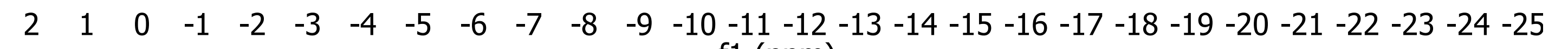
f1 (ppm) 


\section{${ }^{31}$ P NMR}

두

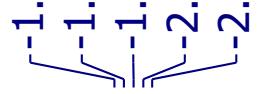

ํํำ 군 누 두

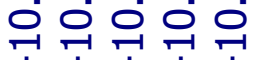

$1 \quad 1$

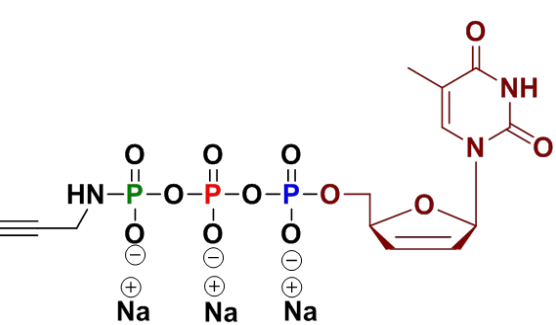

Crude product after precipitation

A (dt)

$-1.97$

$B(d t)$

$-10.35$

$\mathrm{C}(\mathrm{t})$

$-20.62$

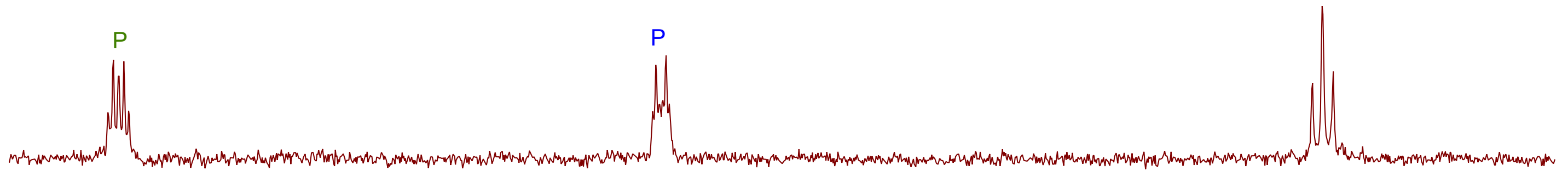

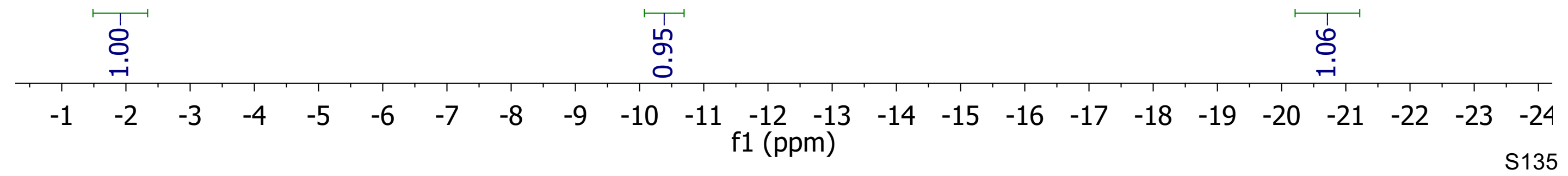




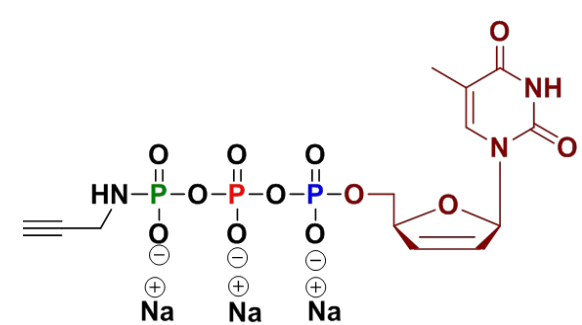

Crude product after precipitation
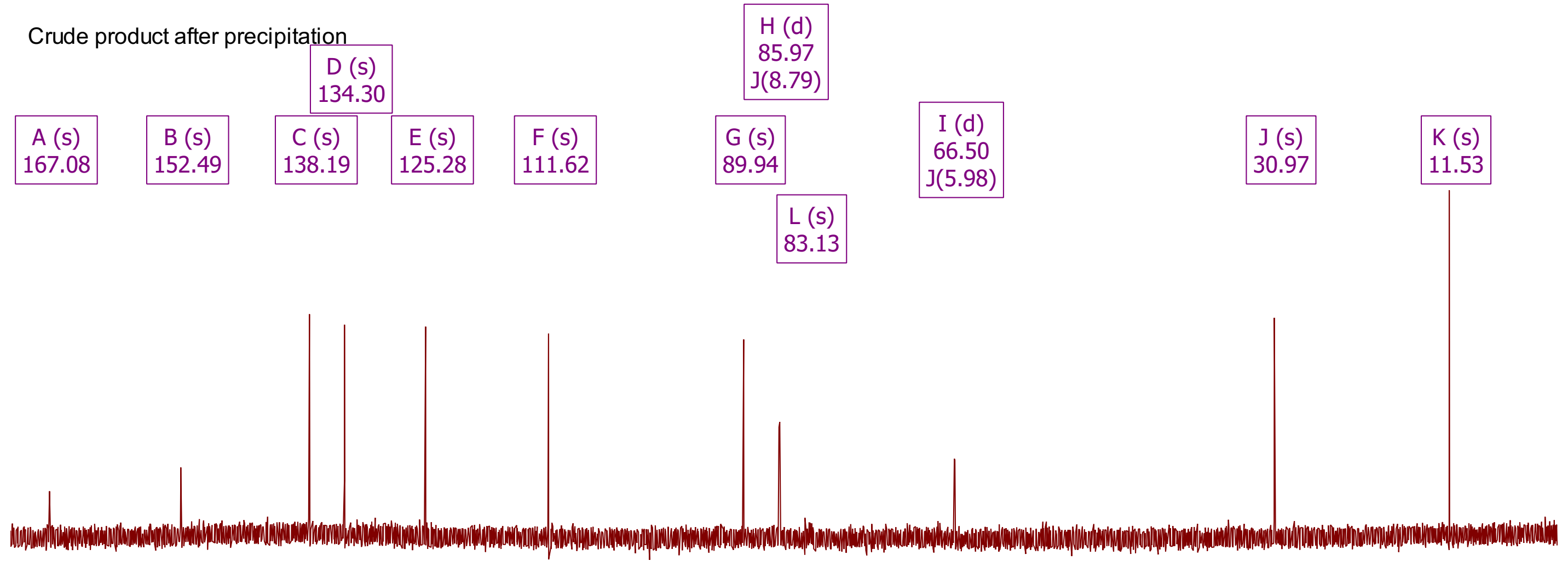

$\begin{array}{rrrrrrrrrrrrrrrrr}.70 & 160 & 150 & 140 & 130 & 120 & 110 & 100 & \begin{array}{c}90 \\ \mathrm{f} 1(\mathrm{ppm})\end{array} & 70 & 60 & 50 & 40 & 30 & 20 & 10 & 0 \\ \mathrm{~s} 136\end{array}$




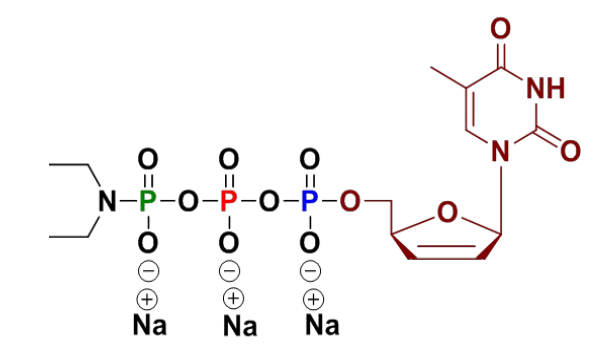

Crude product after precipitation

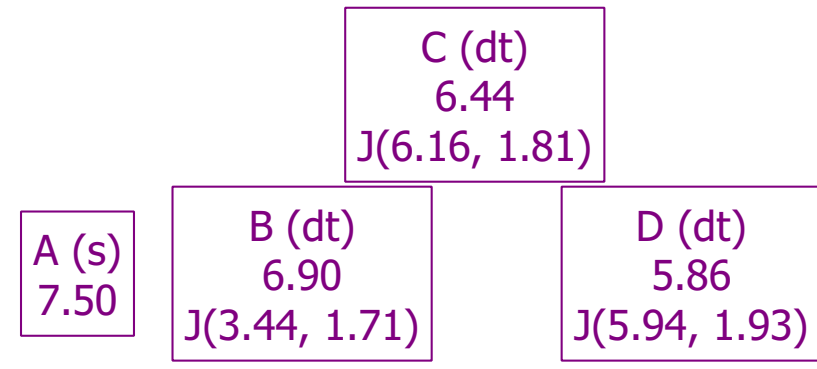

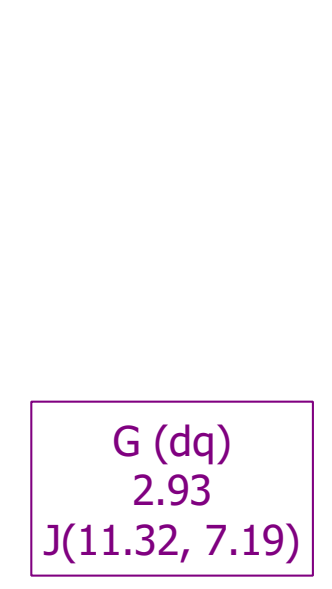
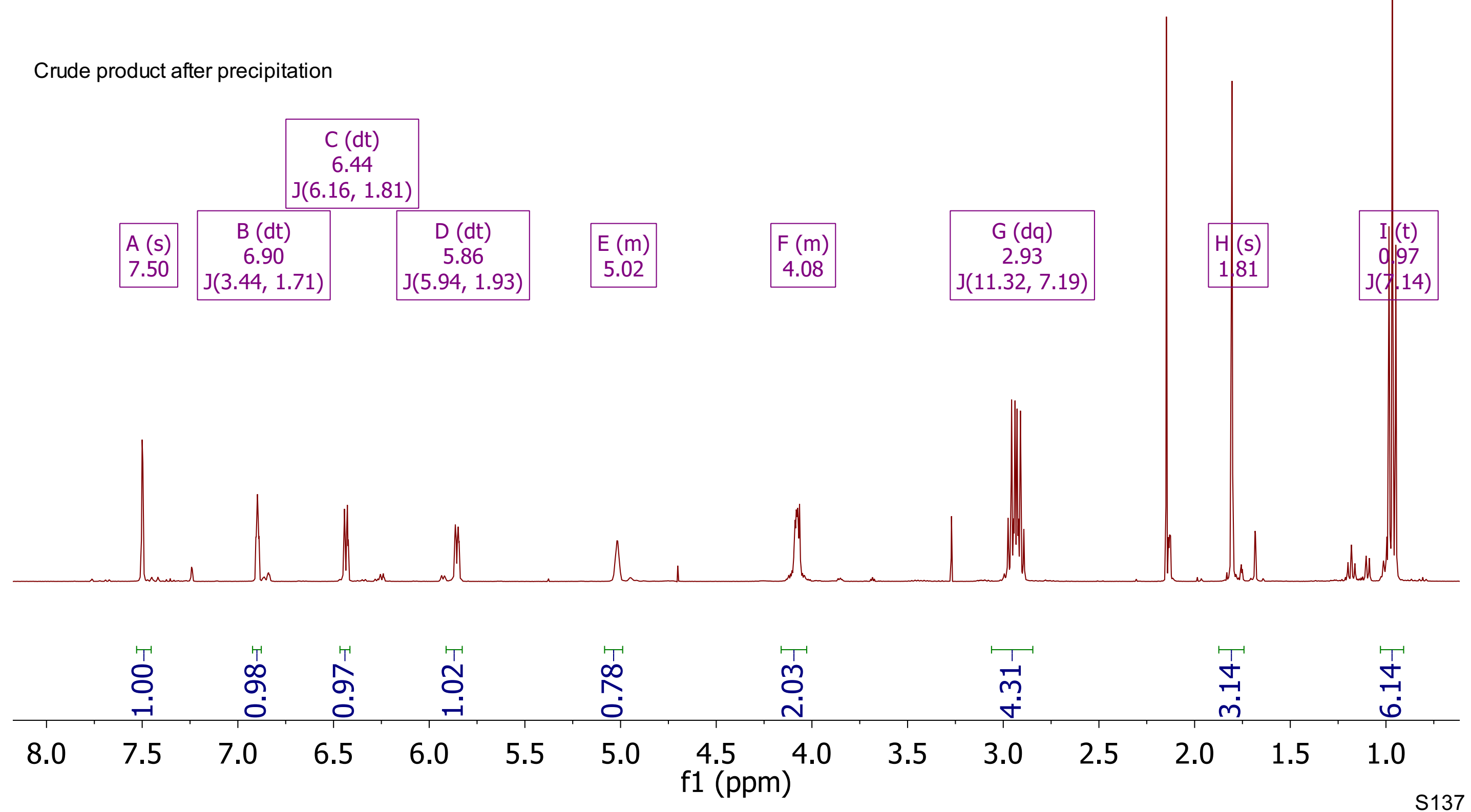


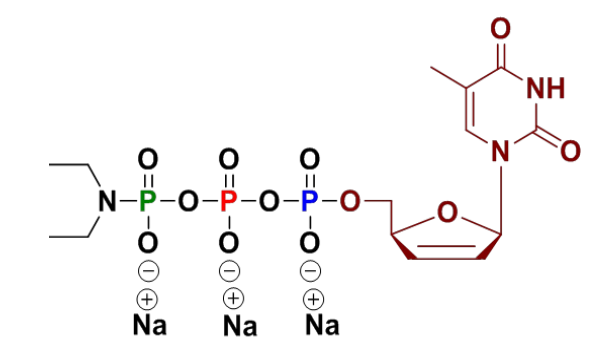

Crude product after precipitation

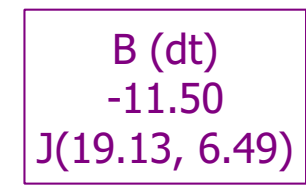

$\mathrm{J}(19.13,6.49)$
A (dd)

$-22.92$

$\mathrm{J}(24.31,18.87)$

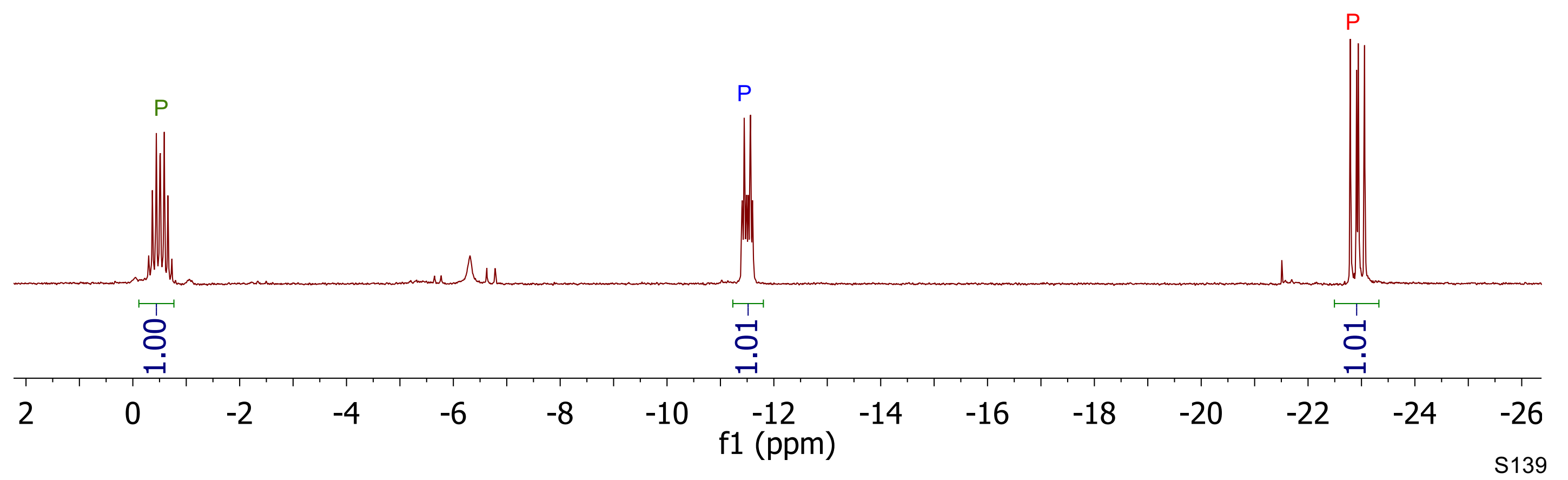




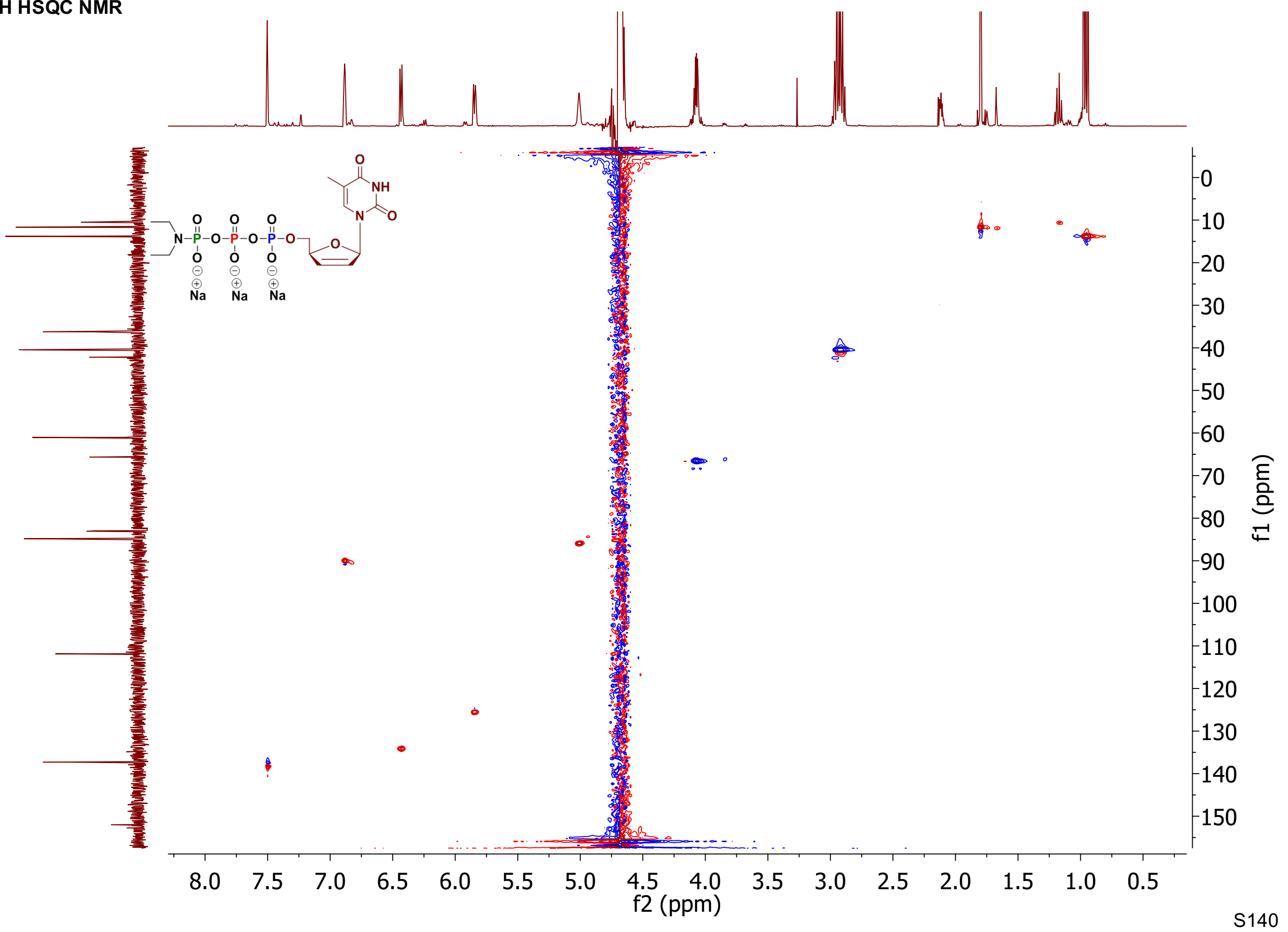




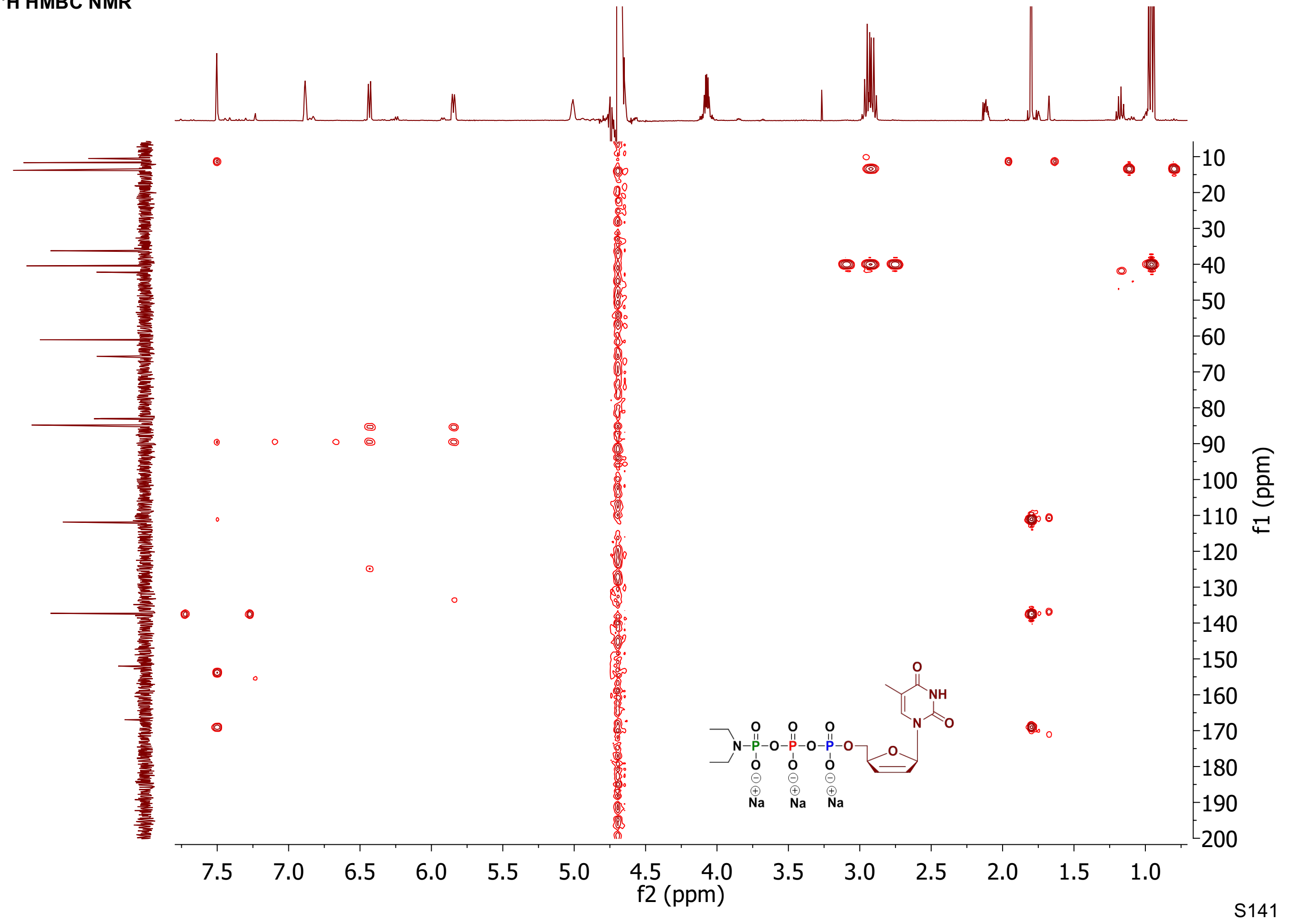




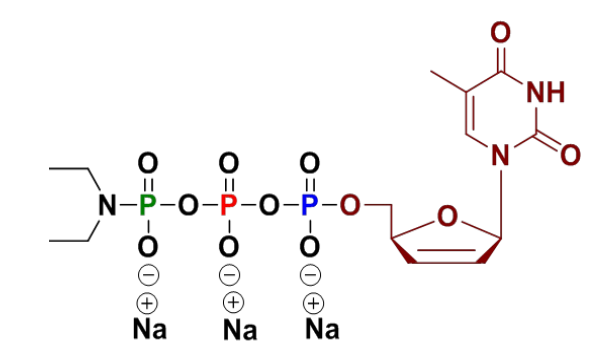

Crude product after precipitation

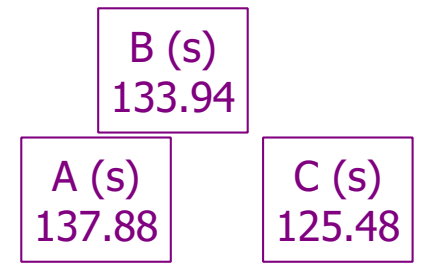

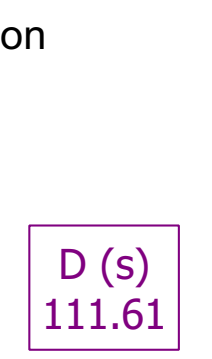

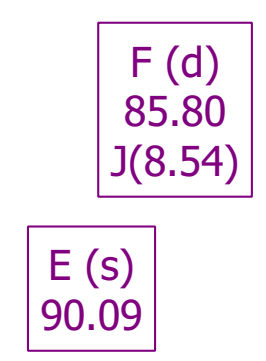

\begin{tabular}{|c|}
\hline$G(d)$ \\
66.58 \\
$J(5.74)$ \\
\hline
\end{tabular}

\begin{tabular}{|c|}
\hline$H(d)$ \\
40.50 \\
$J(3.71)$
\end{tabular}

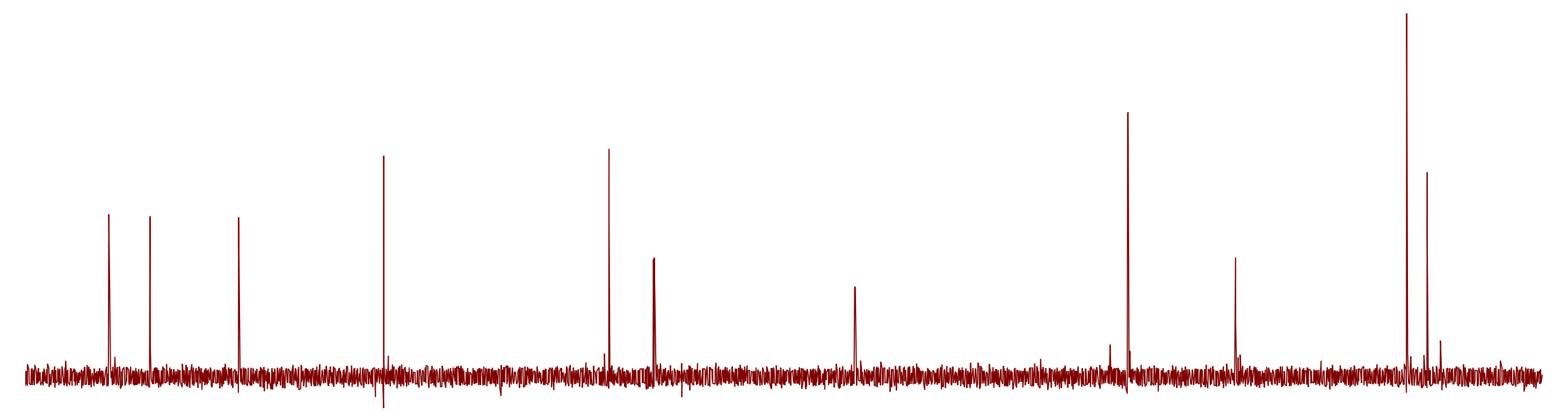

\begin{tabular}{|c|c|c|c|c|c|c|c|c|c|c|c|c|c|c|}
\hline 45 & 135 & 125 & 115 & 105 & 95 & 85 & $\begin{array}{c}75 \\
\mathrm{f} 1(\mathrm{ppm})\end{array}$ & 65 & 55 & 45 & 35 & 25 & 15 & $\begin{array}{l}5 \\
S 142\end{array}$ \\
\hline
\end{tabular}




\section{${ }^{1} \mathrm{H}$ NMR}

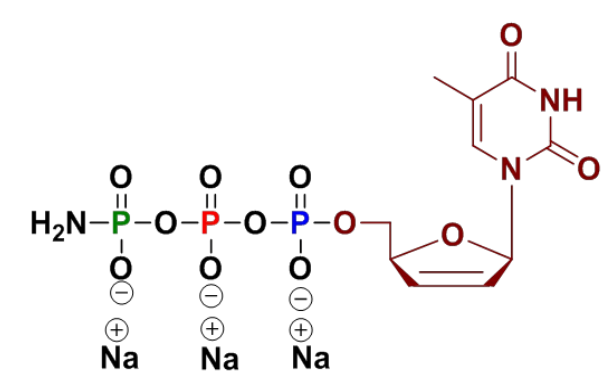

Crude product after precipitation
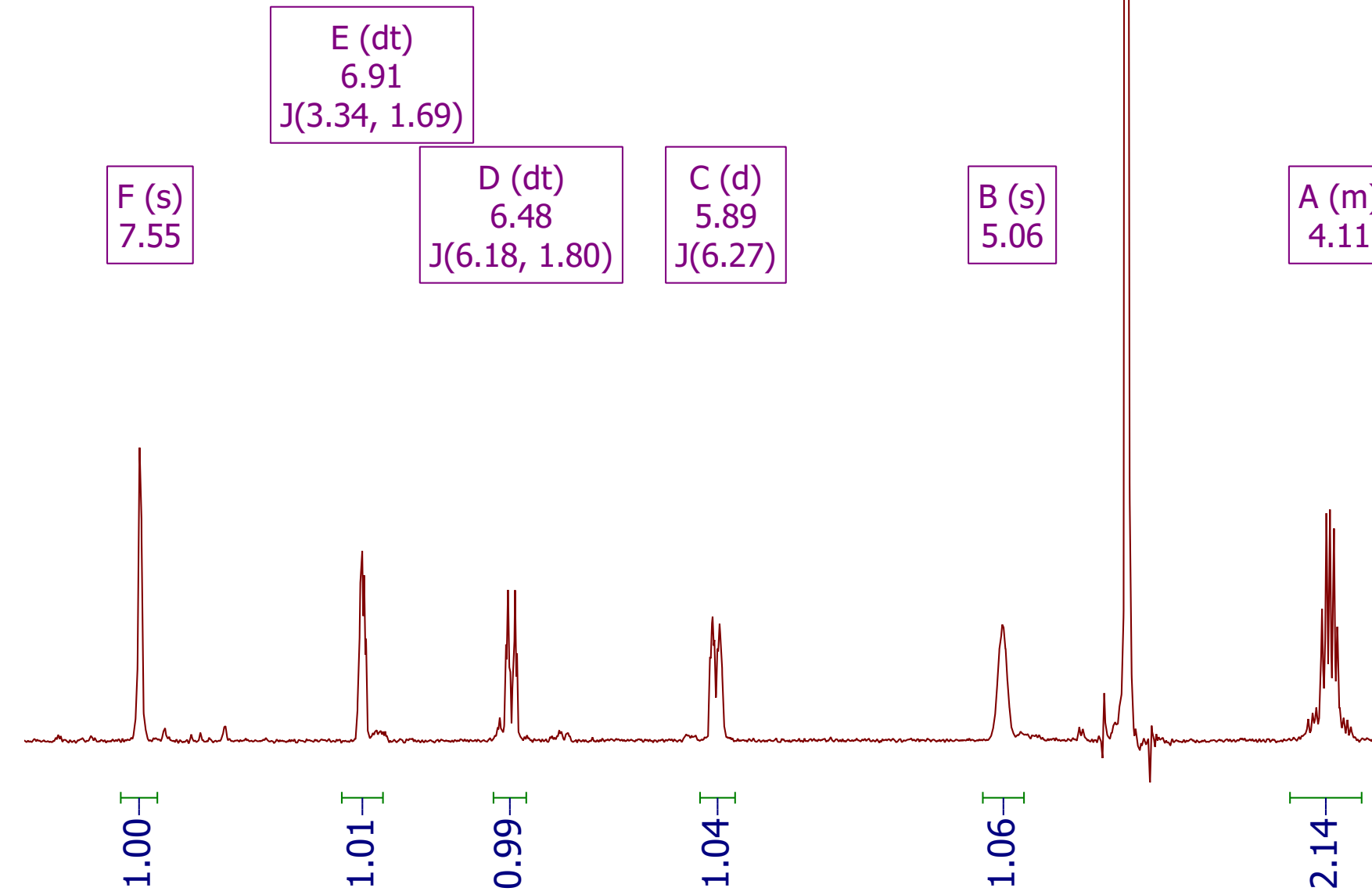

兽

$\begin{array}{llllllllllllllll}7.6 & 7.2 & 6.8 & 6.4 & 6.0 & 5.6 & 5.2 & \begin{array}{c}4.8 \\ \mathrm{f} 1(\mathrm{ppm})\end{array} & 4.4 & 4.0 & 3.6 & 3.2 & 2.8 & 2.4 & 2.0 & 1.6 \\ \mathrm{~s} 143\end{array}$




\section{${ }^{31} \mathrm{P}\left\{{ }^{1} \mathrm{H}\right\} \mathrm{NMR}$}

o $0 \mathrm{~N}$

17

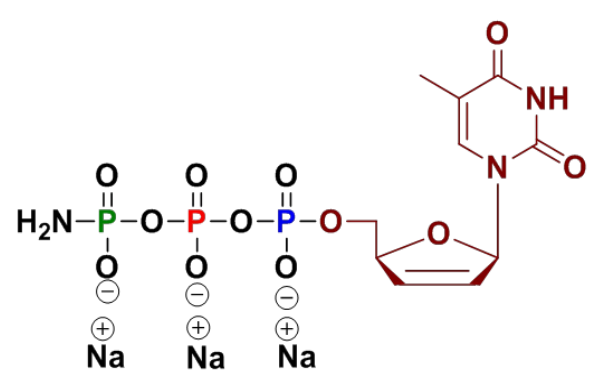

Crude product after precipitation

\begin{tabular}{|c|}
\hline$A(d)$ \\
-1.08 \\
$J(19.26)$ \\
\hline
\end{tabular}
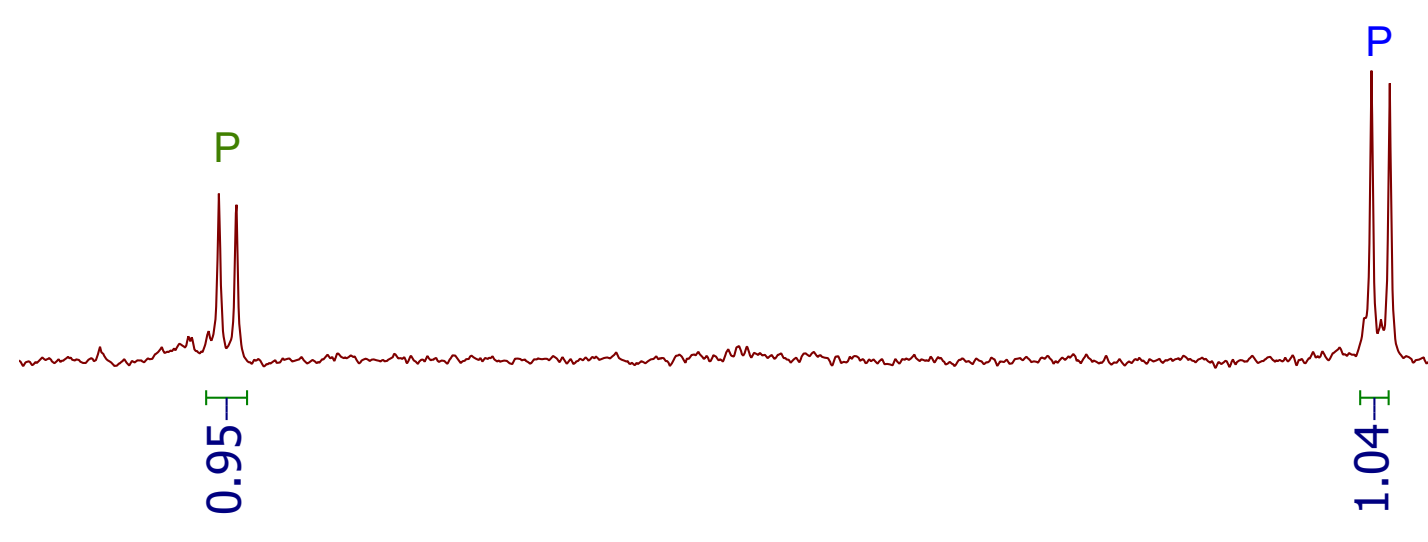

$$
\text { B (d) }
$$$$
-11.53
$$$$
\mathrm{J}(20.07)
$$

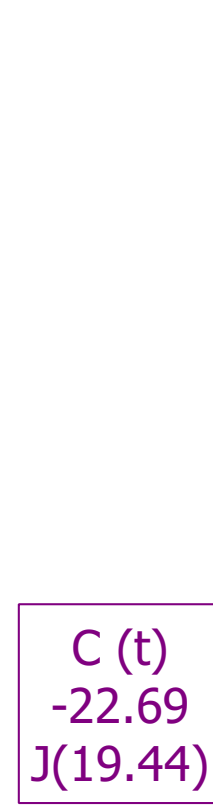

$$
\begin{aligned}
& \begin{array}{llllllllllllllllllllllllllll}
0 & -1 & -2 & -3 & -4 & -5 & -6 & -7 & -8 & -9 & -10 & -11 & -12 & -13 & -14 & -15 & -16 & -17 & -18 & -19 & -20 & -21 & -22 & -23 & -24
\end{array} \\
& \text { f1 (ppm) }
\end{aligned}
$$




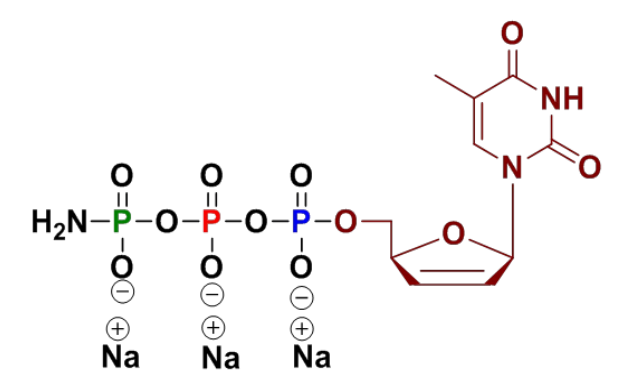

Crude product after precipitation

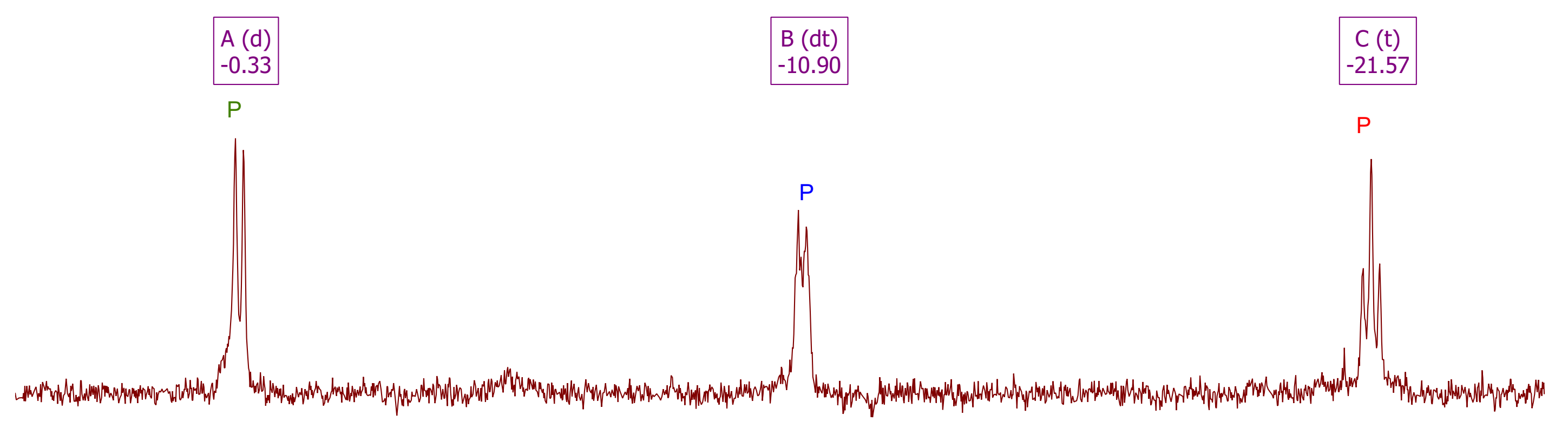

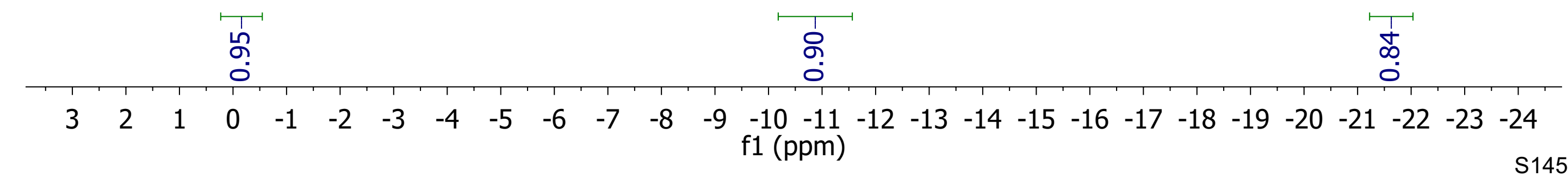




\section{${ }^{1}$ H NMR}

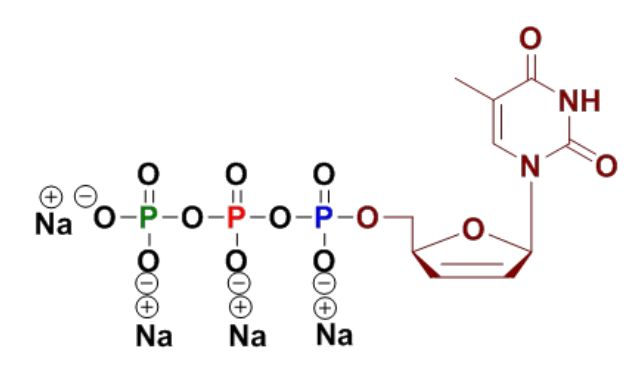

Crude product after precipitation

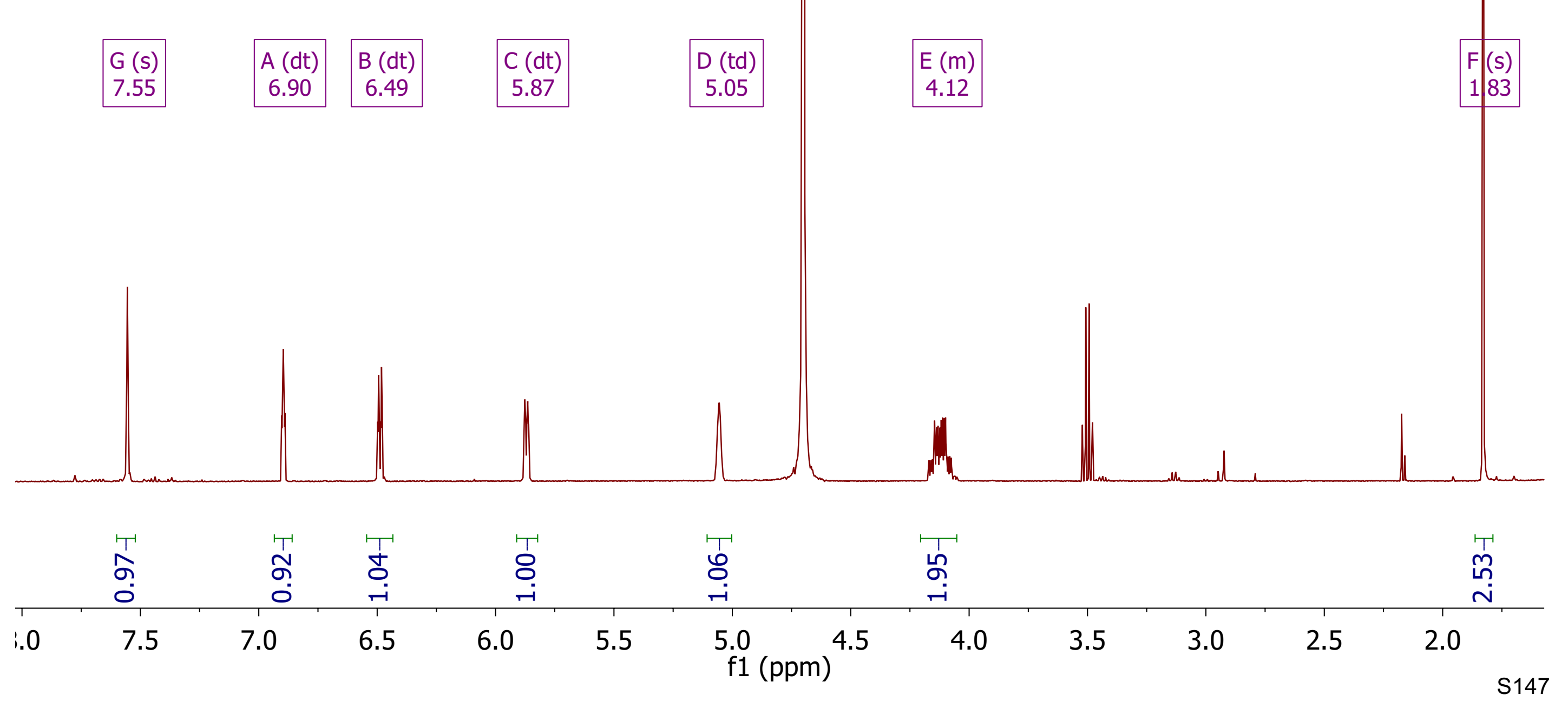




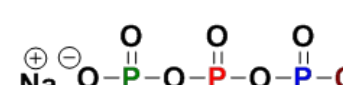

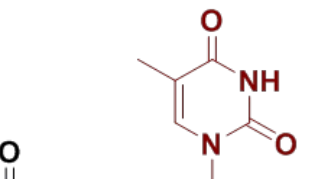

\begin{tabular}{lll}
8 & 8 & 8 \\
\hline & 9 & 8
\end{tabular}

$\mathrm{Na} \mathrm{Na} \mathrm{Na}$

Crude product after precipitation

\begin{tabular}{|c|c|}
\hline \multicolumn{2}{|c|}{$\begin{array}{c}B(d) \\
-10.91 \\
J(19.56)\end{array}$} \\
\hline & $\begin{array}{l}A(d) \\
-11.58 \\
(20.10)\end{array}$ \\
\hline
\end{tabular}

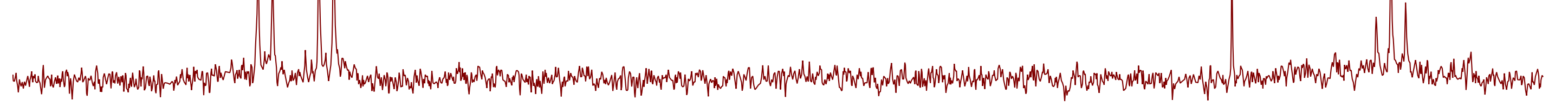

$$
\begin{aligned}
& \text { 马्ष } \\
& \text { '0 }
\end{aligned}
$$

\begin{tabular}{|c|c|c|c|c|c|c|c|c|c|c|c|c|c|c|c|}
\hline-9 & -10 & -11 & -12 & -13 & -14 & -15 & $\begin{array}{rr}-16 & -17 \\
\mathrm{f1} & (\mathrm{ppm})\end{array}$ & -18 & -19 & -20 & -21 & -22 & -23 & -24 & $\begin{array}{r}-2 \\
\text { S148 }\end{array}$ \\
\hline
\end{tabular}




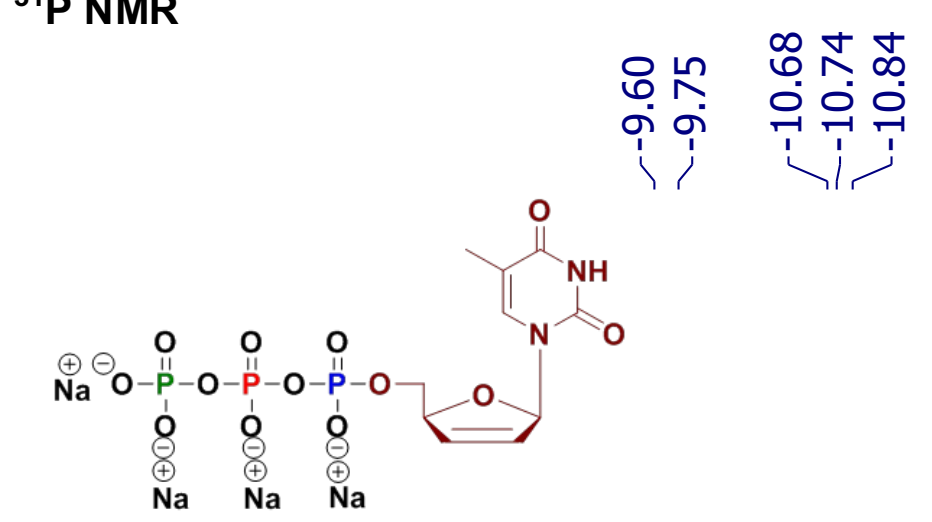

\section{Crude product after precipitation}

$$
\begin{array}{|l|l|}
\hline A(d) & B(d t) \\
-9.68 & -10.74 \\
\hline
\end{array}
$$

\begin{tabular}{|c|c|c|c|c|c|c|c|c|c|c|c|c|c|c|c|c|c|}
\hline & & & & & $\begin{array}{l}1 \\
8 \\
\end{array}$ & & & & & & & & & & & & \\
\hline-6 & -7 & -8 & -9 & -10 & -11 & -12 & -13 & $\begin{array}{r}-14 \\
\mathrm{f} 1\end{array}$ & $\begin{array}{r}-15 \\
\text { opm) }\end{array}$ & -16 & -17 & -18 & -19 & -20 & -21 & -22 & $\begin{array}{l}-23 \\
S 149\end{array}$ \\
\hline
\end{tabular}

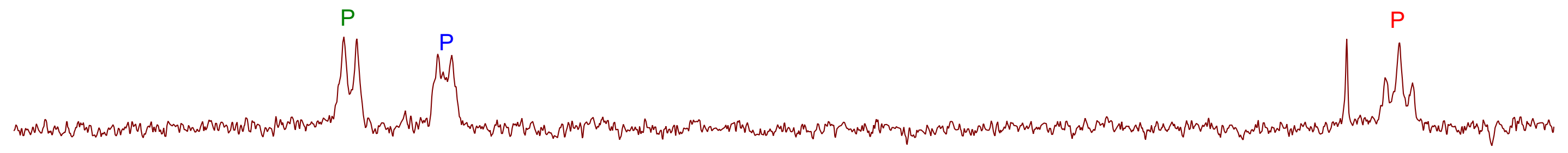




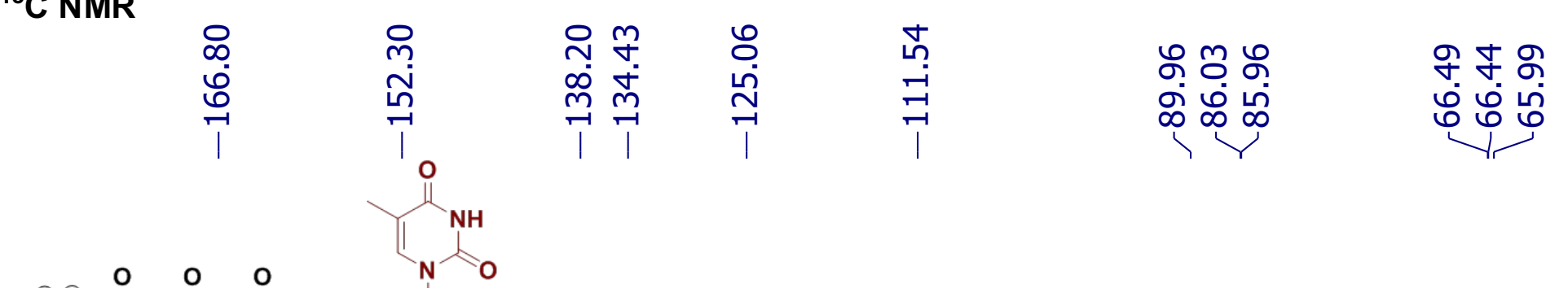

Crude product after precipitation

\begin{tabular}{|c|c|c|c|c|c|c|}
\hline & & $\begin{array}{r}\text { D } \\
134 .\end{array}$ & & & $\begin{array}{l}H(d) \\
86.00\end{array}$ & $\begin{array}{l}\mathrm{K}(\mathrm{s}) \\
65.99\end{array}$ \\
\hline $\begin{array}{c}A(s) \\
166.80\end{array}$ & $\begin{array}{c}B(s) \\
152.30\end{array}$ & $\begin{array}{c}\text { C (s) } \\
138.20\end{array}$ & $\begin{array}{c}E(s) \\
125.06\end{array}$ & $\begin{array}{c}F(s) \\
111.54\end{array}$ & $\begin{array}{l}\text { G (s) } \\
89.96\end{array}$ & $\begin{array}{l}\text { I (d) } \\
66.46\end{array}$ \\
\hline
\end{tabular}

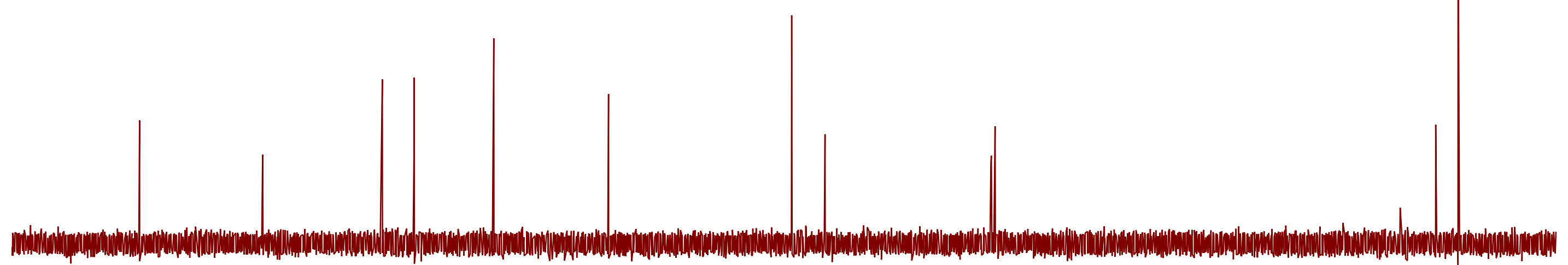

\begin{tabular}{|c|c|c|c|c|c|c|c|c|c|c|c|c|c|c|c|c|c|}
\hline 180 & 170 & 160 & 150 & 140 & 130 & 120 & 110 & 100 & $\begin{array}{c}90 \\
\text { (ppm }\end{array}$ & 80 & 70 & 60 & 50 & 40 & 30 & 20 & 10 \\
\hline
\end{tabular}




\section{${ }^{31} \mathrm{P}\left\{{ }^{1} \mathrm{H}\right\}-N M R$}

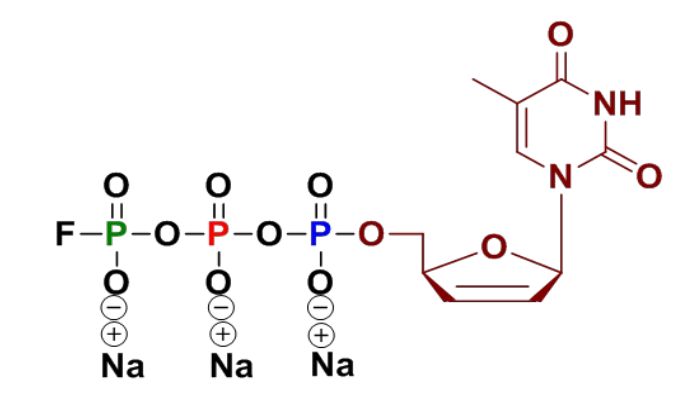

Crude product after precipitation
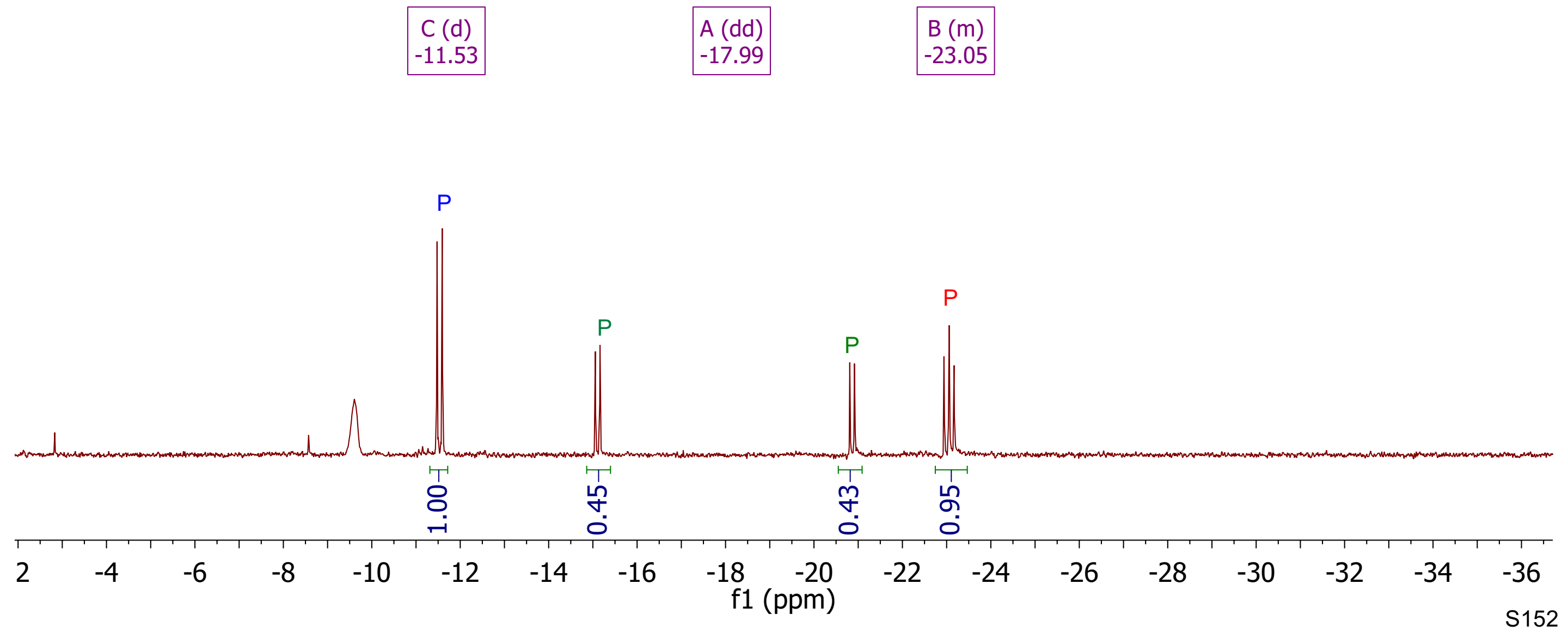


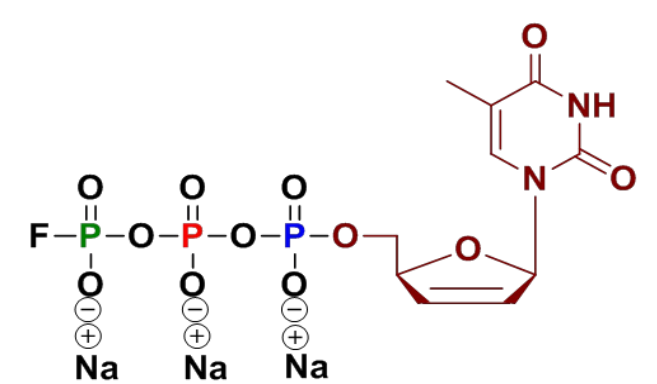

Crude product after precipitation 


\section{${ }^{31} \mathrm{P}\left\{{ }^{19} \mathrm{~F}\right\} N M R$}

ฆั 守 드 ำ ำ ๒ู

ติ ต

감

รั เด

N

간간각걱

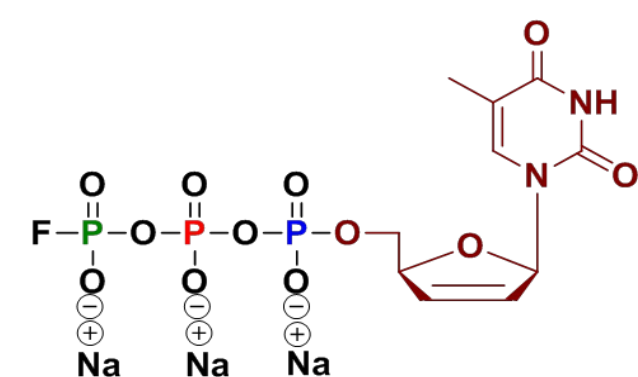

Crude product after precipitation

$$
\text { B (dt) }
$$

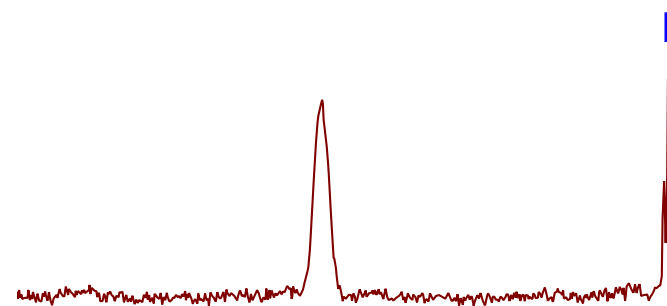

$\mathrm{P}$
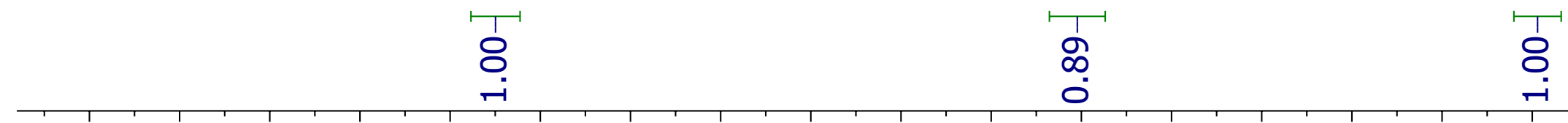

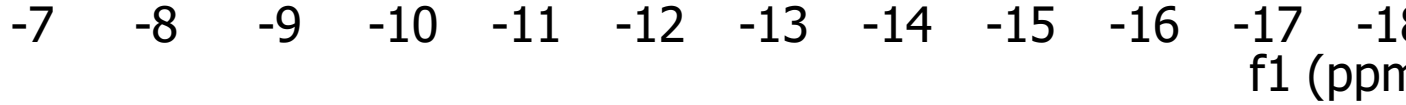




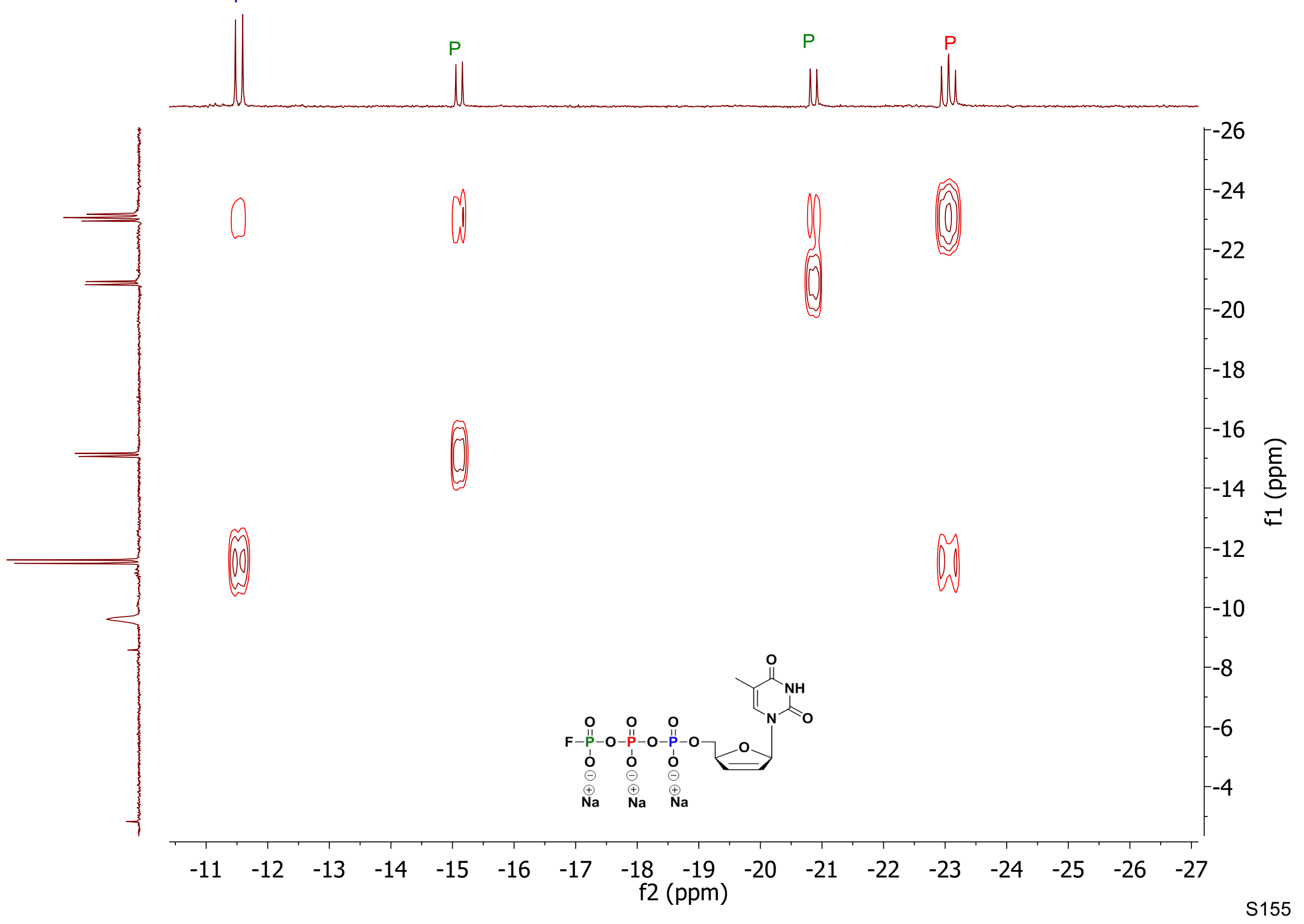




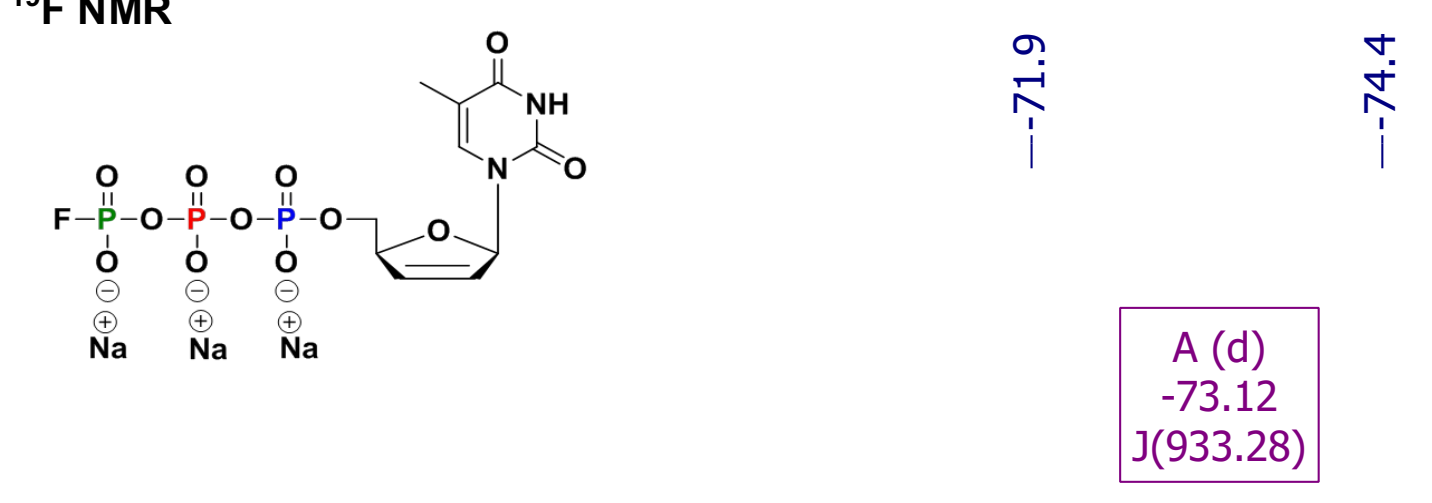
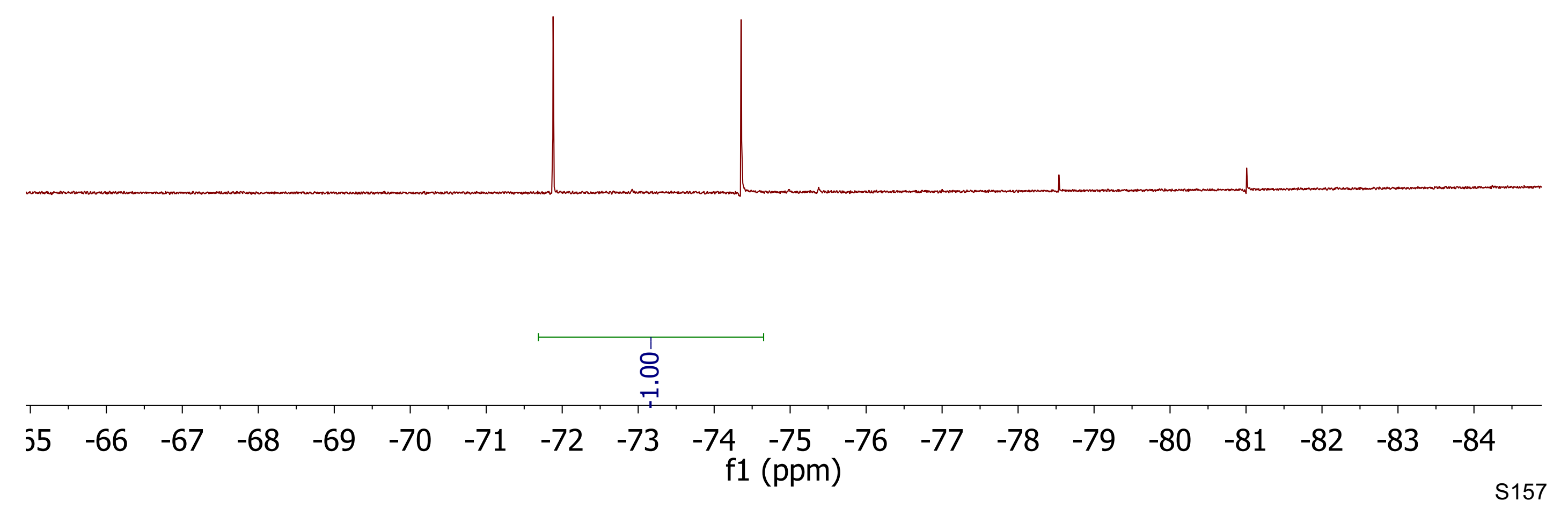


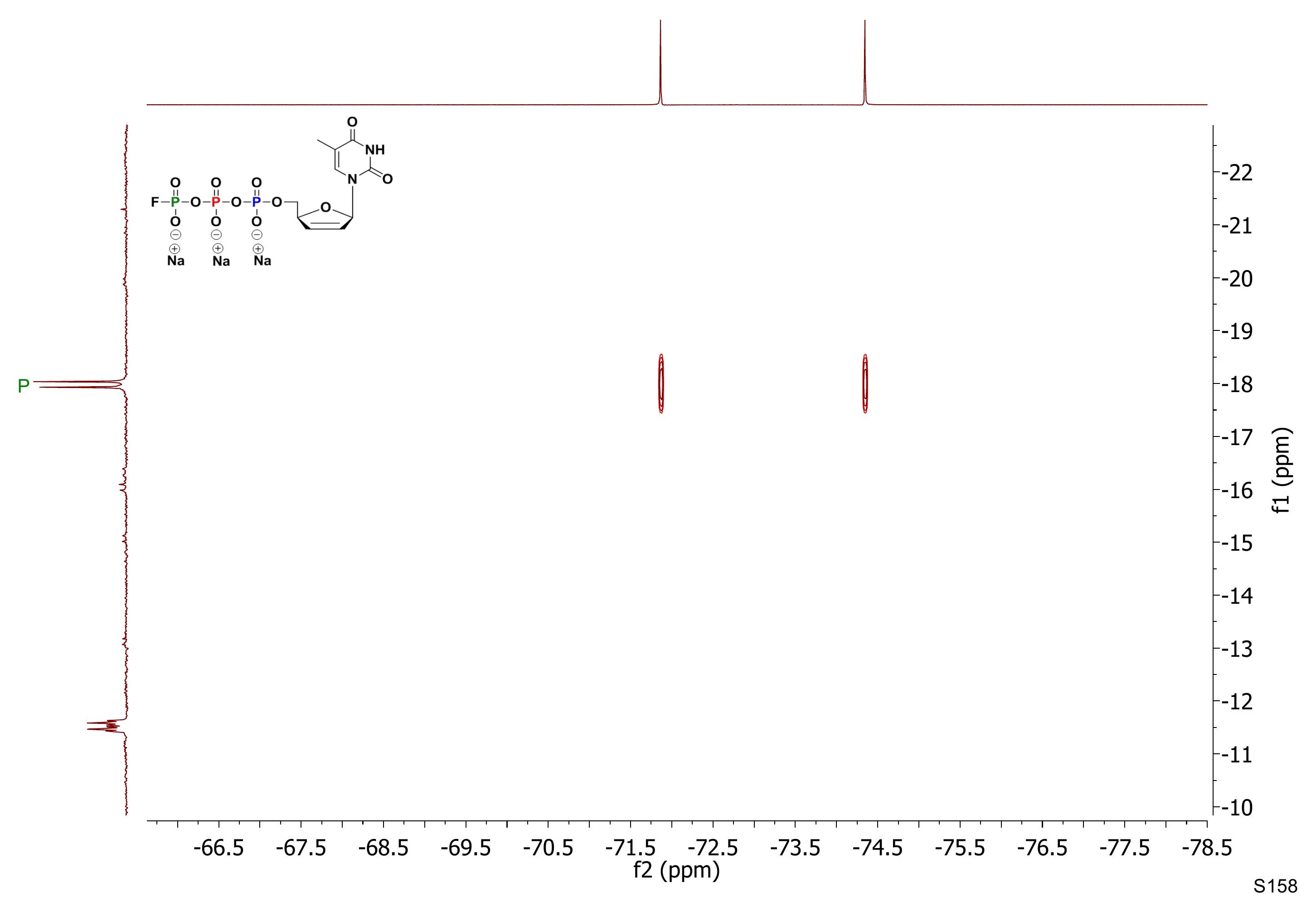




\section{${ }^{31} \mathrm{P}\left\{{ }^{1} \mathrm{H}\right\}-\mathrm{NMR}$}

$P\{\mathrm{H}\}-\mathrm{NMR}$

Crude product after precipitation

\begin{tabular}{|c|c|}
\hline A (d) & B (d) \\
-10.85 & -12.88 \\
\hline
\end{tabular}

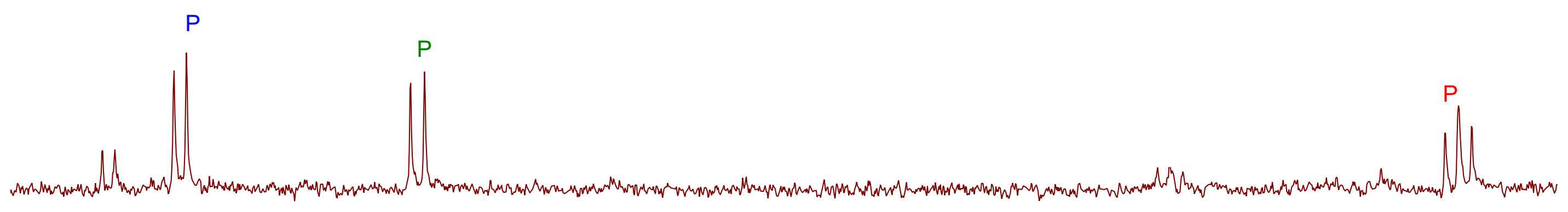

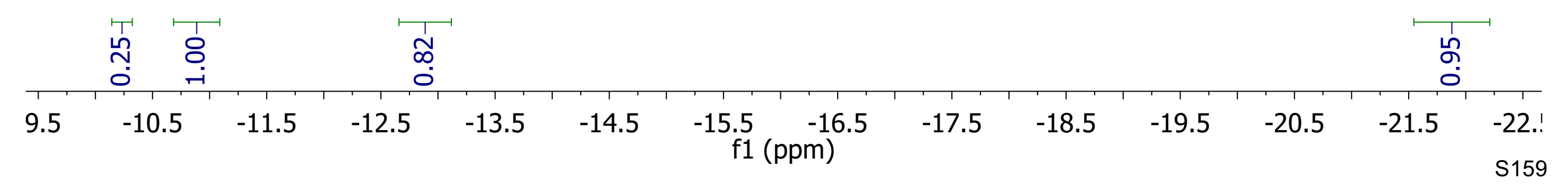


Crude product after precipitation

\begin{tabular}{|c|c|}
\hline$A(d t)$ & $B(d)$ \\
-10.86 & -12.89 \\
\hline
\end{tabular}

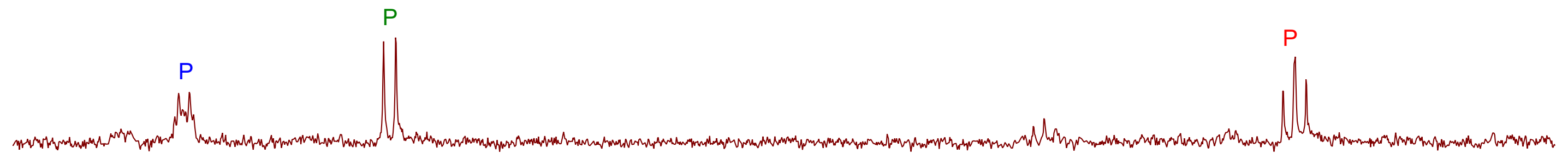

\begin{tabular}{|c|c|c|c|c|c|c|c|c|c|c|c|c|c|c|}
\hline & $\begin{array}{l}1 \\
0 \\
0 \\
-1 \\
1\end{array}$ & & $\begin{array}{l}1 \\
\infty \\
\infty \\
0\end{array}$ & & & & & & & & & 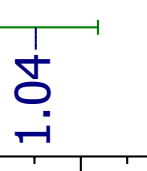 & & \\
\hline-10.0 & -11.0 & -12.0 & -13.0 & -14.0 & -15.0 & -16.0 & $\begin{array}{r}-17.0 \\
(p p m)\end{array}$ & -18.0 & -19.0 & -20.0 & -21.0 & -22.0 & -23.0 & $\begin{array}{r}-24.0 \\
S 160\end{array}$ \\
\hline
\end{tabular}


${ }^{1} \mathrm{H}$ NMR

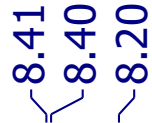

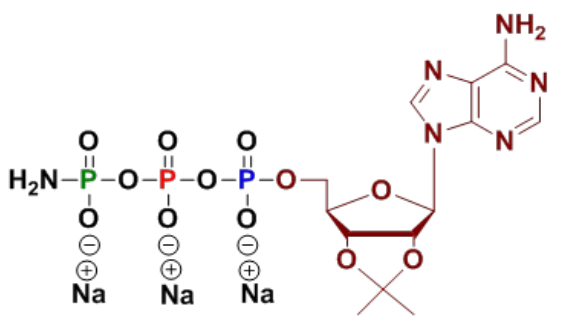

Crude product after precipitation
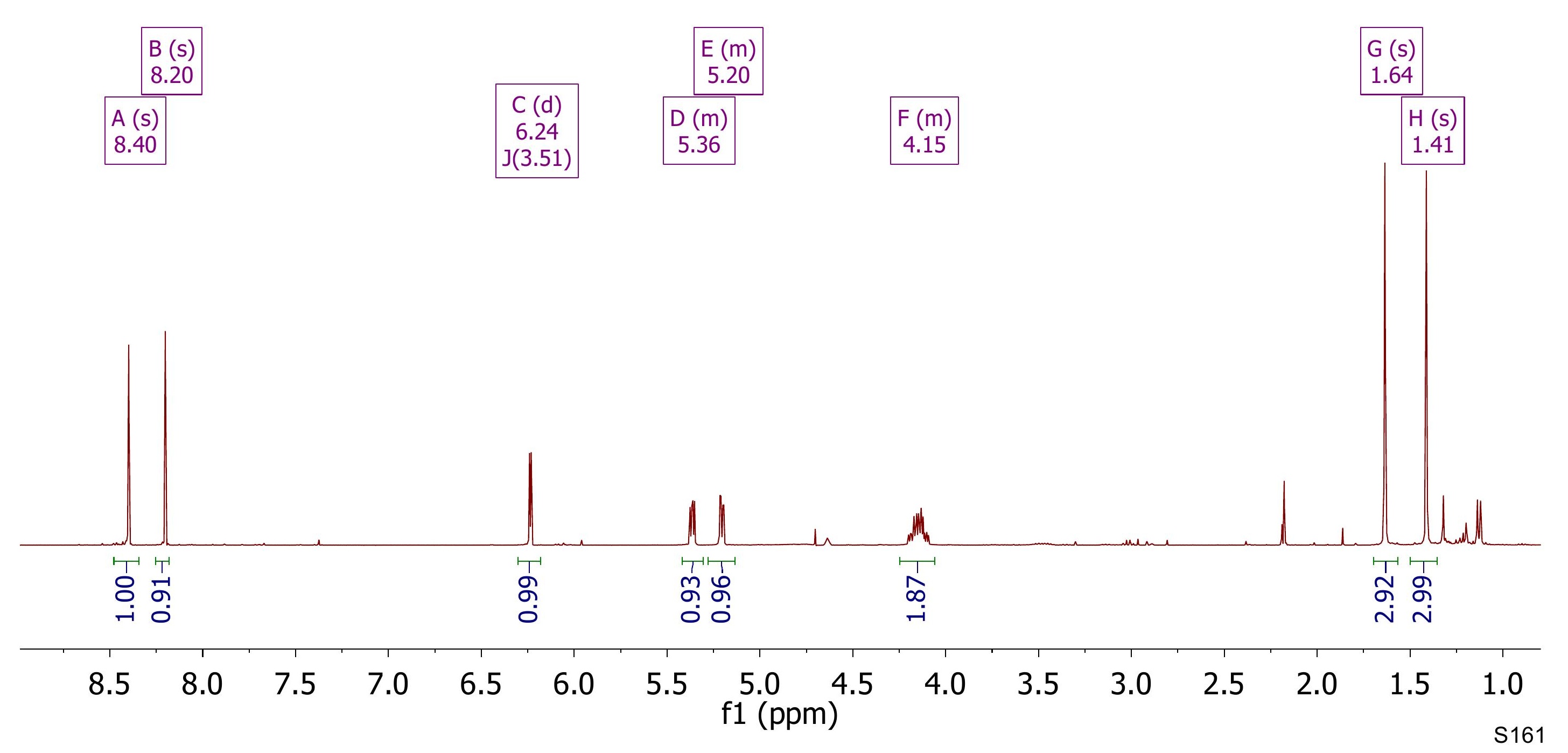

구유

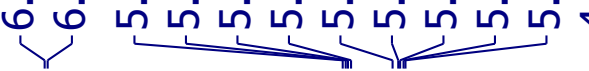


Crude product after precipitation

$$
\begin{array}{|l|}
\hline \text { A (d) } \\
-1.04 \\
\hline
\end{array}
$$

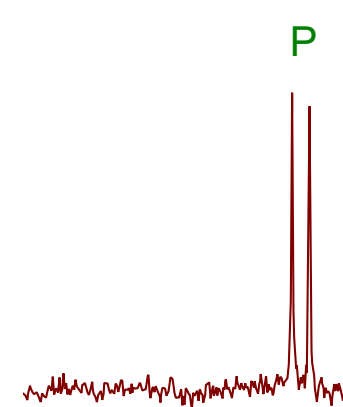




\section{${ }^{31} \mathrm{P}\left\{{ }^{1} \mathrm{H}\right\}$ NMR}

Oִ

$\mathrm{NH}_{2}$

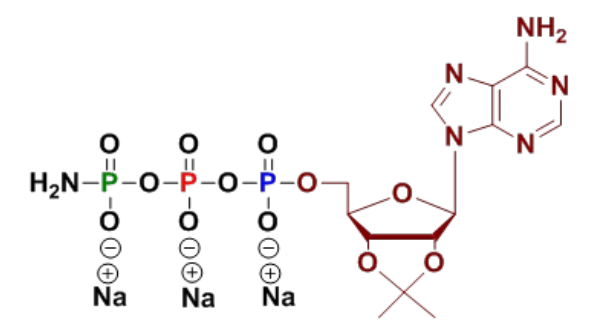

Crude product after precipitation

\begin{tabular}{|c|}
\hline$A(d)$ \\
-0.98 \\
$J(19.12)$ \\
\hline
\end{tabular}
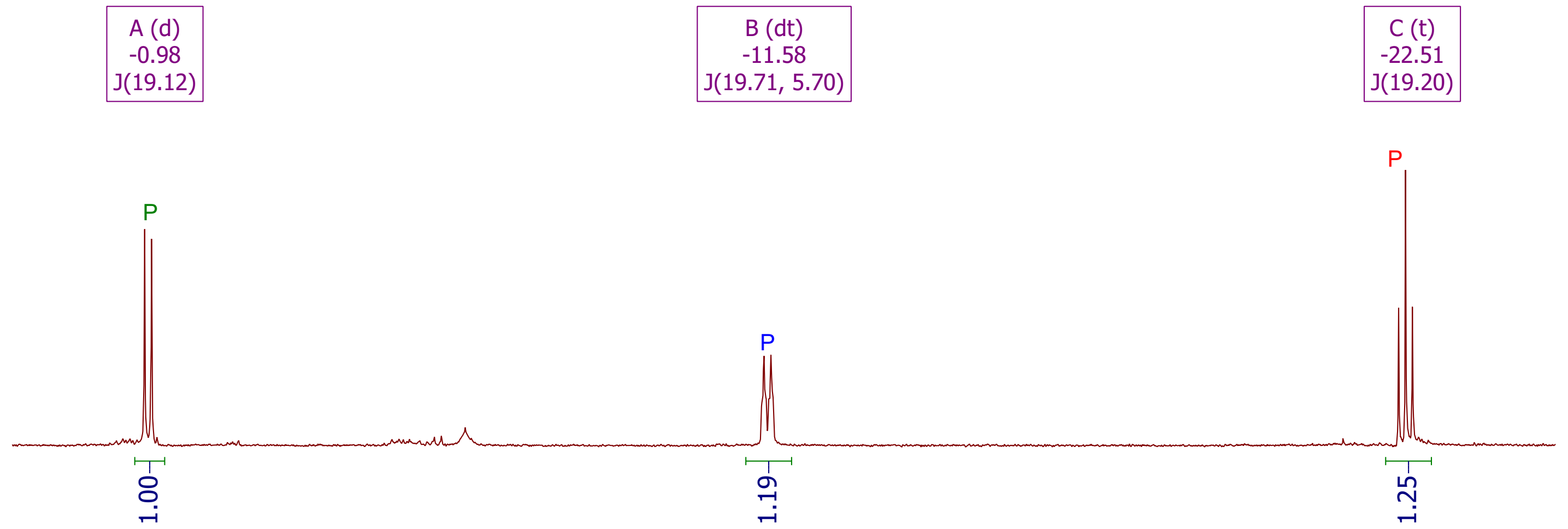

$\begin{array}{llllllllllllllllllllllllllllllll}1 & 0 & -1 & -2 & -3 & -4 & -5 & -6 & -7 & -8 & -9 & -10 & -11 & -12 & -13 & -14 & -15 & -16 & -17 & -18 & -19 & -20 & -21 & -22 & -23 & -24 & -2\end{array}$ f1 (ppm) 


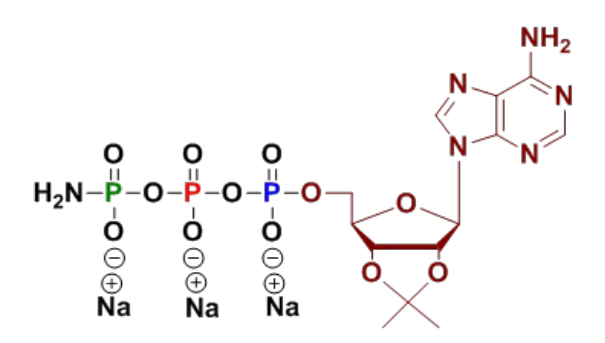

Crude product after precipitation

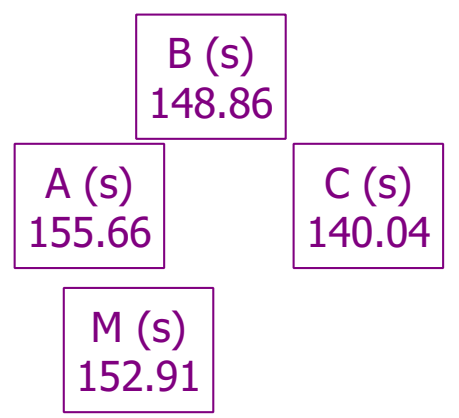

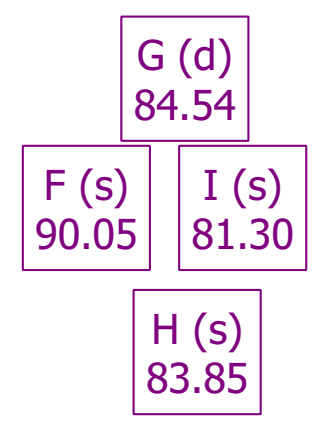

\section{J (d)}

65.78

\begin{tabular}{|c|}
\hline$L(s)$ \\
24.41 \\
\hline$K(s)$ \\
26.15 \\
\hline
\end{tabular}

$\mathrm{L}(\mathrm{s})$

24.41

26.15

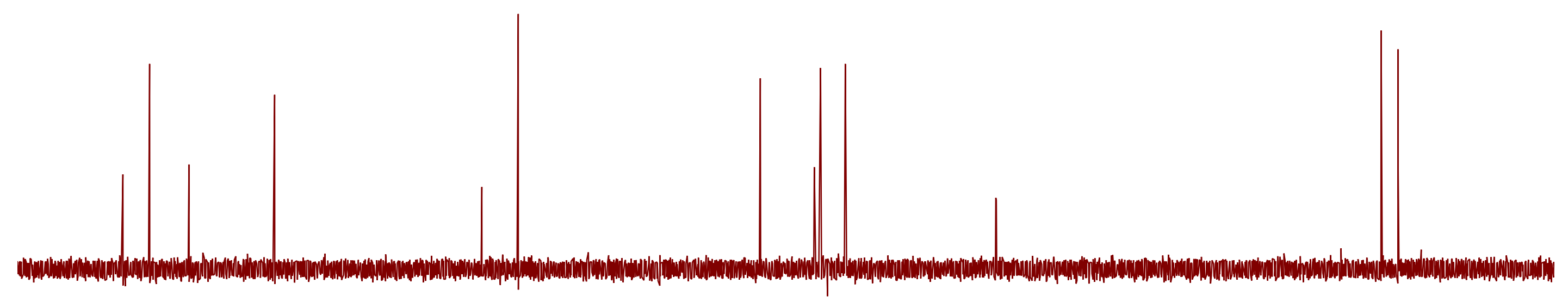

\begin{tabular}{|c|c|c|c|c|c|c|c|c|c|c|c|c|c|c|}
\hline 160 & 150 & 140 & 130 & 120 & 110 & 100 & ${ }_{\mathrm{f1}(\mathrm{ppm})}^{90} 80$ & 70 & 60 & 50 & 40 & 30 & 20 & $\begin{array}{r}10 \\
\text { S164 }\end{array}$ \\
\hline
\end{tabular}



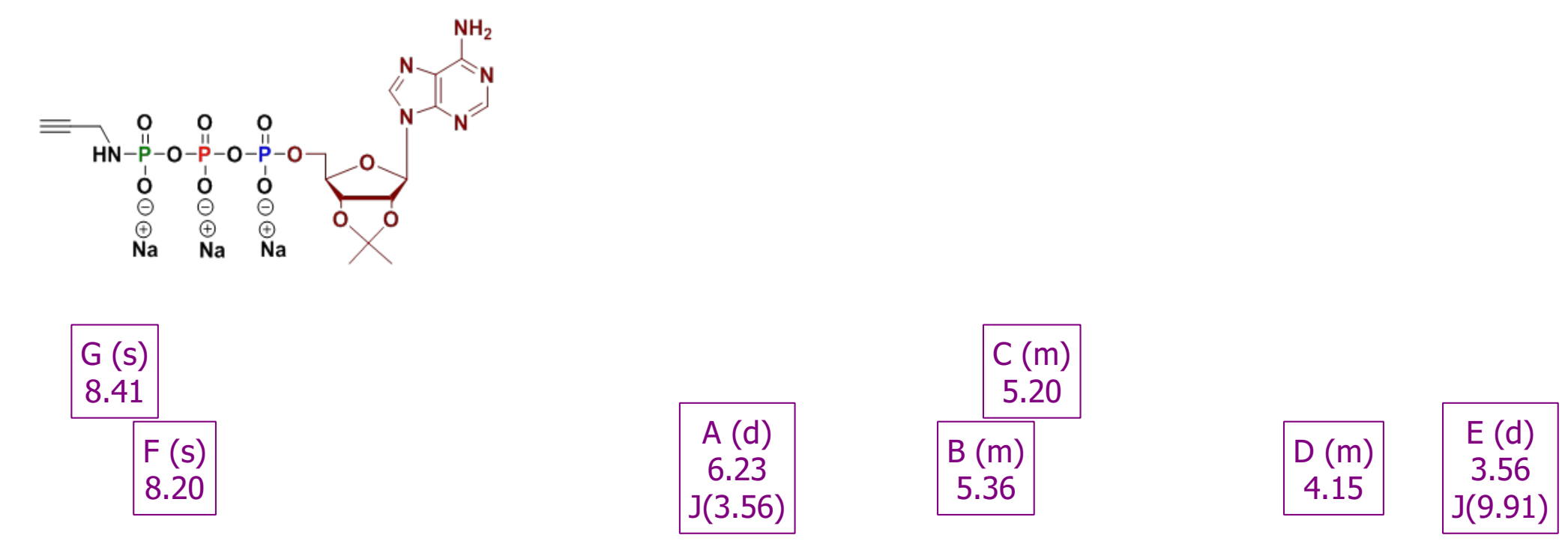

Crude product after precipitation

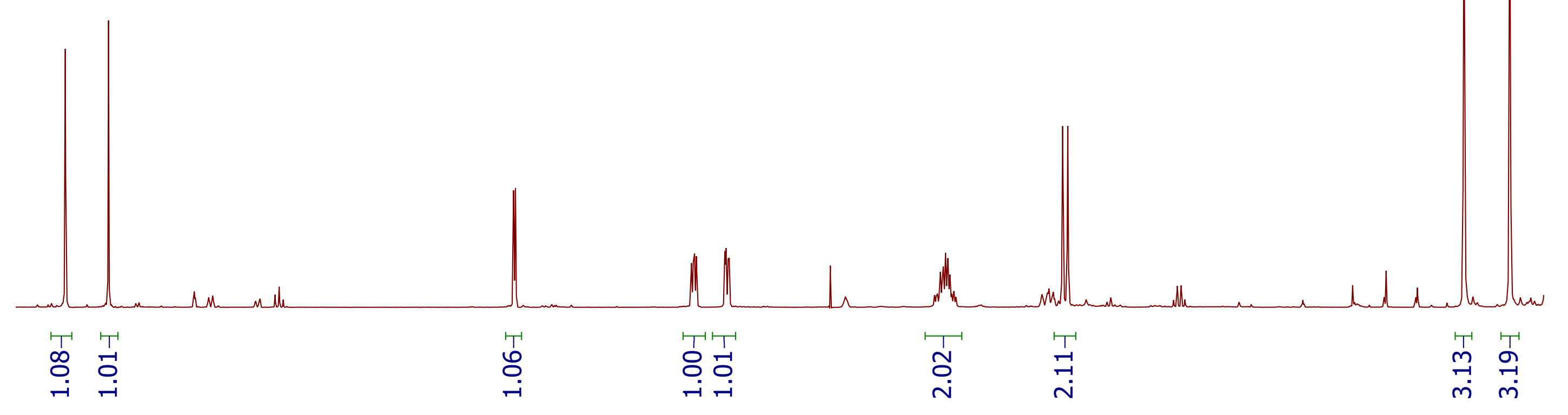

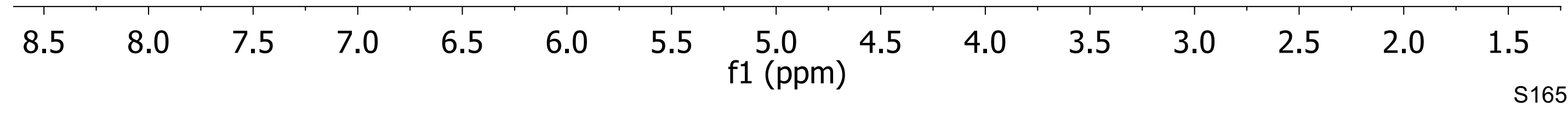




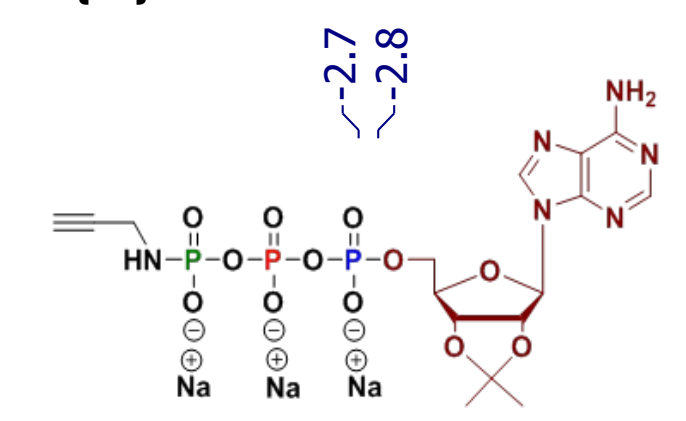

\section{Crude product after precipitation}

A (d)

$-2.76$

$\mathrm{J}(20.87)$
$B(d)$

$-11.60$

$J(19.24)$

P

P

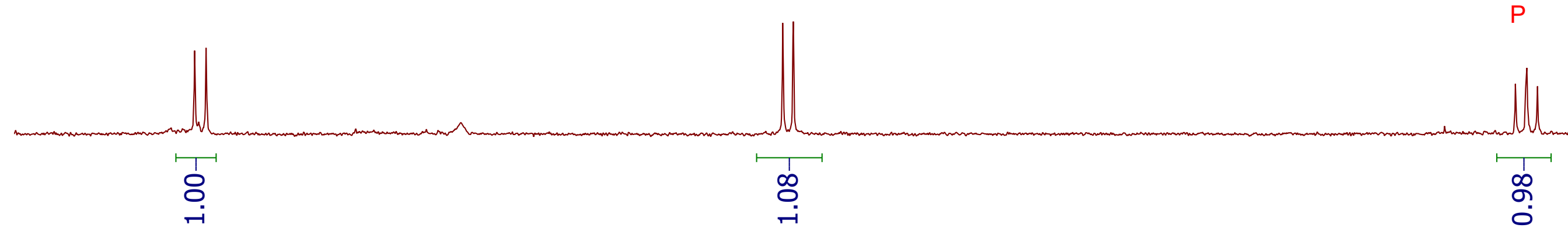

$\begin{array}{lllllllllllllllllllllllll}5 & -1 & -2 & -3 & -4 & -5 & -6 & -7 & -8 & -9 & -10 & -11 & -12 & -13 & -14 & -15 & -16 & -17 & -18 & -19 & -20 & -21 & -22 & -23 & -24\end{array}$ f1 (ppm) 


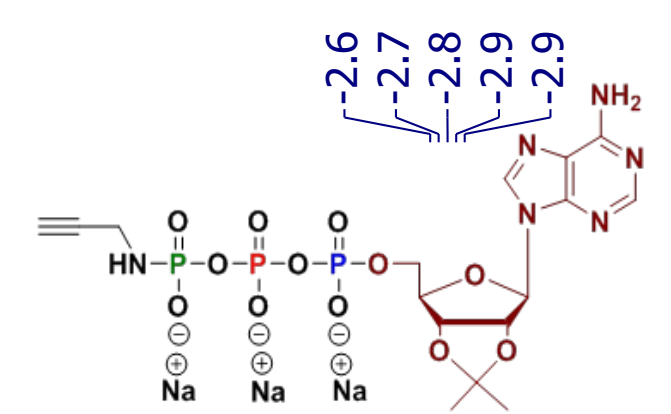

Crude product after precipitation

\begin{tabular}{|c|}
\hline$C(d t)$ \\
-2.76 \\
$J(20.36,10.08)$ \\
\hline
\end{tabular}

$$
\begin{array}{|c|}
\hline B(d) \\
-11.59 \\
J(18.27) \\
\hline
\end{array}
$$

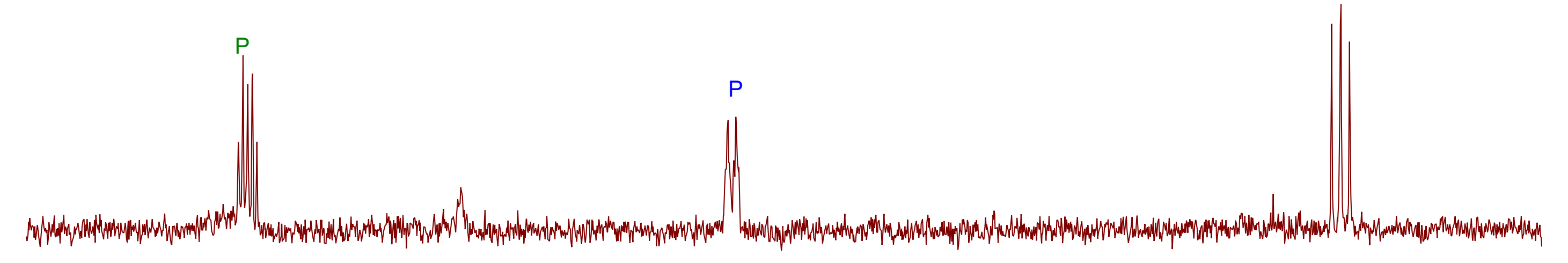

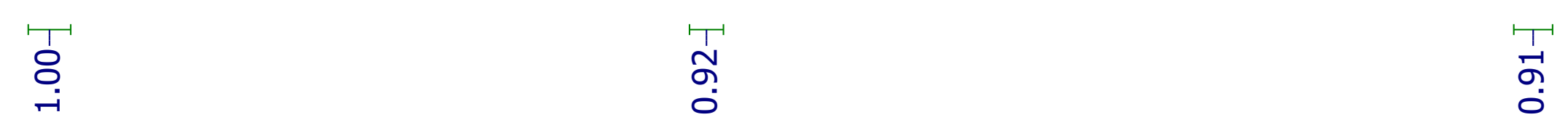

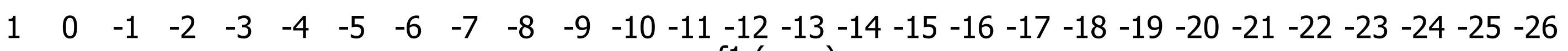
f1 (ppm) 
${ }^{31} \mathrm{P}\left\{{ }^{1} \mathrm{H}\right\} \mathrm{NMR}$

טִ

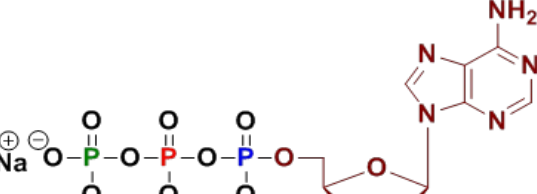

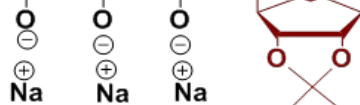

Crude product after precipitation

$$
\begin{array}{|c|}
\hline A(d) \\
-5.55 \\
J(20.12) \\
\hline
\end{array}
$$

P

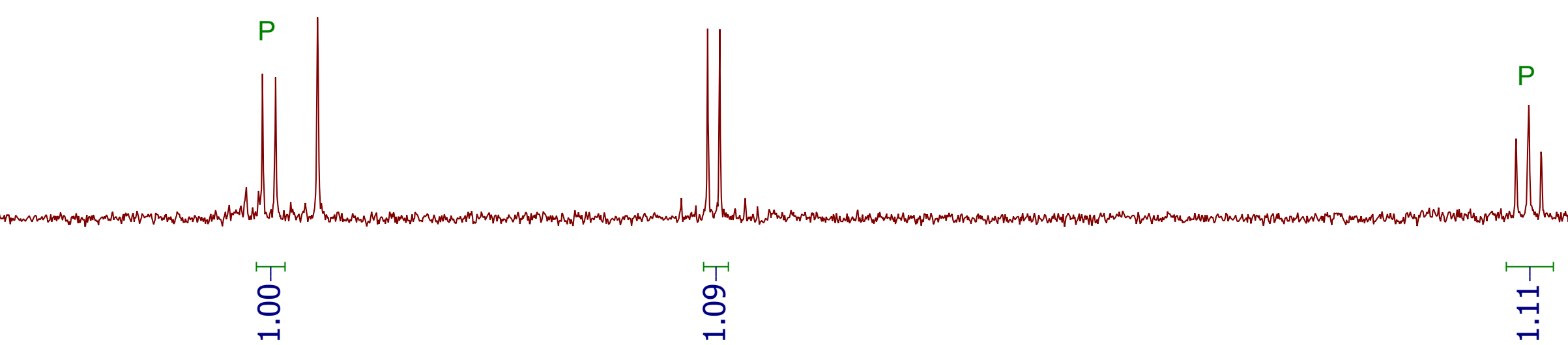

$$
\begin{array}{lllllllllllllllllllllll}
-3 & -4 & -5 & -6 & -7 & -8 & -9 & -10 & -11 & -12 & -13 & -14 & -15 & -16 & -17 & -18 & -19 & -20 & -21 & -22 & -23 & -24 & -25 \\
& & & & & & & & &
\end{array}
$$


Crude product after precipitation

\begin{tabular}{|c|}
\hline$A(d)$ \\
-5.56 \\
$J(20.03)$ \\
\hline
\end{tabular}
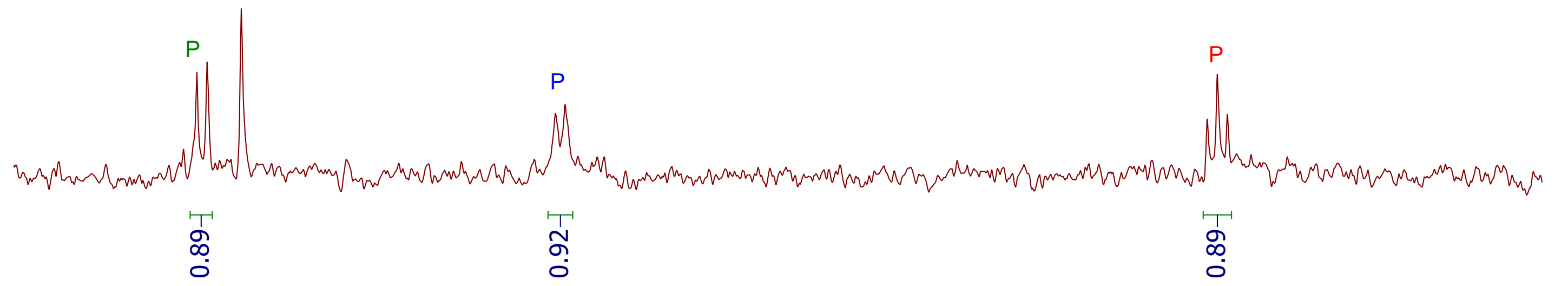

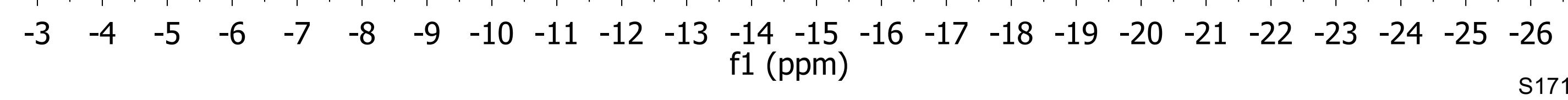




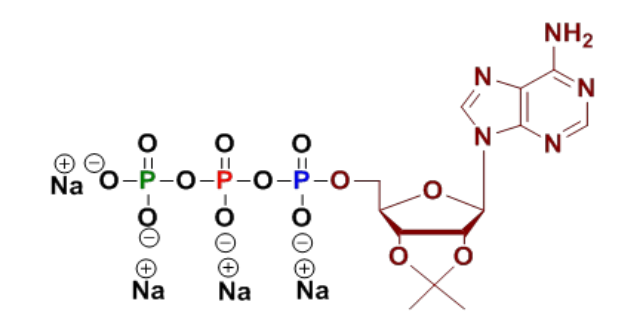

Crude product after precipitation
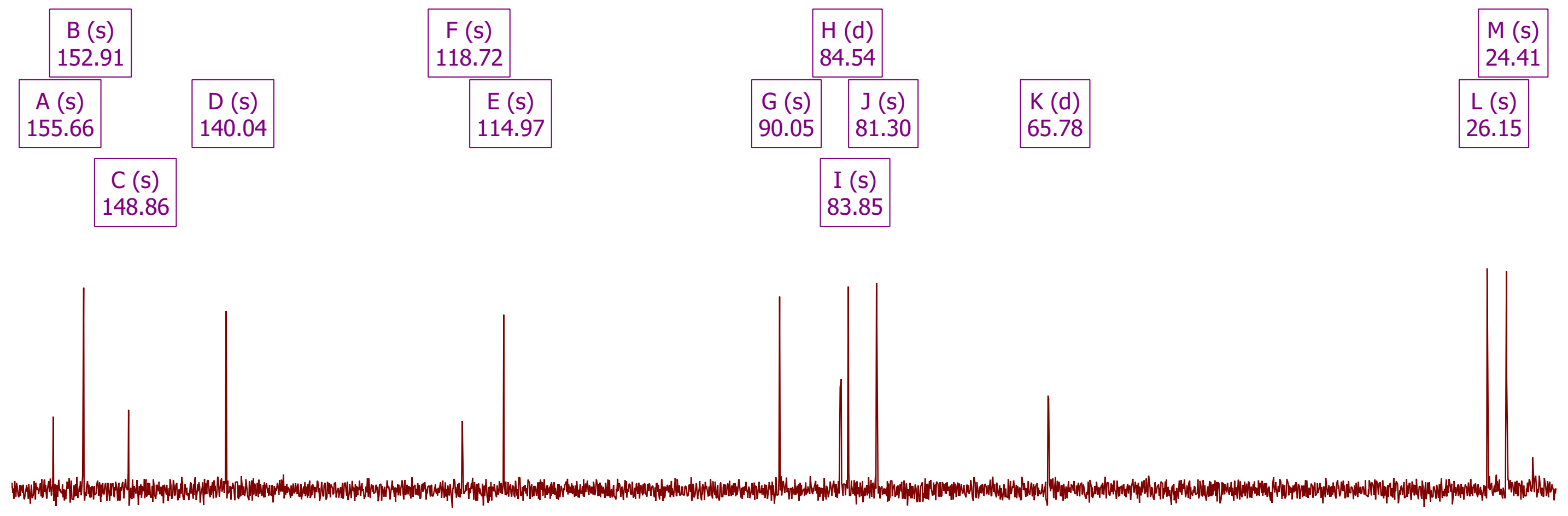


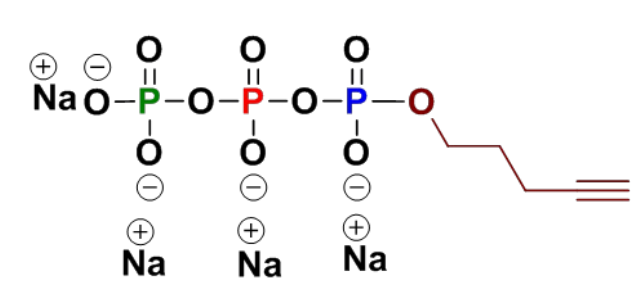

Crude product after precipitation

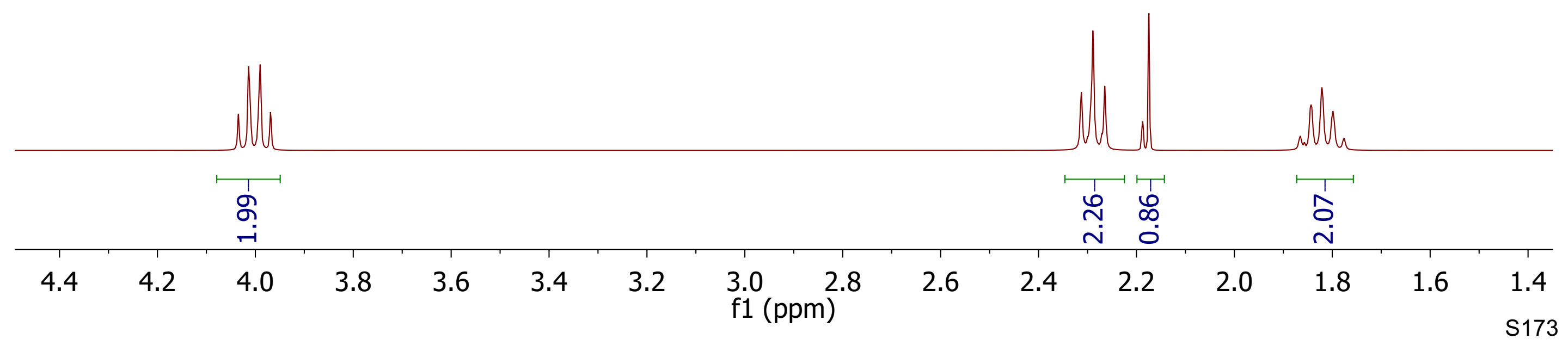




\section{${ }^{31} \mathrm{P}\left\{{ }^{1} \mathrm{H}\right\} \mathrm{NMR}$}

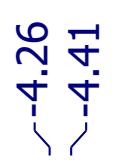

厄) 다

के

ํㅠㅅㅣ 잉

윽 으무

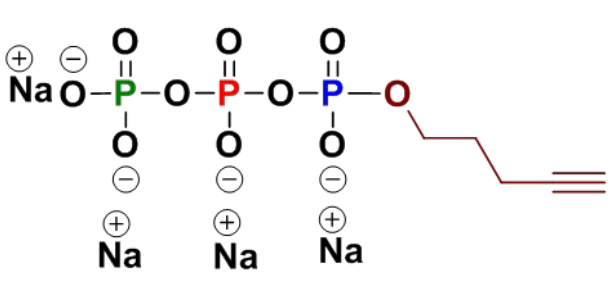

Crude product after precipitation
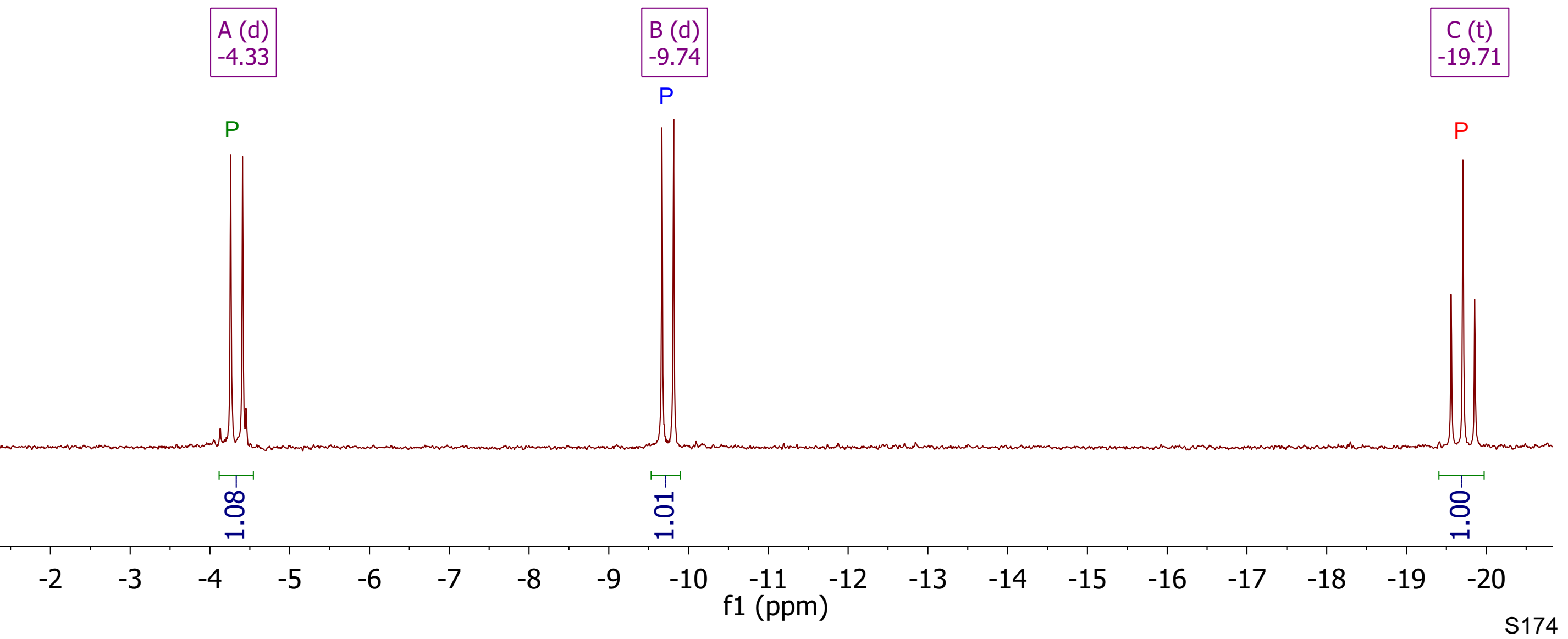


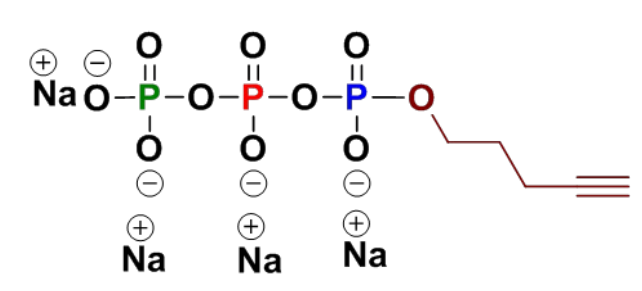

Crude product after precipitation

\begin{tabular}{|c|}
\hline$A(d)$ \\
-4.35 \\
$J(18.00)$ \\
\hline
\end{tabular}
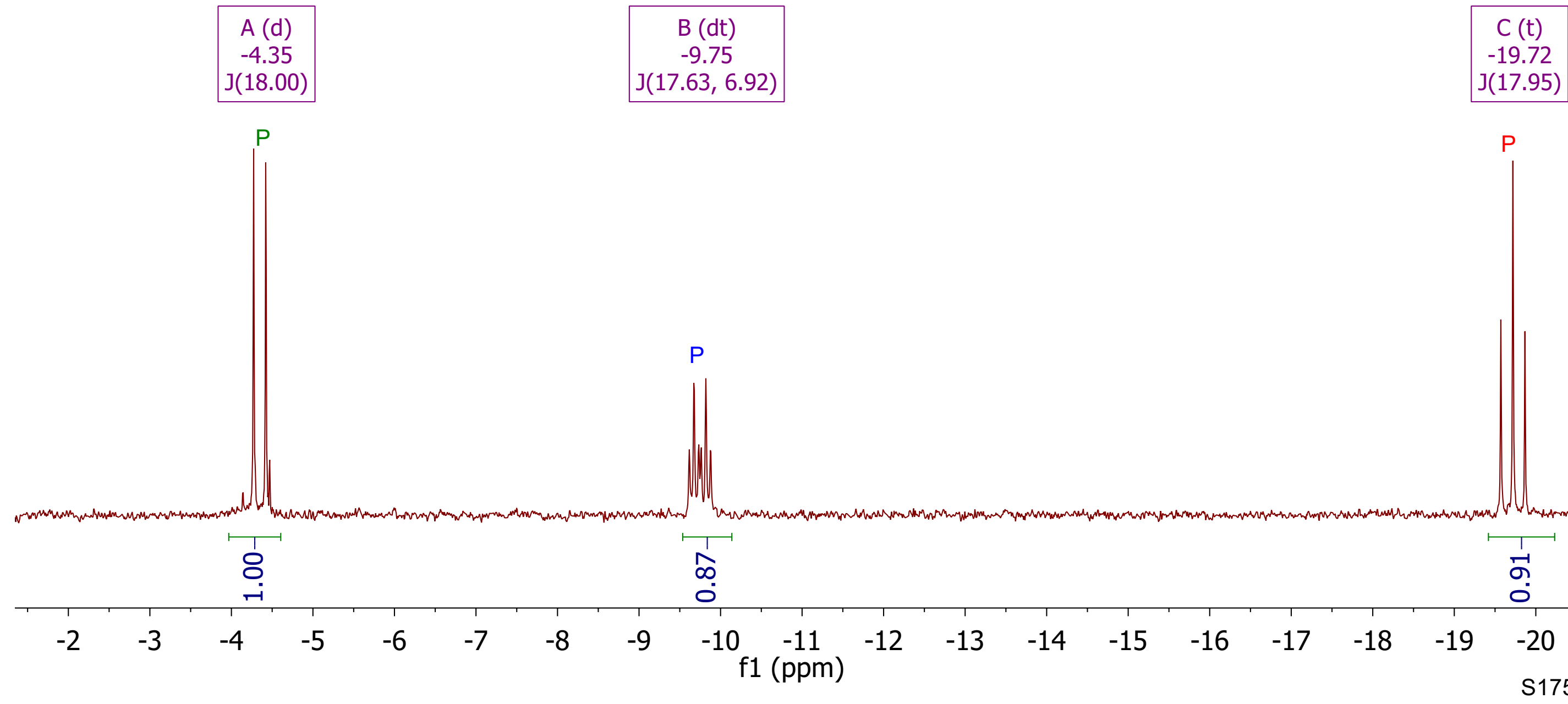


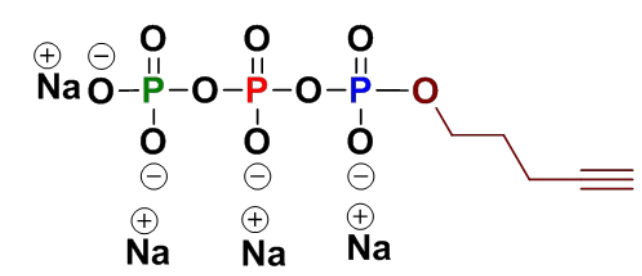

\section{Crude product after precipitation}

\begin{tabular}{|l|l|l|}
\hline$A(s)$ & $B(s)$ & $C(d)$ \\
85.21 & 69.40 & 65.30 \\
\hline
\end{tabular}

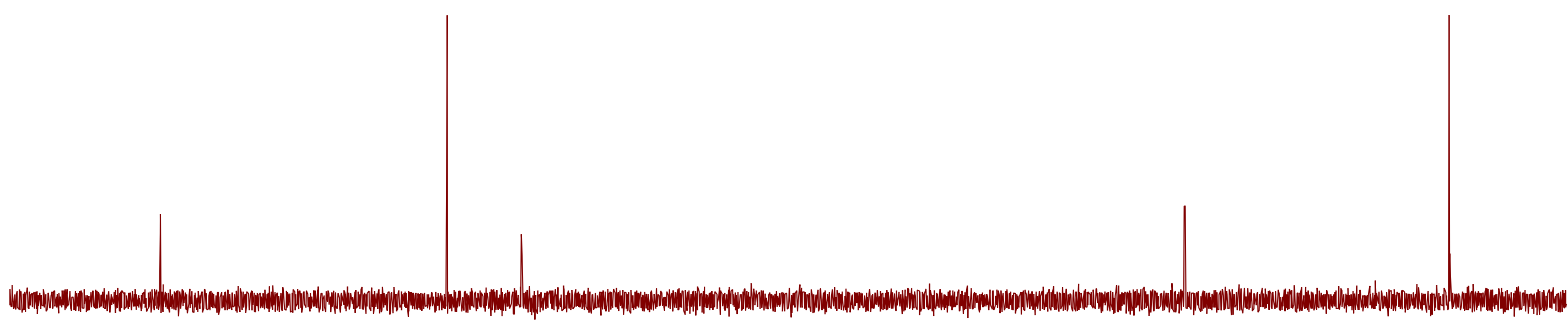

$\begin{array}{lll}65 & 60 & 55 \\ \mathrm{f} 1(\mathrm{ppm}) & 50\end{array}$




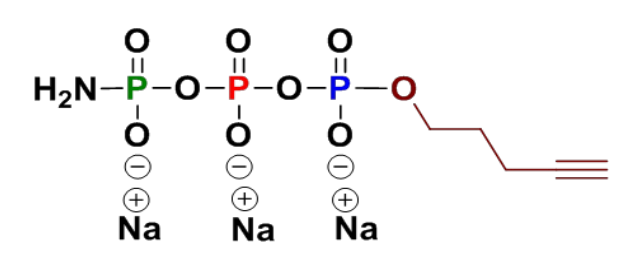

Crude product after precipitation
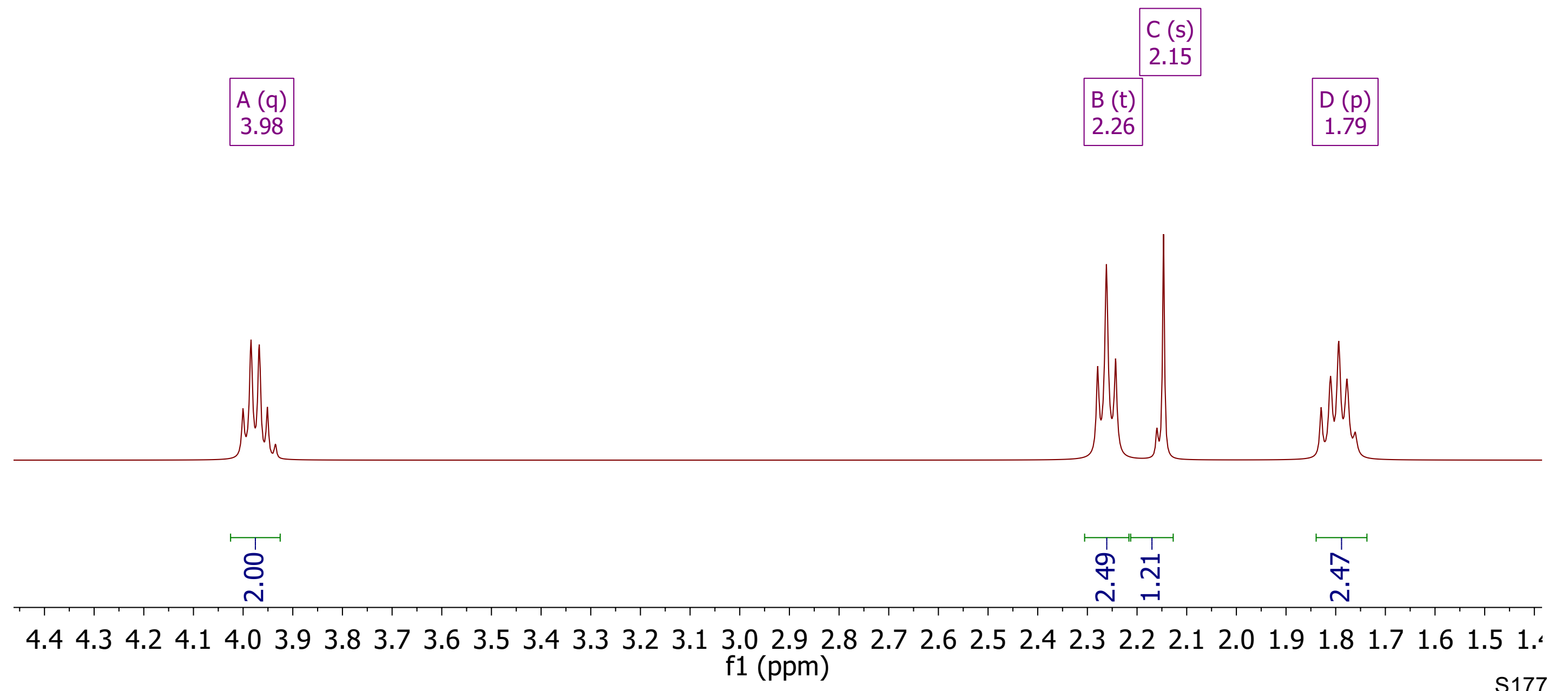
${ }^{31} \mathrm{P}\left\{{ }^{1} \mathrm{H}\right\} \mathrm{NMR}$

彳亍

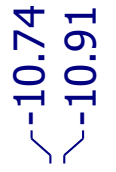

ช ชู

ฟิ กิ

i

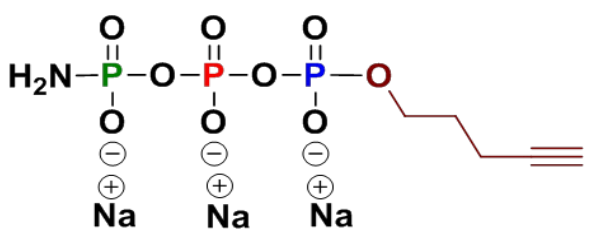

Crude product after precipitation
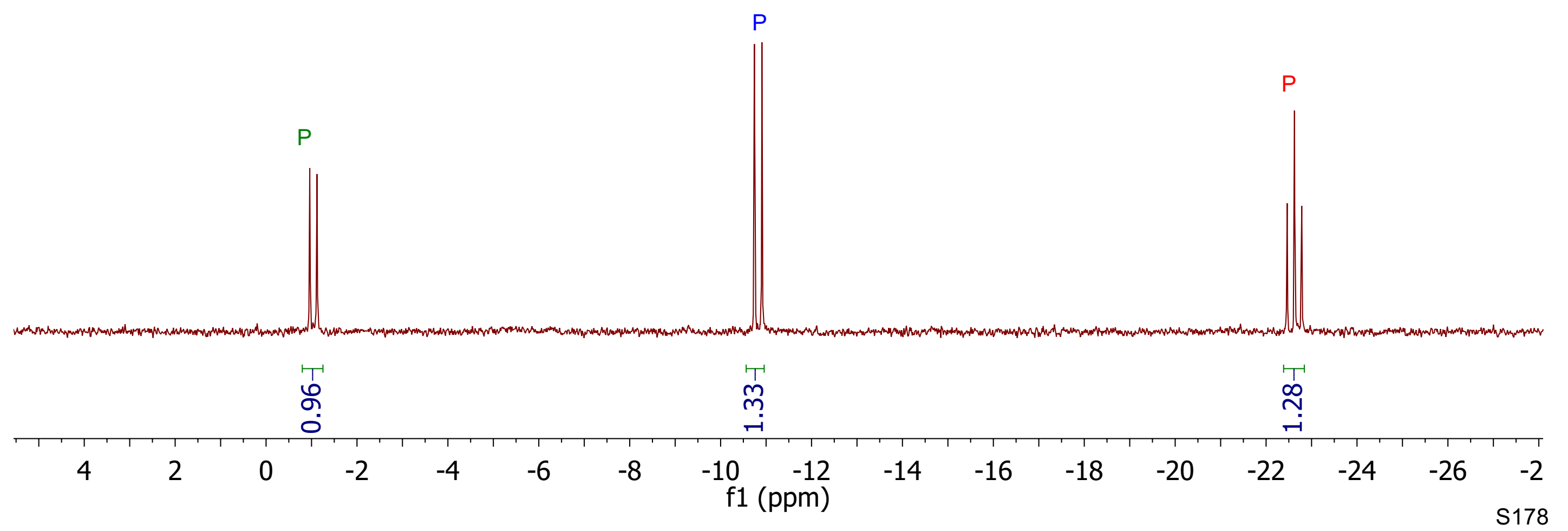


\section{Crude product after precipitation}

$$
\begin{array}{|l|}
\hline \text { A (d) } \\
-0.70 \\
\hline
\end{array}
$$
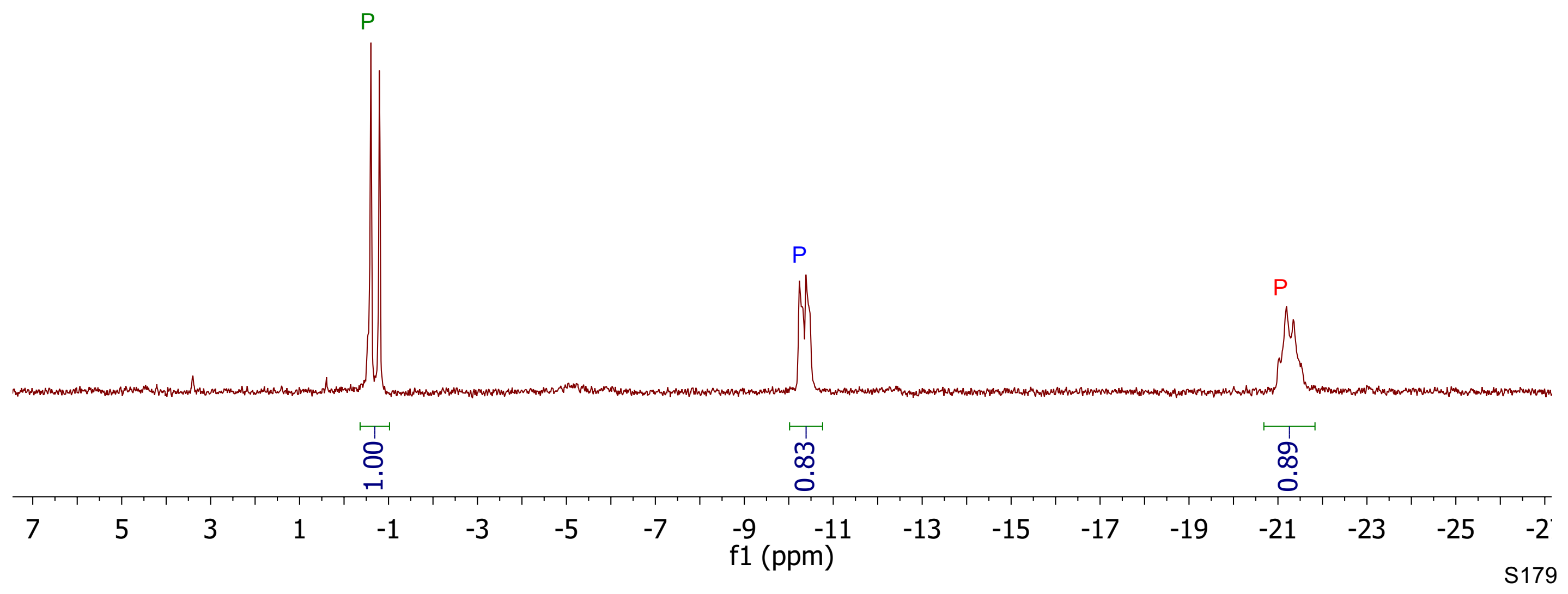
${ }^{31} \mathrm{P}\left\{{ }^{1} \mathrm{H}\right\}-\mathrm{NMR}$

(1)
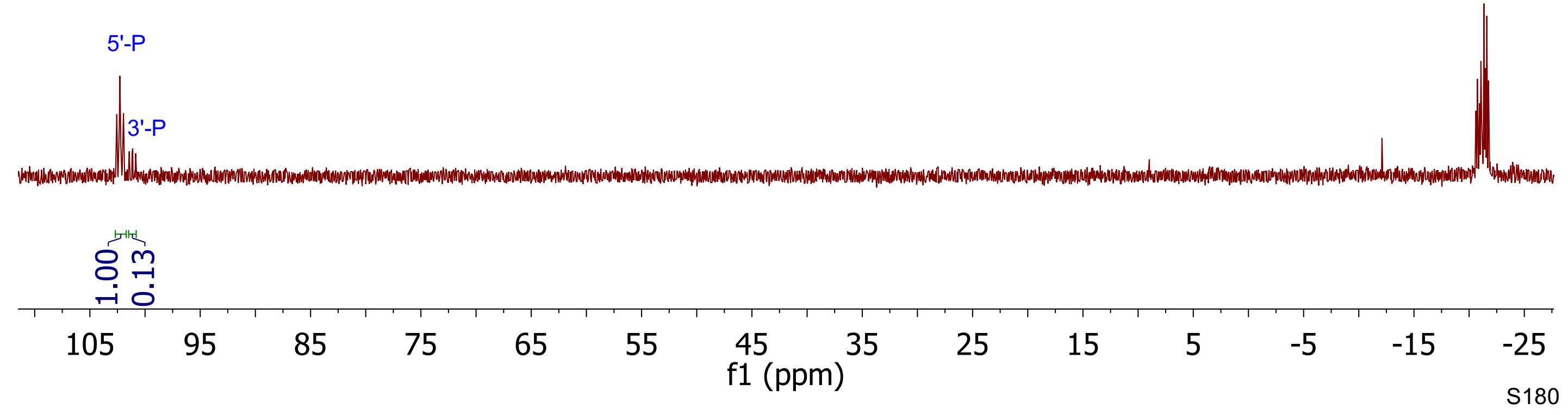


\section{${ }^{1} \mathrm{H}$ NMR}

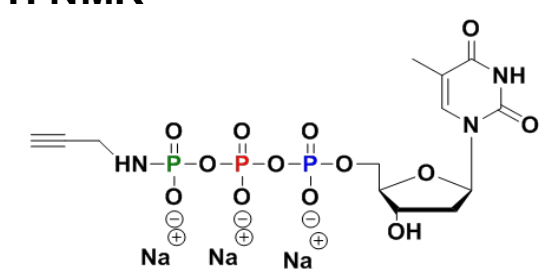

Crude product after precipitation

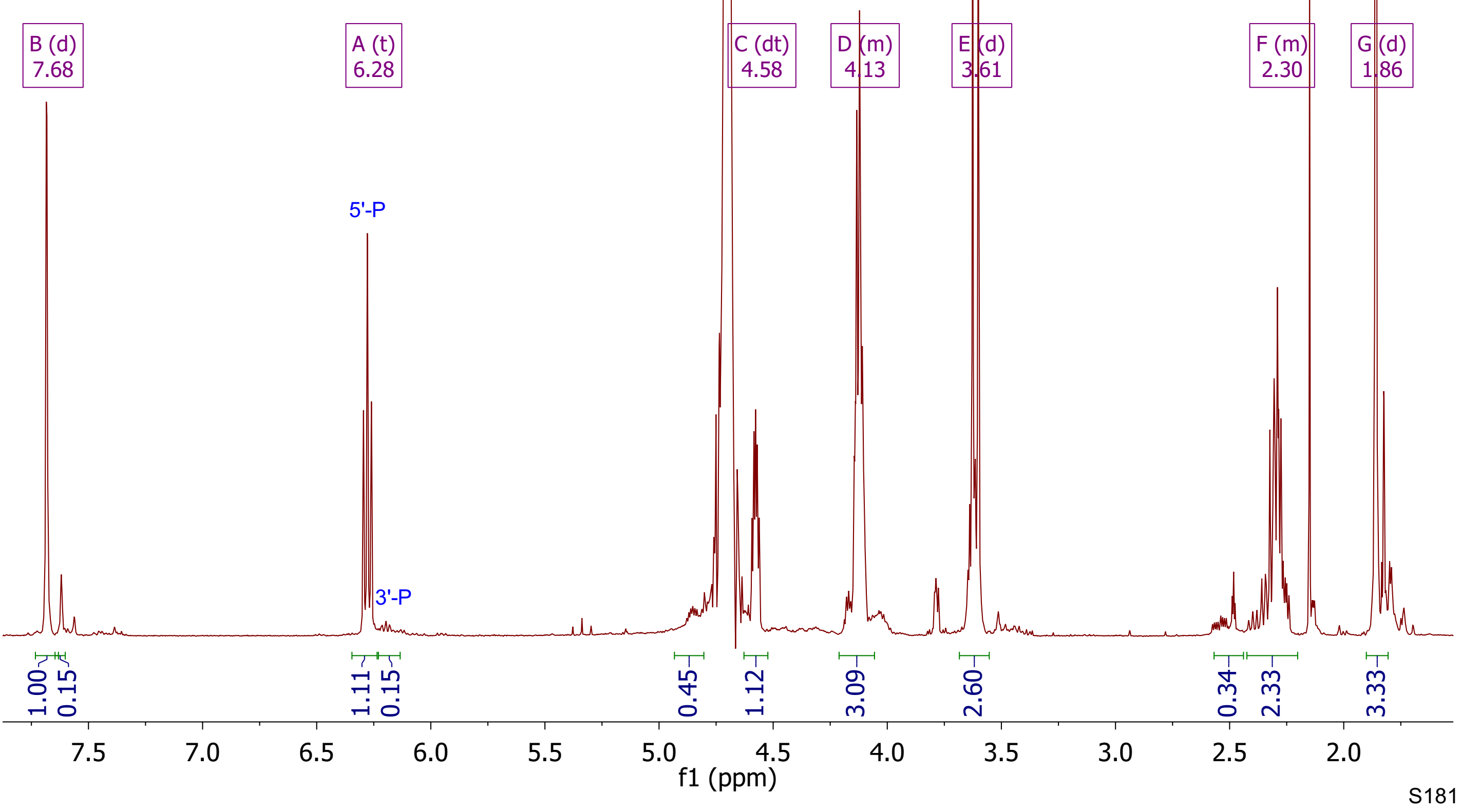


$=\begin{array}{llll}0 & 0 & 0\end{array}$

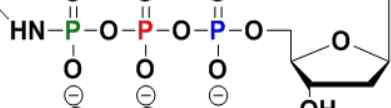

$\stackrel{\ominus}{\ominus} \stackrel{\ominus}{\oplus}$

Crude product after precipitation

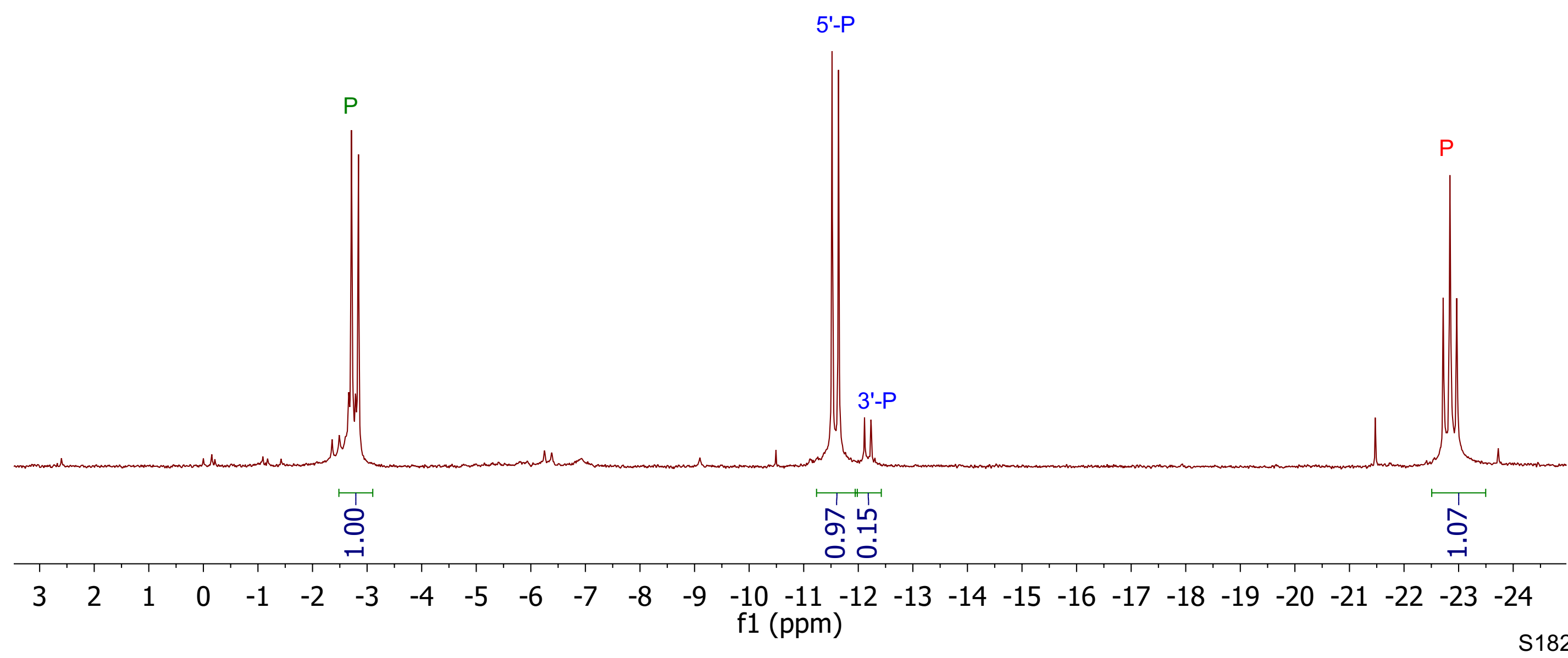


${ }^{31} \mathrm{P}$ NMR

மூำ

กับกั่

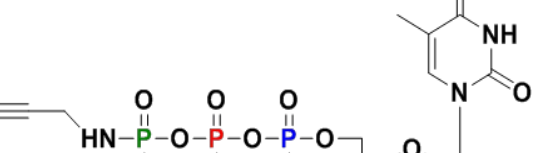

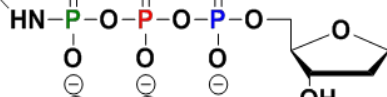

$\mathrm{Na}^{\oplus} \mathrm{Na}$

Crude product after precipitation

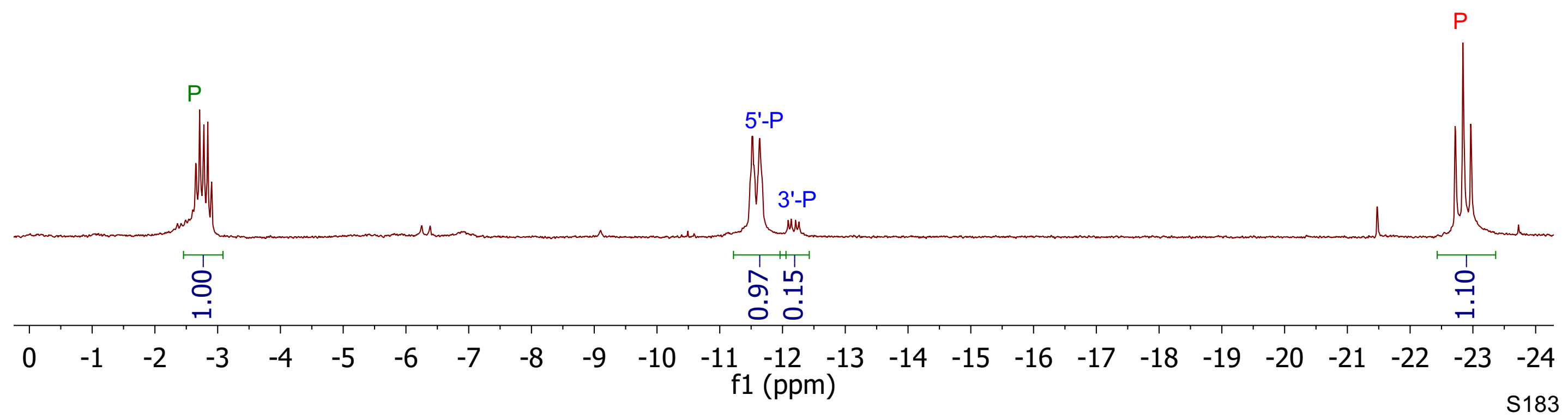




\section{${ }^{1} \mathrm{H}-\mathrm{NMR}$}

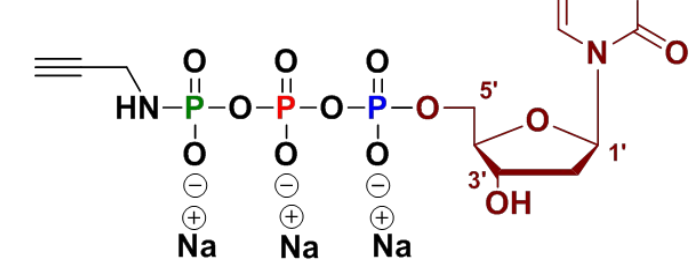

Product after purifucation by MPLC

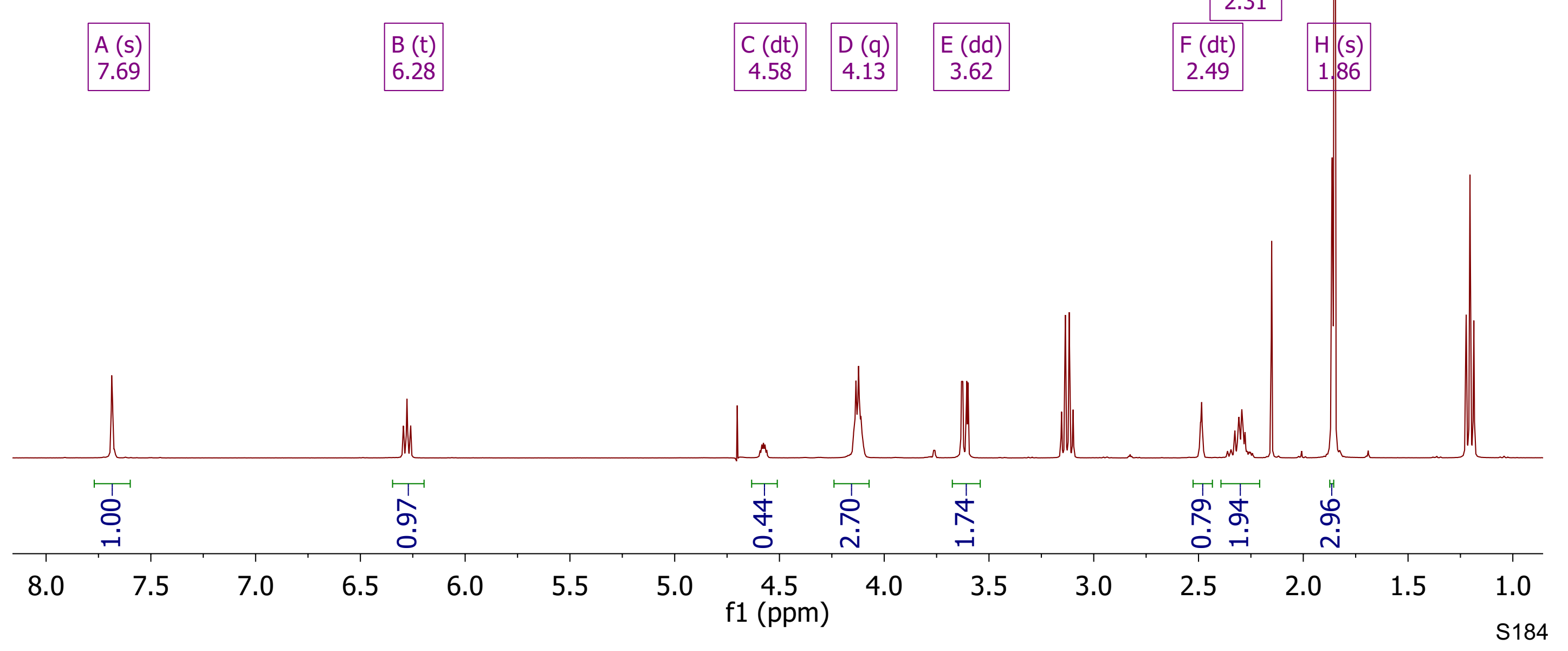




\section{P_- ${ }^{1}$ NMR}

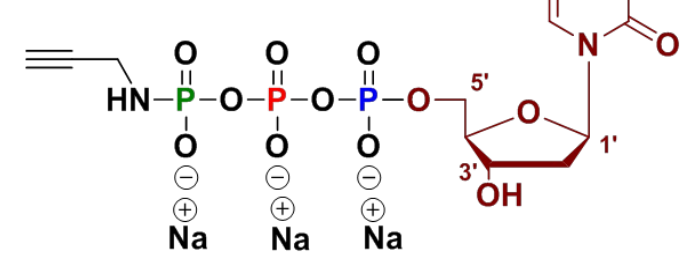

Product after purifucation by MPLC

$$
\begin{array}{l|}
\hline \text { B (dt) } \\
-2.75
\end{array}
$$$$
\text { A (dt) }
$$$$
-11.55
$$

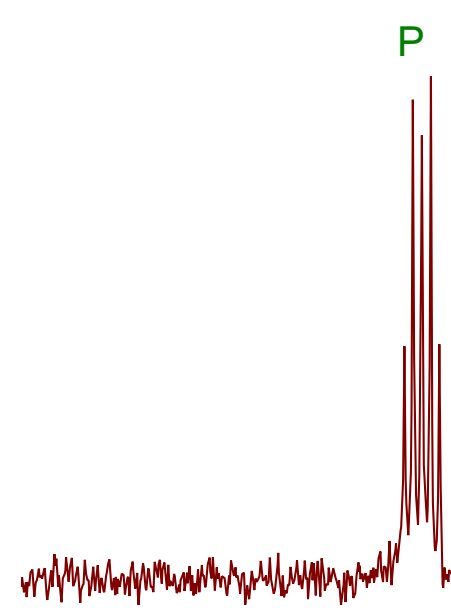

P

$5 '-P$

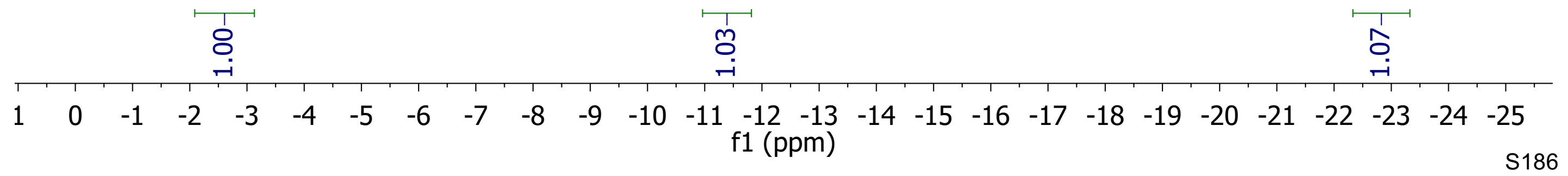

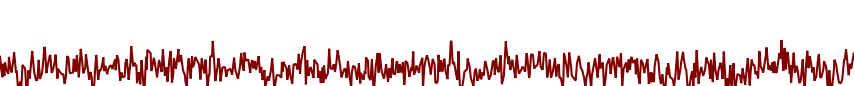

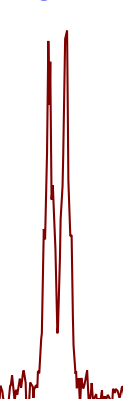

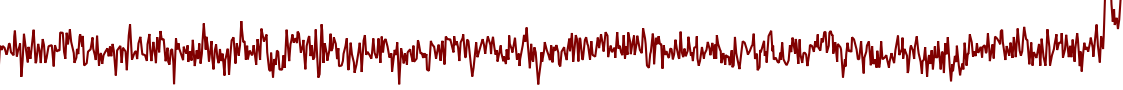

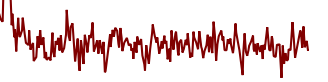




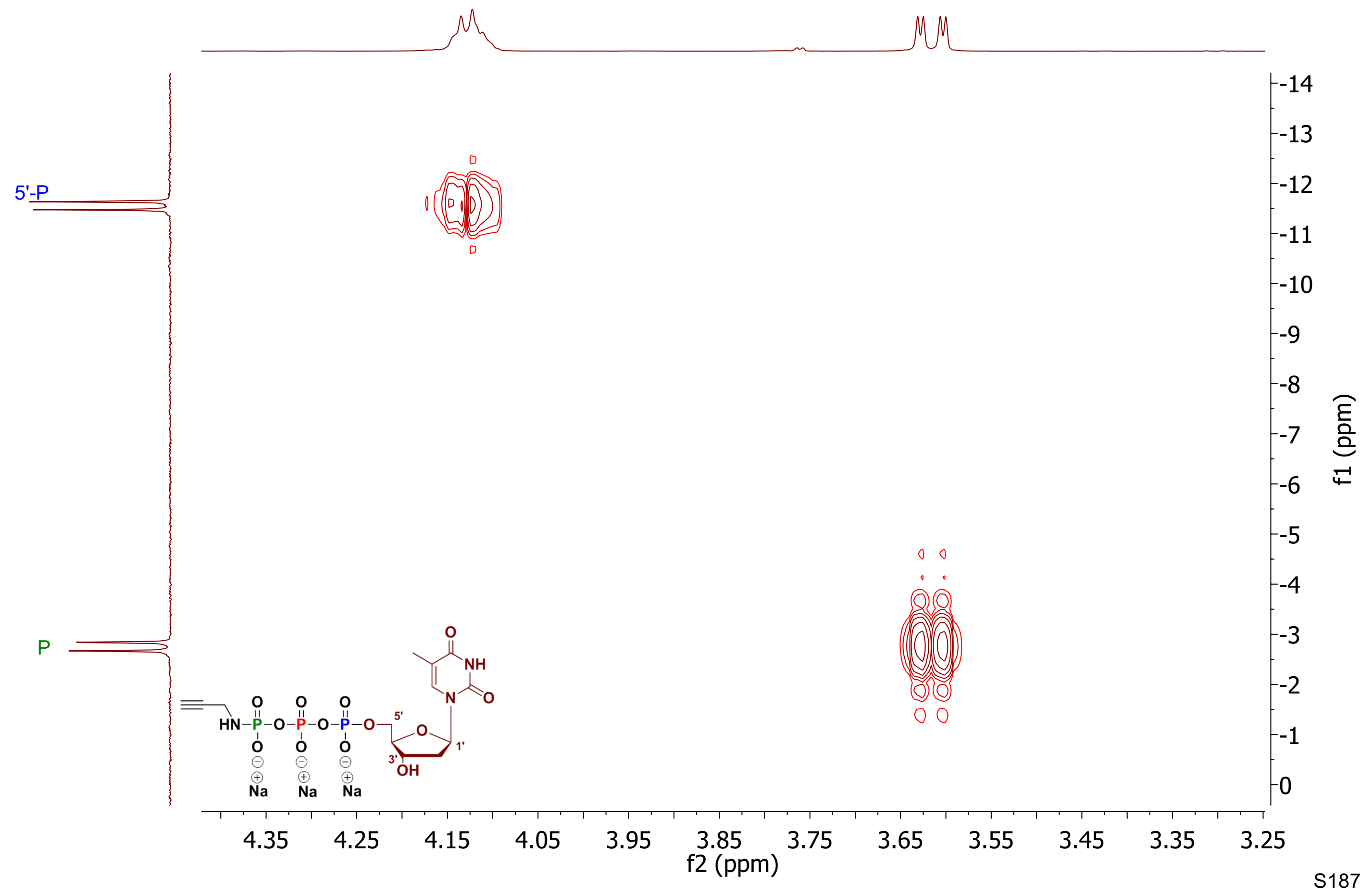




\section{${ }^{13} \mathrm{C}$ NMR}

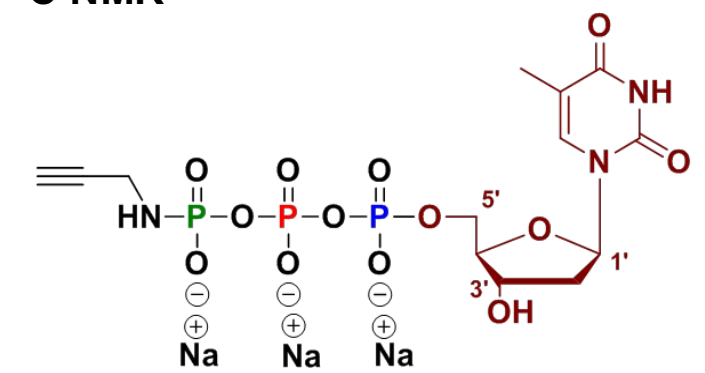

Product after purifucation by MPLC

\begin{tabular}{|c|c|c|c|}
\hline $\begin{array}{c}B(s) \\
166.59\end{array}$ & $\begin{array}{c}\text { C (s) } \\
151.75\end{array}$ & $\begin{array}{c}\text { D (s) } \\
137.38\end{array}$ & \begin{tabular}{|c|}
$E(s)$ \\
111.81
\end{tabular} \\
\hline
\end{tabular}

\begin{tabular}{|c|c|}
\hline $\begin{array}{l}G(d) \\
82.83\end{array}$ & $\begin{array}{c}\mathrm{J}(\mathrm{s}) \\
70.78\end{array}$ \\
\hline $\begin{array}{l}F(d) \\
85.37\end{array}$ & $\begin{array}{c}\text { I (s) } \\
71.35\end{array}$ \\
\hline $\begin{array}{l}\mathrm{H}(\mathrm{s}) \\
84.86\end{array}$ & $\begin{array}{l}K(d) \\
65.35\end{array}$ \\
\hline
\end{tabular}

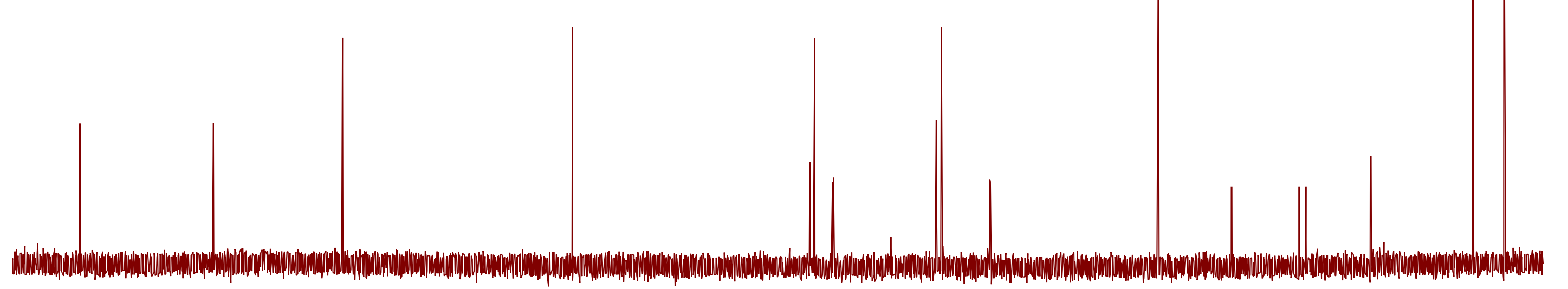

$\begin{array}{llllllllllllllllll}170 & 160 & 150 & 140 & 130 & 120 & 110 & 100 & 90 & 80 & 70 & 60 & 50 & 40 & 30 & 20 & 10 & \text { f1 (ppm) }\end{array}$




\section{${ }^{31} \mathrm{P}\left\{{ }^{1} \mathrm{H}\right\}-\mathrm{NMR}$}

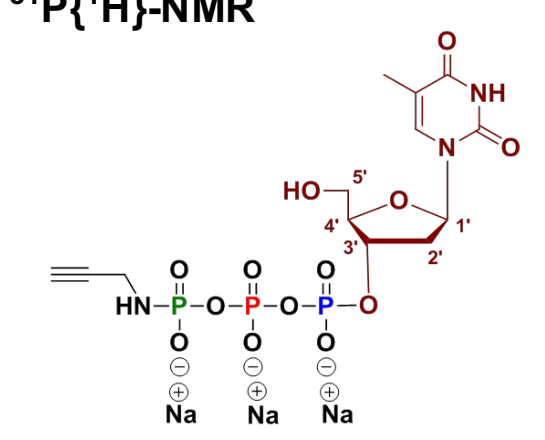

Product after purifucation by MPLC
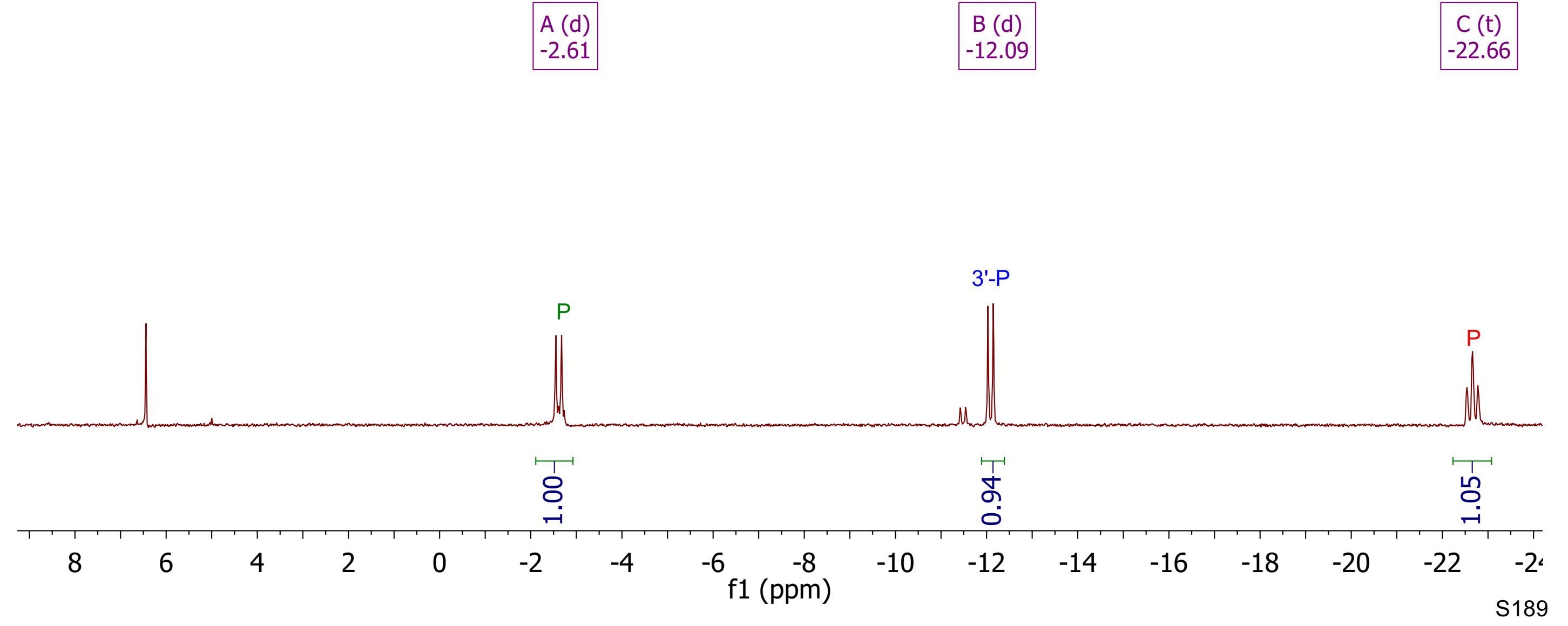
Product after purifucation by MPLC

$$
\begin{array}{|l|}
\hline B(d t) \\
-2.61
\end{array}
$$

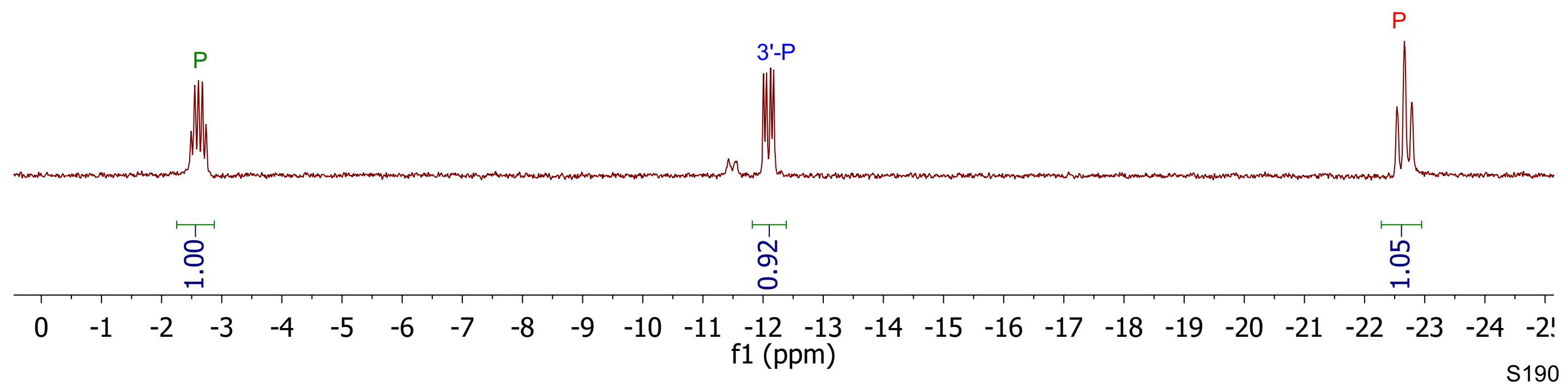




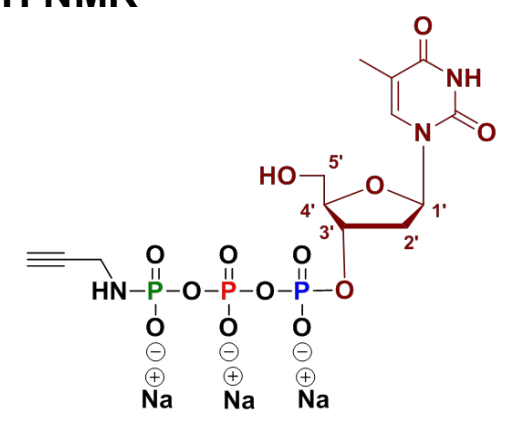

Product after purifucation by MPLC

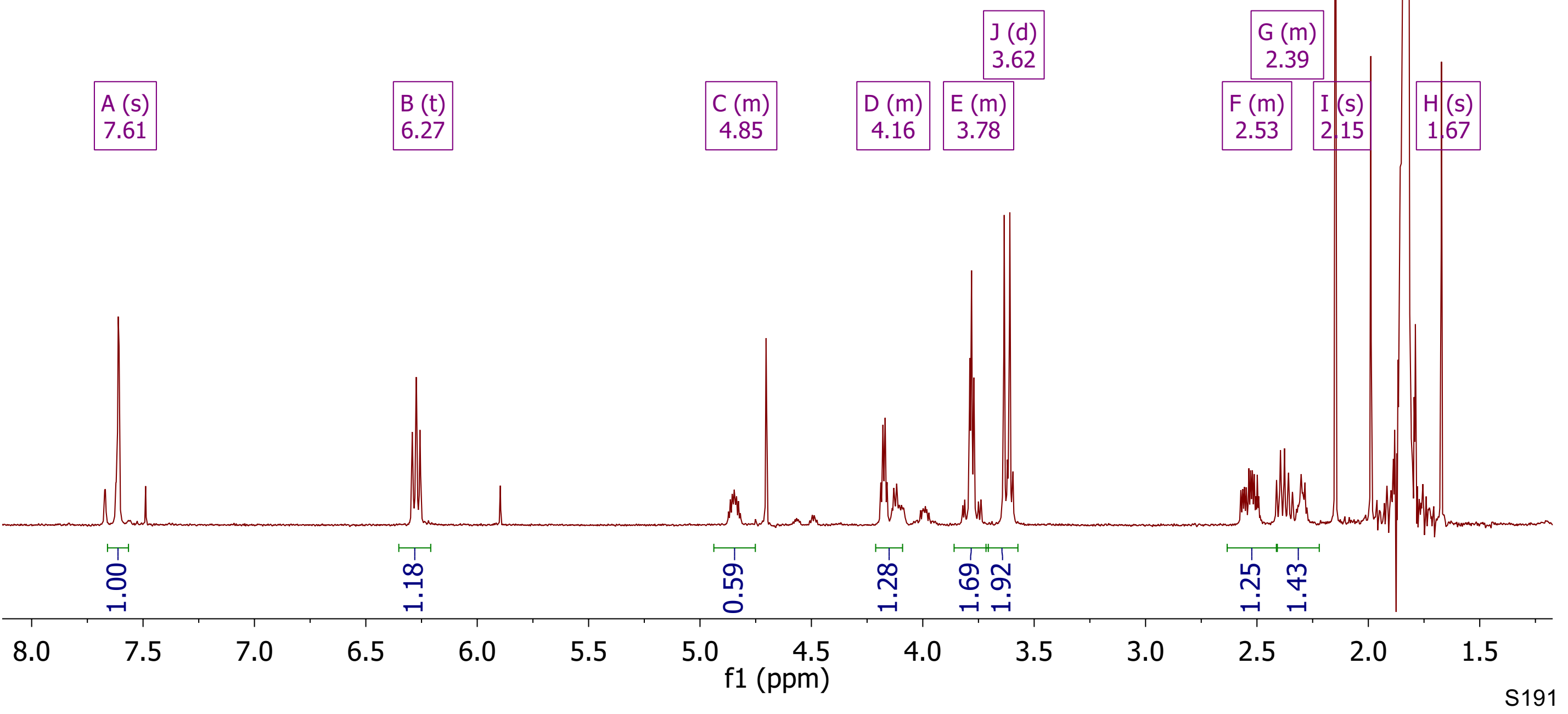




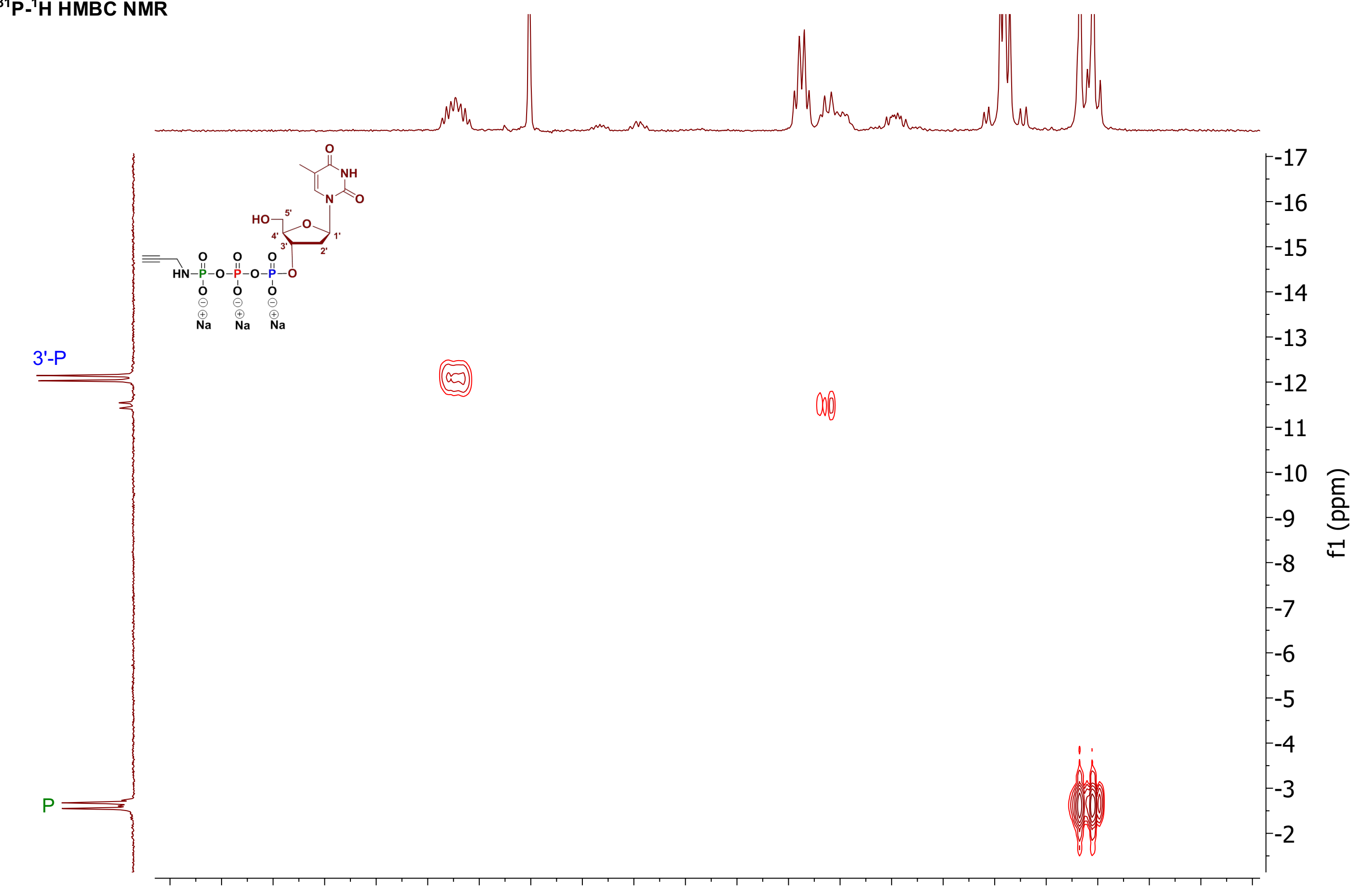

$\begin{array}{llllllllllllllllllllll}5.4 & 5.3 & 5.2 & 5.1 & 5.0 & 4.9 & 4.8 & 4.7 & 4.6 & 4.5 & \begin{array}{c}4.4 \\ \mathrm{f} 2\end{array}(\mathrm{ppm}) & 4.3 & 4.1 & 4.0 & 3.9 & 3.8 & 3.7 & 3.6 & 3.5 & 3.4 & 3.3\end{array}$ 
${ }^{1} \mathrm{H}-\mathrm{NMR}$

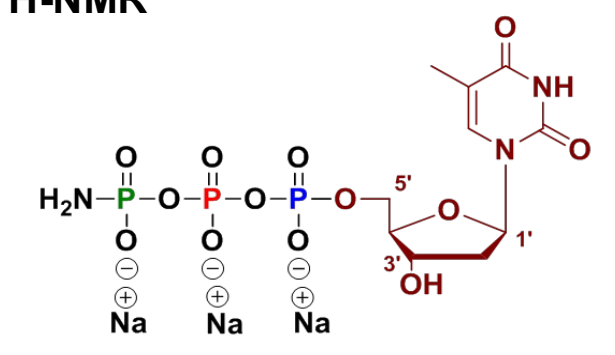

Product after purifucation by MPLC

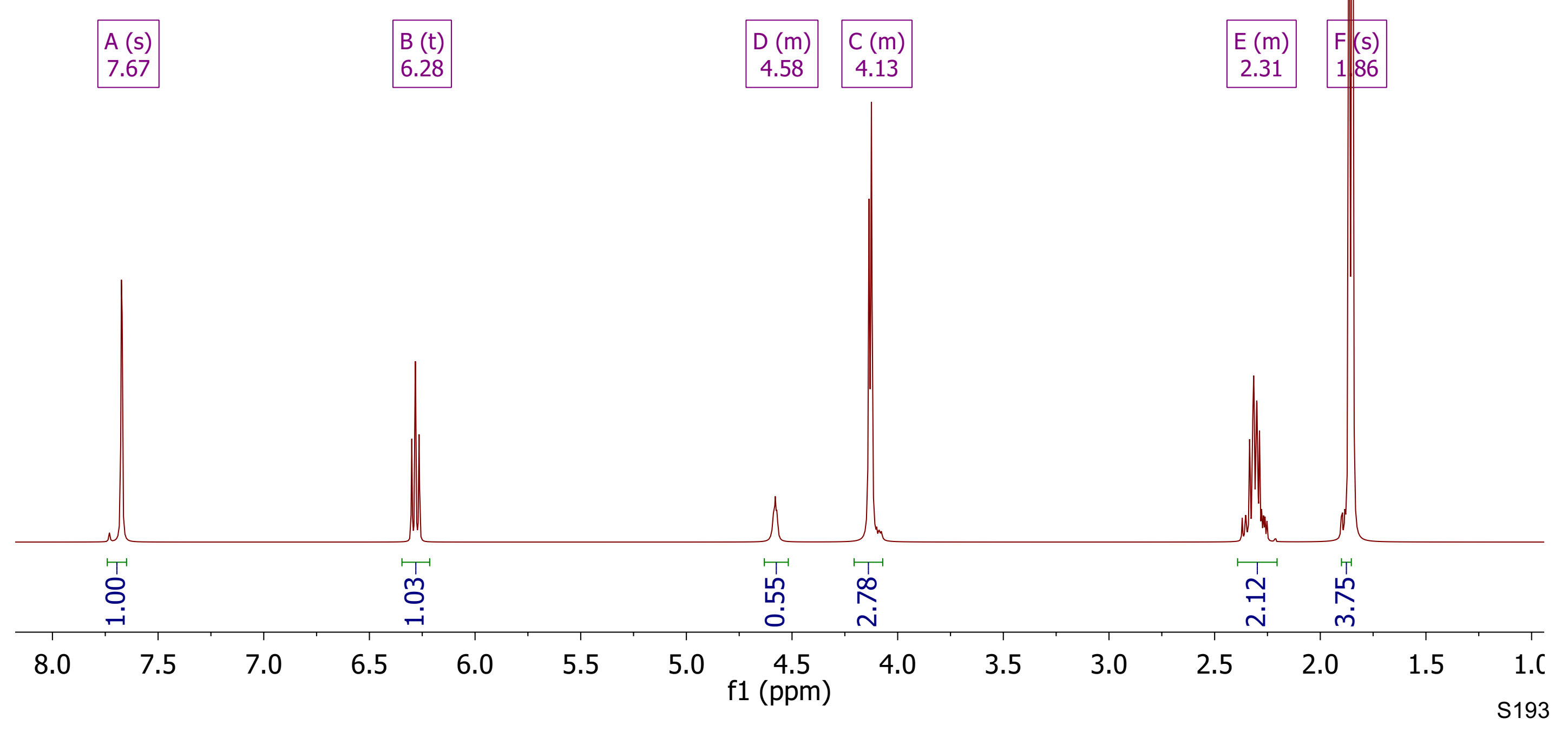




\section{${ }^{31} \mathrm{P}\left\{{ }^{1} \mathrm{H}\right\}-\mathrm{NMR}$}

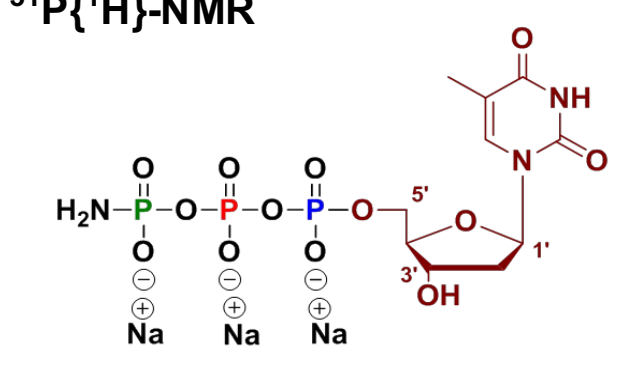

Product after purifucation by MPLC

$$
\begin{array}{|l|}
\hline \text { A (d) } \\
-0.97 \\
\hline
\end{array}
$$$$
\text { B (d) }
$$

$-22.52$
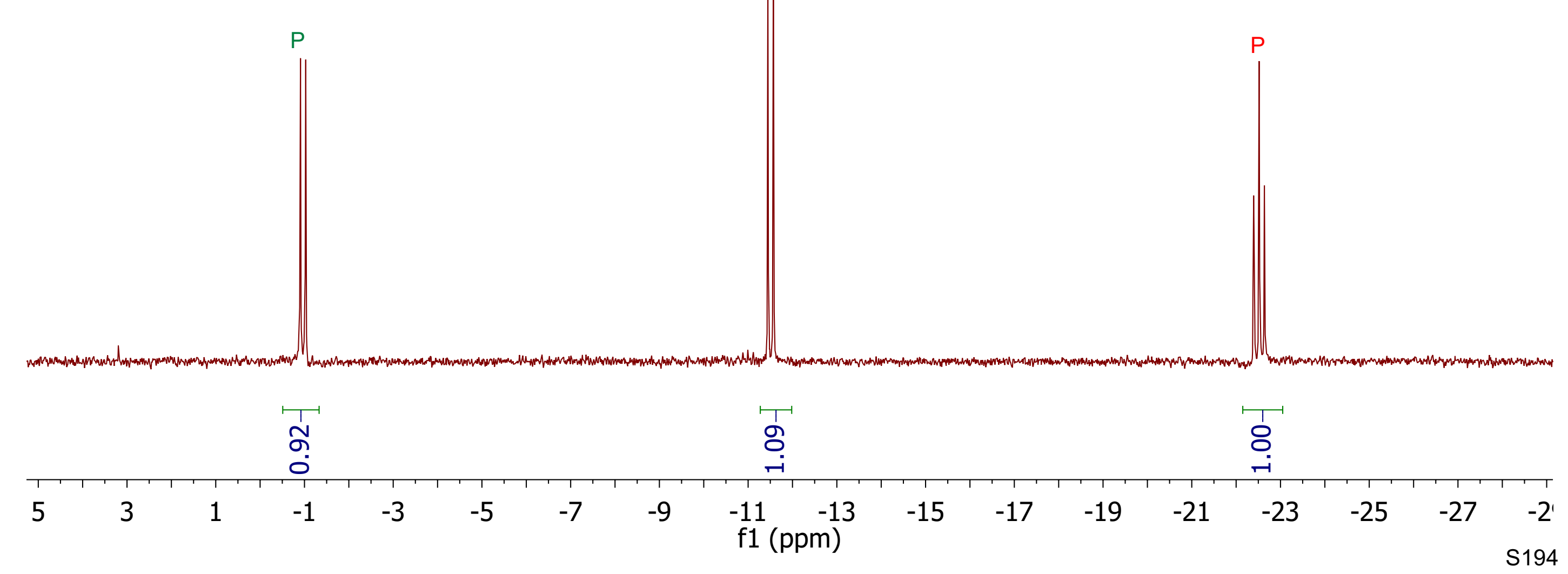


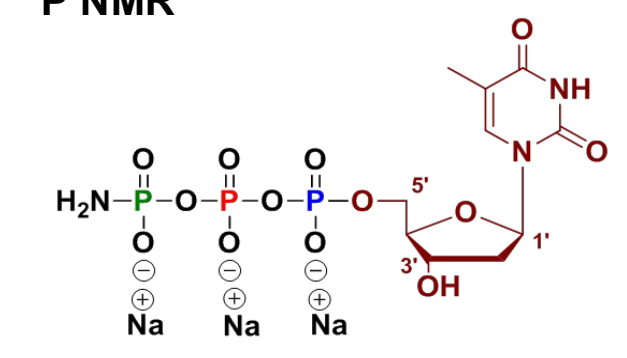

Product after purifucation by MPLC

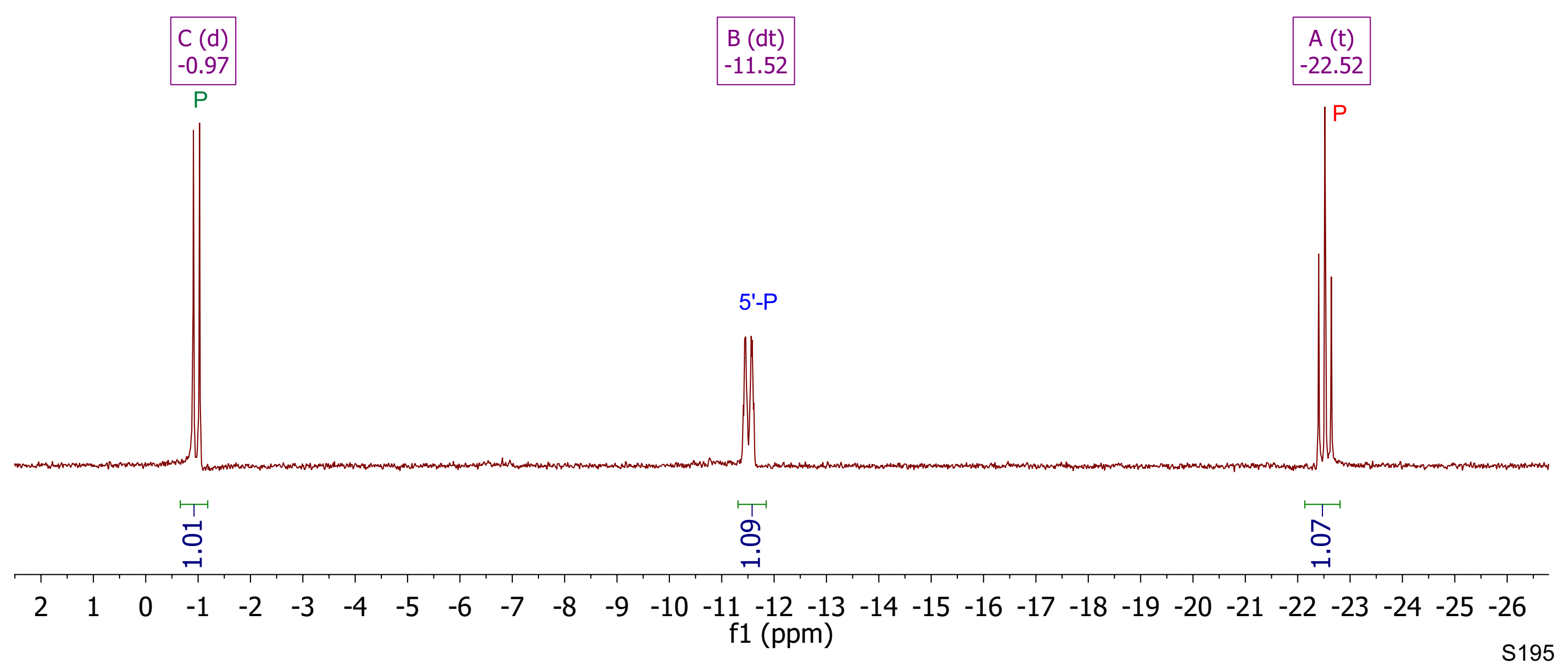




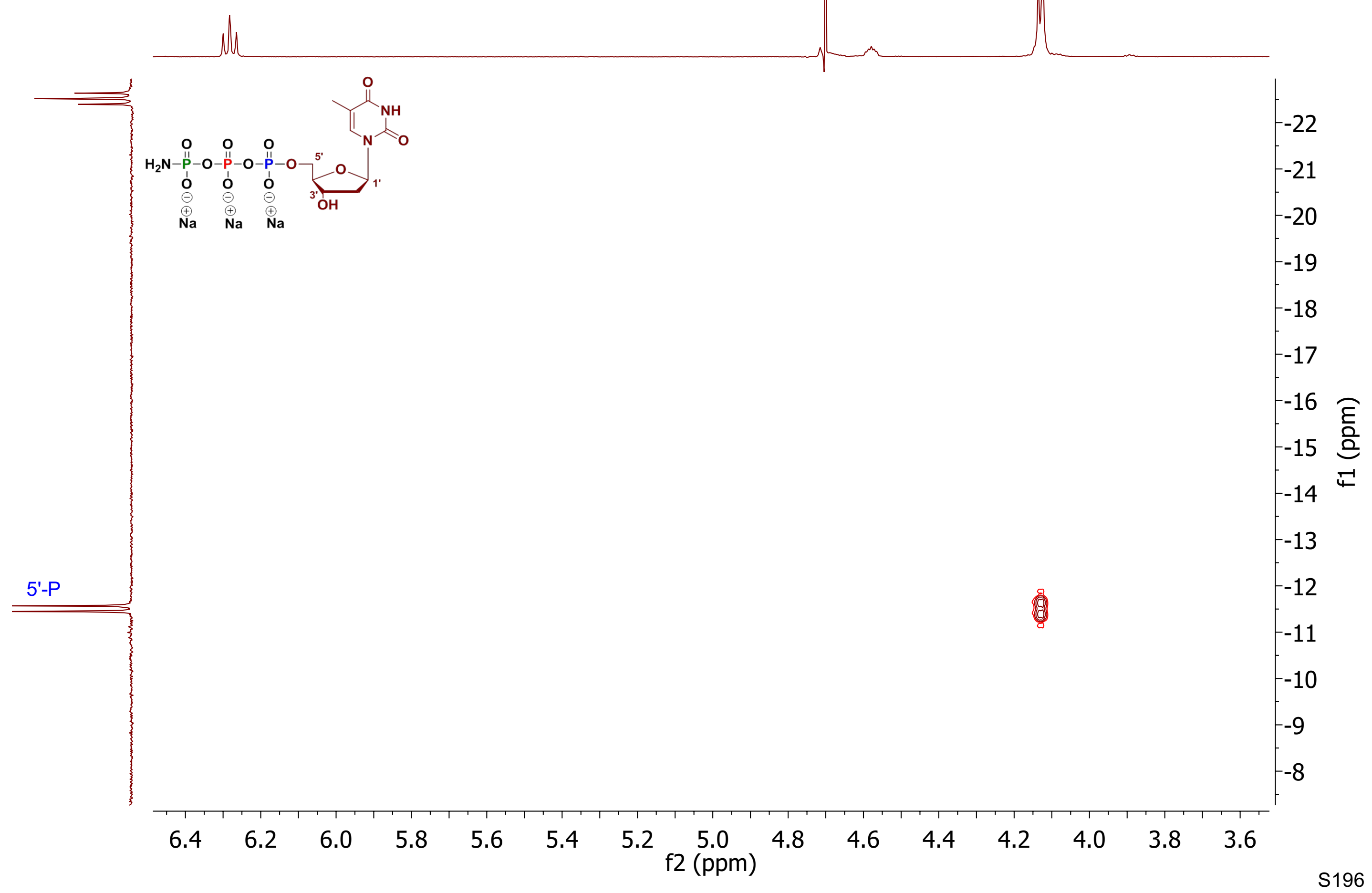




\section{${ }^{31} \mathrm{P}\left\{{ }^{1} \mathrm{H}\right\}-\mathrm{NMR}$}

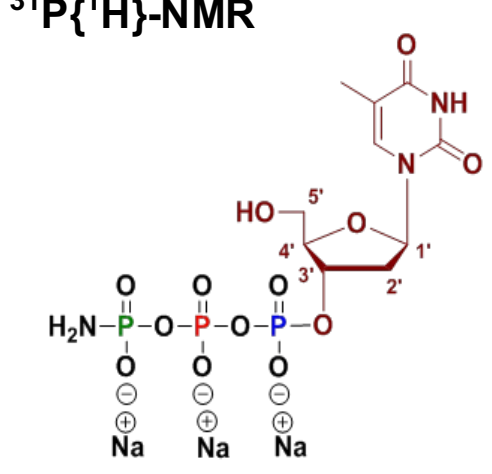

Product after purifucation by MPLC

$$
\begin{array}{|c|}
\hline B(d) \\
-0.87
\end{array}
$$

$-12.04$

$\mathrm{C}(\mathrm{t})$

$-22.44$

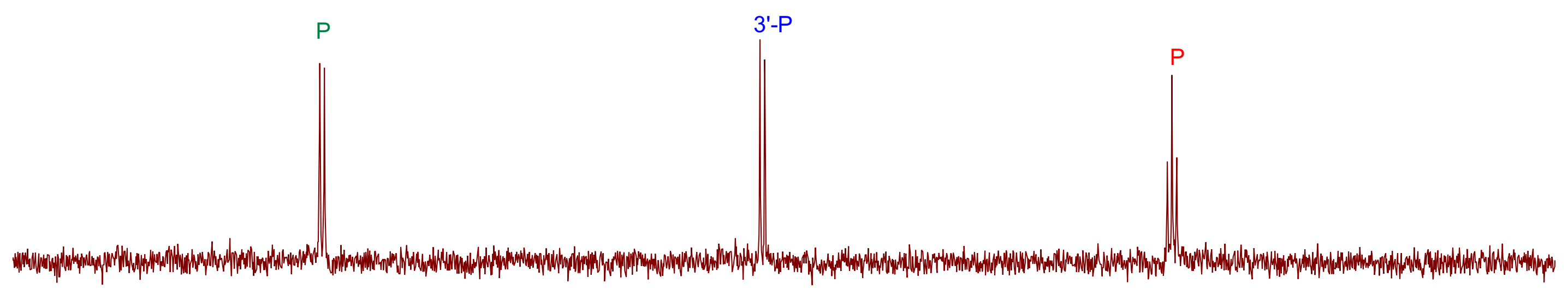

\begin{tabular}{|c|c|c|c|c|c|c|c|c|c|c|c|c|c|c|c|c|c|c|}
\hline & & & & & & & & & '] & & & & $\begin{array}{l}1 \\
\infty \\
0 \\
-1\end{array}$ & & & & & \\
\hline 6 & 4 & 2 & 0 & -2 & -4 & -6 & -8 & -10 & $\begin{array}{l}-12-14 \\
\mathrm{f1}(\mathrm{ppm})\end{array}$ & -16 & -18 & -20 & -22 & -24 & -26 & -28 & -30 & -3 \\
\hline
\end{tabular}




\section{${ }^{31} \mathrm{P}$ NMR}

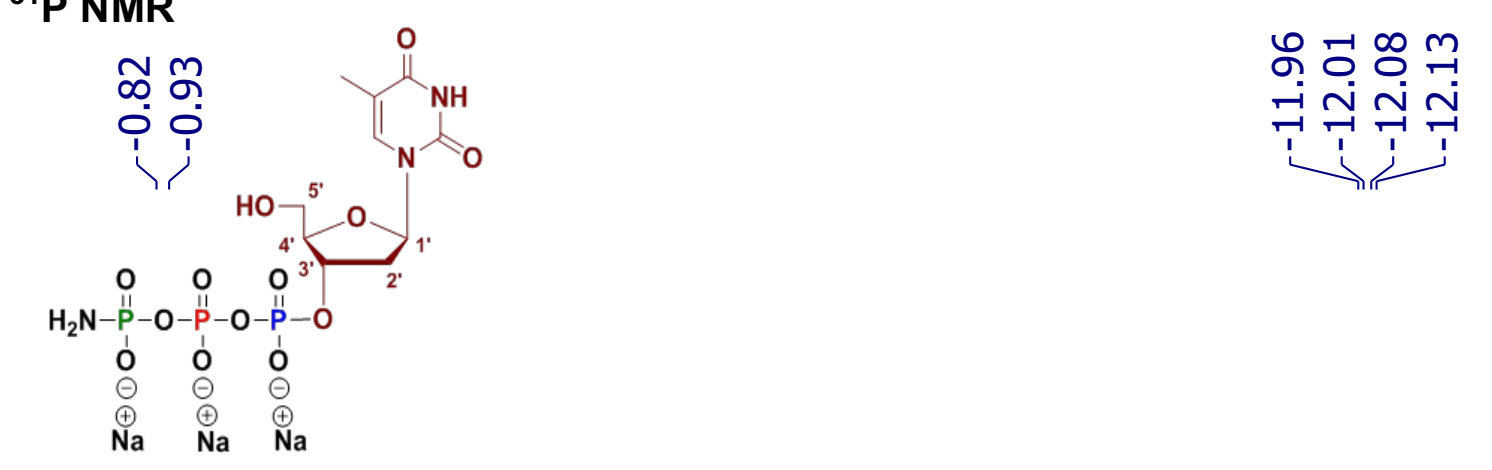

Product after purifucation by MPLC

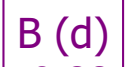

$-0.88$

A (dd)

$-12.05$

P

$\|$

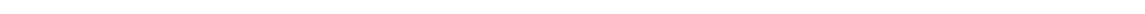

P

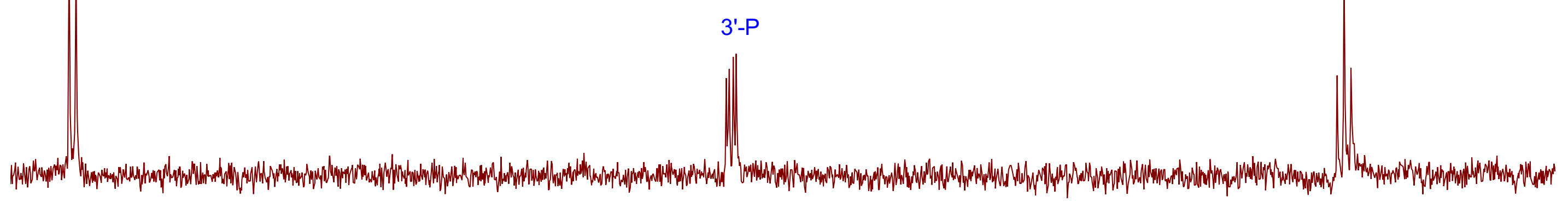

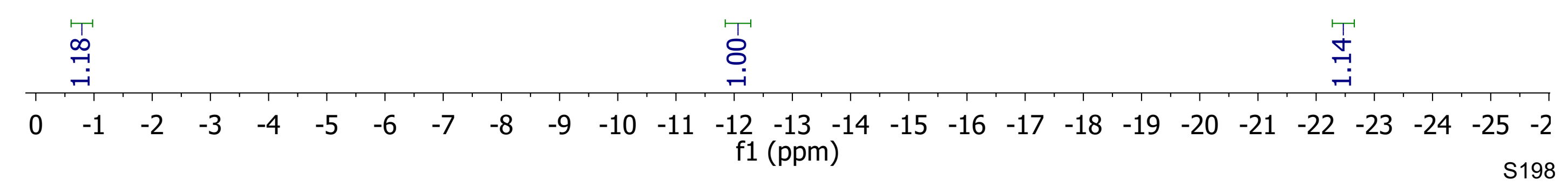


Product after purifucation by MPLC

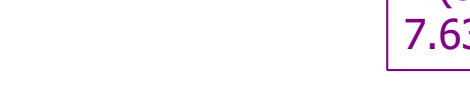

6.29

\begin{tabular}{llll} 
& 4.87 & 4.20 & 3.80 \\
\hline
\end{tabular}
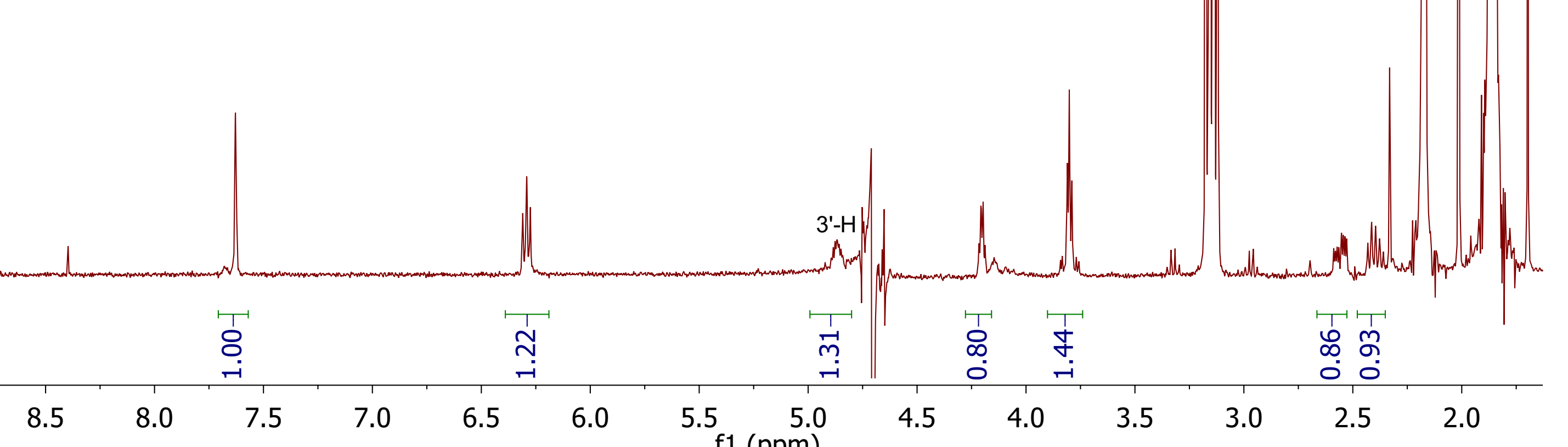

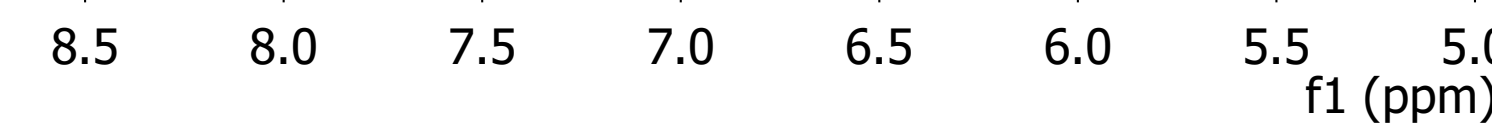


${ }^{31} \mathrm{P}\left\{{ }^{1} \mathrm{H}\right\}-\mathrm{NMR}$

\section{Oxidation}

C (t)

$-23.09$

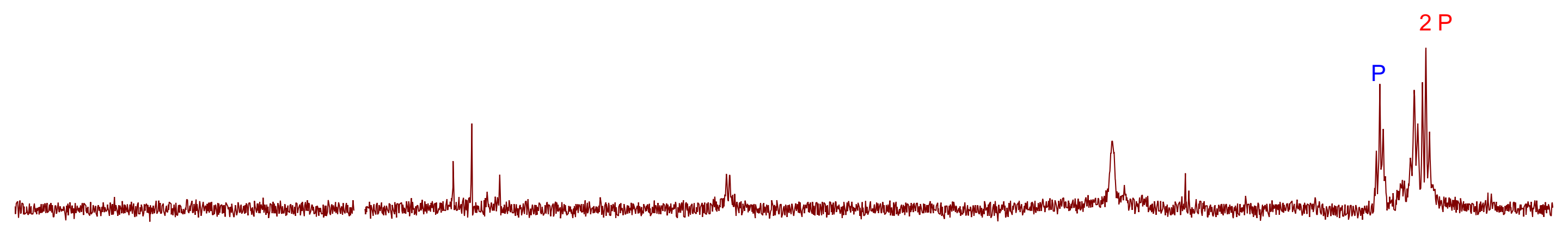

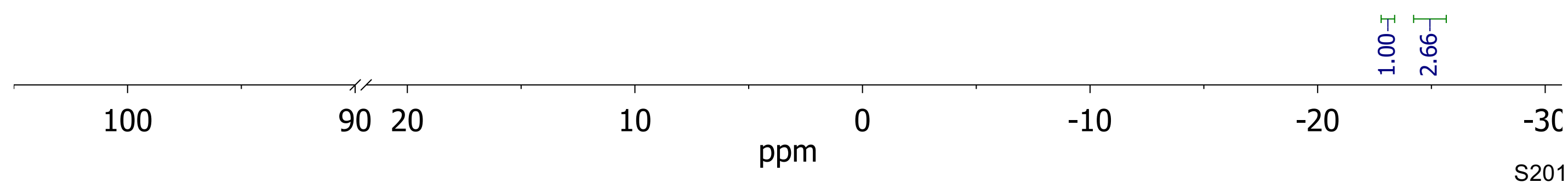




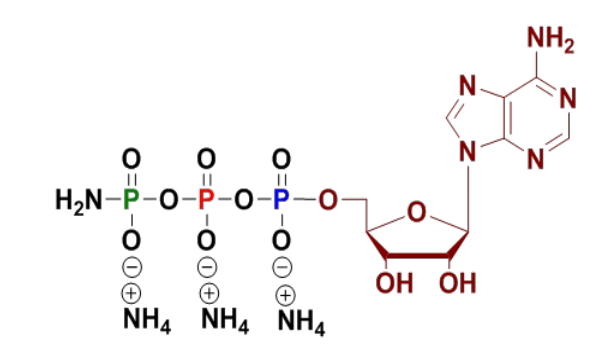

\section{Crude product after precipitation}

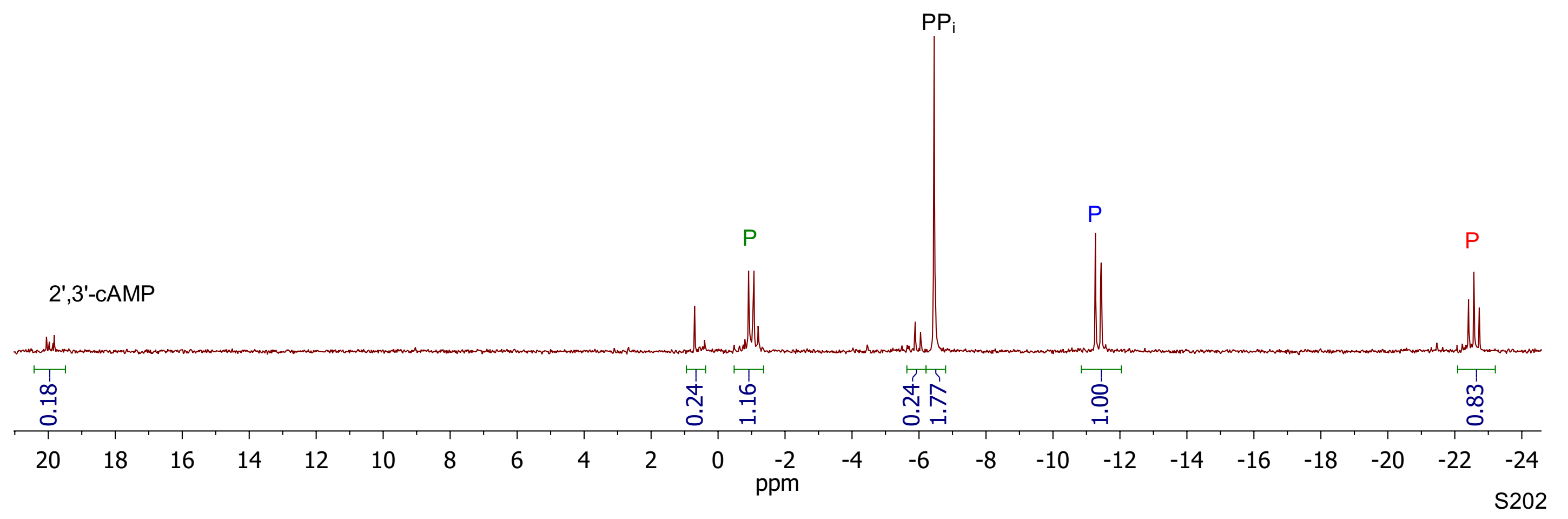




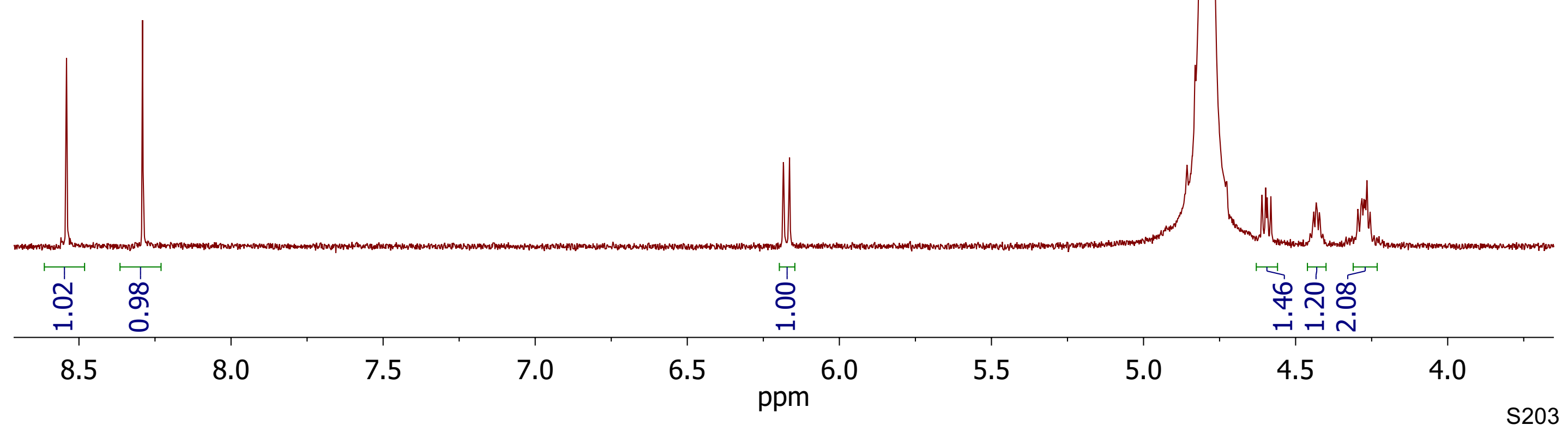




\section{${ }^{31} \mathrm{P}\left\{{ }^{1} \mathrm{H}\right\}-\mathrm{NMR}$}

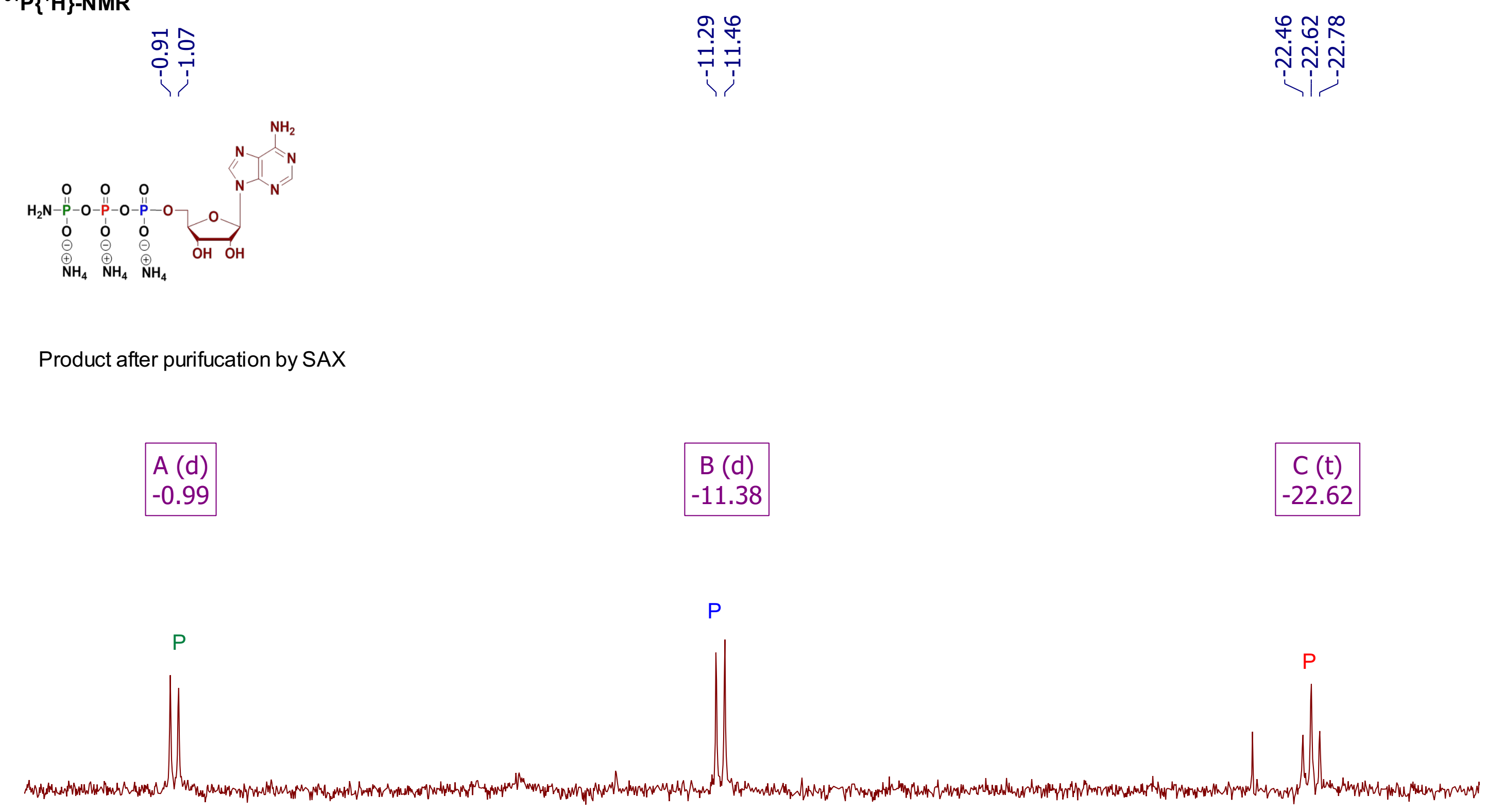

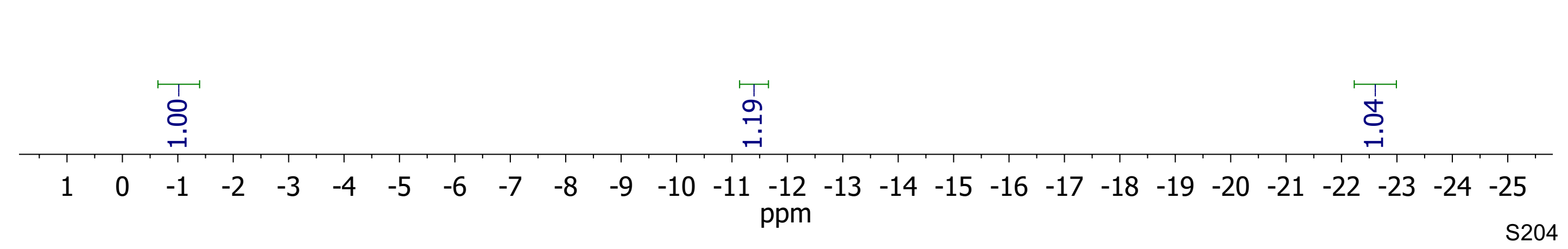


$\begin{array}{lll}\circ & \circ & 0 \\ \ominus & \ominus & \ominus\end{array}$

$\stackrel{\oplus}{\mathrm{NH}_{4}} \quad \stackrel{\oplus}{\mathrm{NH}_{4}} \stackrel{\stackrel{\oplus}{\mathrm{N}} \mathrm{H}_{4}}{4}$

Product after purifucation by SAX
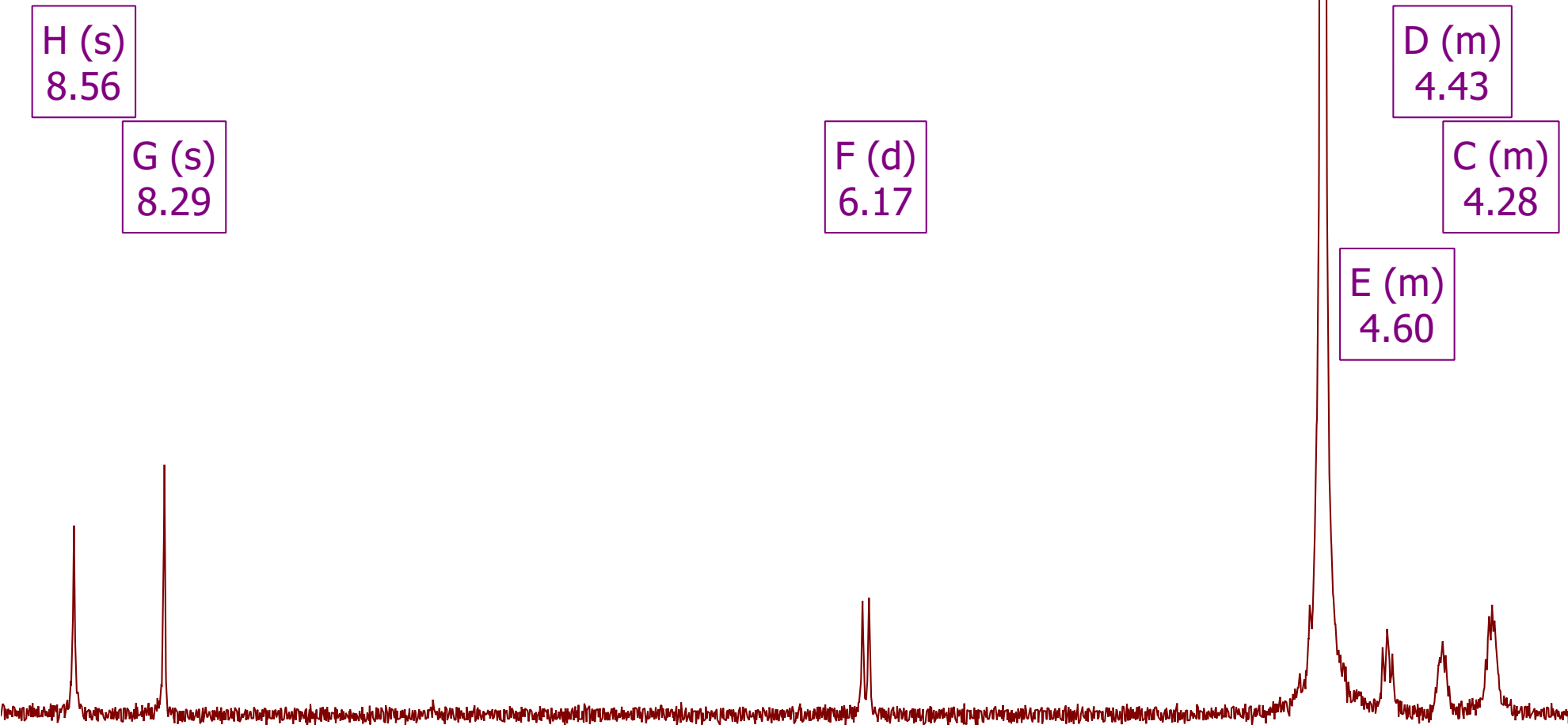

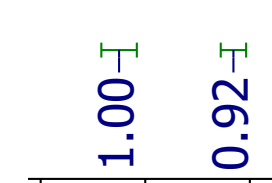

8.0

$\begin{array}{lll}8.0 & 7.5 & 7.0\end{array}$

$\begin{array}{ll}7.0 & 6.5\end{array}$

$6.5 \quad 6.0$

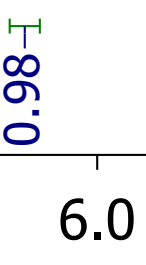

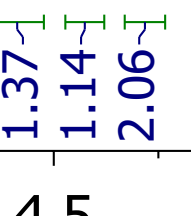

5.5 ppm 


\section{${ }^{31} \mathrm{P}\left\{{ }^{1} \mathrm{H}\right\}-\mathrm{NMR}$}

(1)

\section{Product after purifucation by SAX}

$$
\begin{array}{|l|}
\hline C(d) \\
-2.72 \\
\hline
\end{array}
$$

$$
\begin{array}{|c|}
\hline B(d) \\
-11.38 \\
\hline
\end{array}
$$

\begin{tabular}{|c|c|c|c|c|c|c|c|c|c|c|c|c|c|c|c|c|c|c|}
\hline & & $\vdash$ & & & & & 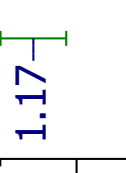 & & & & & & & & & & & \\
\hline 2 & 0 & -2 & -4 & -6 & -8 & -10 & -12 & -14 & $\begin{array}{c}-16 \\
\mathrm{ppm}\end{array}$ & -18 & -20 & -22 & -24 & -26 & -28 & -30 & -32 & $\begin{array}{r}-3 \\
\text { S206 }\end{array}$ \\
\hline
\end{tabular}

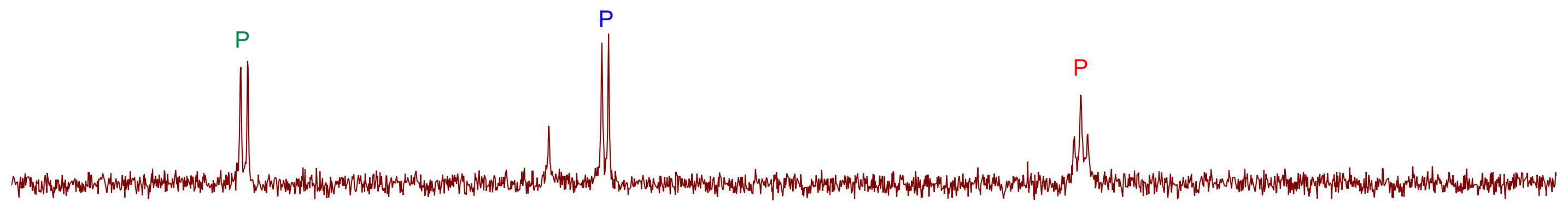




\section{${ }^{31} \mathrm{P}\left\{{ }^{1} \mathrm{H}\right\}-N M R$}

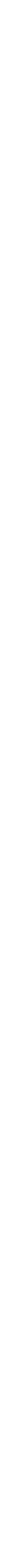




\section{${ }^{31} \mathrm{P}\left\{{ }^{1} \mathrm{H}\right\}-N M R$}

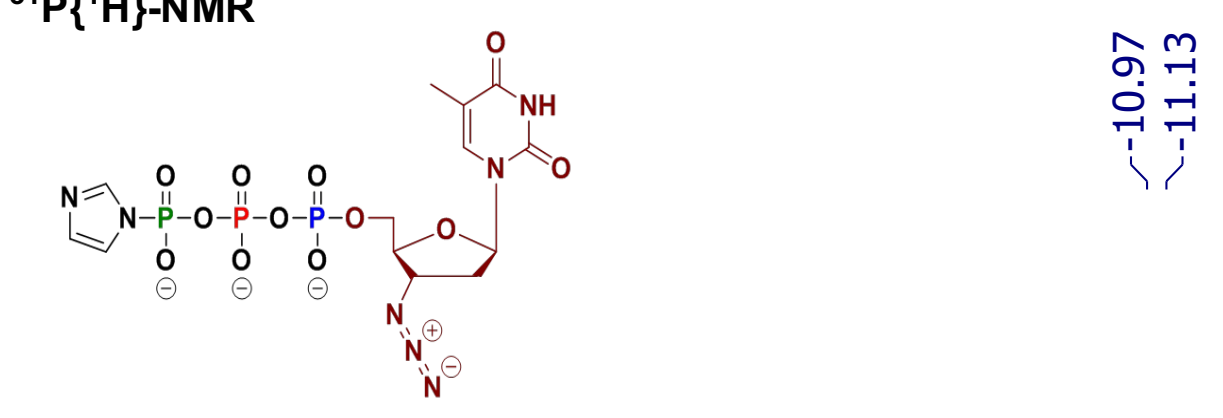

quant. in reaction mixture

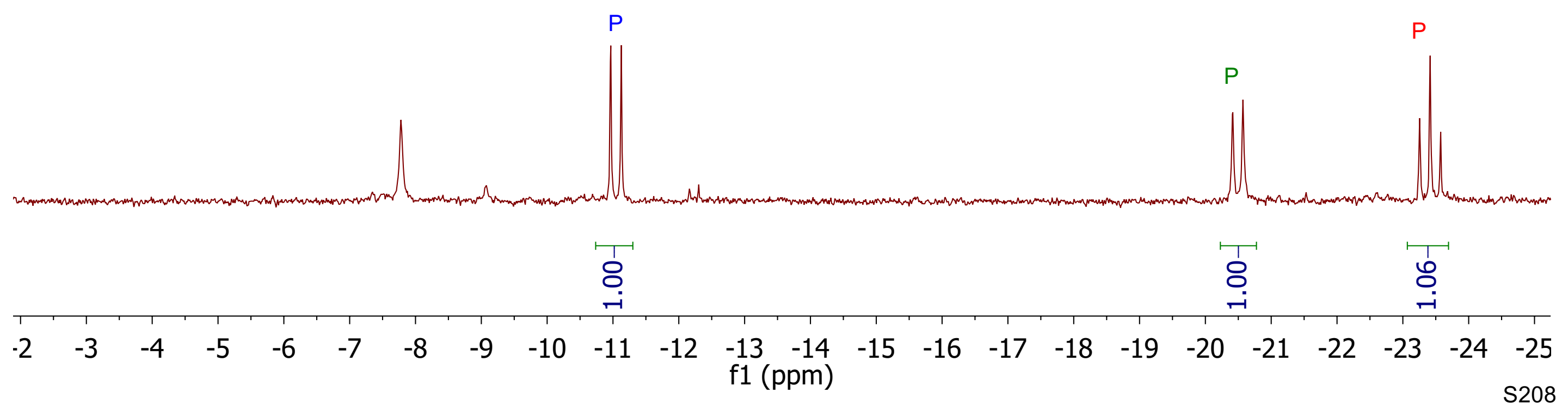




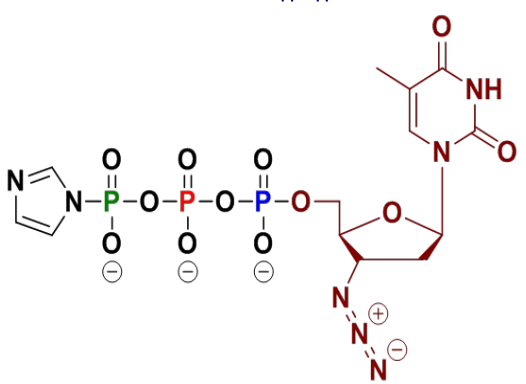

quant. in reaction mixture

$$
\begin{array}{|l|}
\hline \text { C (dt) } \\
-11.05 \\
\hline
\end{array}
$$

$$
B(d)
$$$$
-20.49
$$

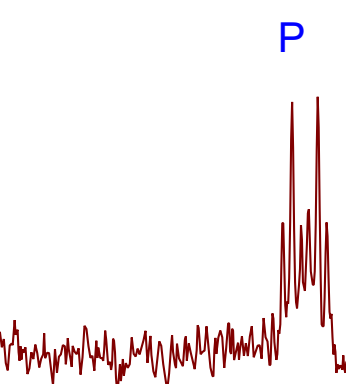

\begin{tabular}{|c|c|c|c|c|c|c|c|c|c|c|c|c|c|c|c|c|}
\hline & $\begin{array}{l}1 \\
8 \\
\\
-i\end{array}$ & & & & & & & & & & & & $\stackrel{1}{\sim}$ & & & \\
\hline-10 & -11 & -12 & -13 & -14 & -15 & -16 & -17 & 1 (ppm) & -19 & -20 & -21 & -22 & -23 & -24 & -25 & -26 \\
\hline
\end{tabular}




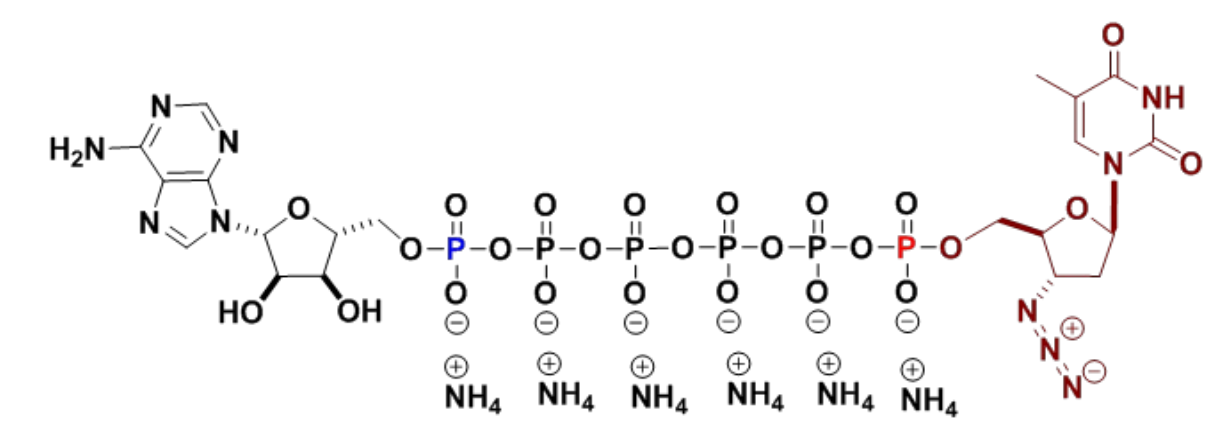

Product after purifucation by SAX

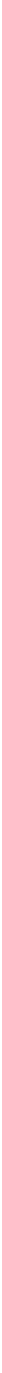




\section{${ }^{31} \mathrm{P}\left\{{ }^{1} \mathrm{H}\right\}-\mathrm{NMR}$}

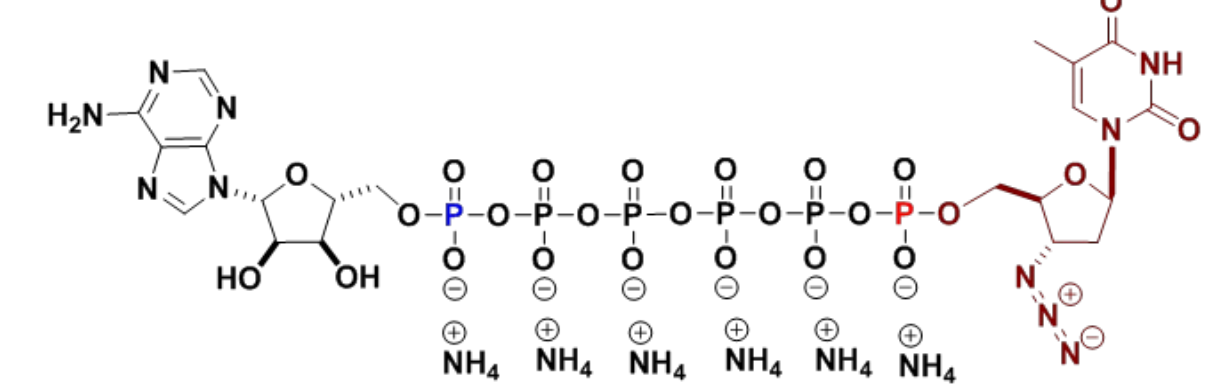

Product after purifucation by SAX

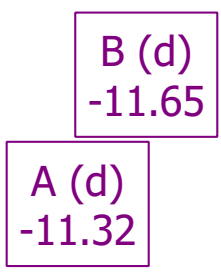

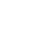

$4 \mathrm{P}$

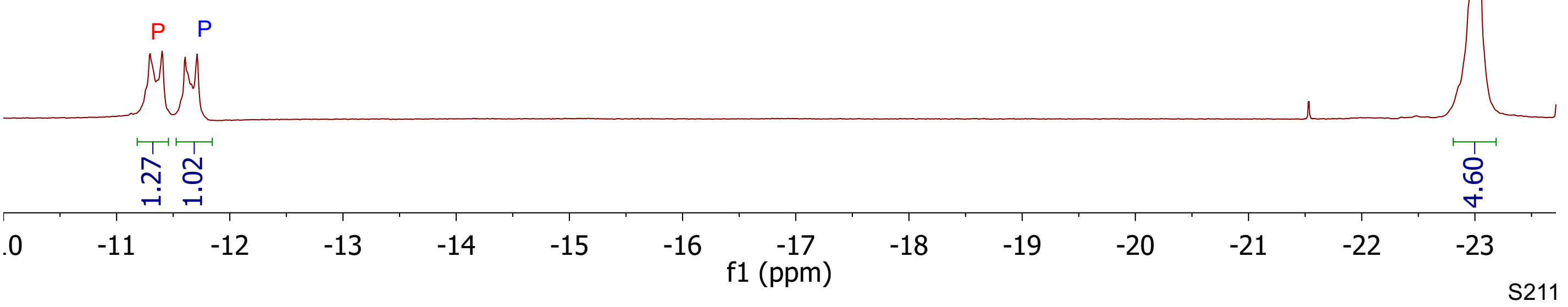




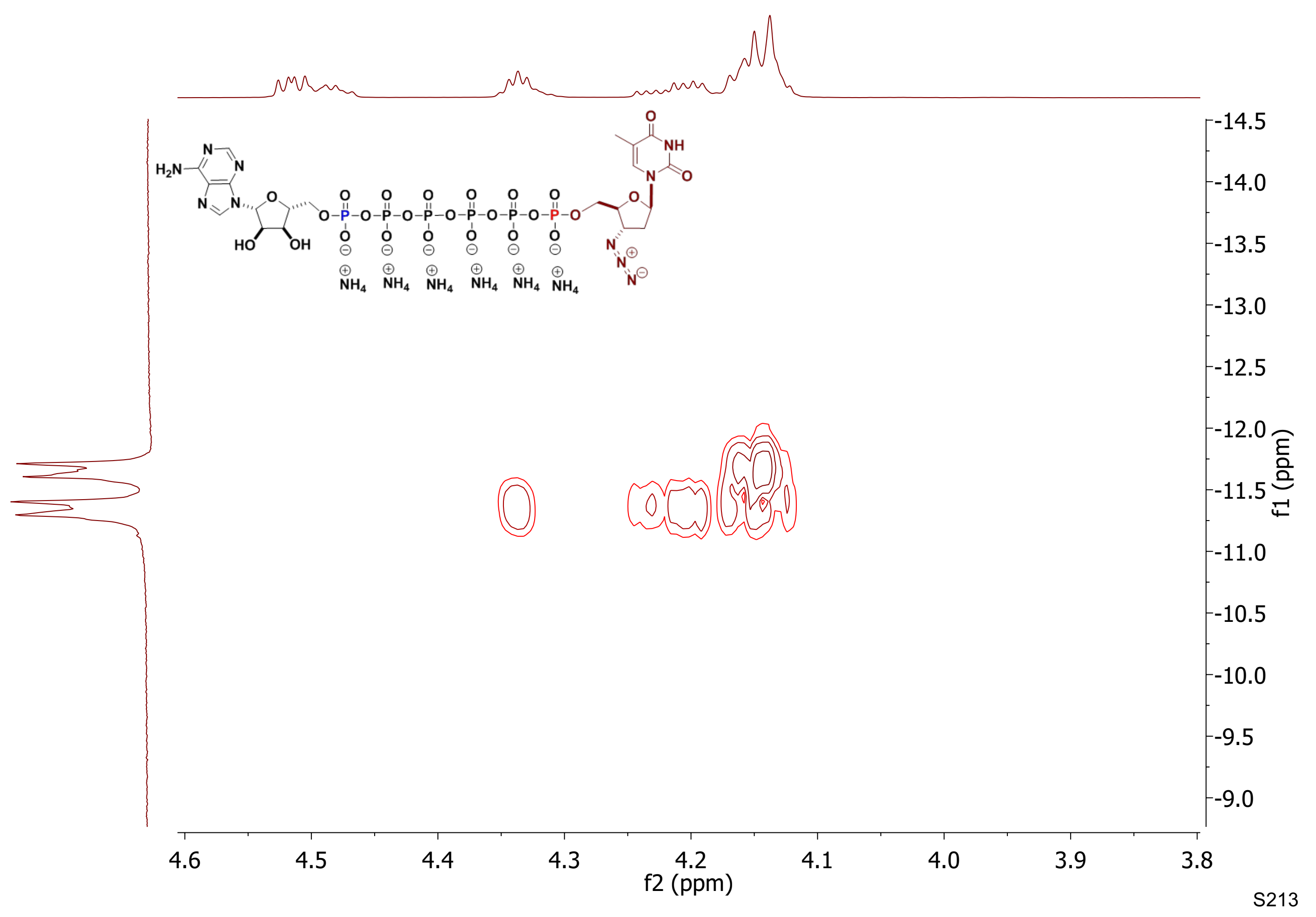




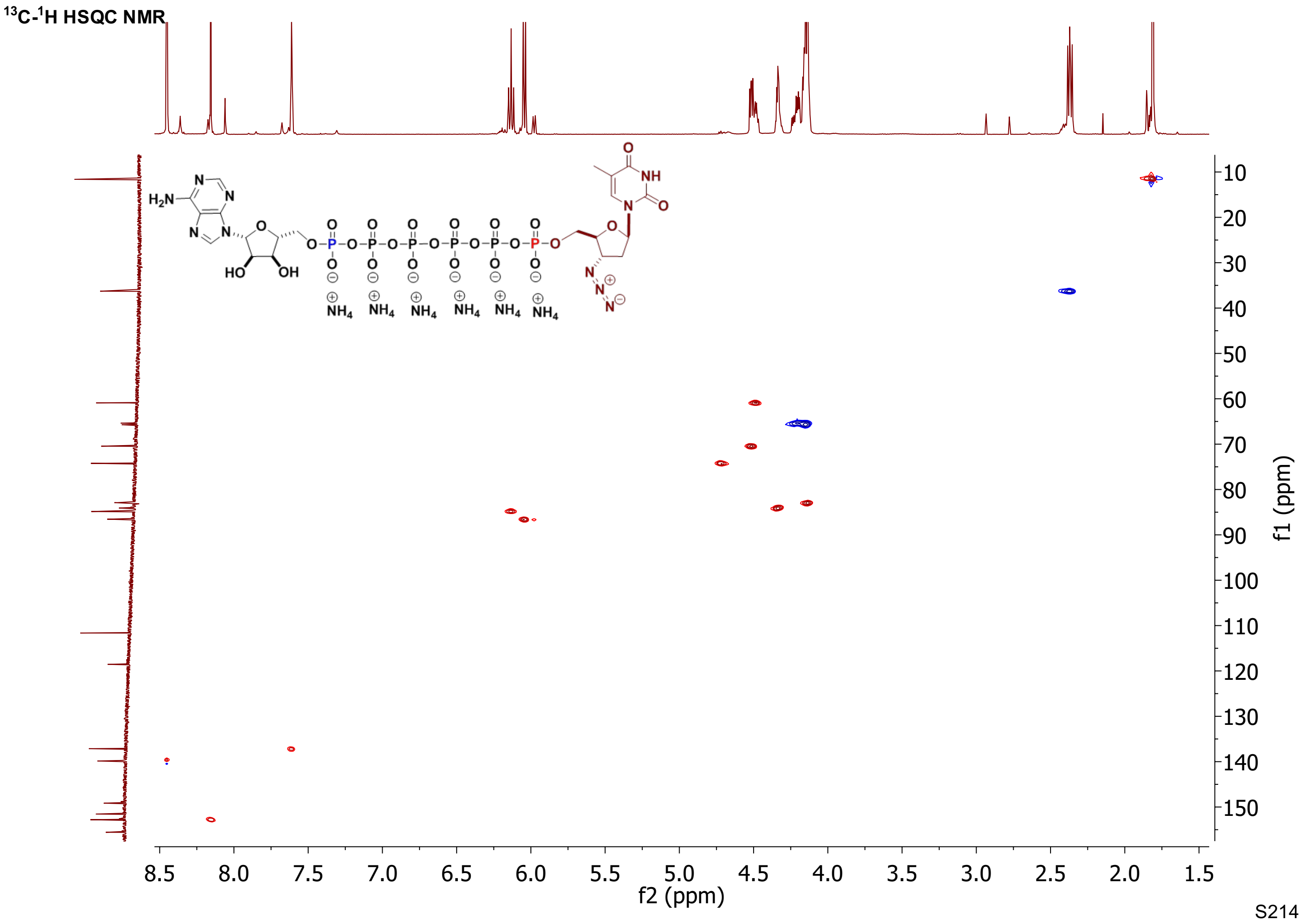




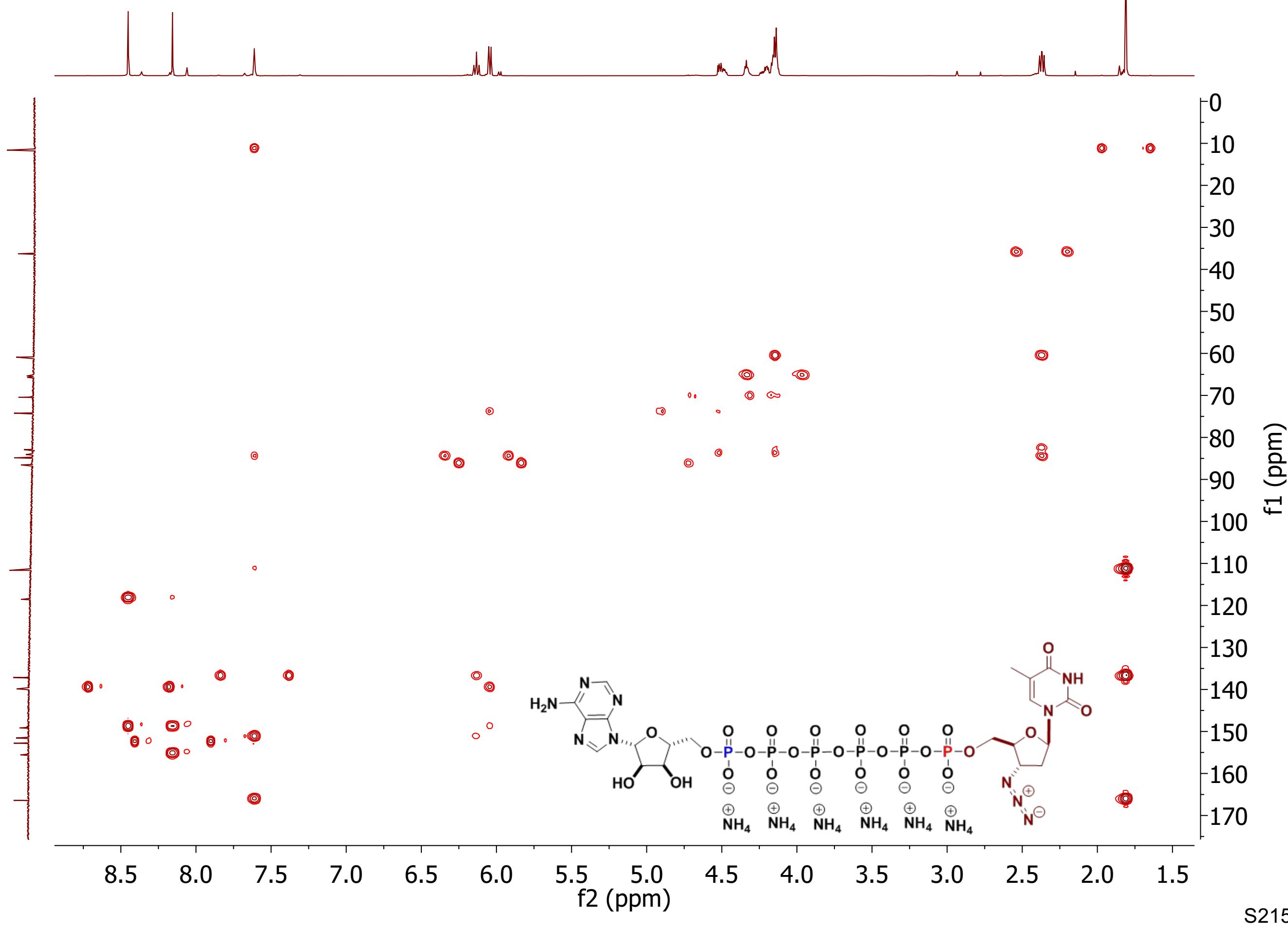




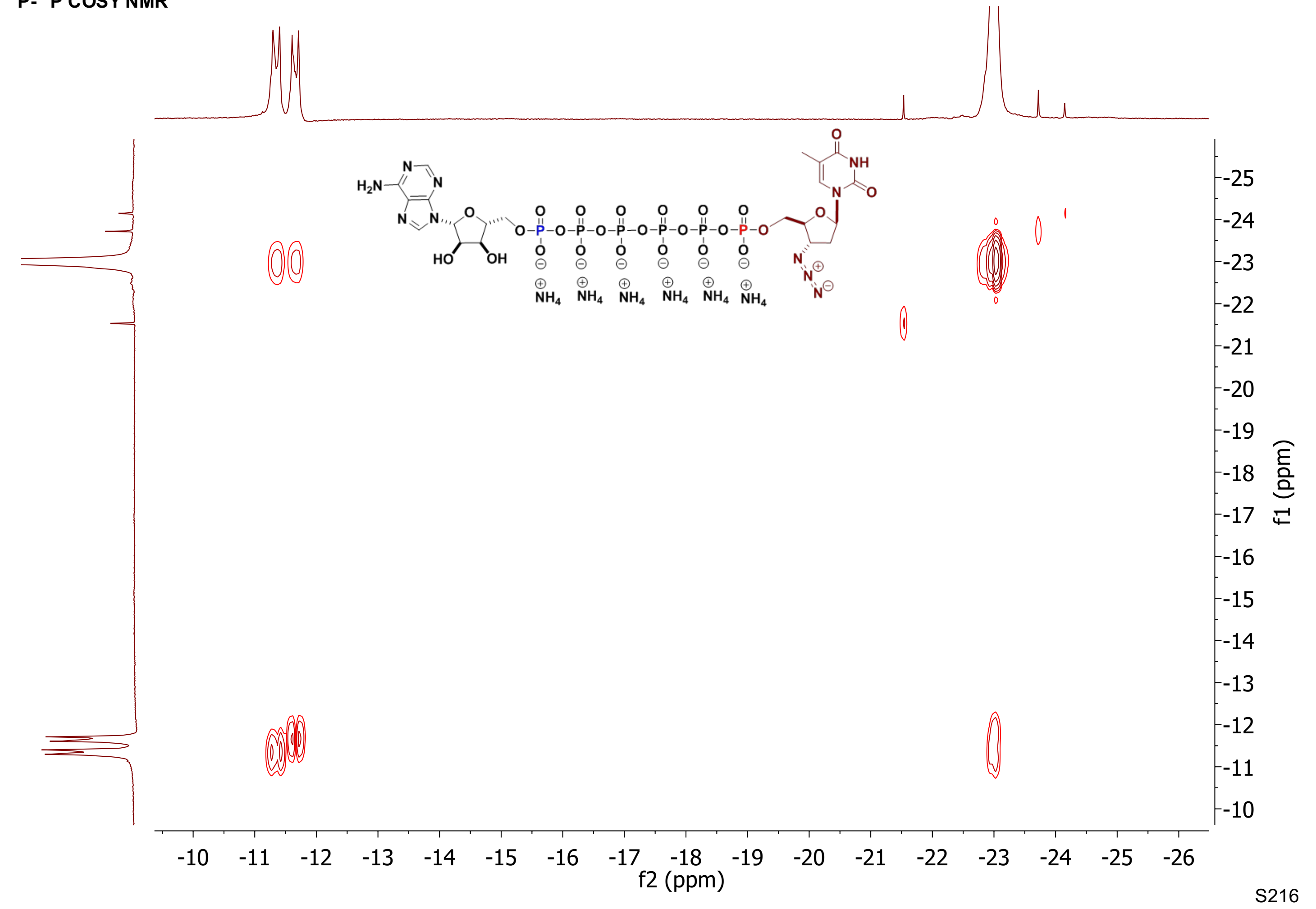




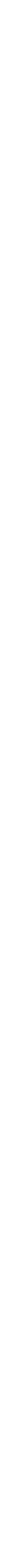




\section{${ }^{31} \mathrm{P}\left\{{ }^{1} \mathrm{H}\right\}-\mathrm{NMR}$}

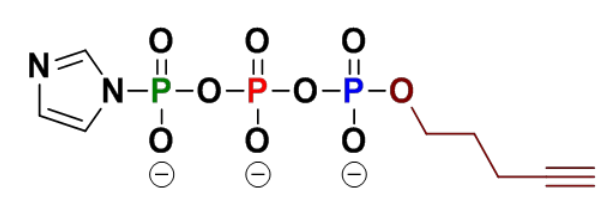

quant. in reaction mixture

$$
\begin{gathered}
A(d) \\
-11.11
\end{gathered}
$$

\begin{tabular}{|c|c|c|c|c|c|c|c|c|c|c|c|c|c|c|c|c|c|}
\hline 9 & -10 & -11 & -12 & -13 & -14 & -15 & -16 & $\begin{array}{r}-17 \\
\mathrm{f} 1\end{array}$ & $\begin{array}{r}-18 \\
\mathrm{ppm})\end{array}$ & -19 & -20 & -21 & -22 & -23 & -24 & -25 & $\begin{array}{l}-26 \\
S 218\end{array}$ \\
\hline
\end{tabular}

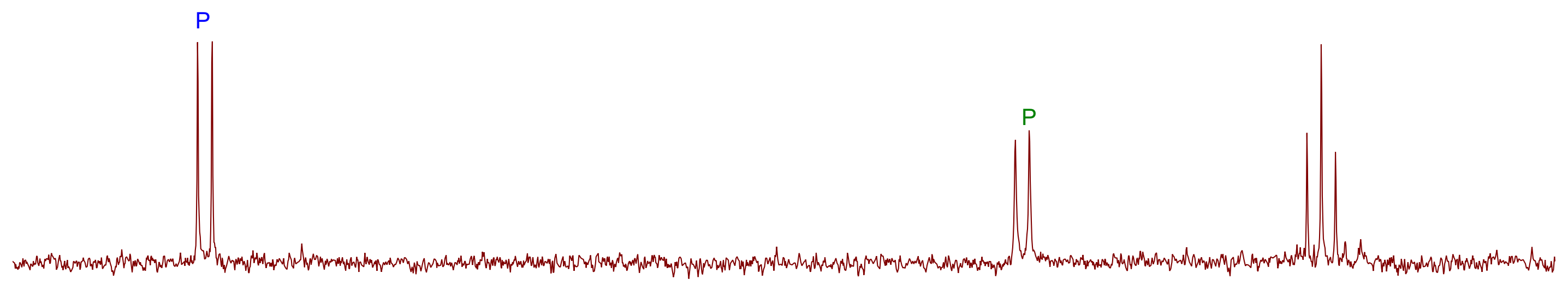




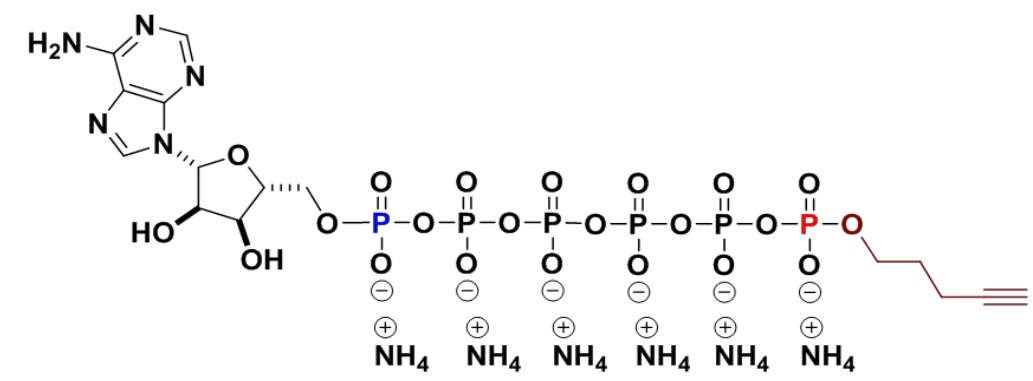

Product after purifucation by SAX
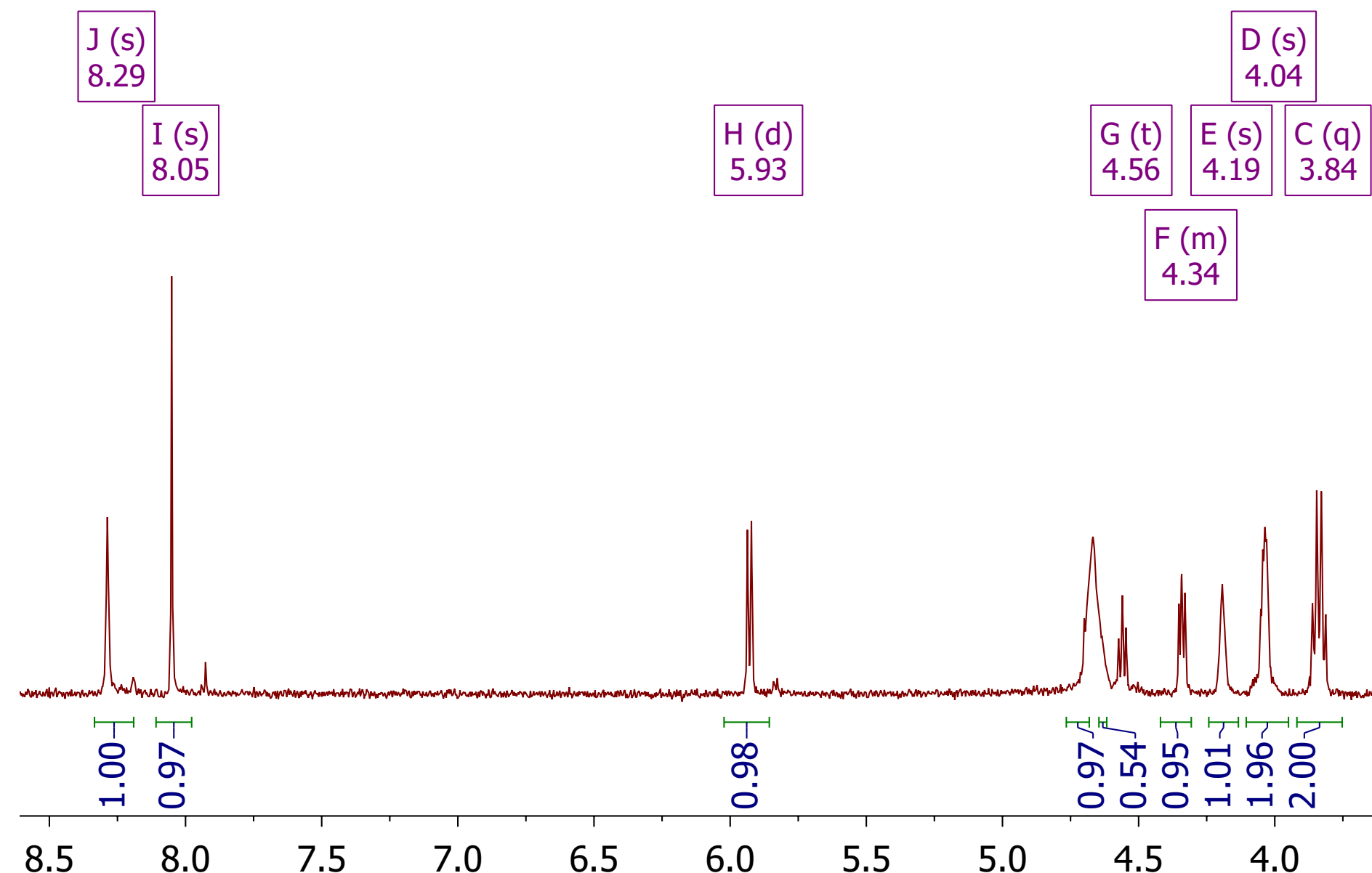

8.0

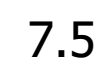

7.0

$6.5 \quad 6.0$ f1 (ppm)

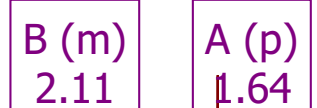

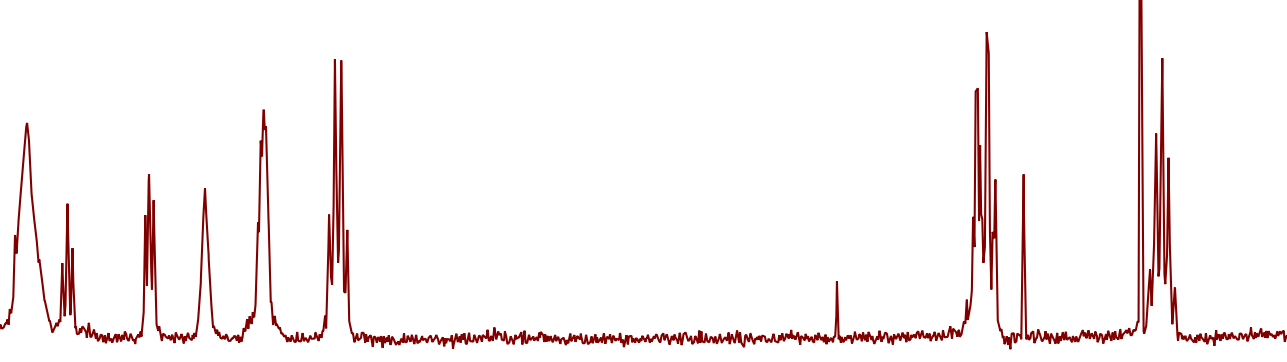

एस⿺𠃊⺊

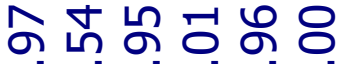
ठ

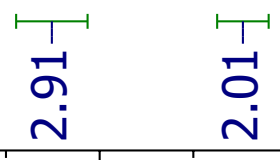

$\begin{array}{llllll}4.0 & 3.5 & 3.0 & 2.5 & 2.0 & 1.5\end{array}$



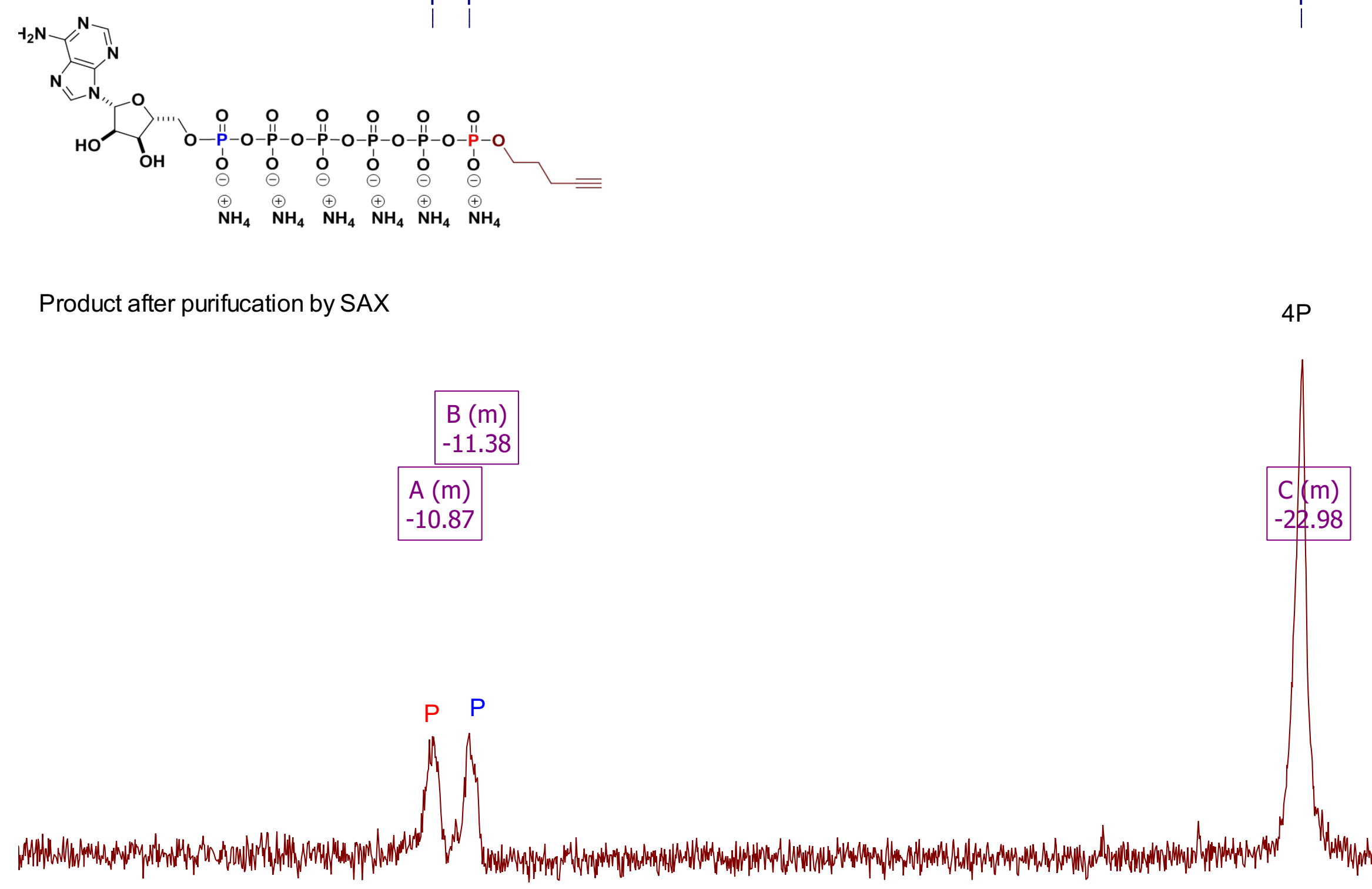

\begin{tabular}{|c|c|c|c|c|c|c|c|c|c|c|c|c|c|c|c|c|c|c|c|c|c|}
\hline & & & & & \begin{tabular}{lll}
1 & 1 \\
0 & 1 \\
0 & 0 \\
\hdashline & 0 \\
& 0 & 0
\end{tabular} & & & & & & & & & & & $\stackrel{1}{\hat{n}}$ & & & & & \\
\hline-6 & -7 & -8 & -9 & -10 & -11 & -12 & -13 & -14 & -15 & $\begin{array}{cc}-16 & -17 \\
\mathrm{f} 1 & (\mathrm{ppm})\end{array}$ & -18 & -19 & -20 & -21 & -22 & -23 & -24 & -25 & -26 & -27 & -28 \\
\hline
\end{tabular}




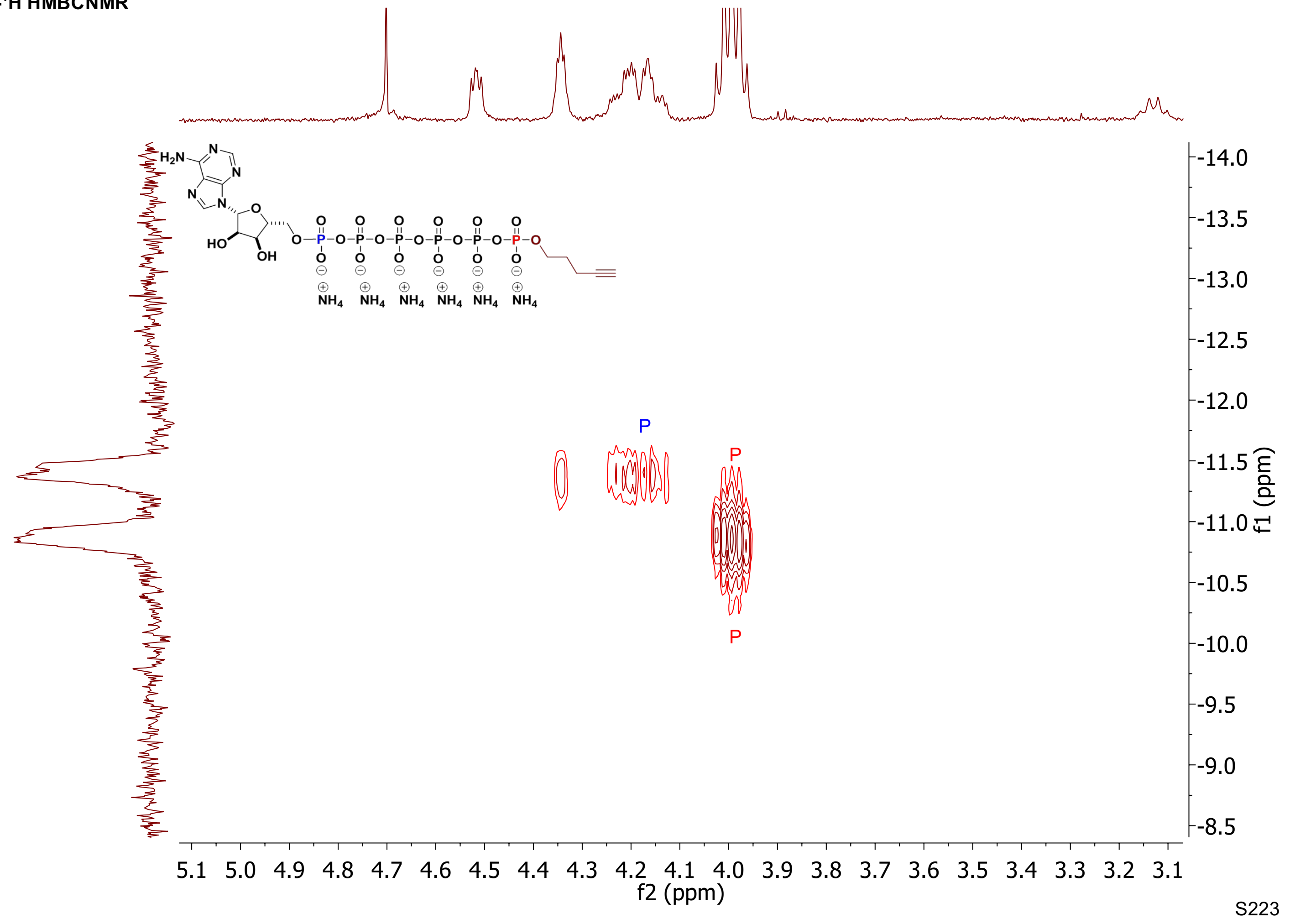




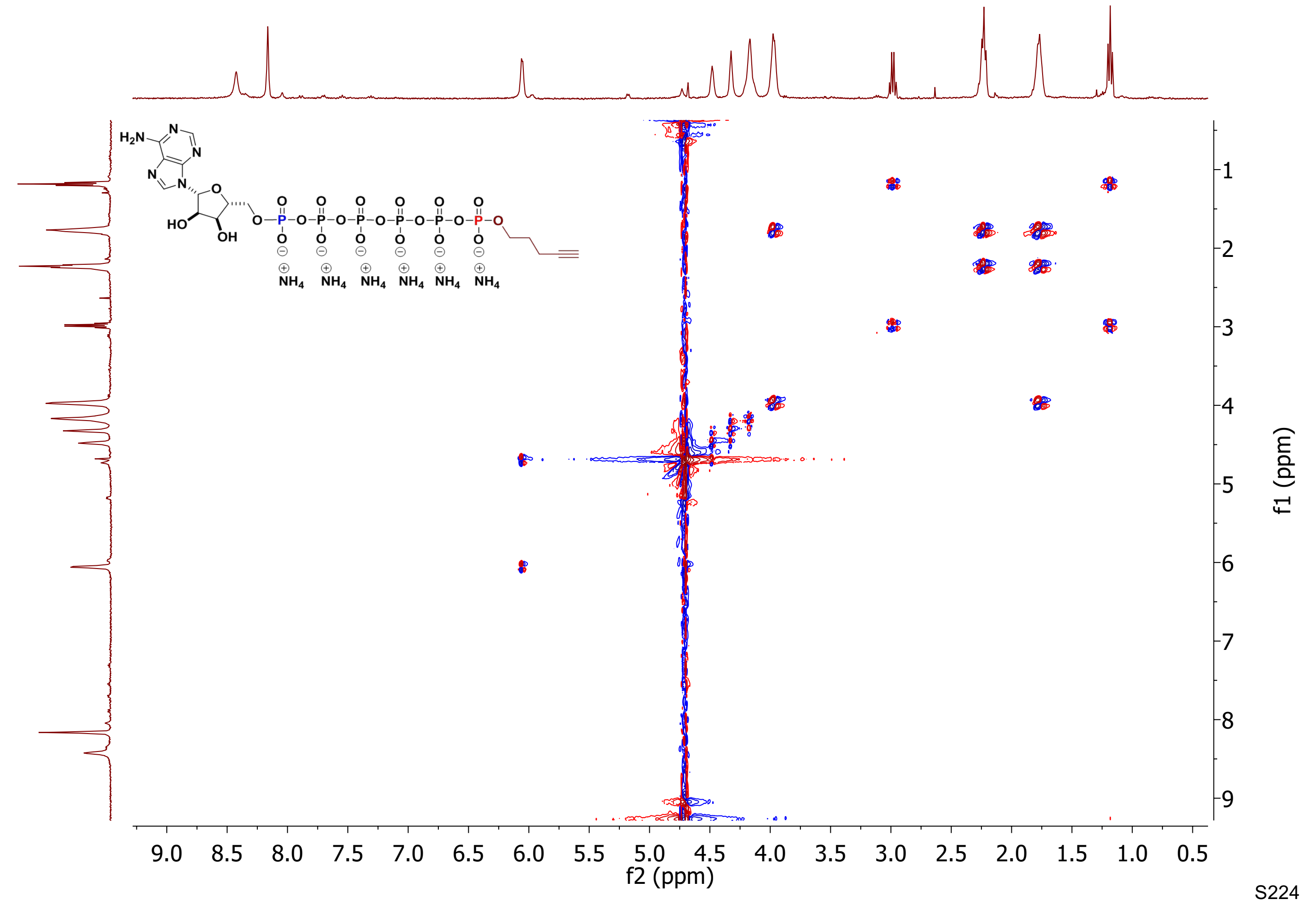




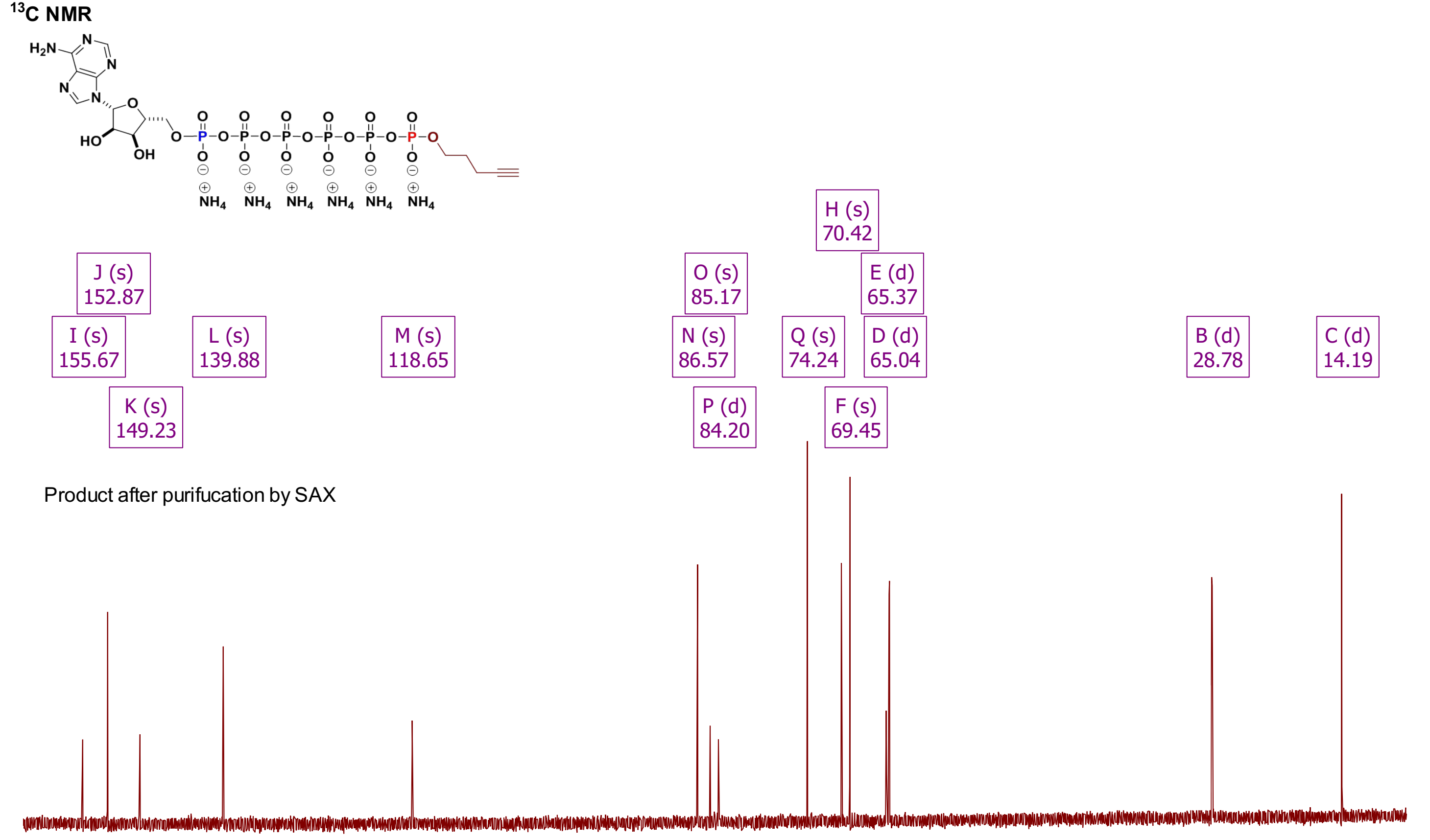




\section{Synthesis of triphosphates based on $\mathrm{c}-\mathrm{Py}_{\mathrm{CH} 2} \mathrm{PA}\left(\mathrm{A}_{2}\right)$}

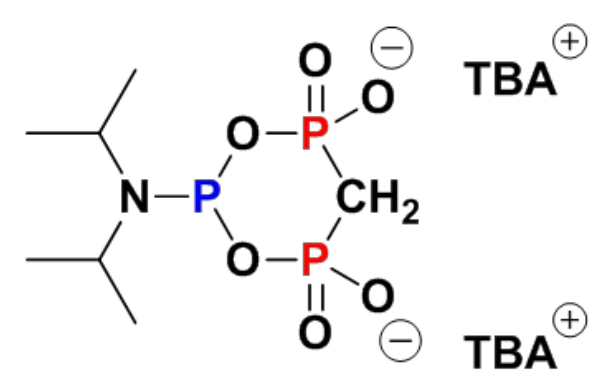




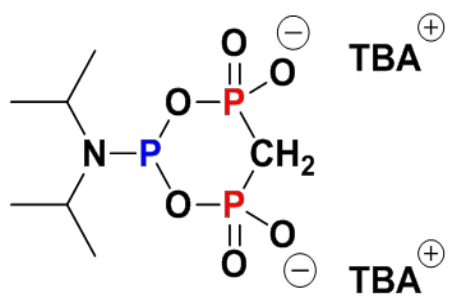

$$
\begin{array}{|c|}
A(t) \\
125.71 \\
J(9.02)
\end{array}
$$
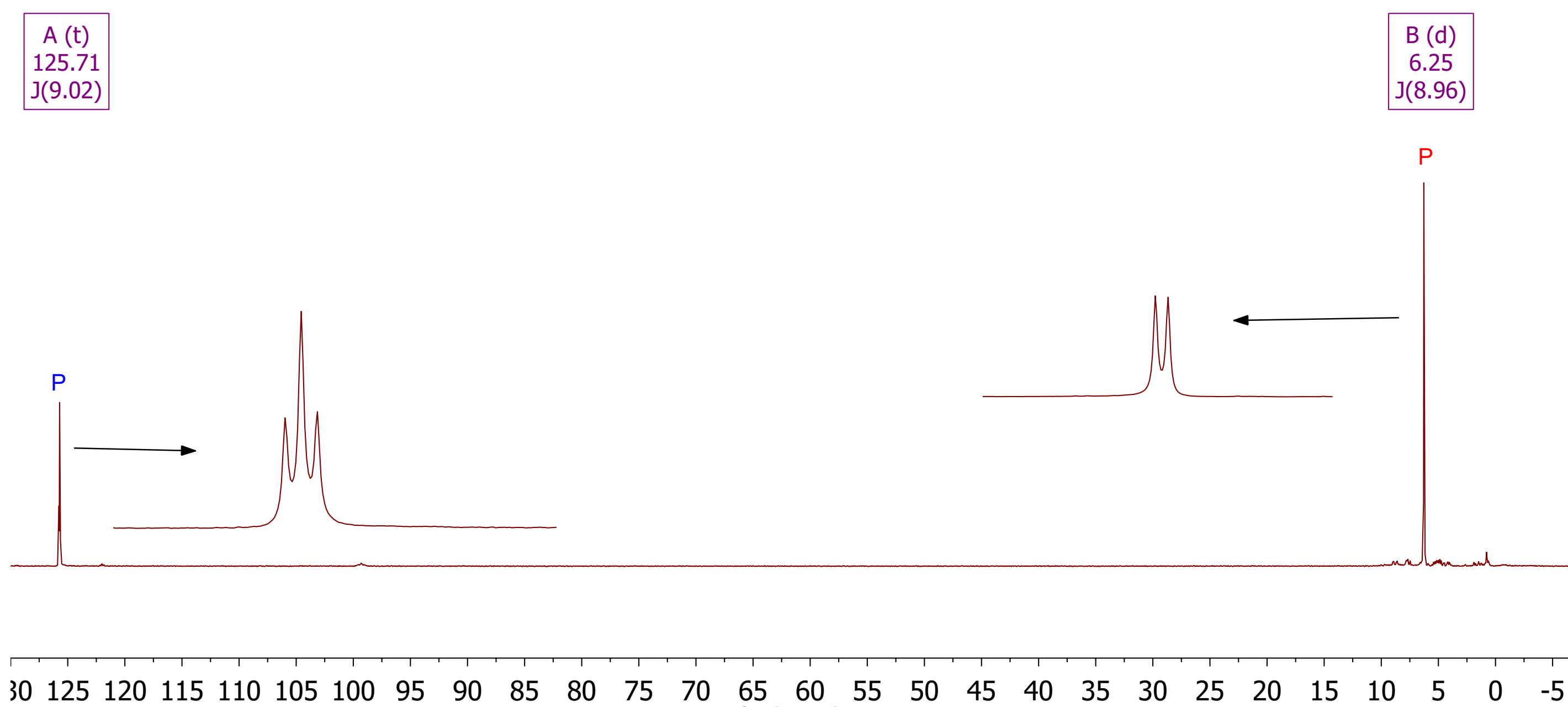


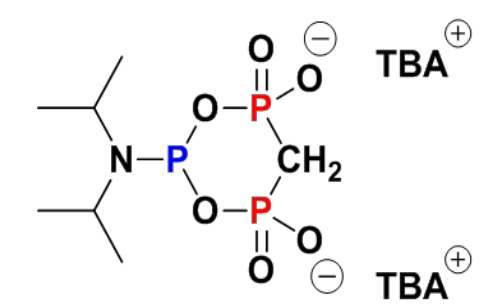

\begin{tabular}{|c|}
\hline$A(p)$ \\
125.71 \\
$J(10.12)$ \\
\hline
\end{tabular}

\begin{tabular}{|c|}
\hline$B(t d)$ \\
6.25 \\
$J(18.61,8.73)$ \\
\hline
\end{tabular}
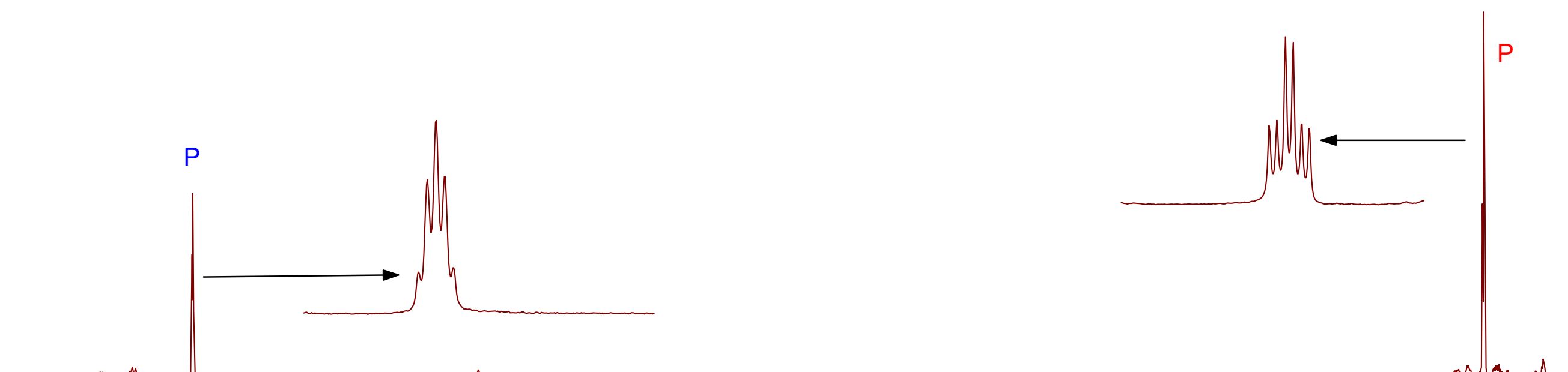


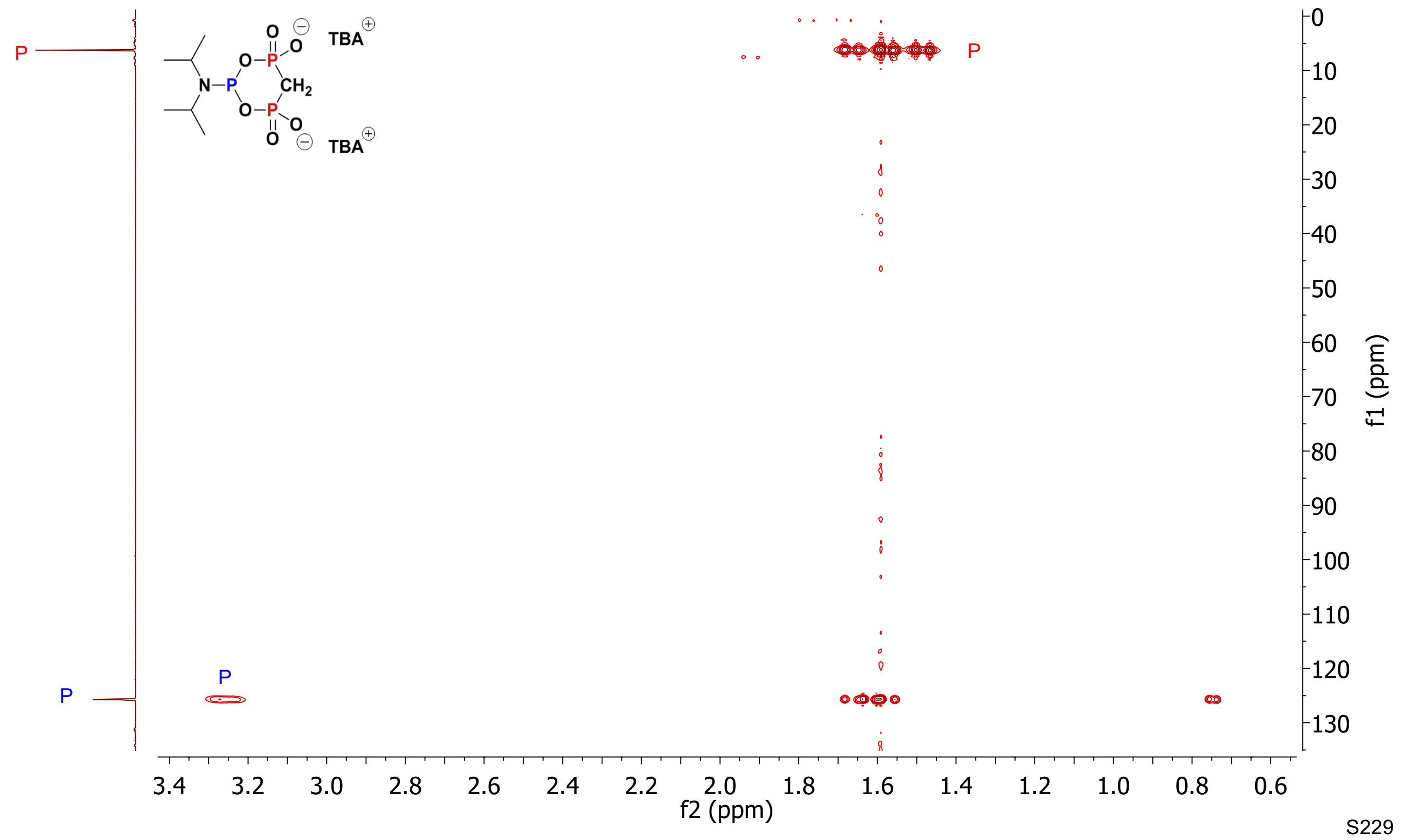




\section{${ }^{31} \mathrm{P}\left\{{ }^{1} \mathrm{H}\right\}$ NMR}

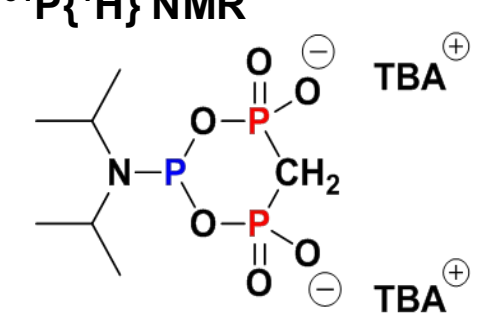

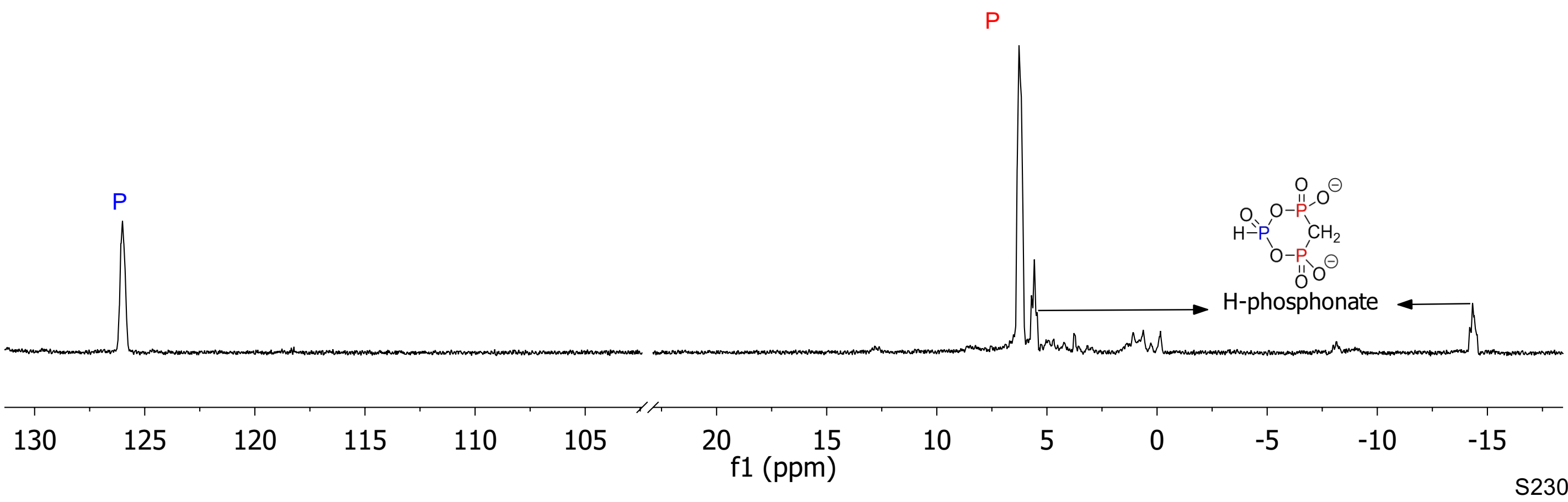



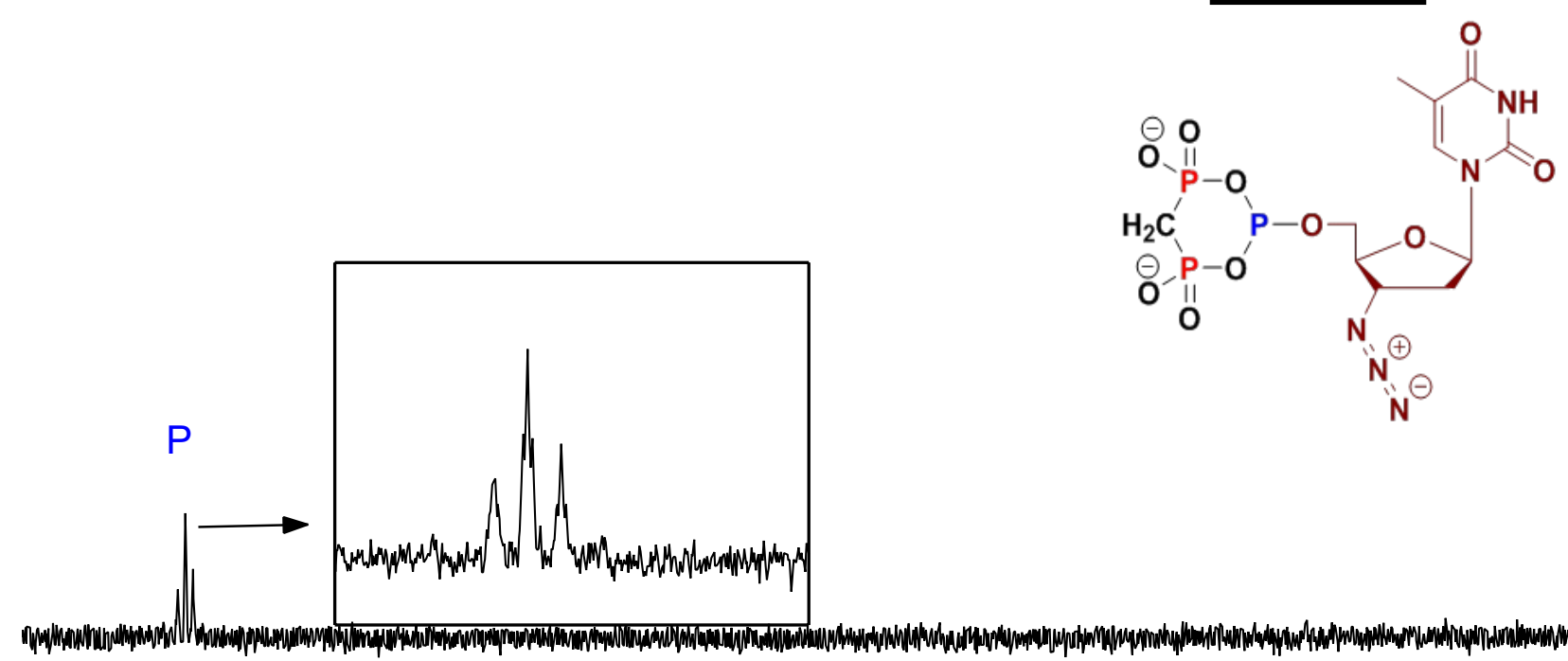

${ }^{31} \mathrm{P}\left\{{ }^{1} \mathrm{H}\right\}$ NMR

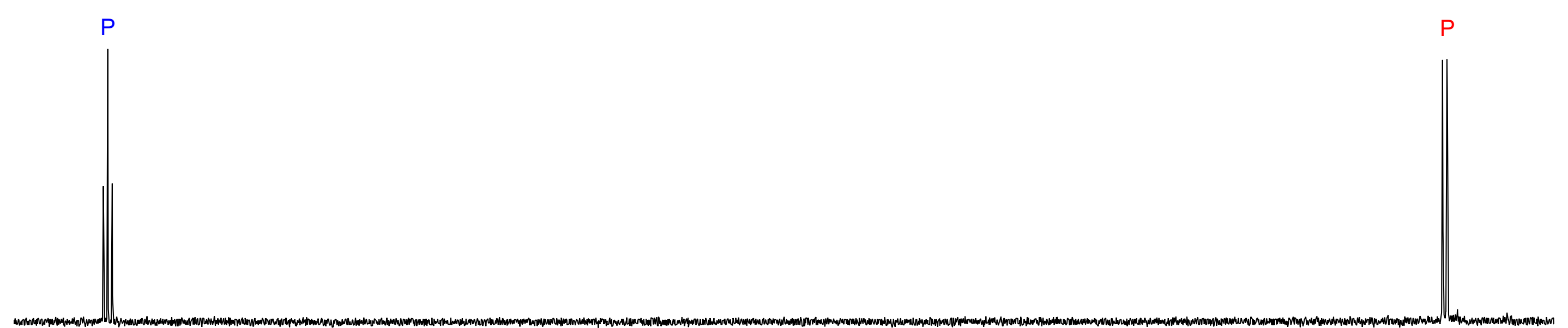

$\begin{array}{lllllllllllllllllllllll}110 & 105 & 100 & 95 & 90 & 85 & 80 & 75 & 70 & 65 & 60 & \begin{array}{c}55 \\ \mathrm{f} 1(\mathrm{ppm})\end{array} & 50 & 45 & 40 & 35 & 30 & 25 & 20 & 15 & 10 & 5 & 0\end{array}$



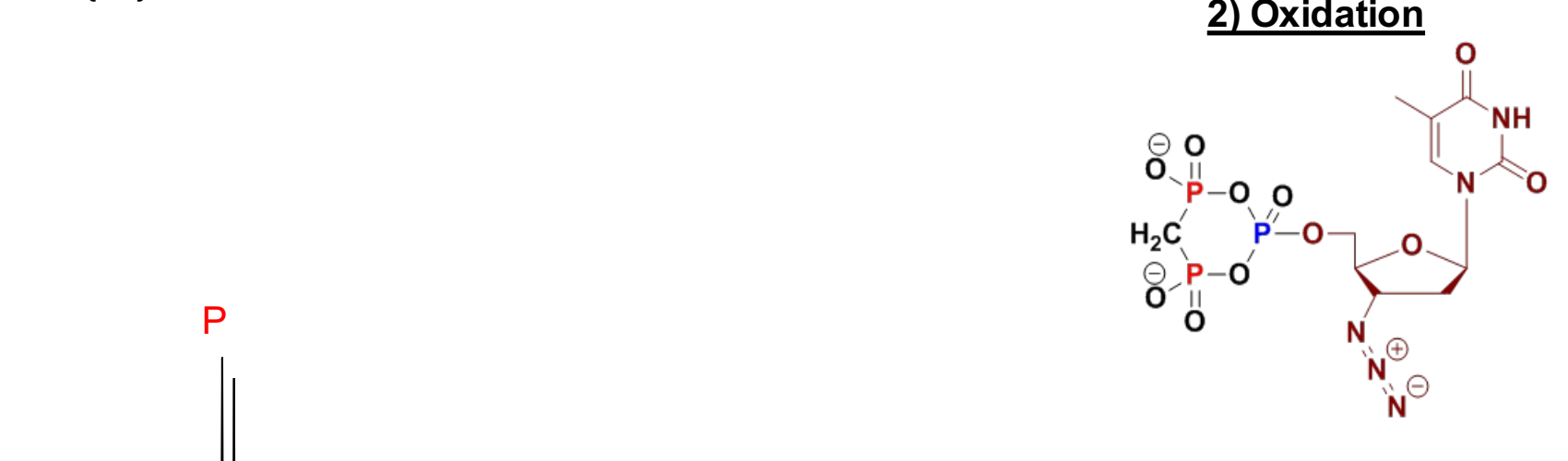

${ }^{31} \mathrm{P}$ NMR

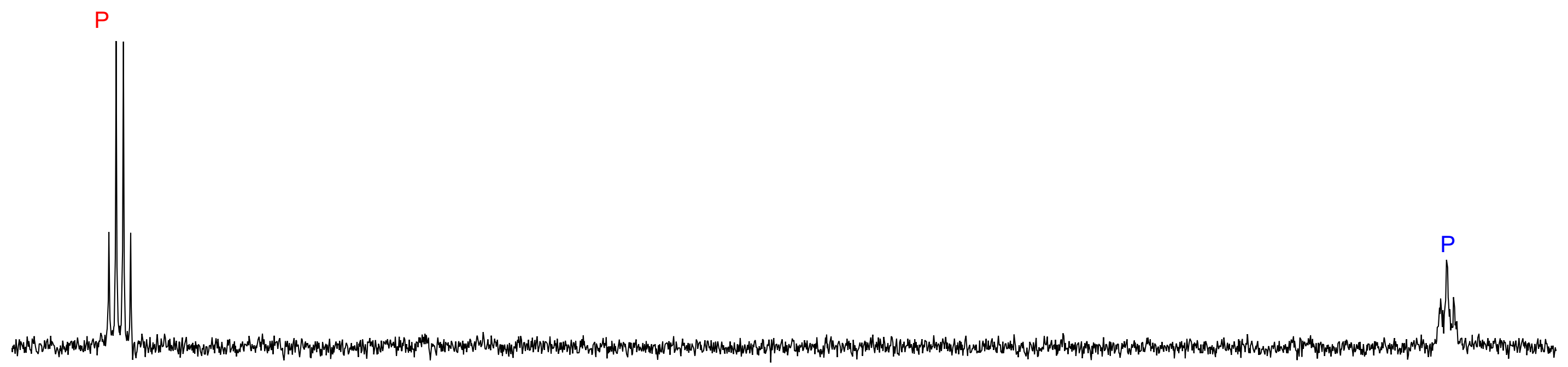

$\begin{array}{lllllll}6 & 4 & 2 & 0 & -2 & -4 & -6\end{array}$

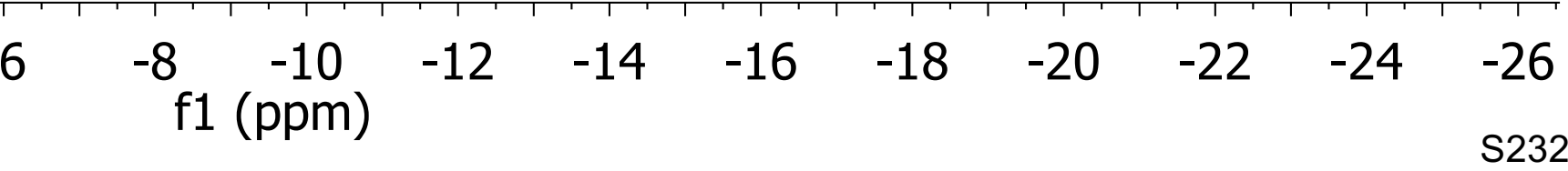



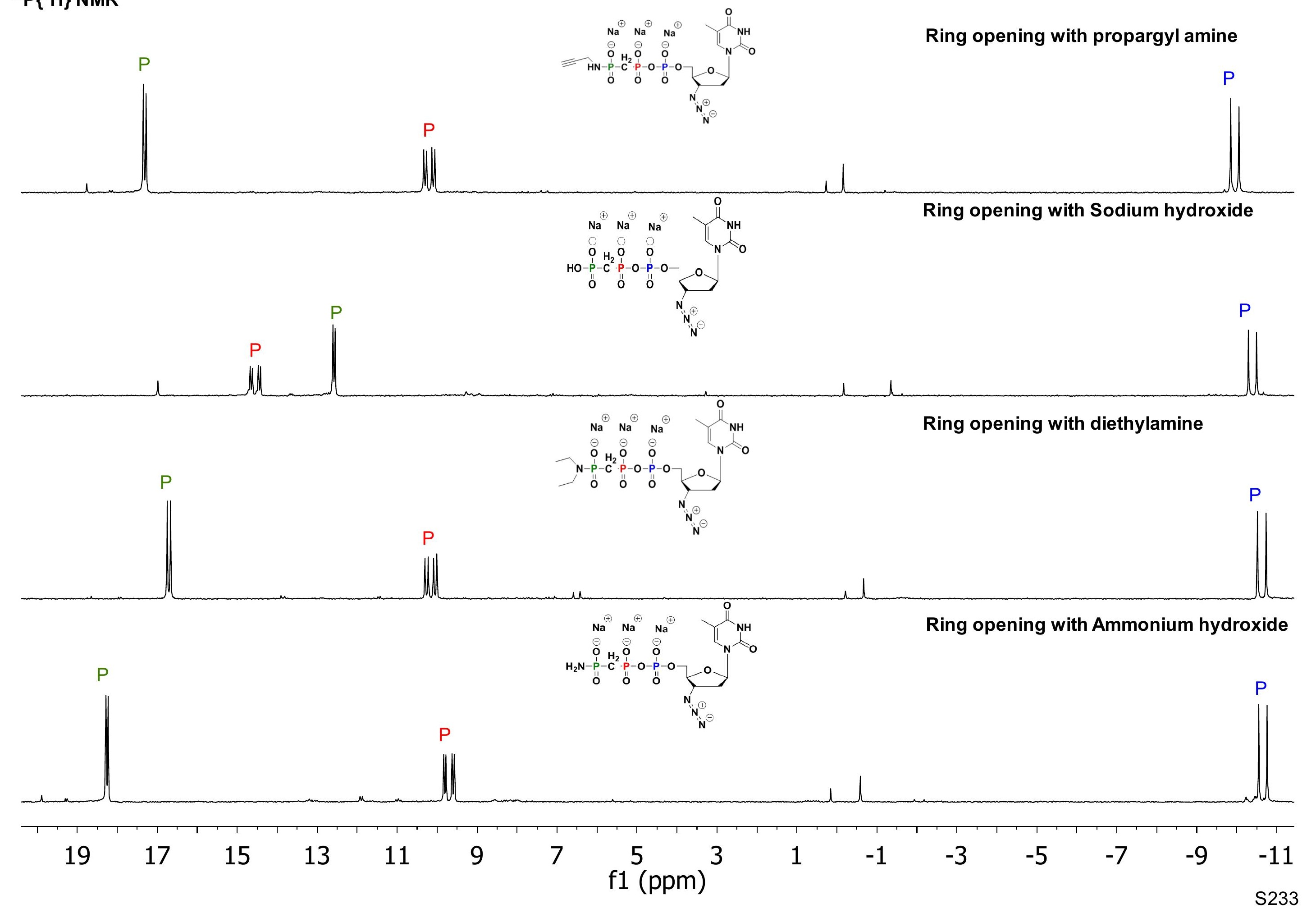


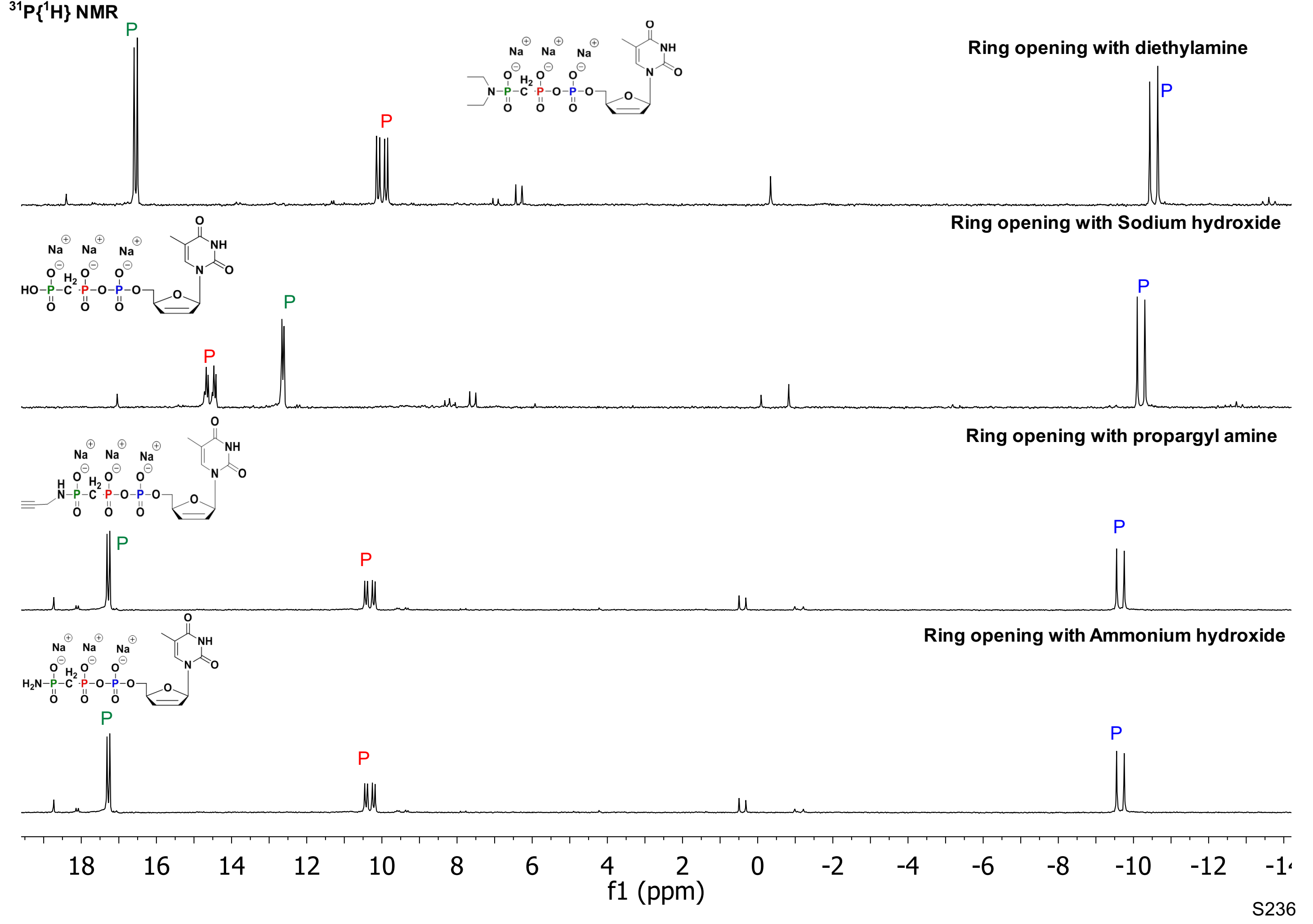




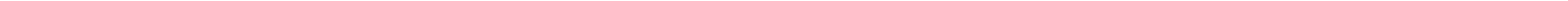




\section{${ }^{31} \mathrm{P}\left\{{ }^{1} \mathrm{H}\right\}$ NMR}
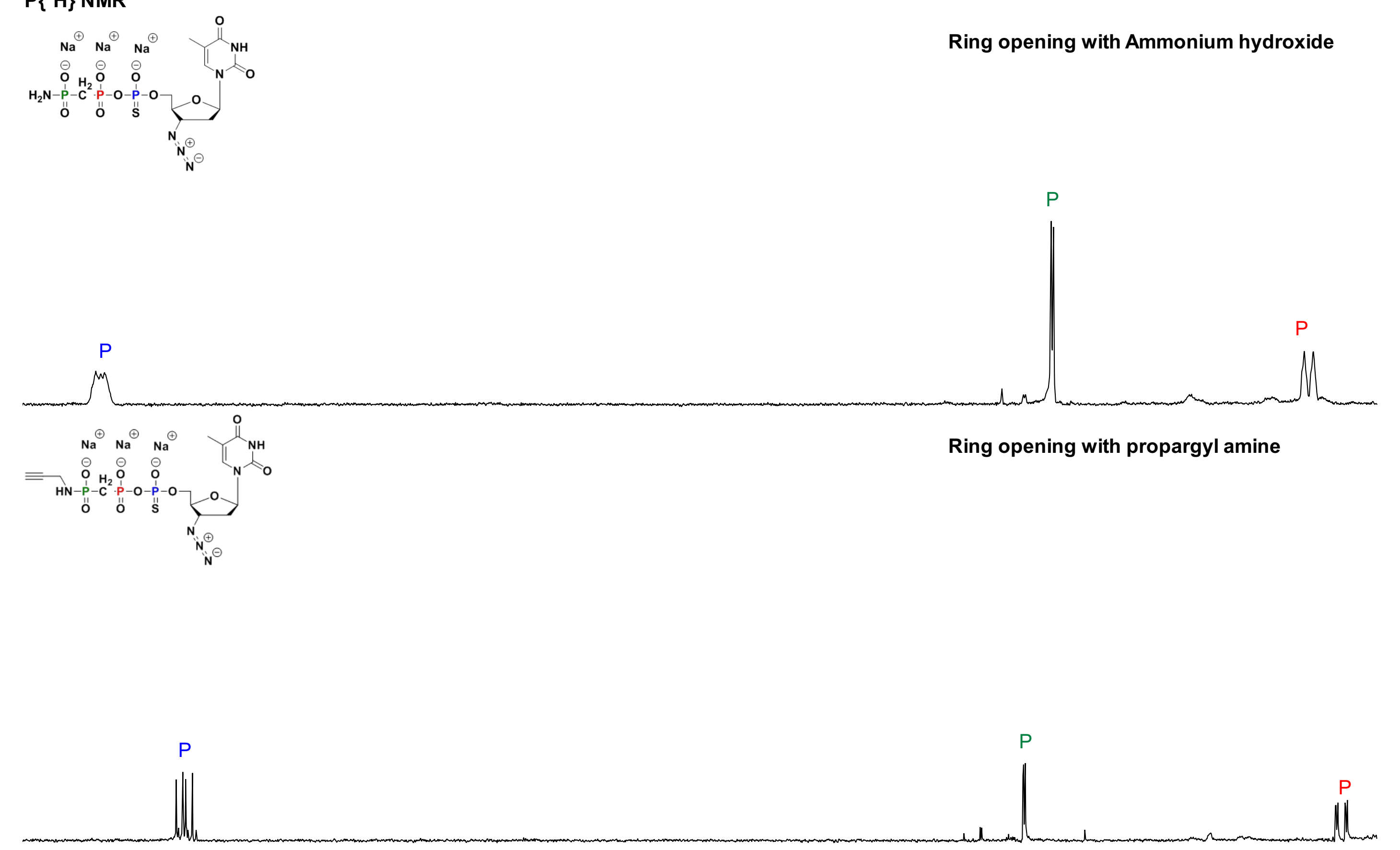


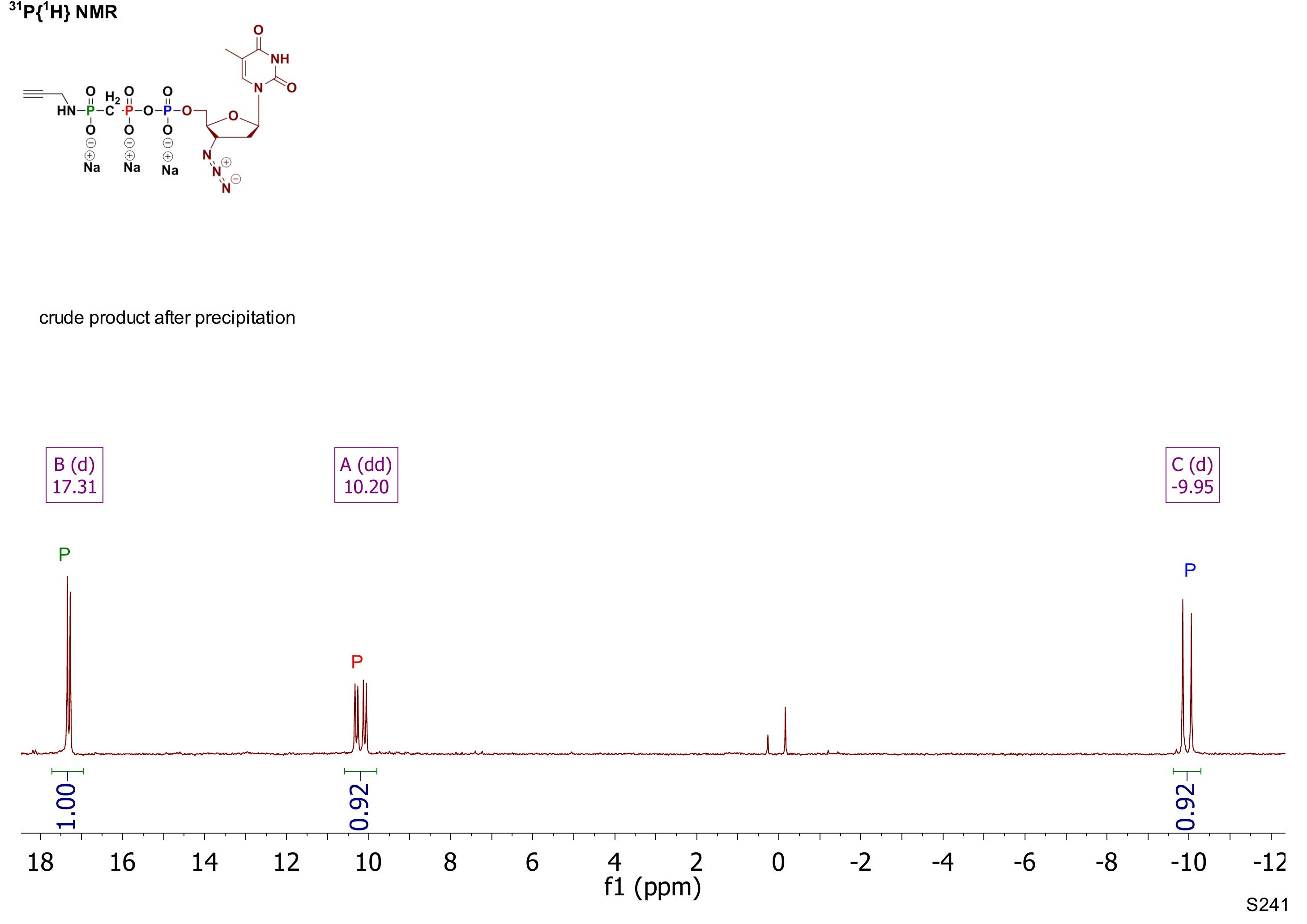




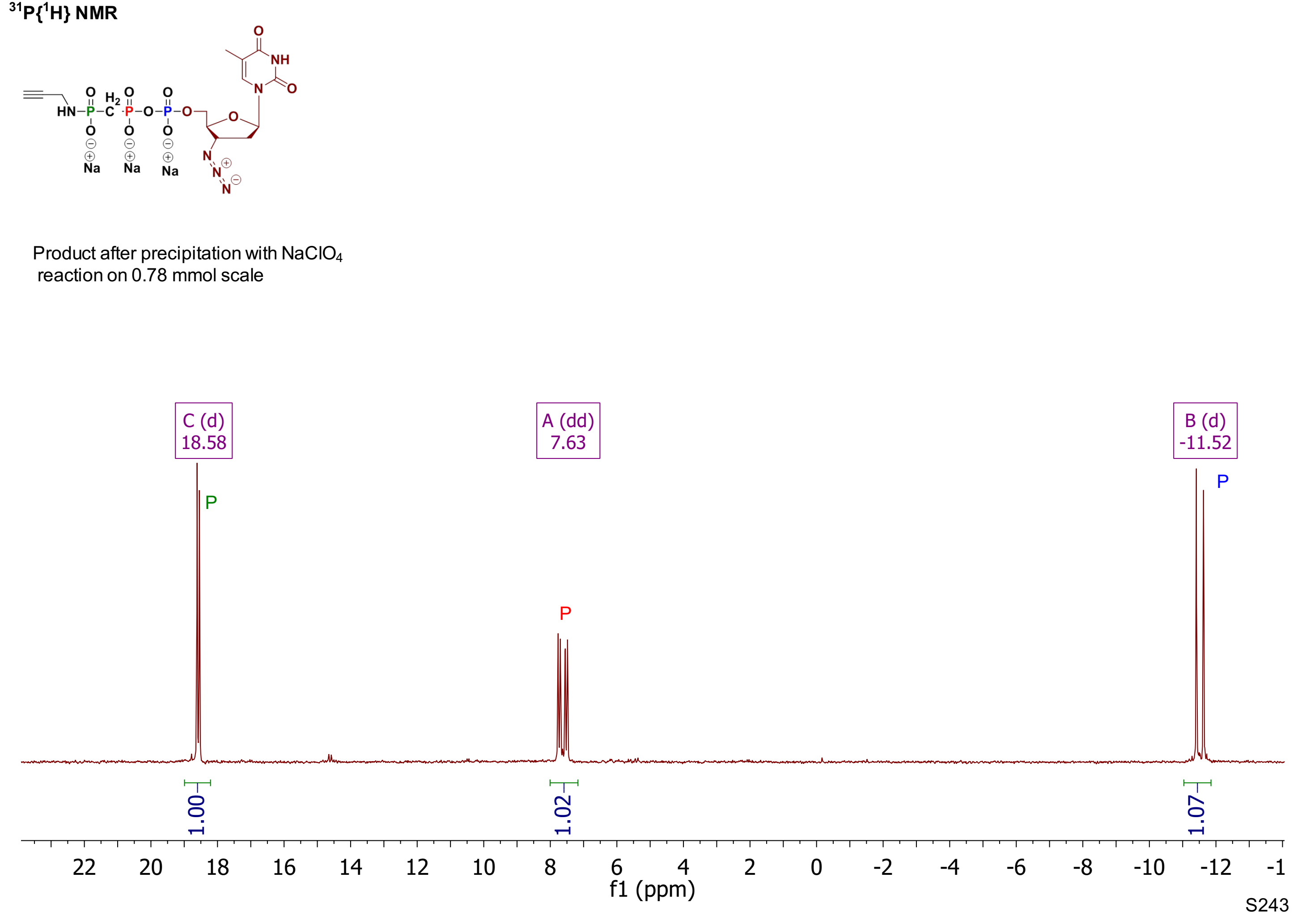




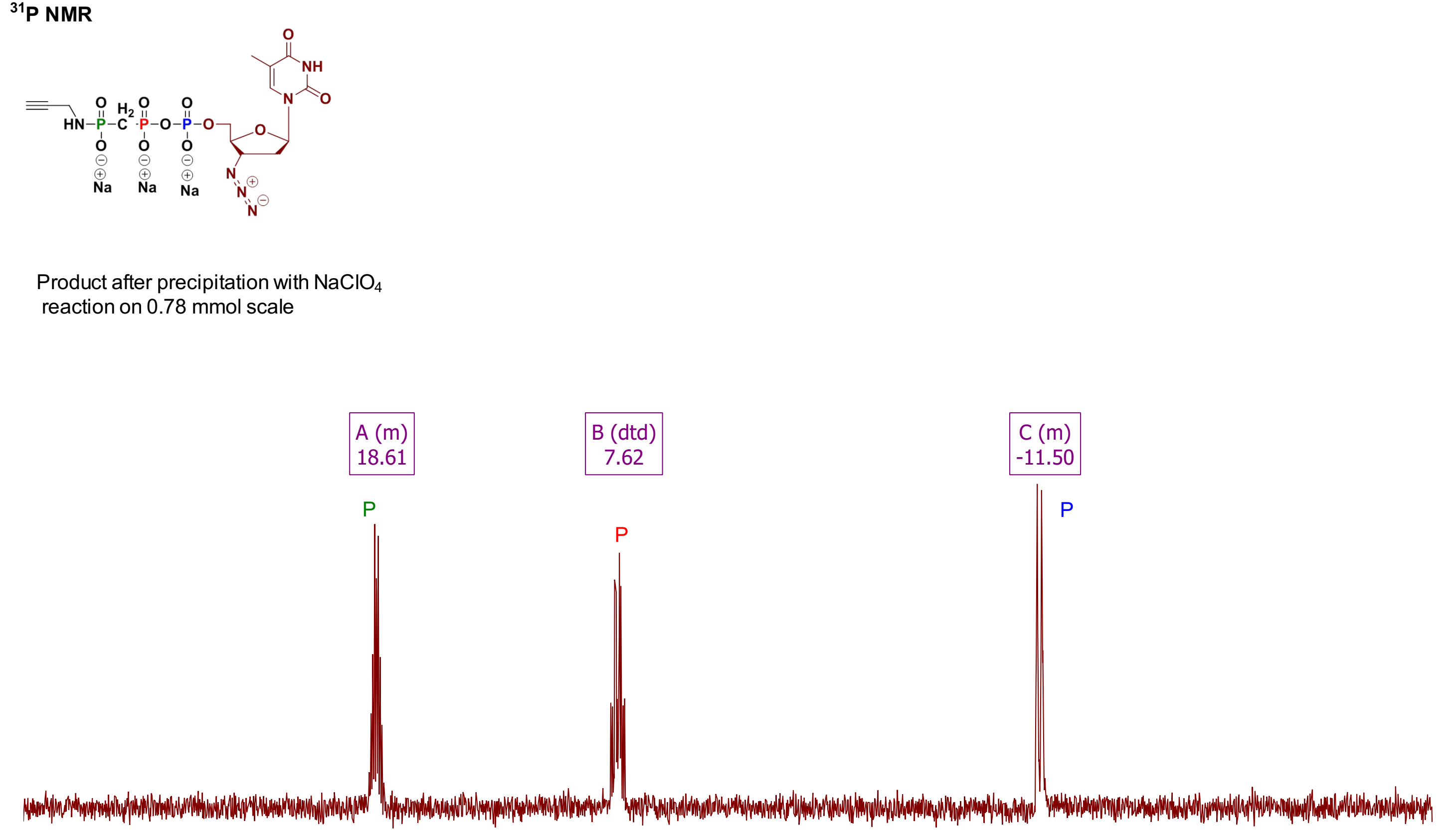




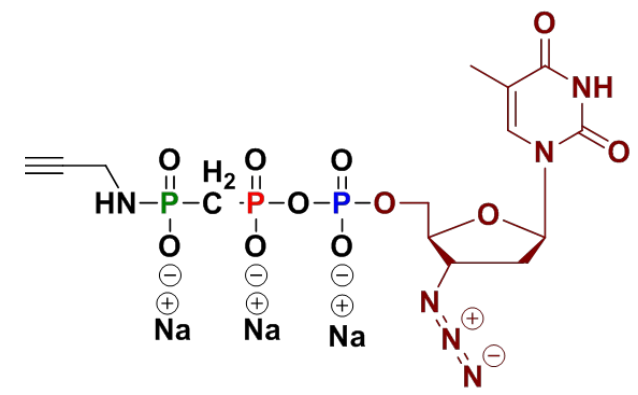

crude product after precipitation

\begin{tabular}{|c|c|c|}
\hline K (s) & J (s) & I (d) \\
166.81 & 151.88 & 137.26 \\
\hline
\end{tabular}

\begin{tabular}{|c|}
\hline$G(s)$ \\
84.87 \\
\hline$F(d)$ \\
82.97
\end{tabular}
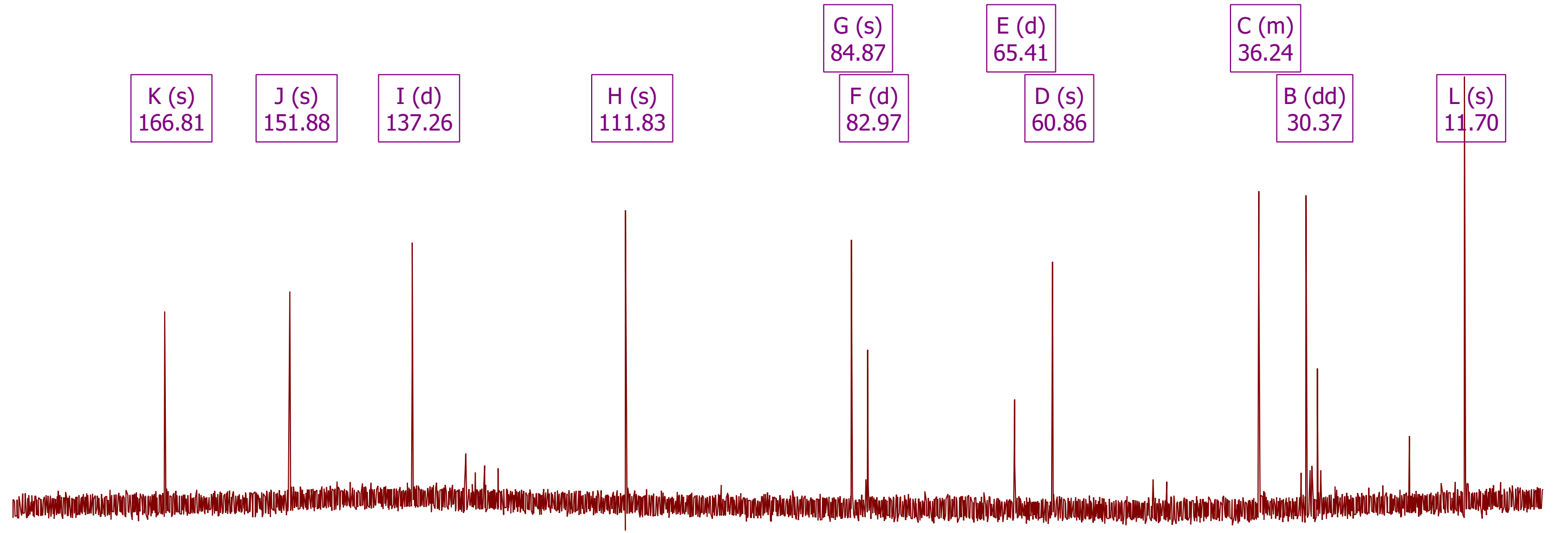

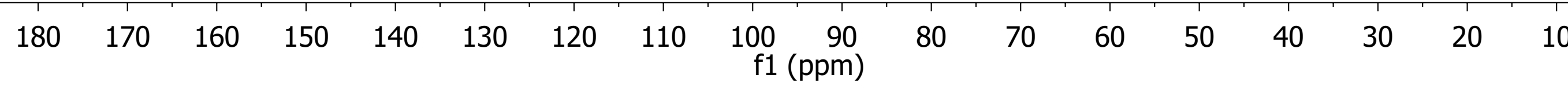




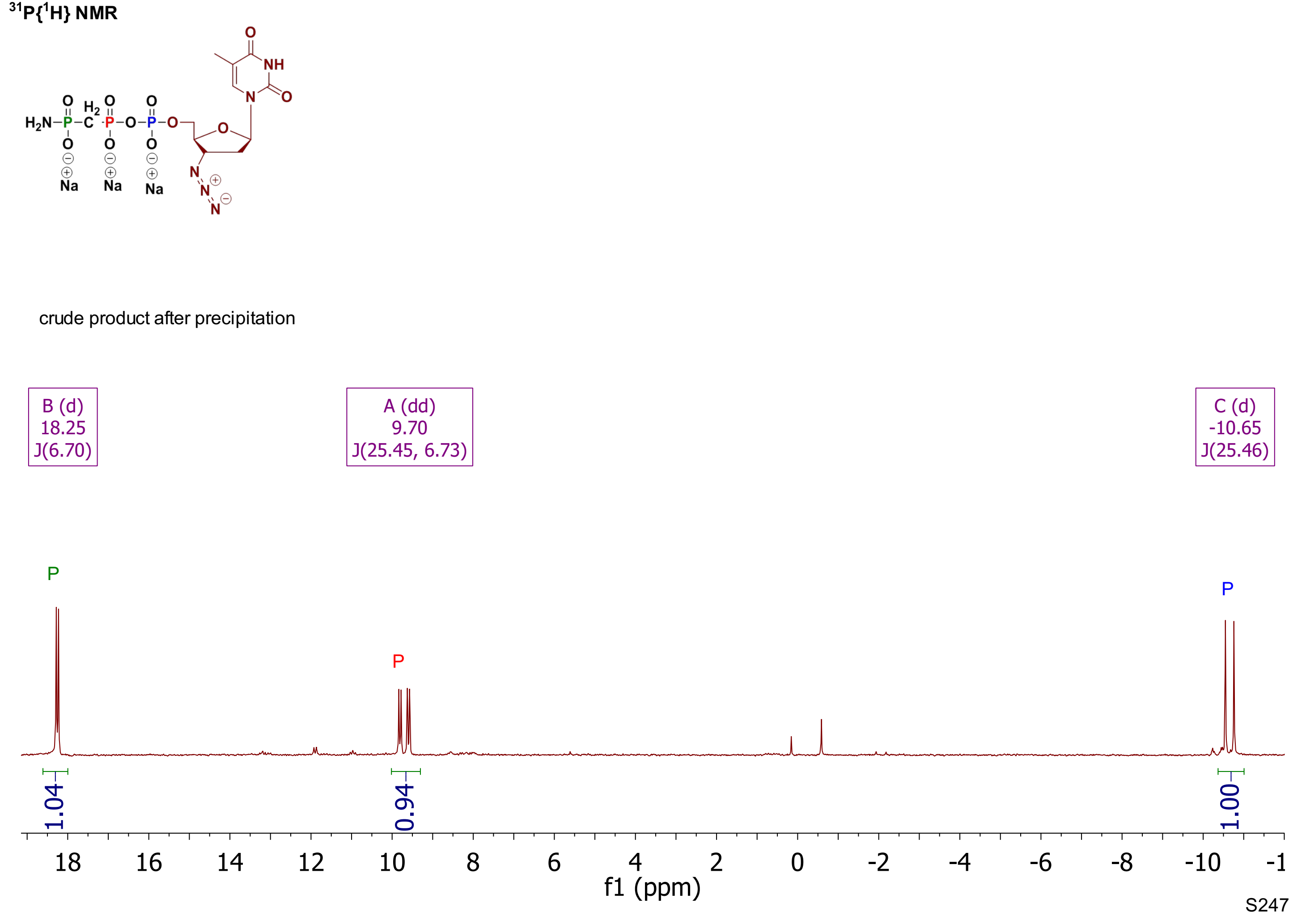




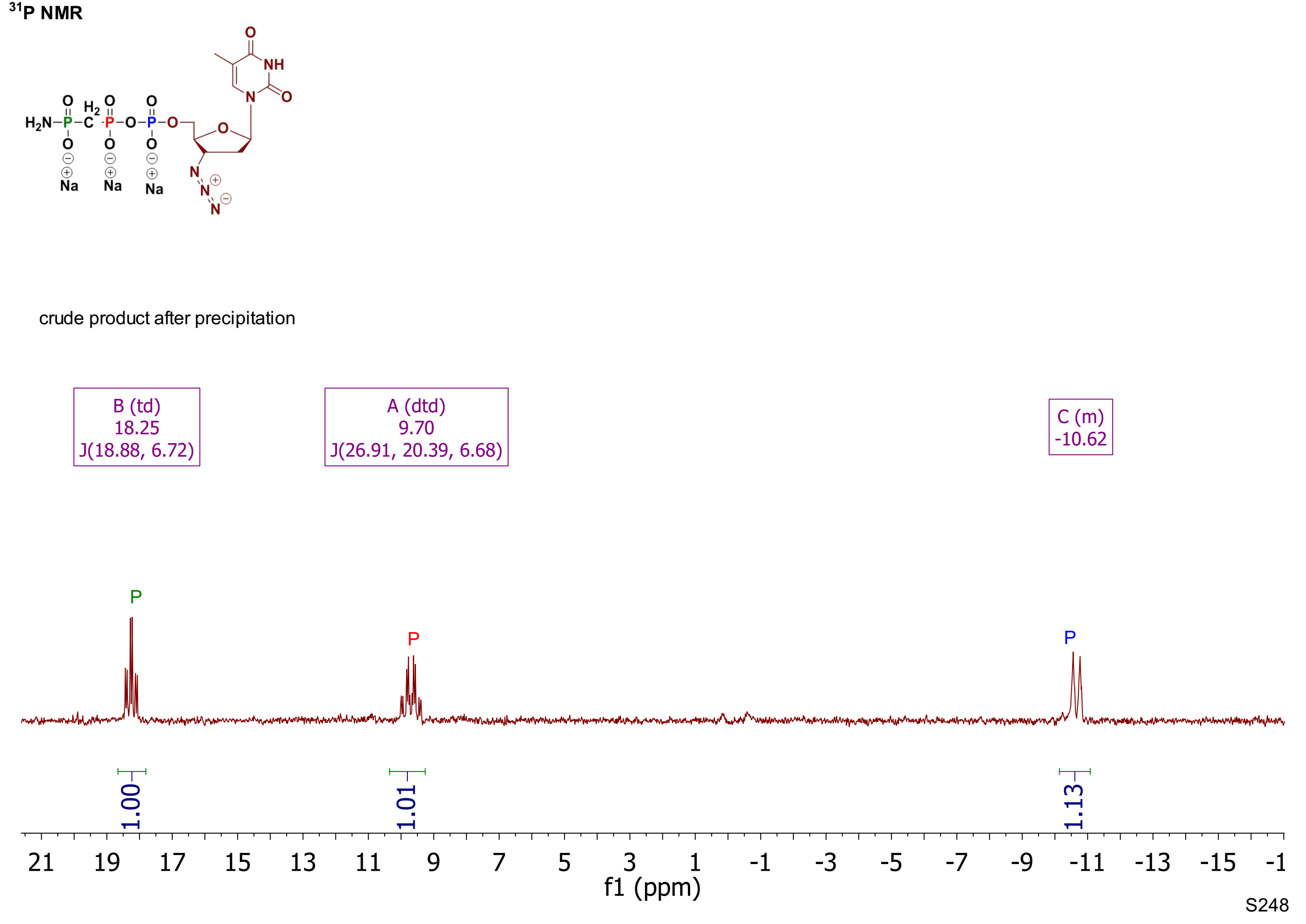




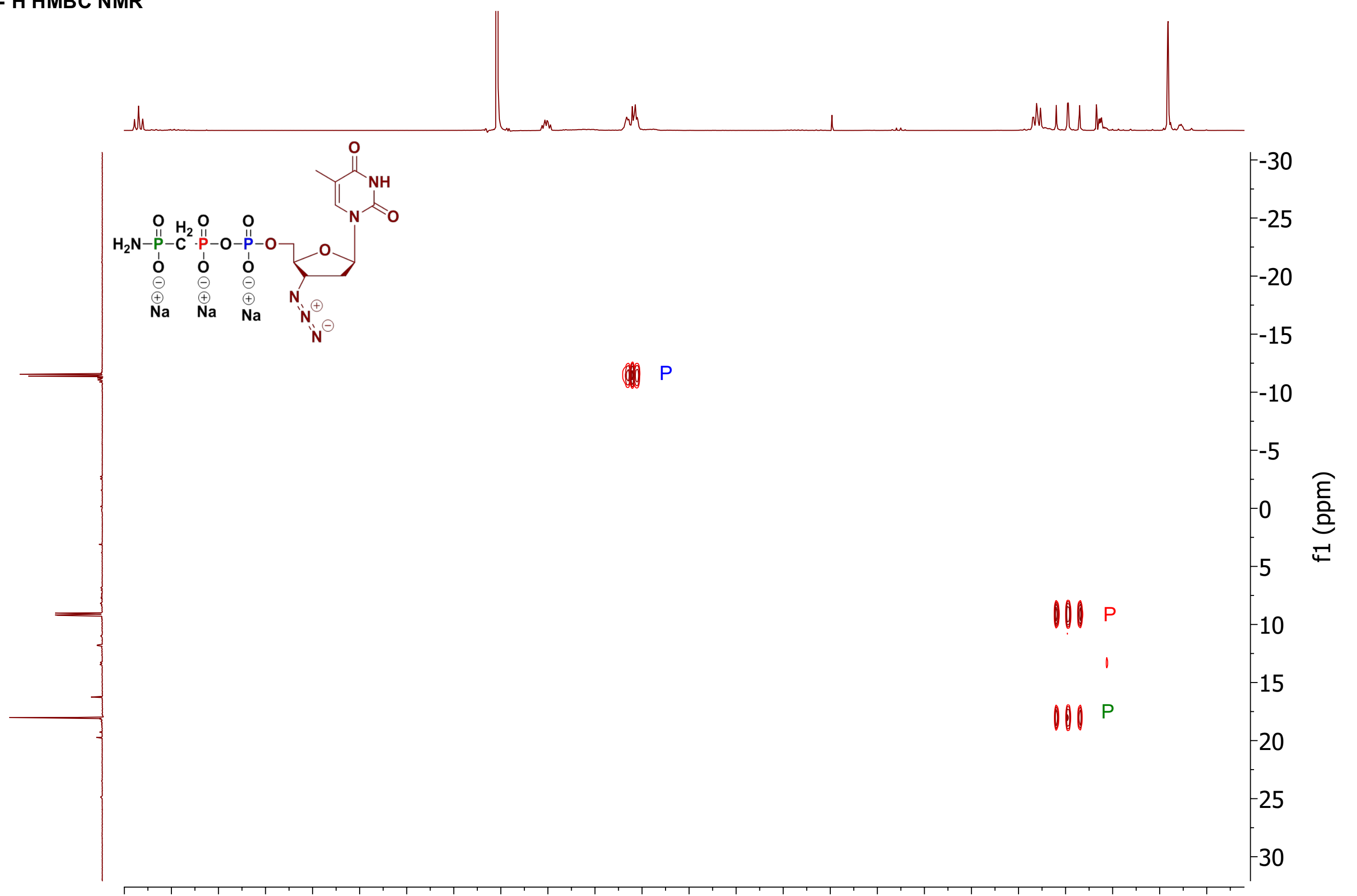




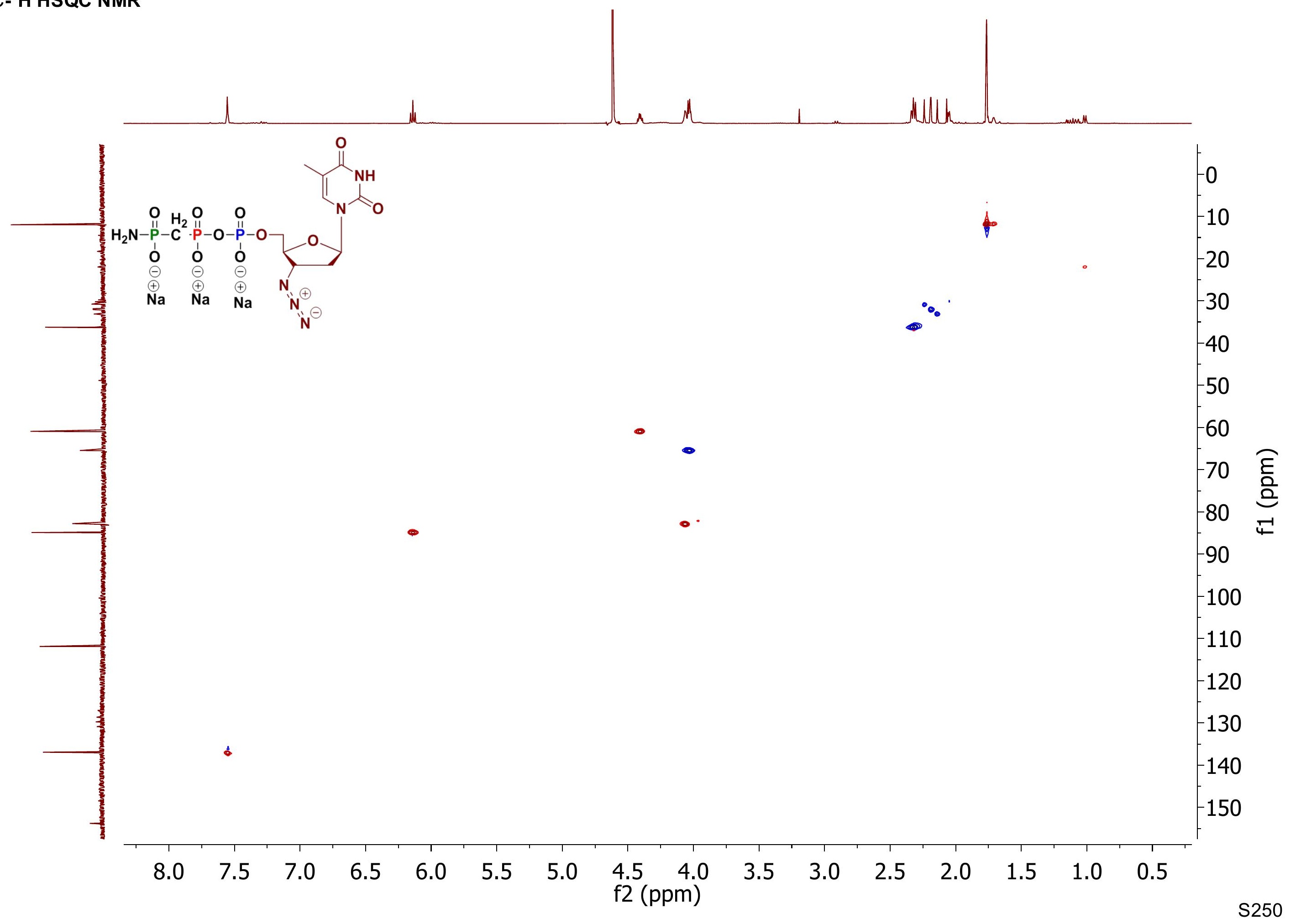




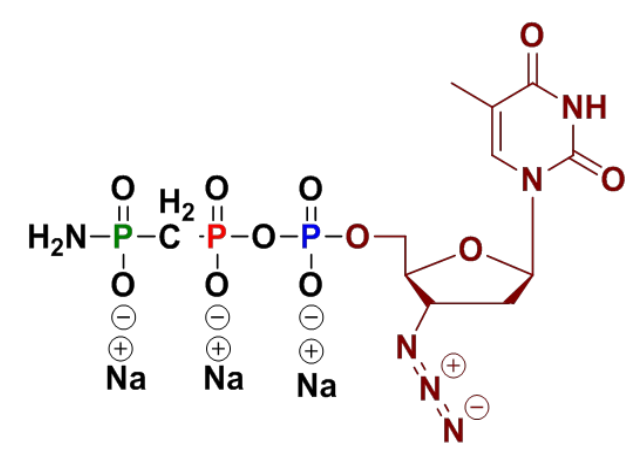

crude product after precipitation

\begin{tabular}{|c|c|c|}
\hline$C(s)$ & $A(s)$ & $B(s)$ \\
169.32 & 153.79 & 136.93 \\
\hline
\end{tabular}

\begin{tabular}{|c|}
\hline$F(d)$ \\
82.82 \\
\hline$E(s)$ \\
84.90 \\
\hline
\end{tabular}
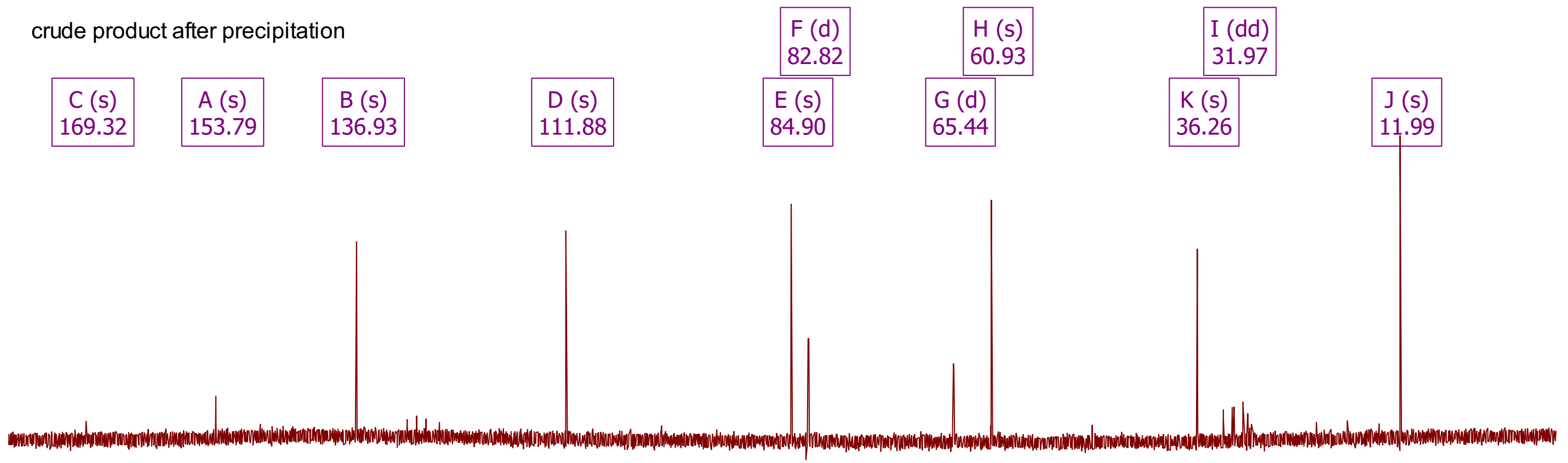

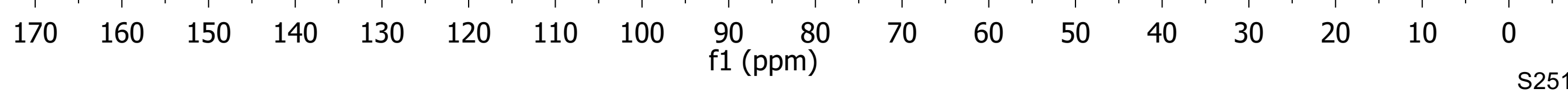




\section{${ }^{31} \mathrm{P}\left\{{ }^{1} \mathrm{H}\right\}$ NMR}

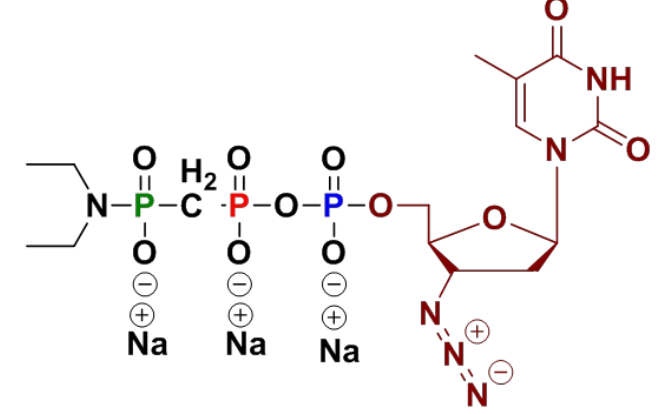

crude product after precipitation

\begin{tabular}{|c|}
\hline$C(d)$ \\
16.71 \\
\hline
\end{tabular}$\quad$\begin{tabular}{c|}
$B(d d)$ \\
10.16 \\
\hline
\end{tabular}

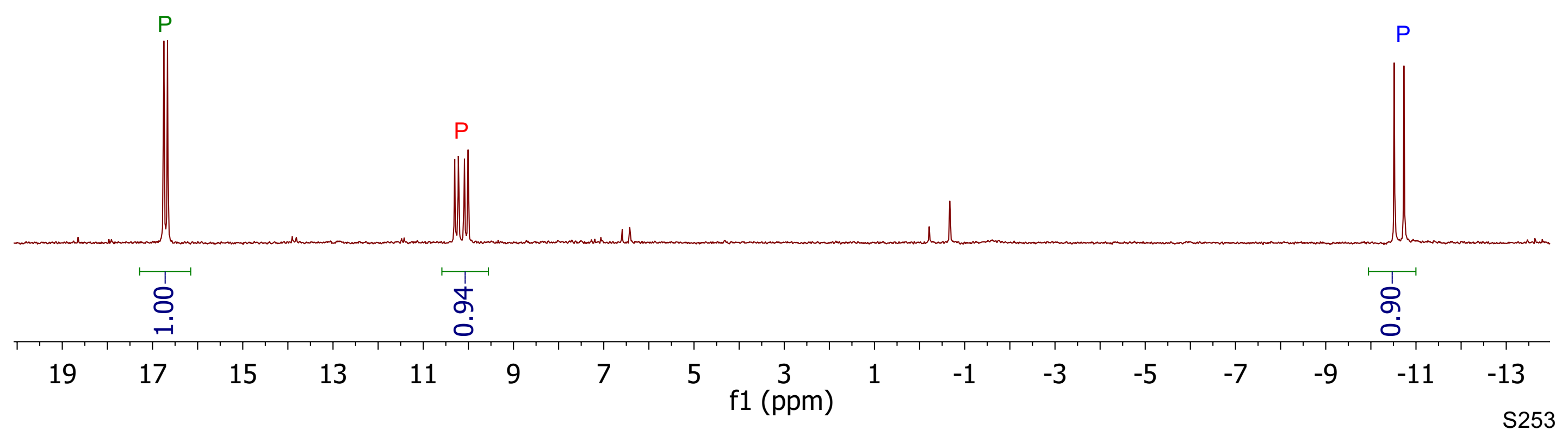




$$
{ }^{31} \text { P NMR }
$$

crude product after precipitation

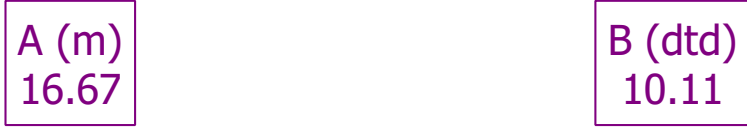

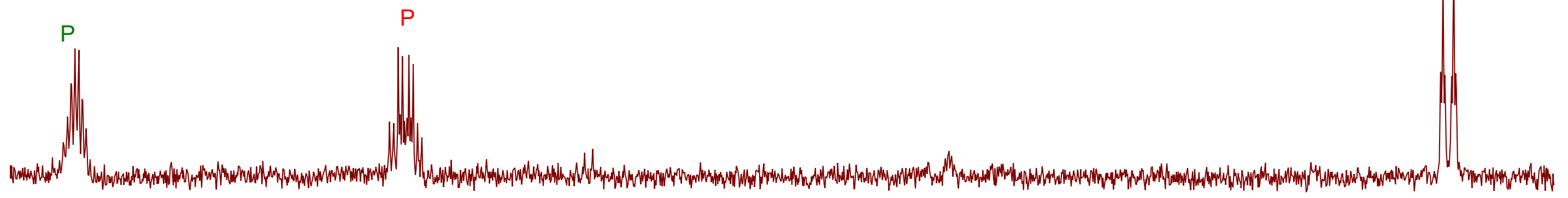

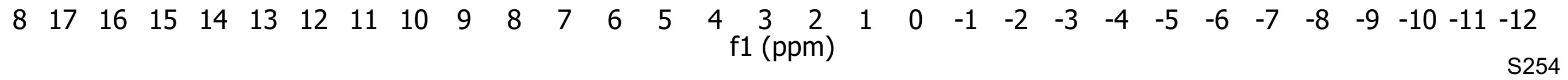




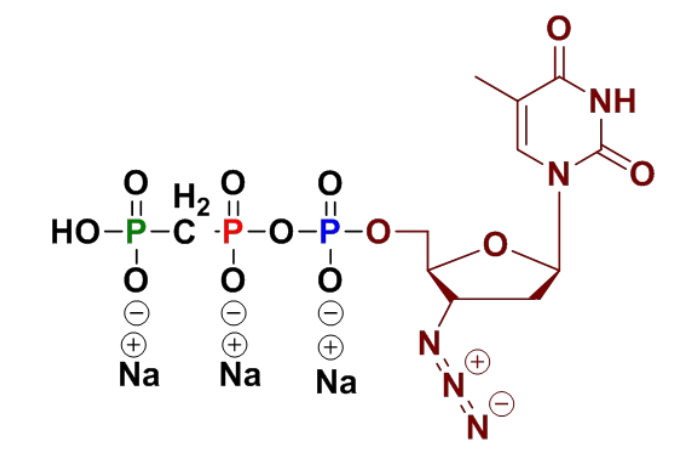

crude product after precipitation

$$
\begin{array}{|l|}
\hline \text { A (s) } \\
7.58 \\
\hline
\end{array}
$$
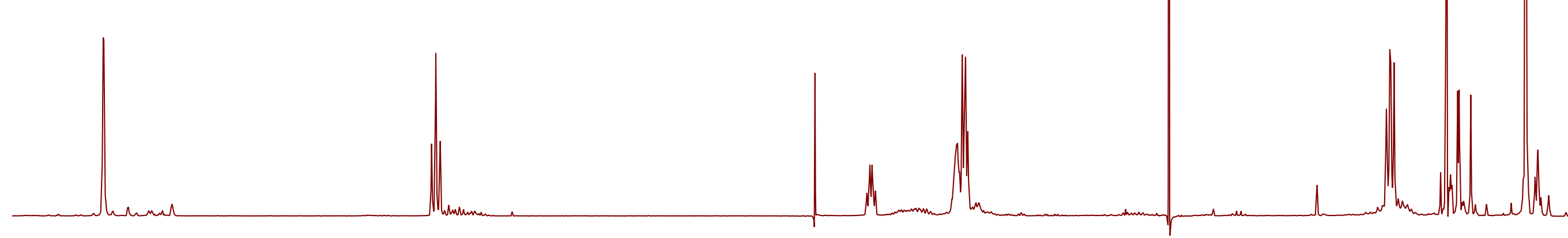

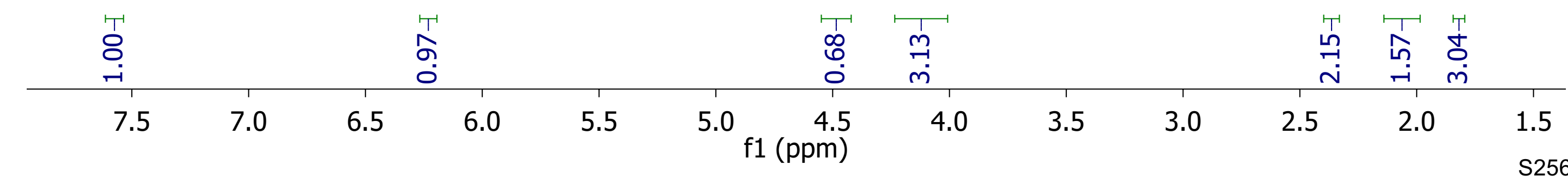

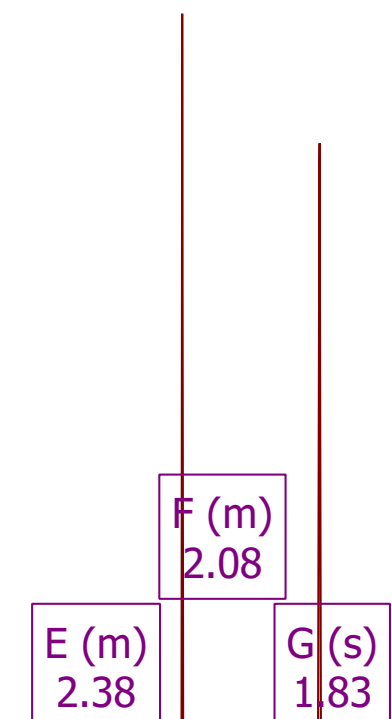




\section{${ }^{31} \mathrm{P}\left\{{ }^{1} \mathrm{H}\right\}$ NMR}

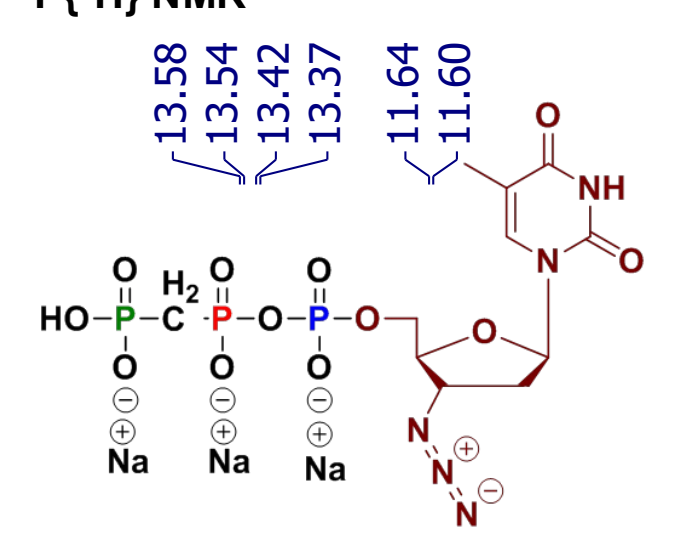

crude product after precipitation

\begin{tabular}{|c|c|}
\hline$A(d d)$ & $B(d)$ \\
13.48 & 11.62 \\
\hline
\end{tabular}

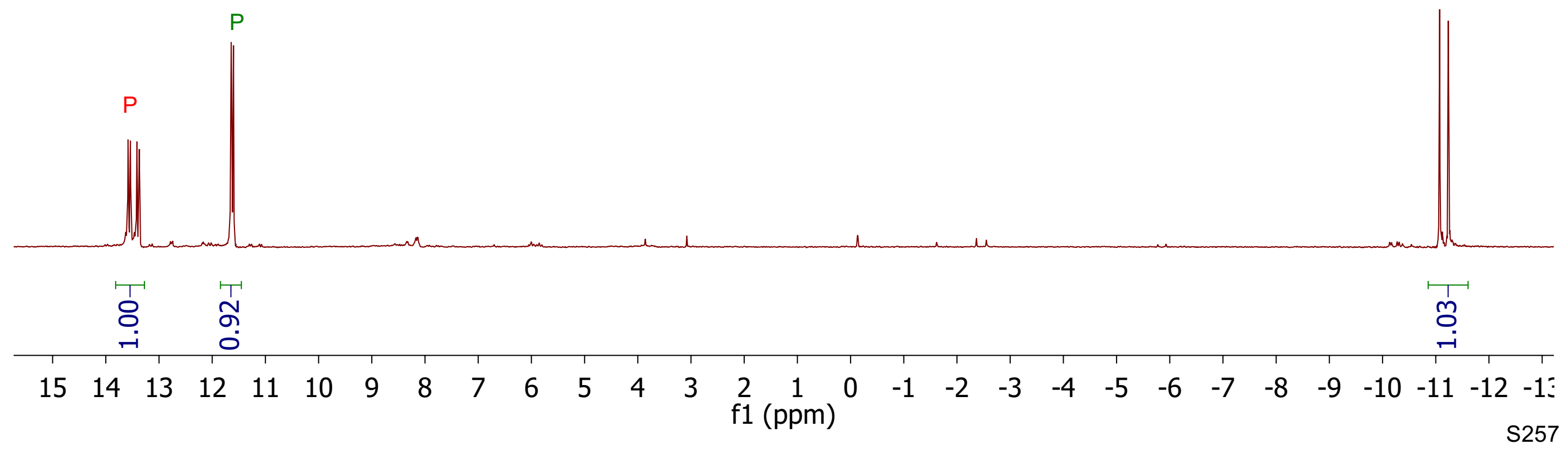




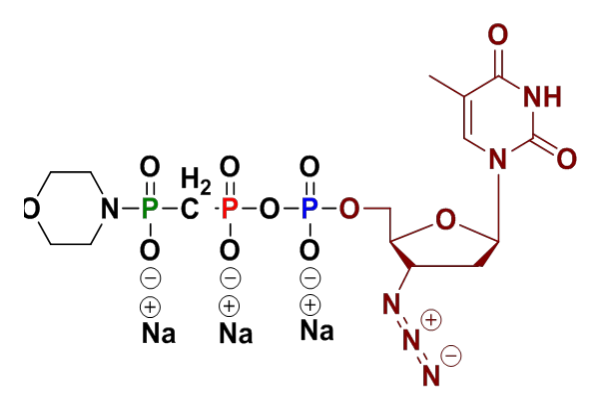

crude product after precipitation

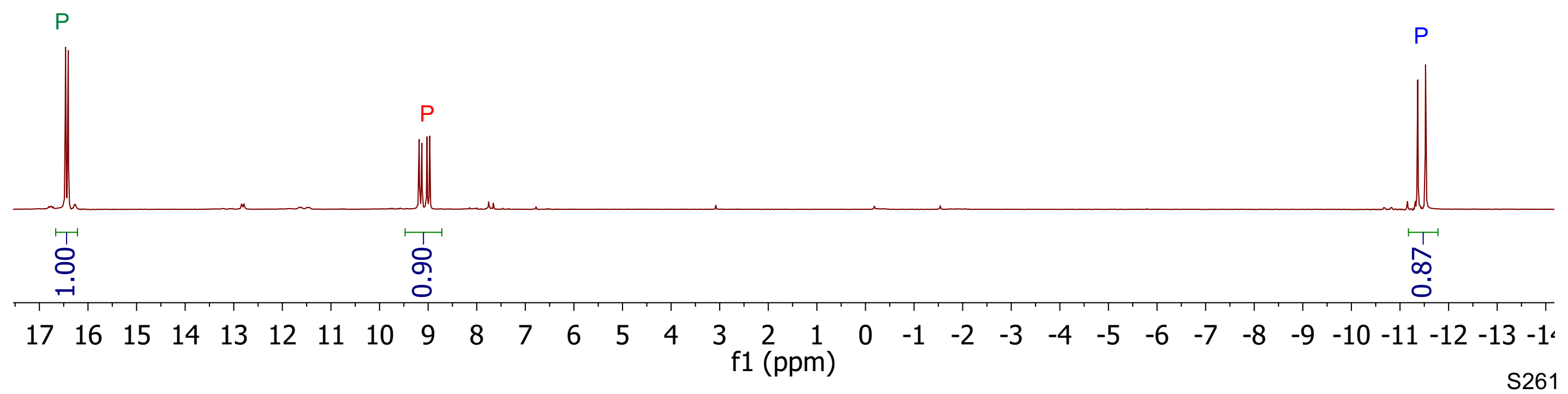




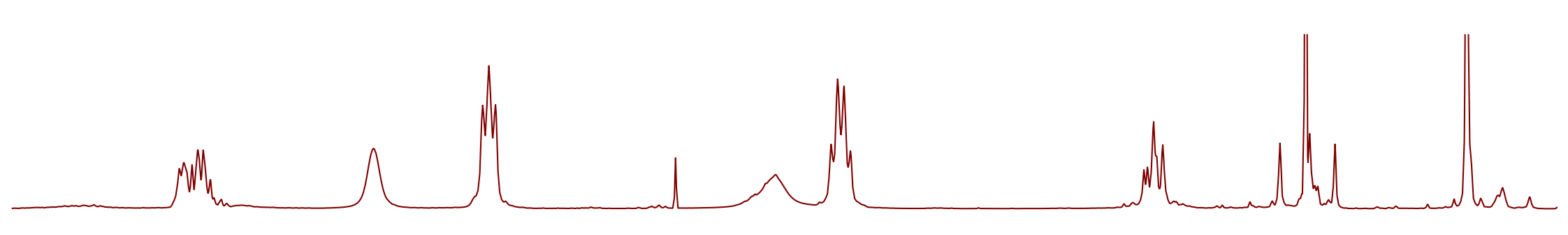

P

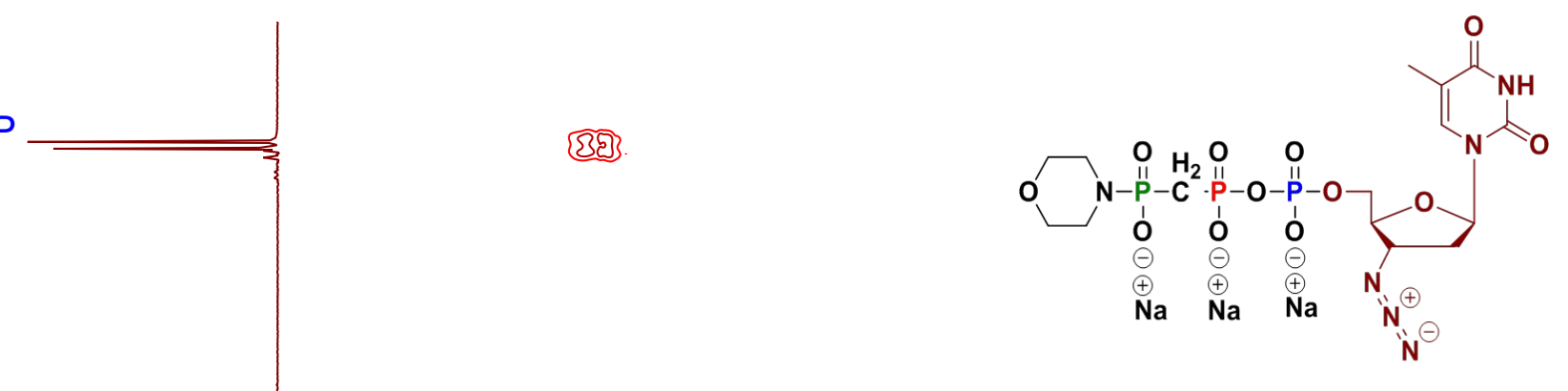

$-14$

$-12$

$-10$

$-8$

$-6$

$-4$

$-2$

$-0$

-2 छิ

4 든

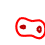

() 


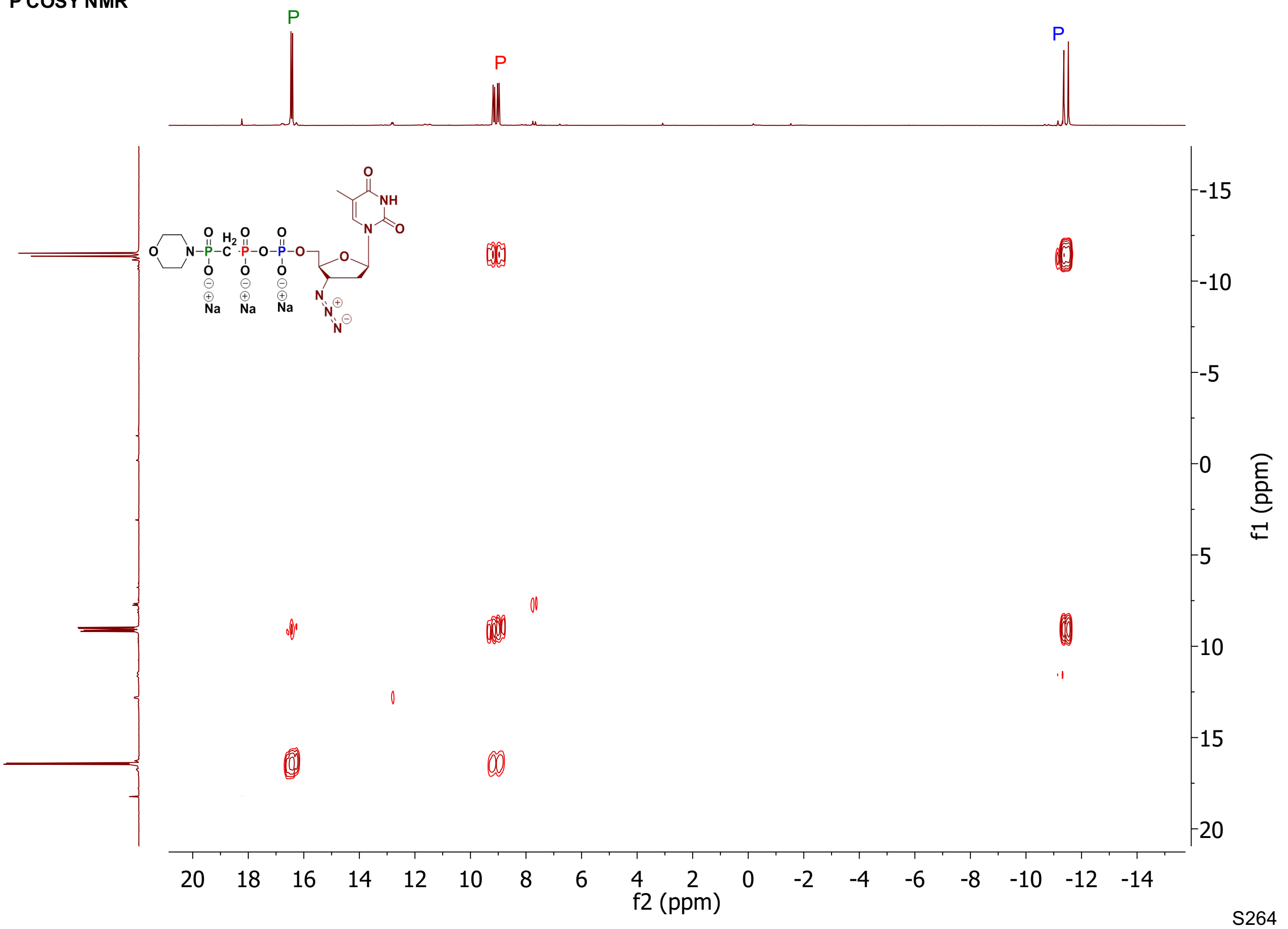




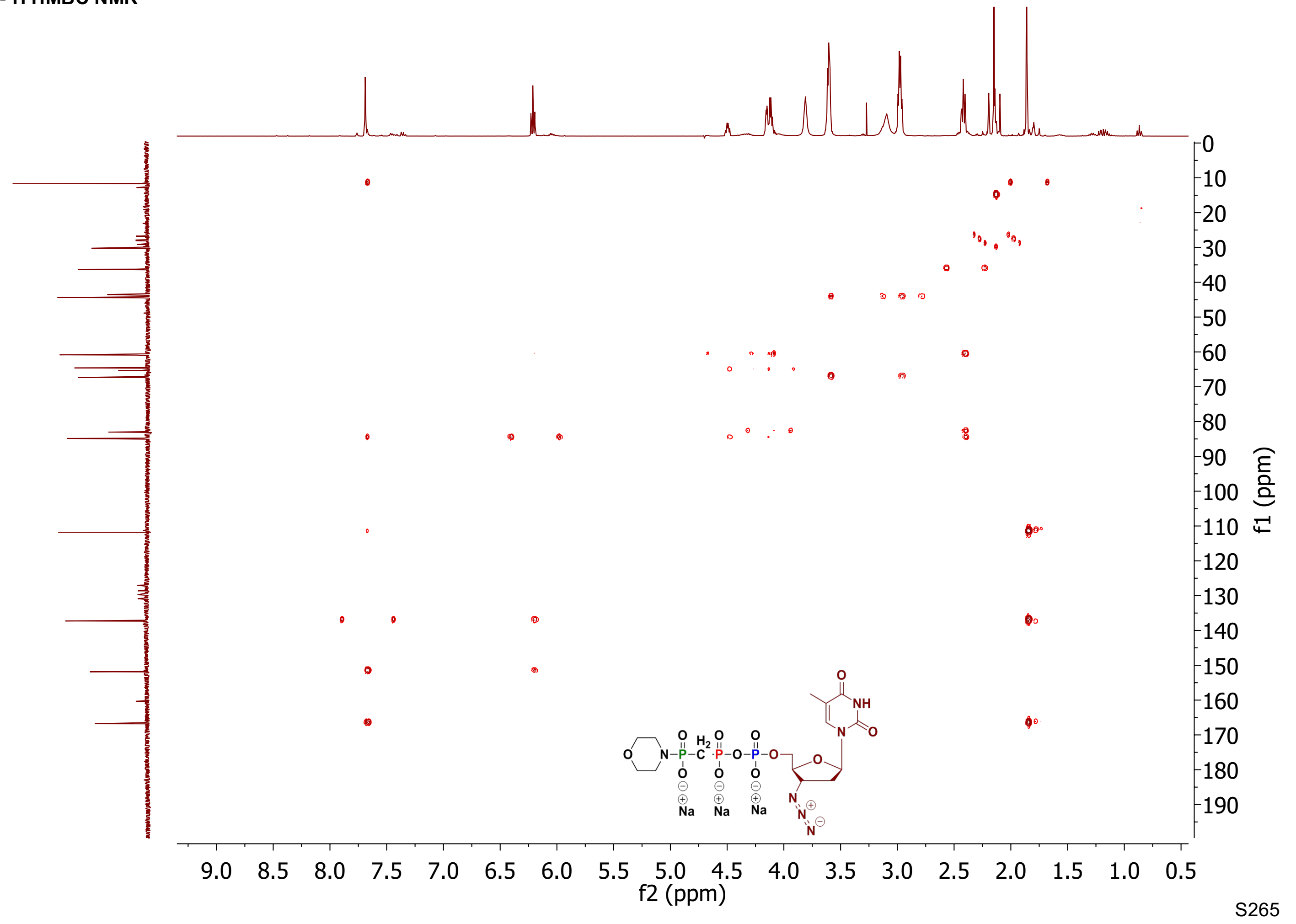




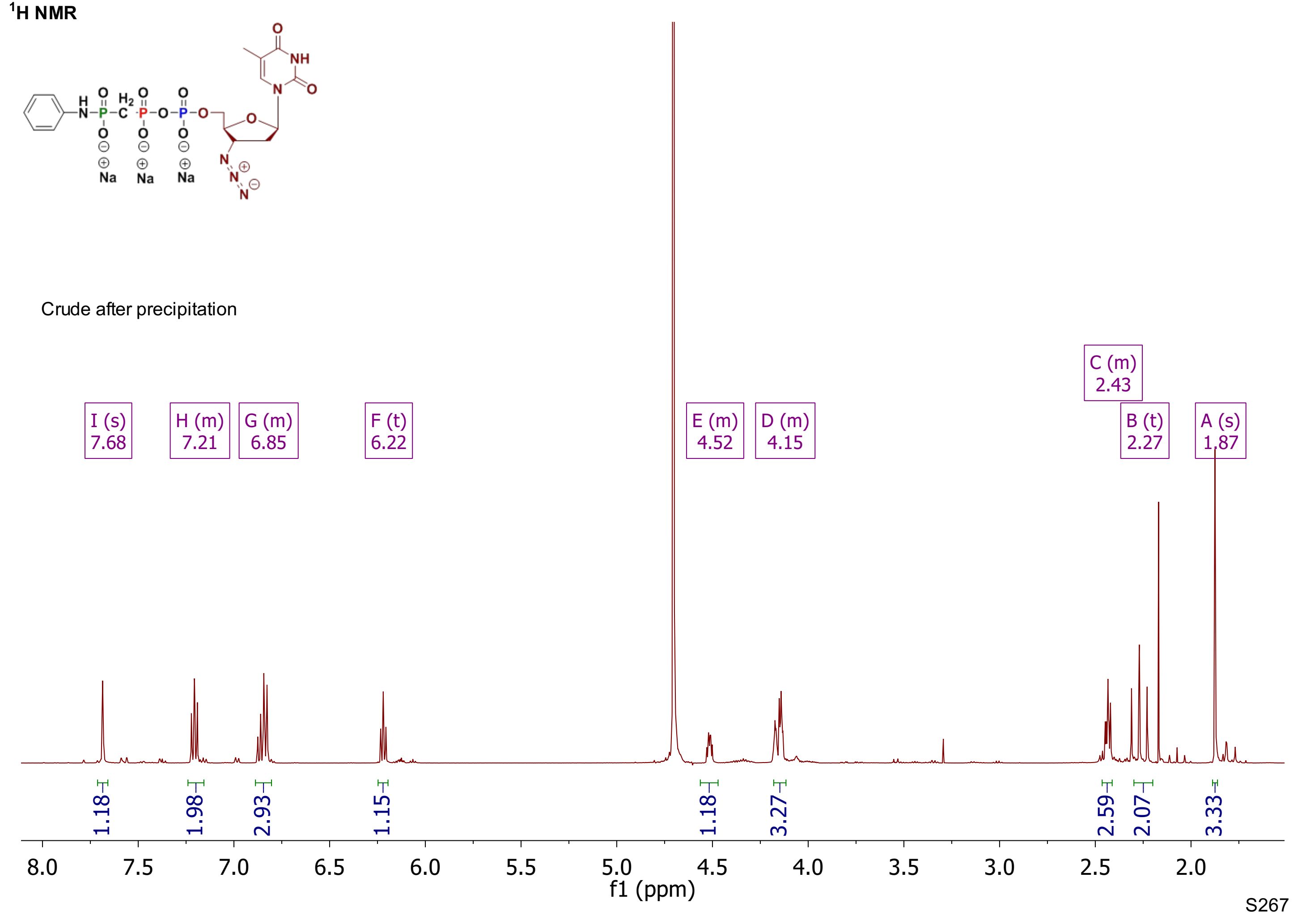




$$
{ }^{31} \mathrm{P}\left\{{ }^{1} \mathrm{H}\right\} \text { NMR }
$$

crude product after precipitation

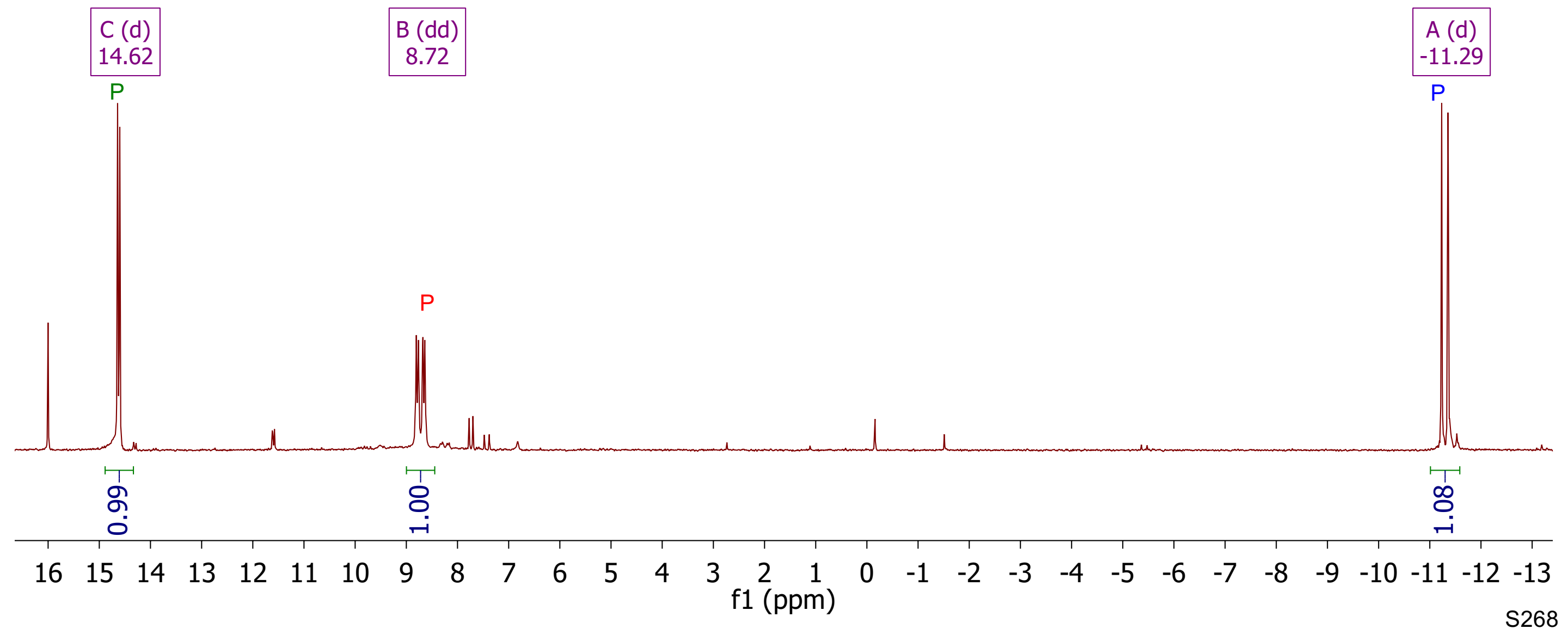




$$
{ }^{31} \mathbf{P} \text { NMR }
$$

crude product after precipitation

\begin{tabular}{|c|c|}
\hline$A(t d)$ & $B(d t d)$ \\
14.62 & 8.72 \\
\hline
\end{tabular}

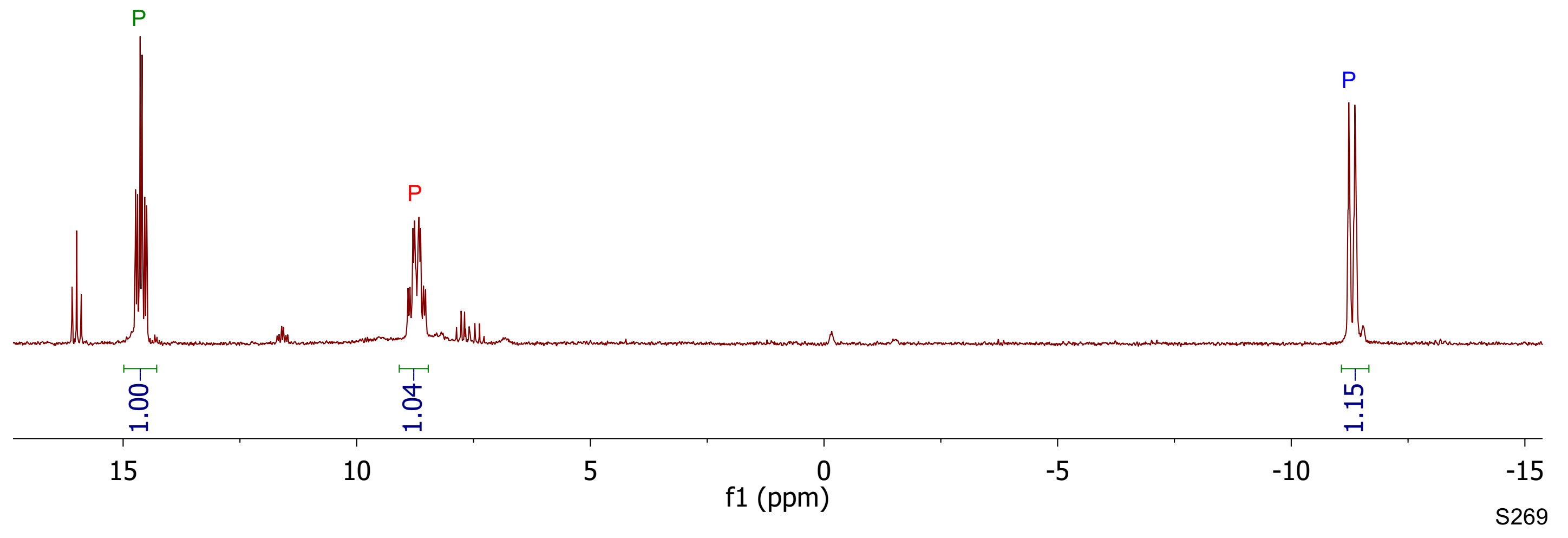




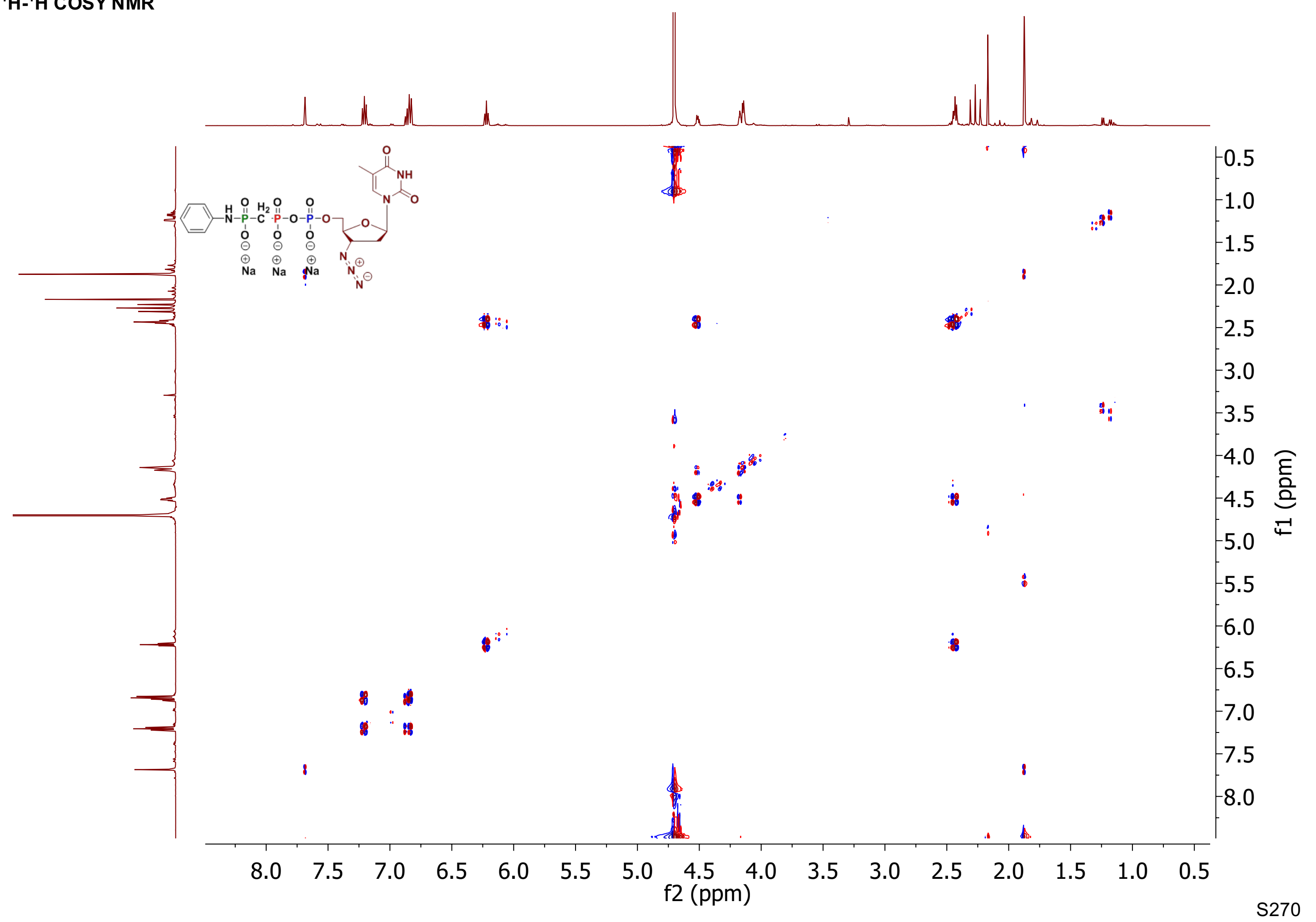




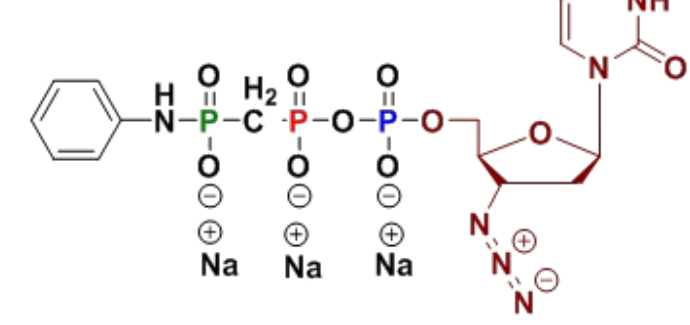

crude product after precipitation

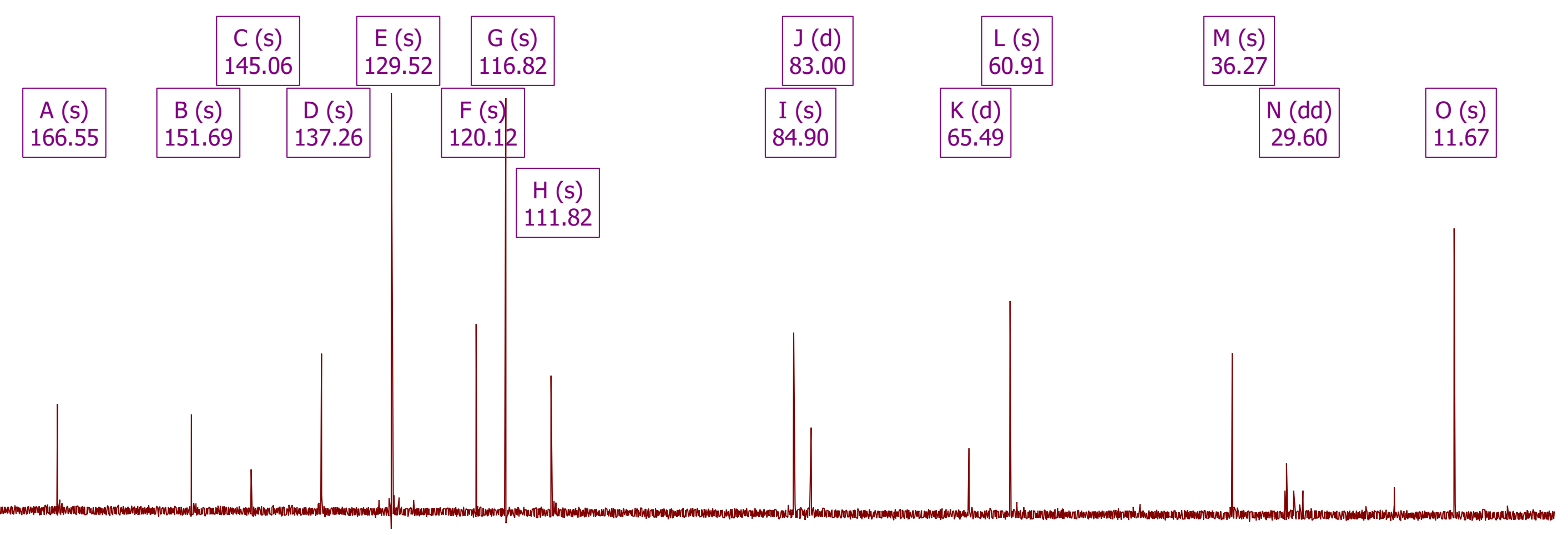

\begin{tabular}{|c|c|c|c|c|c|c|c|c|c|c|c|c|c|c|c|}
\hline & 160 & 150 & 140 & 130 & 120 & 110 & 100 & $\left.{ }_{\mathrm{f1}}^{90}{ }^{80} \mathrm{ppm}\right)^{80}$ & 70 & 60 & 50 & 40 & 30 & 20 & 10 \\
\hline
\end{tabular}




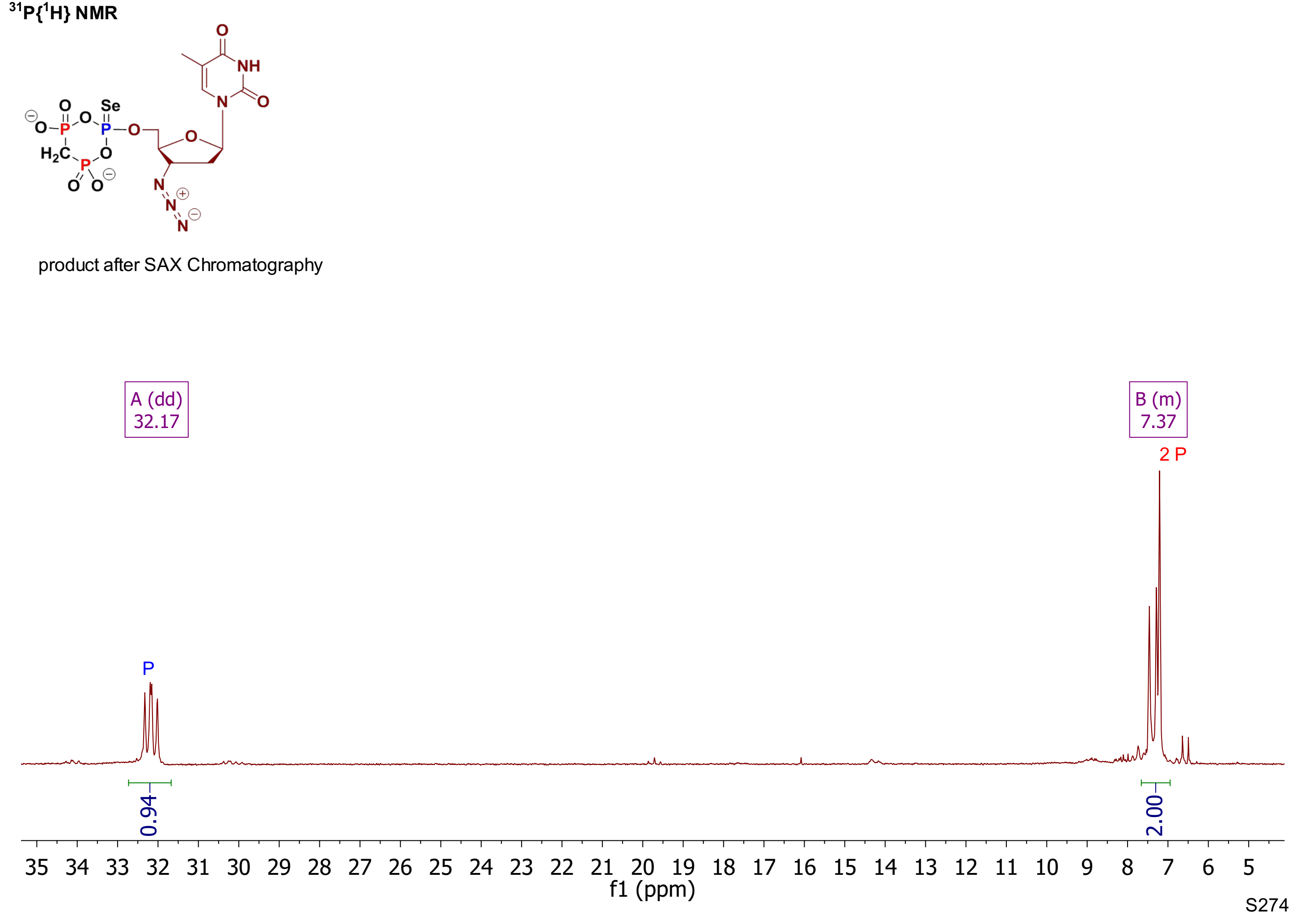




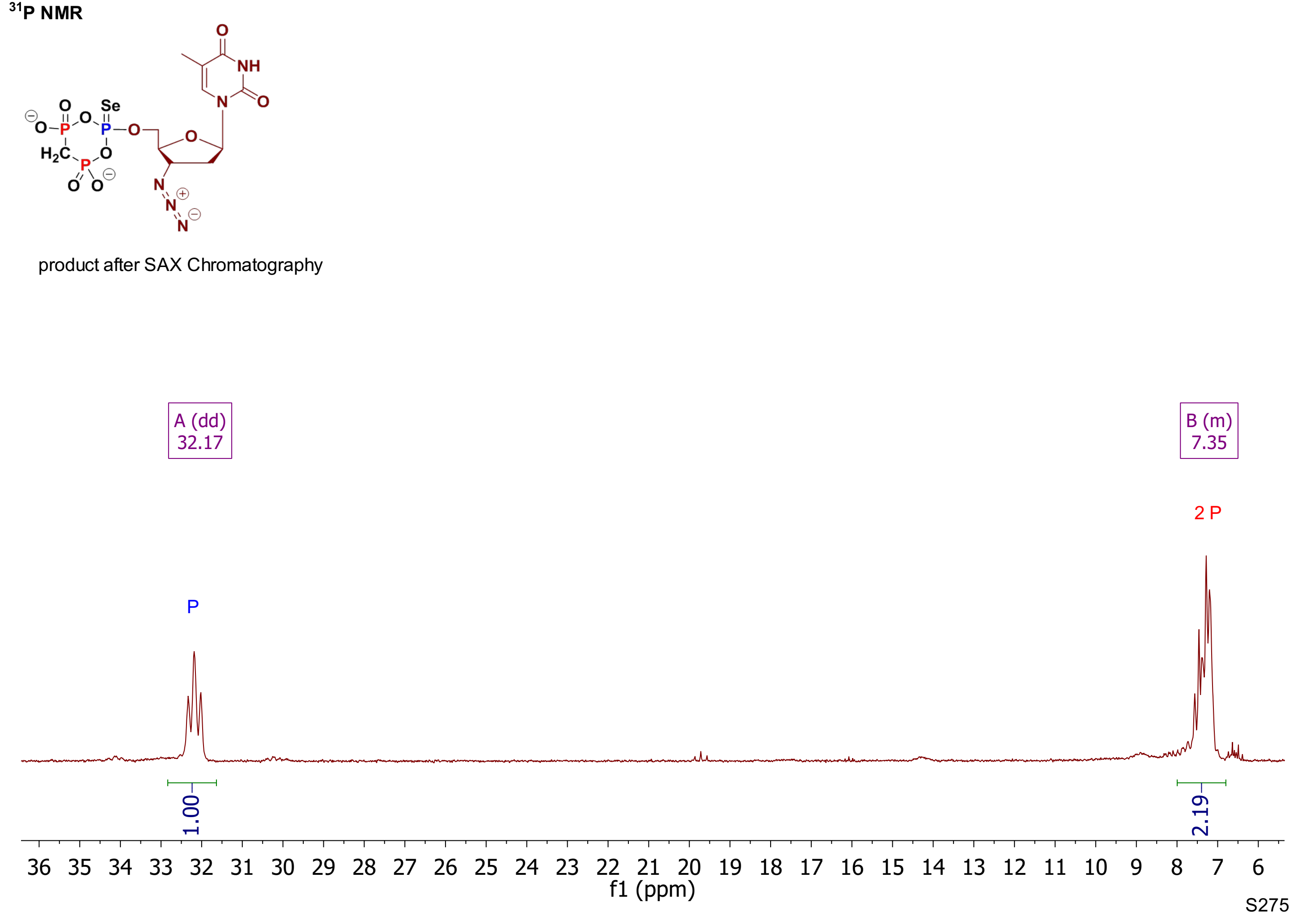




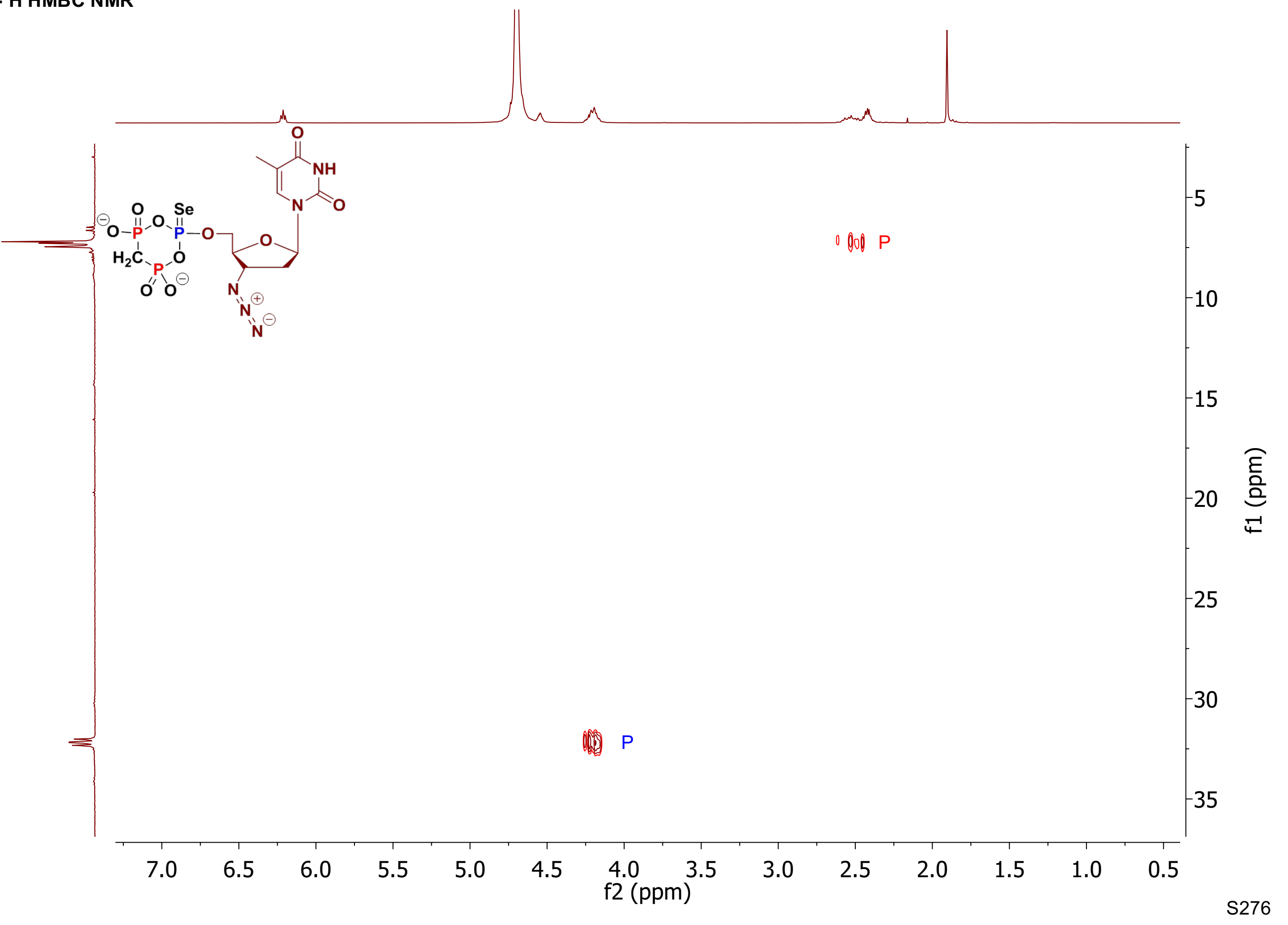




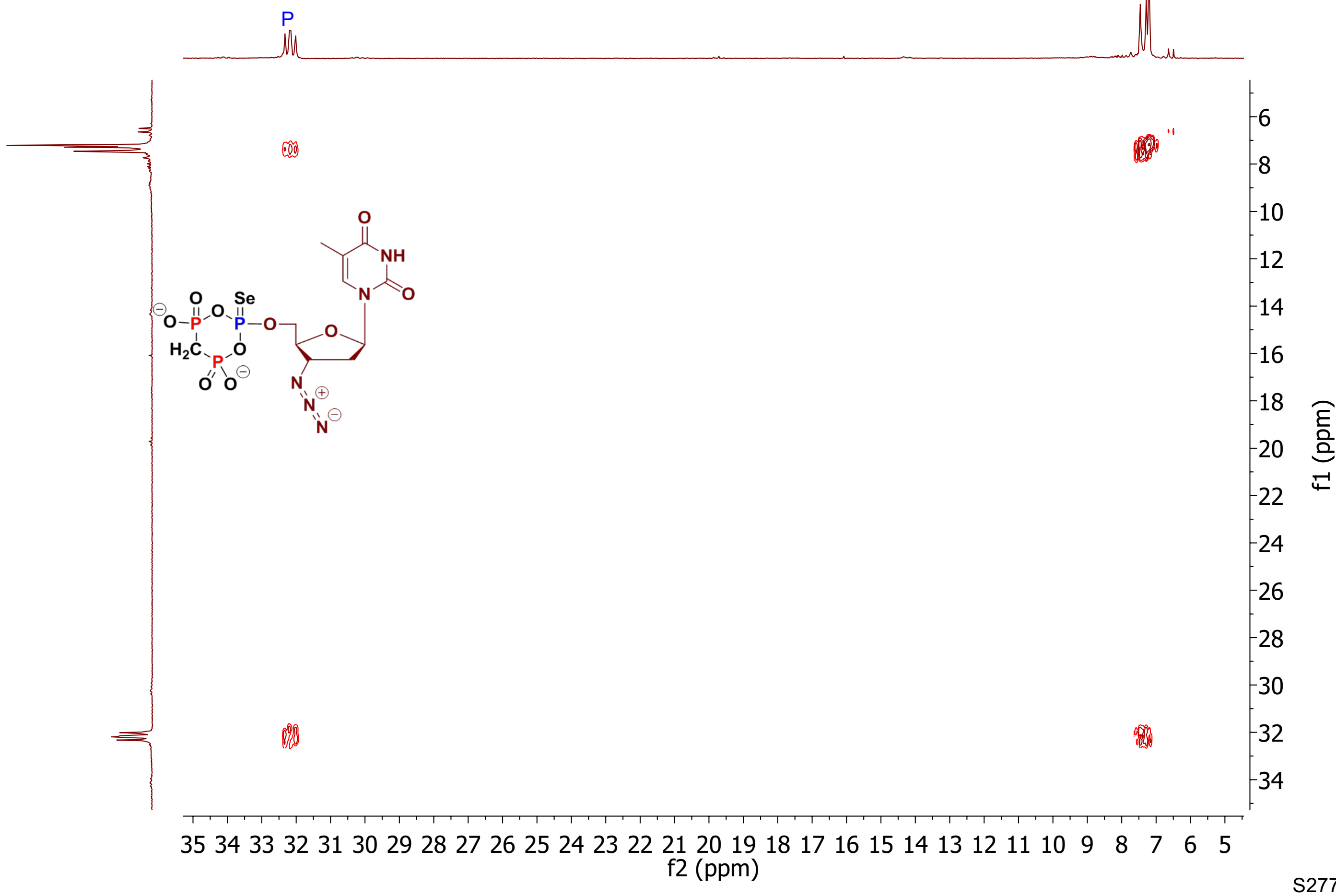




\section{${ }^{1} \mathrm{H}-{ }^{1} \mathrm{H}$ HMBC NMR}

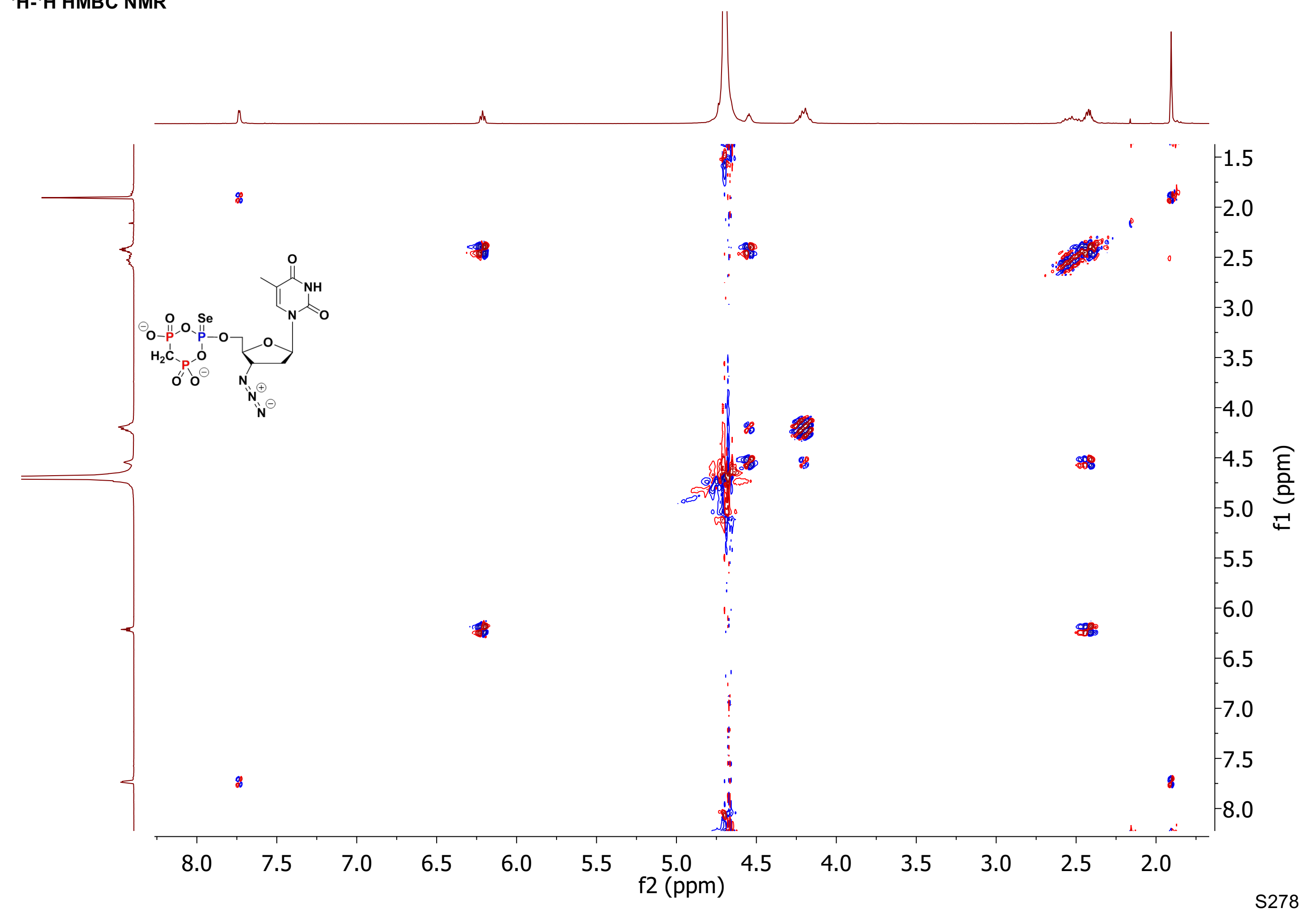




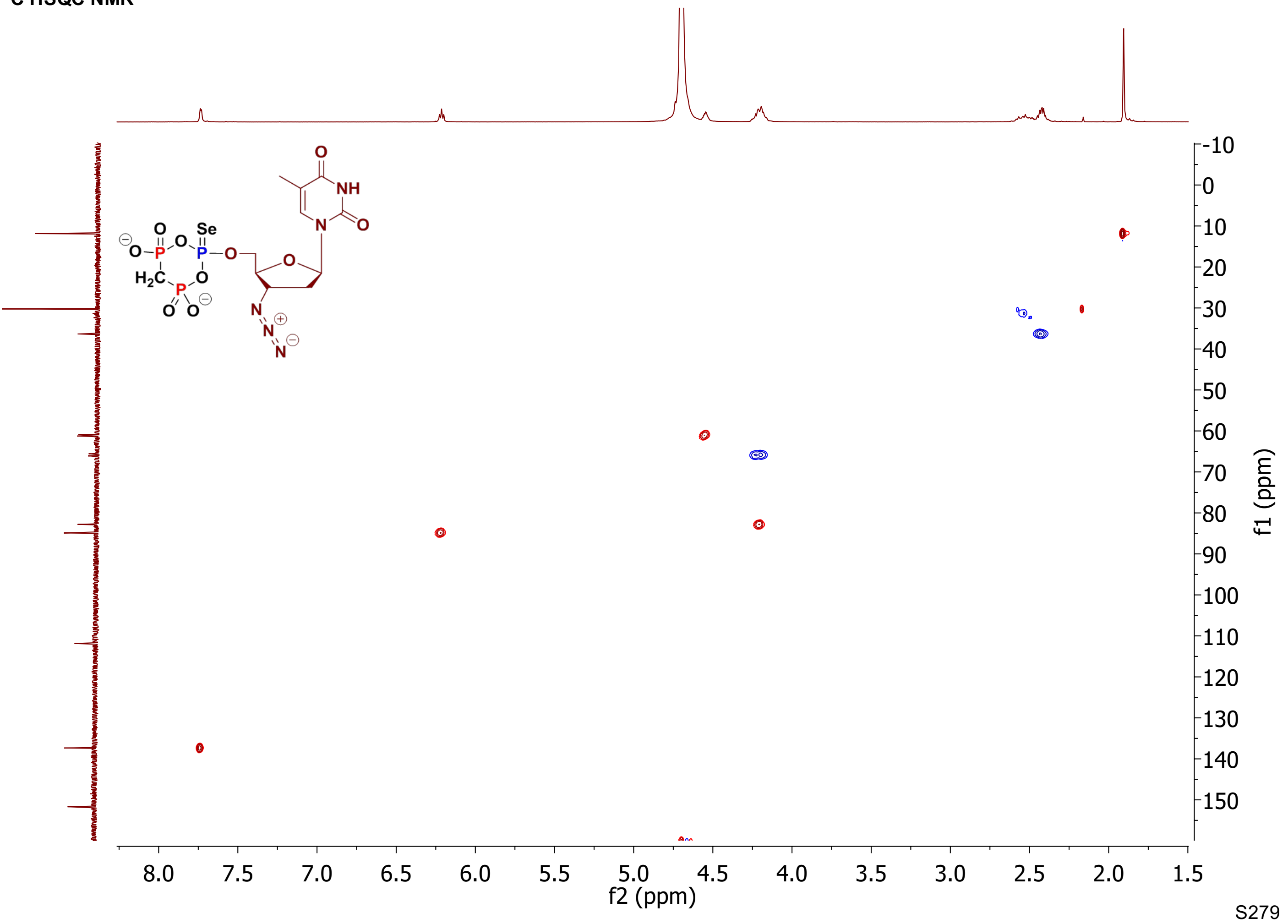




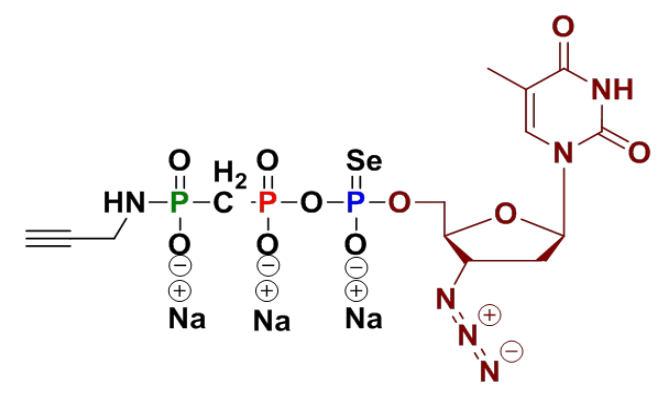

product after SAX Chromatography
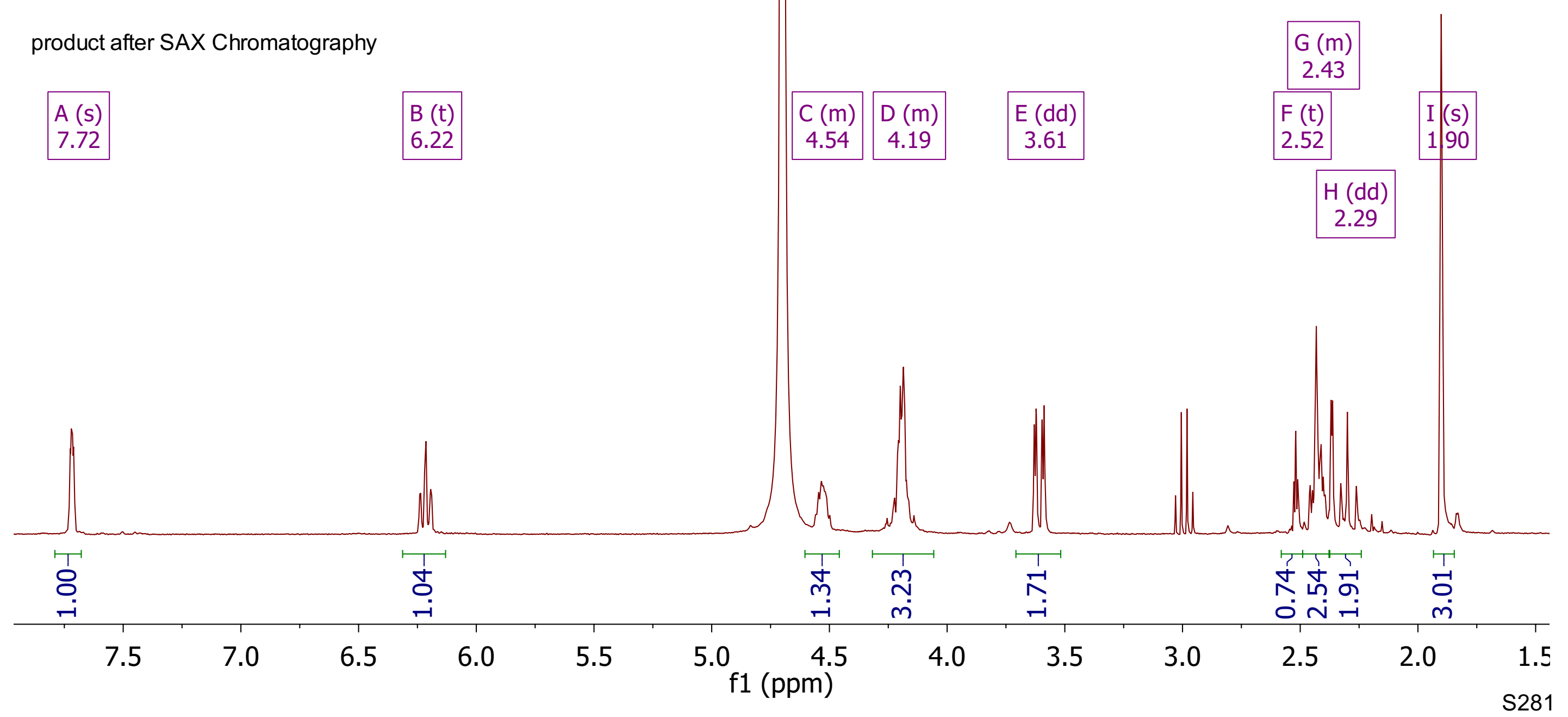


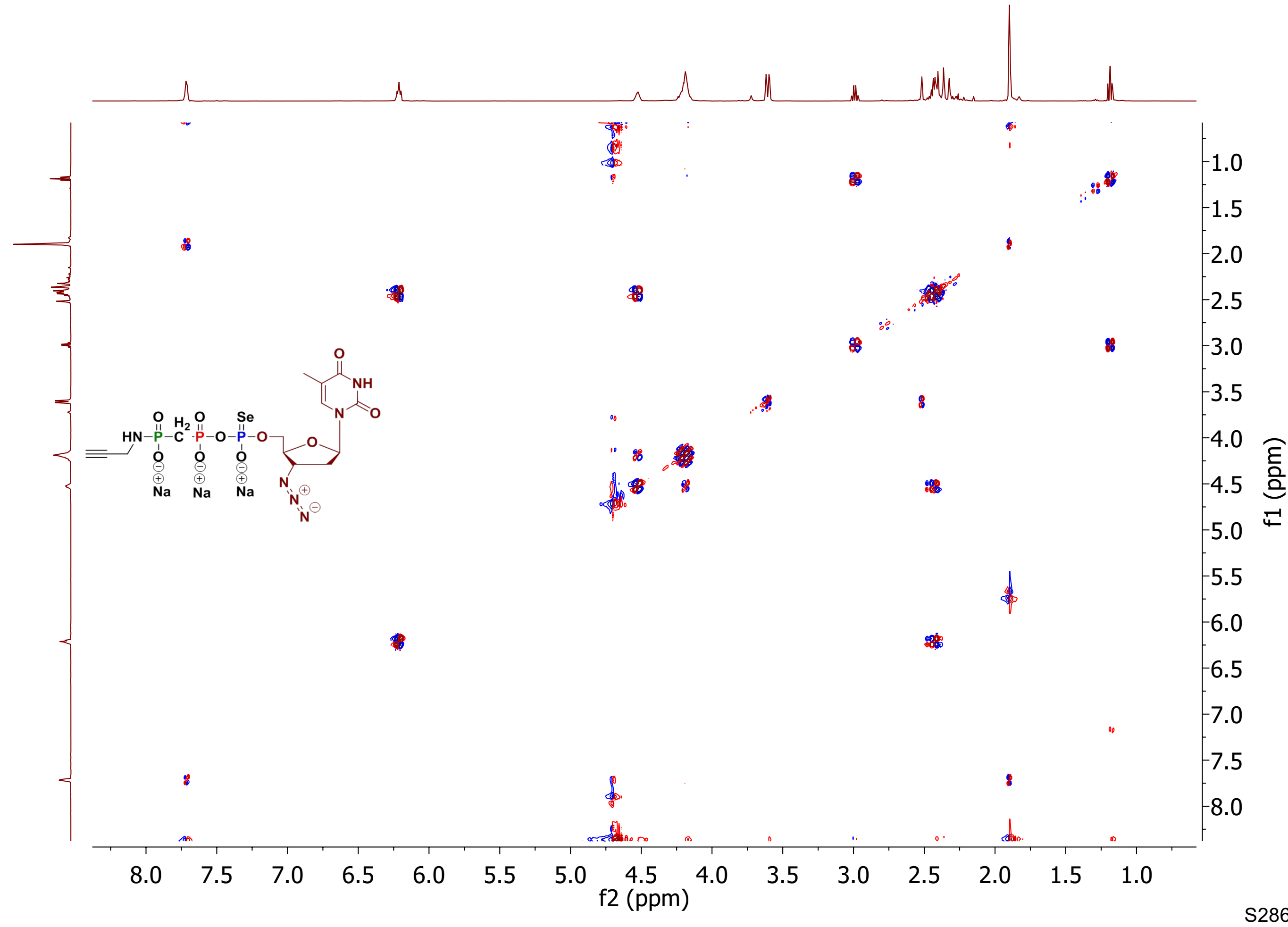




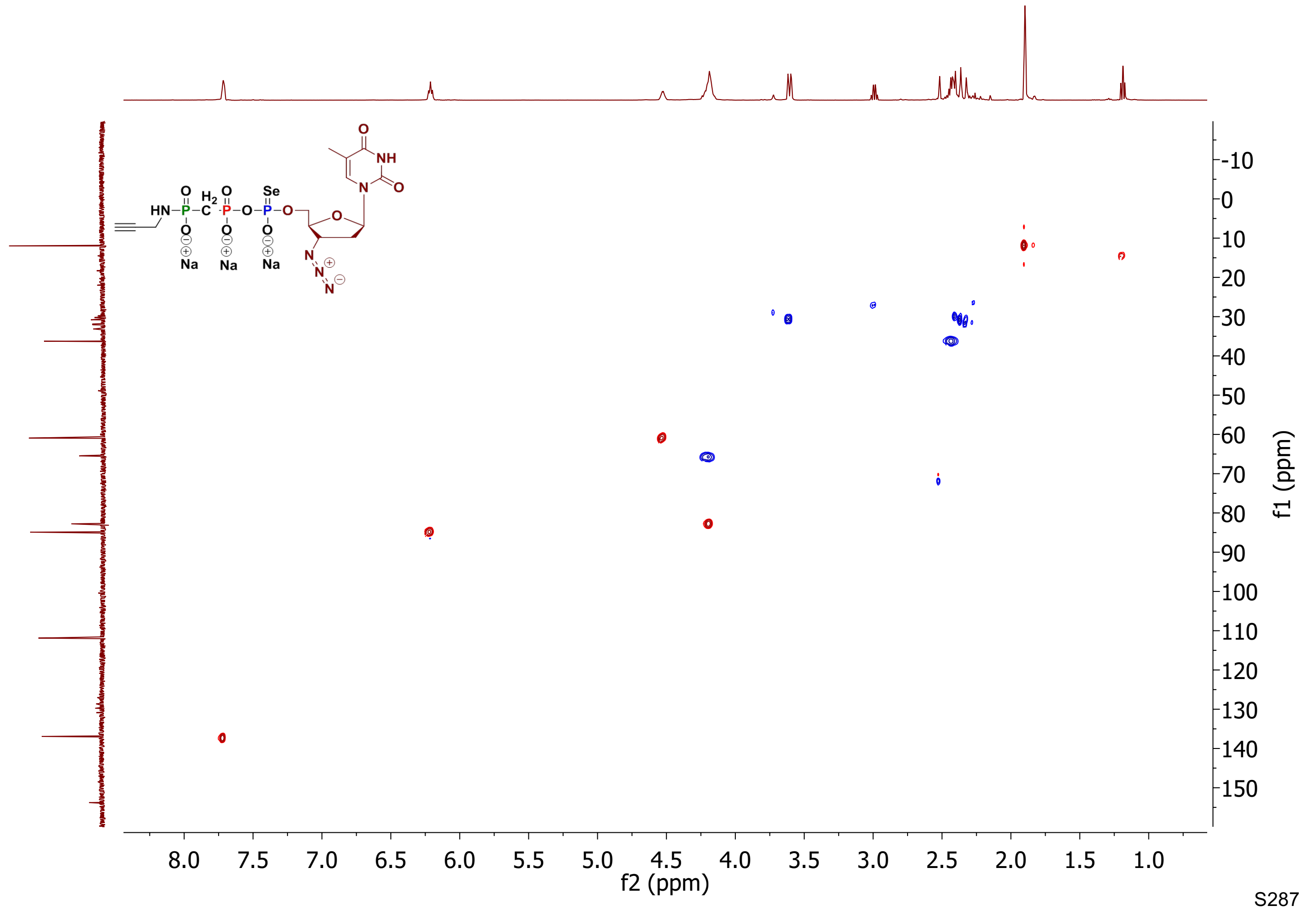


after SAX Chromatography

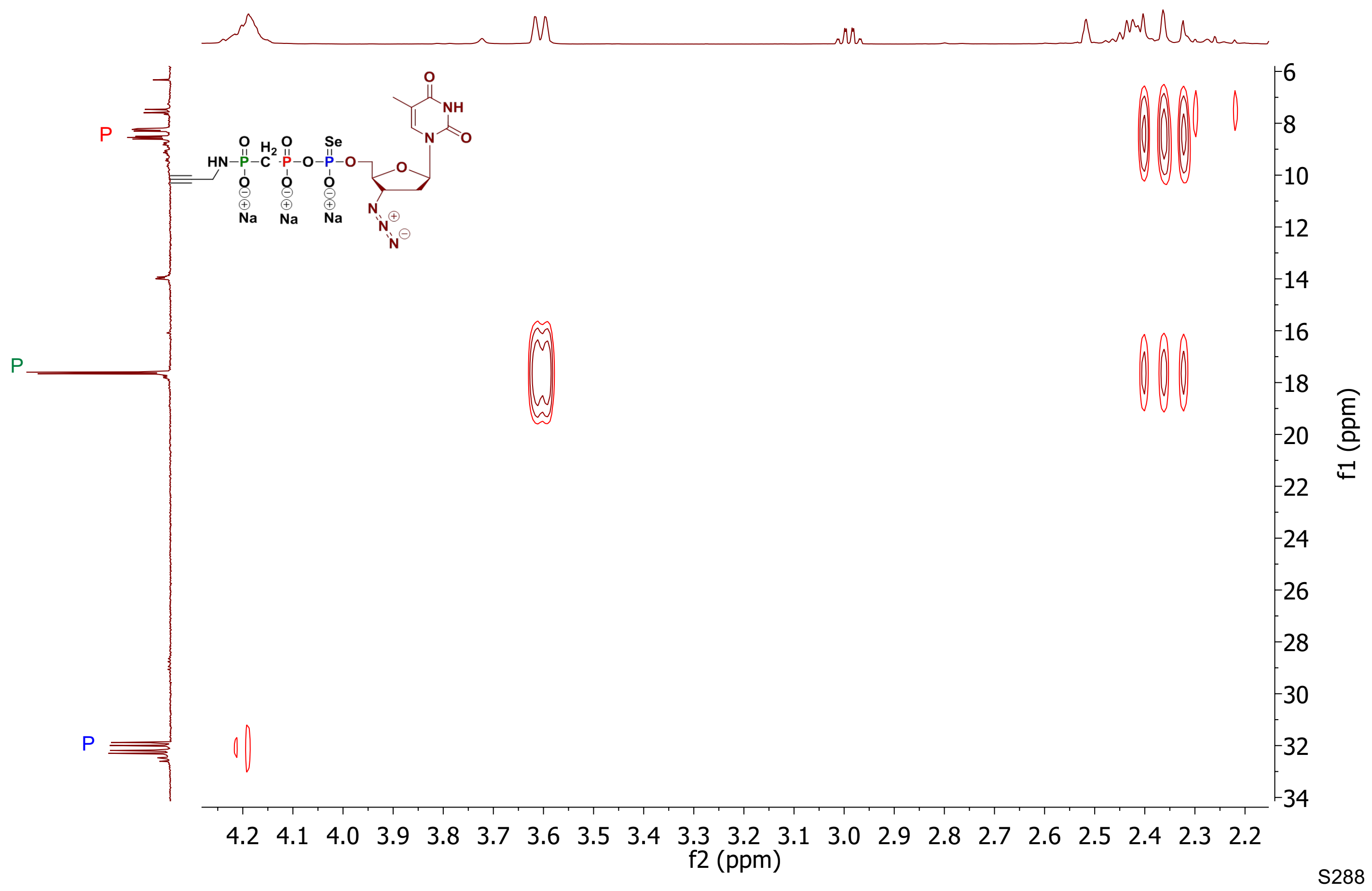




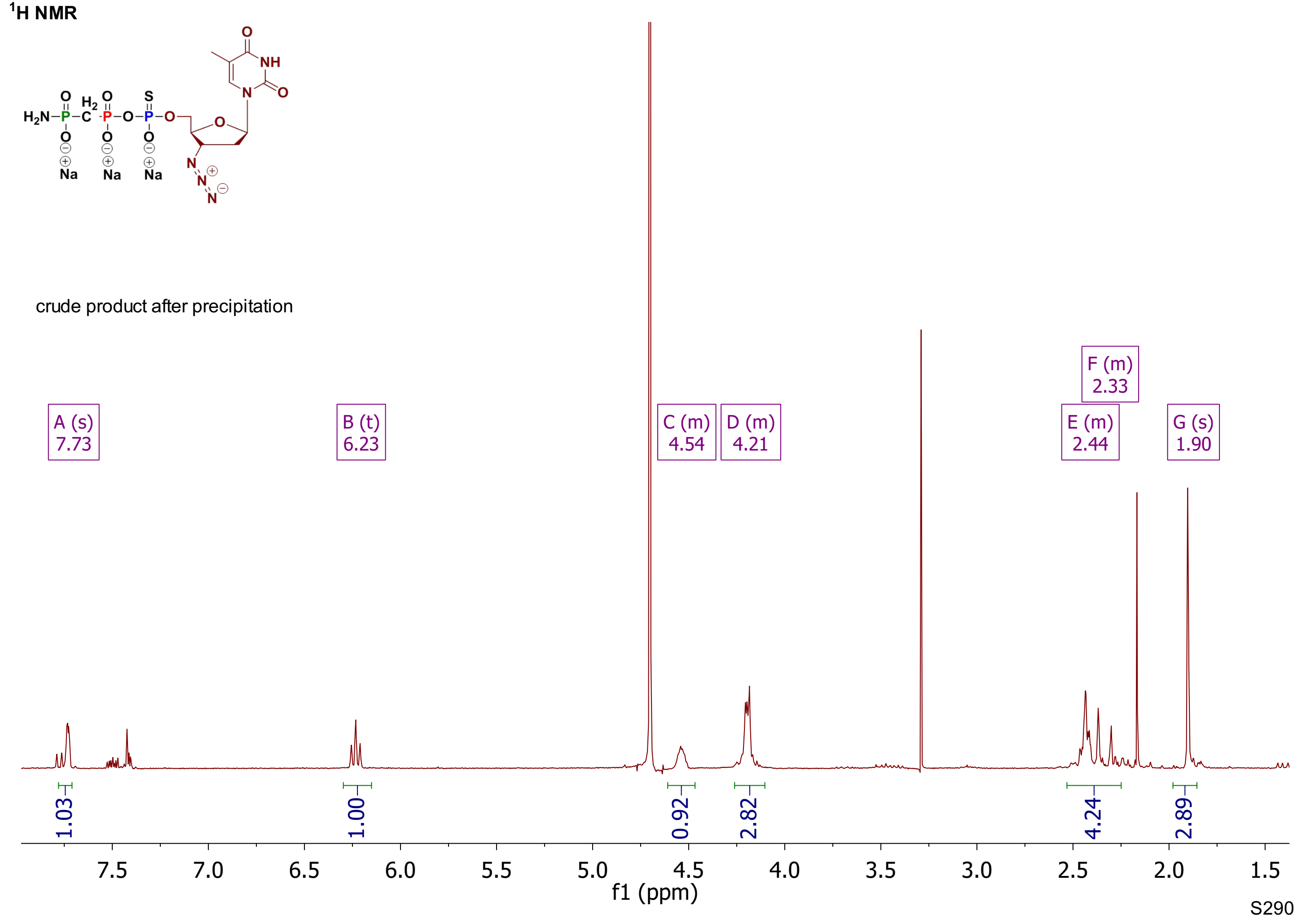




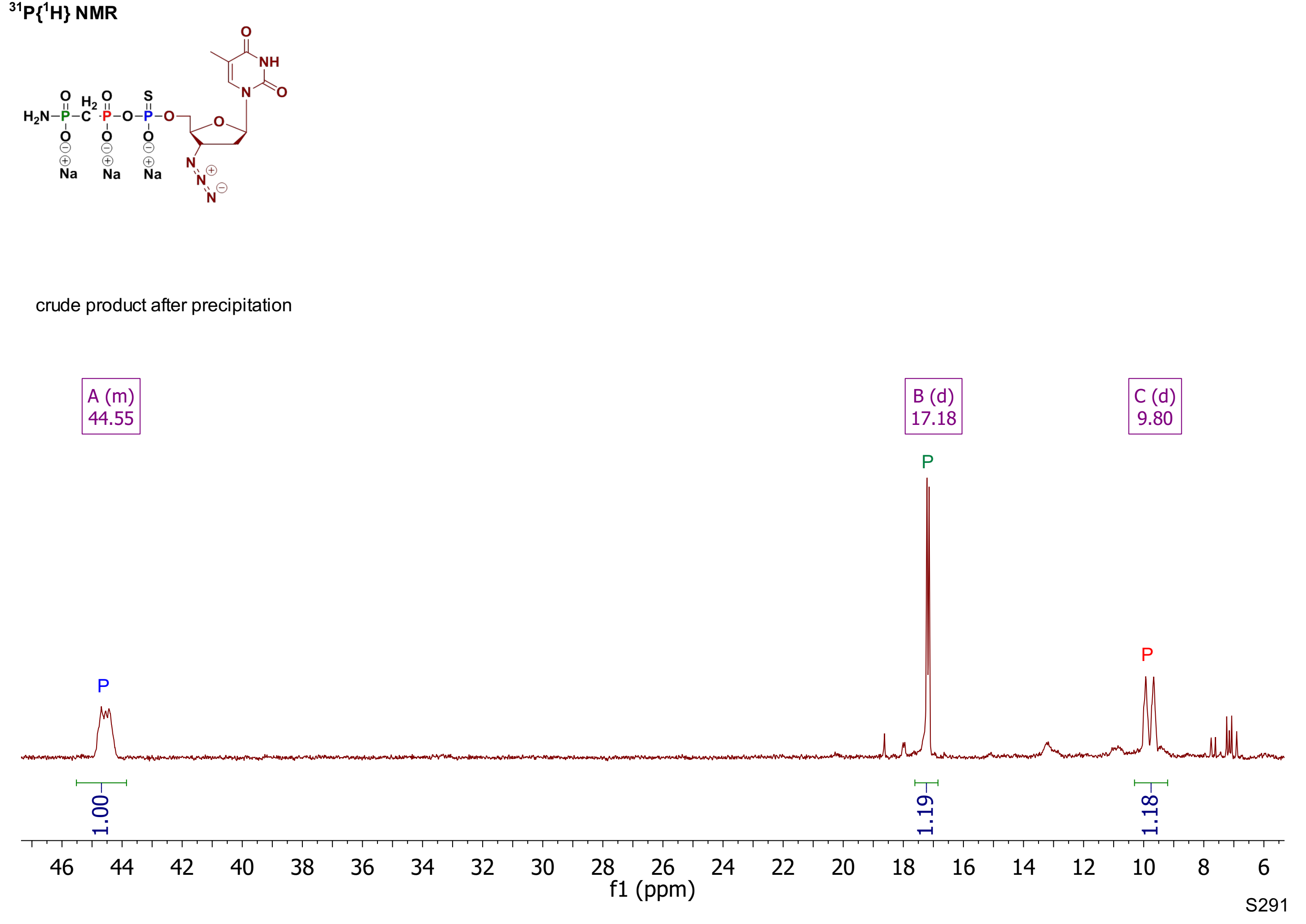




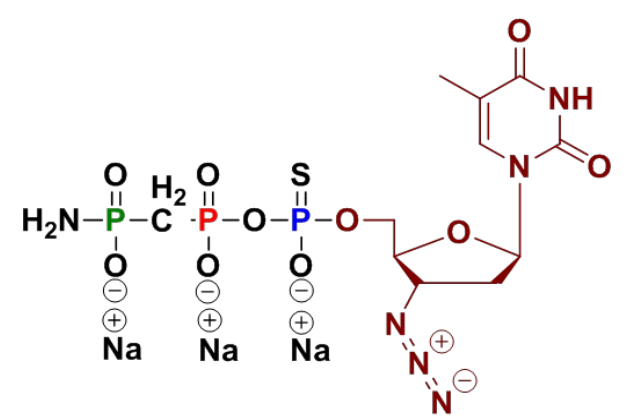

crude product after precipitation

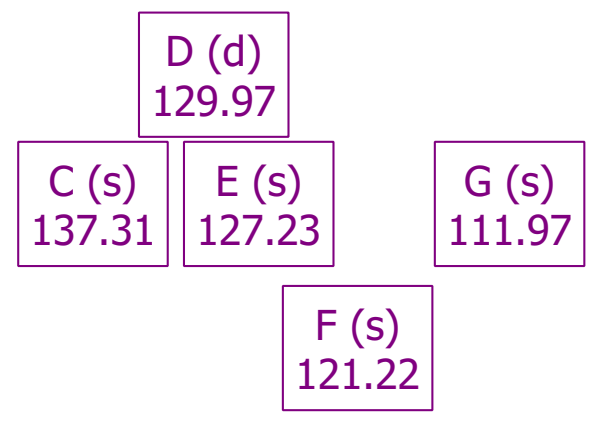

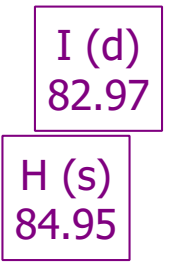

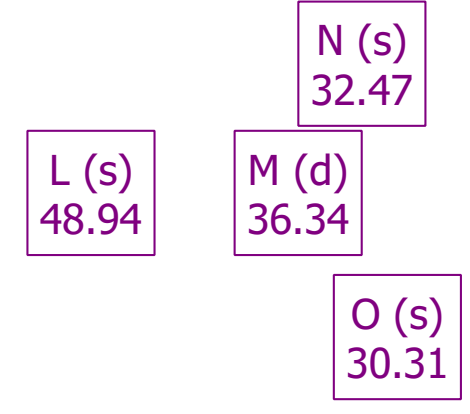

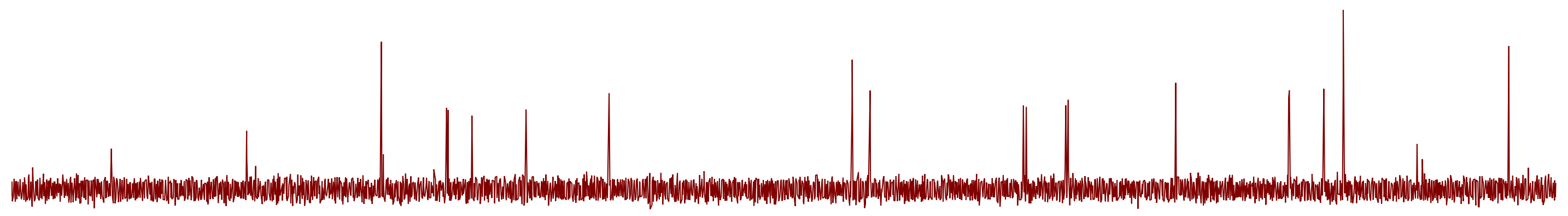




\section{${ }^{1} \mathrm{H}$ NMR}

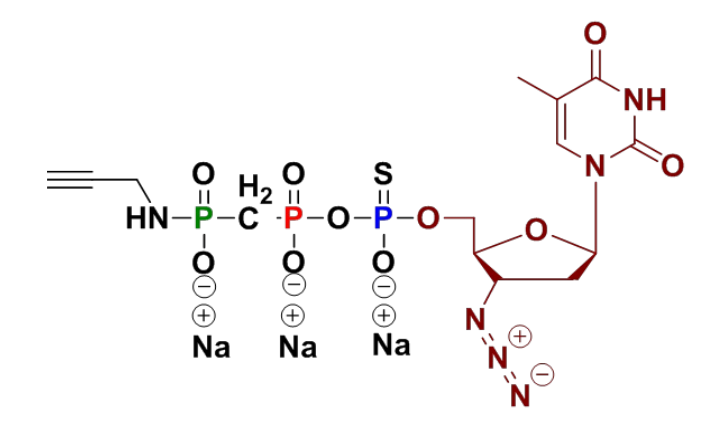

crude product after precipitation

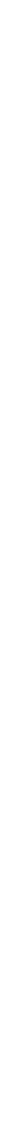

8.2 8.0 7.8 7.6 7.4 7.2 7.0 6.8 6.6 6.4 6.2 6.0 5.8 5.6 5.4 5.2 5.0 4.8 4.6 4.4 4.2 4.0 3.8 3.6 3.4 3.2 3.0 2.82 .62 .42 .22 .01 .81 . f1 (ppm) 


\section{${ }^{31} P\left\{{ }^{1} H\right\} N M R$}

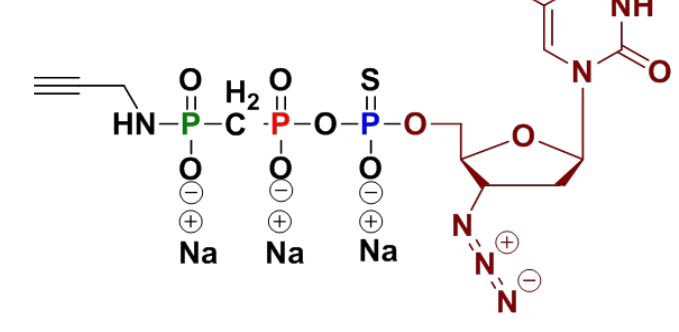

crude product after precipitation

A (dd)
42.14

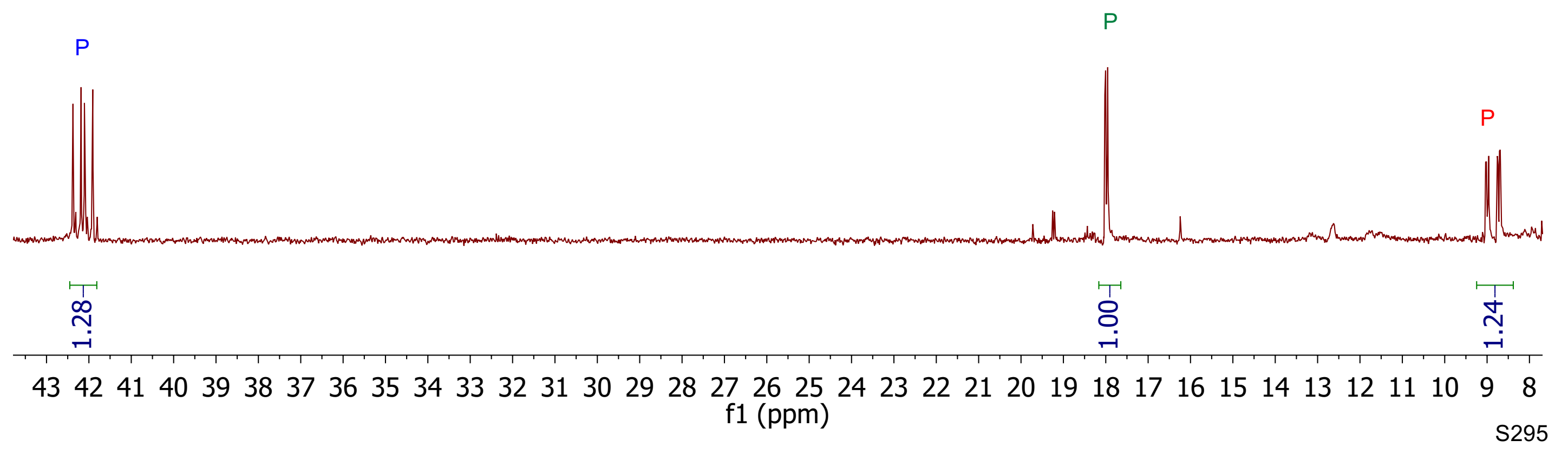




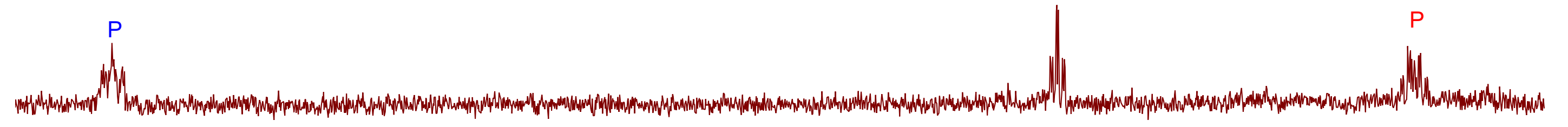

\begin{tabular}{|c|c|c|c|c|c|c|}
\hline 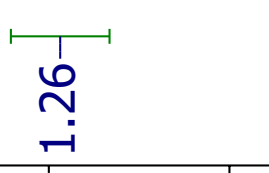 & & & & & & \\
\hline 40 & 35 & 30 & $\begin{array}{c}25 \\
\mathrm{f} 1(\mathrm{ppm})\end{array}$ & 20 & 15 & 10 \\
\hline
\end{tabular}




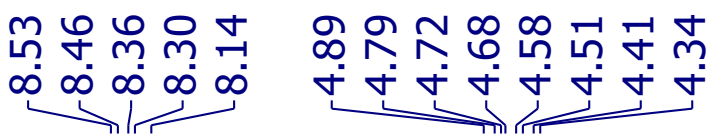

0

II

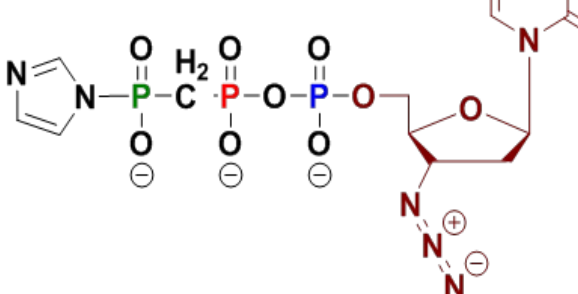

quntat. in reaction mixture

$A(m)$
8.33

$$
\begin{gathered}
\text { B (dtd) } \\
4.58
\end{gathered}
$$

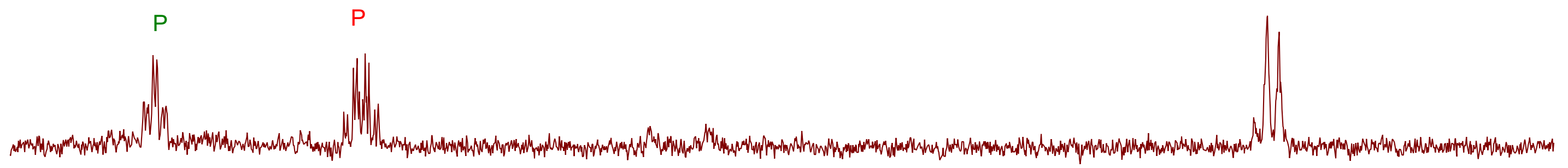

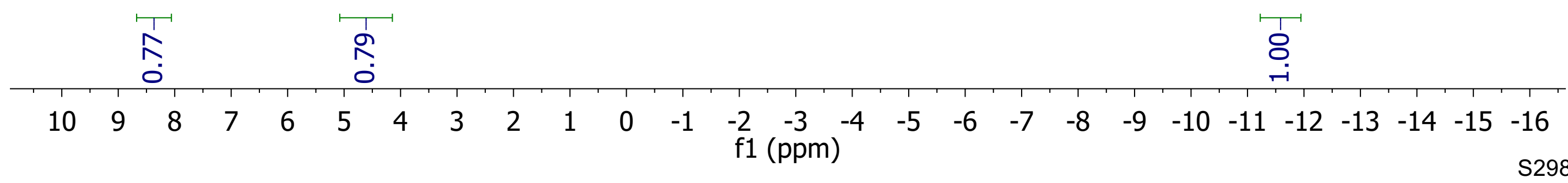




\section{${ }^{1} \mathrm{H}$ NMR}

(1)

product after SAX purification

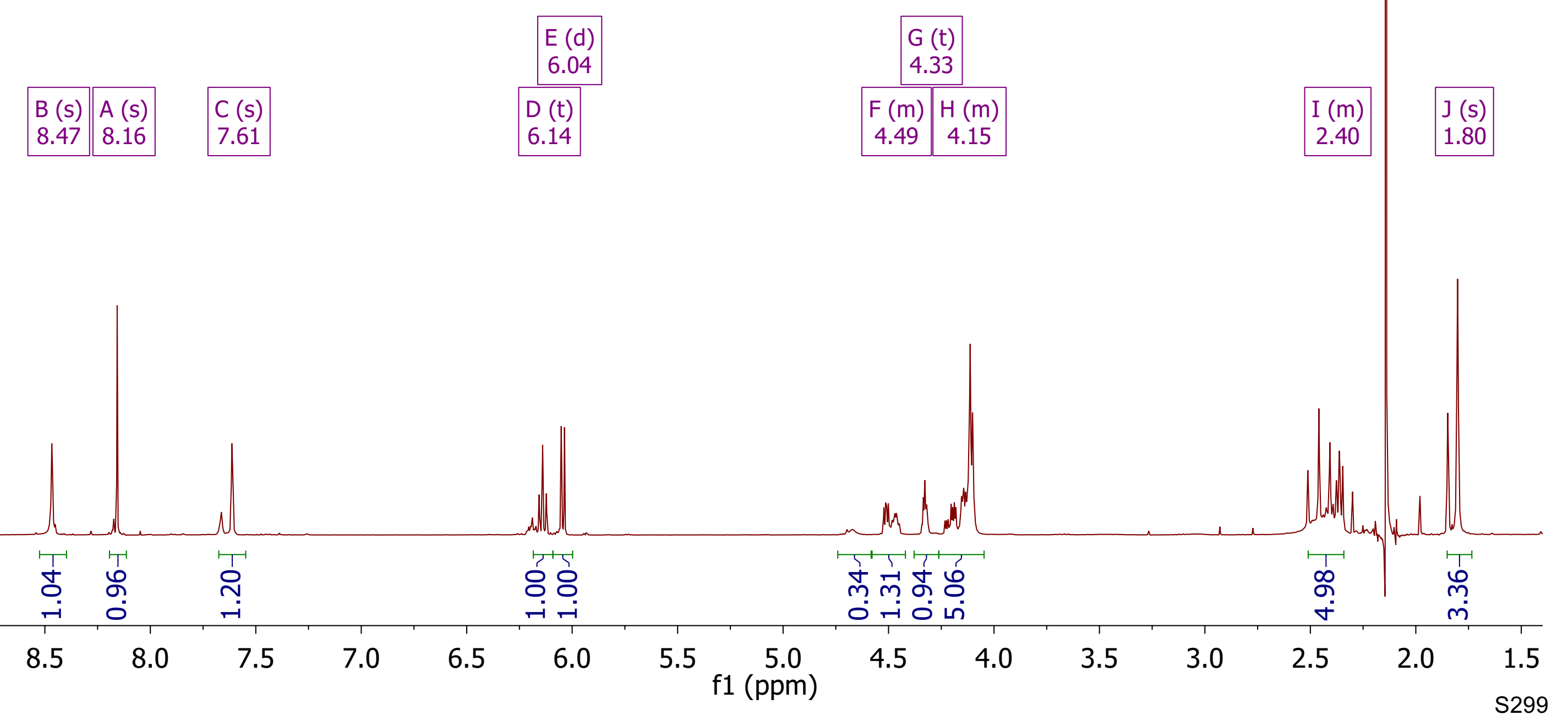




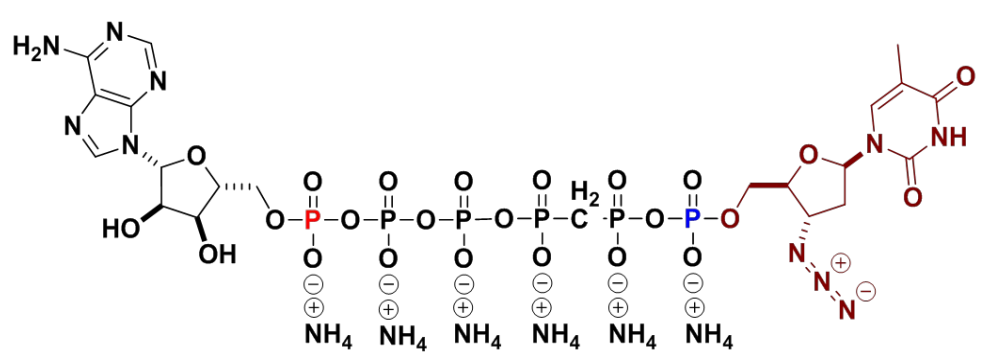

product after $\mathrm{SAX}$ purification

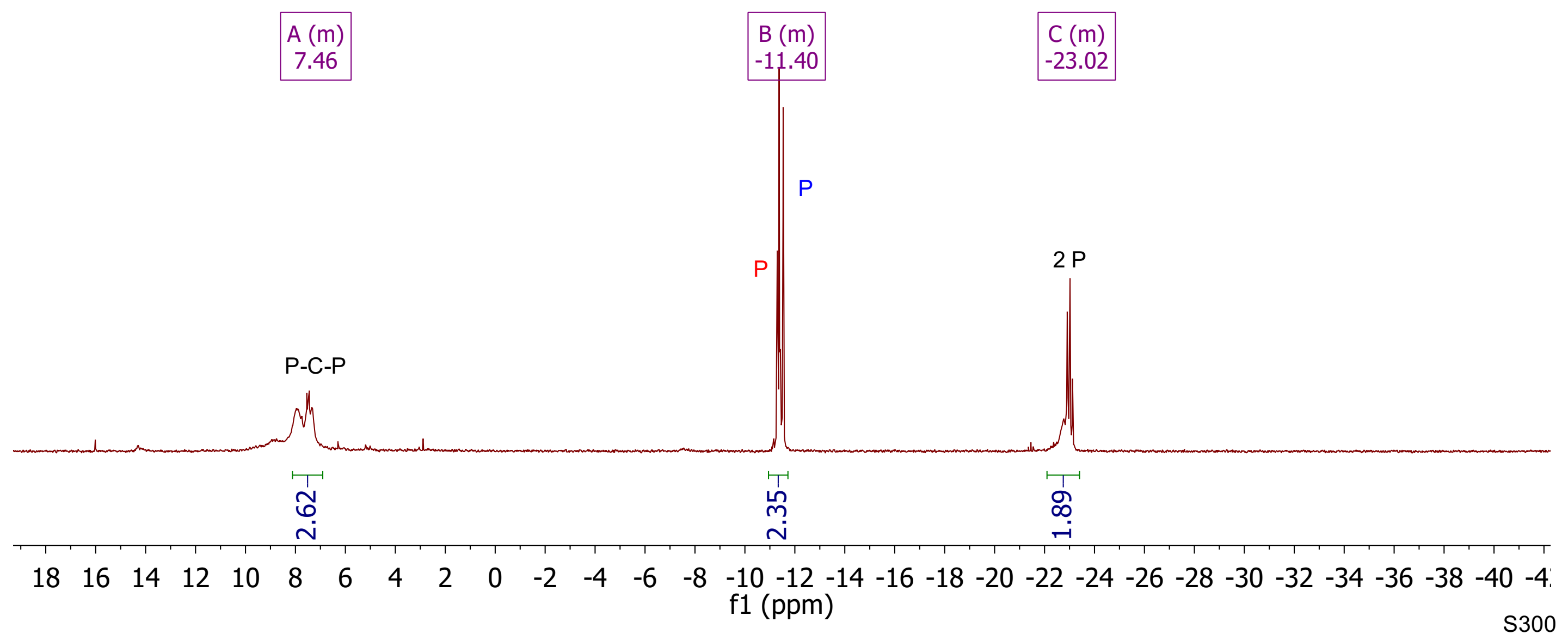




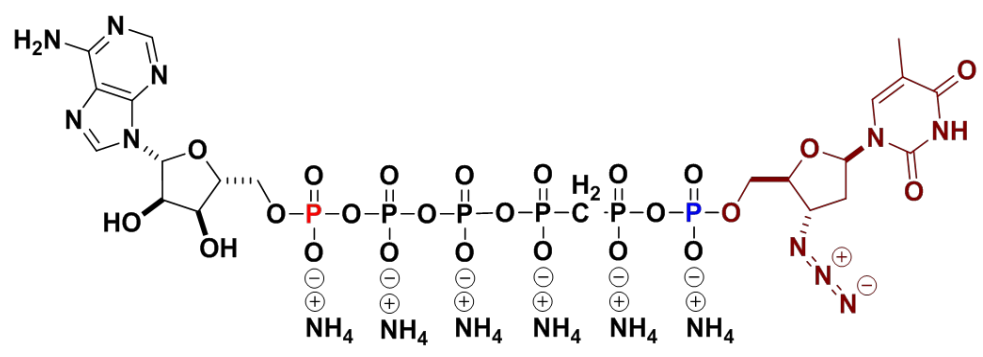

product after $\mathrm{SAX}$ purification

$$
\begin{aligned}
& \text { A (m) } \\
& 7.54 \\
& \hline
\end{aligned}
$$
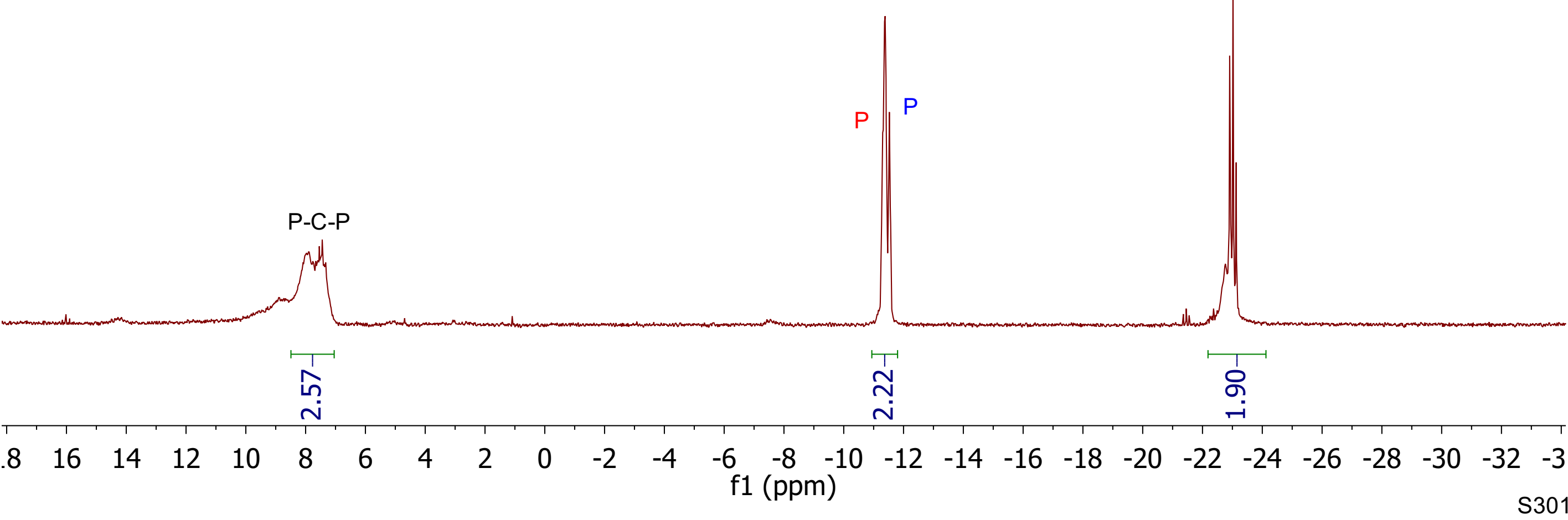


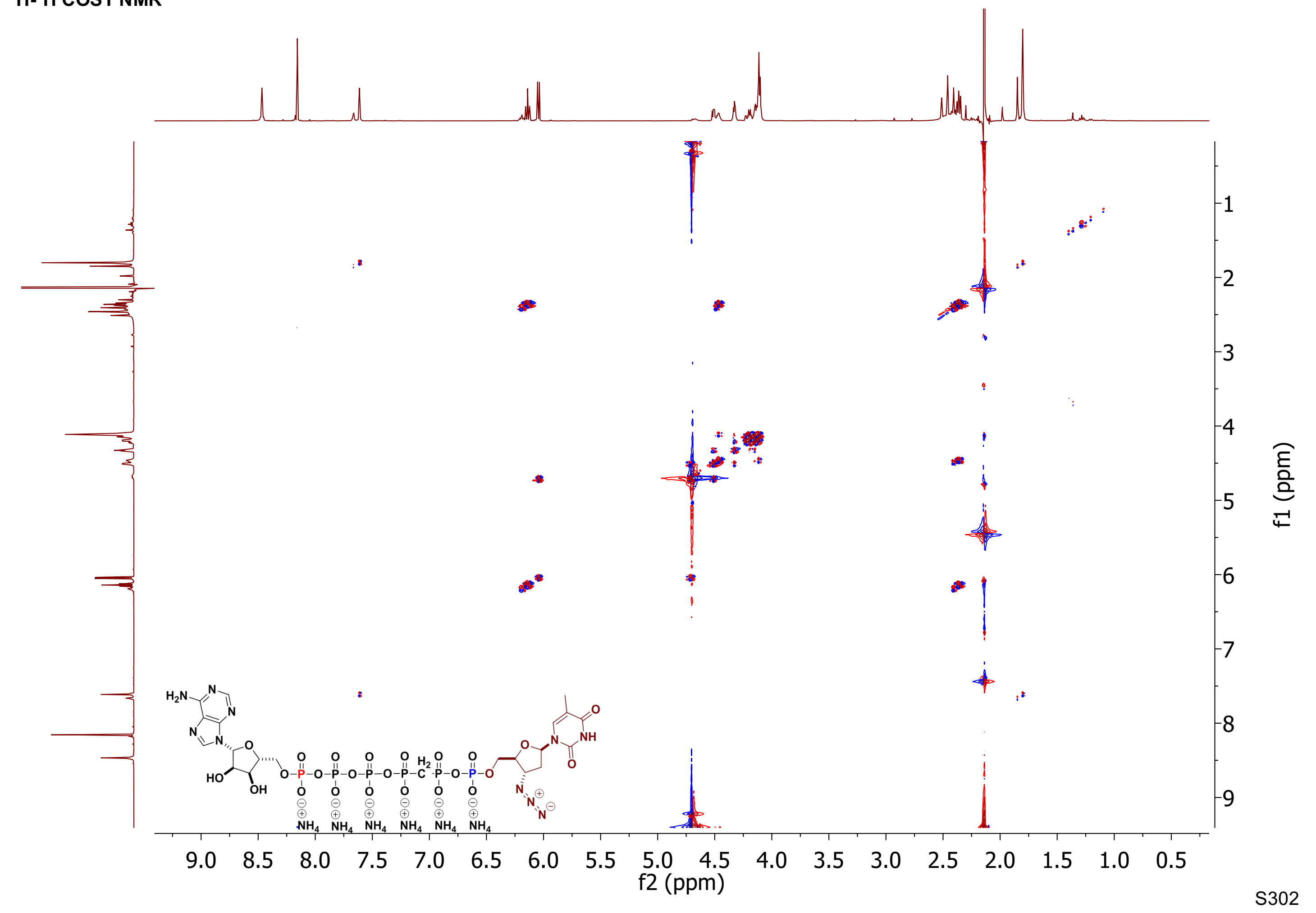




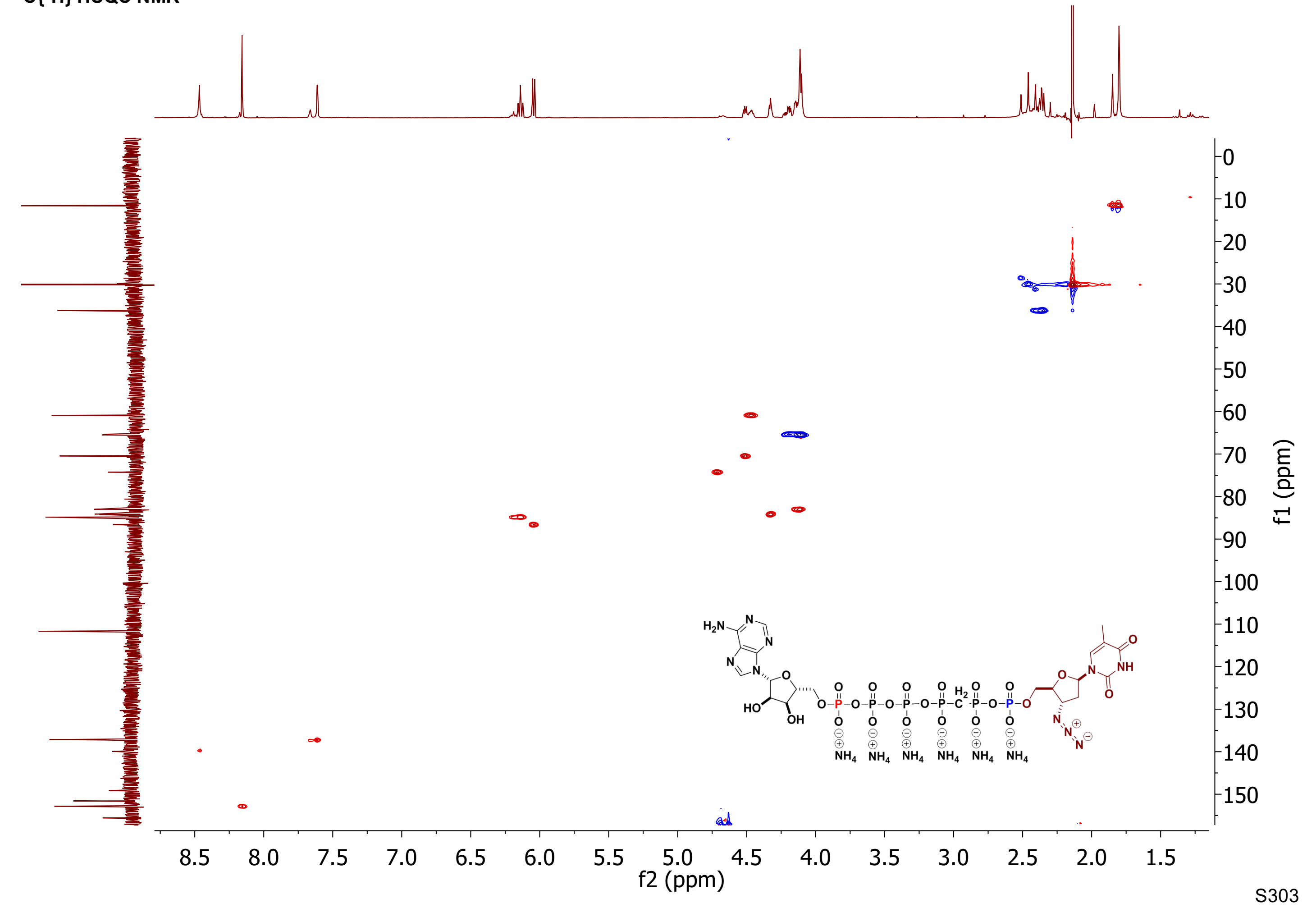




\section{${ }^{13} \mathrm{C}\left\{{ }^{1} \mathrm{H}\right\}$ HMBC NMR}

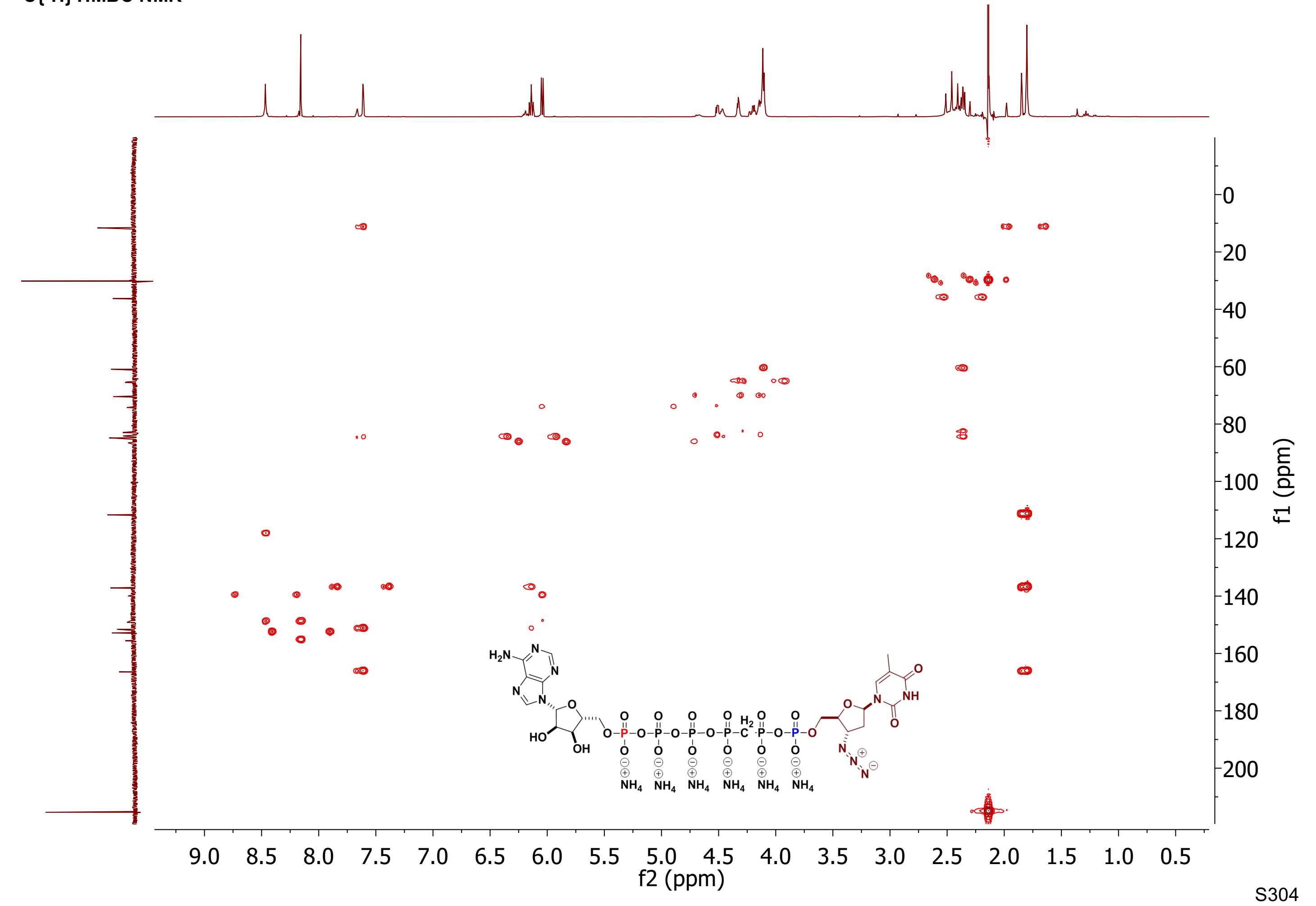




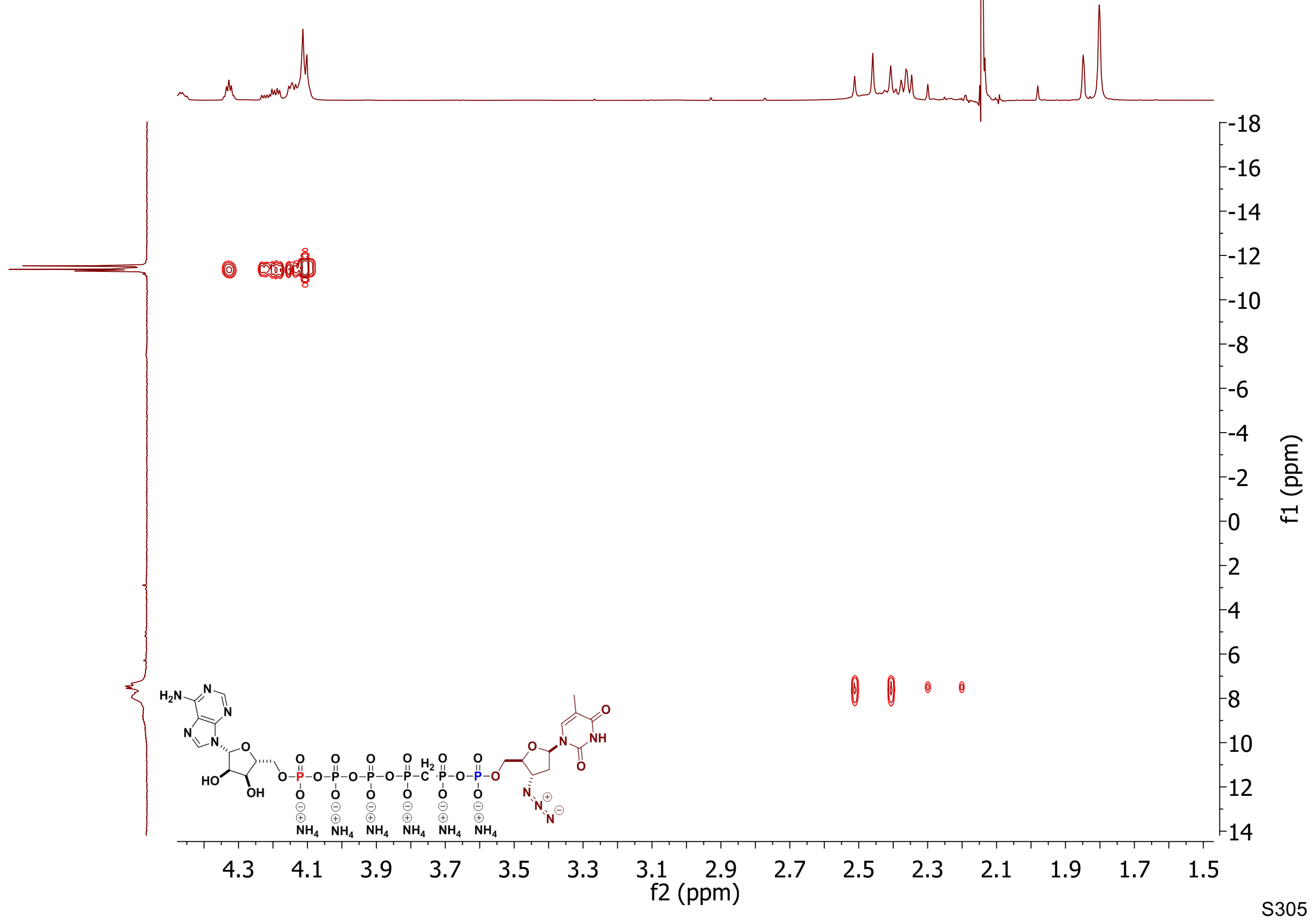




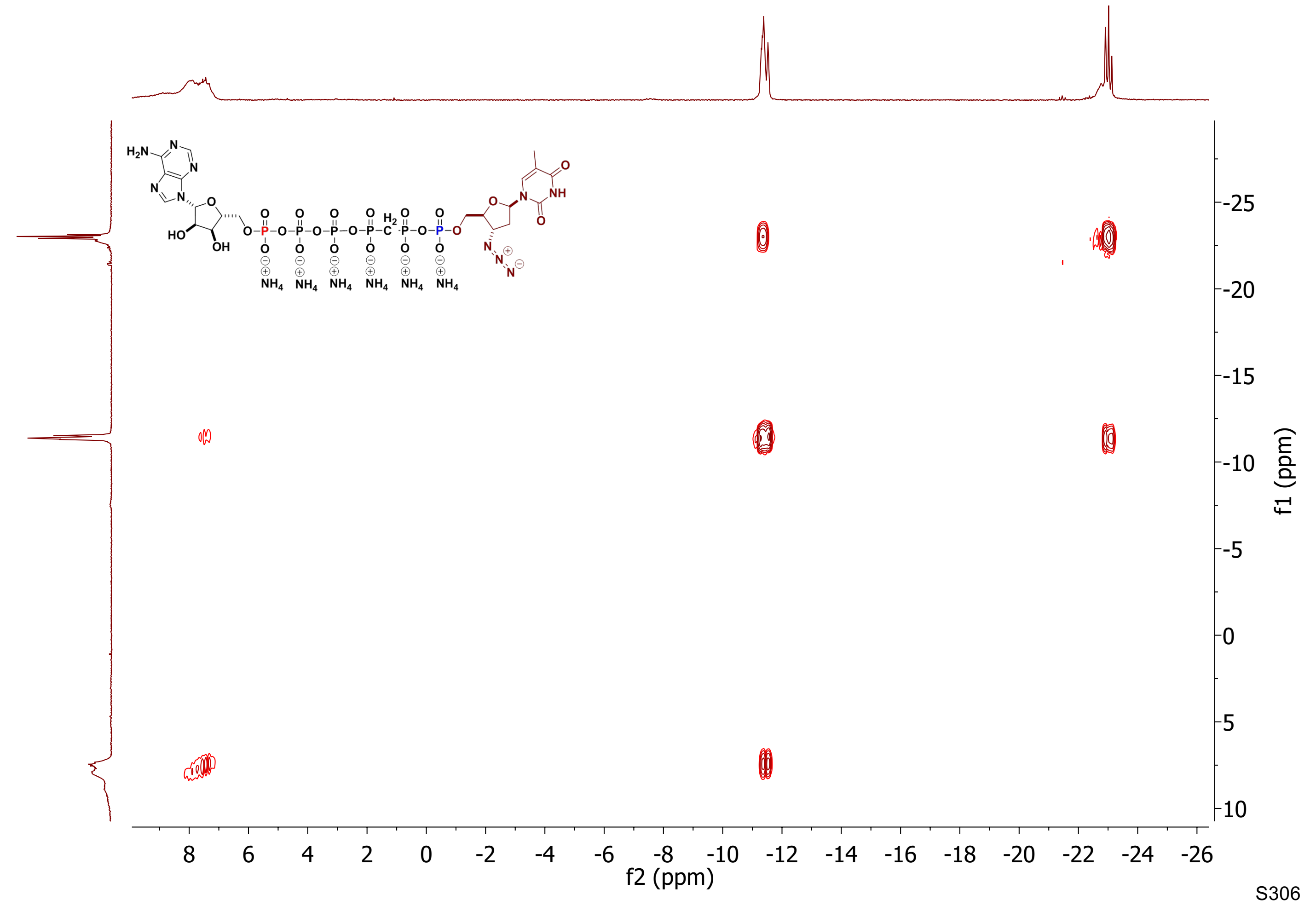




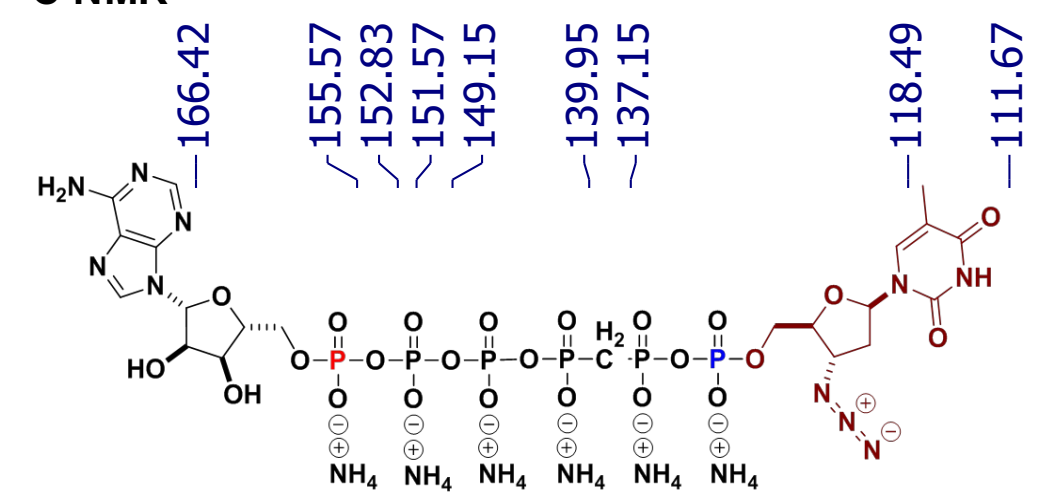

product after SAX purification

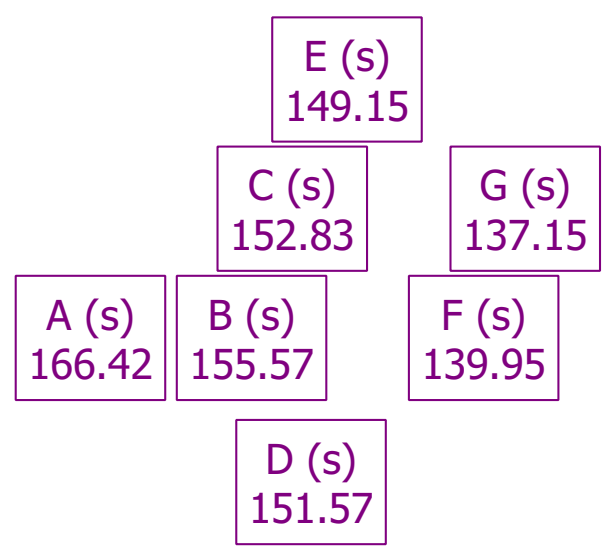

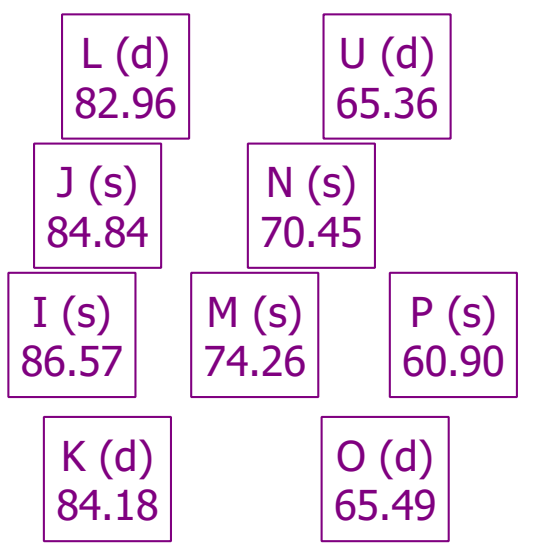

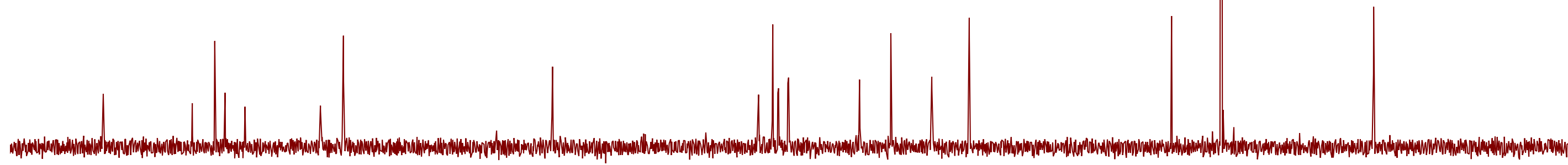

$\begin{array}{lllllllllllllllllll}170 & 160 & 150 & 140 & 130 & 120 & 110 & 100 & \underset{\mathrm{f} 1(\mathrm{ppm})}{80} & 70 & 60 & 50 & 40 & 30 & 20 & 10 & 0 & -10 \\ \mathrm{~s} 307\end{array}$




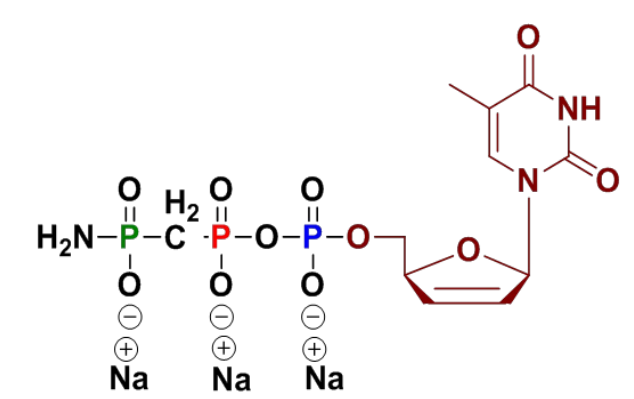

crude product after precipitation

\begin{tabular}{|c|c|c|c|c|c|}
\hline $\begin{array}{l}A(s) \\
7.53\end{array}$ & $\begin{array}{c}B(m) \\
6.89\end{array}$ & $\begin{array}{c}C(m) \\
6.45\end{array}$ & $\begin{array}{c}\mathrm{D}(\mathrm{m}) \\
5.87\end{array}$ & $\begin{array}{c}E(m) \\
5.03\end{array}$ & $\begin{array}{c}F(d t) \\
4.07\end{array}$ \\
\hline
\end{tabular}

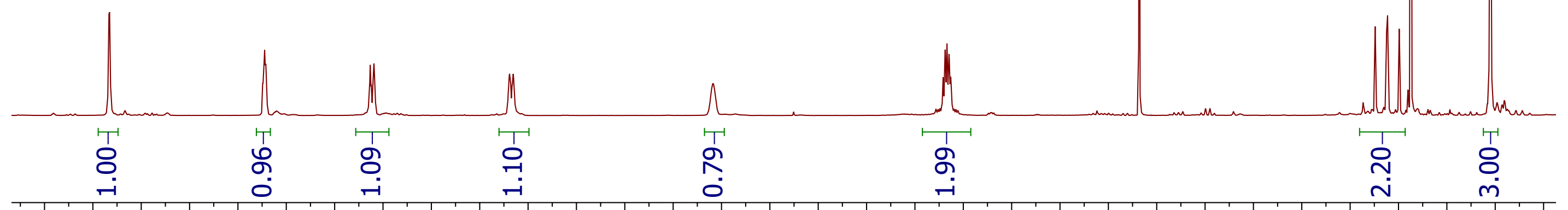

7.87 .67 .47 .27 .06 .86 .66 .46 .26 .05 .85 .65 .45 .25 .04 .84 .64 .44 .24 .03 .83 .63 .43 .23 .02 .82 .62 .42 .22 .01 .81 .6 f1 (ppm) 


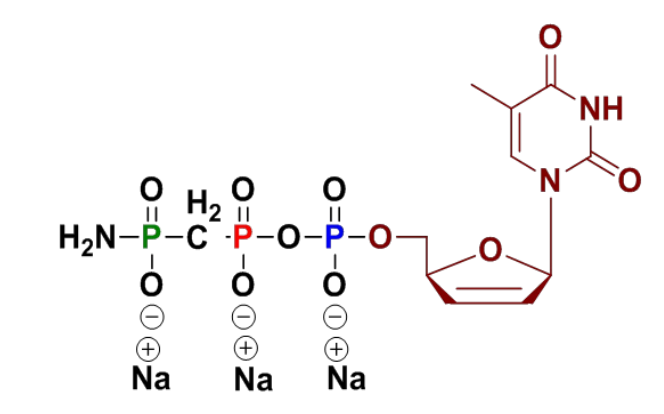

crude product after precipitation

$$
\begin{array}{|l|}
\hline A(d) \\
17.94 \\
\hline
\end{array}
$$

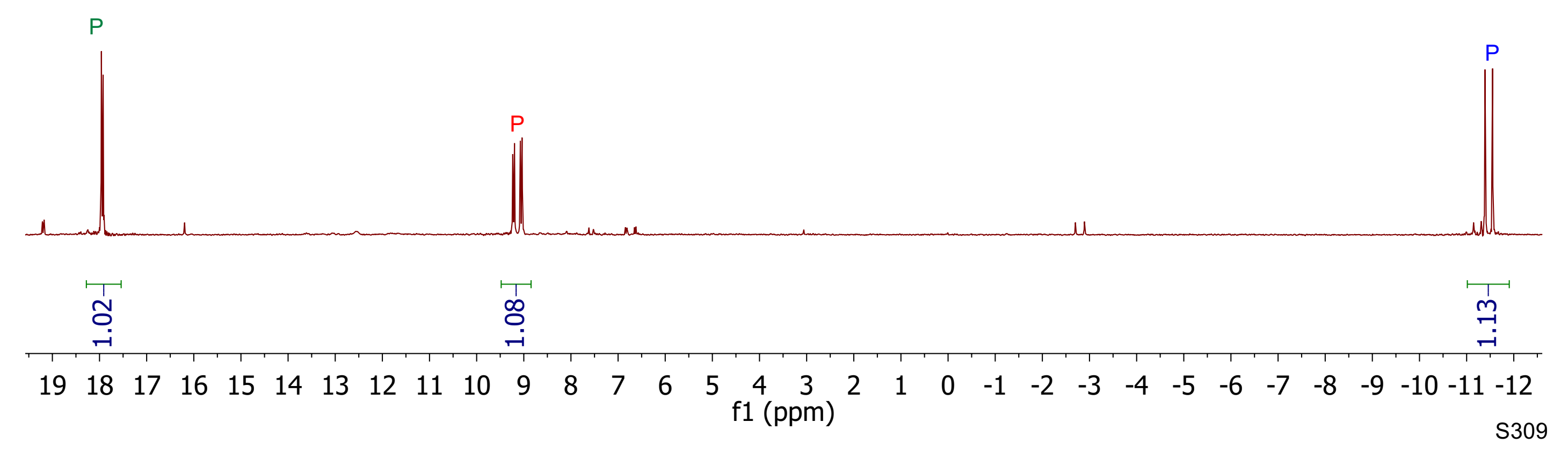




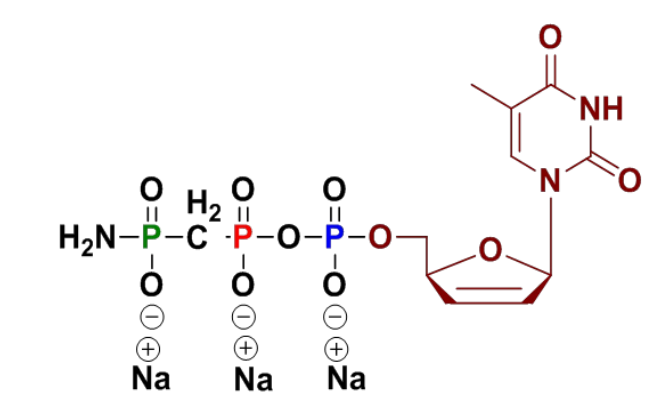

crude product after precipitation

$$
\text { A (td) }
$$$$
17.99
$$

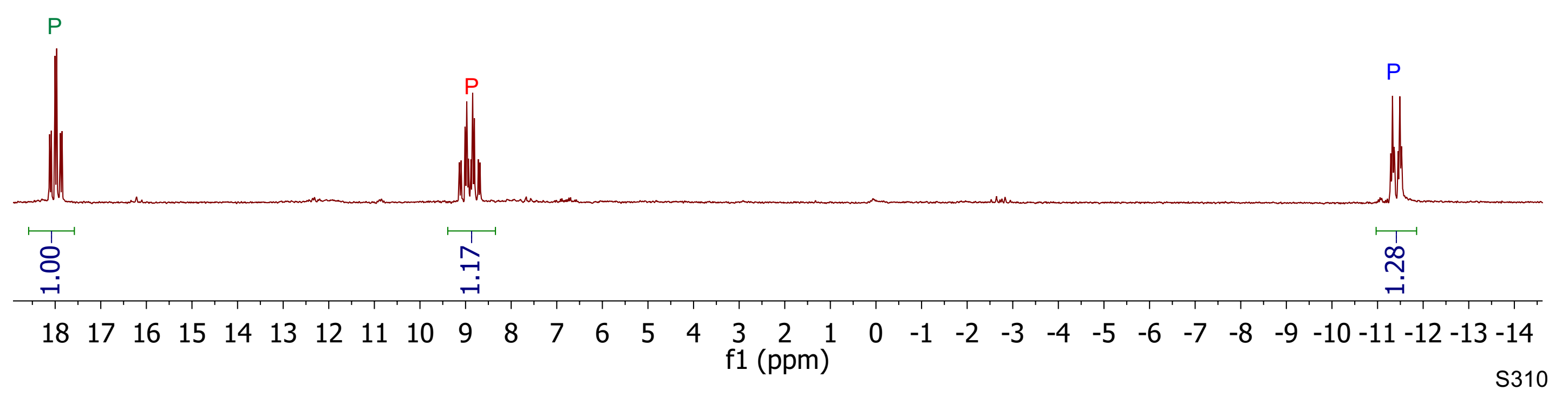




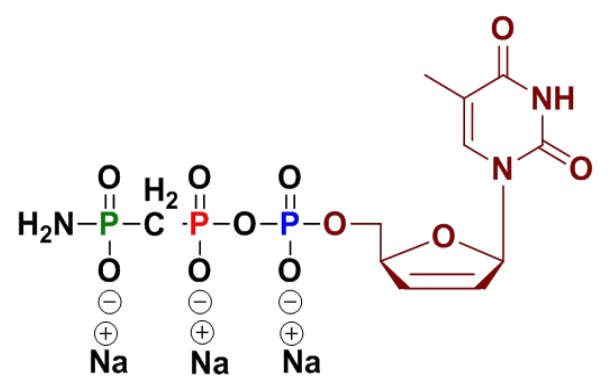

crude product after precipitation

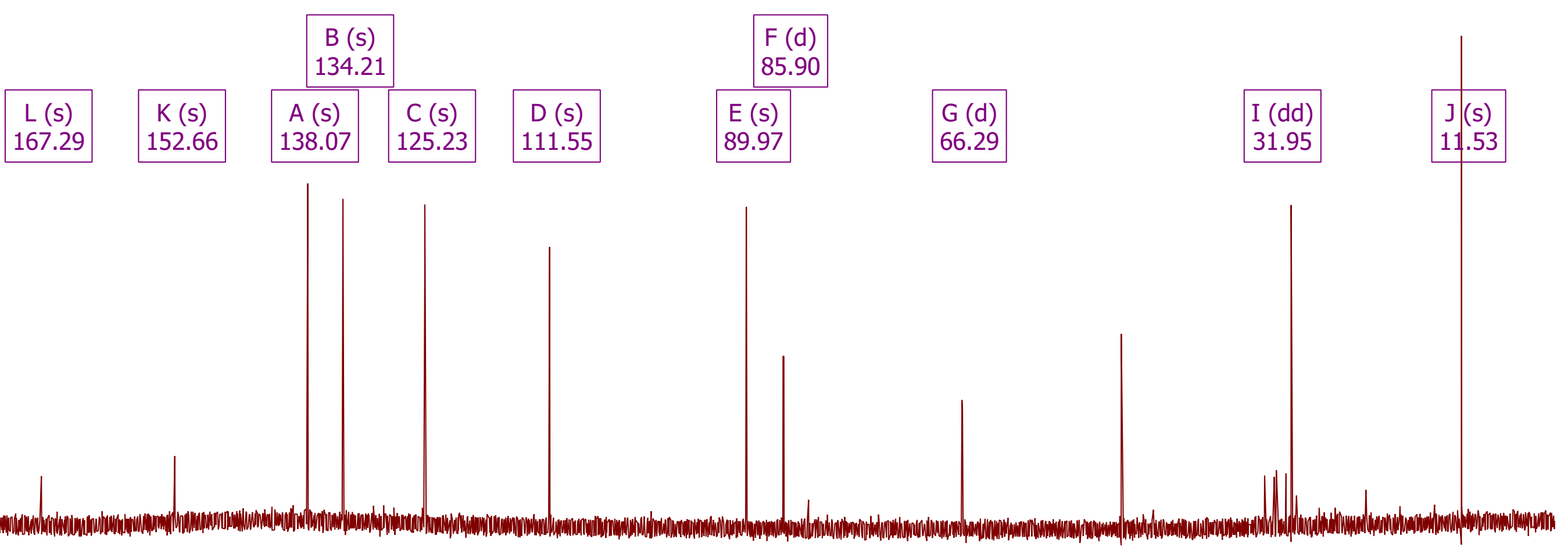

\begin{tabular}{|c|c|c|c|c|c|c|c|c|c|c|c|c|c|c|c|c|c|}
\hline .80 & 170 & 160 & 150 & 140 & 130 & 120 & 110 & 100 & $\begin{array}{c}90 \\
\mathrm{ppm}\end{array}$ & 80 & 70 & 60 & 50 & 40 & 30 & 20 & 10 \\
\hline
\end{tabular}




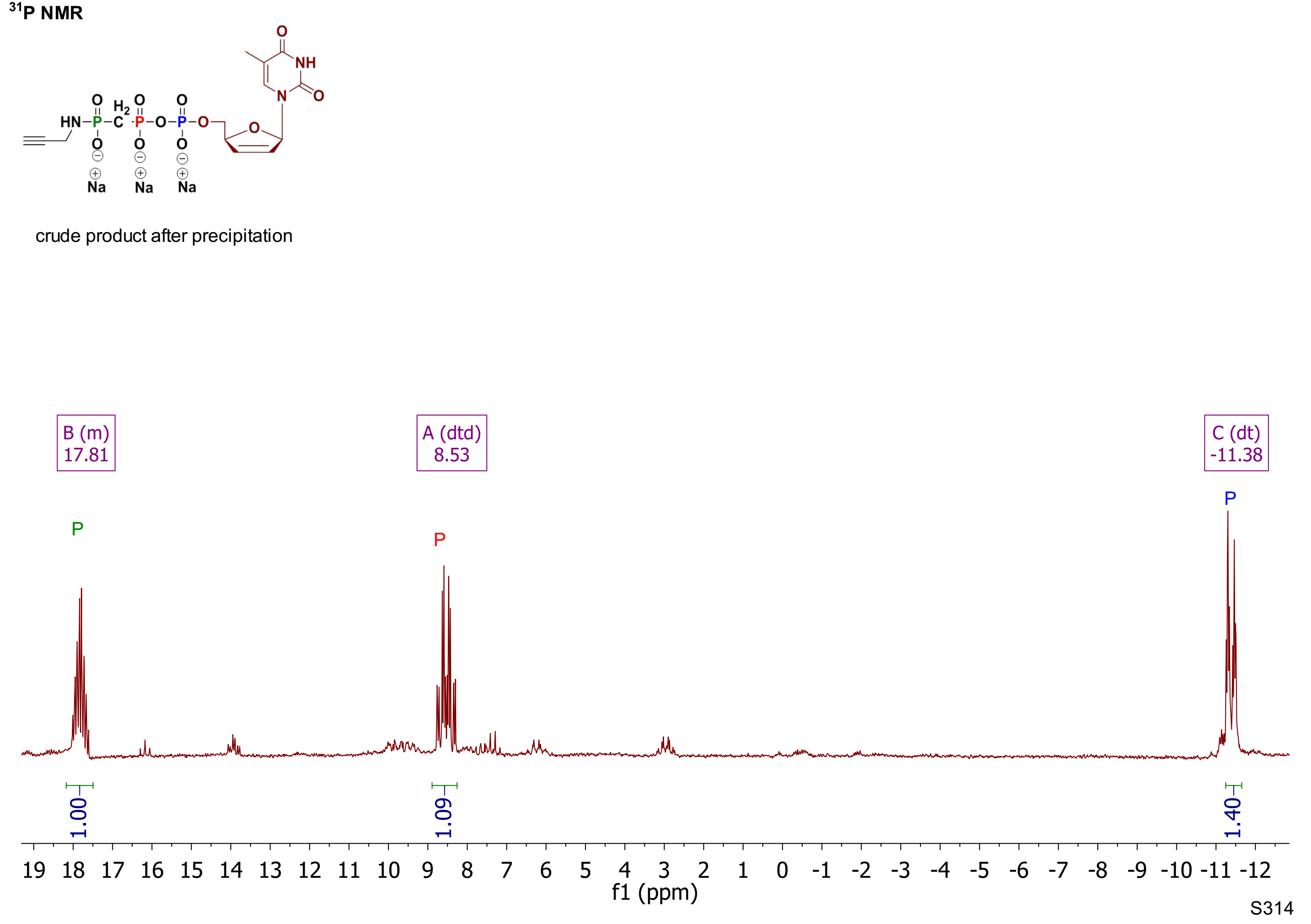




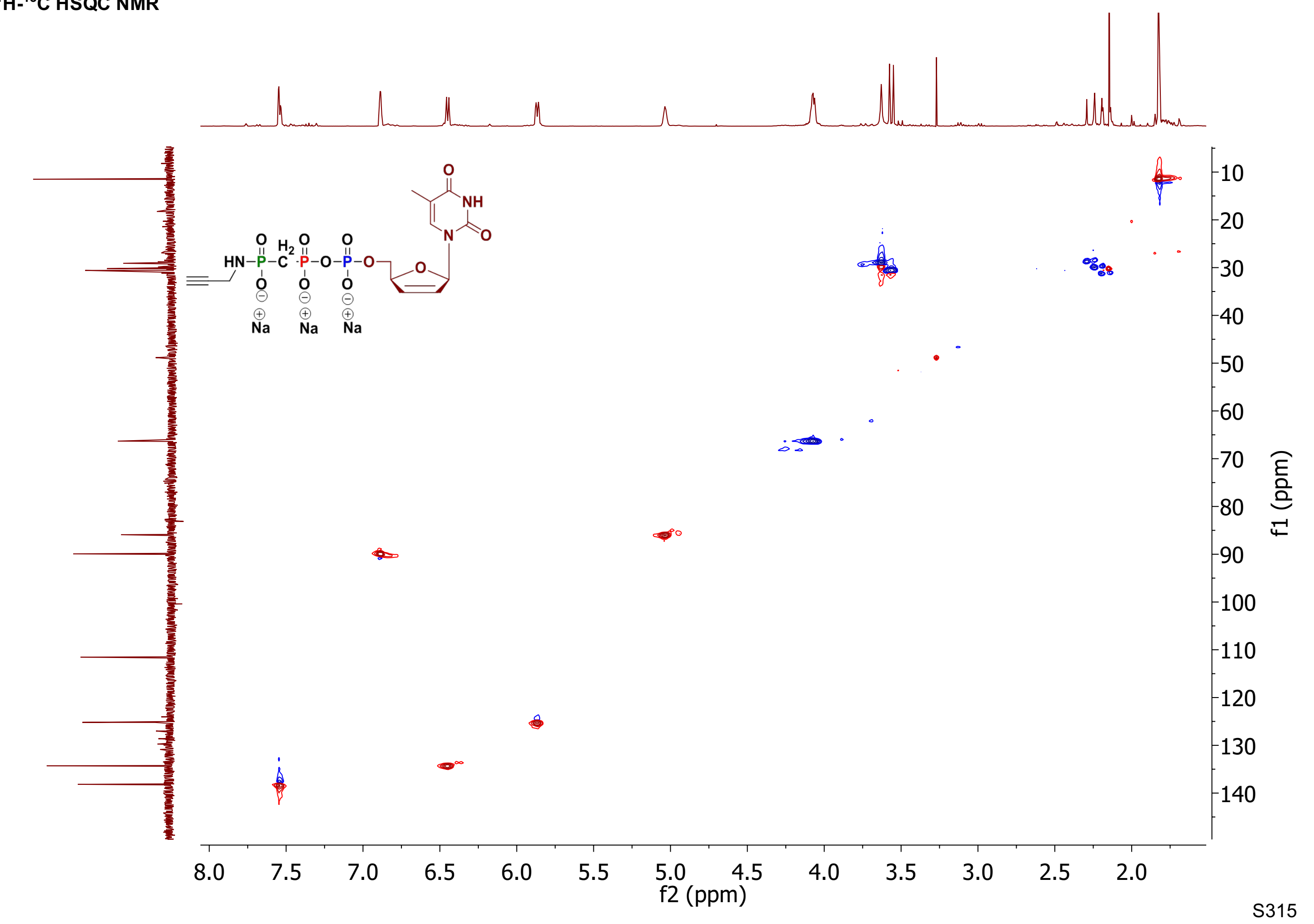




\section{${ }^{1} \mathrm{H}-{ }^{13} \mathrm{C}$ HMBC NMR}

after precipitation

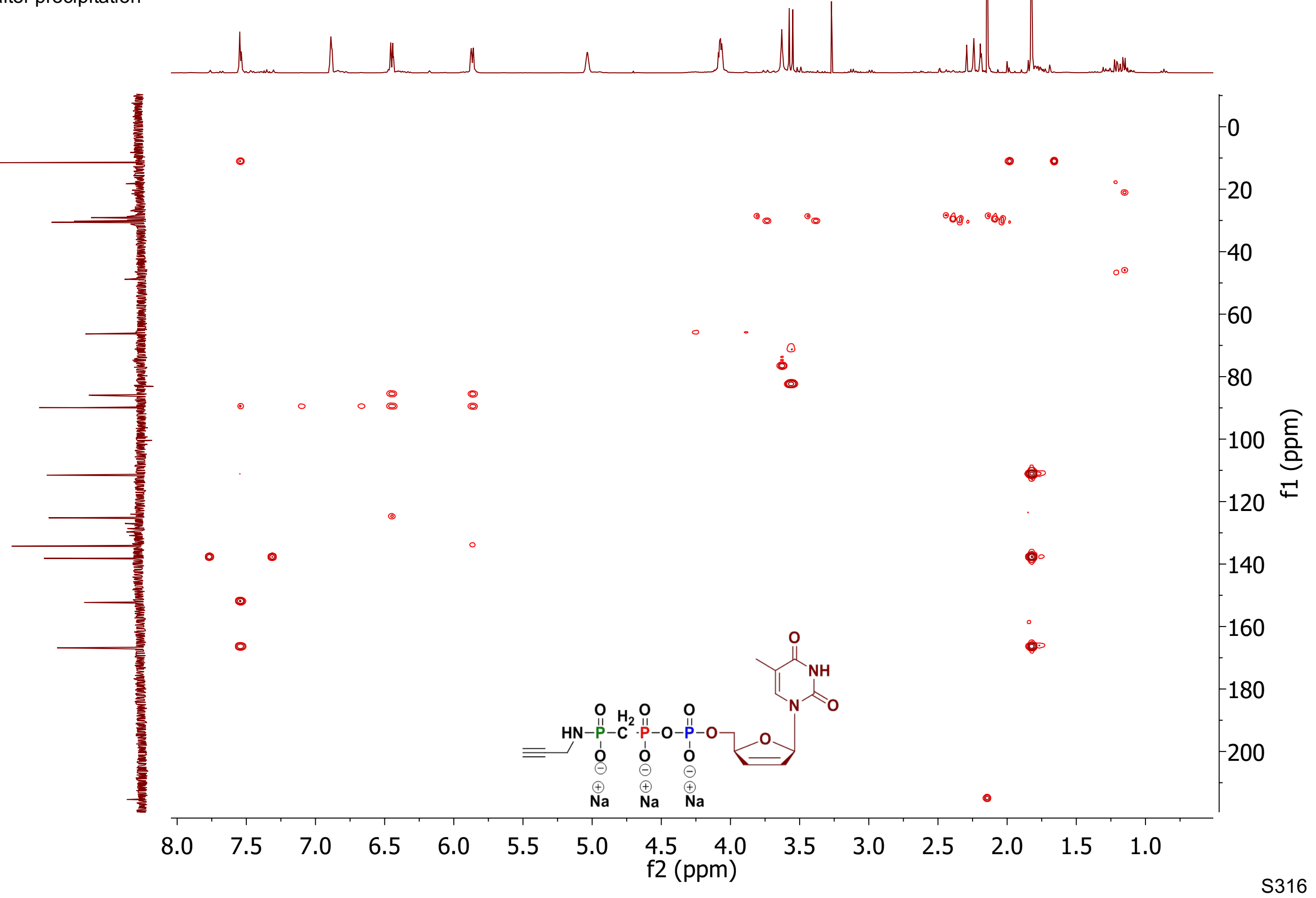




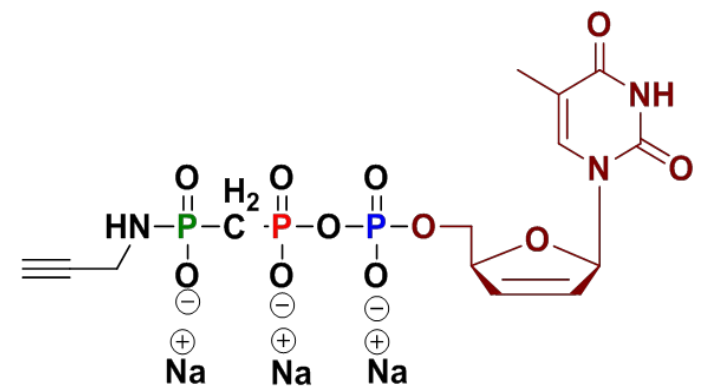

crude product after precipitation

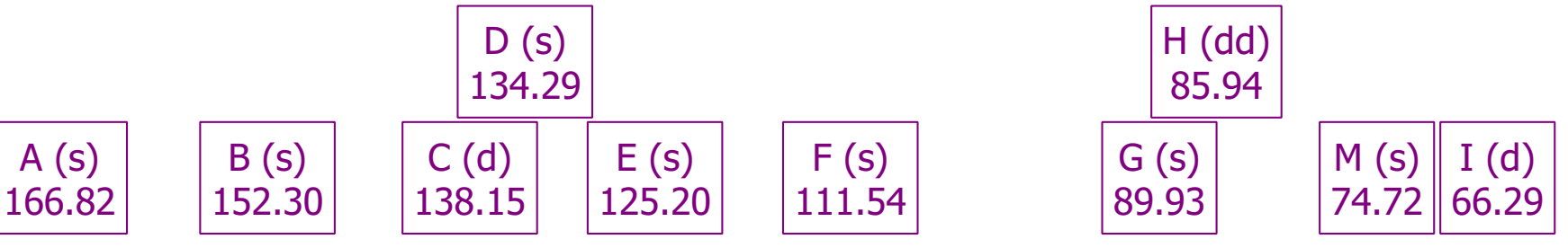

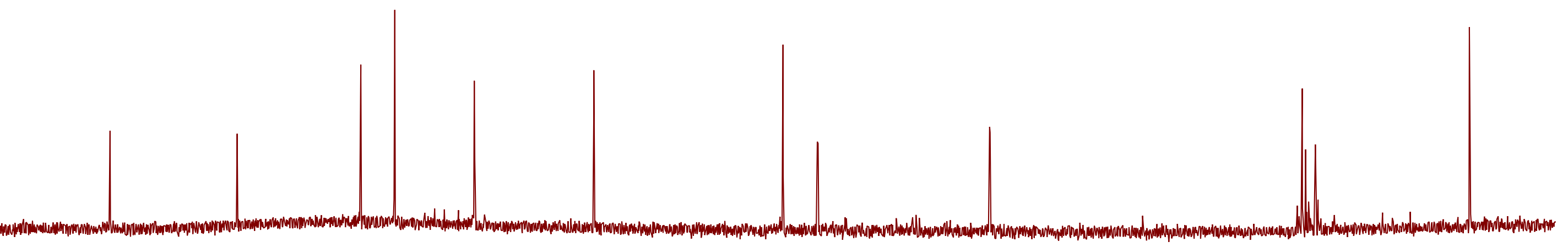

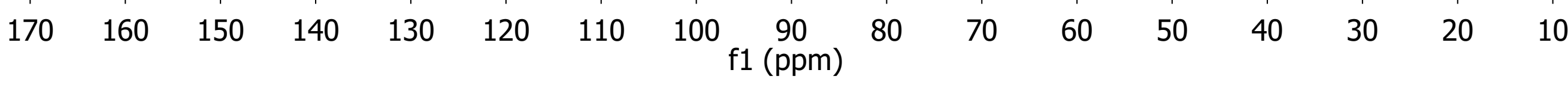




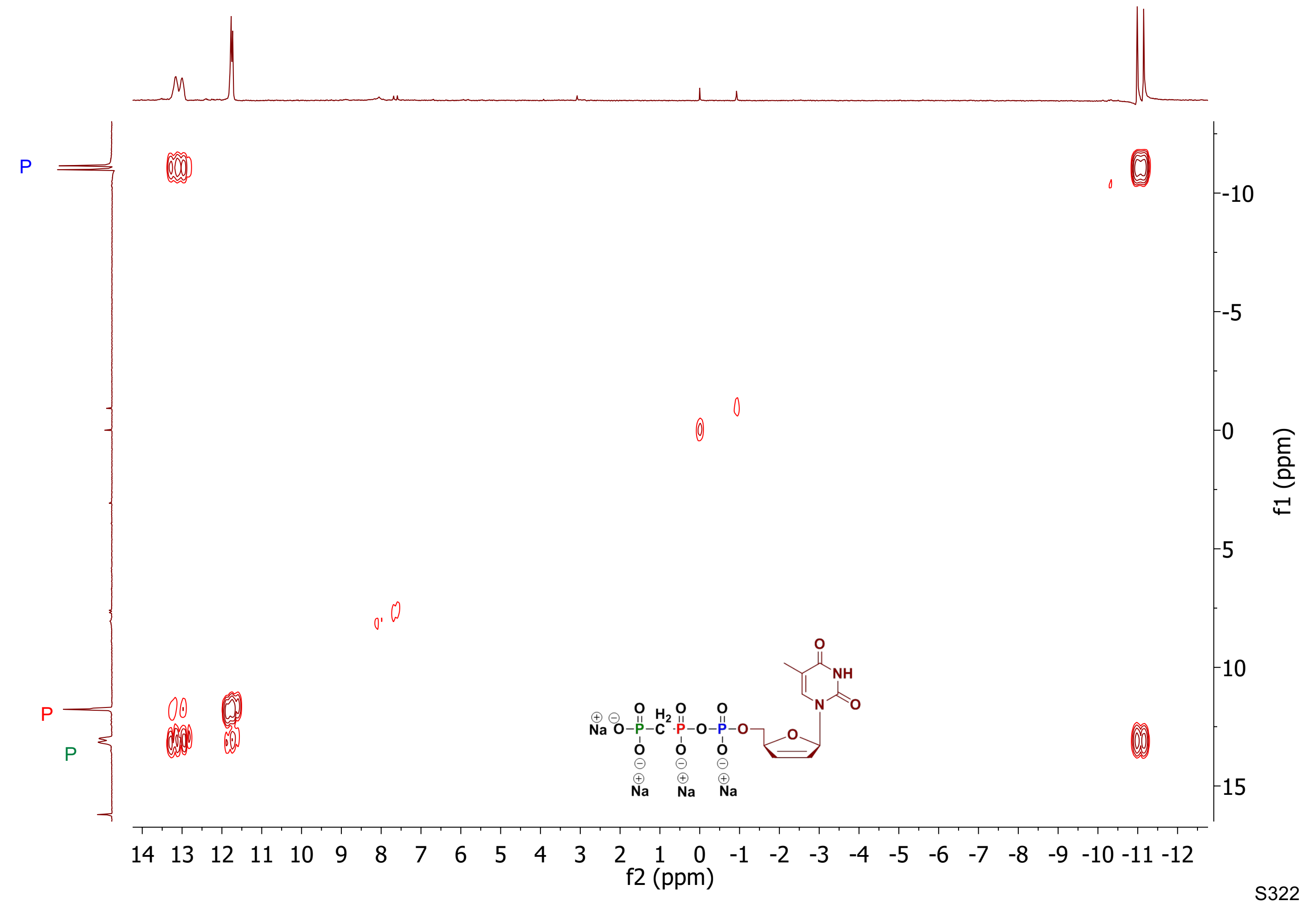




\section{${ }^{31} P\left\{{ }^{1} H\right\} N M R$}

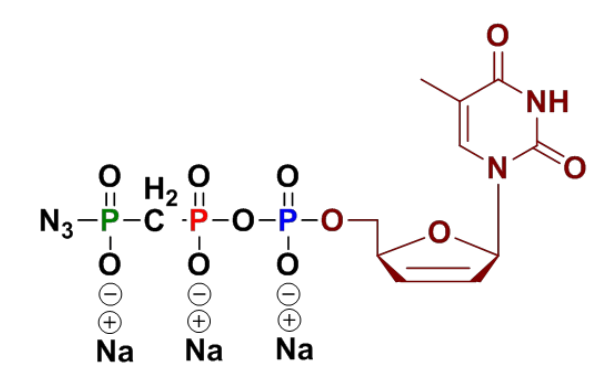

Ring opening with $\mathrm{LiN}_{3}$

crude product after precipitation

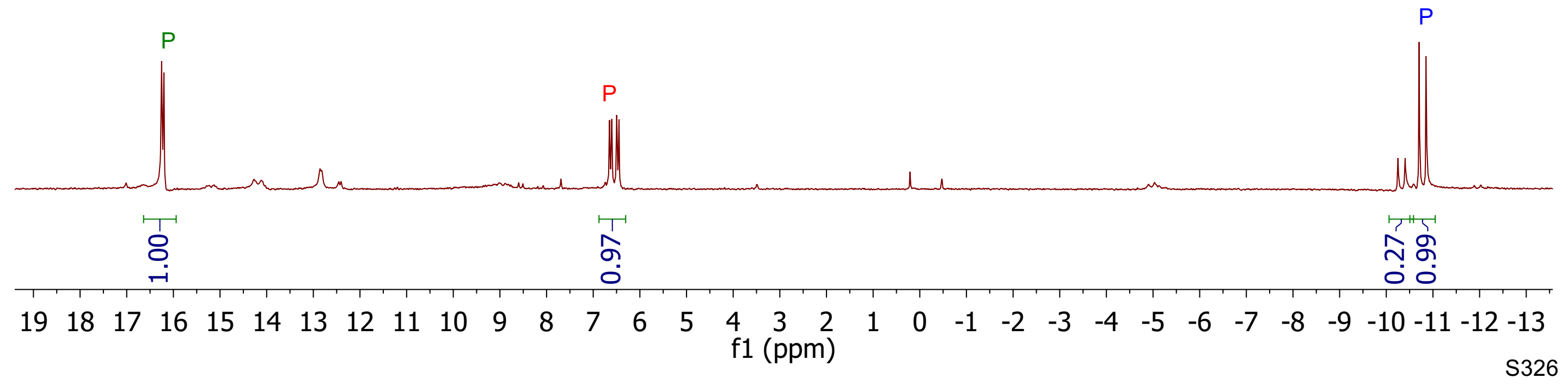




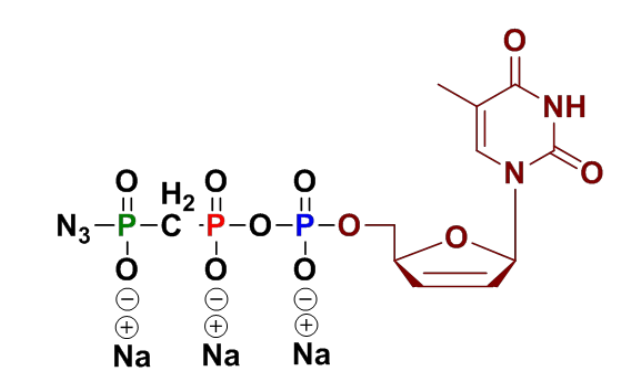

Ring opening with $\mathrm{LiN}_{3}$

crude product after precipitation

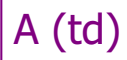
16.22

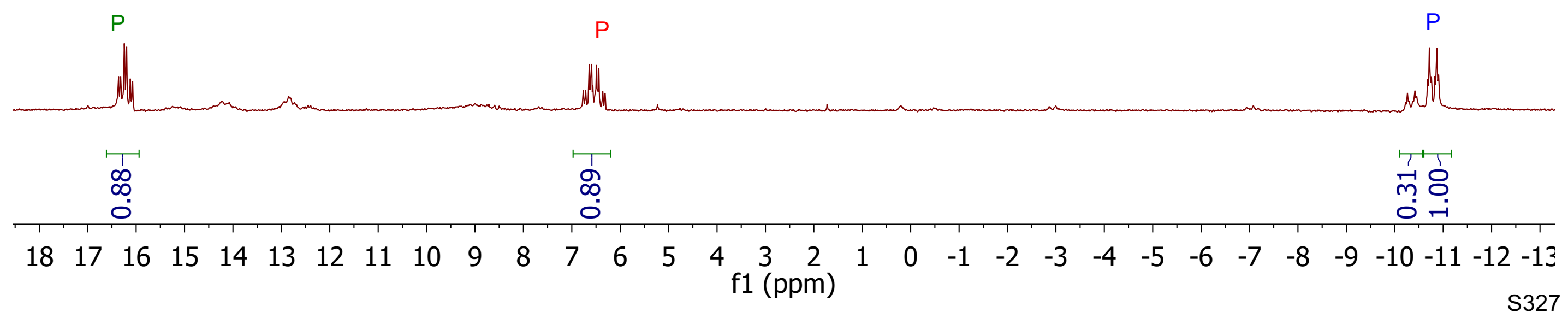




\section{${ }^{31} \mathrm{P}\left\{{ }^{1} \mathrm{H}\right\}$ NMR $\quad$ Spiking experiments demonstrate that $\mathrm{P}-\mathrm{N}_{3}(\mathbf{3 5})$ is decomposed in $\mathrm{P}-\mathrm{OH}(\mathbf{3 4})$ over the time}

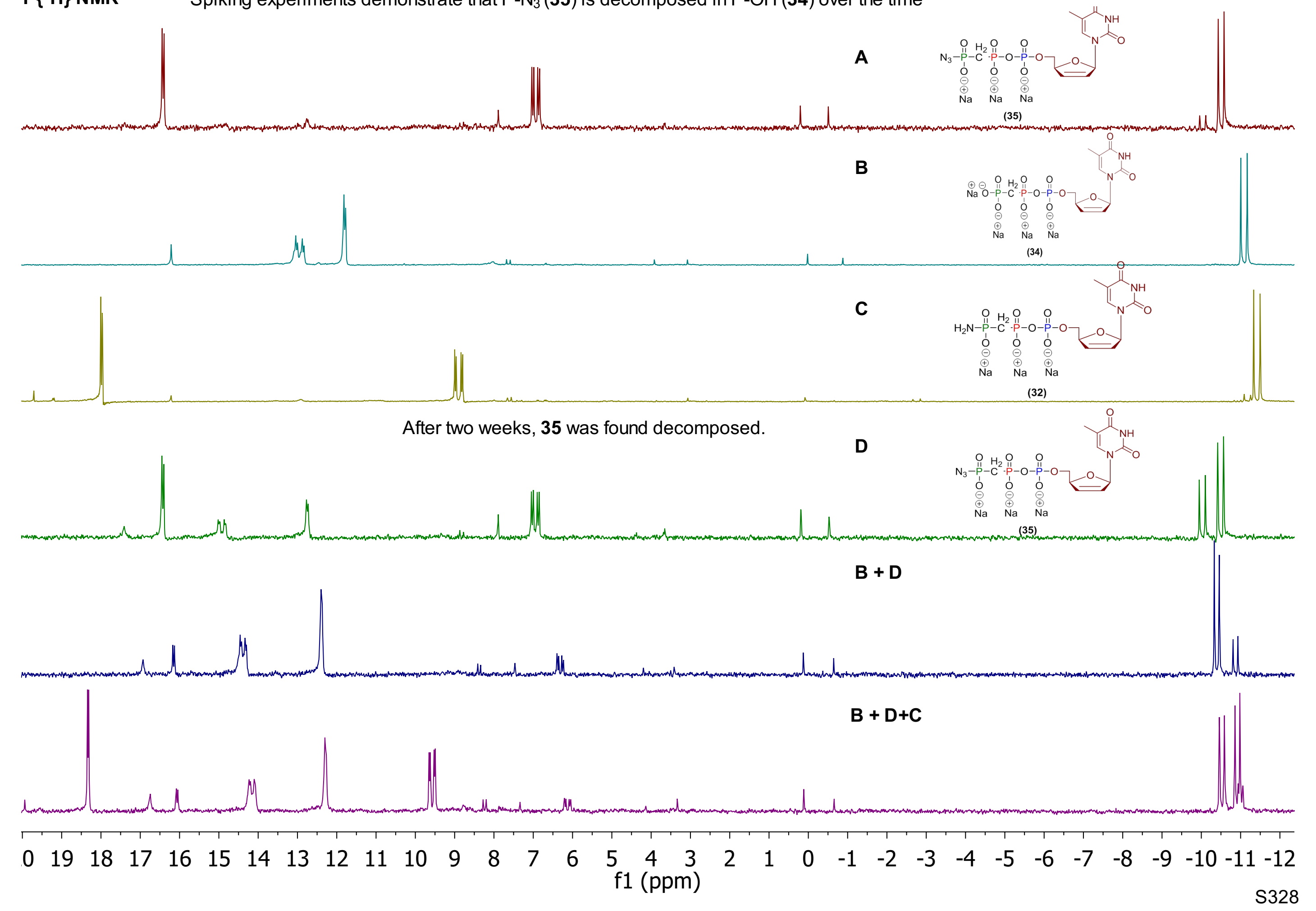




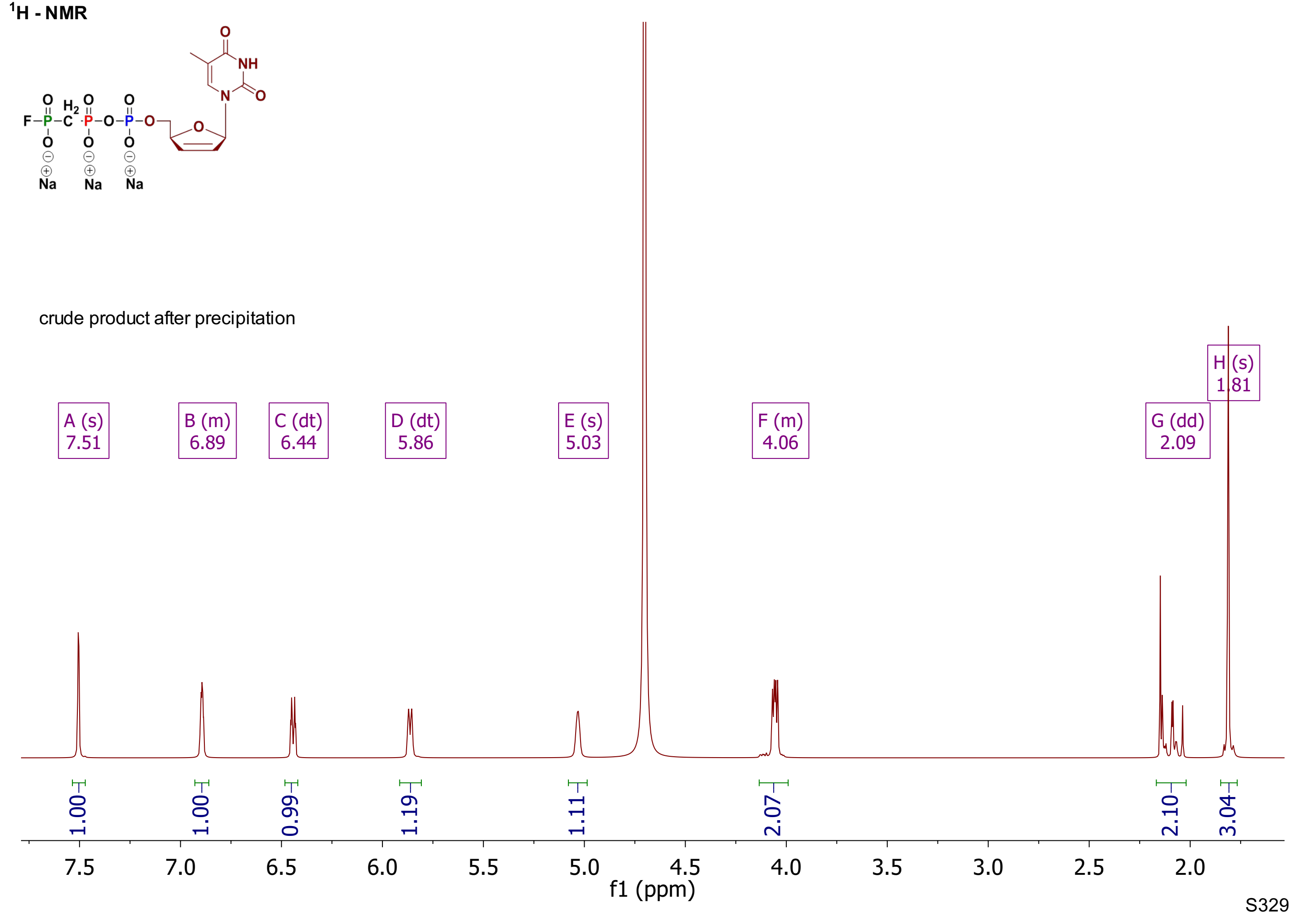




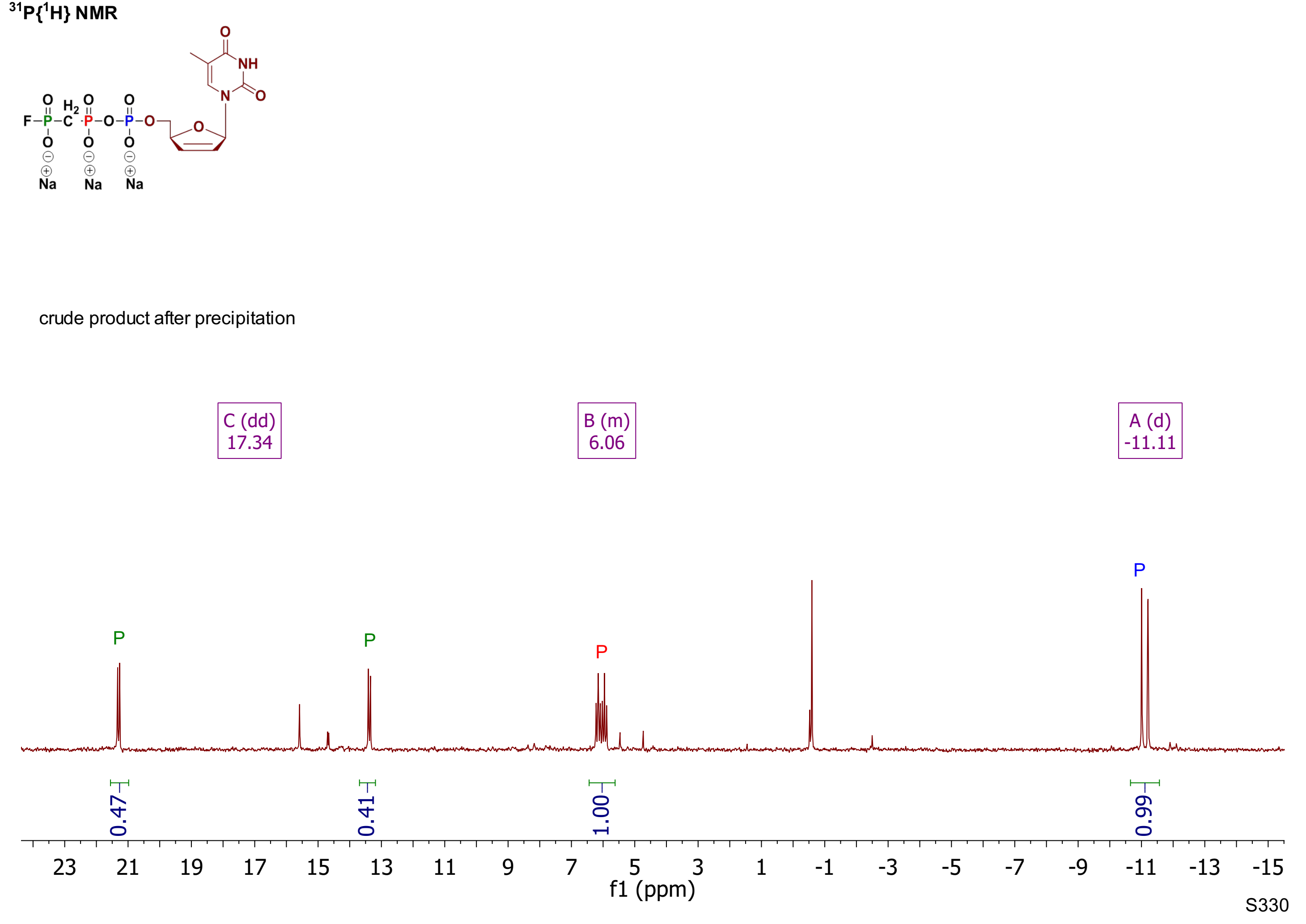




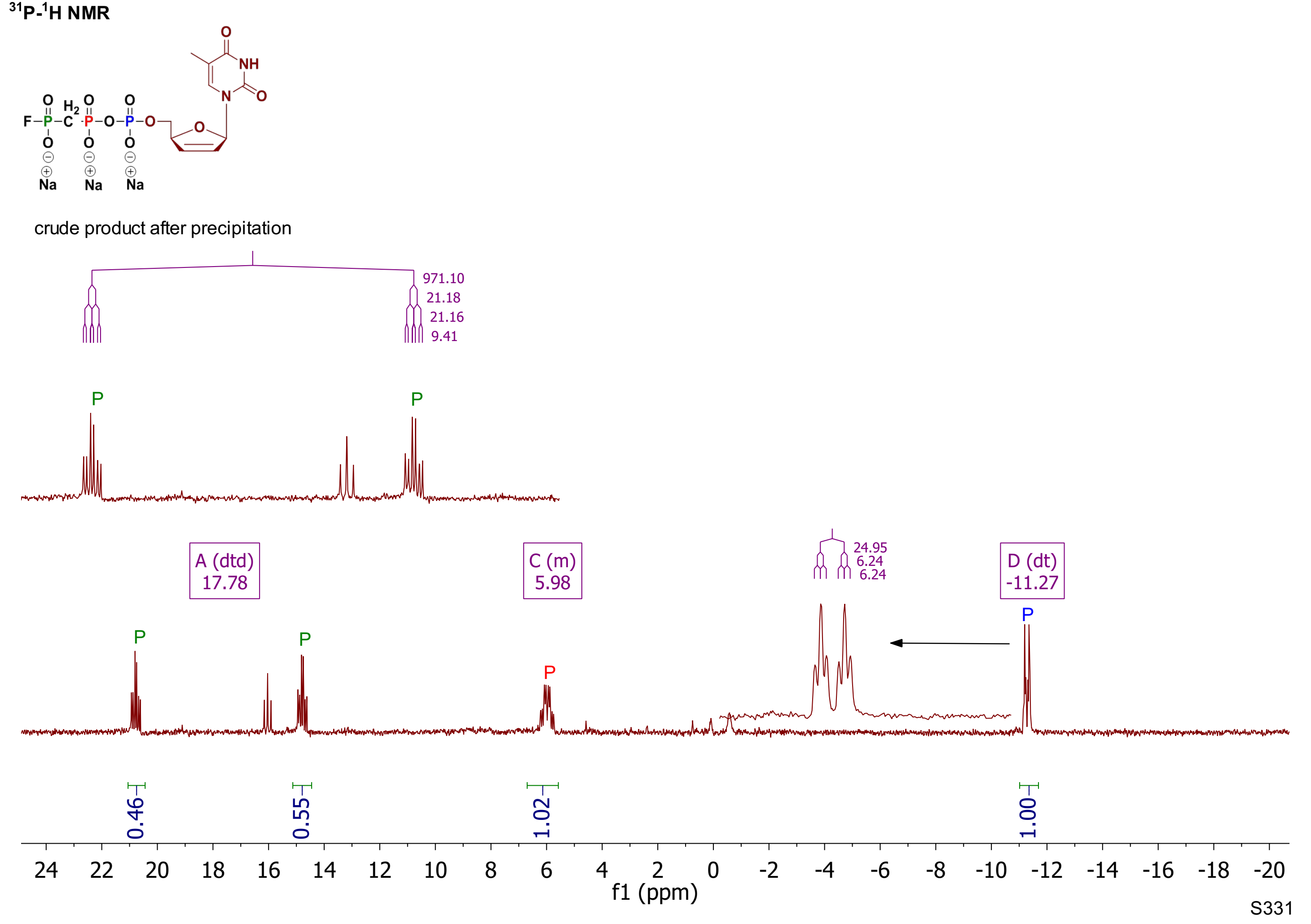




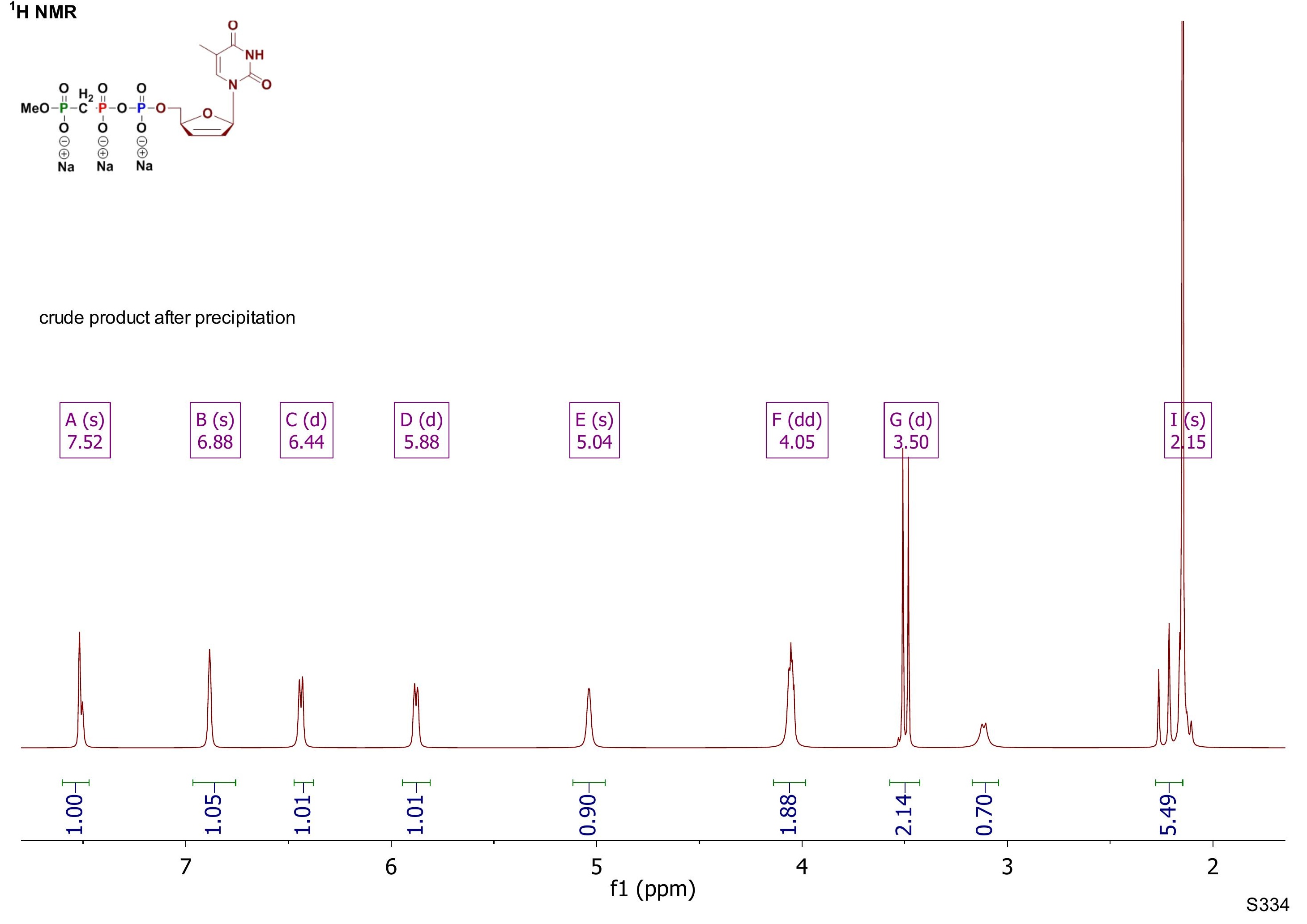




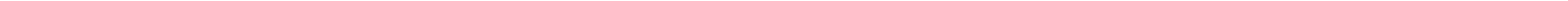




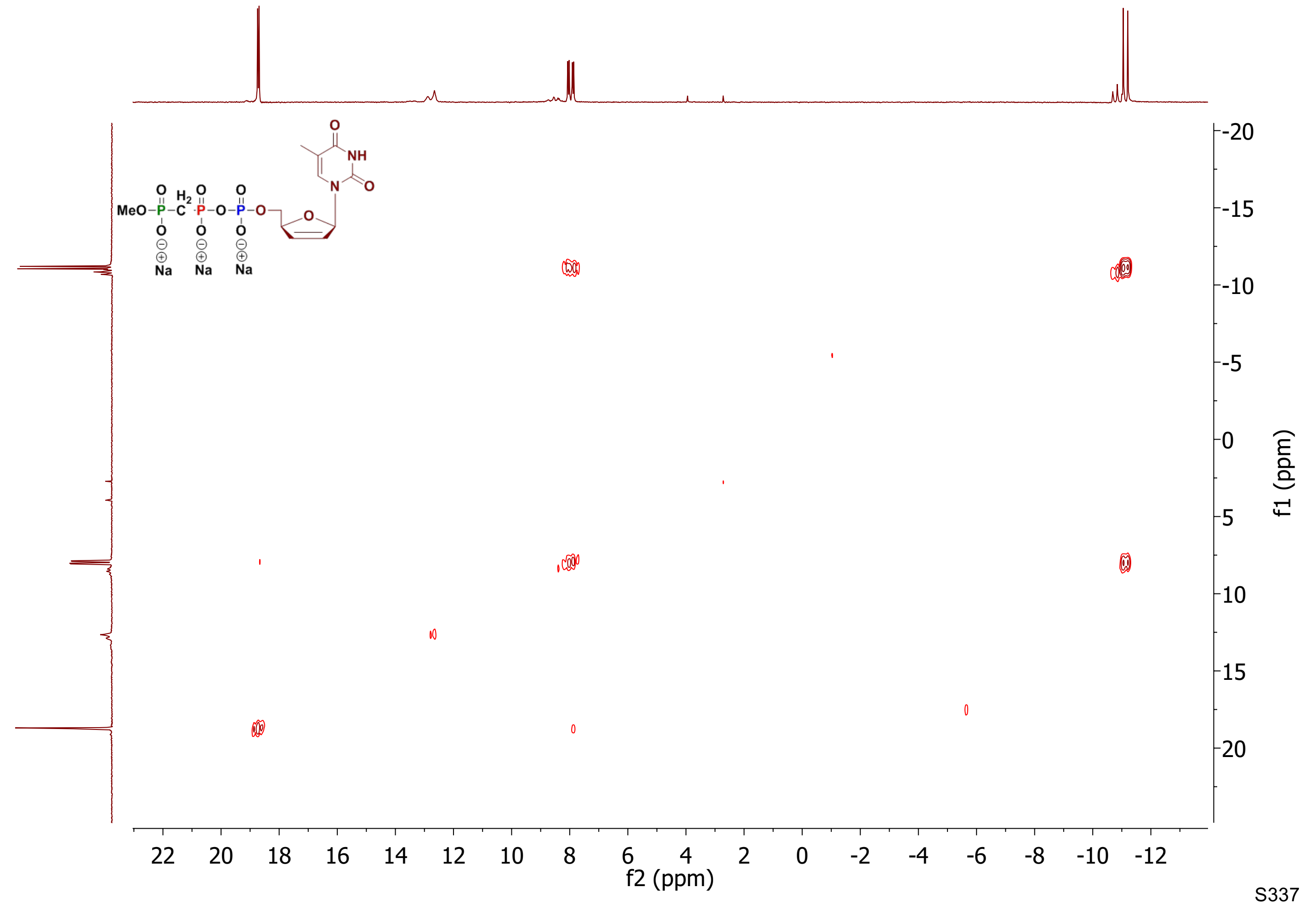




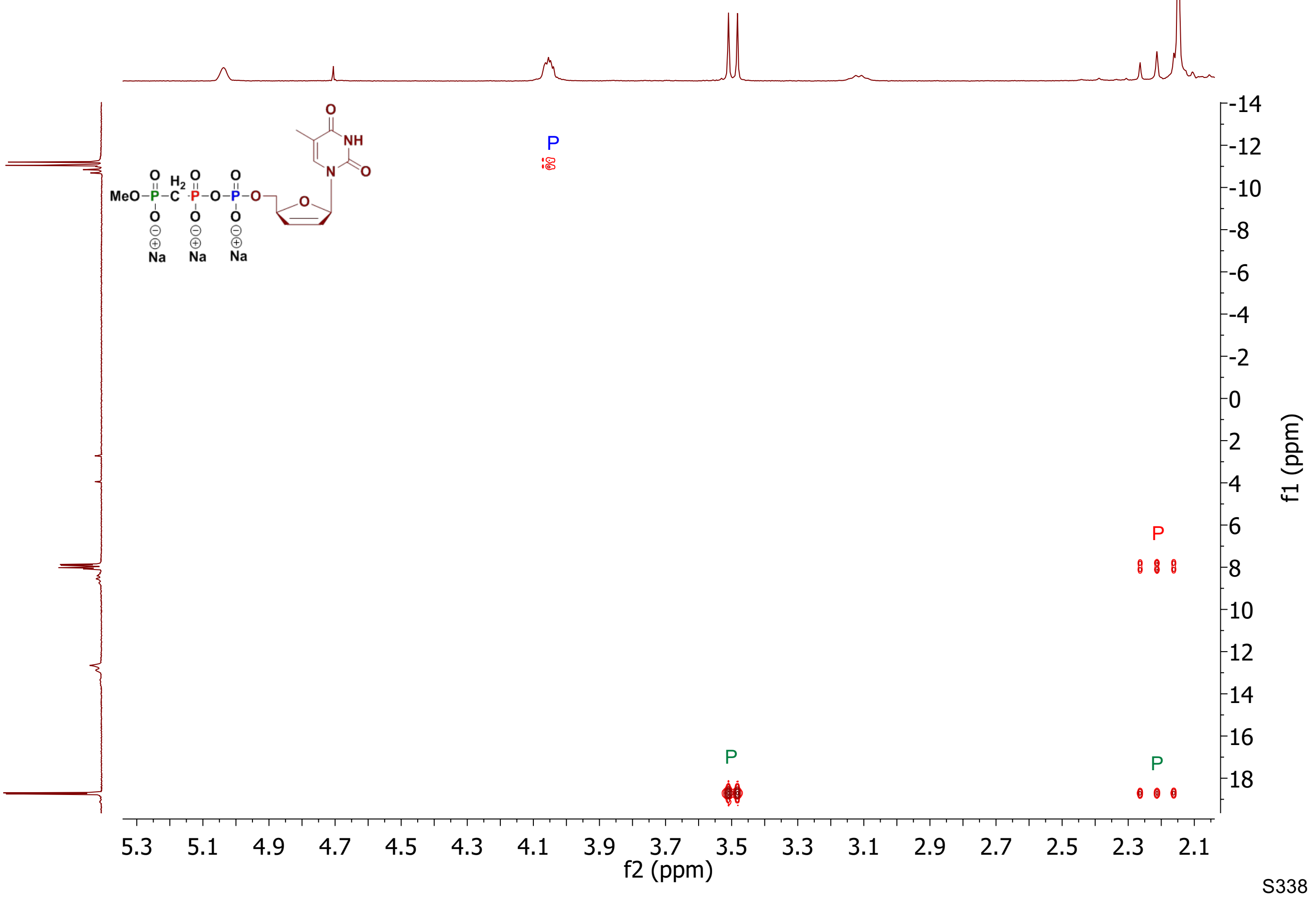




\section{${ }^{1} \mathrm{H}$ NMR}

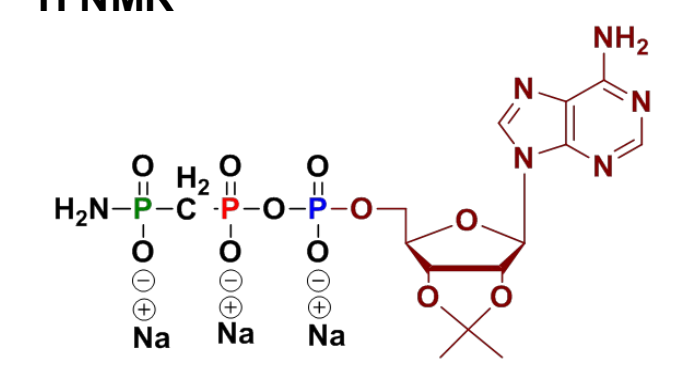

crude product after precipitation
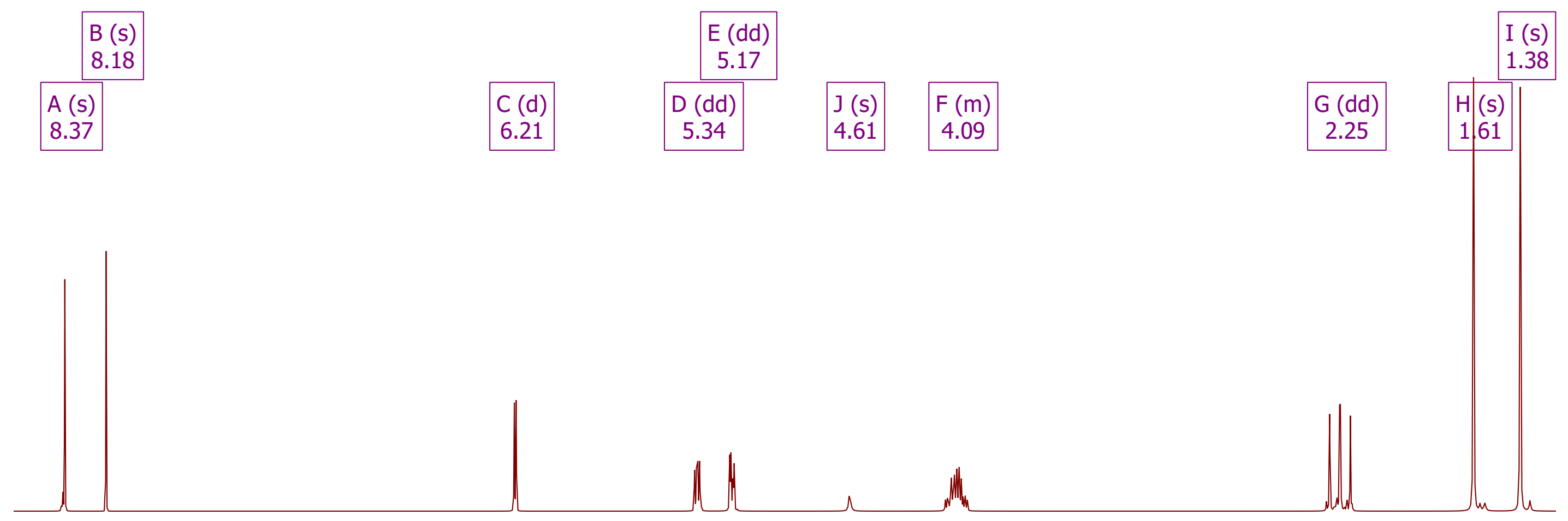

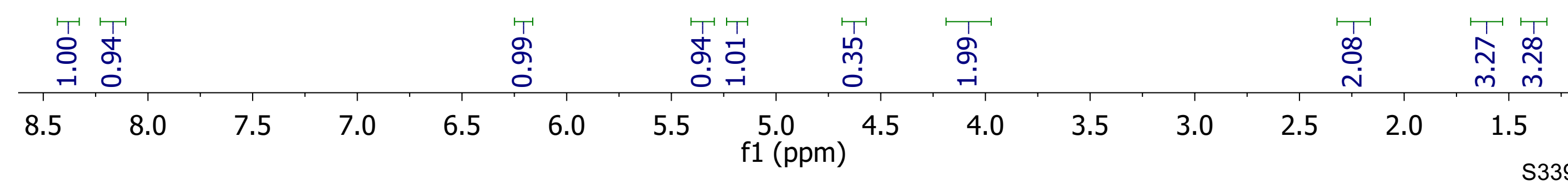




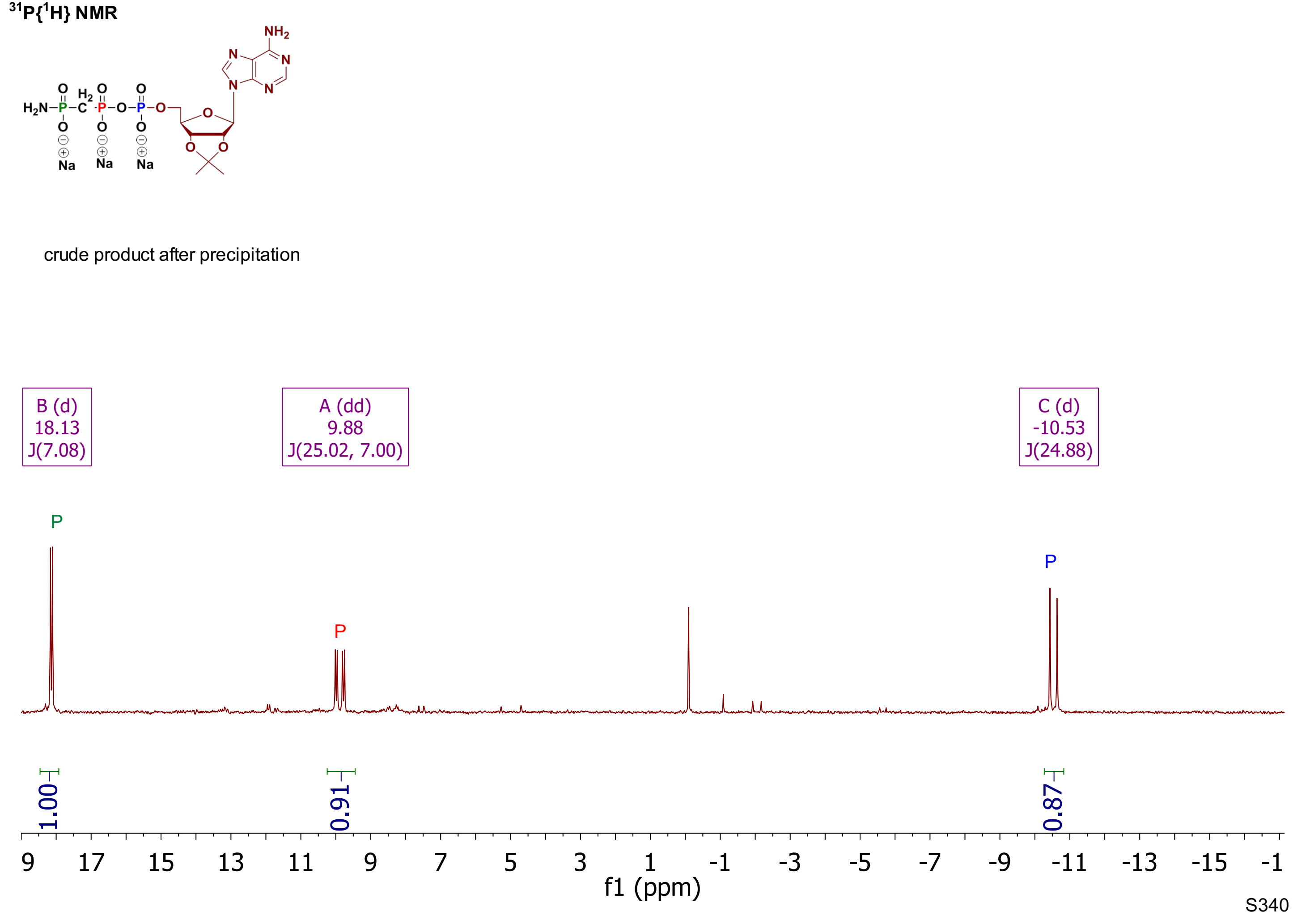




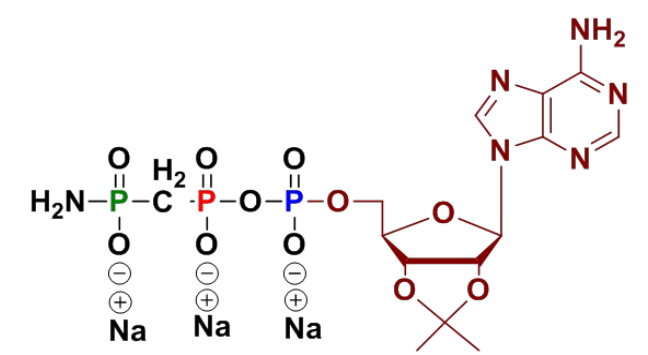

crude product after precipitation
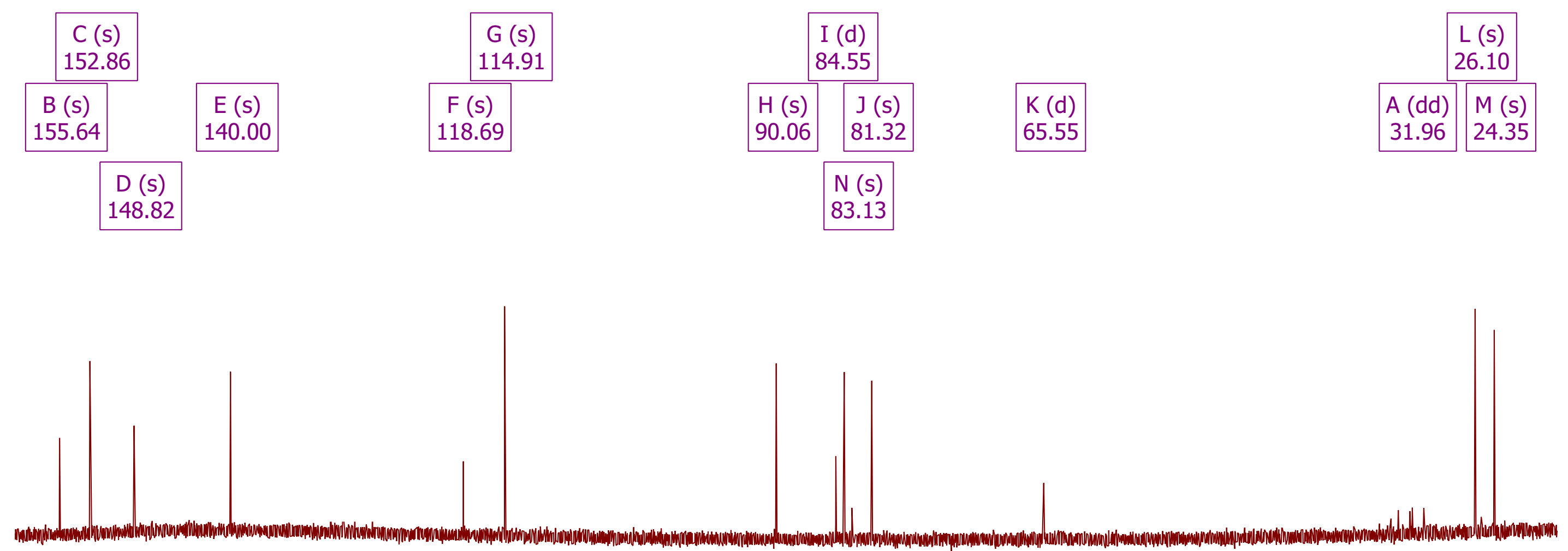


\section{${ }^{1} \mathrm{H}$ NMR}

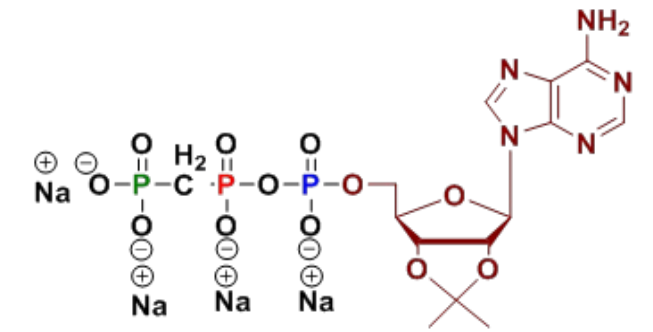

crude product after precipitation

\begin{tabular}{|l|}
\hline \multicolumn{1}{|c|}{$\begin{array}{r}\mathrm{B}(\mathrm{s}) \\
8.18\end{array}$} \\
\hline $\mathrm{A}(\mathrm{s})$ \\
8.39 \\
\hline
\end{tabular}

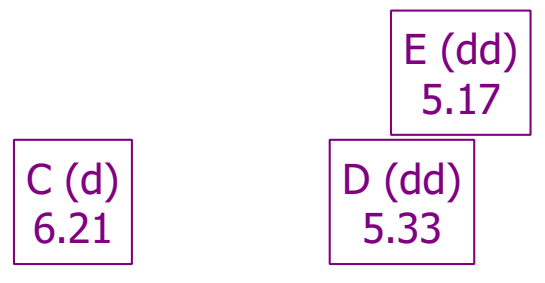

F (m) 4.09

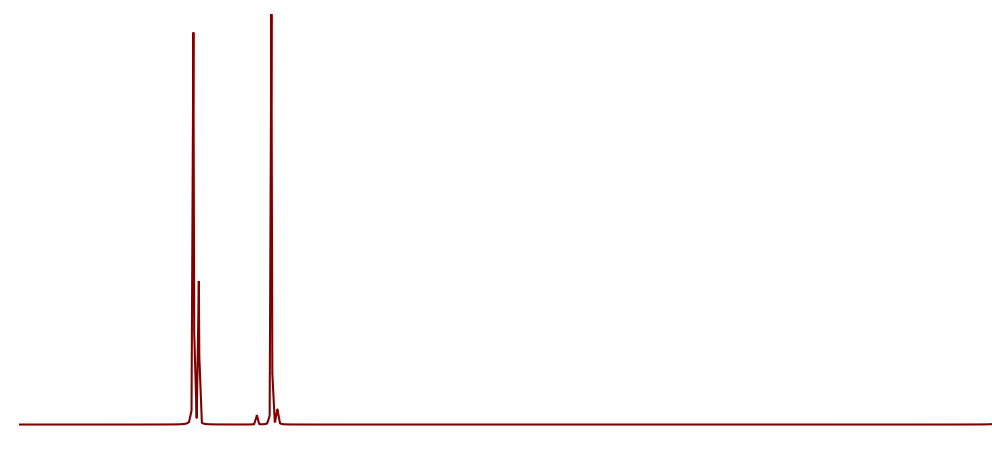

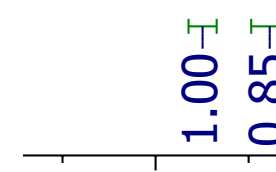
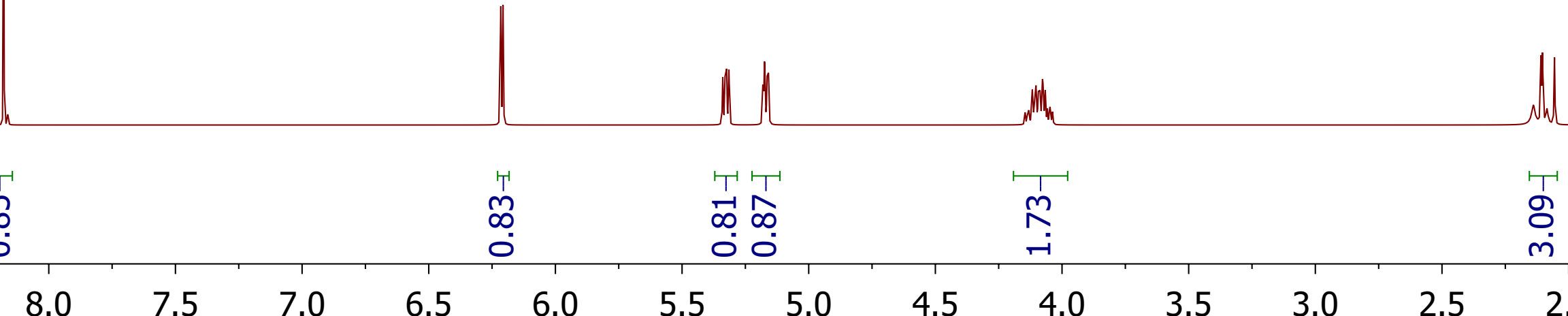

\section{5}

8.0

7.5

$6.5 \quad 6.0$

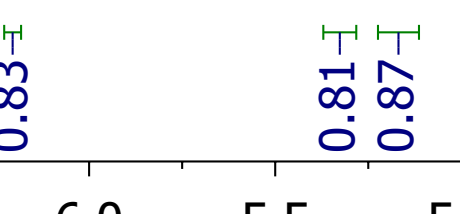

$\begin{array}{lc}5.5 & 5.0 \\ \mathrm{f} 1(\mathrm{ppm})\end{array}$

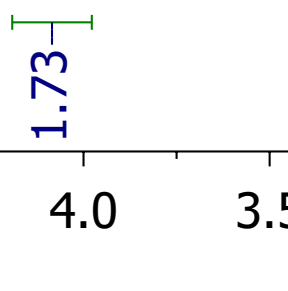

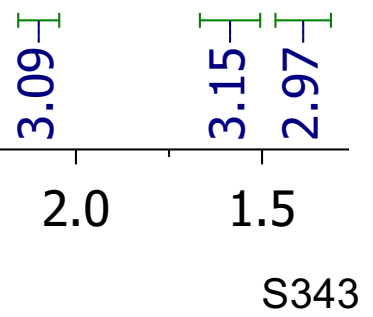




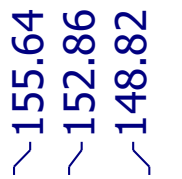

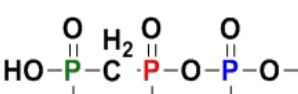

$\stackrel{\ominus}{\Theta}_{\oplus} \stackrel{\circ}{\ominus}_{\oplus} \stackrel{\circ}{\ominus}_{\oplus}$

$\mathrm{Na}{ }^{\oplus a}{ }^{\oplus}$

crude product after precipitation

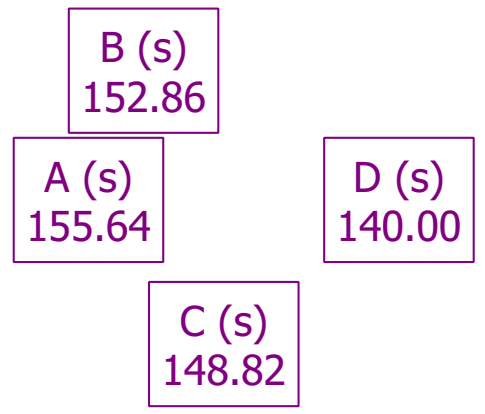

$$
S_{N}^{N}
$$$$
N^{N}
$$

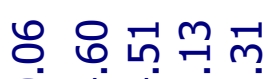

வ்

กิก กิ

חن

 m으용으 mึ ?

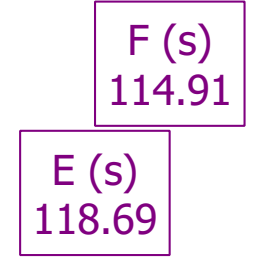

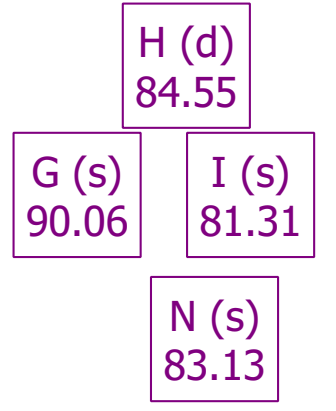

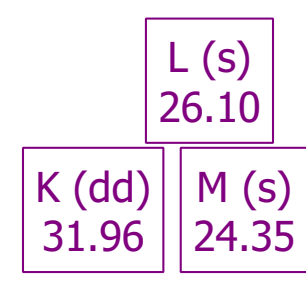

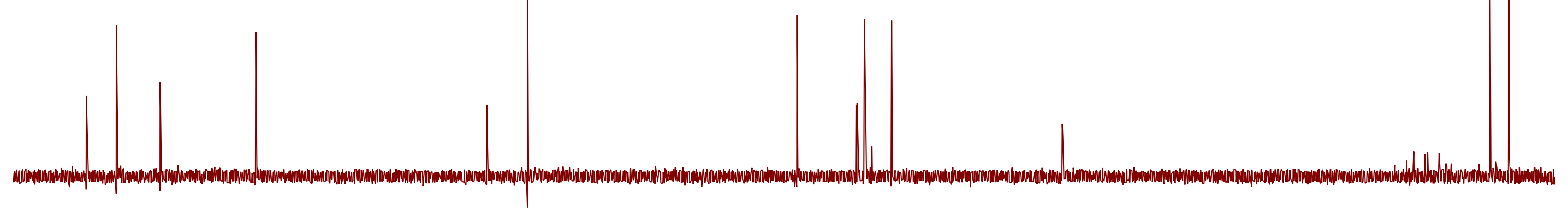




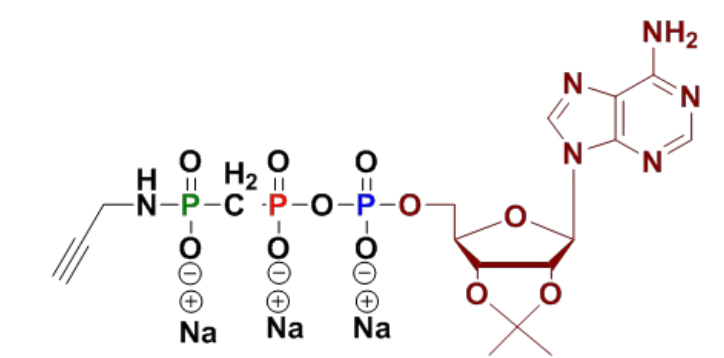

crude product after precipitation
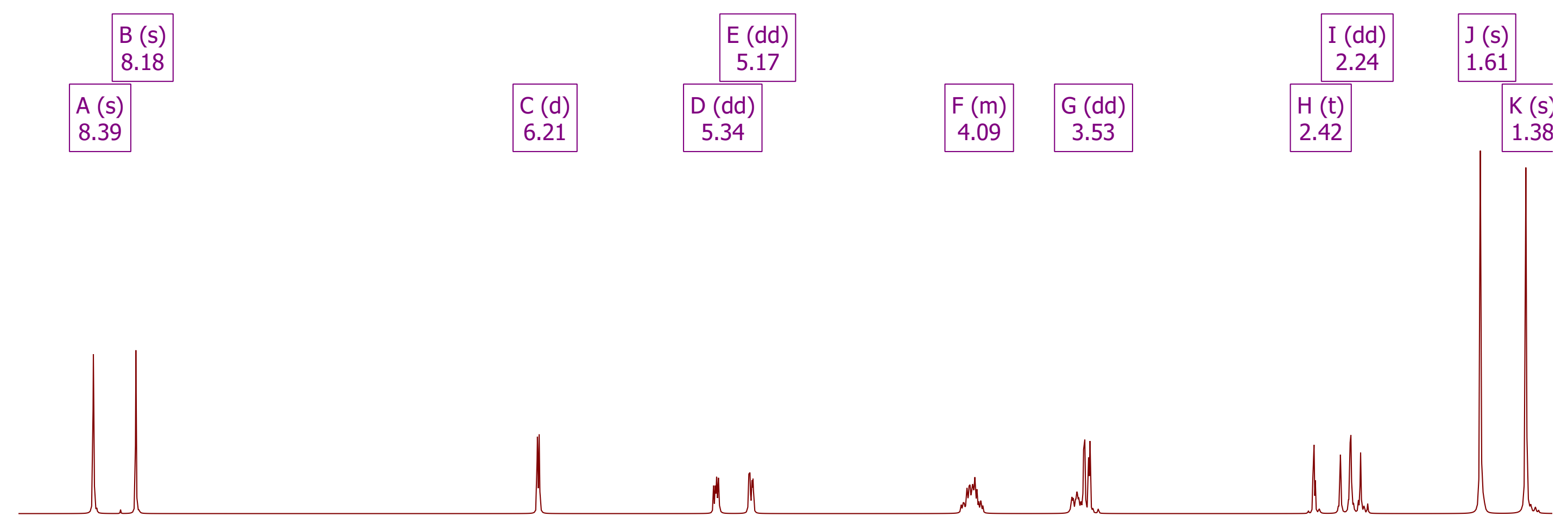

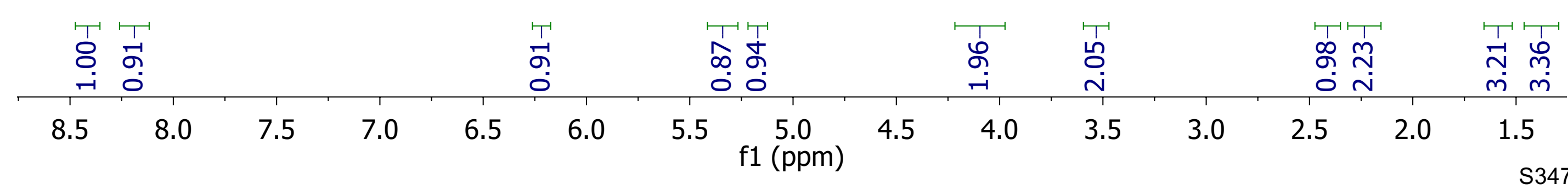




\section{${ }^{31} P\left\{{ }^{1} H\right\} N M R$}

도용

NंÑ

우남웅

$\infty \infty \infty$

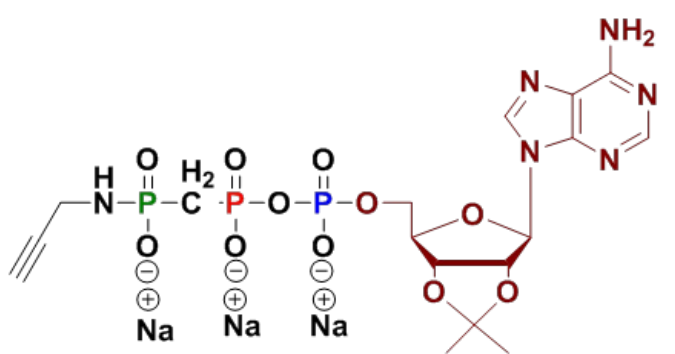

crude product after precipitation

$$
\begin{aligned}
& C(d) \\
& 17.73 \\
& \hline
\end{aligned}
$$

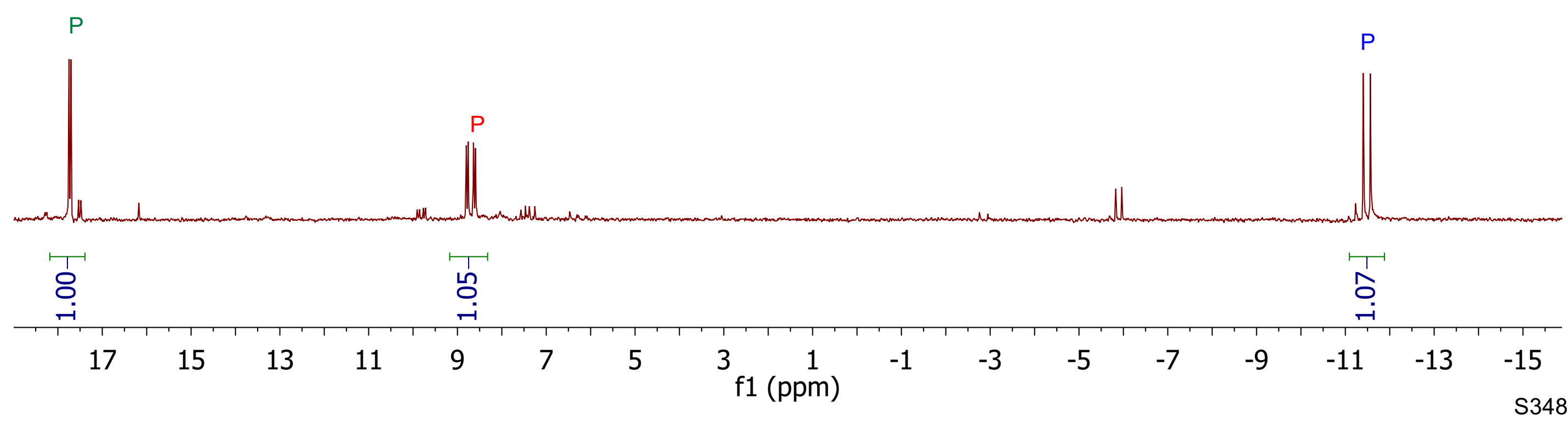




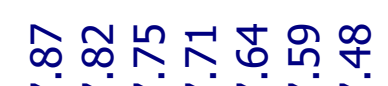

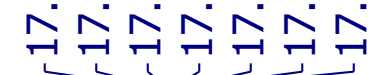

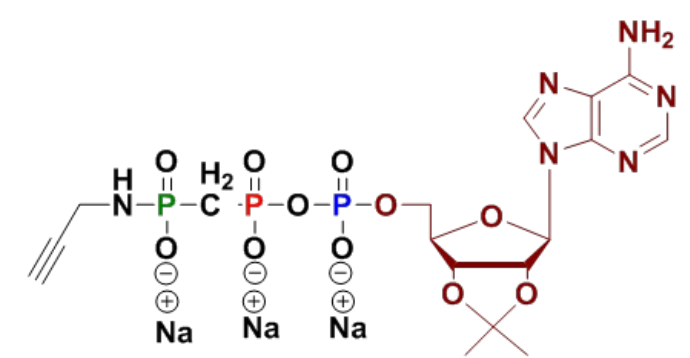

crude product after precipitation

$$
\begin{array}{|l|}
\hline B(m) \\
17.73 \\
\hline
\end{array}
$$

\begin{tabular}{|c|c|c|c|c|c|c|c|c|c|c|c|c|c|c|c|c|}
\hline '† & & & & & & & & & & & & & & & $\underset{\sim}{\stackrel{T}{\sim}}$ & \\
\hline 18 & 16 & 14 & 12 & 10 & 8 & 6 & 4 & $\begin{array}{l}2 \\
\mathrm{f} 1(\mathrm{ppm})\end{array}$ & 0 & -2 & -4 & -6 & -8 & -10 & -12 & -14 \\
\hline
\end{tabular}

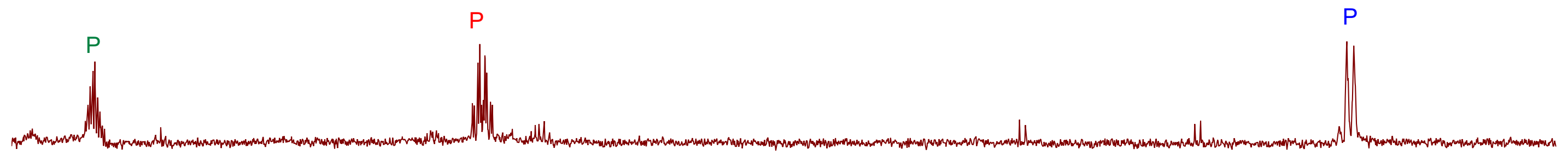




\section{${ }^{31} P\left\{{ }^{1} H\right\} N M R$}

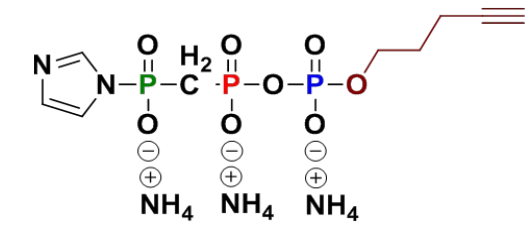

quantat. in reaction mixture

\begin{tabular}{|c|c|}
\hline$B(d)$ & $A(d d)$ \\
8.80 & 4.30 \\
\hline
\end{tabular}

C (d) $-10.94$

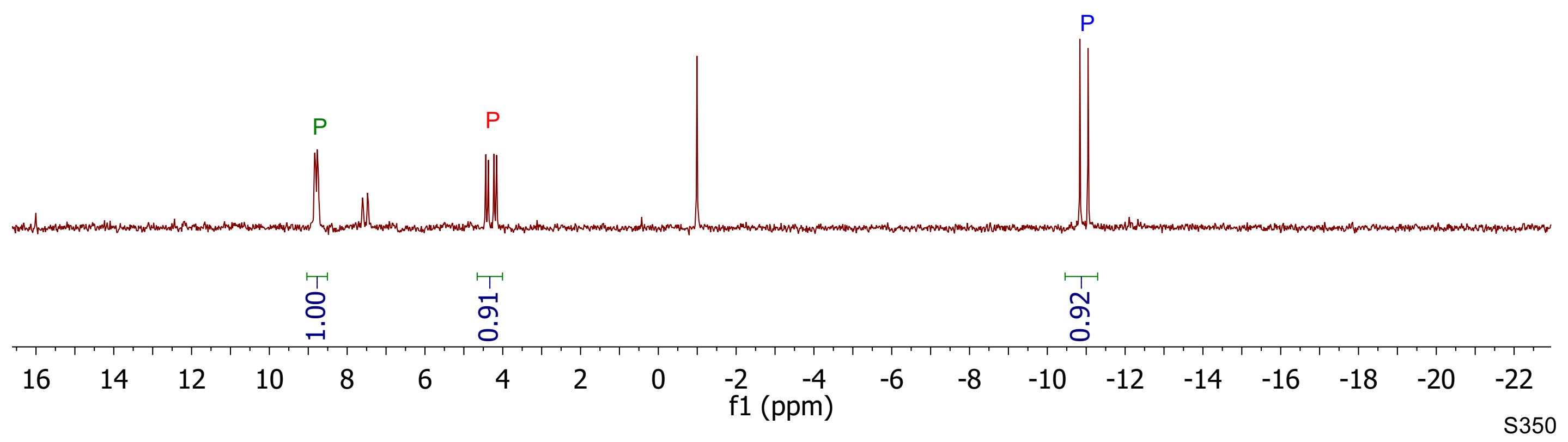




\section{${ }^{1} \mathrm{H}\left\{{ }^{31} \mathrm{P}\right\}$ NMR}
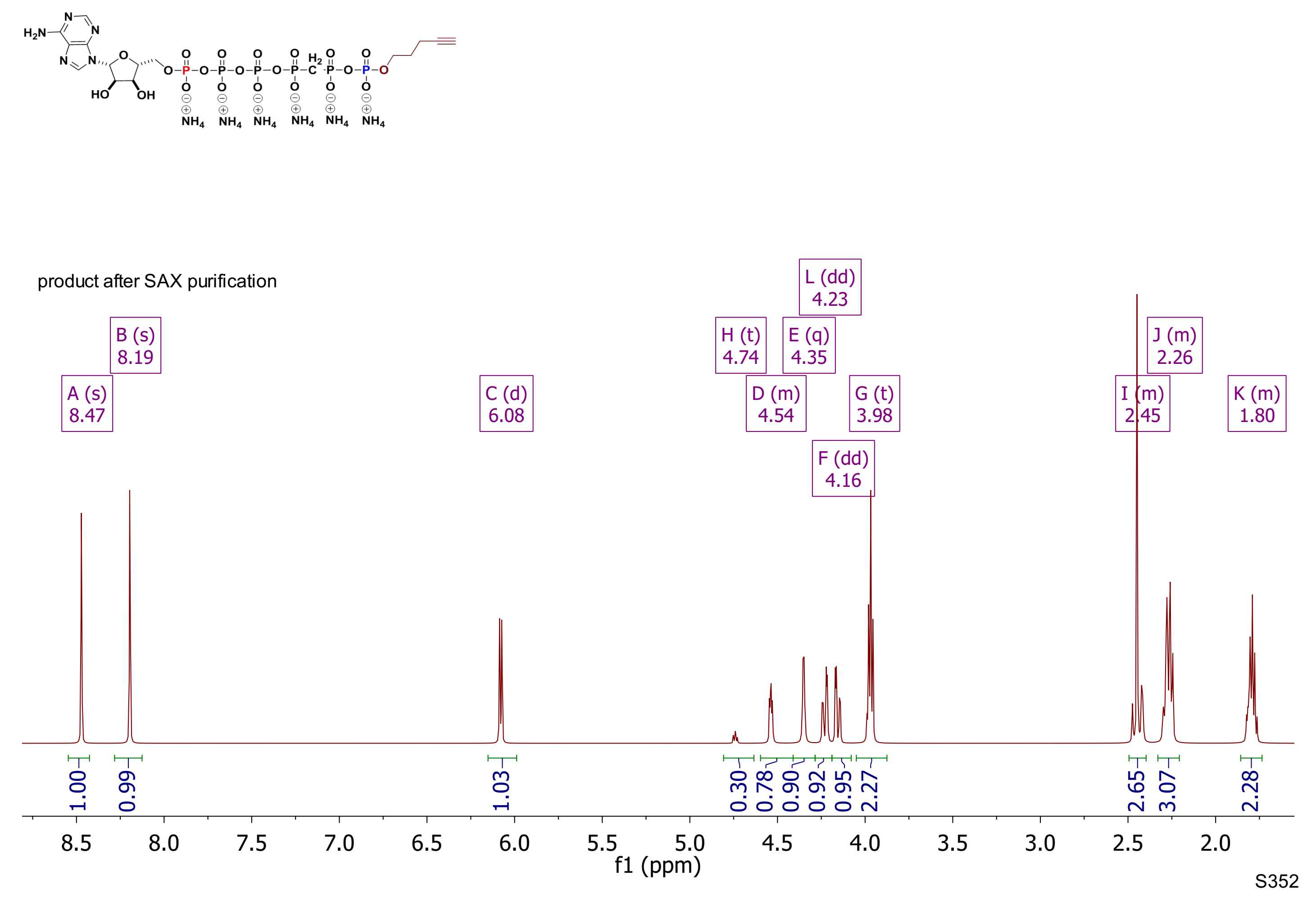


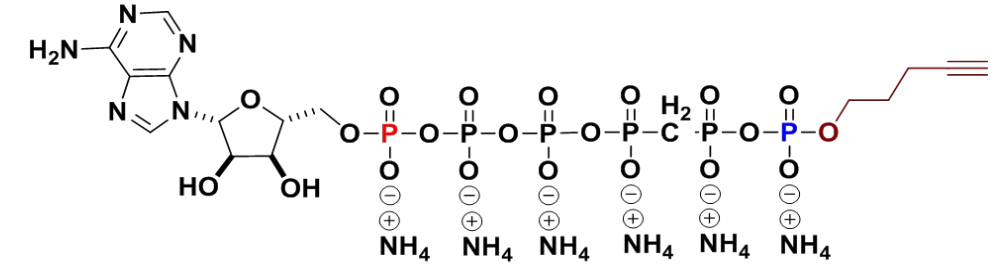

product after SAX purification
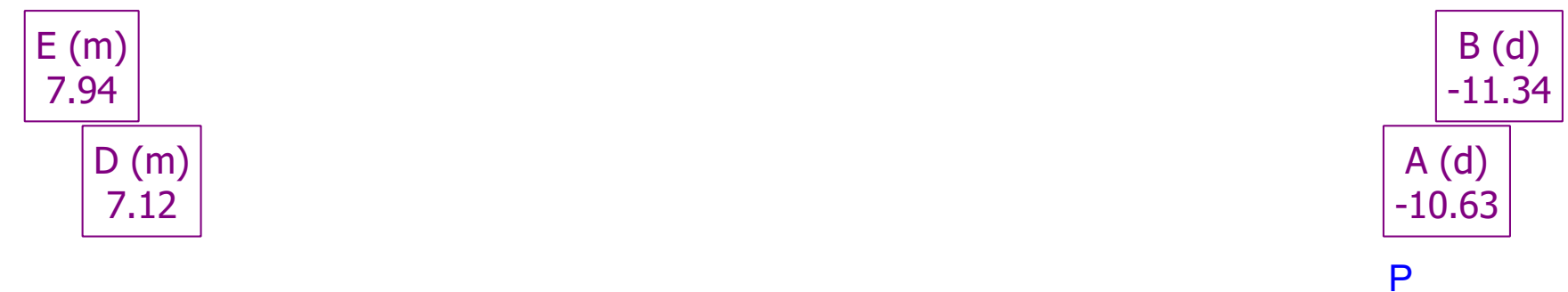

- P-C-P

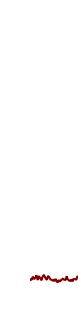

กั้ ชั

$\begin{array}{ccccccccc}7 & 5 & 3 & 1 & -1 & -3 & -5 & -7 & -9 \\ & & & & & & \mathrm{f} 1(\mathrm{ppm})\end{array}$

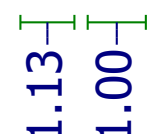

$\begin{array}{llllll}-11 & -13 & -15 & -17 & -19 & -21\end{array}$

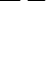

$-23$
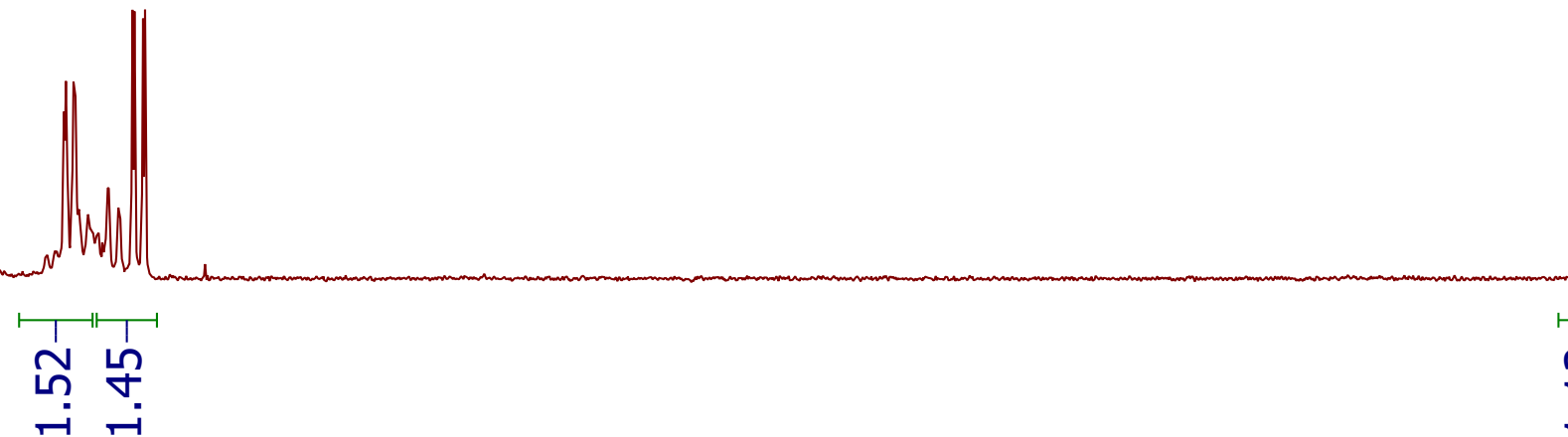


\section{${ }^{31} \mathrm{P}-{ }^{31} \mathrm{P}$ COSY NMR}

product after SAX purification

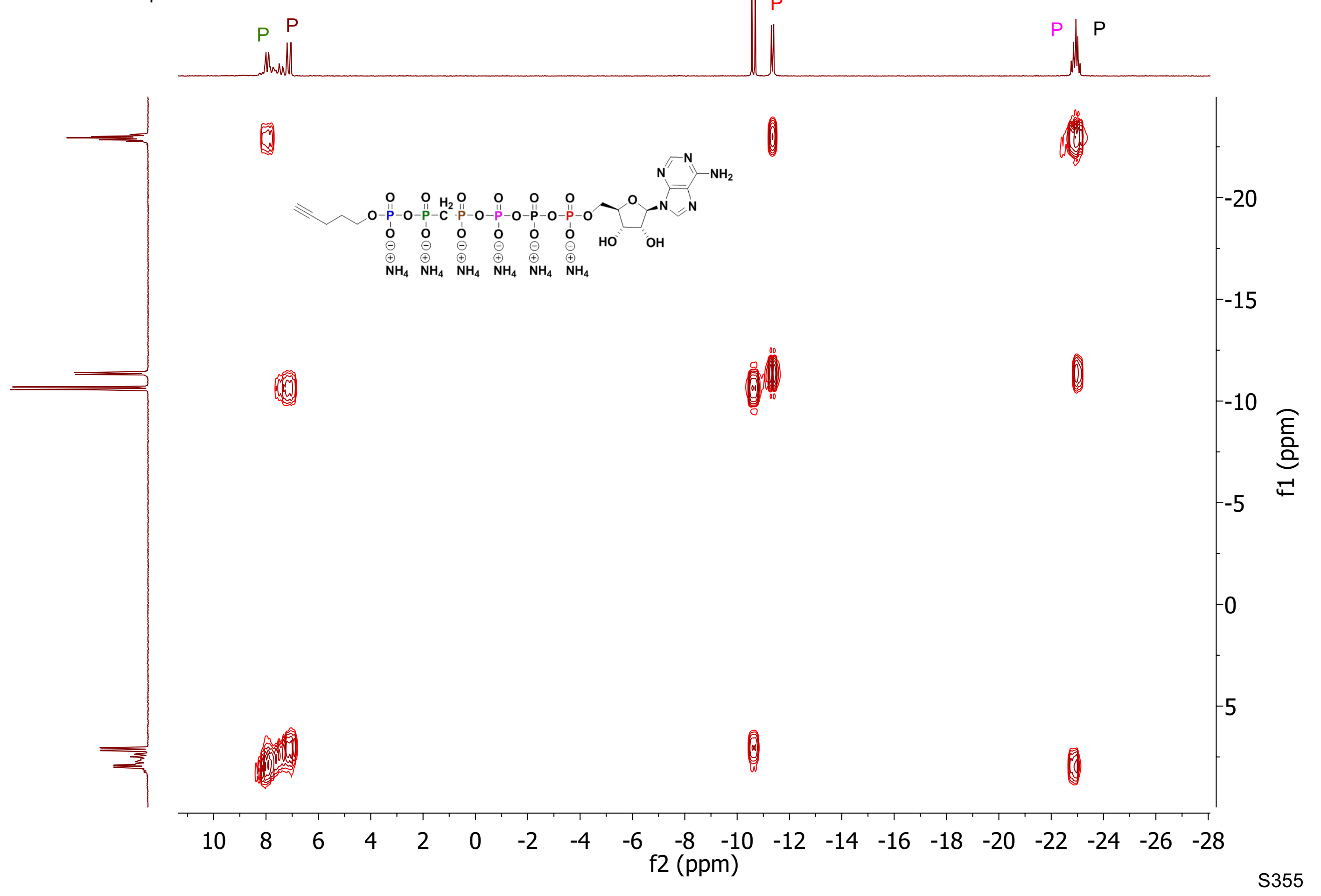




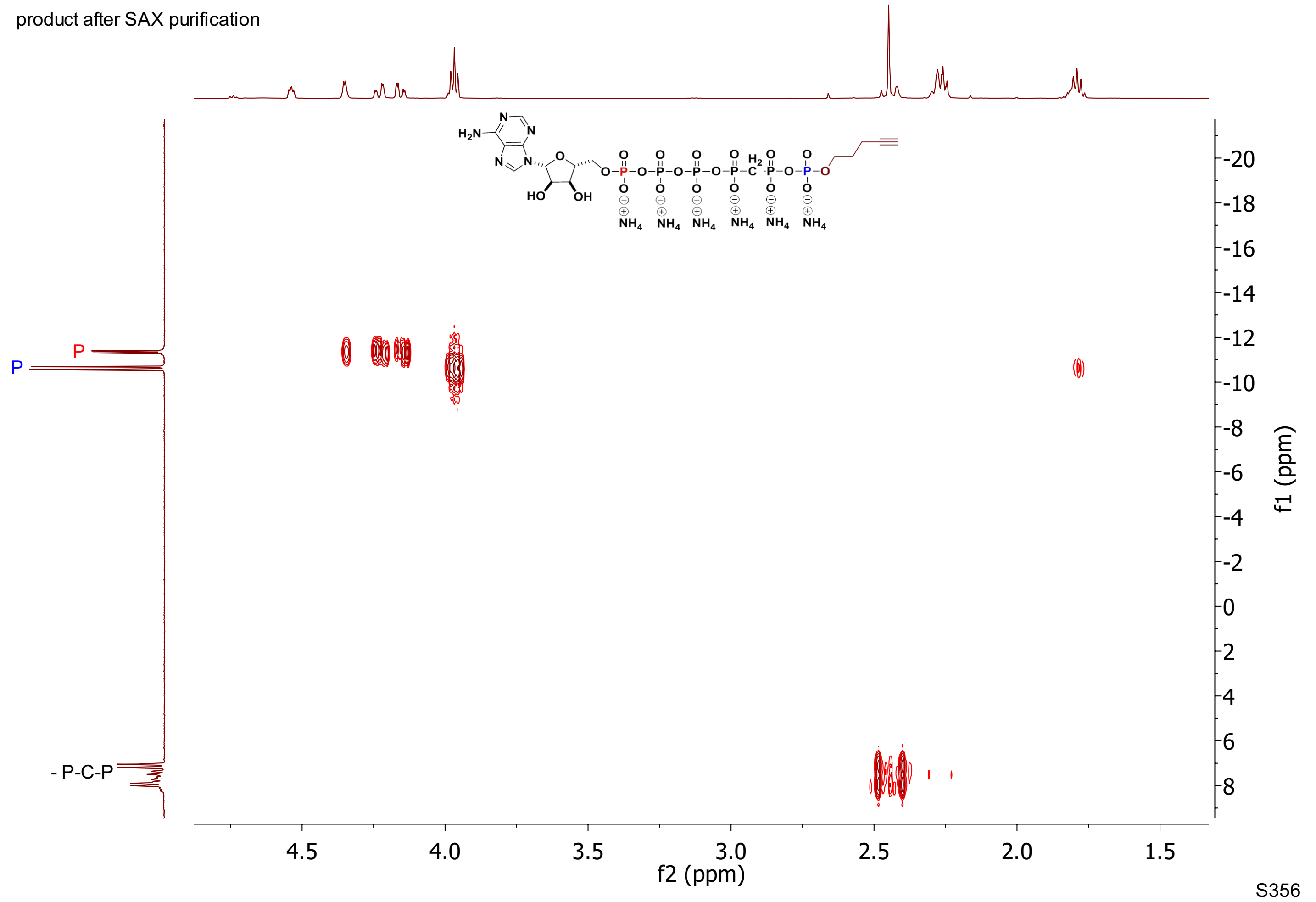




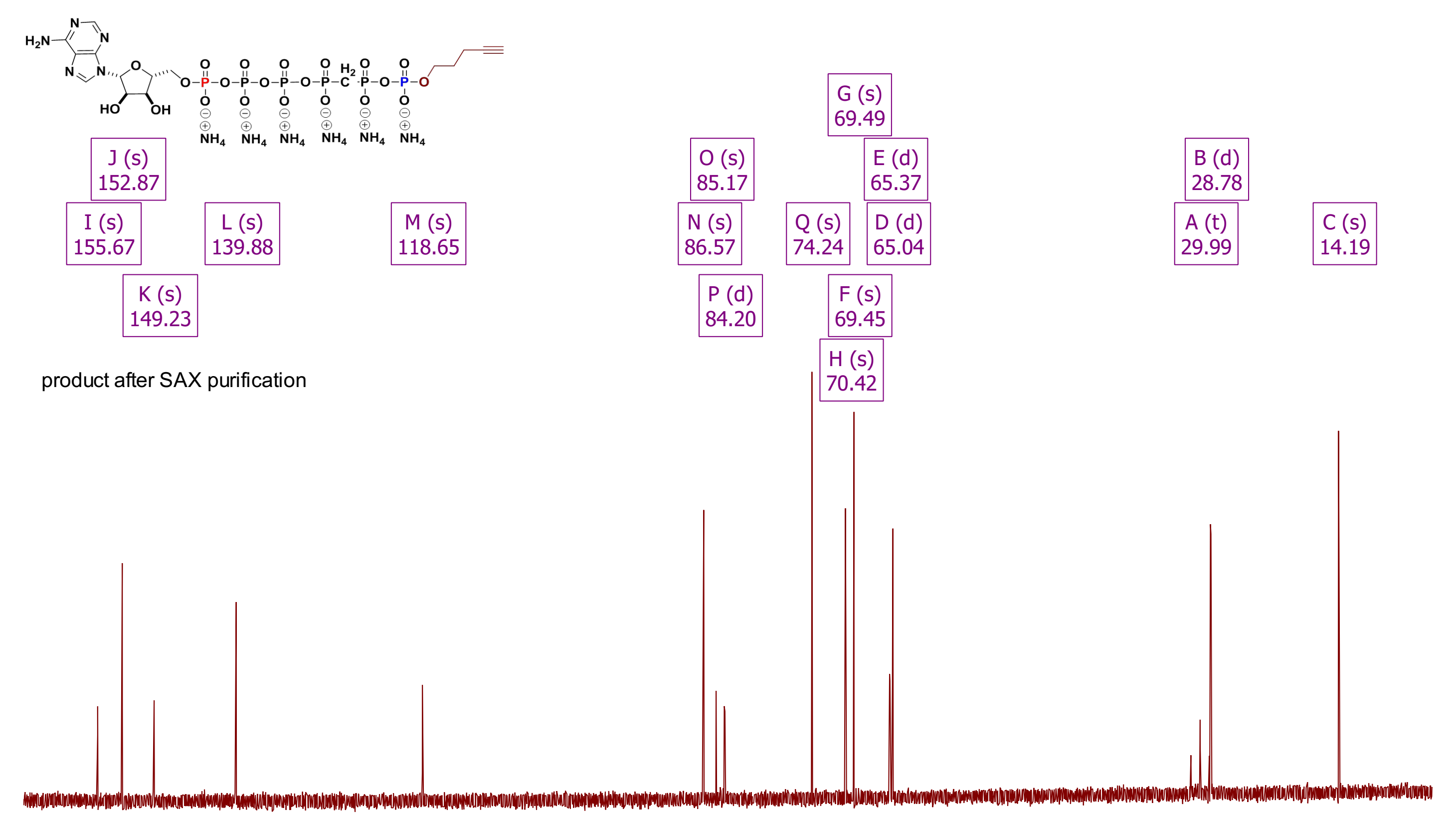

\begin{tabular}{|c|c|c|c|c|c|c|c|c|c|c|c|c|c|c|}
\hline 160 & 150 & 140 & 130 & 120 & 110 & 100 & $\begin{array}{lr}90 & 80 \\
& \mathrm{f} 1(\mathrm{ppm})\end{array}$ & 70 & 60 & 50 & 40 & 30 & 20 & $\begin{array}{l}10 \\
\text { S357 }\end{array}$ \\
\hline
\end{tabular}




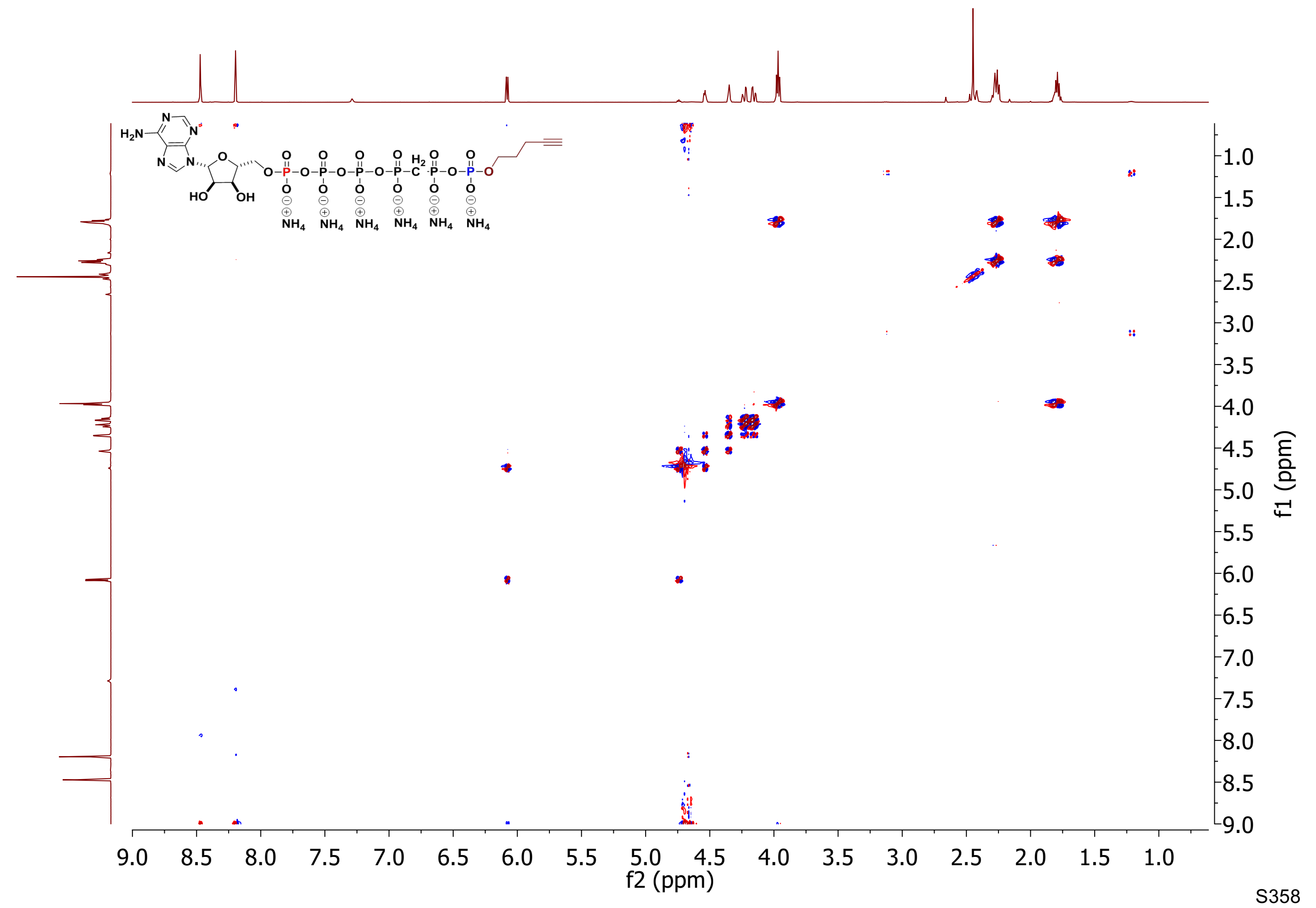




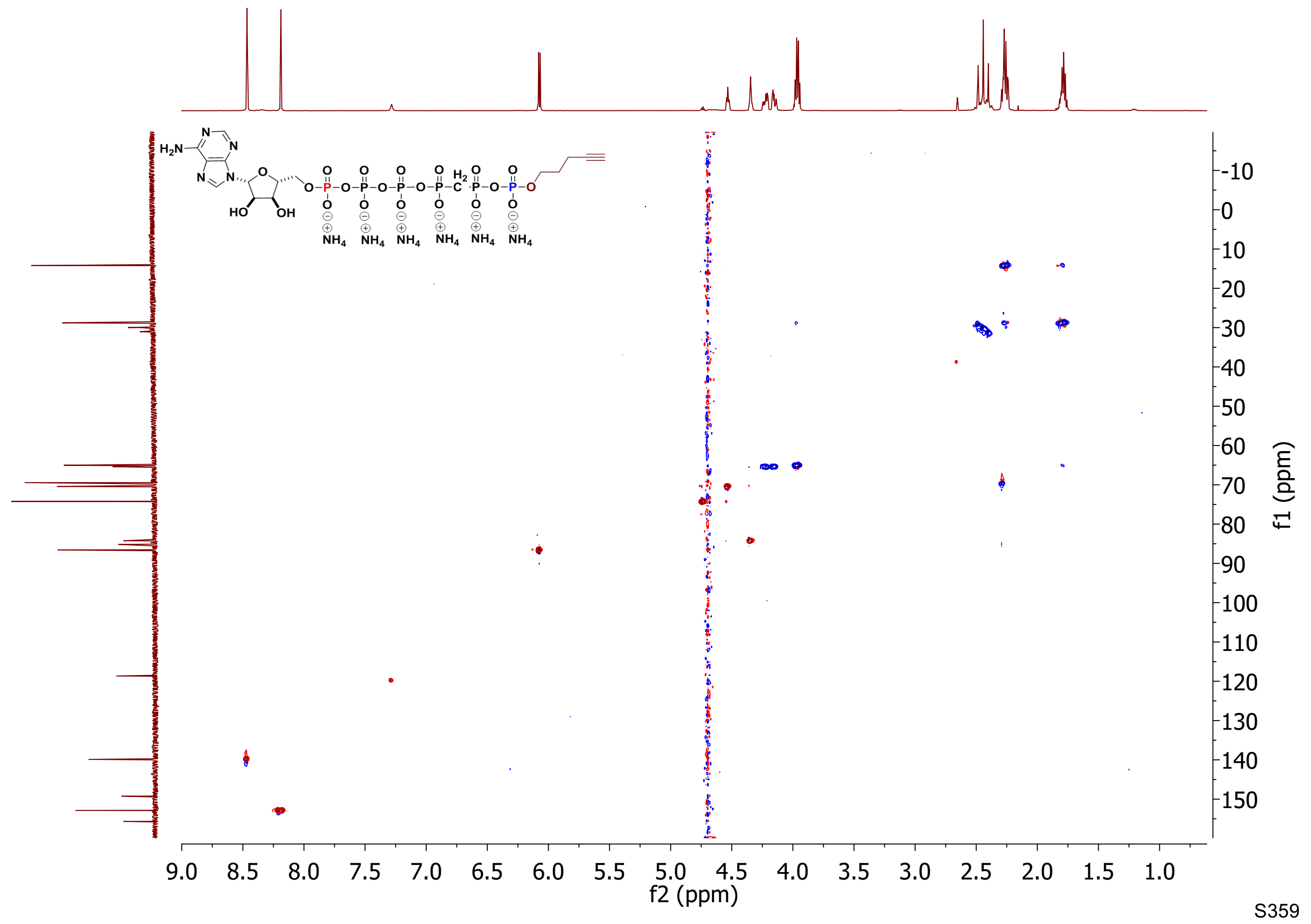




\section{Synthesis of triphosphates based on $c-\mathrm{Py}_{\mathrm{CF} 2} \mathrm{PA}\left(\mathrm{A}_{3}\right)$}

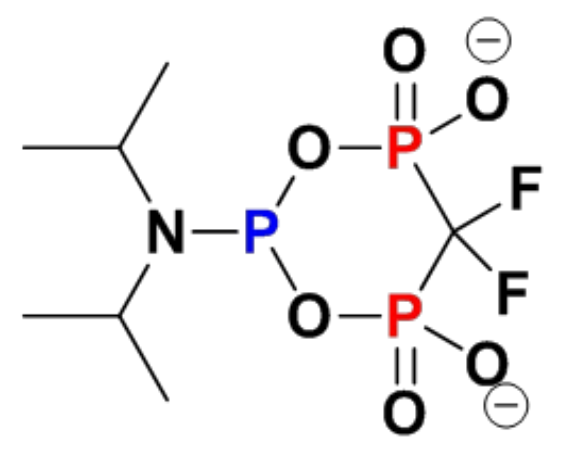



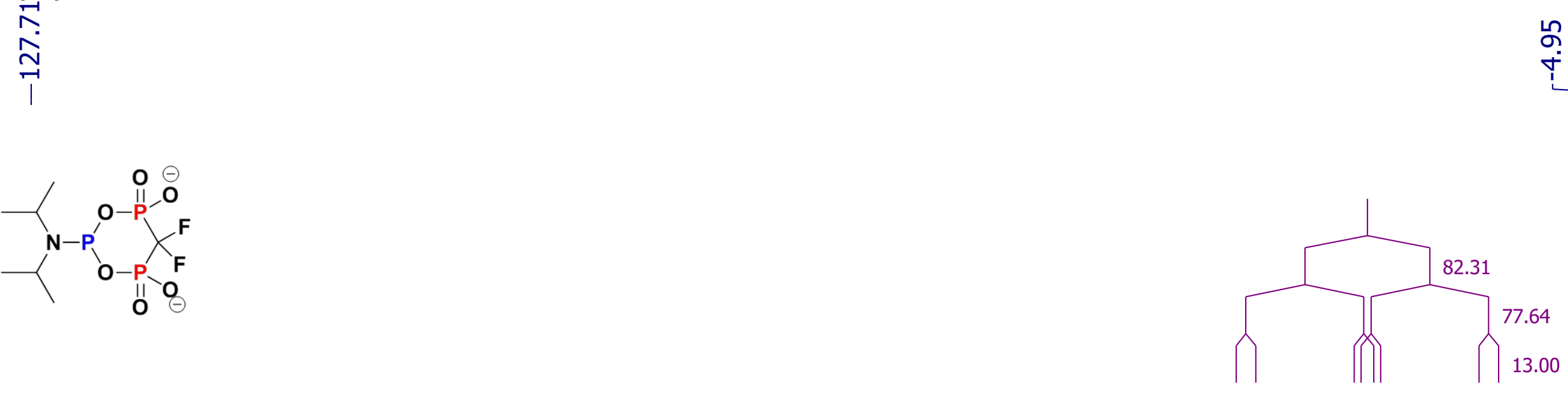

$\mathrm{P}$
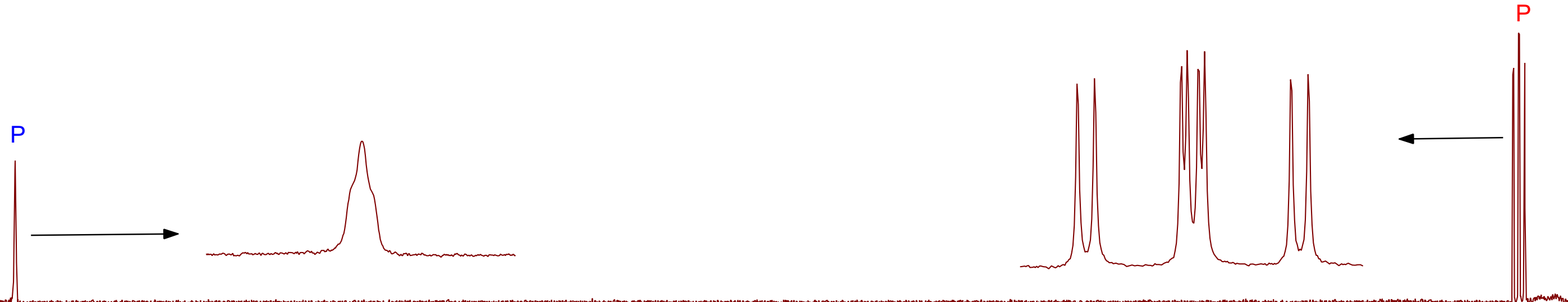

$\mathrm{P}$

กิ

1
0
ㄱ

$\begin{array}{lllllllllllllllllllllllllllll}30 & 125 & 120 & 115 & 110 & 105 & 100 & 95 & 90 & 85 & 80 & 75 & 70 & 65 & 60 & 55 & 50 & 45 & 40 & 35 & 30 & 25 & 20 & 15 & 10 & 5 & 0 & -5 & -1\end{array}$ f1 (ppm) 


\section{${ }^{31} \mathrm{P}\left\{{ }^{19} \mathrm{~F}\right\}$ NMR}

응

ิㅗㅊ

$r$
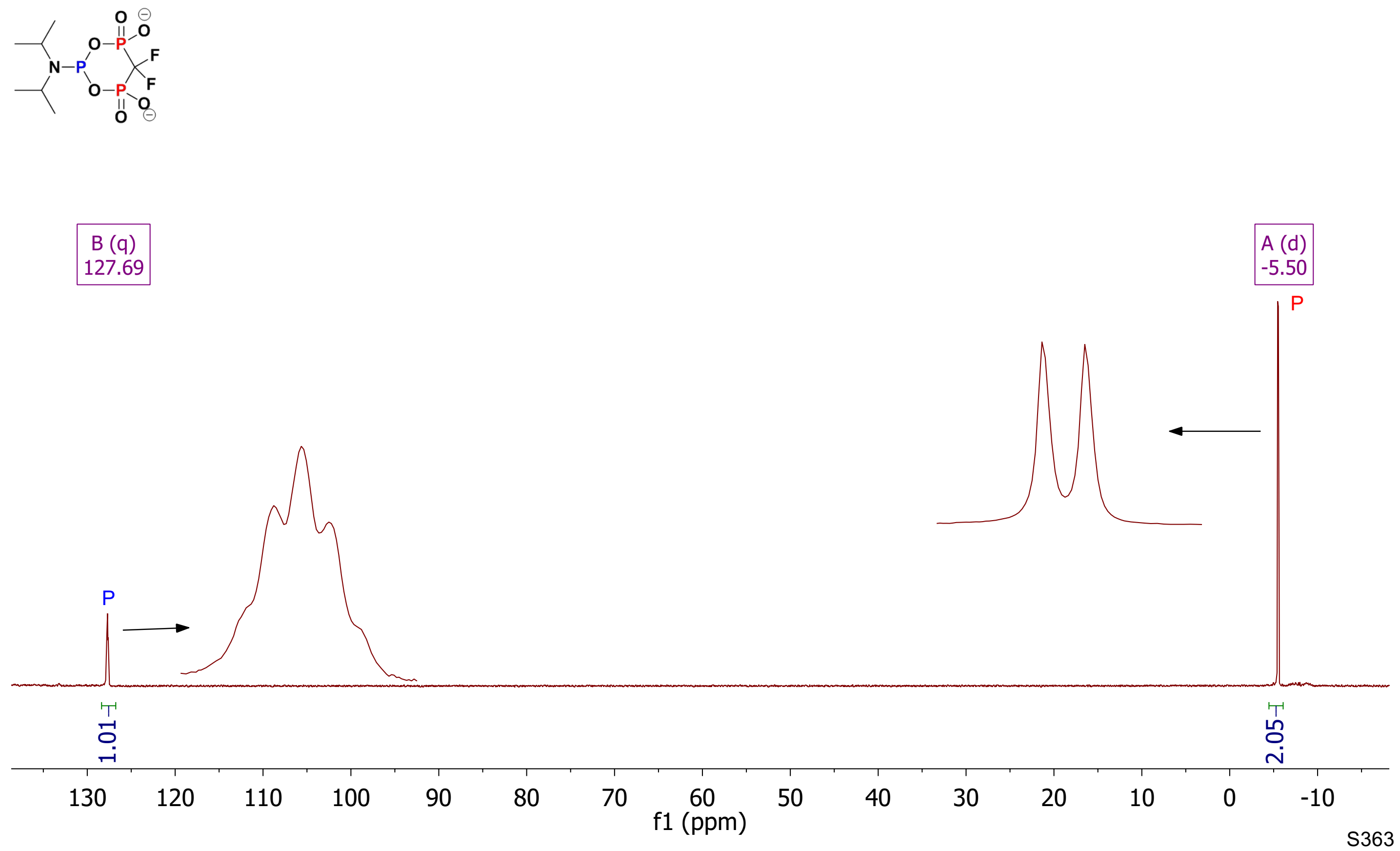


\section{${ }^{31} \mathrm{P}\left\{{ }^{1} \mathrm{H}\right\} \mathrm{NMR}$}

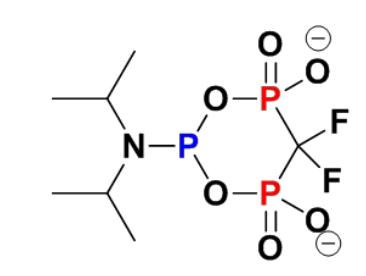

$\underline{\text { Stability studies }}$

After 2 week

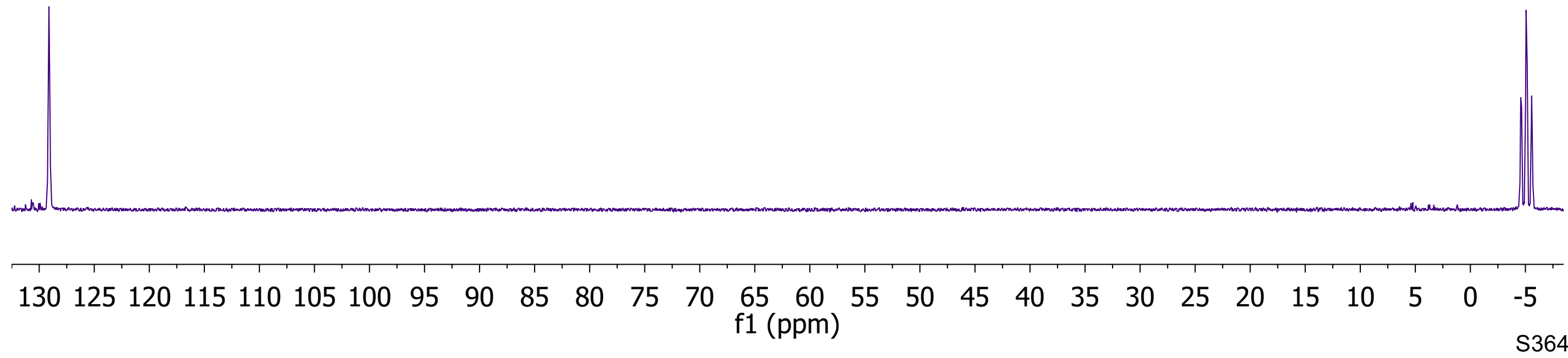



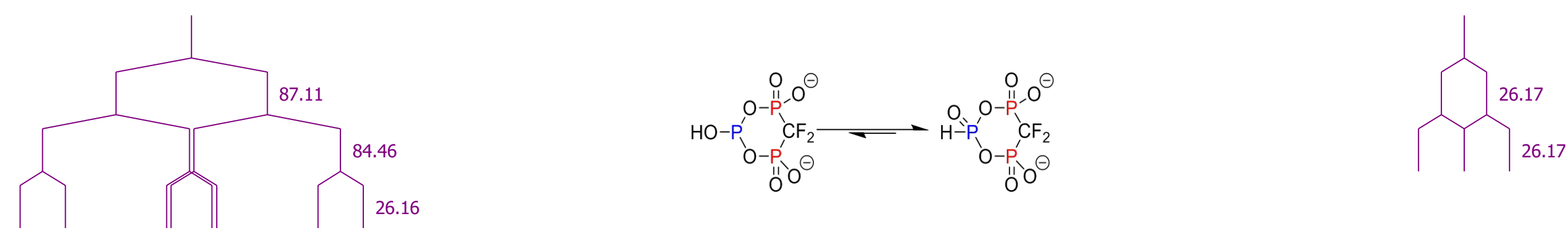

$$
\begin{gathered}
\hline \text { B (ddd) } \\
-8.60
\end{gathered}
$$

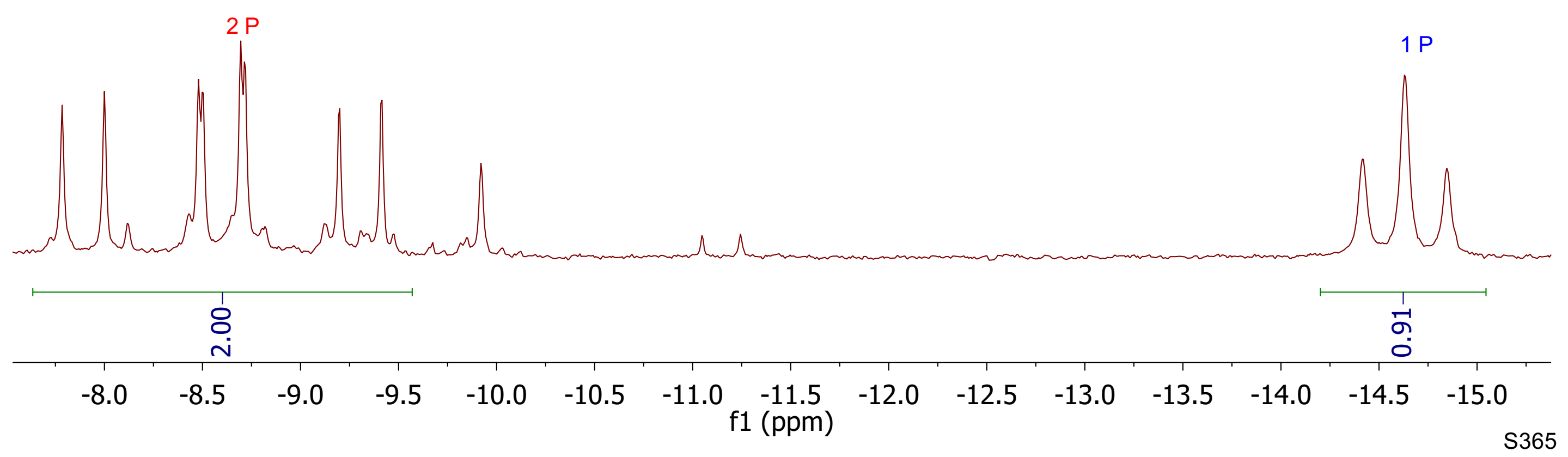



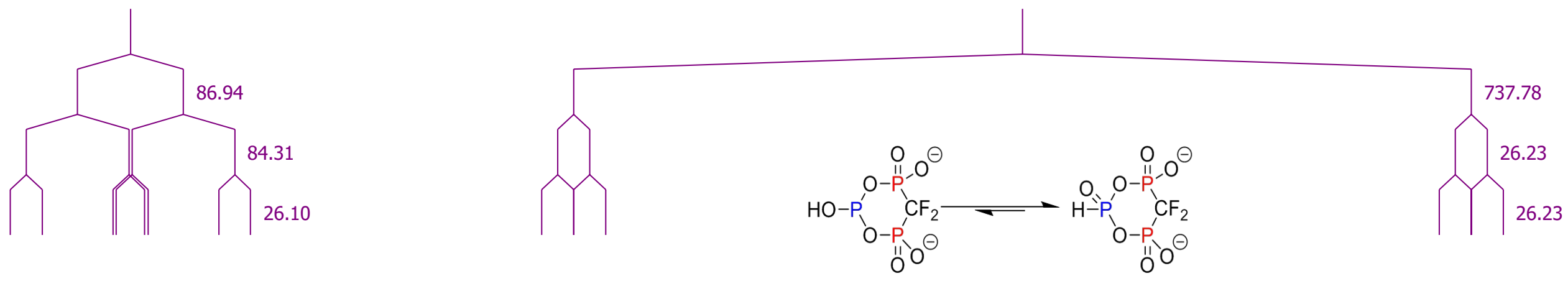

$$
\begin{gathered}
B \text { (ddd) } \\
-8.60
\end{gathered}
$$

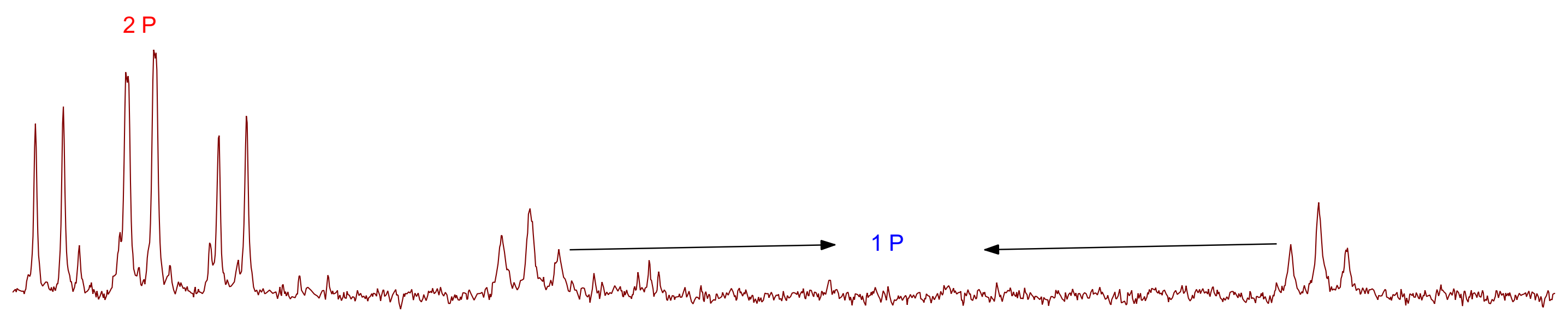

\begin{tabular}{|c|c|c|c|c|c|c|c|c|c|c|c|}
\hline & & & & & & & & & & & \\
\hline-8.0 & -9.0 & -10.0 & -11.0 & -12.0 & $\begin{array}{r}-13.0 \\
f 1\end{array}$ & $\begin{array}{l}-14.0 \\
\mathrm{~m})\end{array}$ & -15.0 & -16.0 & -17.0 & -18.0 & -19.0 \\
\hline
\end{tabular}




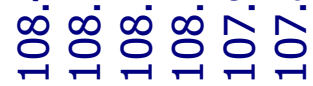
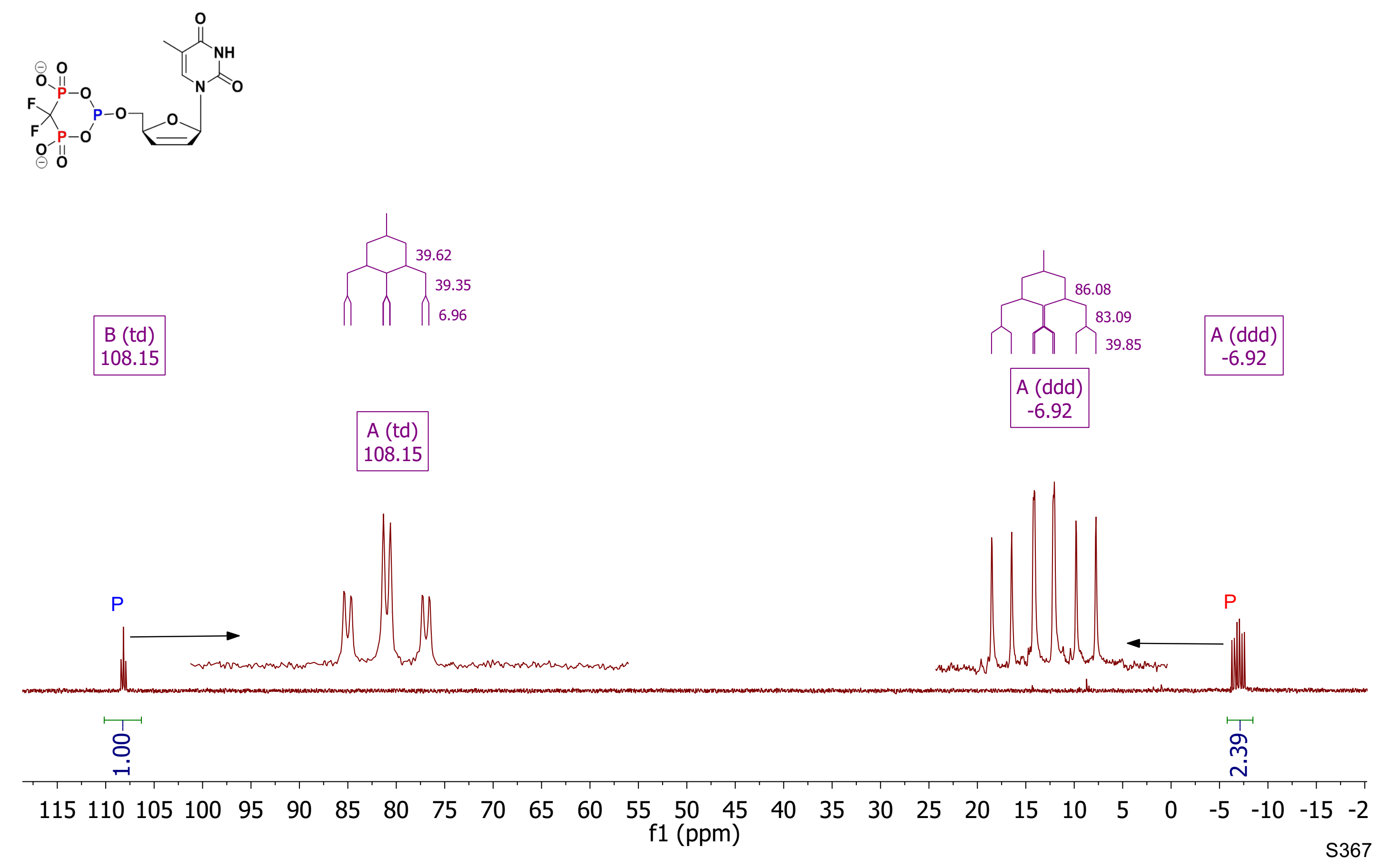


\section{${ }^{31} \mathrm{P}$ NMR}

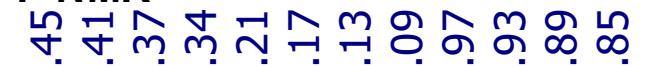

ᄋ
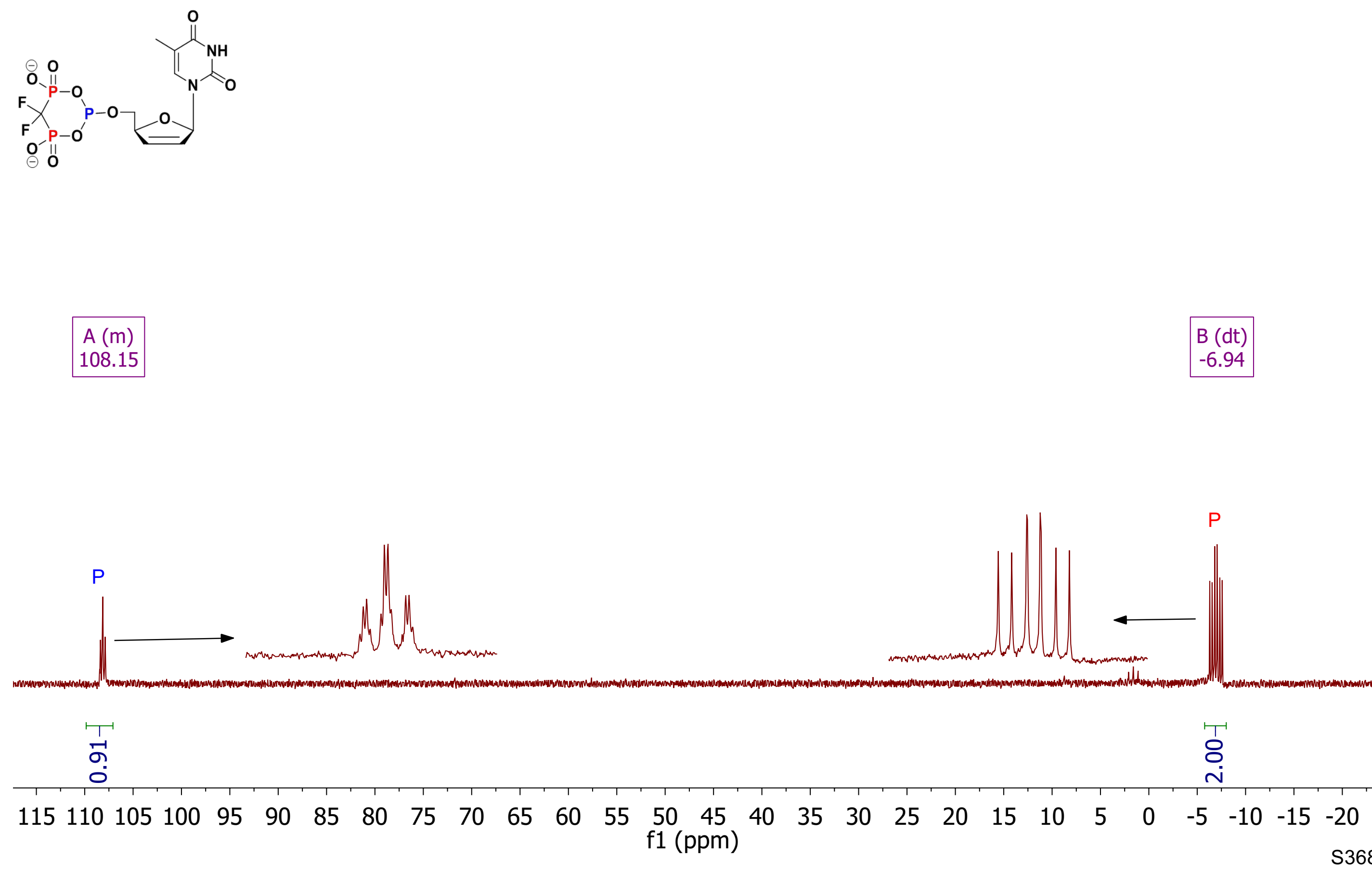
${ }^{31} \mathrm{P}\left\{{ }^{19} \mathrm{~F}\right\}$ NMR

초ำำㄴำำ

$m \stackrel{m}{m} \stackrel{m}{\rightarrow} \stackrel{m}{=}$

각극극
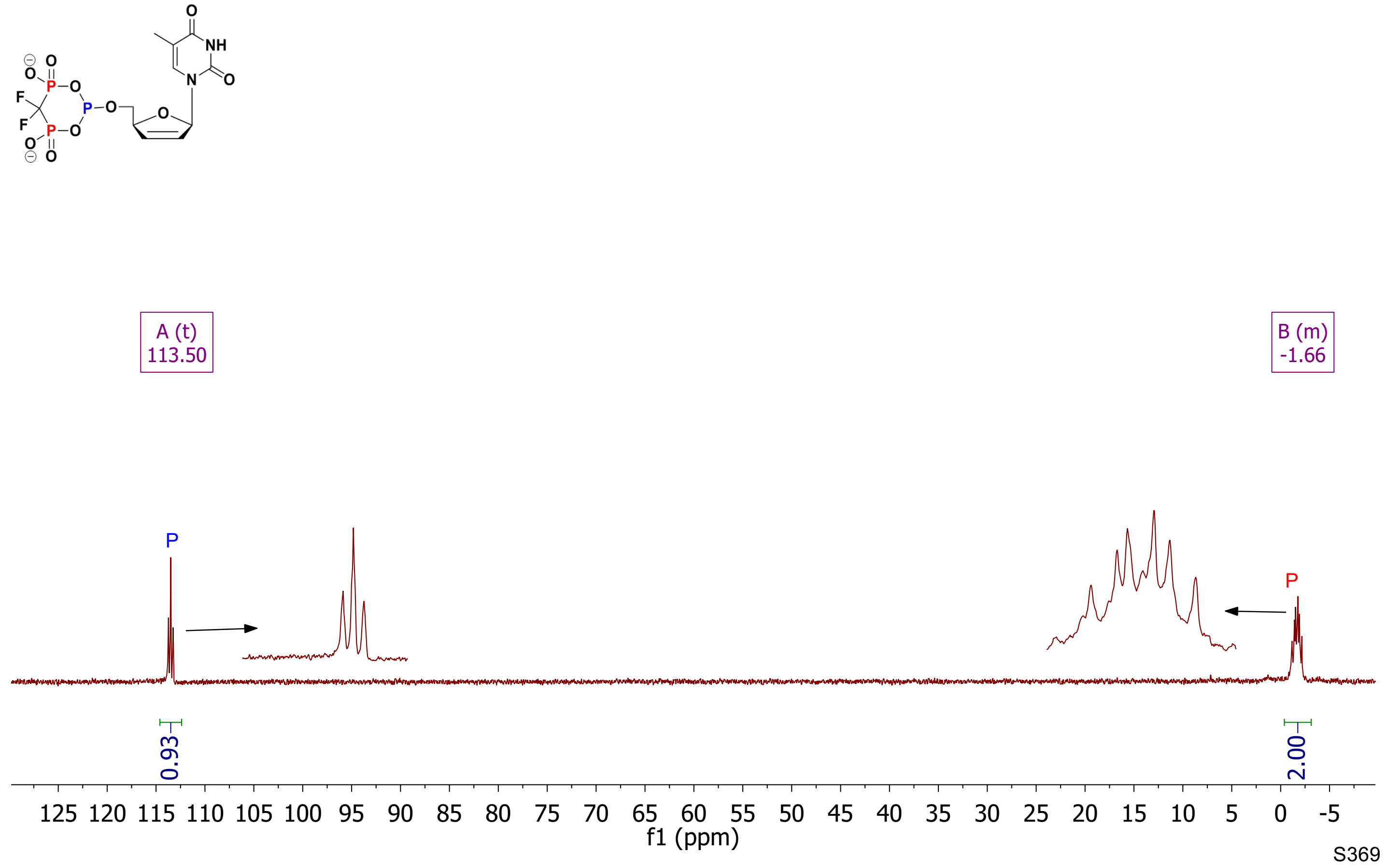


\section{${ }^{31} \mathrm{P}\left\{{ }^{1} \mathrm{H}\right\}$ NMR}

○ํํำ

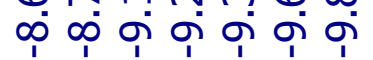

กิซ ㄷำ

กั่ กั่
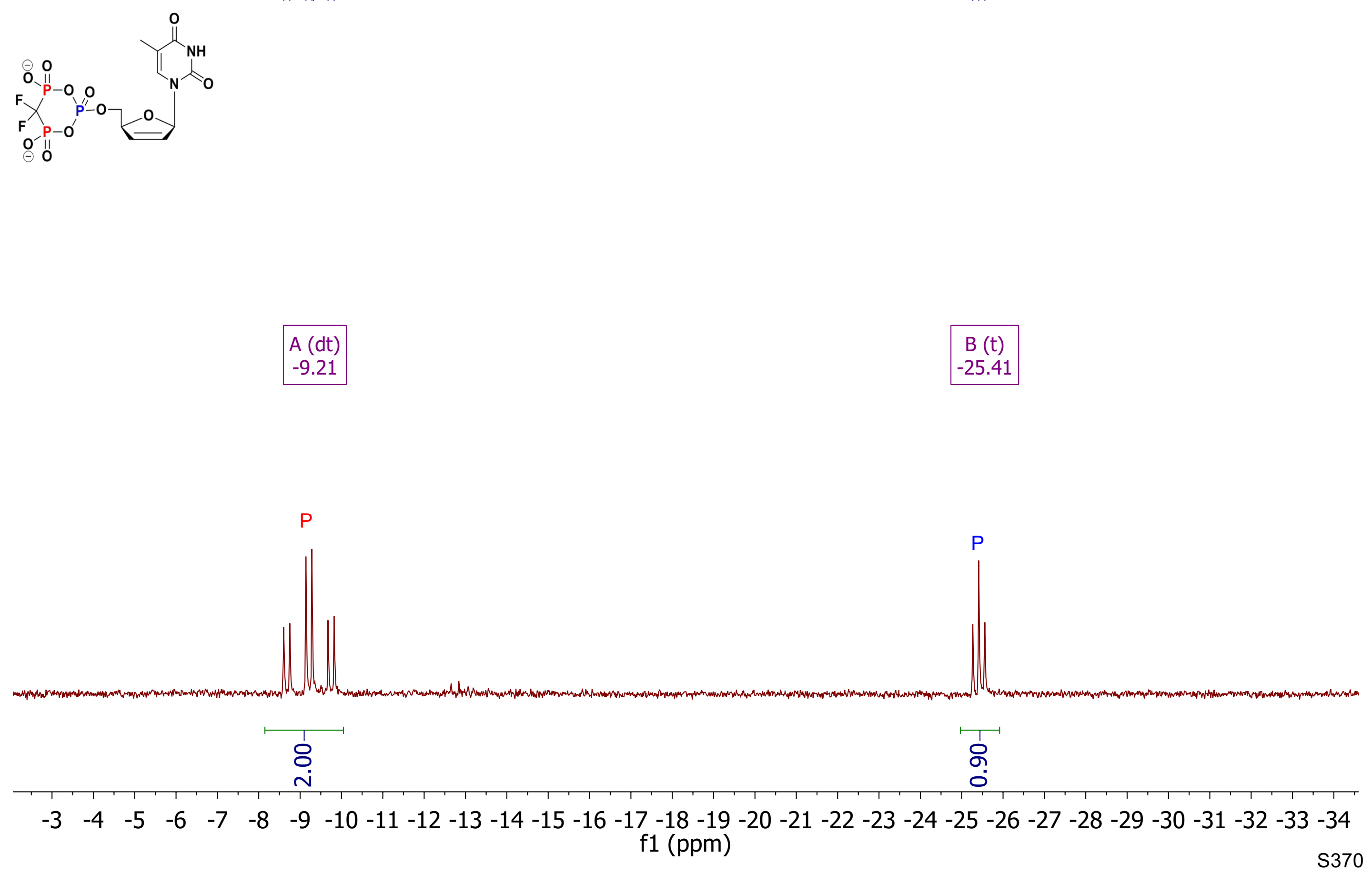

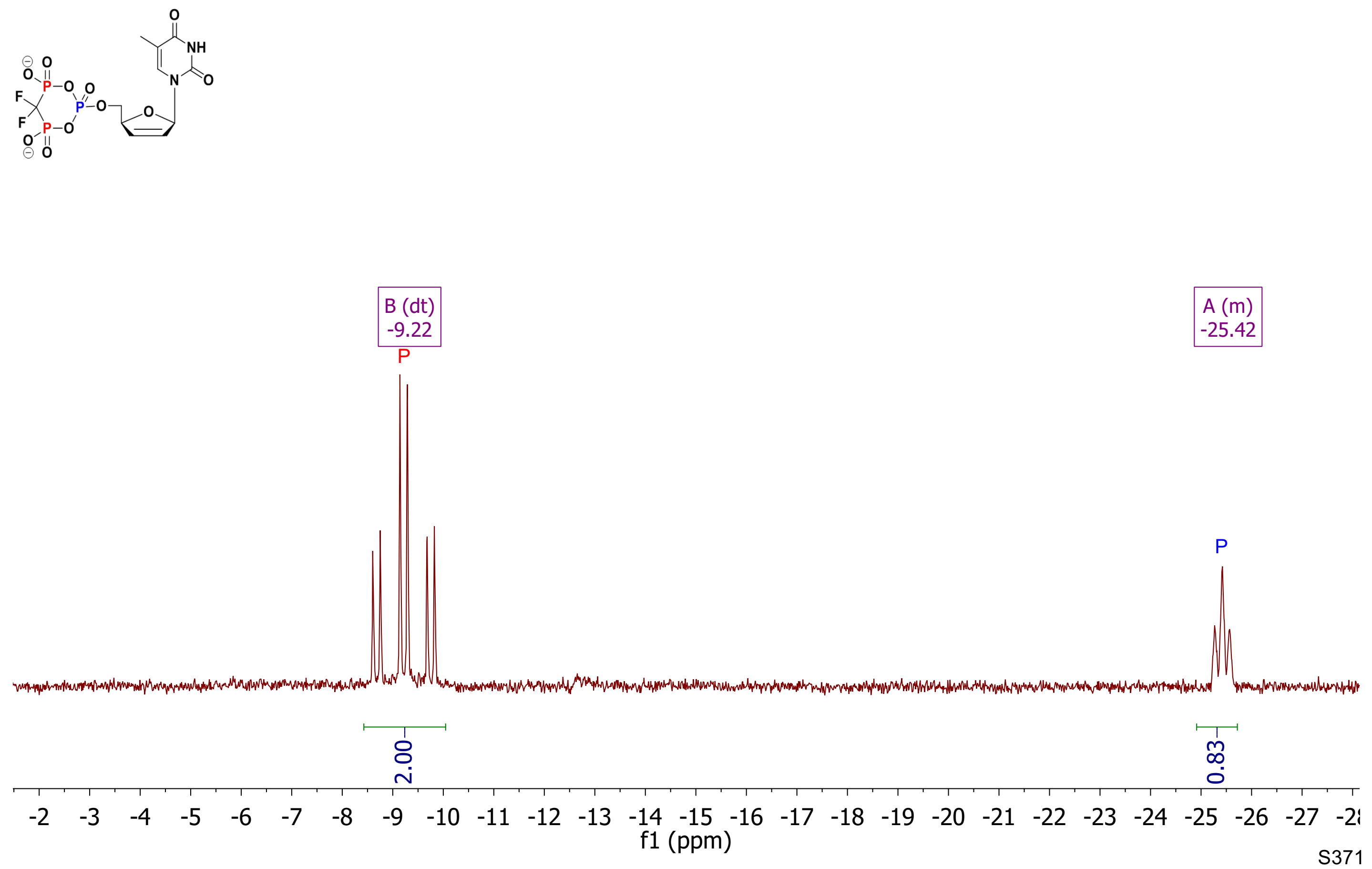


\section{${ }^{31} \mathrm{P}\left\{{ }^{19} \mathrm{~F}\right\} \mathrm{NMR}$}

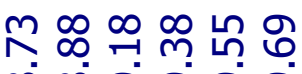

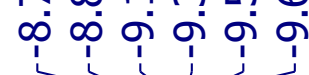
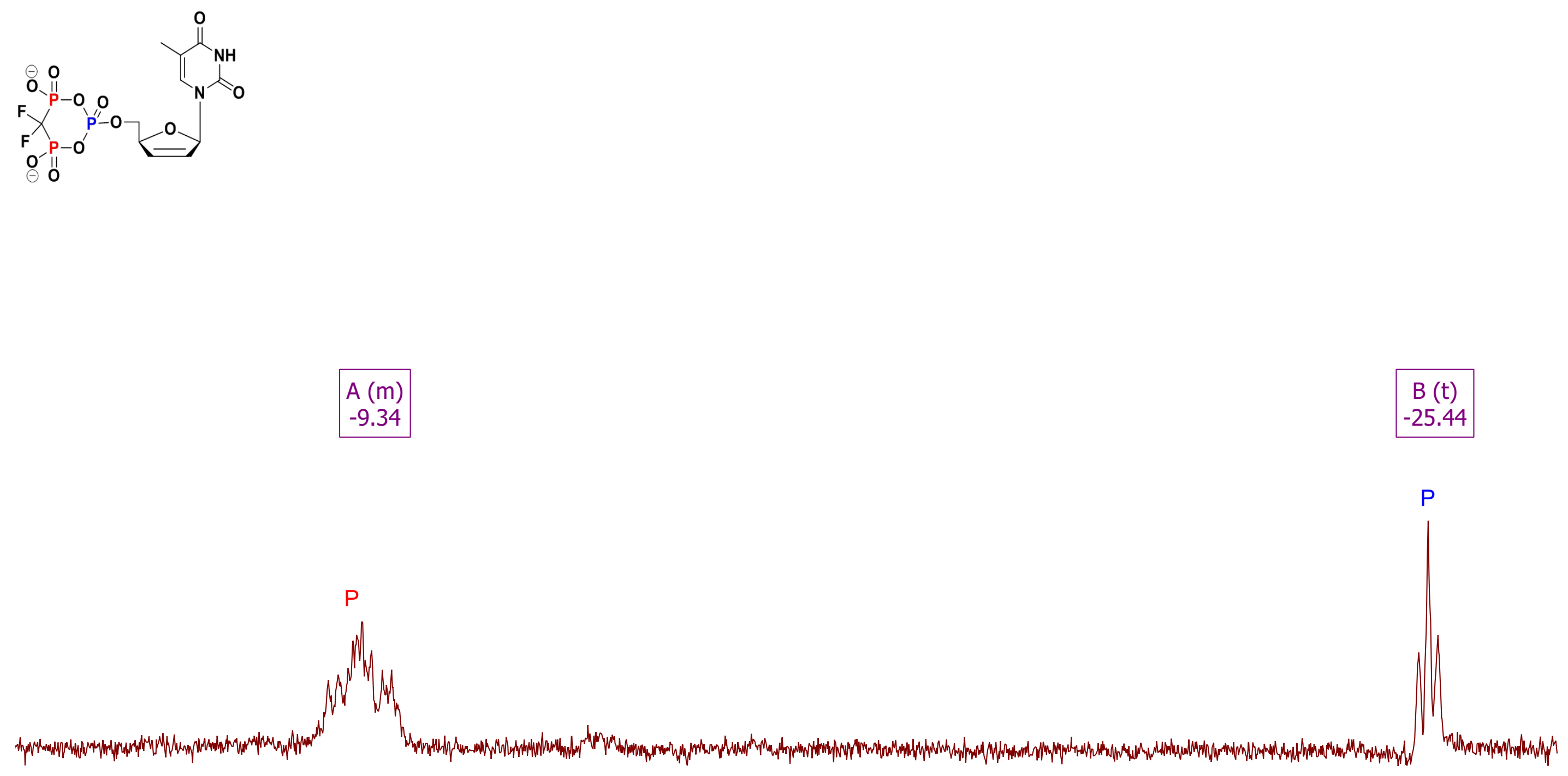

\begin{tabular}{|c|c|c|c|c|c|c|c|c|c|c|c|c|c|c|c|c|c|c|c|c|c|c|}
\hline 4 & -5 & -6 & -7 & -8 & -9 & -10 & -11 & -12 & -13 & -14 & $\begin{array}{cc}-15 & -16 \\
\mathrm{f} 1 & (\mathrm{ppm})\end{array}$ & -17 & -18 & -19 & -20 & -21 & -22 & -23 & -24 & -25 & -26 & $\begin{array}{l}1 \\
-27 \\
\text { S372 }\end{array}$ \\
\hline
\end{tabular}




\section{${ }^{1}$ H NMR}

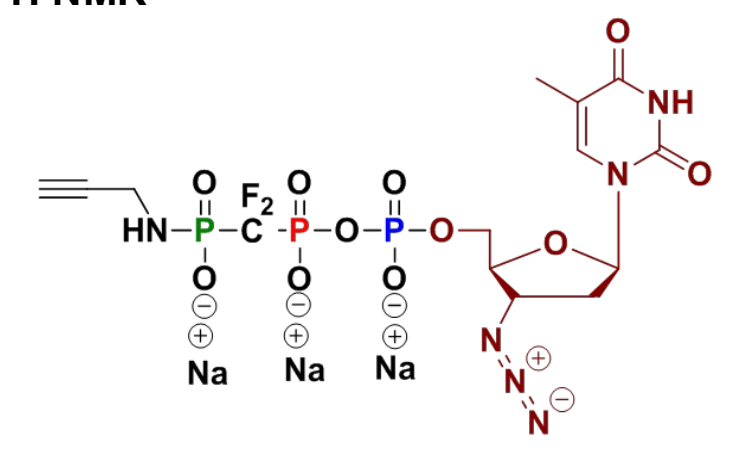

crude product after precipitation

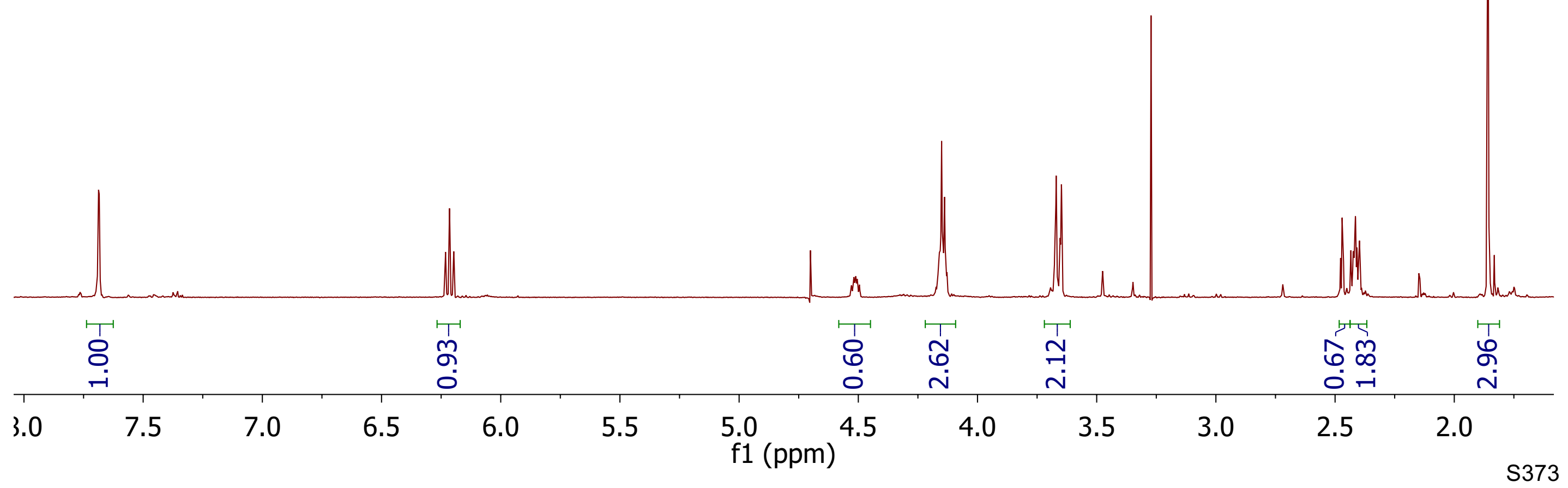




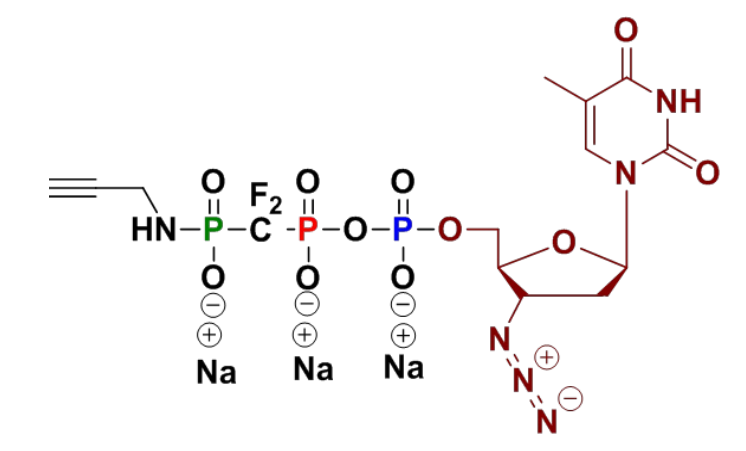

crude product after precipitation

$$
\begin{array}{|c|}
\hline A(m) \\
6.24 \\
\hline
\end{array}
$$

P

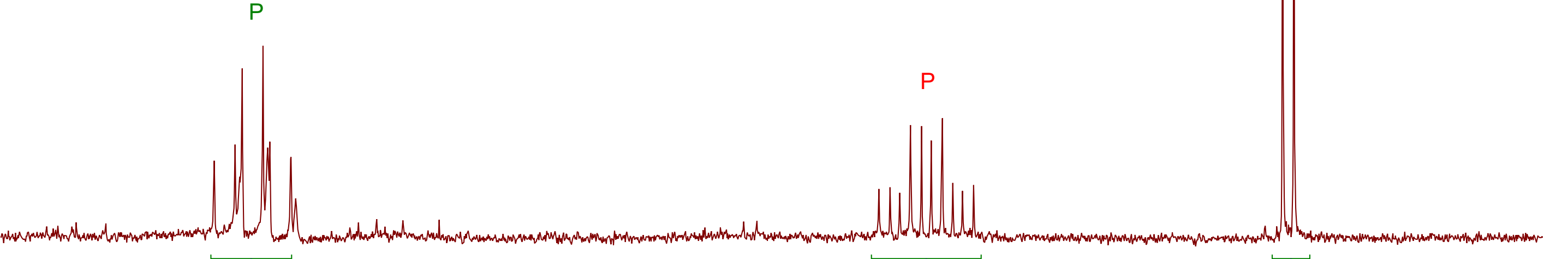

$\begin{array}{lllll}5 & 4 & 3 & 2 & 1\end{array}$

\begin{tabular}{|c|}
\hline B (tdd) \\
-5.38
\end{tabular}

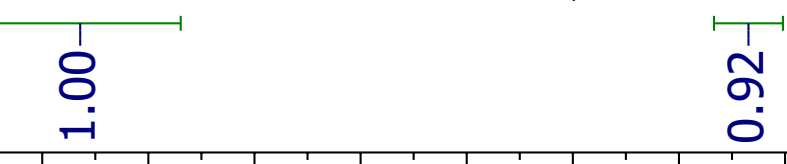

\section{$C(d)$}

$-11.61$ 


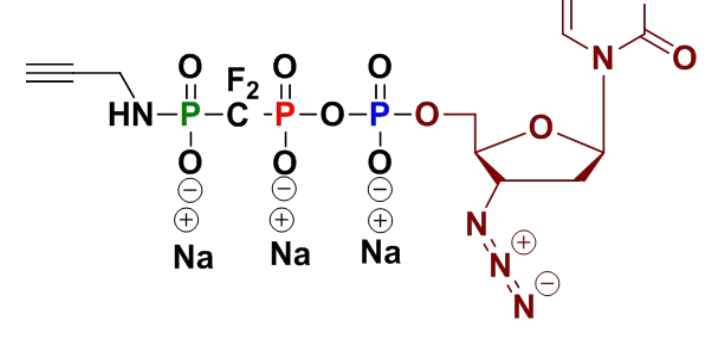

crude product after precipitation

$$
\begin{gathered}
A(m) \\
6.21 \\
\hline
\end{gathered}
$$

$$
\begin{gathered}
B(t d d) \\
-5.41 \\
\hline
\end{gathered}
$$

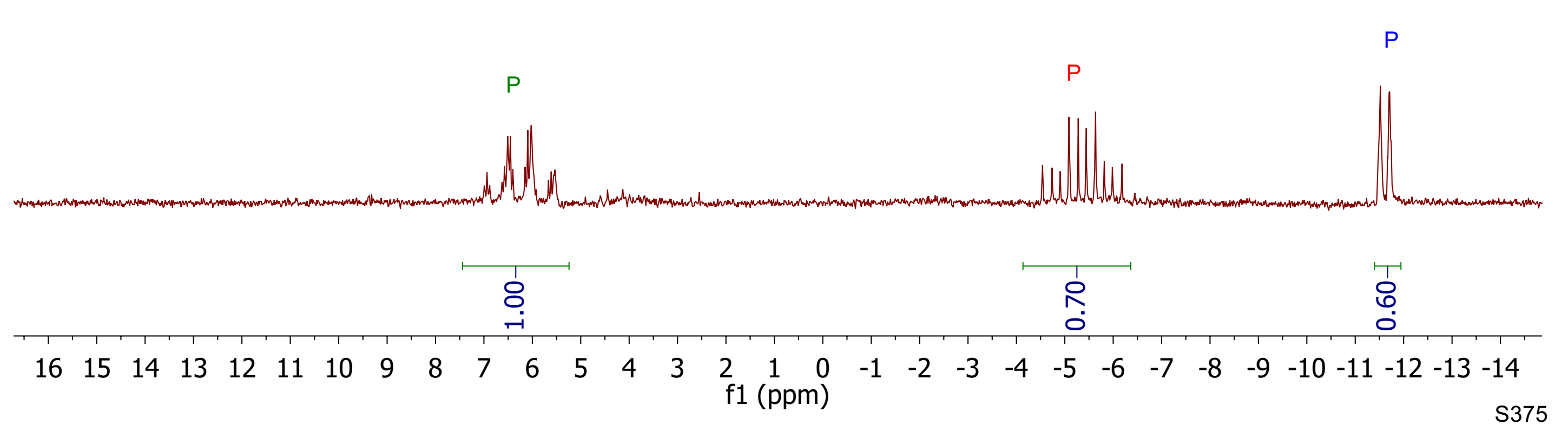




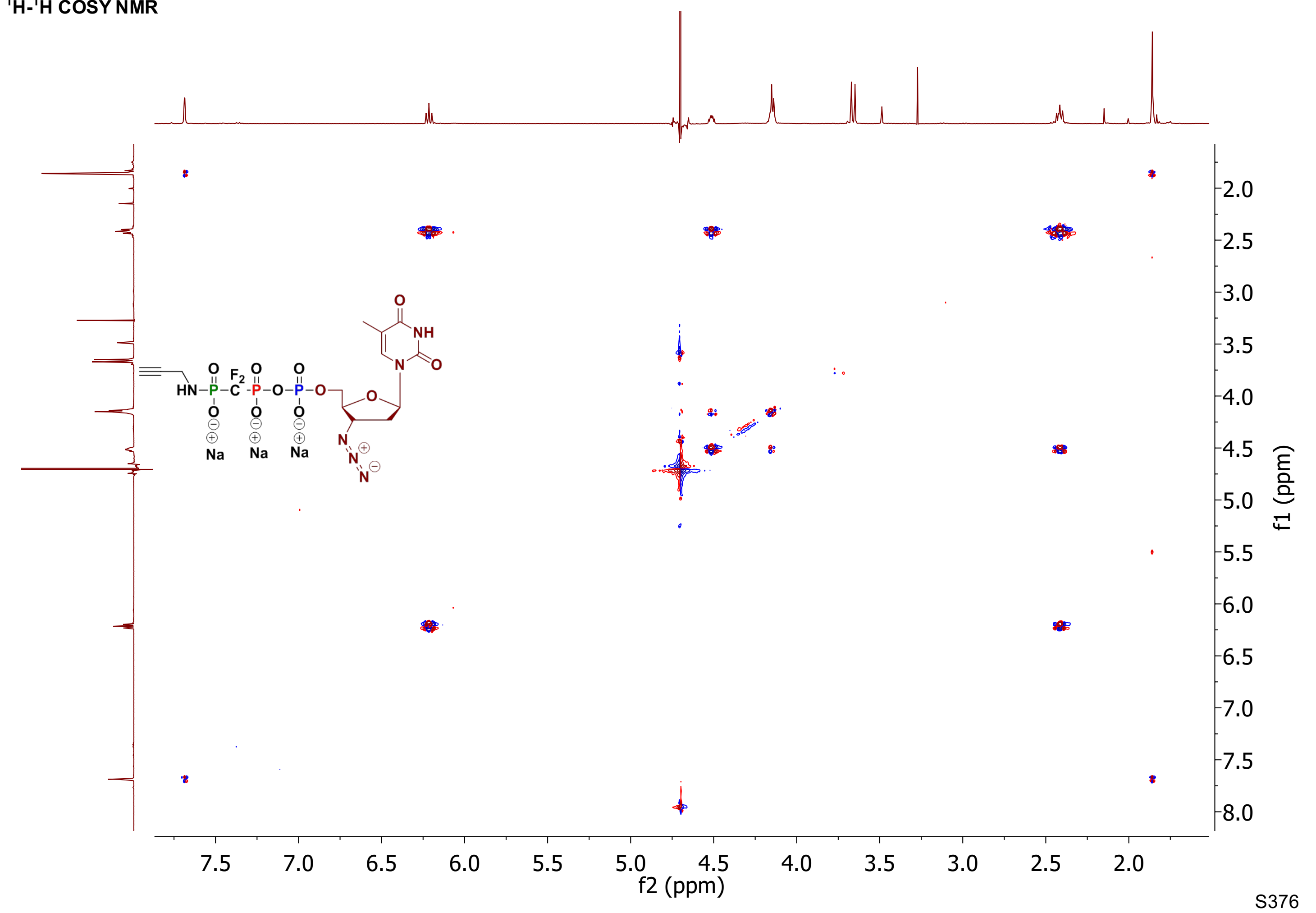



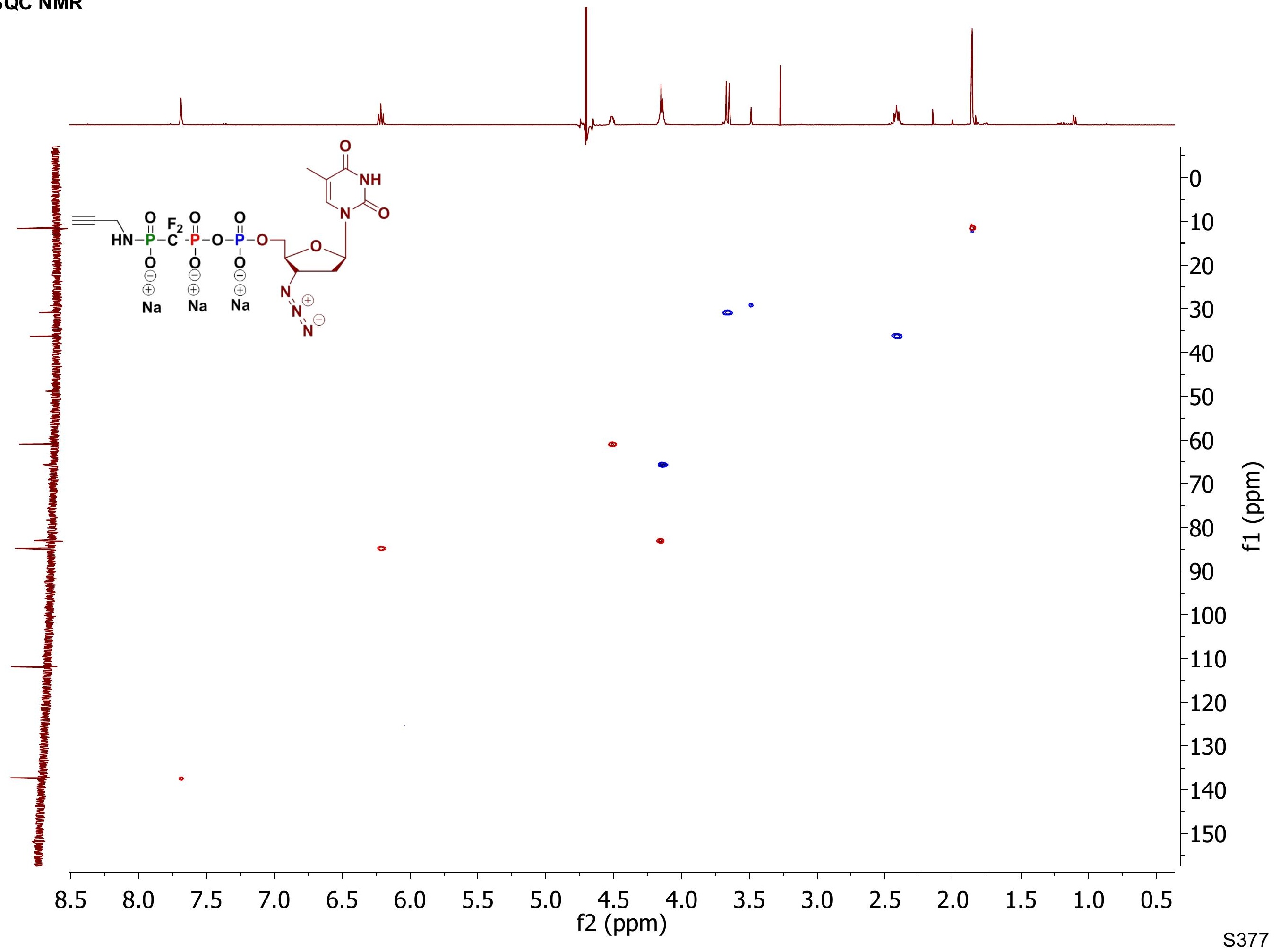


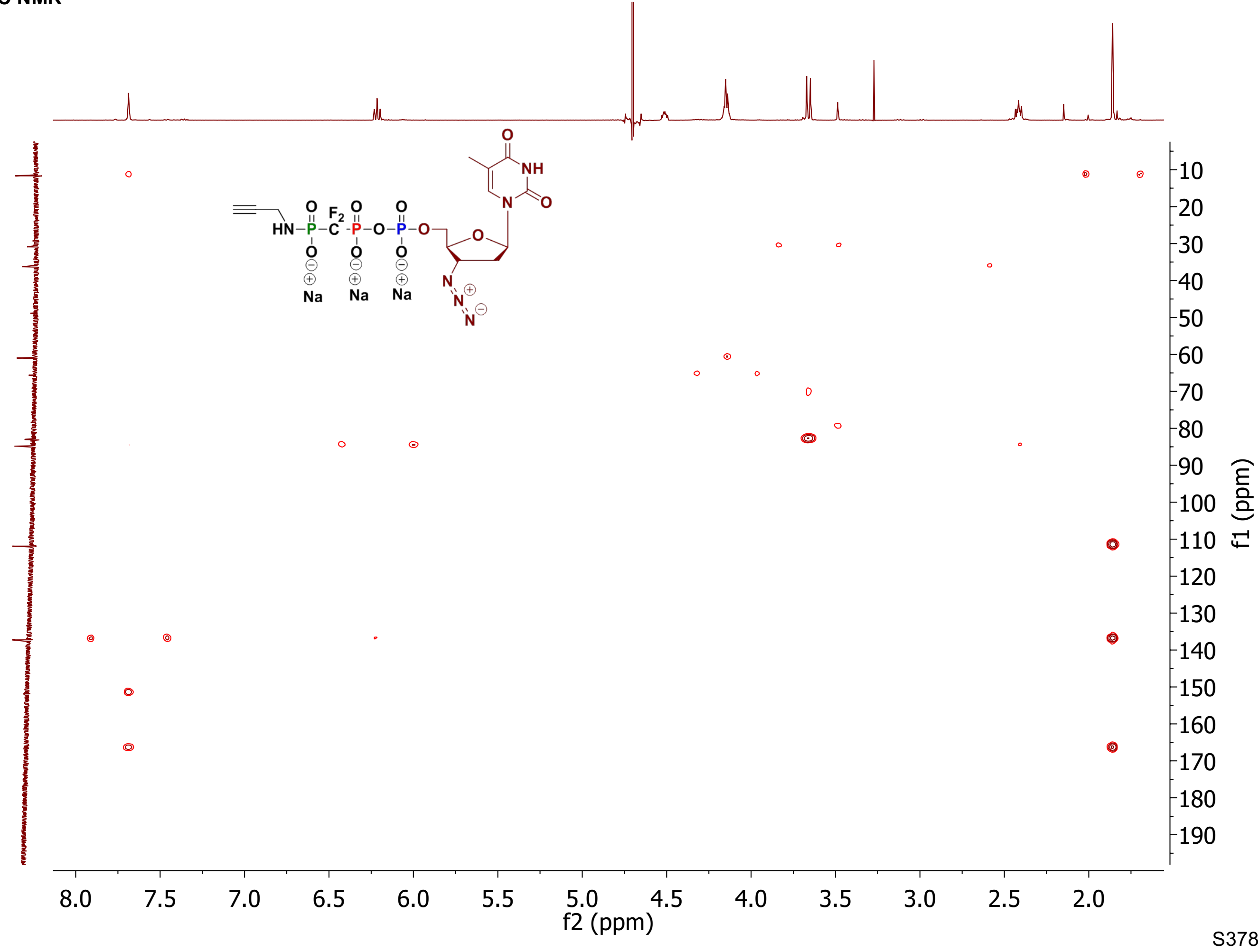




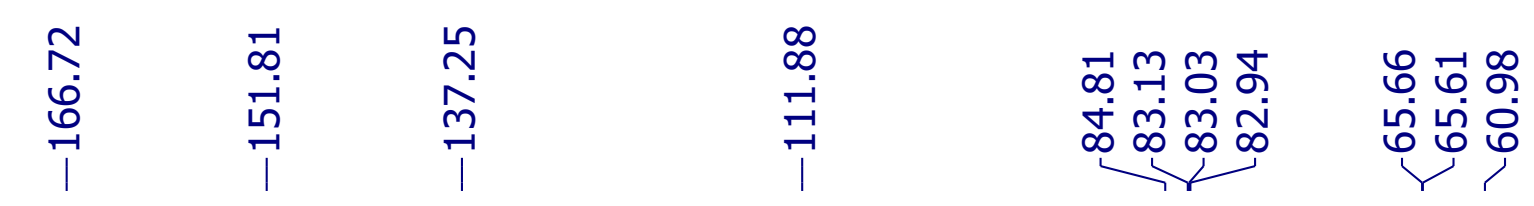

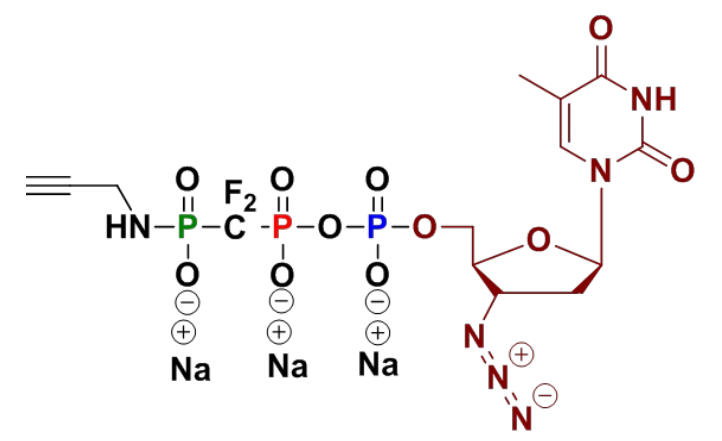

crude product after precipitation

\begin{tabular}{|c|c|c|}
\hline$L(s)$ & $\mathrm{K}(\mathrm{s})$ \\
166.72 & $\mathrm{~J}(\mathrm{~s})$ \\
131.81 & 137.25 \\
\hline
\end{tabular}
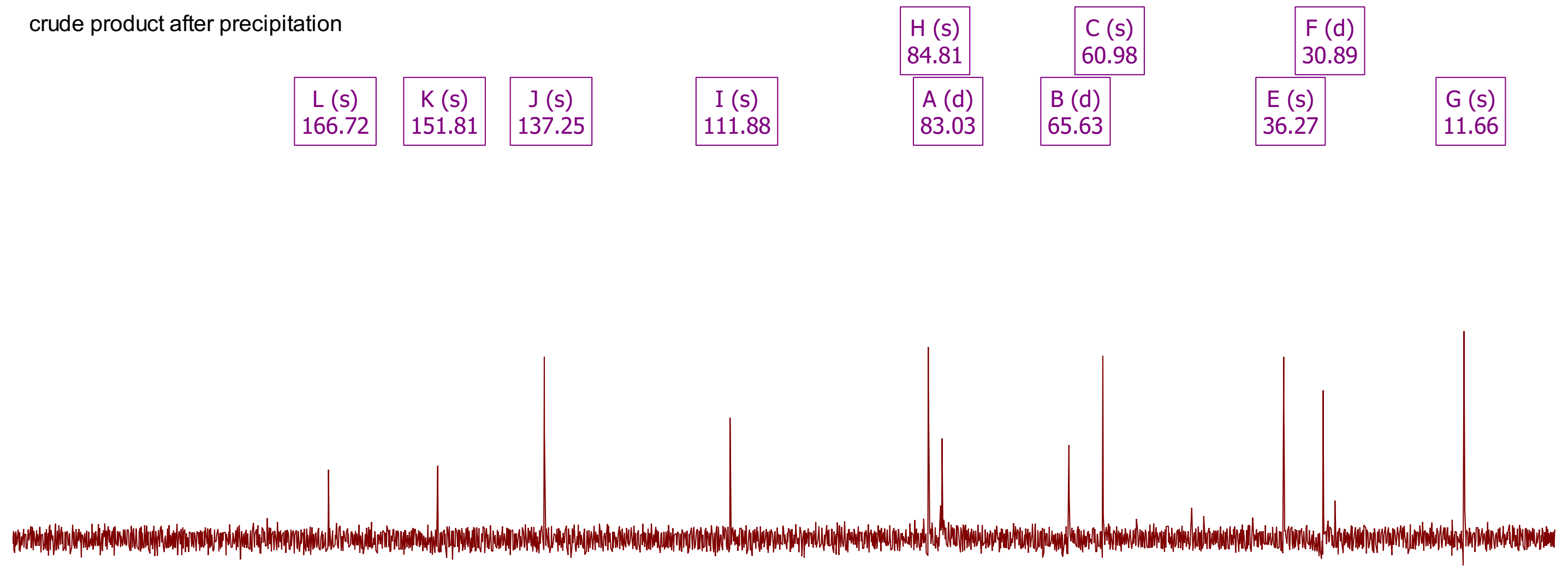


\section{${ }^{1} \mathrm{H}$ NMR}

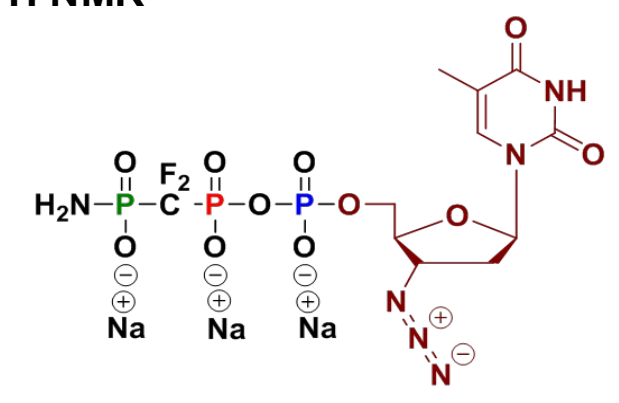

crude product after precipitation

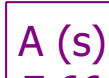

$$
\text { B (t) }
$$

7.66

6.21

$$
\begin{array}{|c|c|}
\hline C(m) & D(m) \\
4.51 & 4.17 \\
\hline
\end{array}
$$
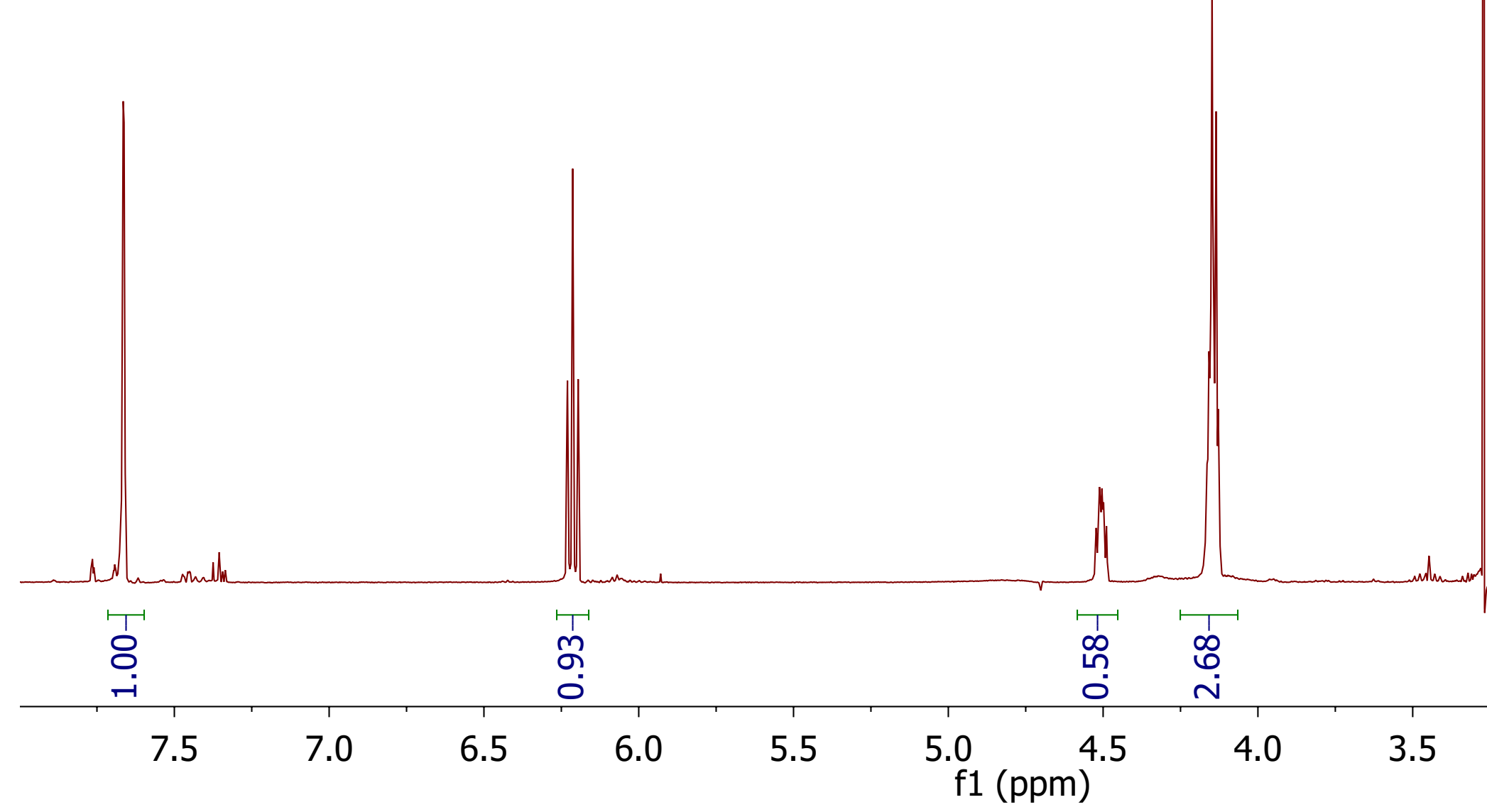


\section{${ }^{31} \mathrm{P}\left\{{ }^{1} \mathrm{H}\right\}$ NMR}

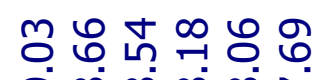

कi

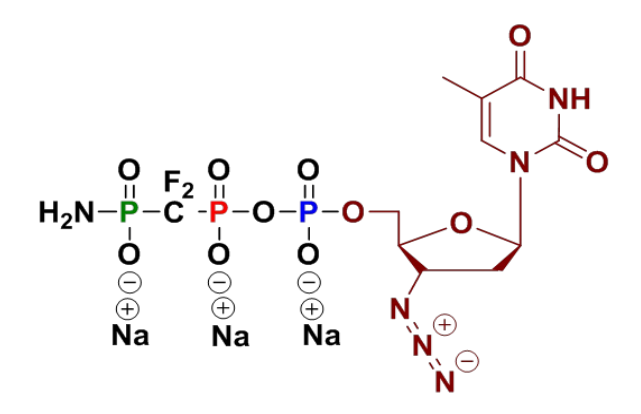

crude product after precipitation

$$
\begin{array}{|l|}
\hline C(t d) \\
8.36 \\
\hline
\end{array}
$$

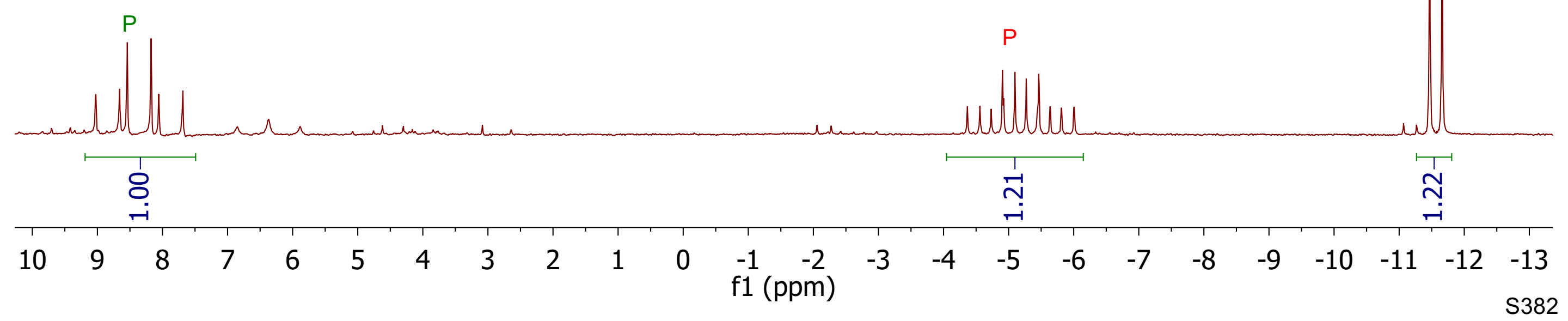


${ }^{31} \mathrm{P}$ NMR

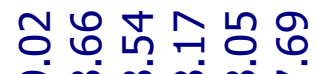

बi $\infty \infty^{\circ} \infty$

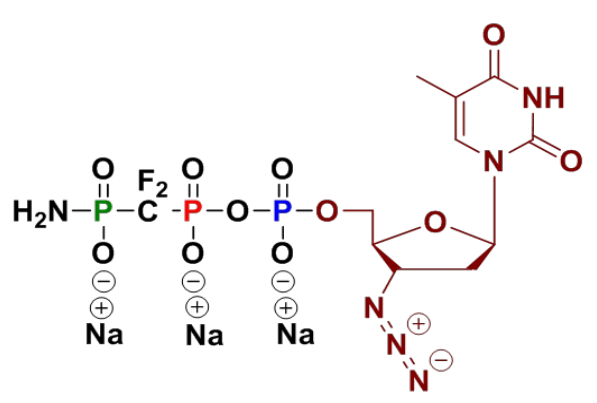

crude product after precipitation

A (td)

8.36

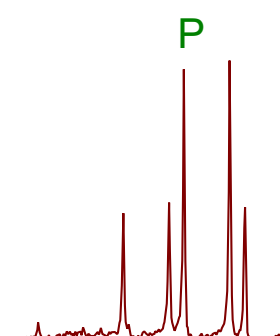

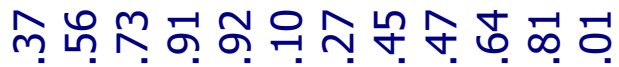

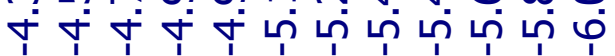

$$
\begin{gathered}
\text { B (tdd) } \\
-5.19
\end{gathered}
$$

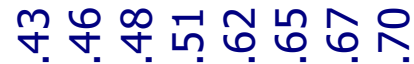
각걱각각각걱

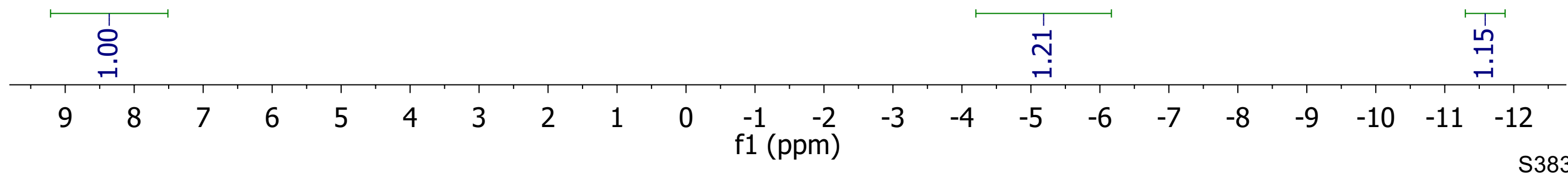




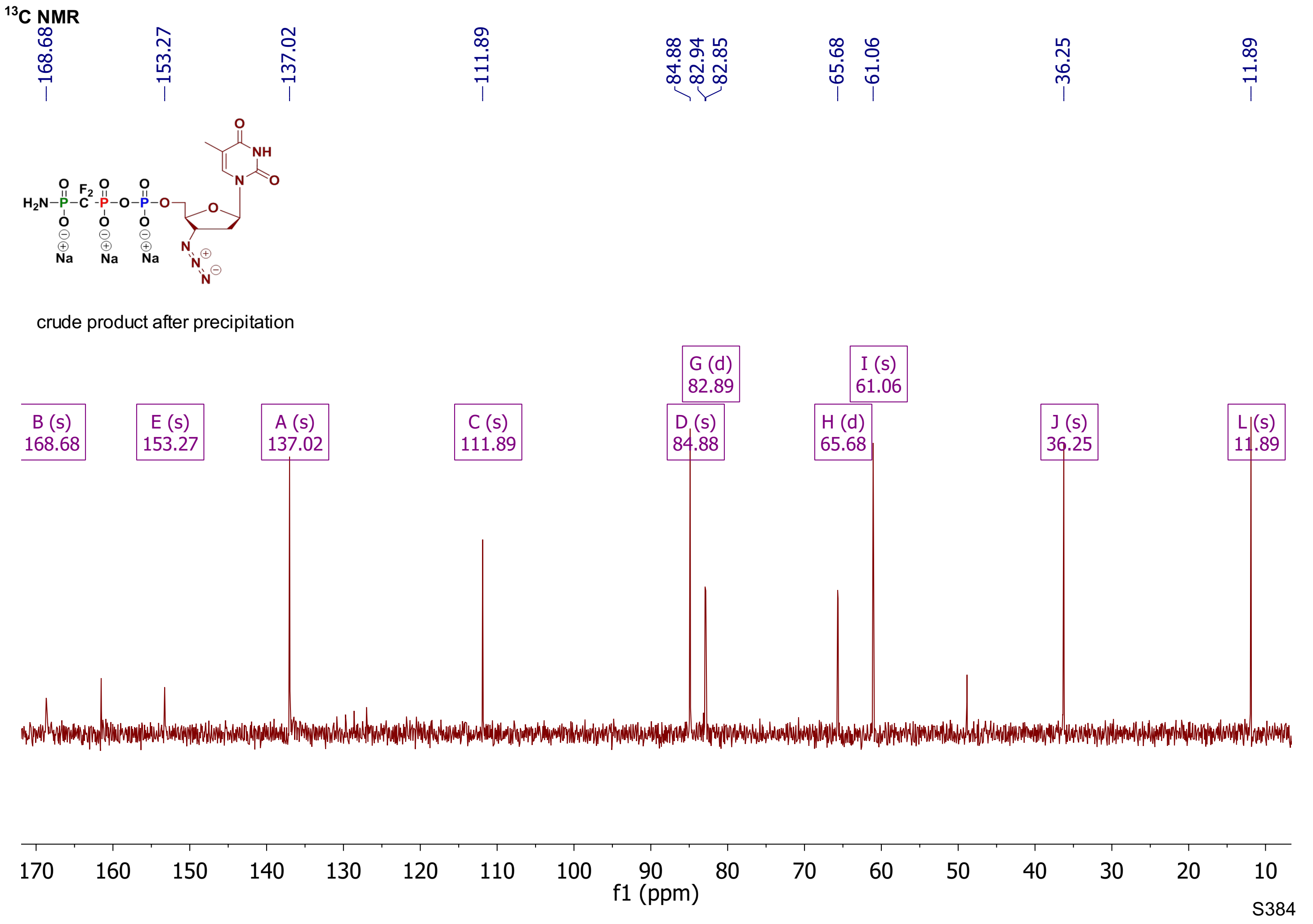




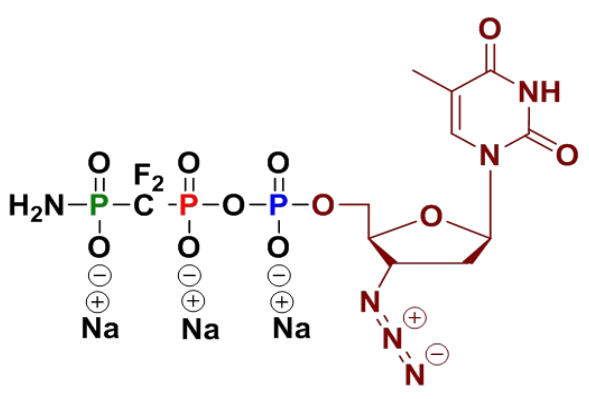

crude product after precipitation

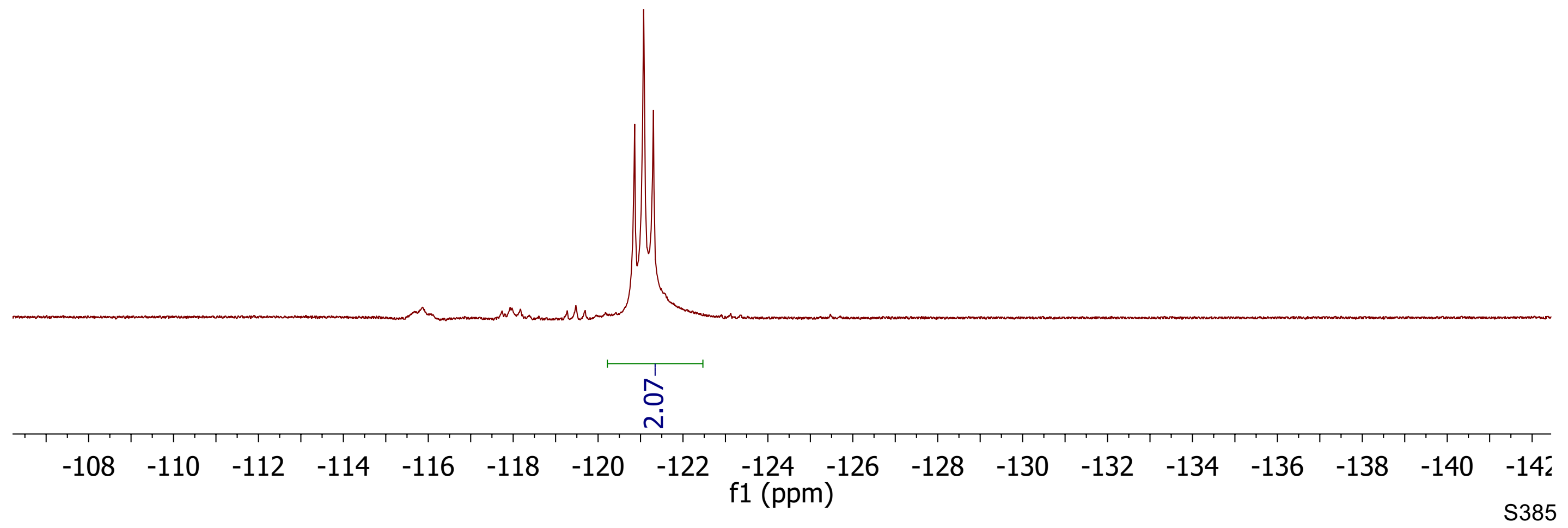




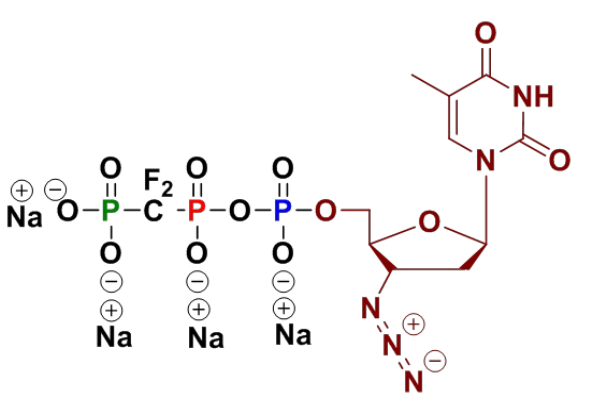

crude product after precipitation

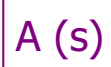

7.65

\begin{tabular}{|c|c|}
\hline$C(m)$ & $D(m)$ \\
4.51 & 4.15 \\
\hline
\end{tabular}
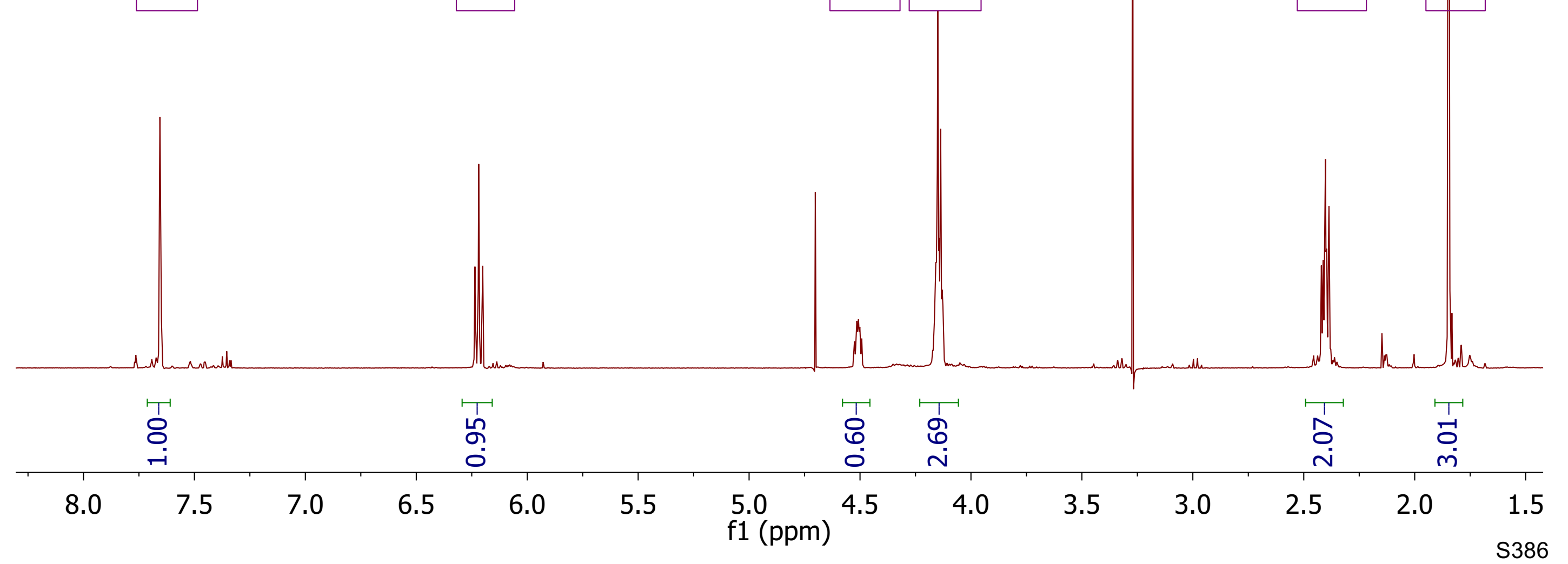
ํํㄱㅋㄷㅅㅡำ ष

๓ 수수수

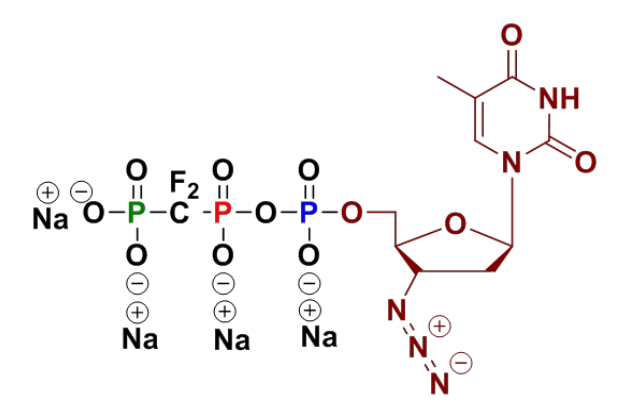

crude product after precipitation
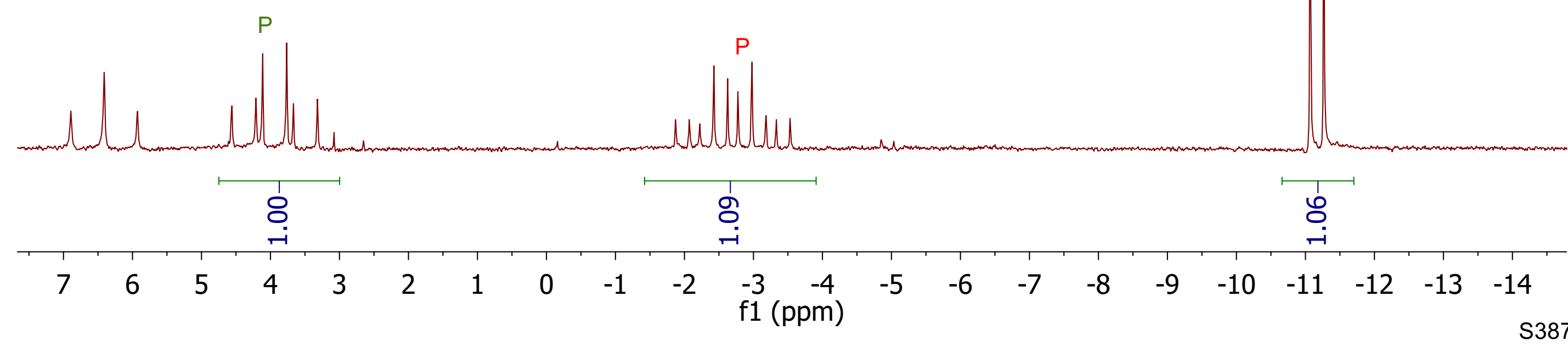


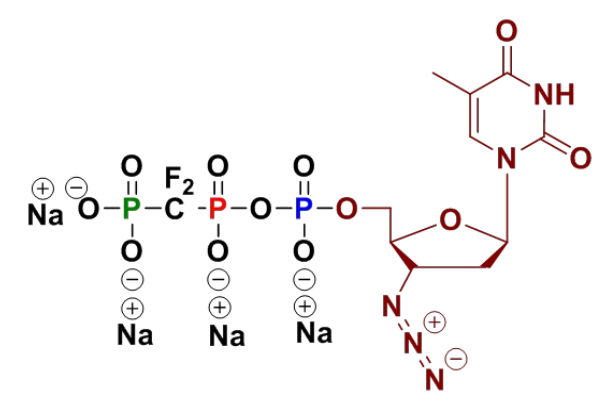

crude product after precipitation

\begin{tabular}{|c|c|c|c|c|c|c|c|c|c|c|}
\hline & & & & & $\begin{array}{l}1 \\
0 \\
0 \\
\end{array}$ & & & & & \\
\hline-113.5 & -114.5 & -115.5 & -116.5 & -117.5 & $\begin{array}{l}-118.5 \\
\mathrm{f} 1 \text { (ppm) }\end{array}$ & -119.5 & -120.5 & -121.5 & -122.5 & $\begin{array}{r}-123 \\
\text { S389 }\end{array}$ \\
\hline
\end{tabular}




\section{${ }^{1} \mathrm{H}$ NMR}

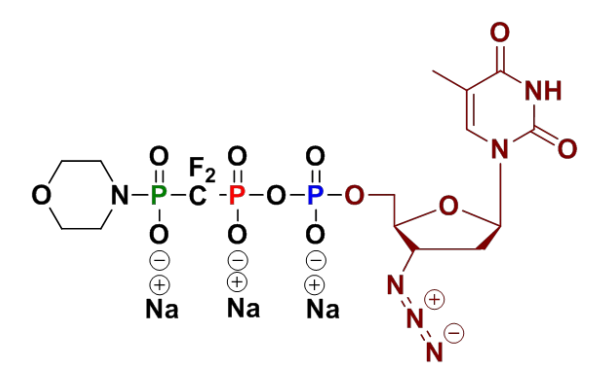

crude product after precipitation
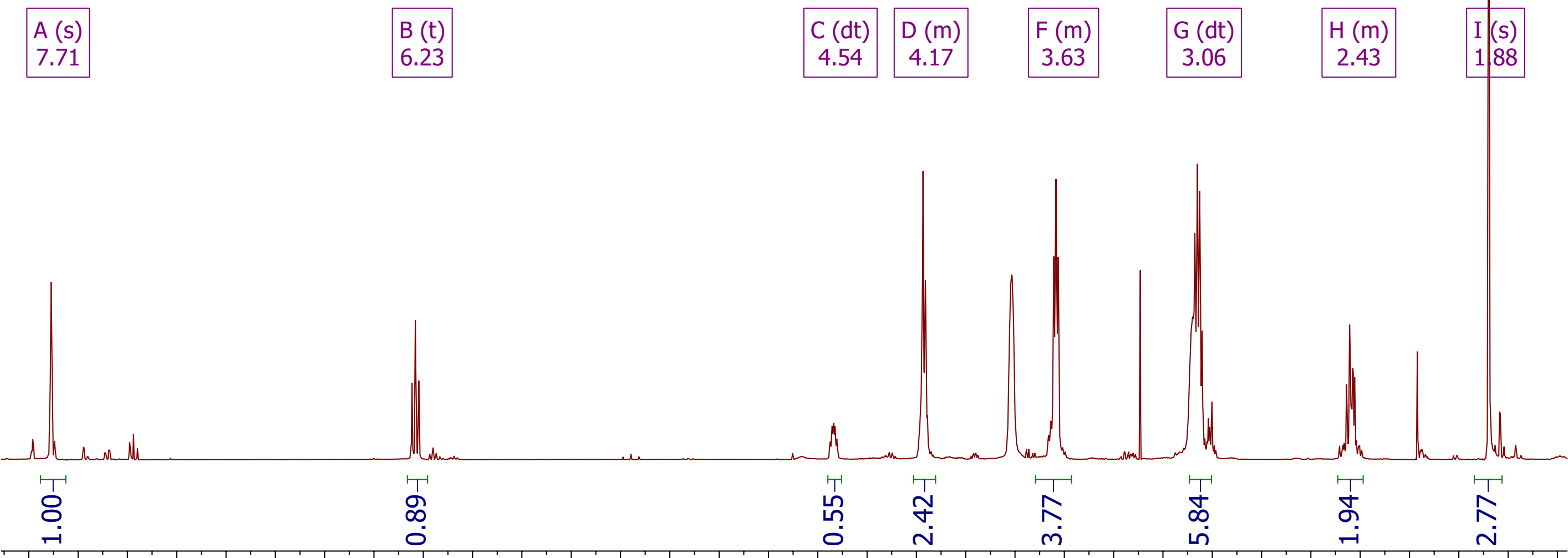

7.87 .67 .47 .27 .06 .86 .66 .46 .26 .05 .85 .65 .45 .25 .04 .84 .64 .44 .24 .03 .83 .63 .43 .23 .02 .82 .62 .42 .22 .01 .81 .6 f1 (ppm) 


\section{${ }^{31} \mathrm{P}\left\{{ }^{1} \mathrm{H}\right\}$ NMR}

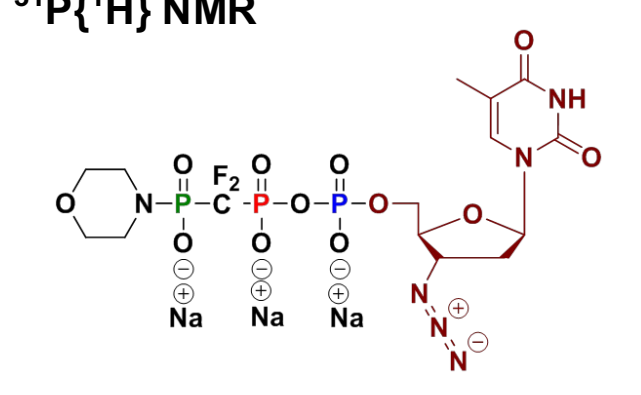

crude product after precipitation

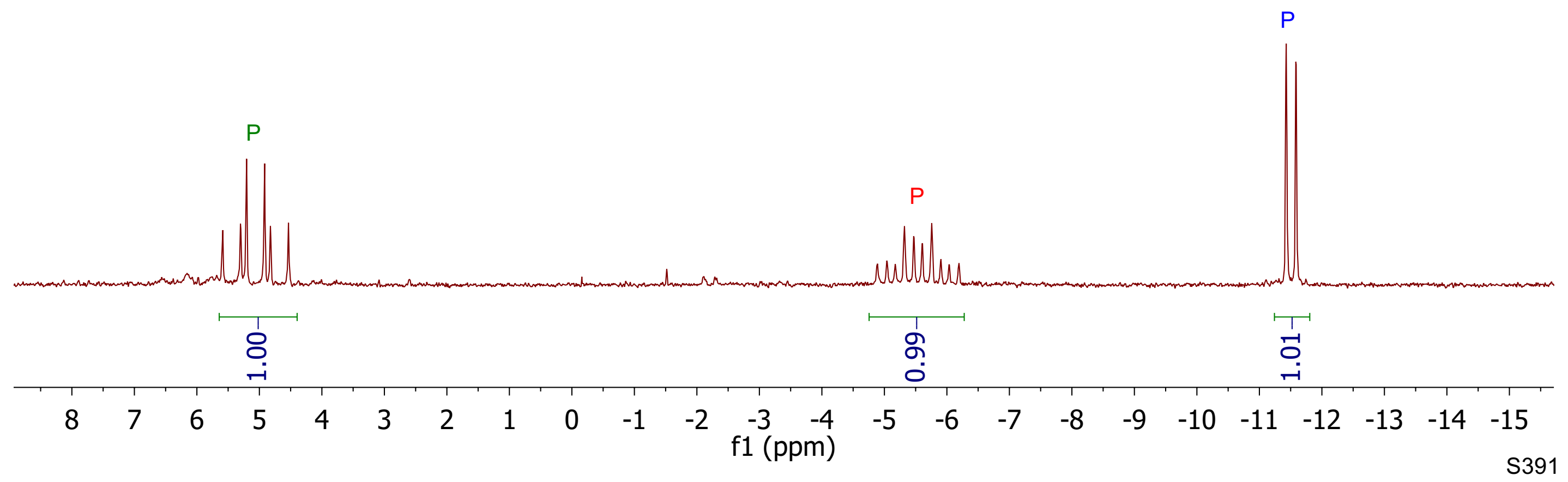




\section{${ }^{31} \mathrm{P}$ NMR}

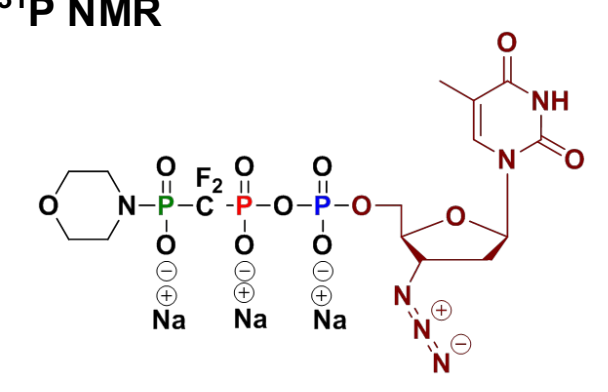

crude product after precipitation

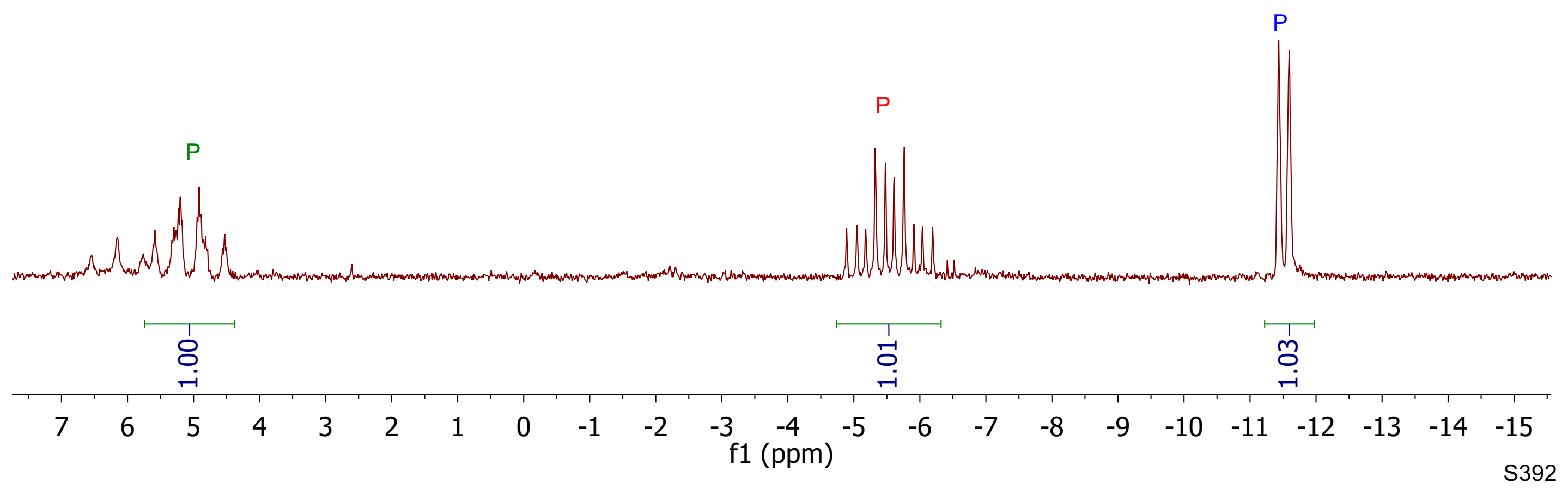




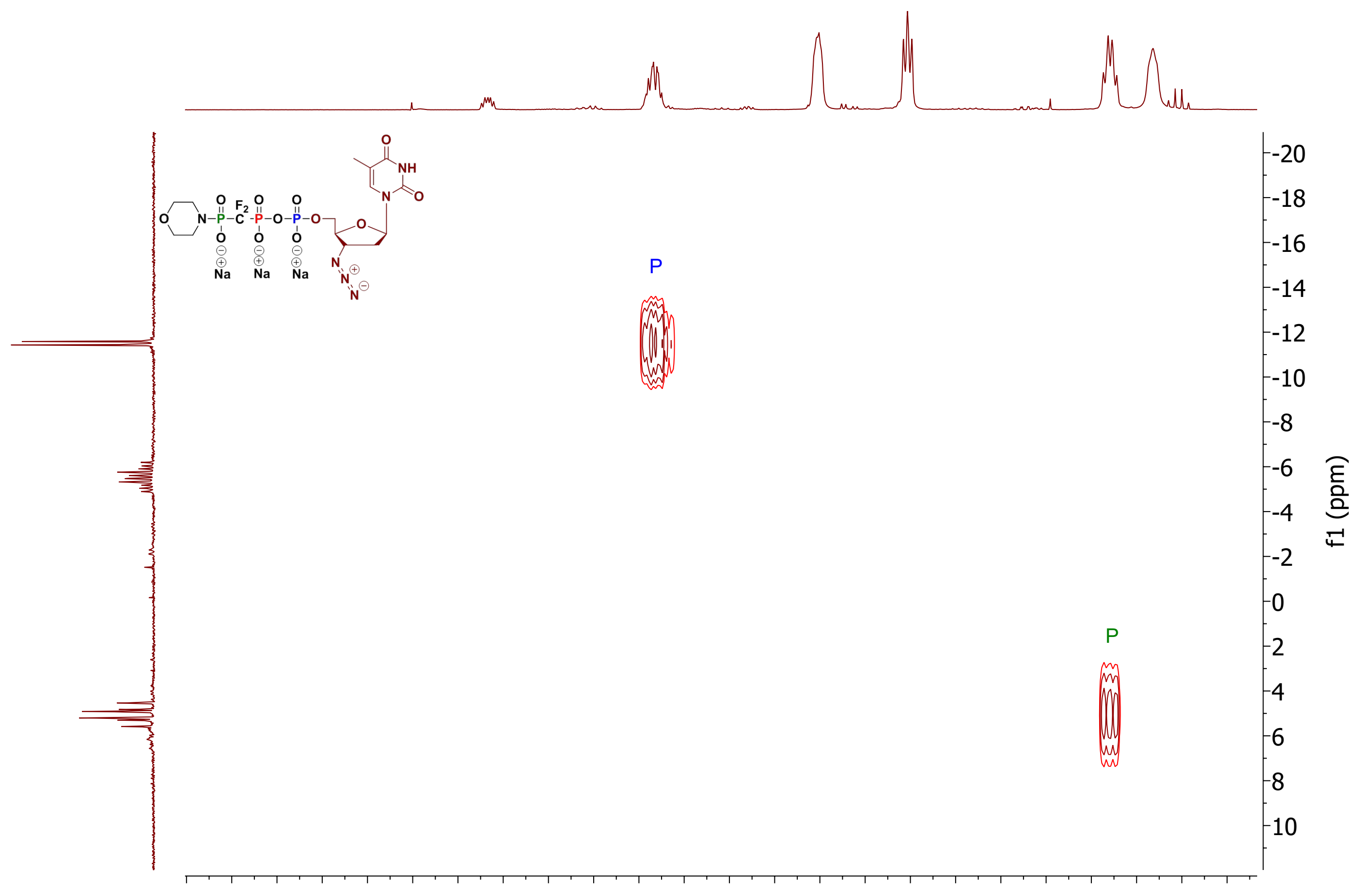

$\begin{array}{llllllllllllllllllllllll}5.2 & 5.1 & 5.0 & 4.9 & 4.8 & 4.7 & 4.6 & 4.5 & 4.4 & 4.3 & 4.2 & 4.1 & 4.0 & 3.9 & 3.8 & 3.7 & 3.6 & 3.5 & 3.4 & 3.3 & 3.2 & 3.1 & 3.0 & 2.9\end{array}$ f2 (ppm) 


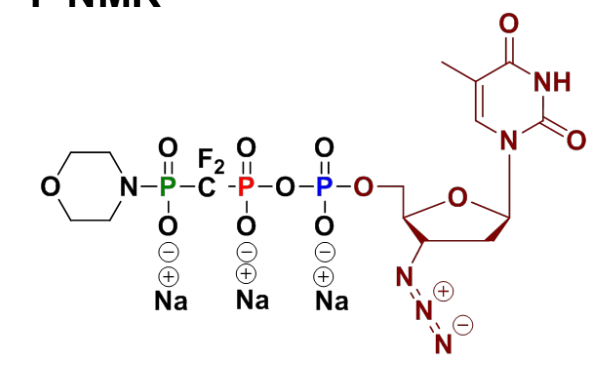

crude product after precipitation

A (ddd)

$-118.14$

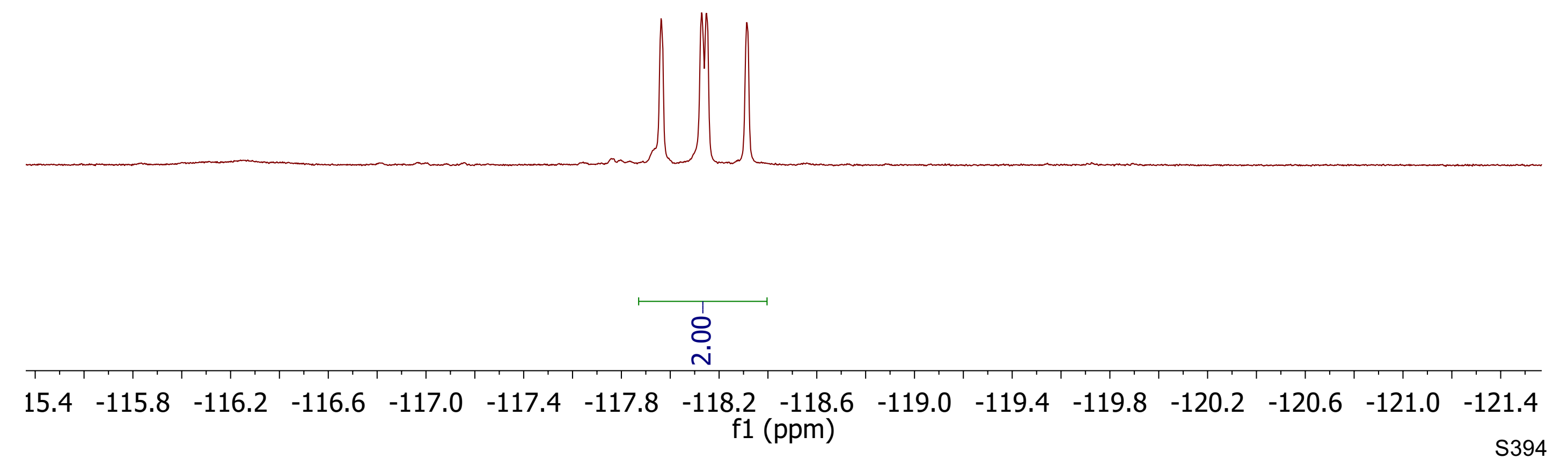




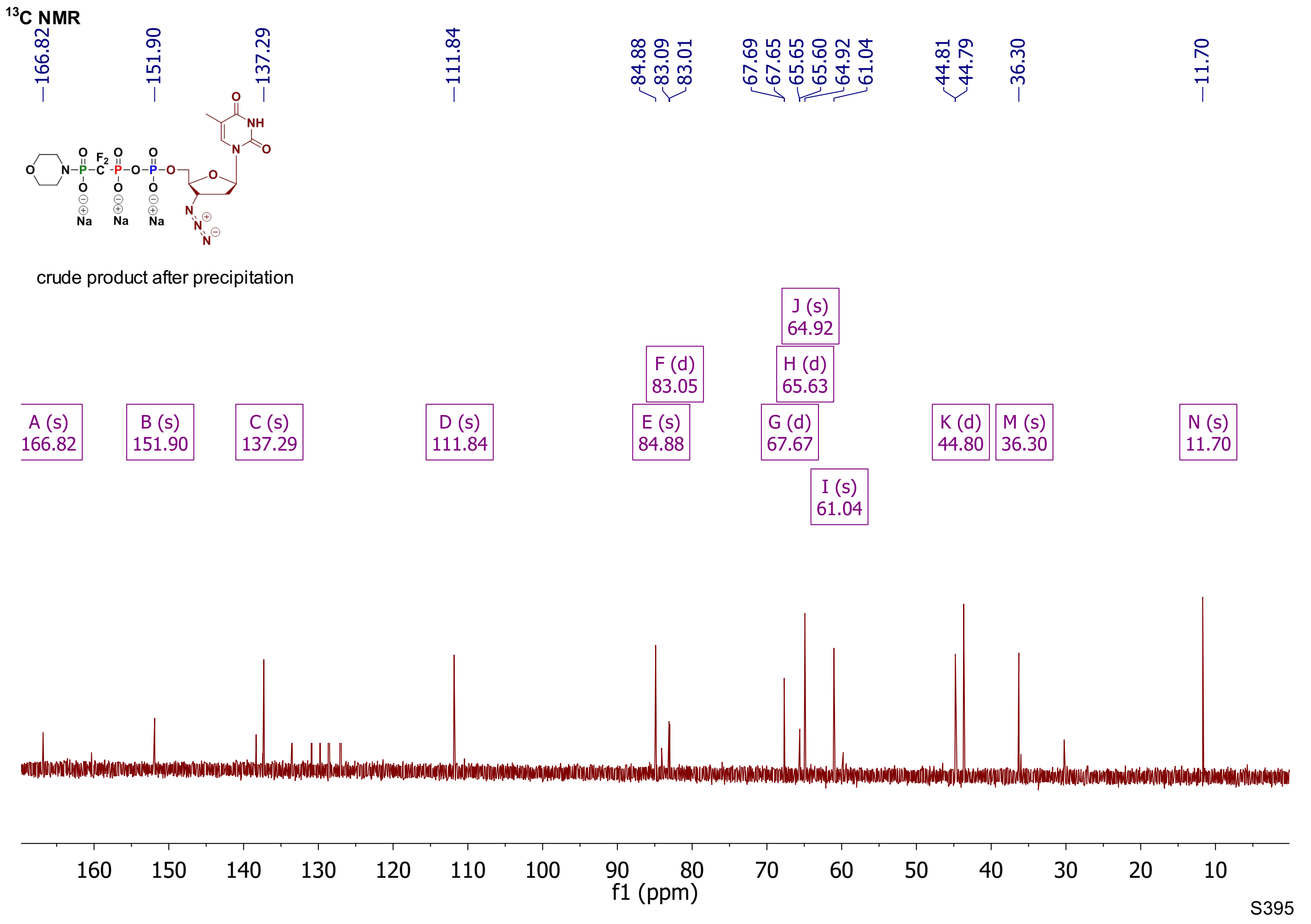




\section{${ }^{1} \mathrm{H}$ NMR}

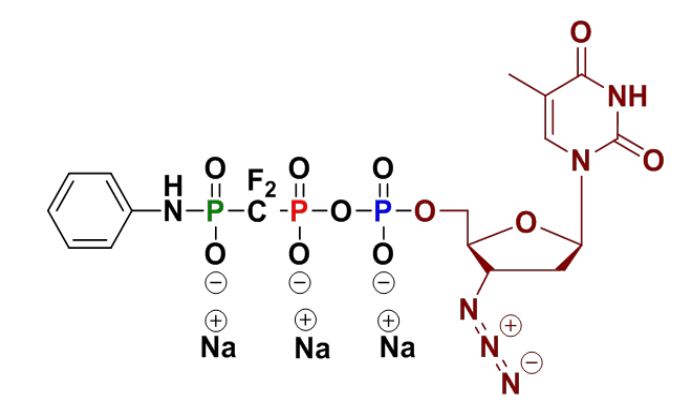

crude product after precipitation

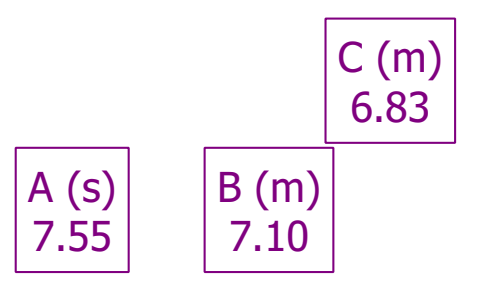

\begin{tabular}{|l|}
\hline$D(t)$ \\
6.13 \\
\hline
\end{tabular}

\begin{tabular}{|c|c|}
\hline$E(d t)$ & $G(m)$ \\
4.34 & 4.02 \\
\hline
\end{tabular}
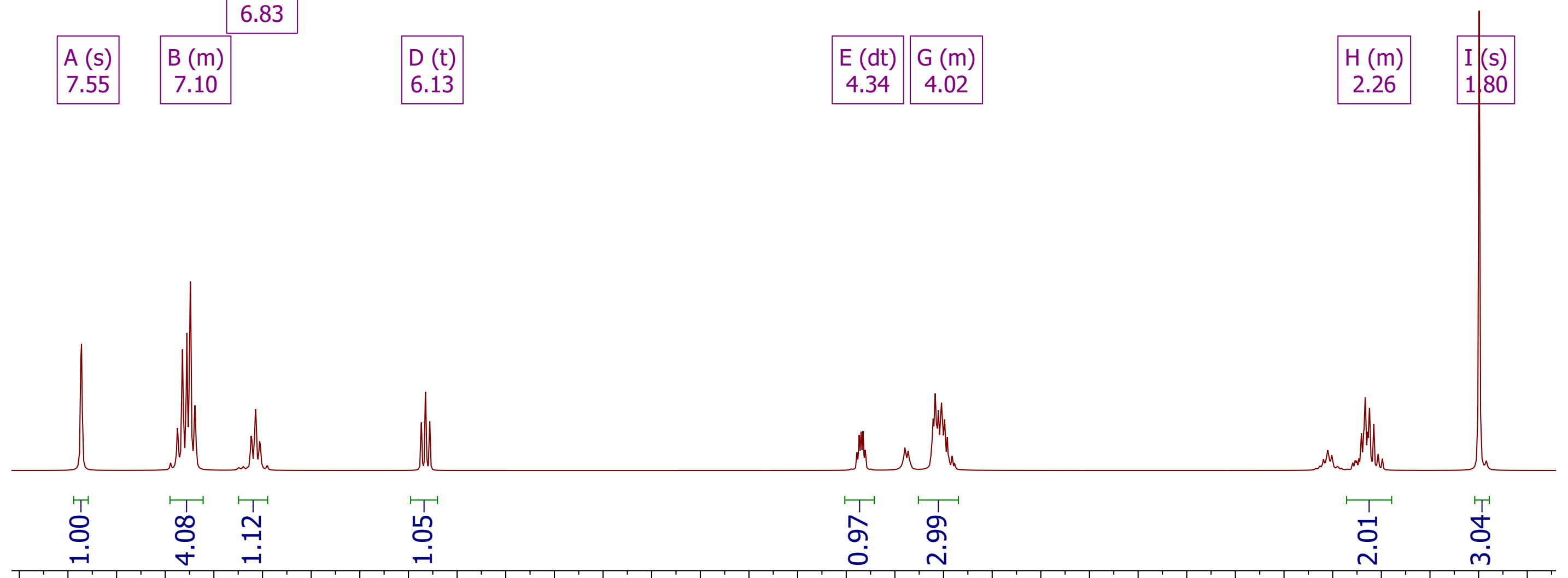

'.8 7.6 7.4 7.2 7.0 6.8 6.6 6.4 6.2 6.0 5.8 5.6 5.4 5.2 5.04 .84 .64 .44 .24 .03 .83 .63 .43 .23 .02 .82 .62 .42 .22 .01 .81 .6 f1 (ppm) 


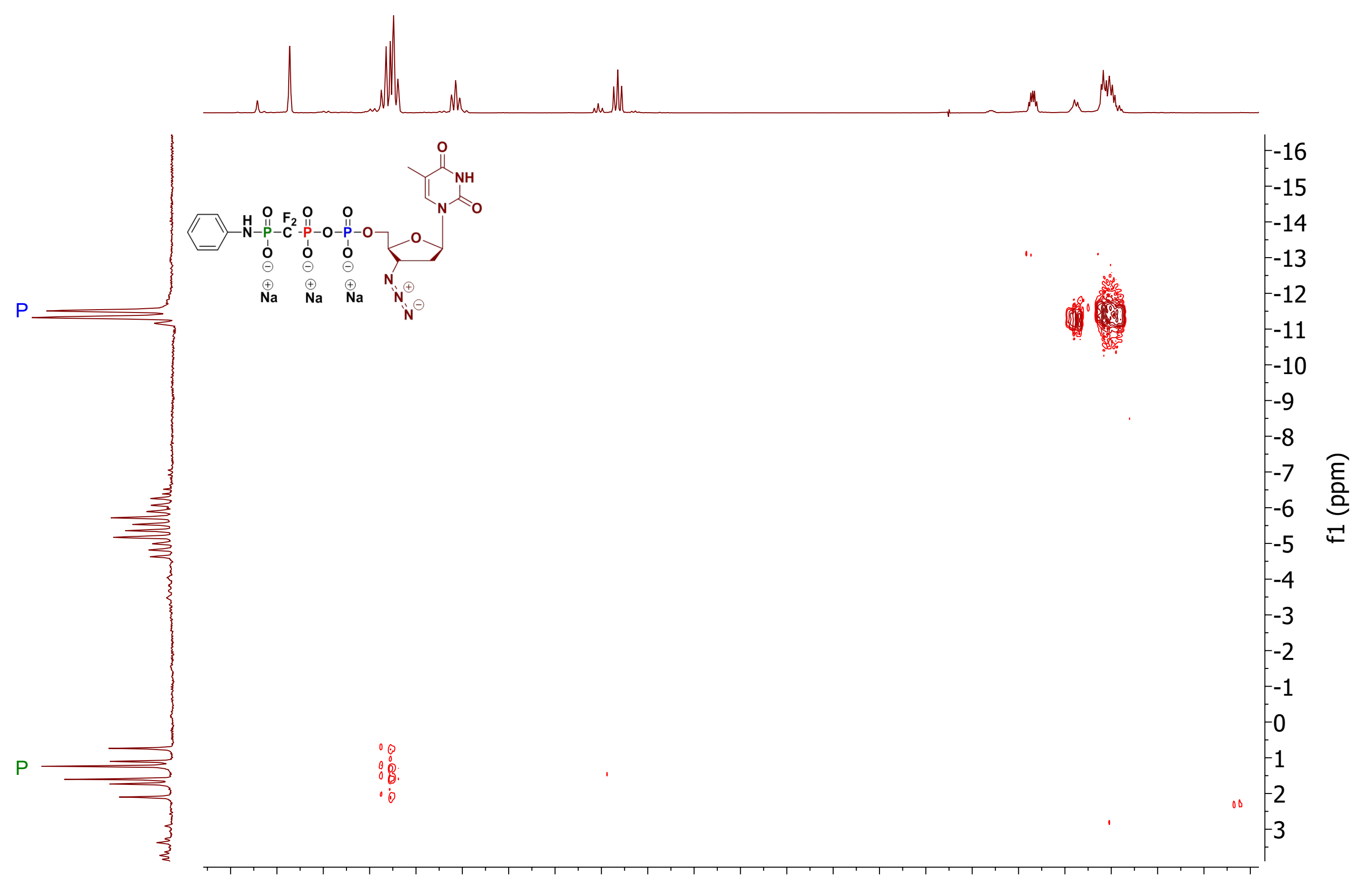

$\begin{array}{lllllllllllllllllllllll}7.8 & 7.6 & 7.4 & 7.2 & 7.0 & 6.8 & 6.6 & 6.4 & 6.2 & 6.0 & 5.8 & 5.6 & 5.4 & 5.2 & 5.0 & 4.8 & 4.6 & 4.4 & 4.2 & 4.0 & 3.8 & 3.6 & 3.4\end{array}$ f2 (ppm) 


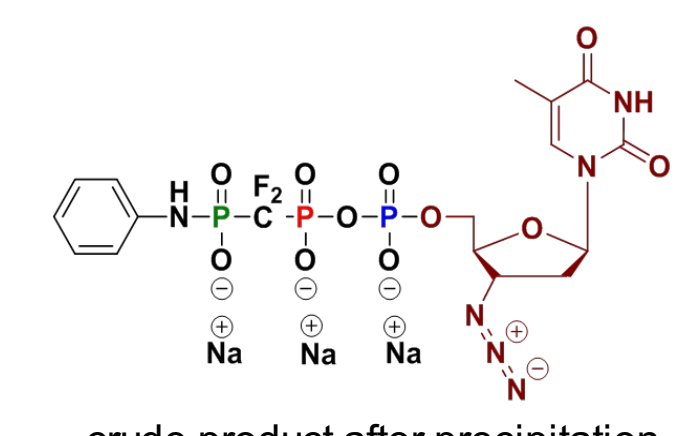

crude product after precipitation

A (ddd)
-119.24

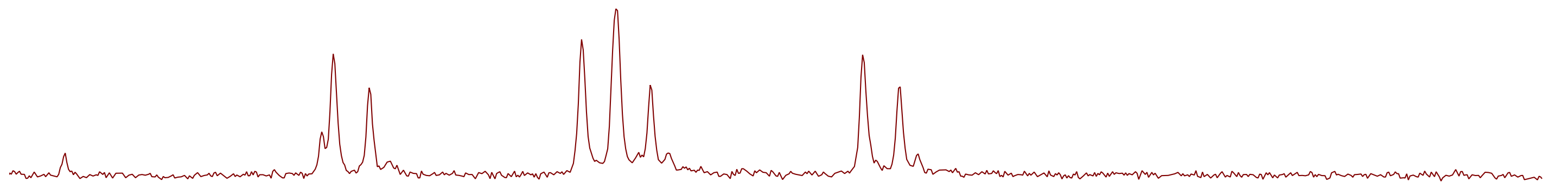

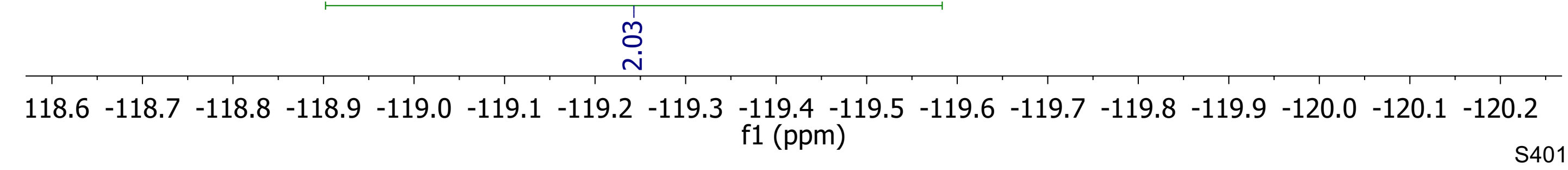




\section{${ }^{1} \mathrm{H}$ NMR}

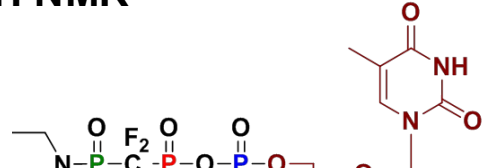

$\left[\begin{array}{ccc}8 & 8 & \ddots \\ 1 & 0 & 0 \\ 2 & 1 & 1\end{array}\right]$

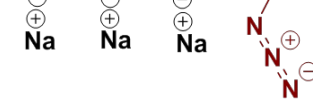

crude product after precipitation

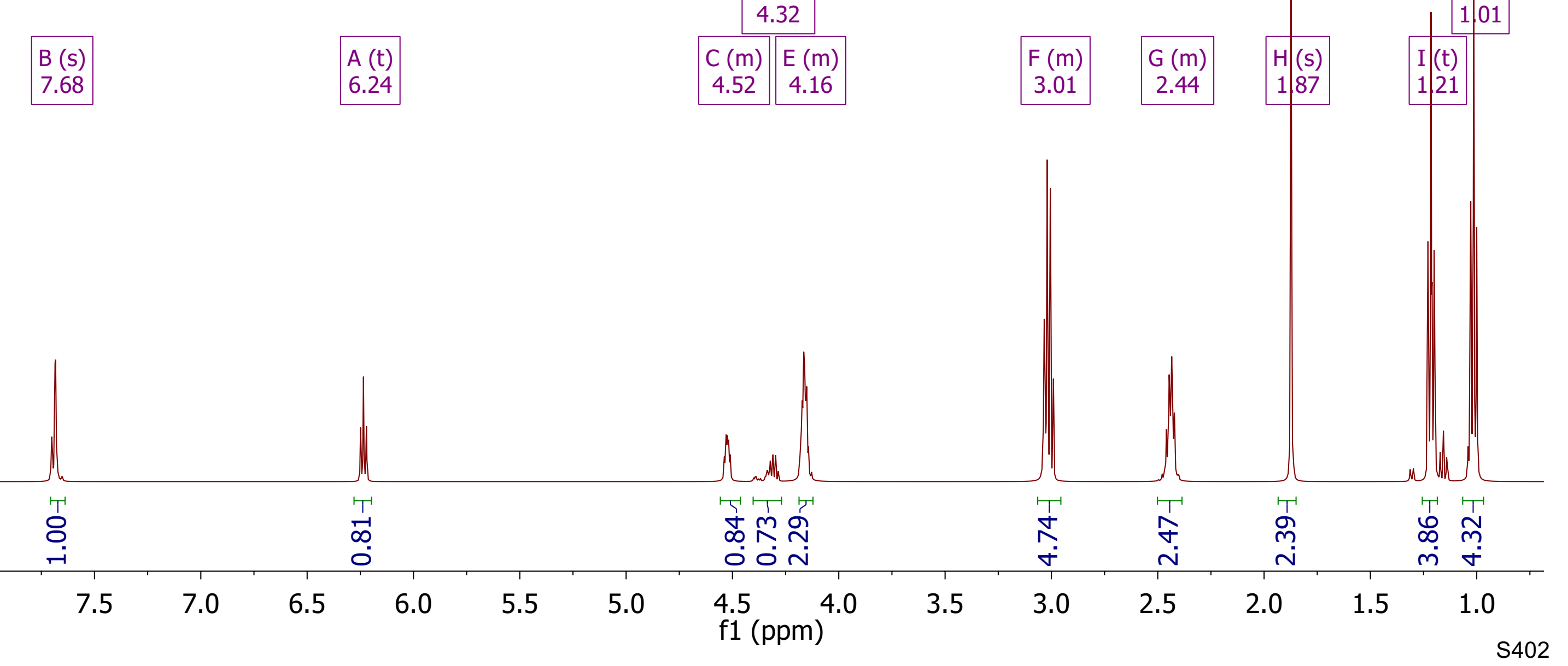




\section{${ }^{31} \mathrm{P}\left\{{ }^{1} \mathrm{H}\right\} \mathrm{NMR}$}

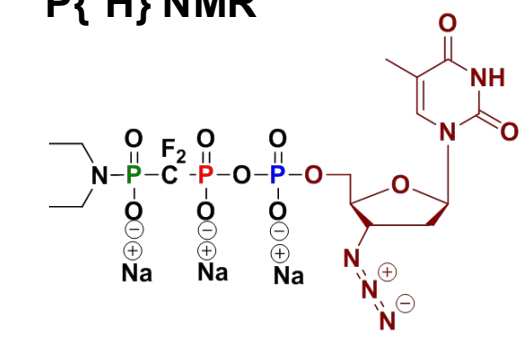

crude product after precipitation

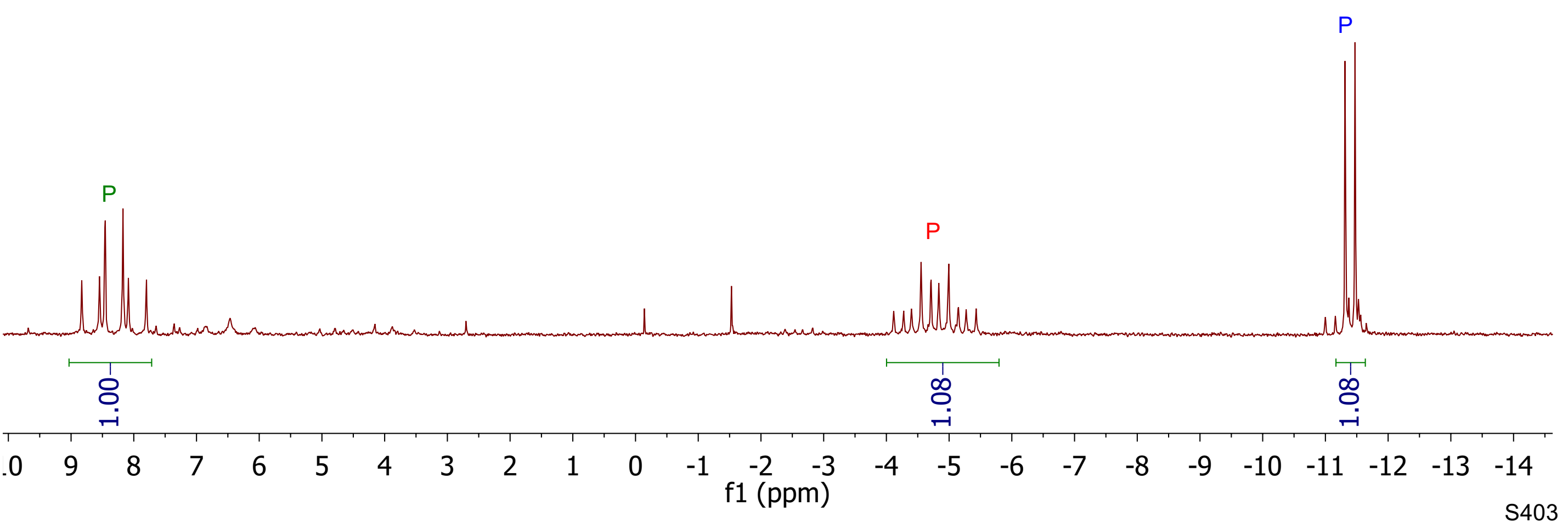


31
31

crude product after precipitation

C (m)

8.31
B (tdd)

$-4.78$

P

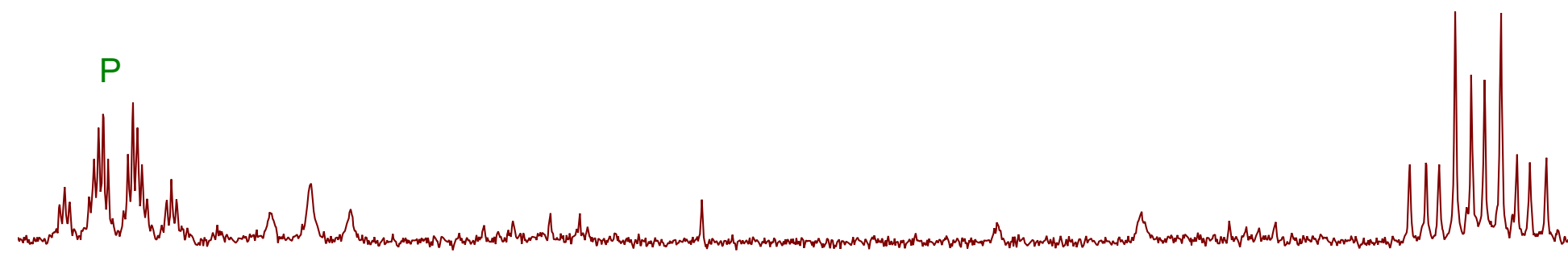

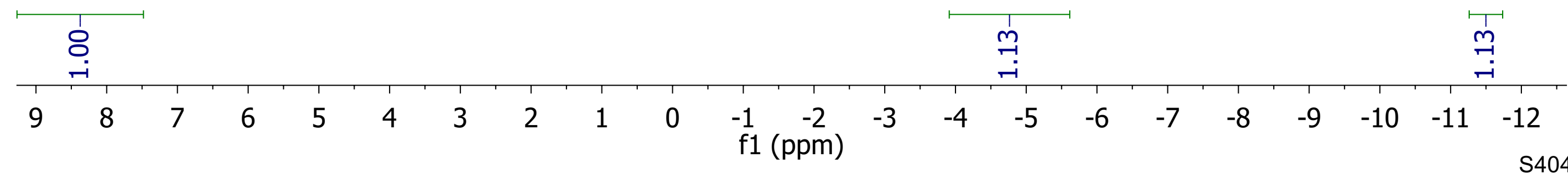




\section{${ }^{19} \mathrm{~F}$ NMR}

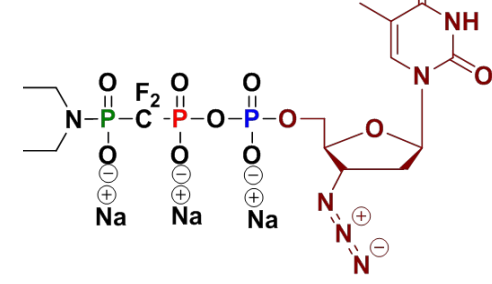

crude product after precipitation
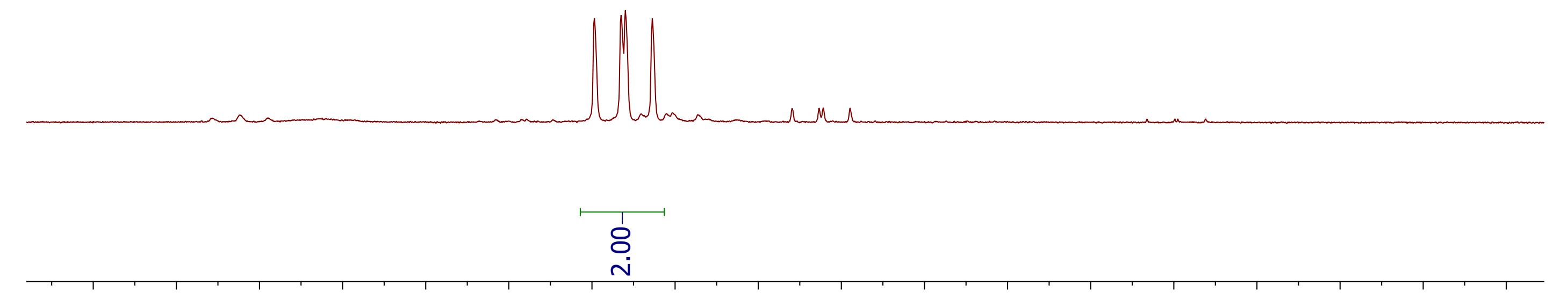

-114.5 -115.0 -115.5 -116.0 -116.5 -117.0 -117.5 -118.0 -118.5 -119.0 -119.5 -120.0 -120.5 -121.0 -121.5 -122.0 -122.5 -123.0 f1 (ppm) 


\section{${ }^{31} \mathrm{P}\left\{{ }^{1} \mathrm{H}\right\}$ NMR}

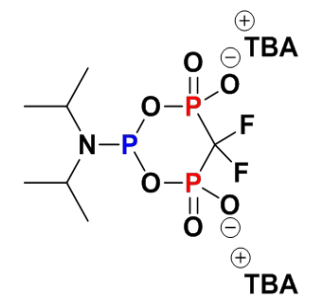

Amonhwumum
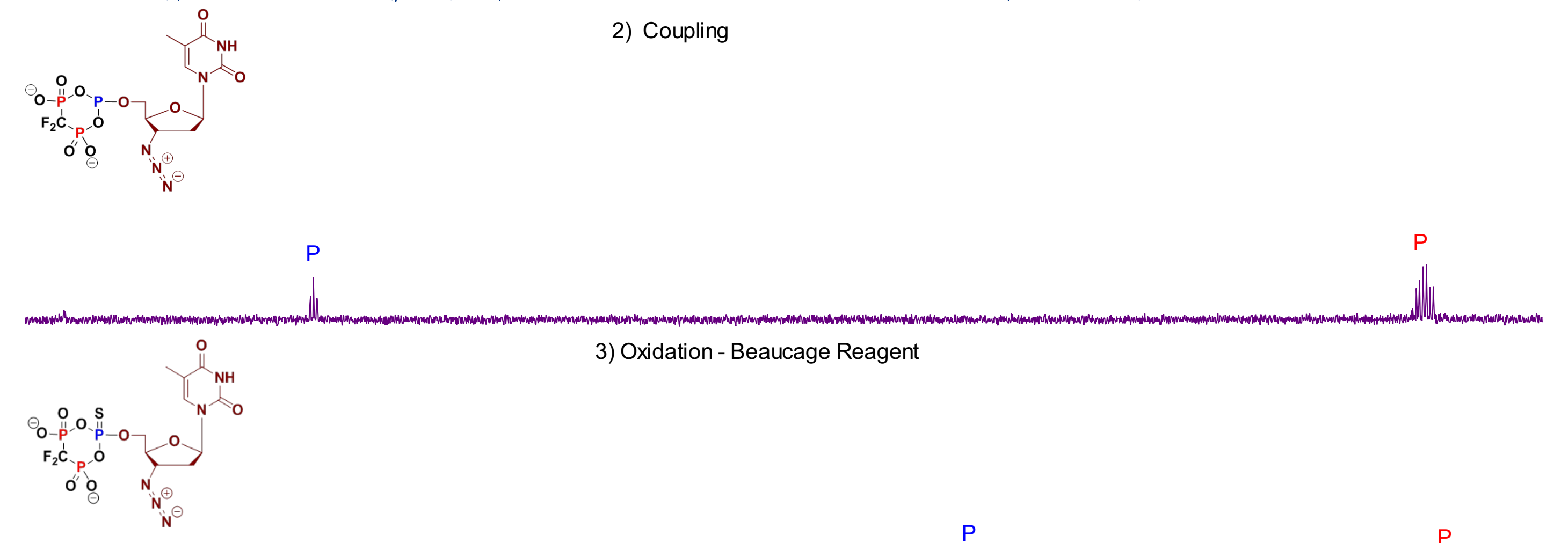

3) Oxidation - Beaucage Reagent

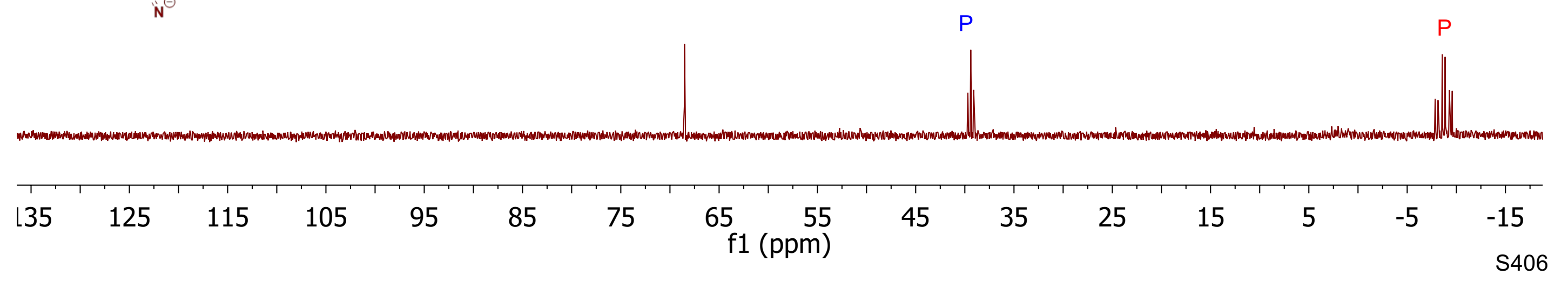




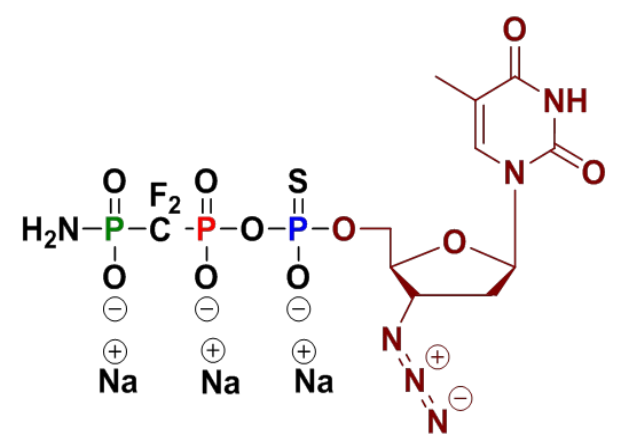

crude product after precipitation

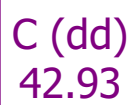

$\mathrm{P}$

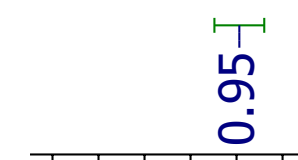

$46 \quad 44$

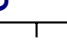

4038

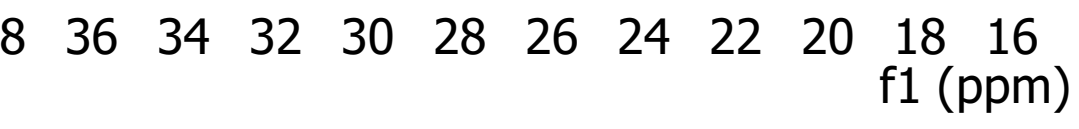




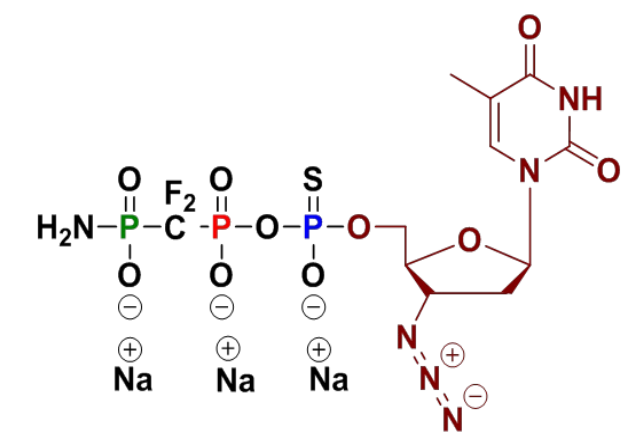

crude product after precipitation

$$
\begin{aligned}
& \hline \text { B (m) } \\
& 42.93
\end{aligned}
$$

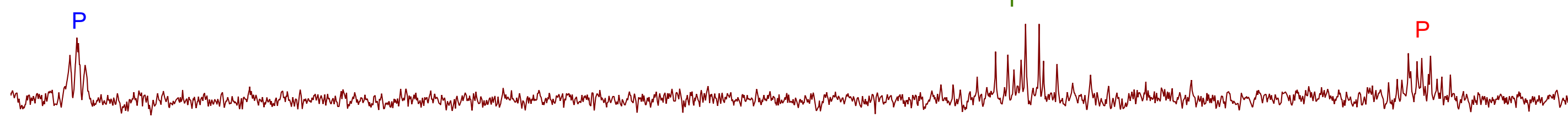

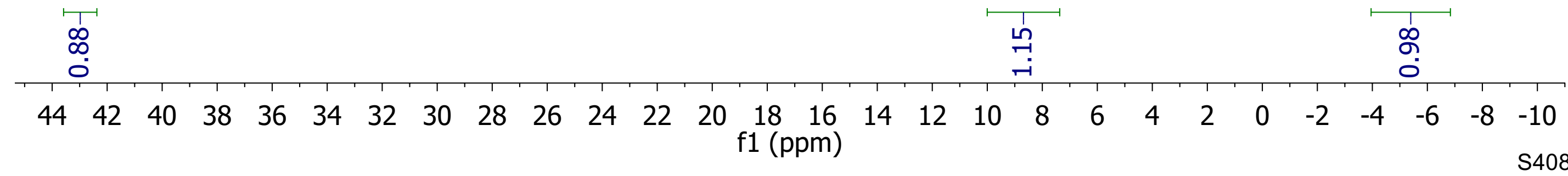




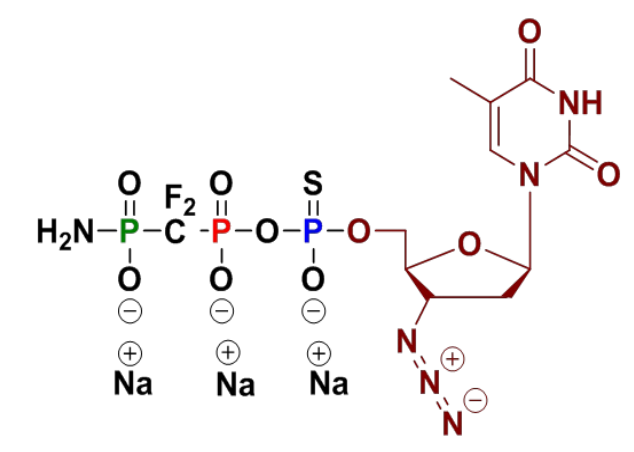

crude product after precipitation

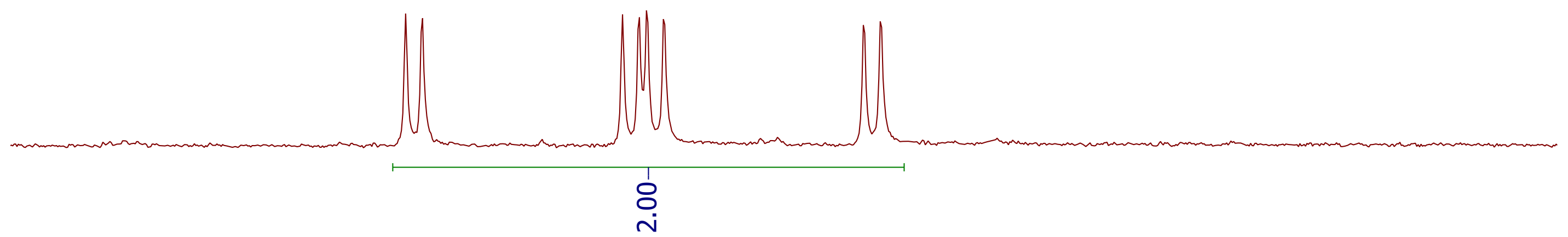

\begin{tabular}{llllllllll}
\hline-120.3 & -120.5 & -120.7 & -120.9 & -121.1 & -121.3 & -121.5 & -121.7 & -121.9 & -122.1 \\
\hline
\end{tabular}




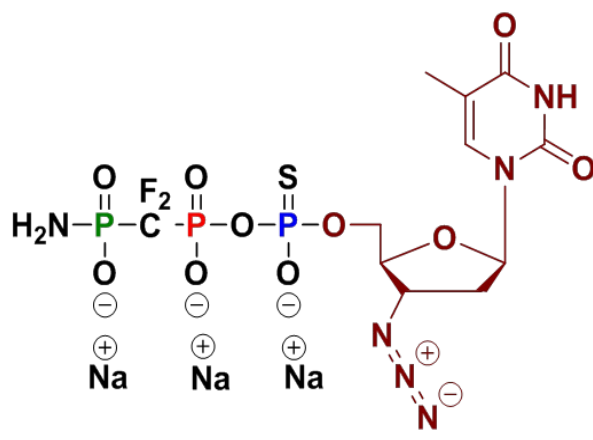

crude product after precipitation

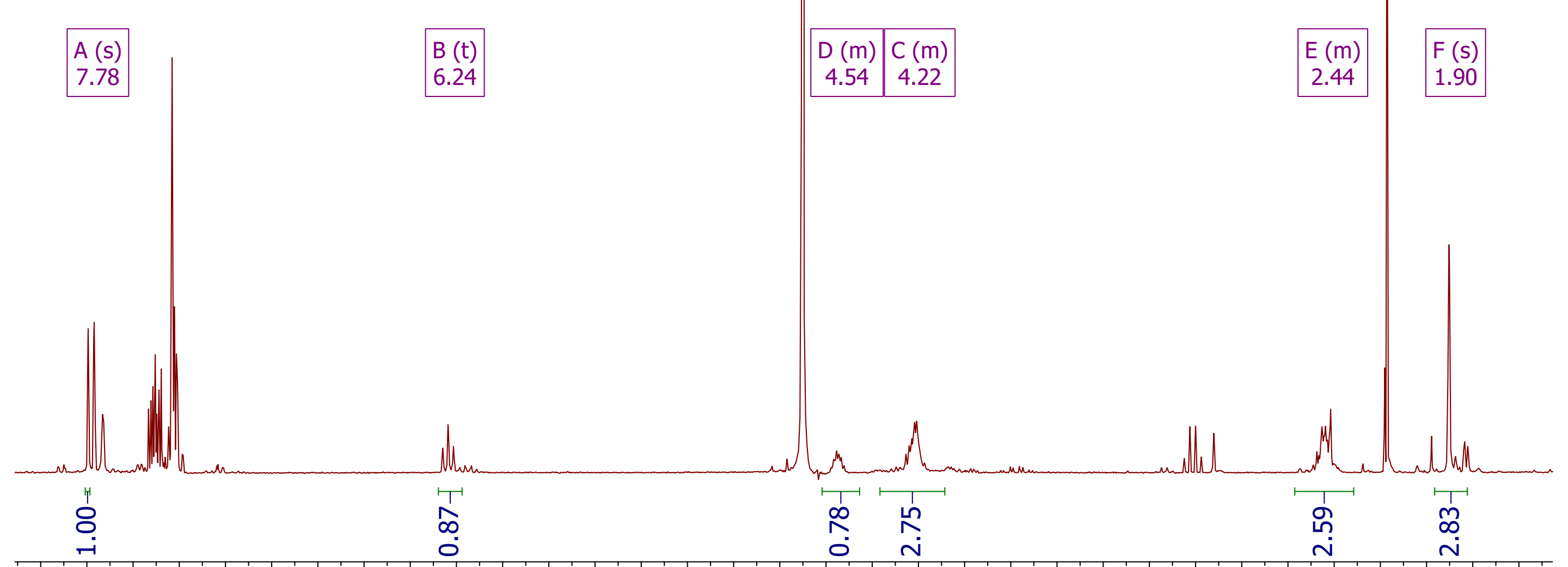




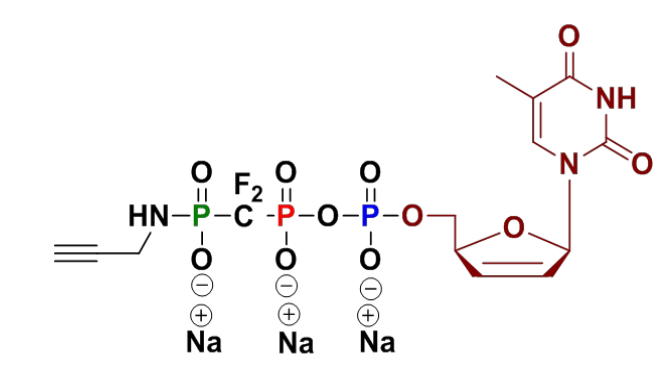

crude product after precipitation
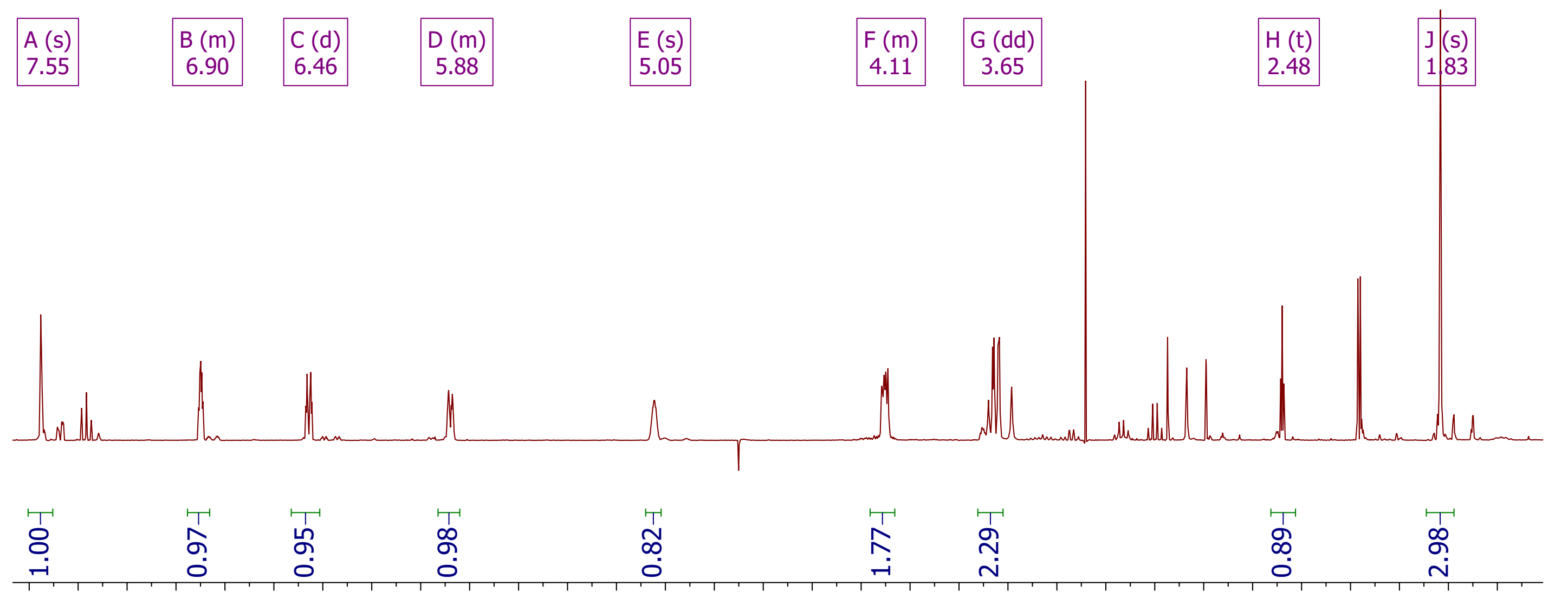

7.6 7.4 7.2 7.0 $6.86 .66 .46 .26 .05 .85 .65 .45 .25 .04 .8 \quad 4.64 .44 .2 \quad 4.03 .83 .63 .43 .23 .02 .82 .62 .42 .22 .01 .81 .6$ f1 (ppm) 


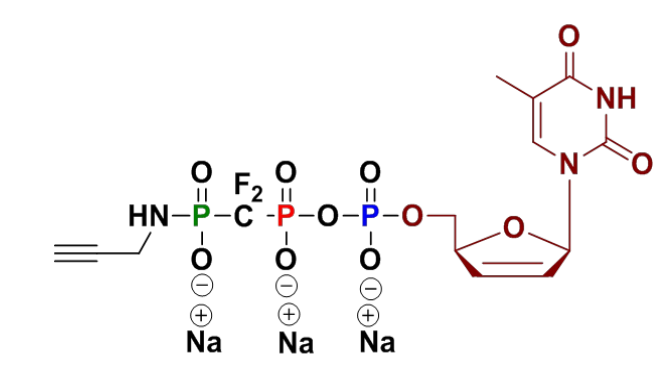

crude product after precipitation

\begin{tabular}{|c|}
\hline$C(\mathrm{~m})$ \\
6.30 \\
\hline
\end{tabular}

$$
\begin{array}{|c|}
\hline \text { A (tdd) } \\
-5.25
\end{array}
$$

P

P
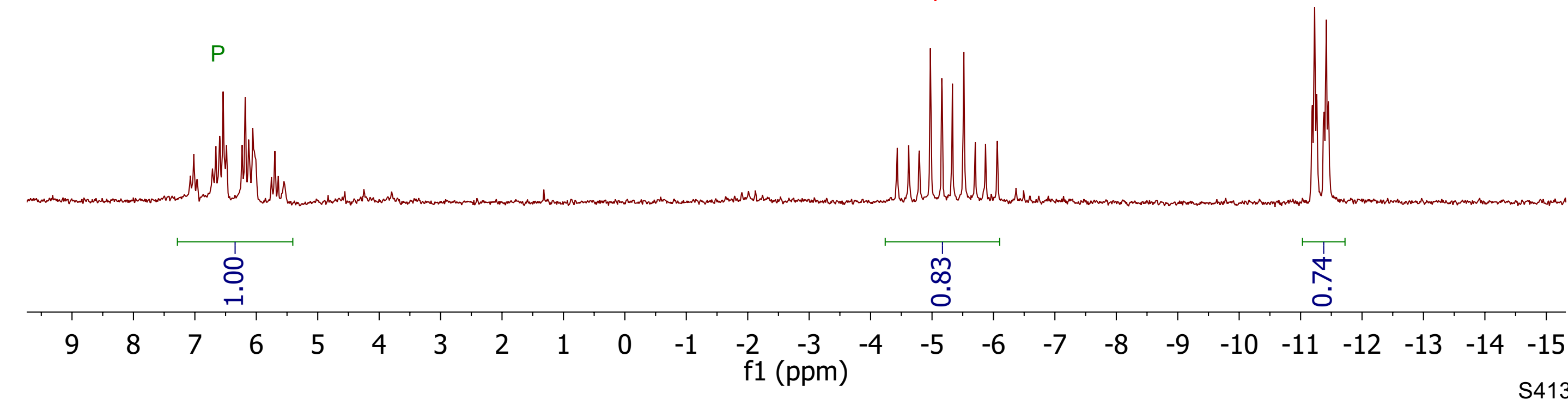


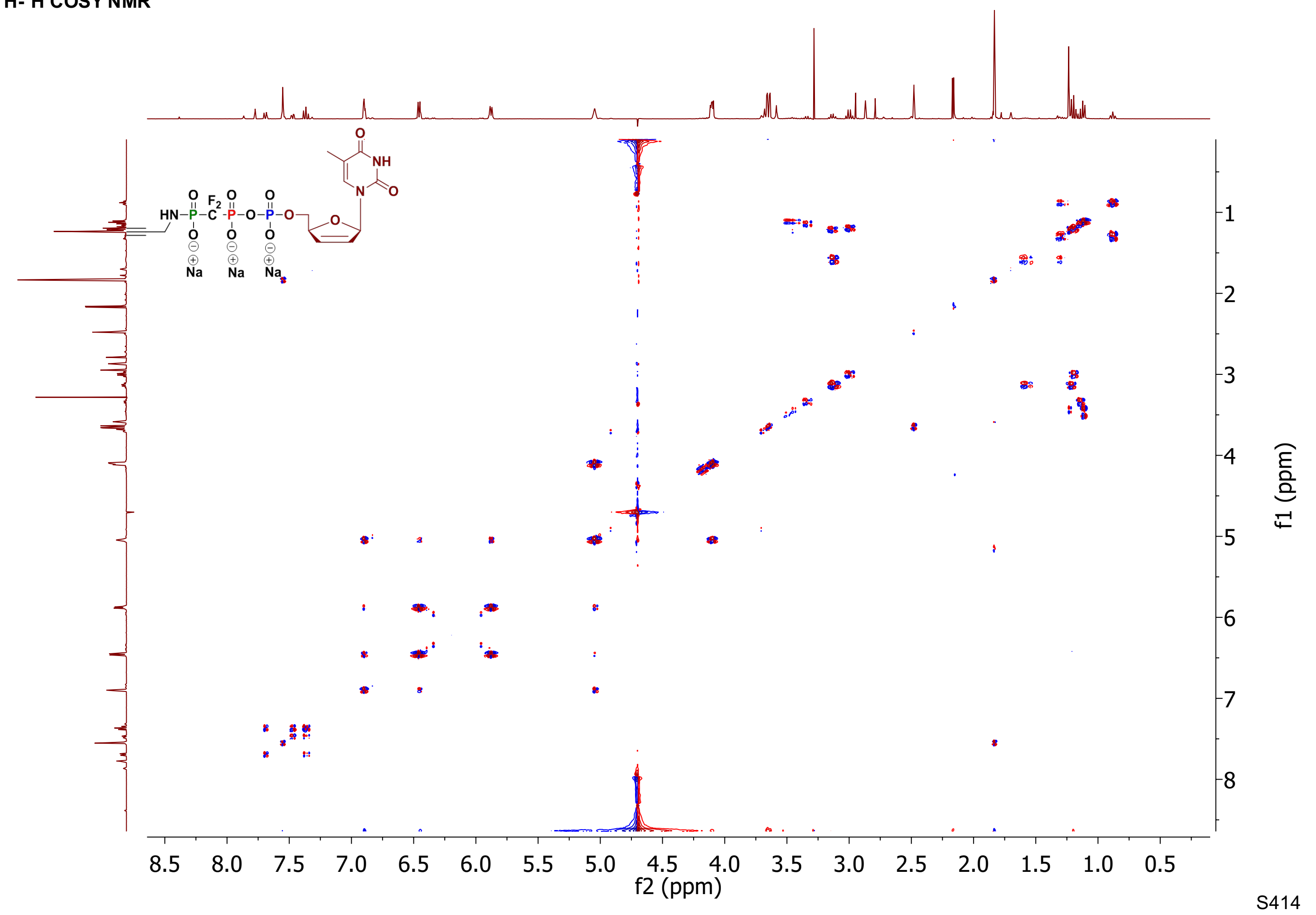




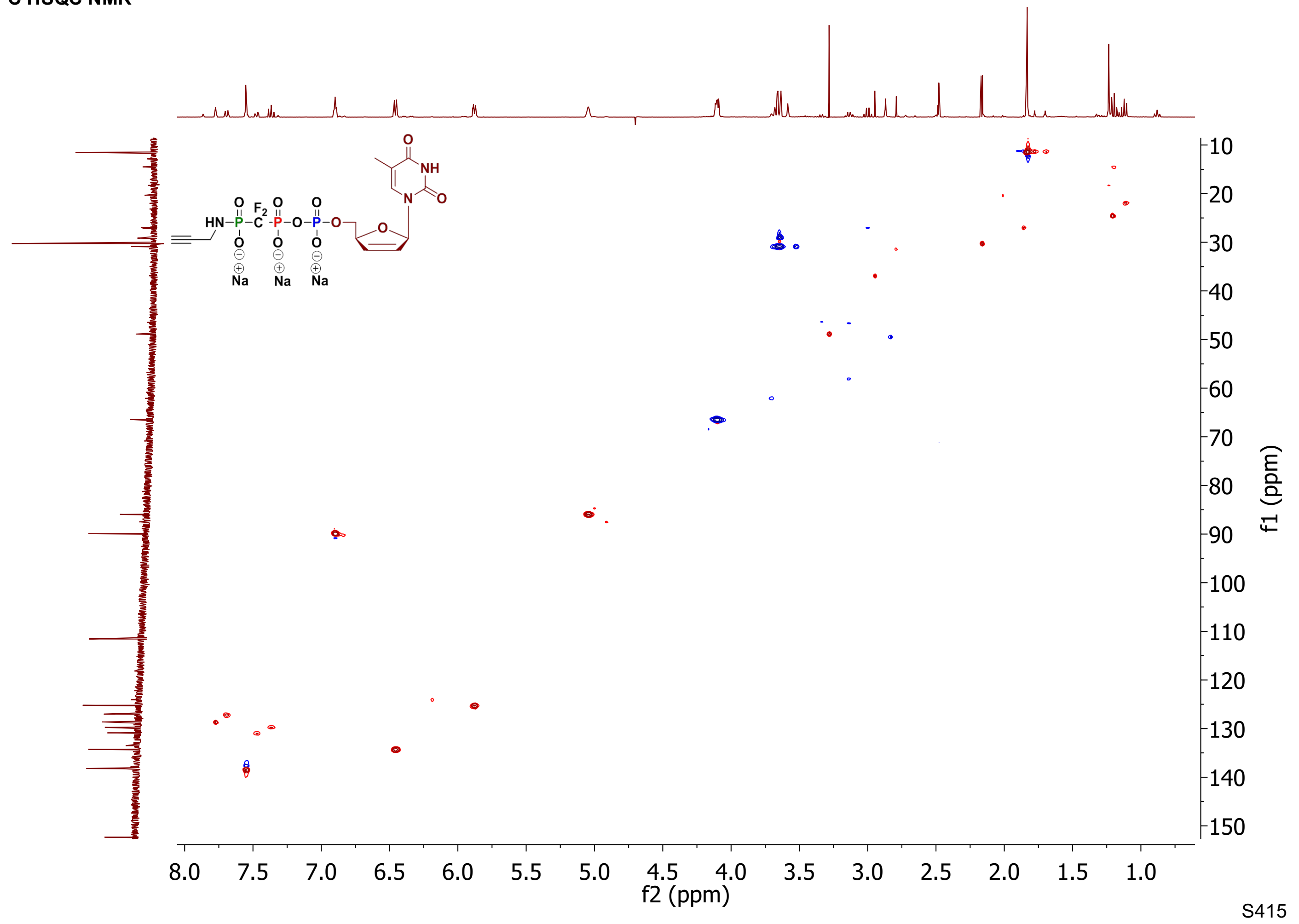




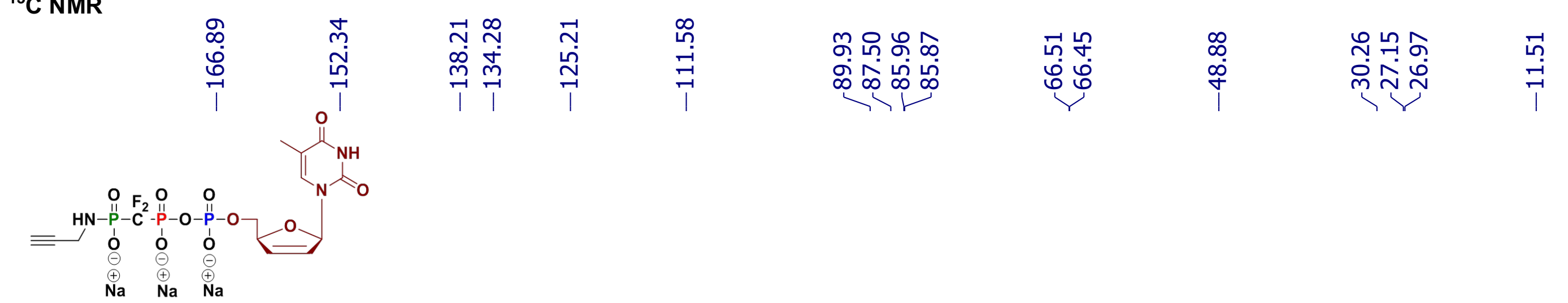

crude product after precipitation

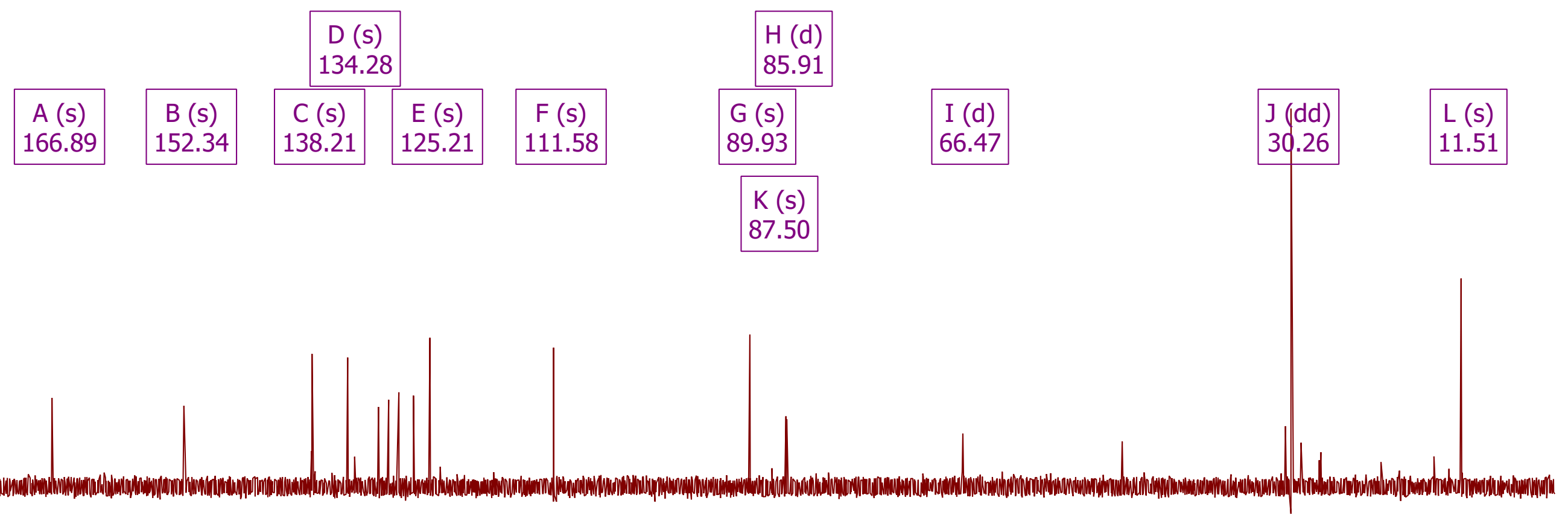

\begin{tabular}{|c|c|c|c|c|c|c|c|c|c|c|c|c|c|c|c|c|}
\hline 180 & 170 & 160 & 150 & 140 & 130 & 120 & 110 & $\begin{array}{lr}100 & 90 \\
\mathrm{f1} & (\mathrm{ppm})\end{array}$ & 80 & 70 & 60 & 50 & 40 & 30 & 20 & $\begin{array}{l}10 \\
\text { S416 }\end{array}$ \\
\hline
\end{tabular}




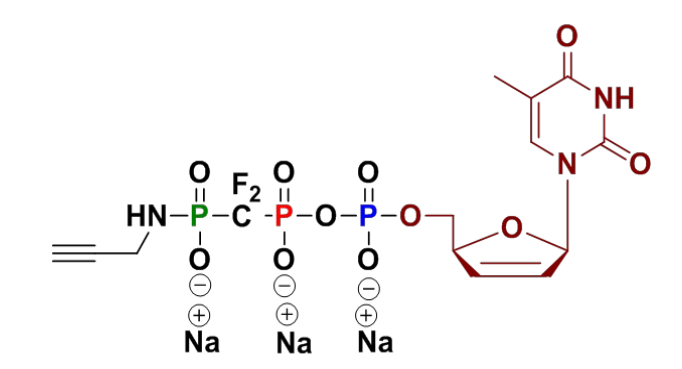

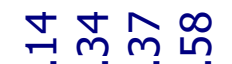

จํ

군

crude product after precipitation

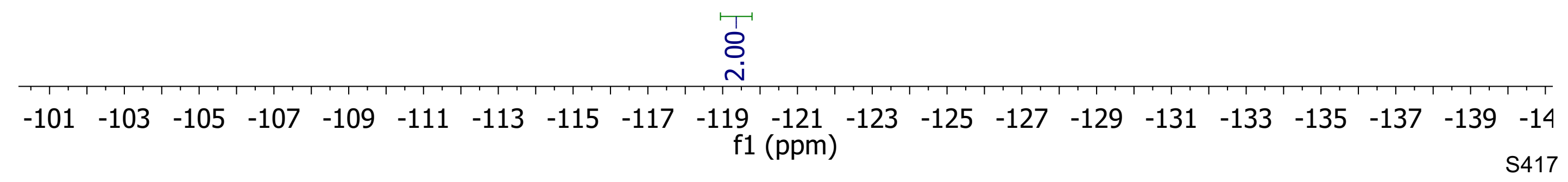




\section{${ }^{1} \mathrm{H}$ NMR}

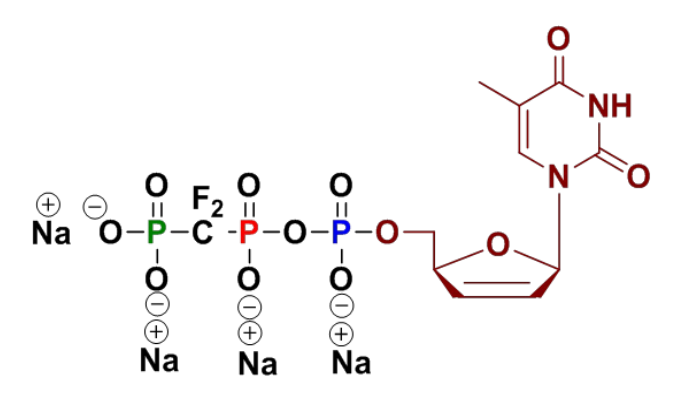

crude product after precipitation

\begin{tabular}{|c|c|c|c|c|c|}
\hline $\begin{array}{l}G(d) \\
7.54\end{array}$ & $\begin{array}{c}A(d t) \\
6.89\end{array}$ & $\begin{array}{c}B(d t) \\
6.47\end{array}$ & $\begin{array}{c}C(d t) \\
5.86\end{array}$ & $\begin{array}{l}D(s) \\
5.05\end{array}$ & $\begin{array}{c}E(d t) \\
4.10\end{array}$ \\
\hline
\end{tabular}

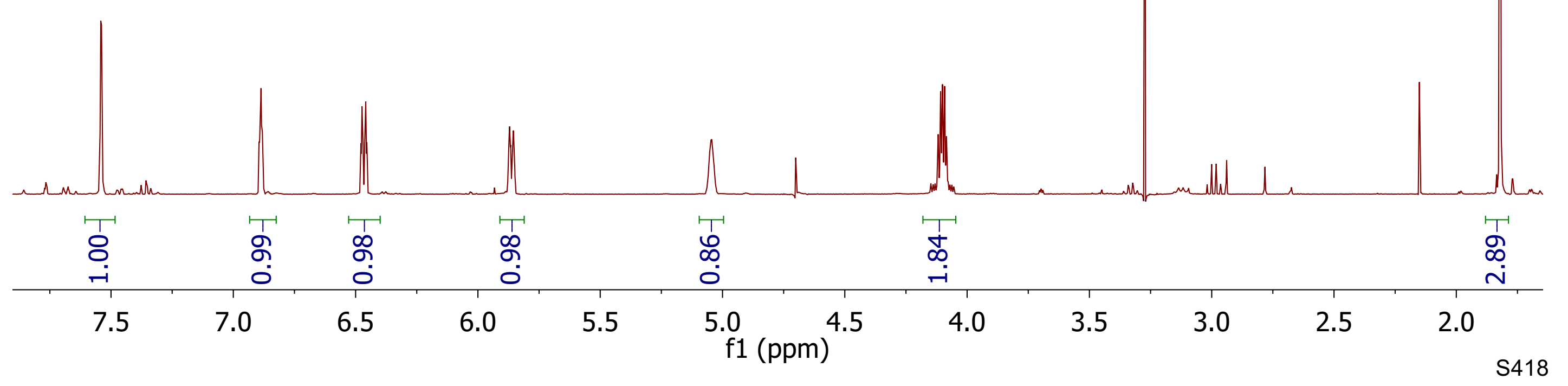




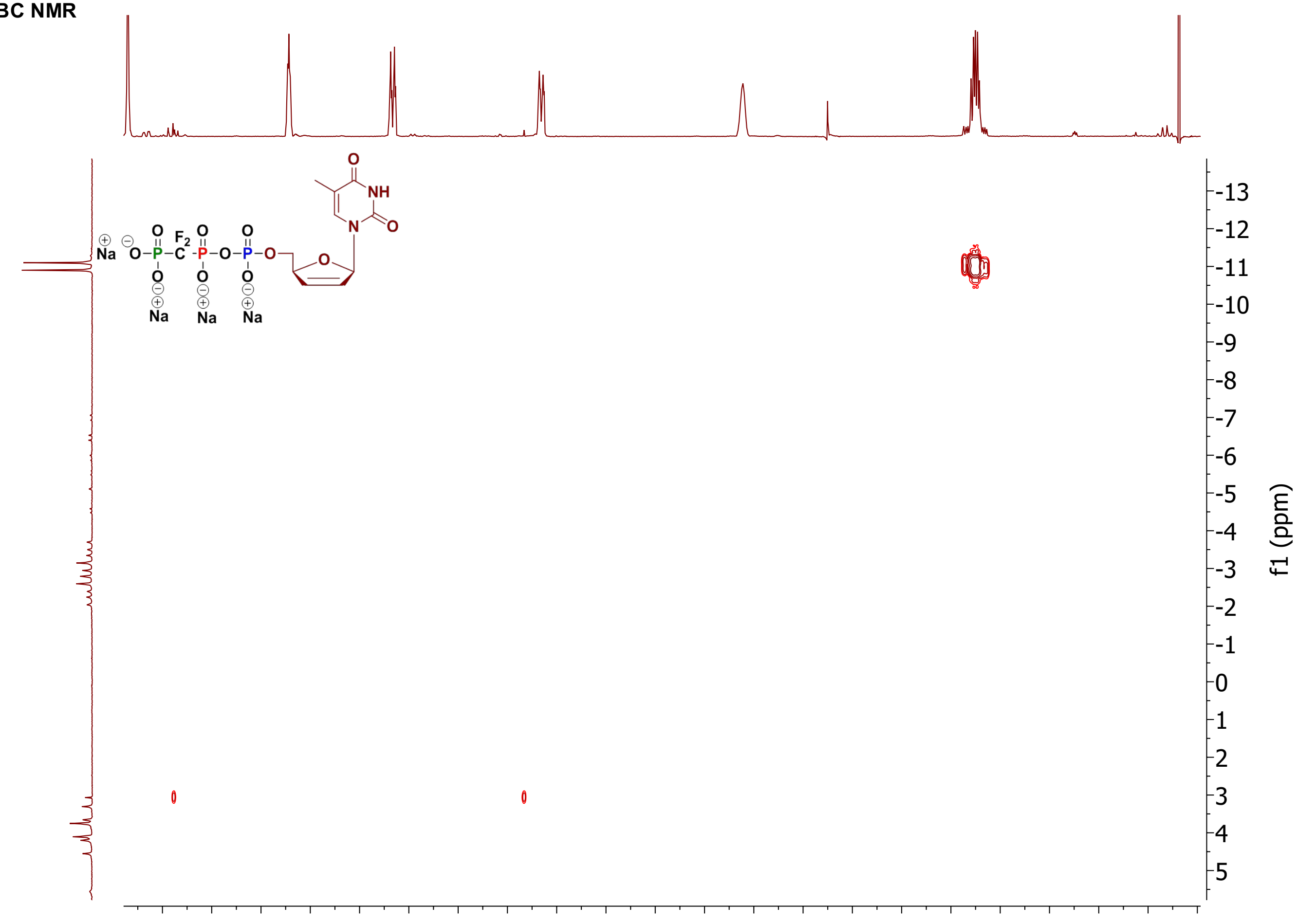

$\begin{array}{llllllllllllllllllllll}7.4 & 7.2 & 7.0 & 6.8 & 6.6 & 6.4 & 6.2 & 6.0 & 5.8 & 5.6 & 5.4 & 5.2 & 5.0 & 4.8 & 4.6 & 4.4 & 4.2 & 4.0 & 3.8 & 3.6 & 3.4 & 3.2\end{array}$ f2 (ppm) 


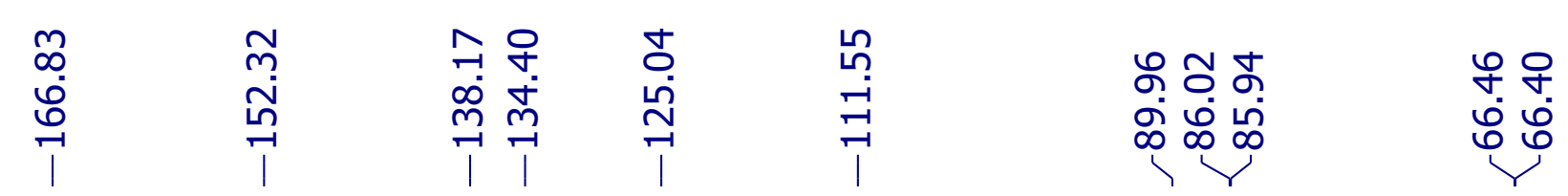

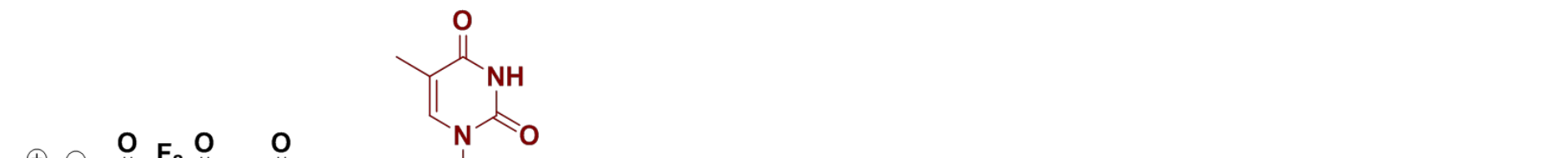

\begin{tabular}{|c|c|c|c|c|c|c|}
\hline \multirow[b]{2}{*}{$\begin{array}{c}\text { A (s) } \\
166.83\end{array}$} & \multirow[b]{2}{*}{$\begin{array}{c}B(s) \\
152.32\end{array}$} & $\begin{array}{c}D(s) \\
134.40\end{array}$ & & & $\begin{array}{l}H(d) \\
85.98\end{array}$ & \\
\hline & & $\begin{array}{c}C(s) \\
138.17\end{array}$ & $\begin{array}{c}F(s) \\
125.04\end{array}$ & $\begin{array}{c}E(s) \\
111.55\end{array}$ & $\begin{array}{l}\text { G (s) } \\
89.96\end{array}$ & $\begin{array}{l}\text { I (d) } \\
66.43\end{array}$ \\
\hline
\end{tabular}

\begin{tabular}{|c|c|c|c|c|c|c|c|c|c|c|c|c|c|c|c|c|c|c|}
\hline 180 & 170 & 160 & 150 & 140 & 130 & 120 & 110 & 100 & $\begin{array}{c}90 \\
\text { (ppn }\end{array}$ & 80 & 70 & 60 & 50 & 40 & 30 & 20 & 10 & $\begin{array}{c}0 \\
5422\end{array}$ \\
\hline
\end{tabular}




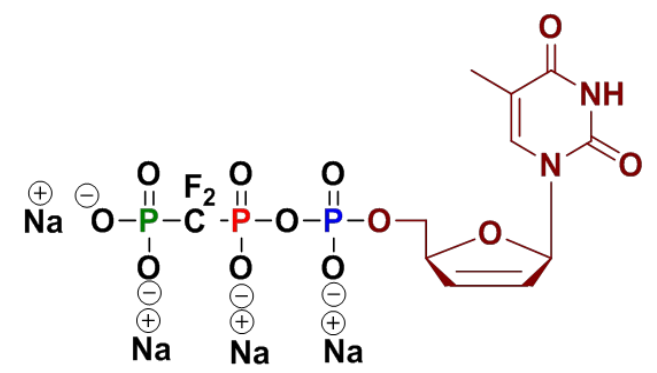

crude product after precipitation 


\section{${ }^{1} \mathrm{H}$ NMR}

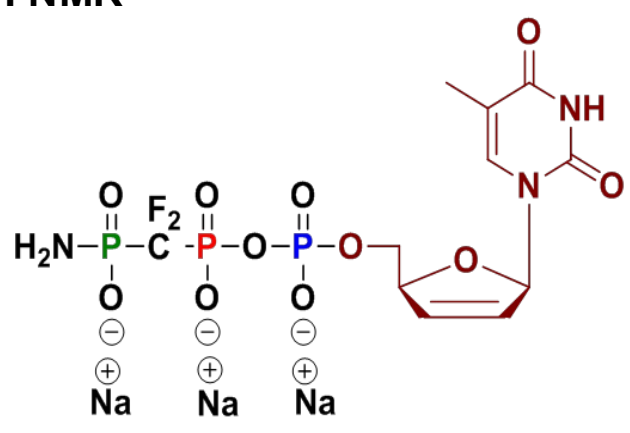

\begin{tabular}{|l|l|l|l|}
\hline$A(s)$ \\
7.53 & $B(m)$ & $C(d)$ \\
6.89
\end{tabular}$\quad \begin{gathered}D(d) \\
6.45\end{gathered} \quad$\begin{tabular}{ll}
$E(s)$ & $F(m)$ \\
5.03 & \\
\hline
\end{tabular}

crude product after precipitation

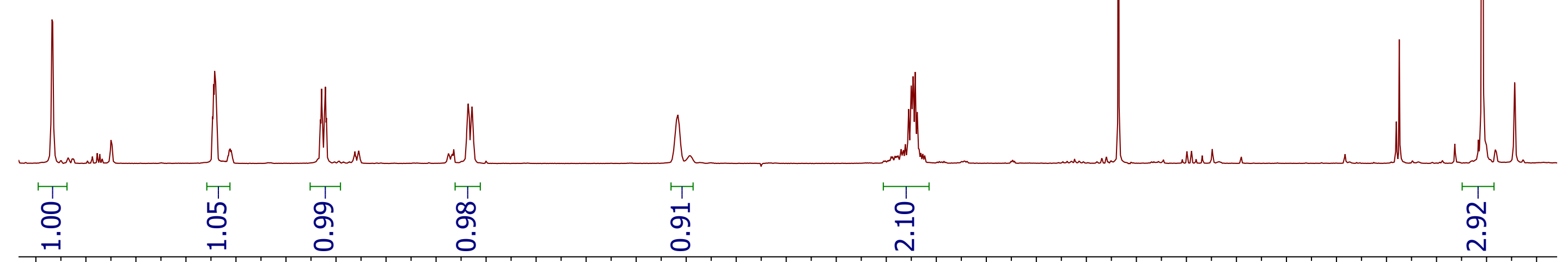
f1 (ppm) 


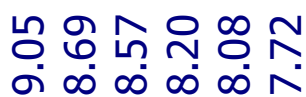

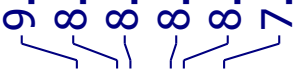

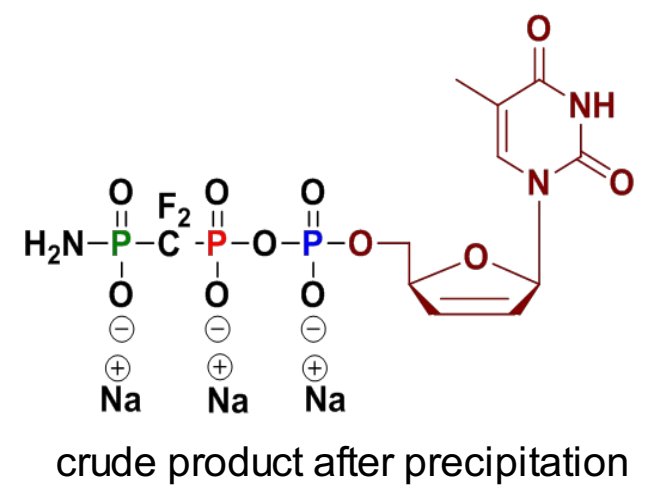

$$
\begin{array}{|c|}
\hline \text { A (td) } \\
8.39
\end{array}
$$
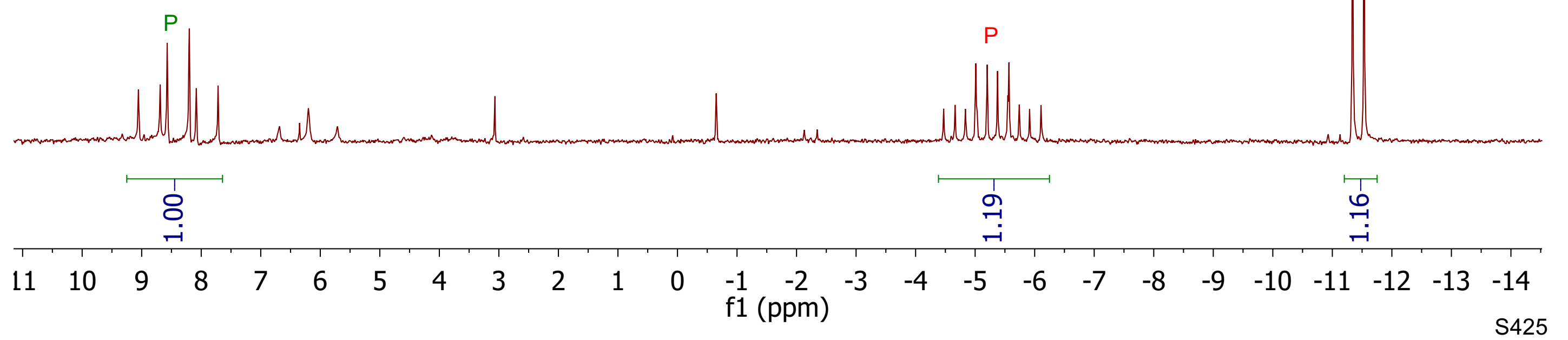


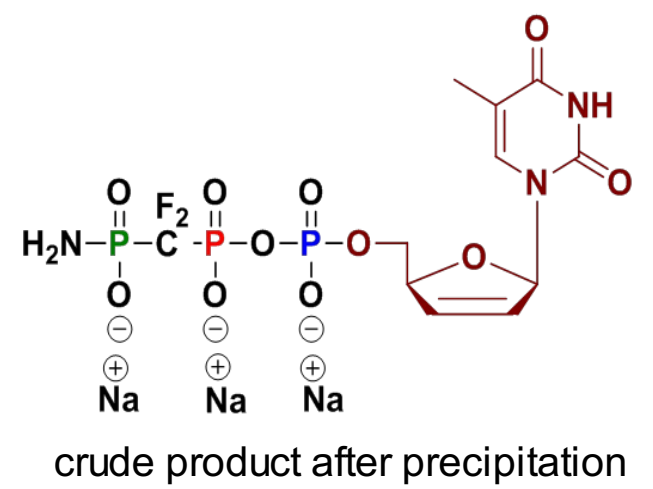

$$
\begin{array}{|c|}
\hline A(t d) \\
8.38 \\
\hline
\end{array}
$$

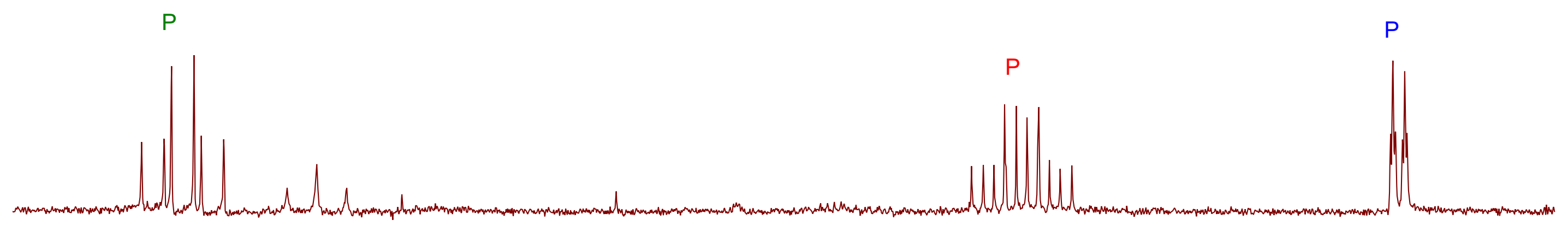

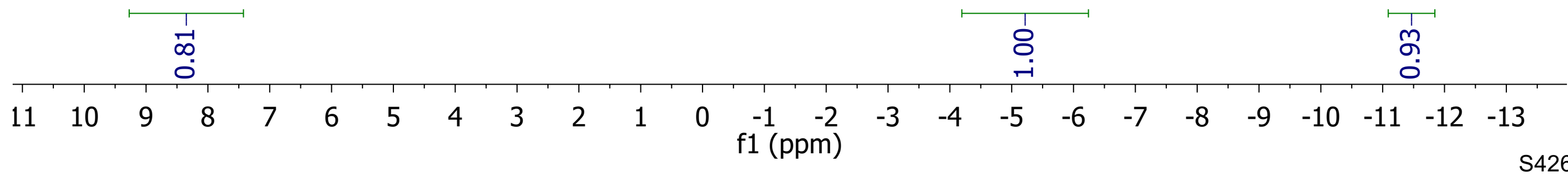




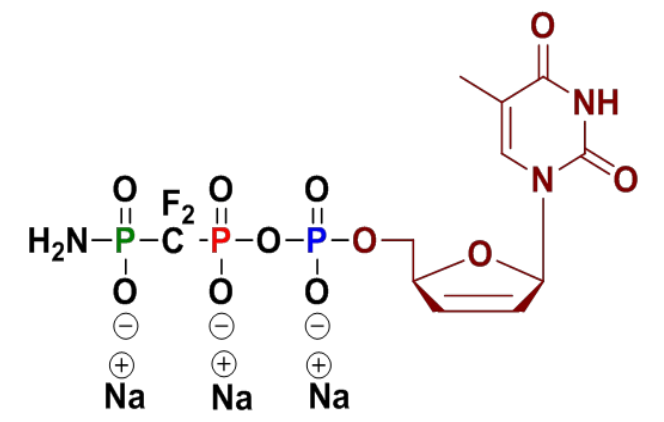

crude product after precipitation

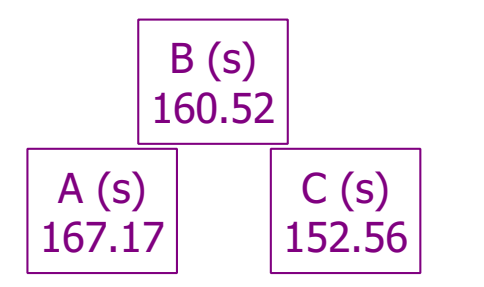

\begin{tabular}{|c|c|c|}
\hline $\begin{array}{l}\text { E (s } \\
134 .\end{array}$ & & \\
\hline $\begin{array}{c}D(s) \\
138.14\end{array}$ & $\begin{array}{c}\mathrm{F}(\mathrm{s}) \\
125.15\end{array}$ & $\begin{array}{c}\mathrm{G}(\mathrm{s}) \\
111.55\end{array}$ \\
\hline
\end{tabular}
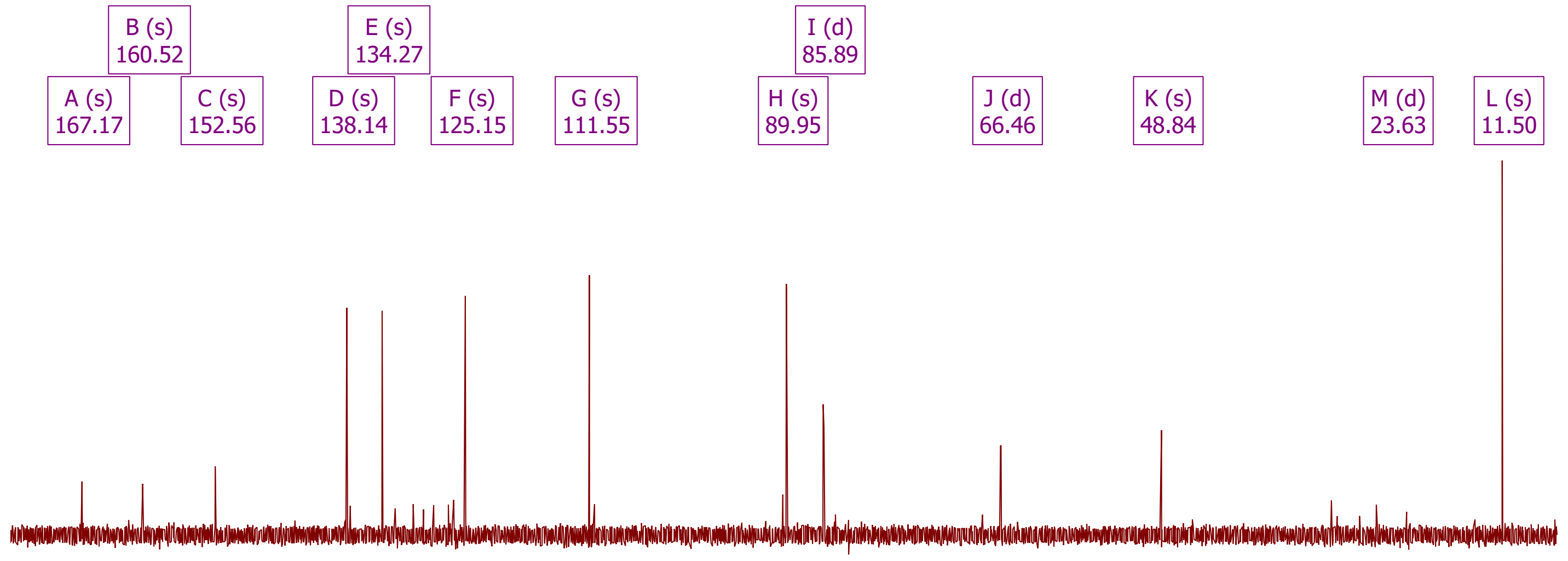

\begin{tabular}{|c|c|c|c|c|c|c|c|c|c|c|c|c|c|c|c|}
\hline 170 & 160 & 150 & 140 & 130 & 120 & 110 & 100 & $\begin{array}{c}90 \\
\mathrm{f} 1(\mathrm{ppm})\end{array}$ & 80 & 70 & 60 & 50 & 40 & 30 & 20 \\
\hline
\end{tabular}




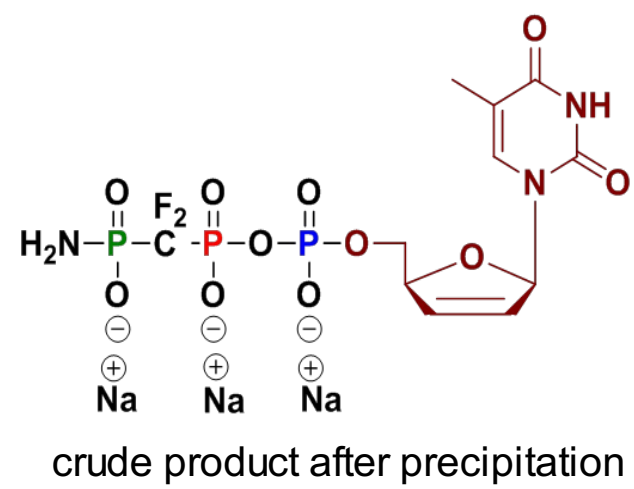




\section{${ }^{1} \mathrm{H}$ NMR}

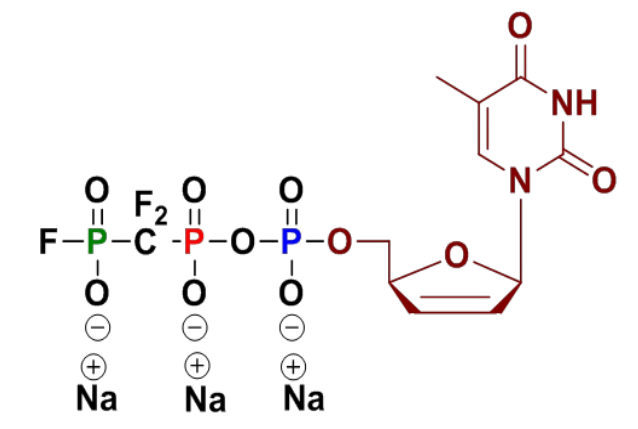

crude product after precipitation

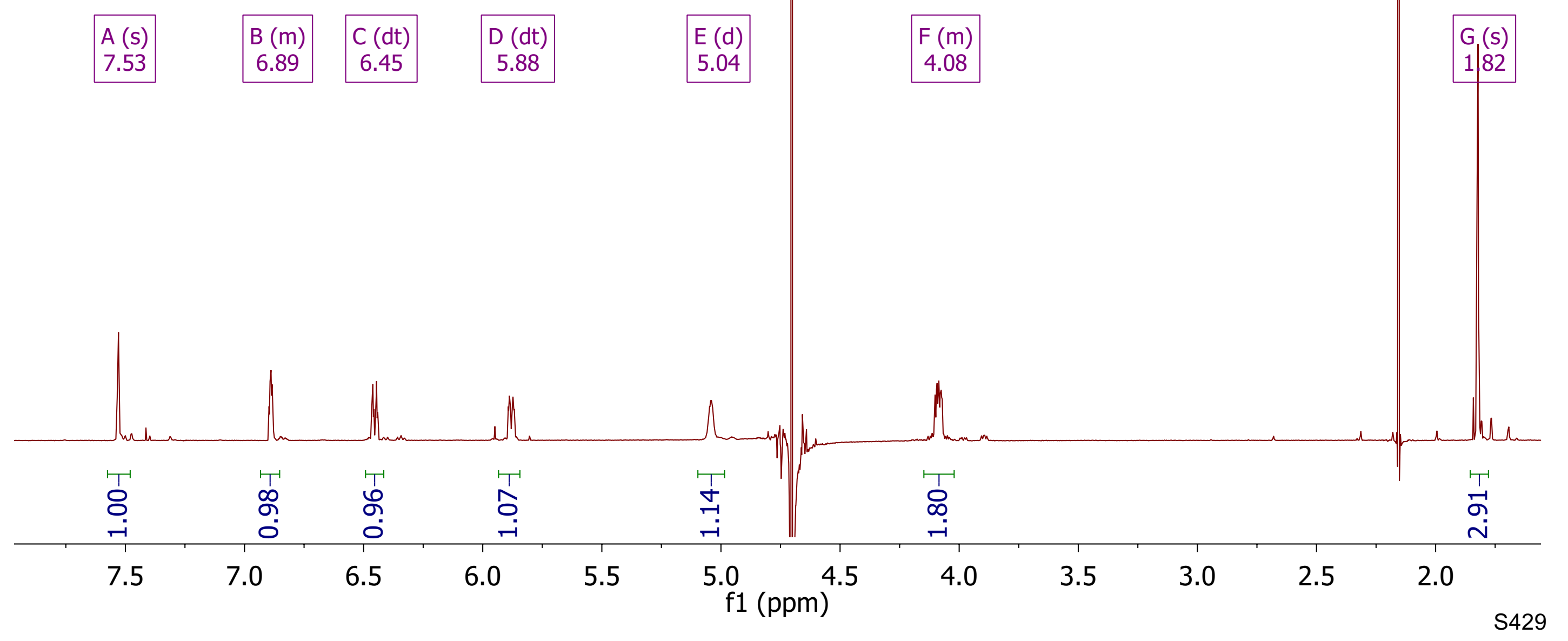




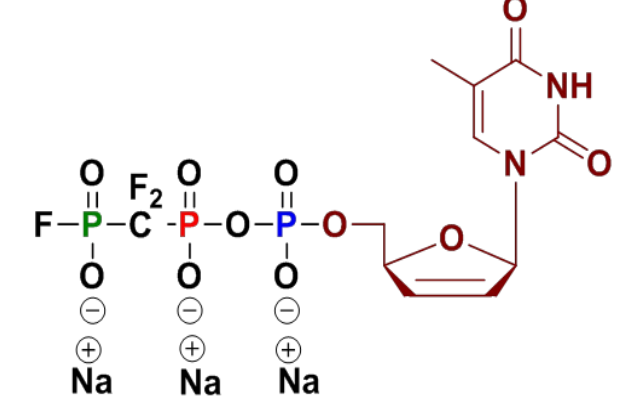

\begin{tabular}{l|l|} 
crude product after precipitation & $A(m)$ \\
1.13
\end{tabular}

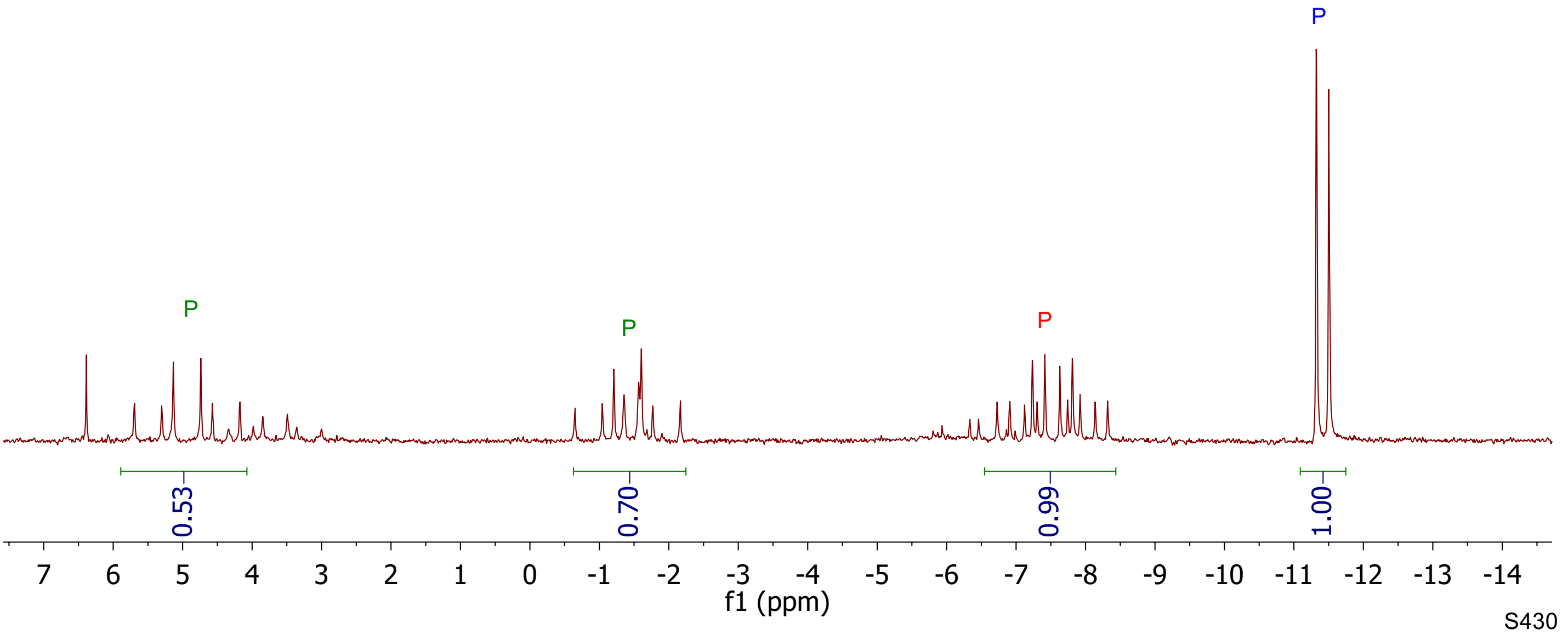




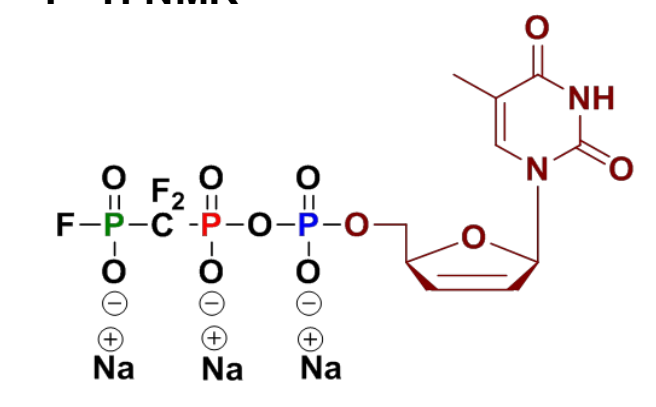

crude product after precipitation

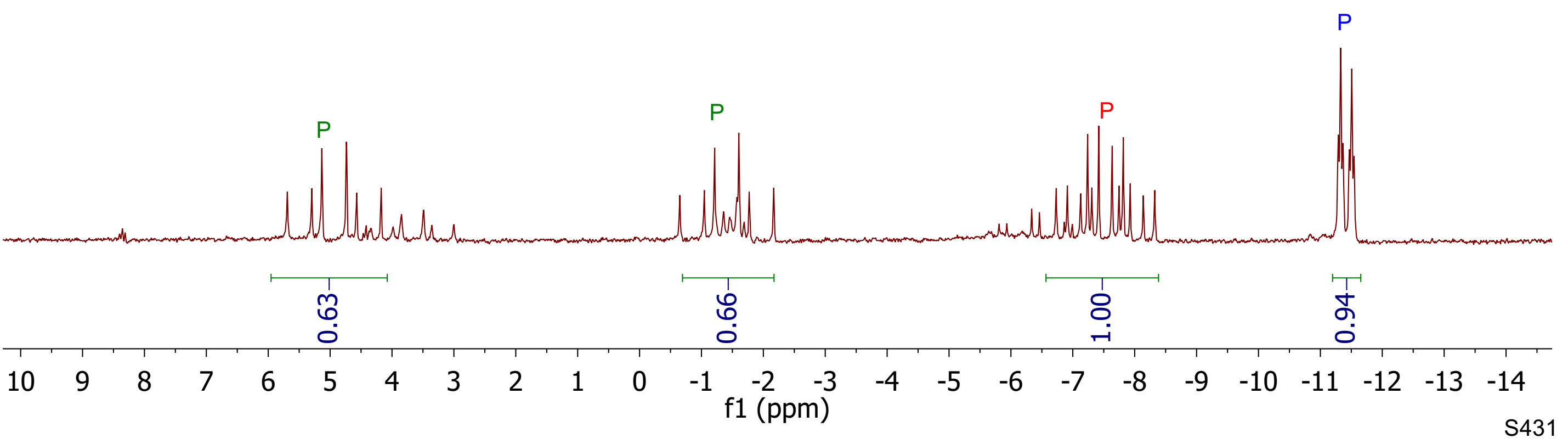




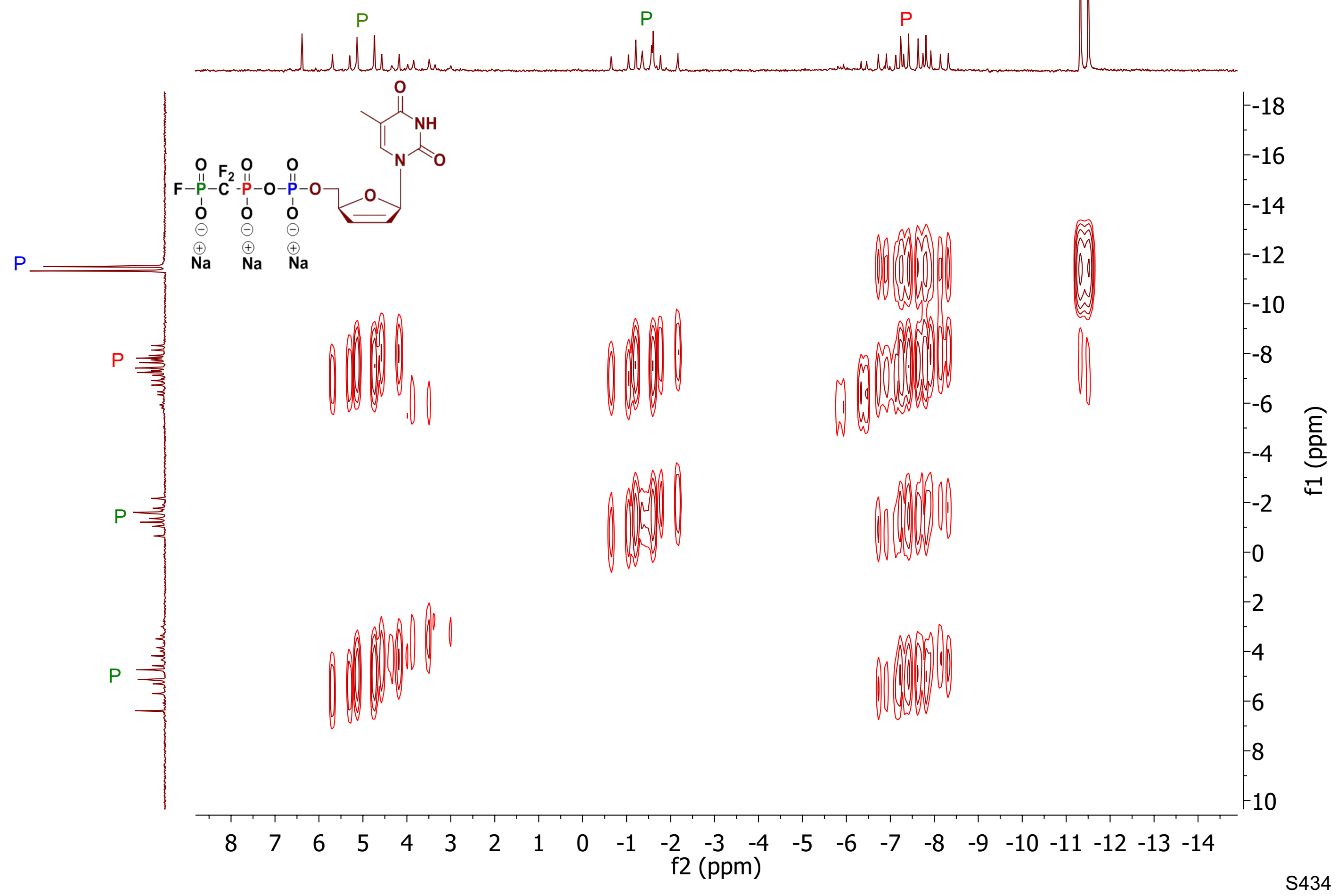




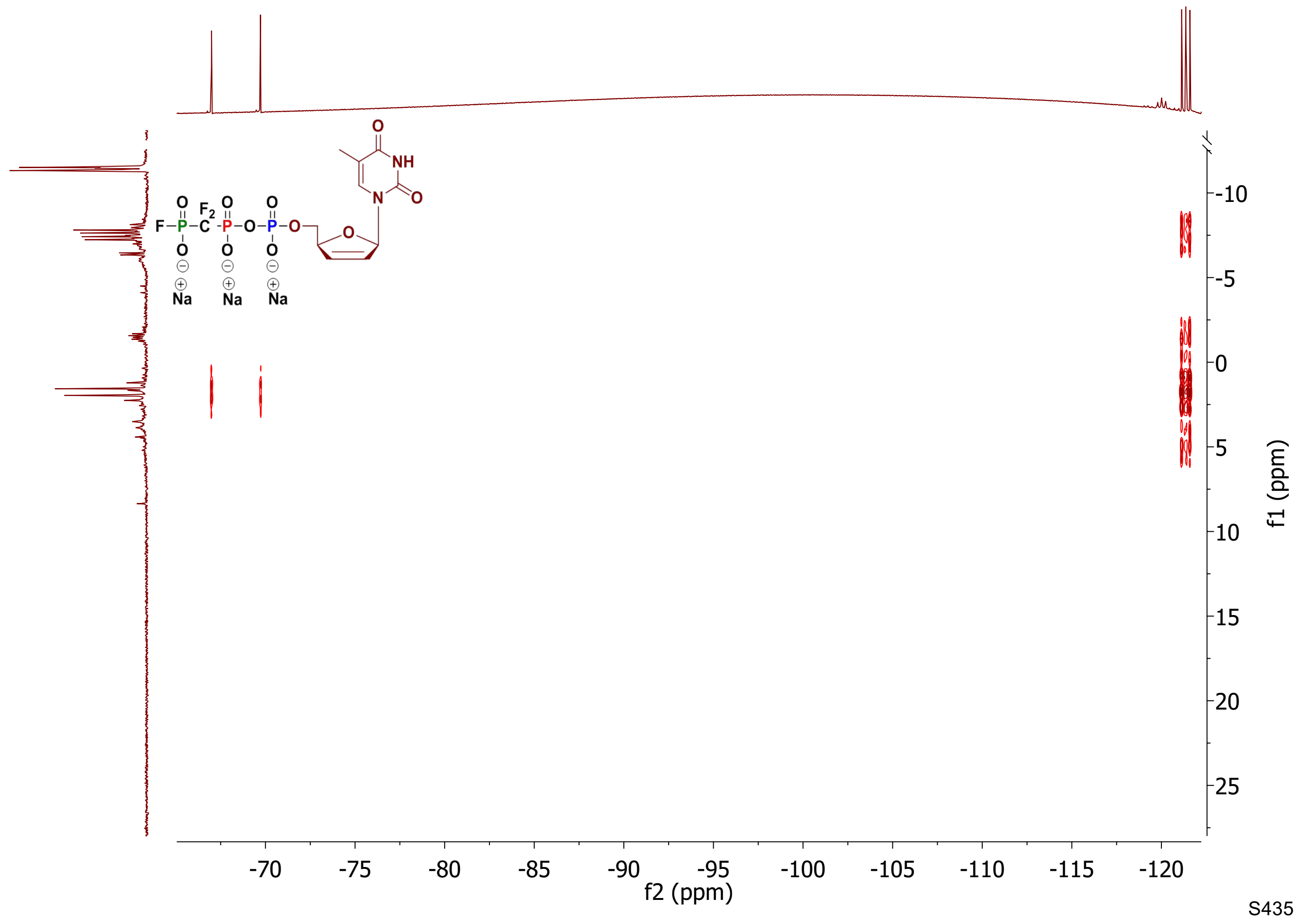


${ }^{13} \mathrm{C} . \mathrm{NMR}$

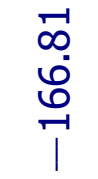

กัก

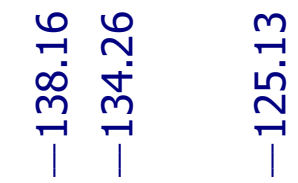

독

รั ๙ู ฒ

ஓ ம்

ตู

ตั

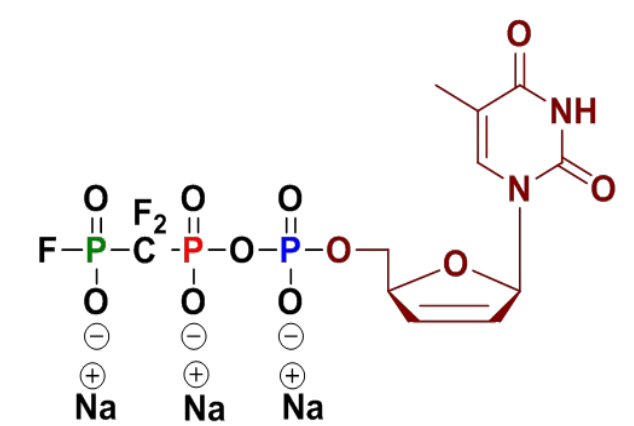

$\mathrm{Na} \mathrm{Na} \mathrm{Na}$

crude product after precipitation

\begin{tabular}{|c|c|c|c|c|c|c|}
\hline \multirow[b]{2}{*}{$\begin{array}{c}\text { B (s) } \\
166.81\end{array}$} & \multirow[b]{2}{*}{$\begin{array}{c}C(s) \\
152.33\end{array}$} & $\begin{array}{c}E(s) \\
134.26\end{array}$ & & & $\begin{array}{c}I(d) \\
85.88\end{array}$ & \\
\hline & & $\begin{array}{c}D(s) \\
138.16\end{array}$ & $\begin{array}{c}F(s) \\
125.13\end{array}$ & $\begin{array}{c}G(s) \\
111.51\end{array}$ & $\begin{array}{l}H(s) \\
89.99\end{array}$ & $\begin{array}{l}J(d) \\
66.60\end{array}$ \\
\hline
\end{tabular}

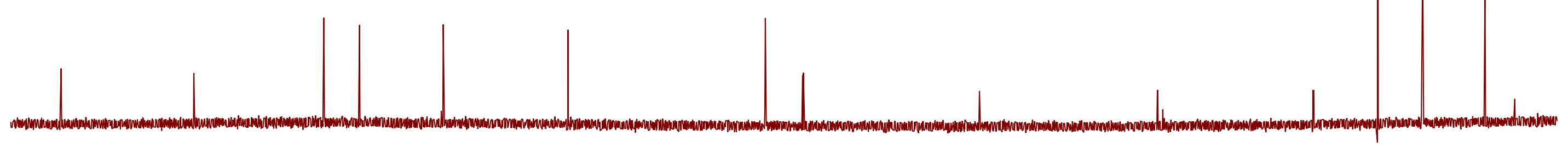

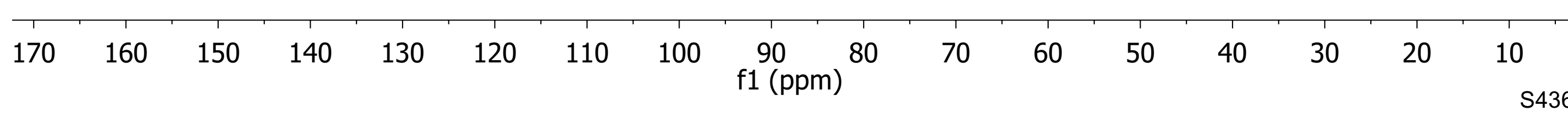




\section{${ }^{1} \mathrm{H}$ NMR}

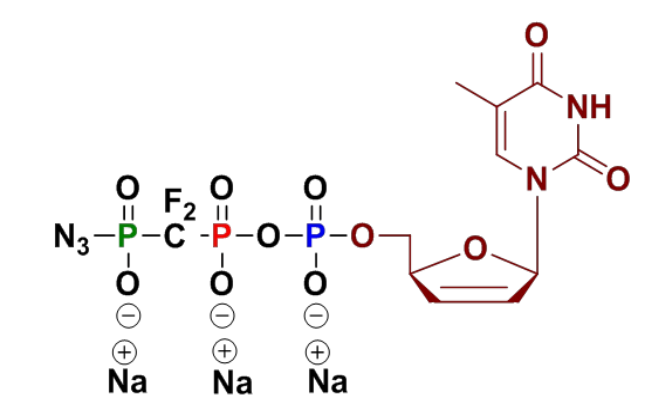

crude product after precipitation

\begin{tabular}{|c|c|c|c|c|c|}
\hline $\begin{array}{l}A(s) \\
7.53\end{array}$ & $\begin{array}{c}B(m) \\
6.89\end{array}$ & $\begin{array}{l}\text { C (d) } \\
6.45\end{array}$ & $\begin{array}{l}\mathrm{D}(\mathrm{d}) \\
5.86\end{array}$ & $\begin{array}{l}E(s) \\
5.03\end{array}$ & $\begin{array}{l}F(m) \\
4.09\end{array}$ \\
\hline
\end{tabular}

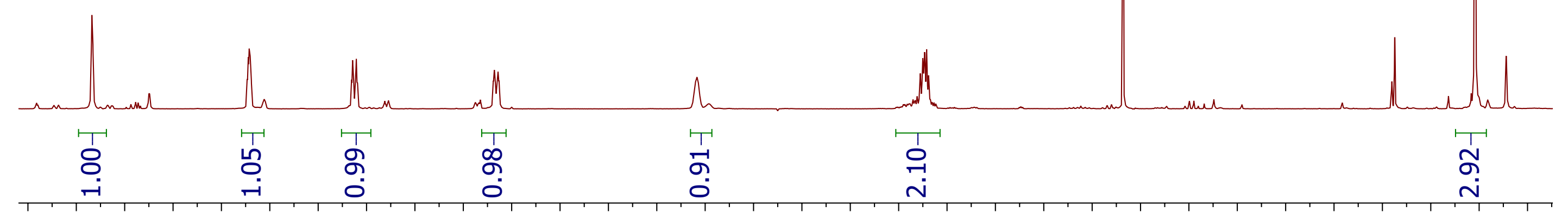

'.8 7.6 7.4 7.2 7.0 6.86 .66 .46 .26 .05 .85 .65 .45 .25 .04 .84 .64 .44 .24 .03 .83 .63 .43 .23 .02 .82 .62 .42 .22 .01 .81 .6 f1 (ppm) 


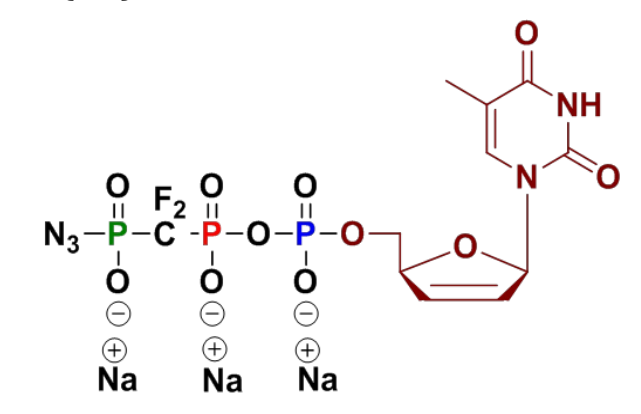

crude product after precipitation

$$
\begin{array}{|l|}
\hline B(m) \\
-7.24 \\
\hline
\end{array}
$$

P

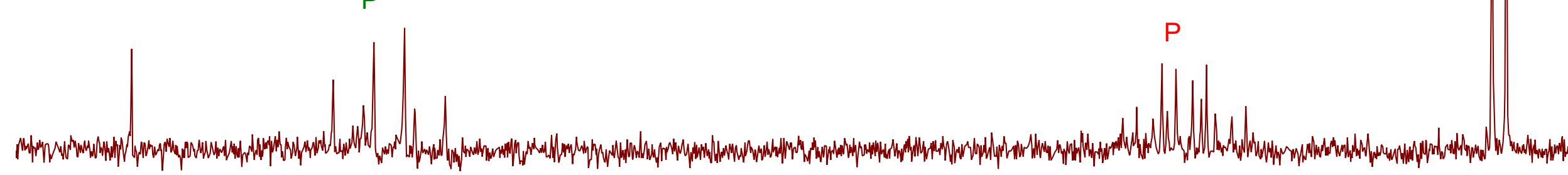

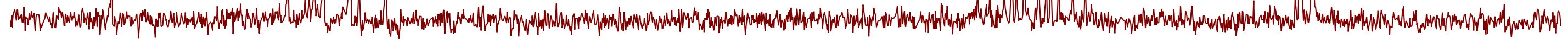

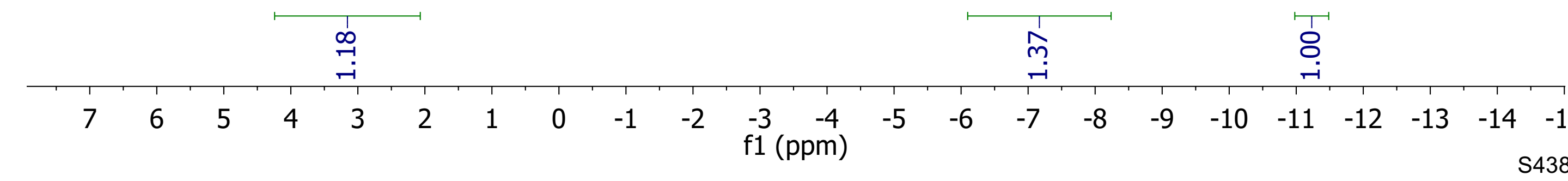


${ }^{19} \mathrm{~F} \mathrm{NMR}$

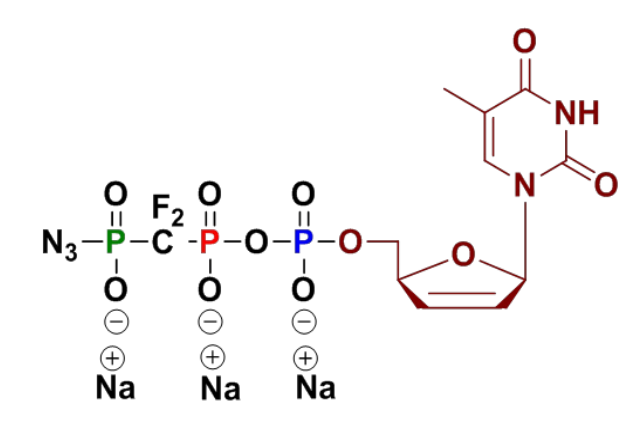

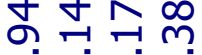

$\infty$ 의 $\sigma$

가국

crude product after precipitation

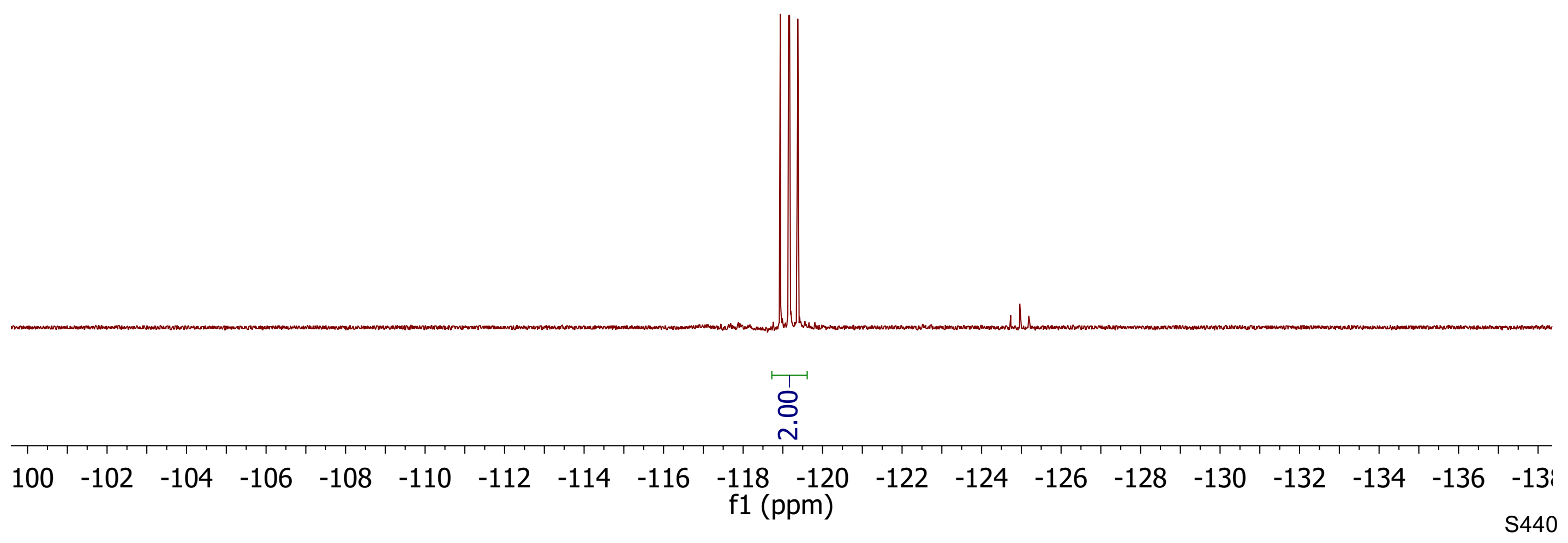


quantat. in reaction mixture

$$
\begin{aligned}
& B(t d) \\
& -4.94
\end{aligned}
$$

\section{C (d)} $-12.34$

$P$

P

P 


\section{${ }^{31} \mathrm{P}$ NMR}

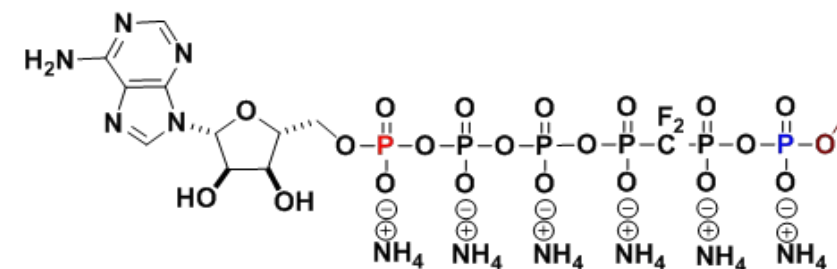

product purified by SAX

$C(\mathrm{~m})$

$-6.66$

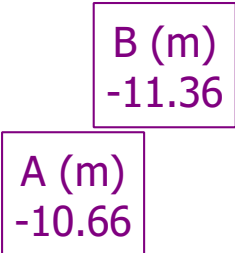

- P-CF $-\mathrm{P}$ -

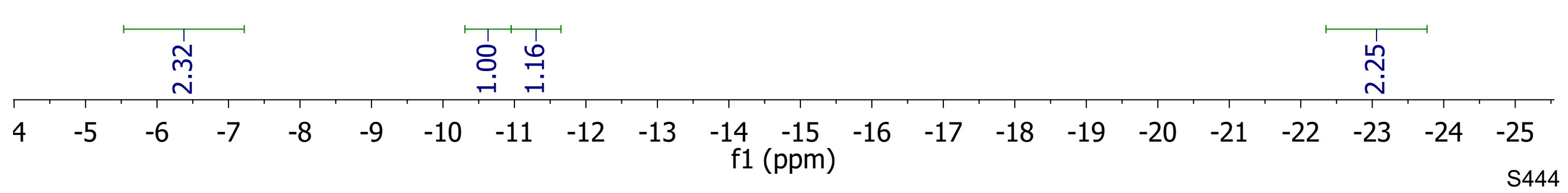




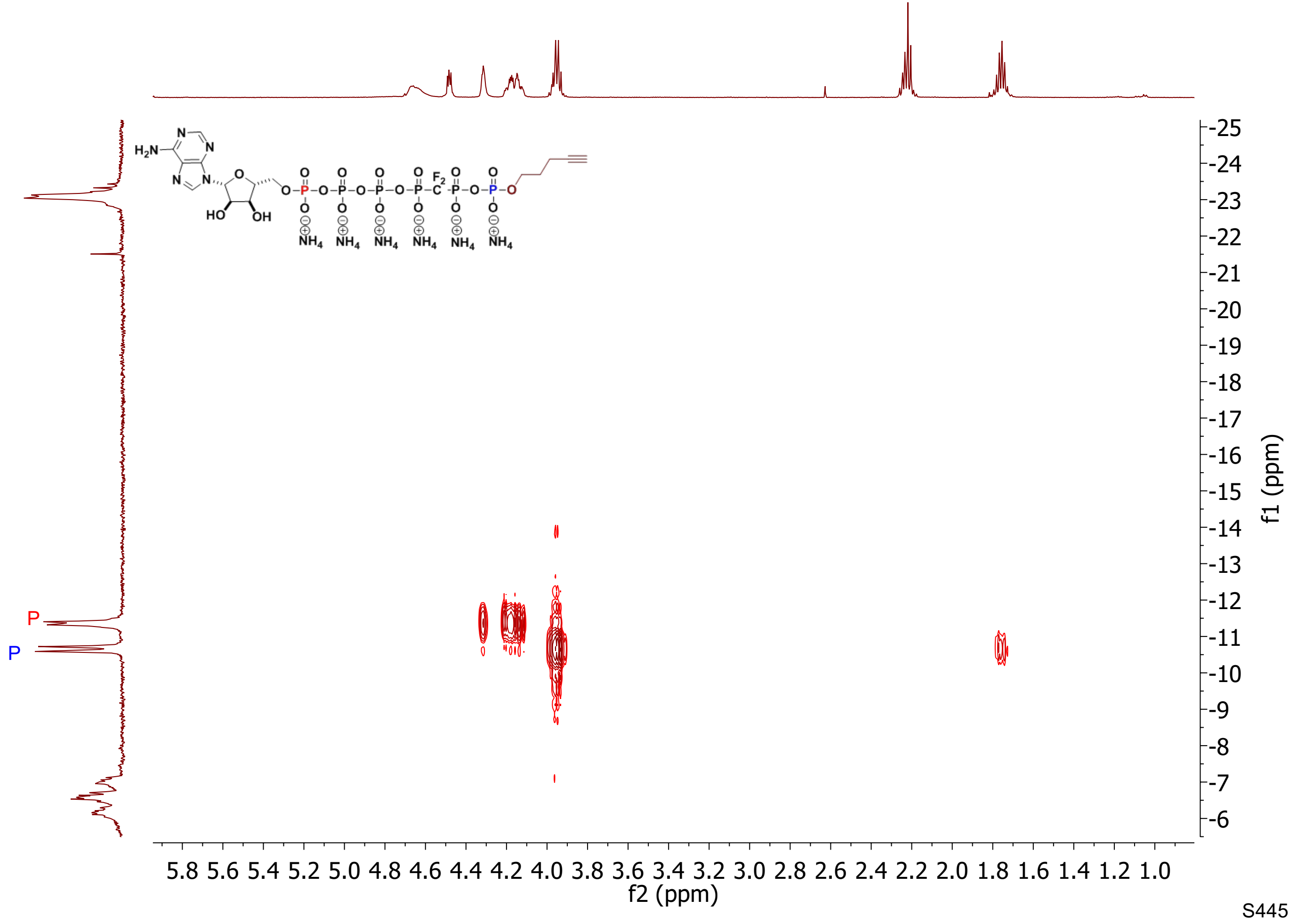




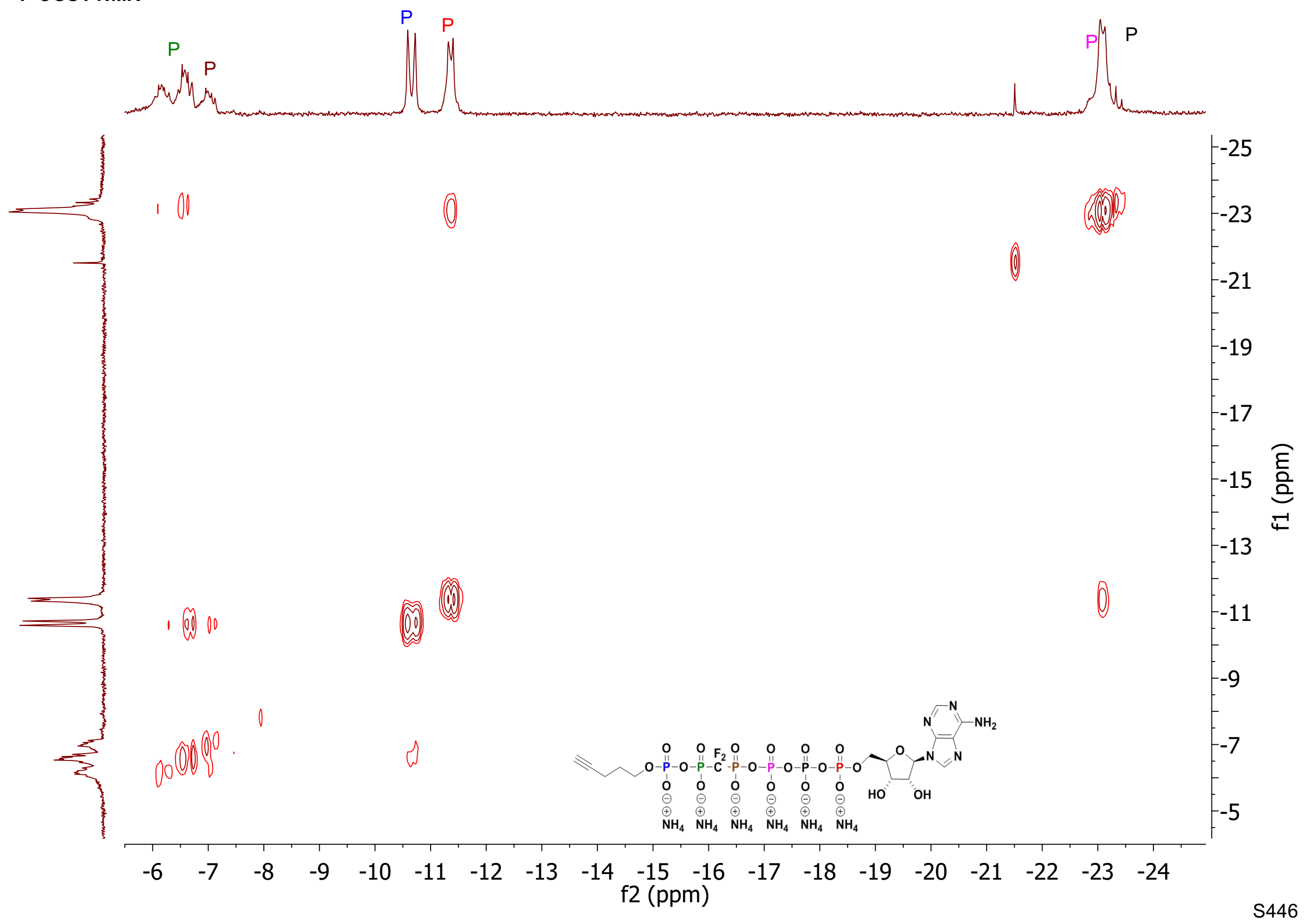




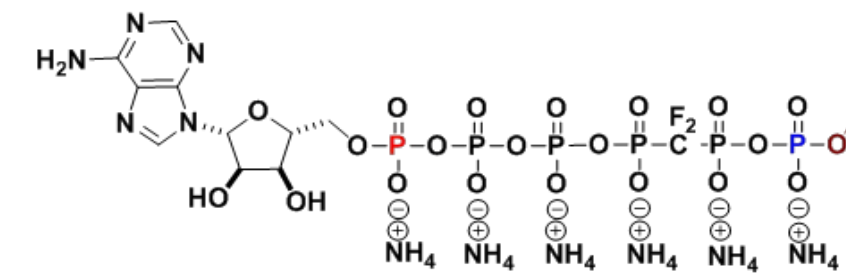

product purified by SAX
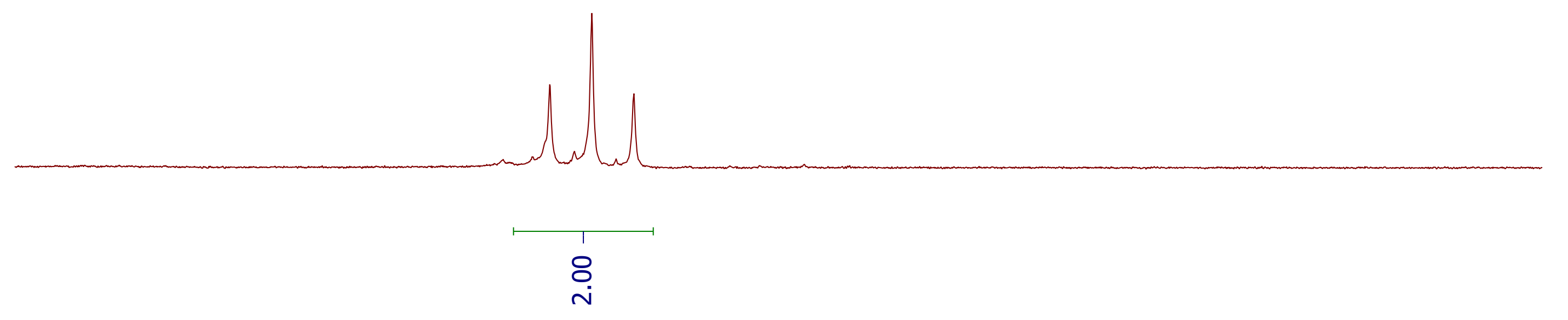

$\begin{array}{lllll}-118.5 & -119.0 & -119.5 & -120.0 & -120.5\end{array}$

$-121.0-121.5$

f1 (ppm)

$-122.0 \quad-122.5$

$-123.0$

$-123.5$

$-124.0$

-12
$S 447$ 


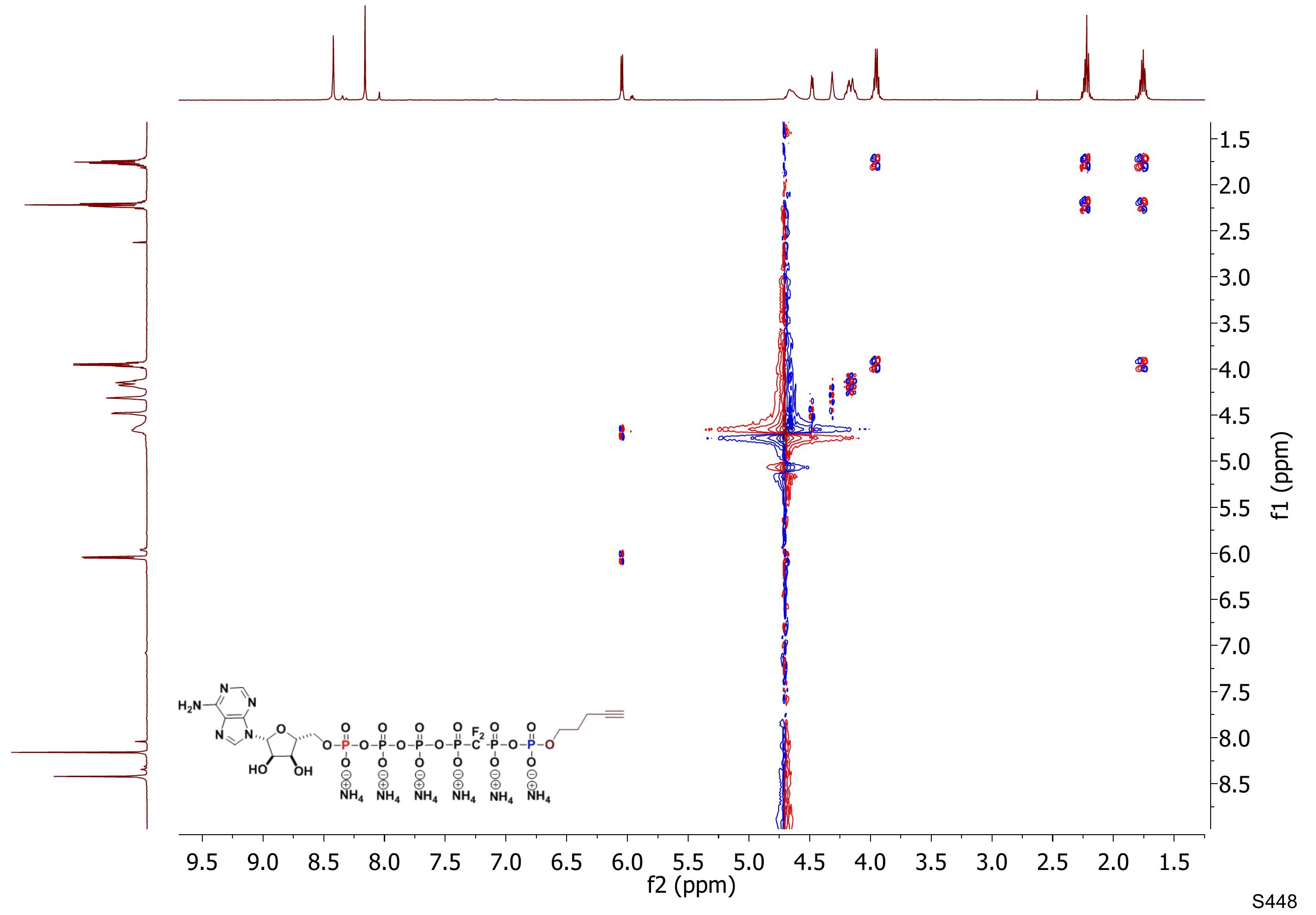




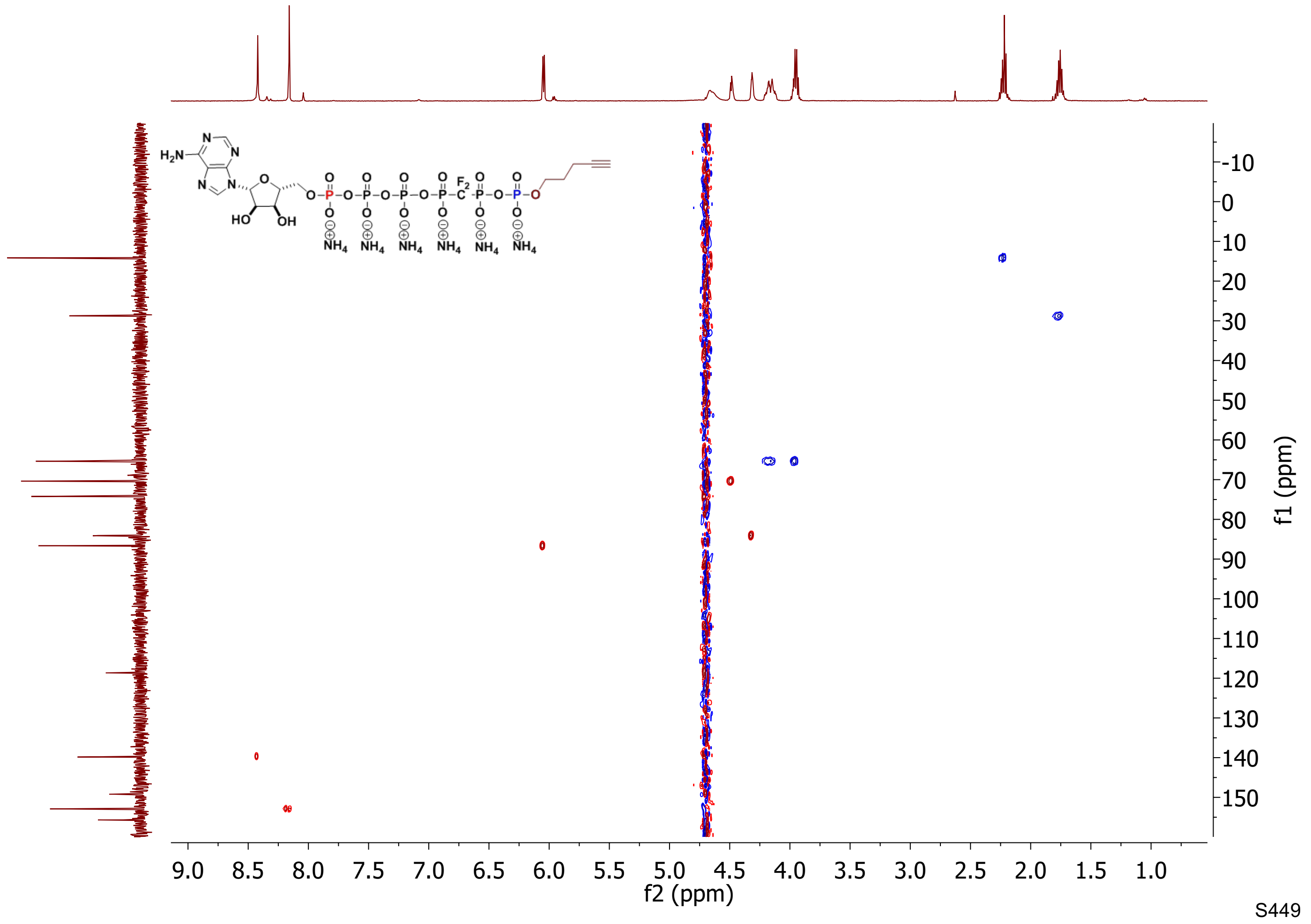


${ }^{13} \mathrm{C} \quad \mathrm{NMR}$

œ 뭉 유 ํํㅇํำ ํํำ

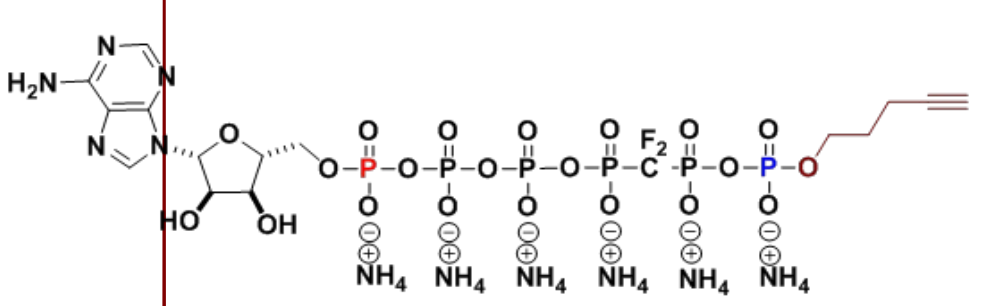
product purified by SAX
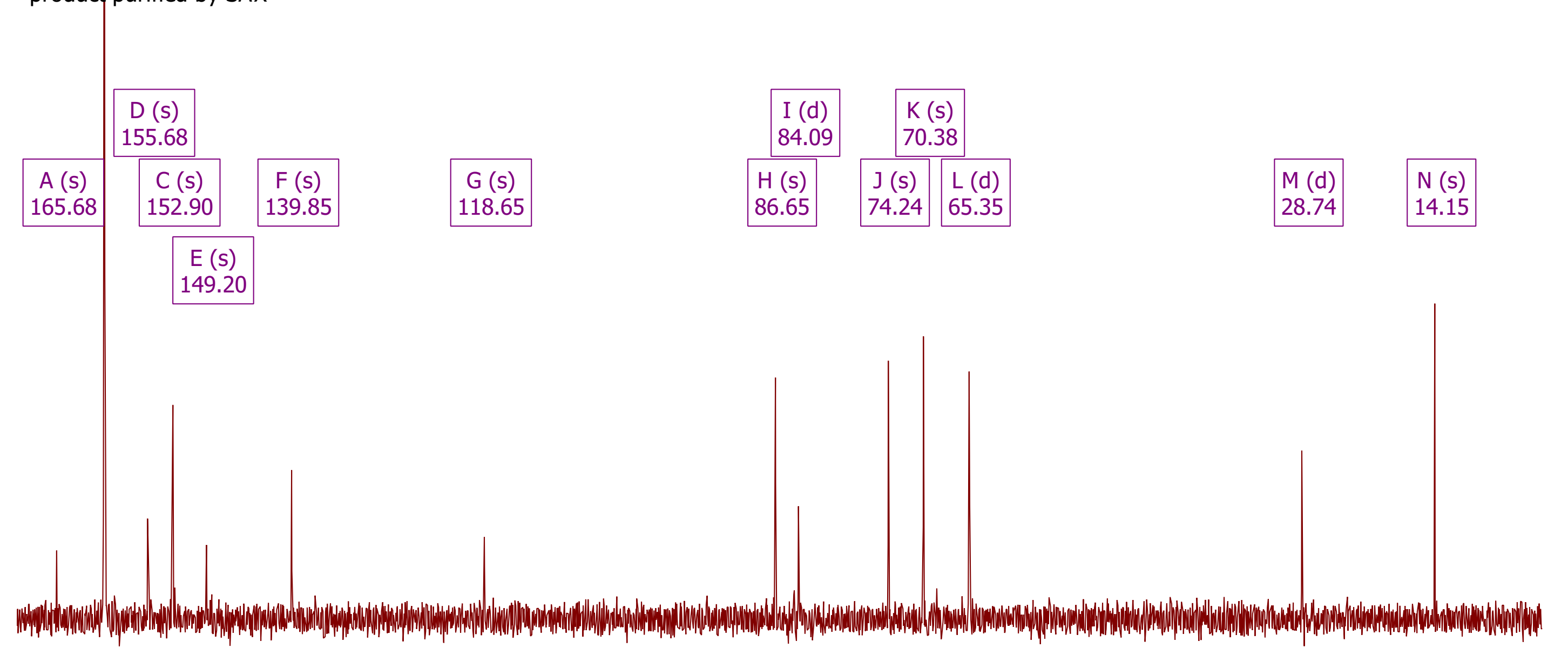

$\begin{array}{lllllllllllllllll}70 & 160 & 150 & 140 & 130 & 120 & 110 & 100 & 90 & 80 & 70 & 60 & 50 & 40 & 30 & 20 & 10\end{array}$




\section{Synthesis of triphosphates based}

on c-Py ${ }_{\mathrm{CC} 2} \mathrm{PA}\left(\mathrm{A}_{4}\right)$

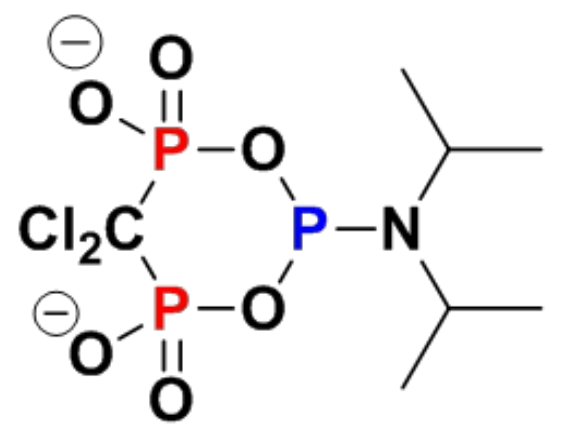



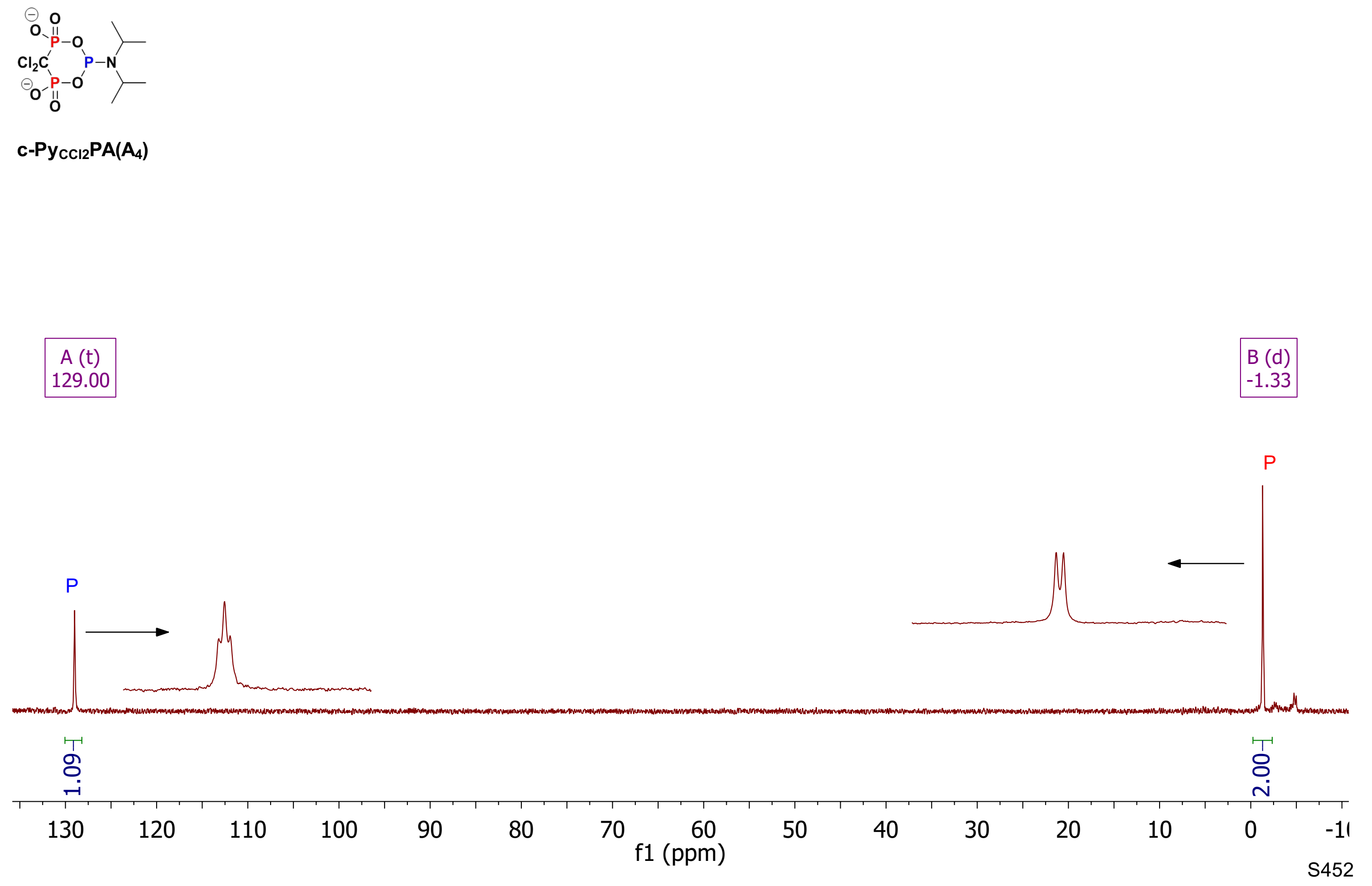

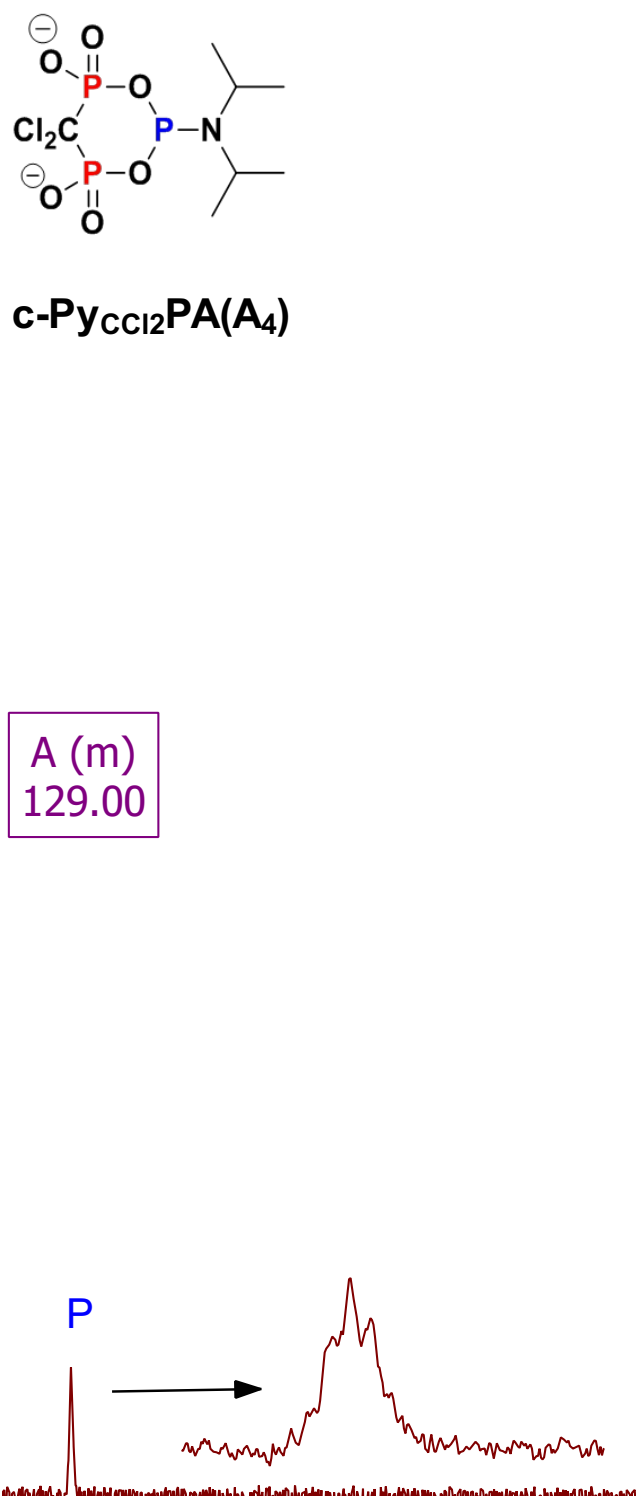

(1)

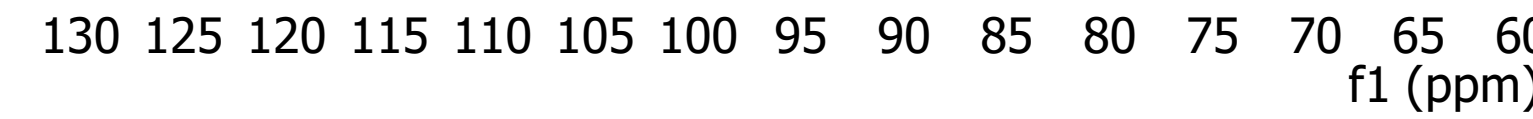

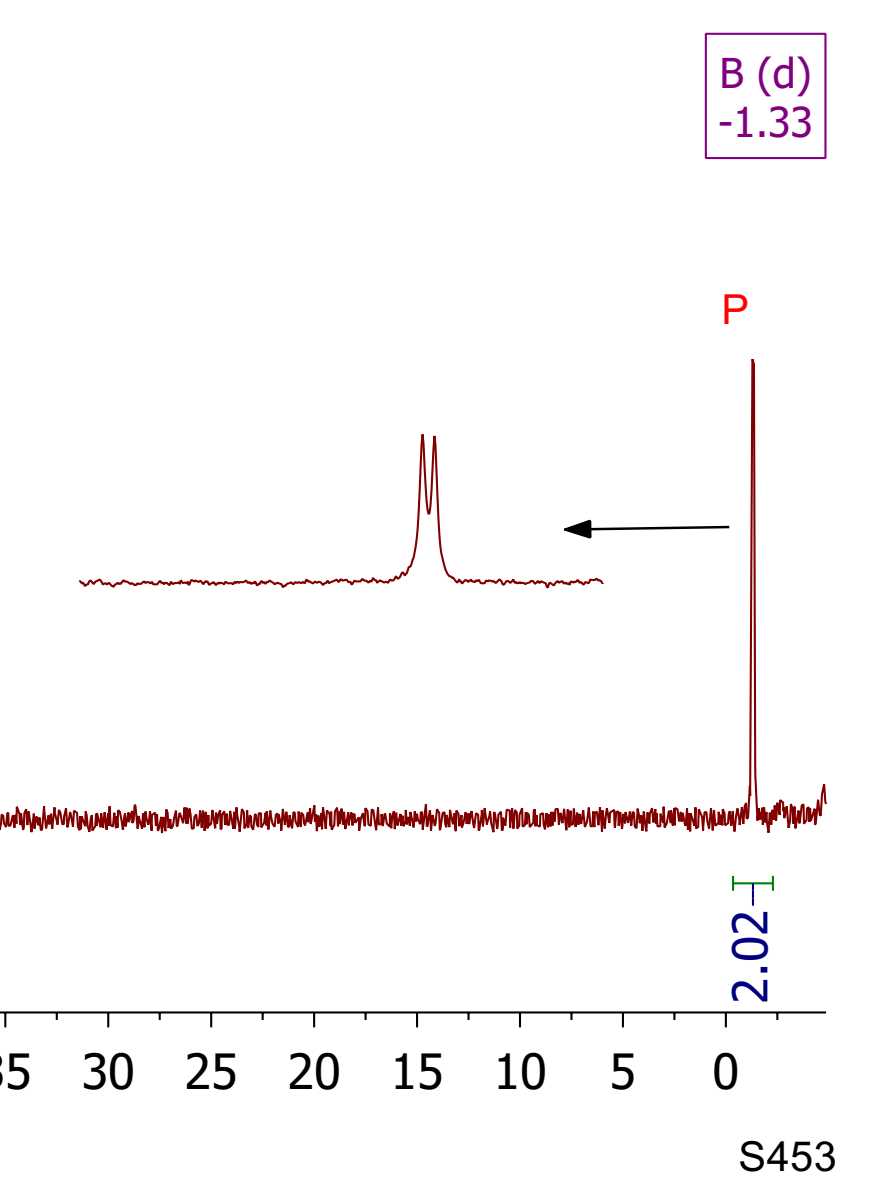




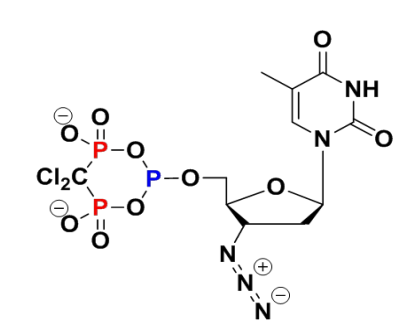

\section{1) Coupling}
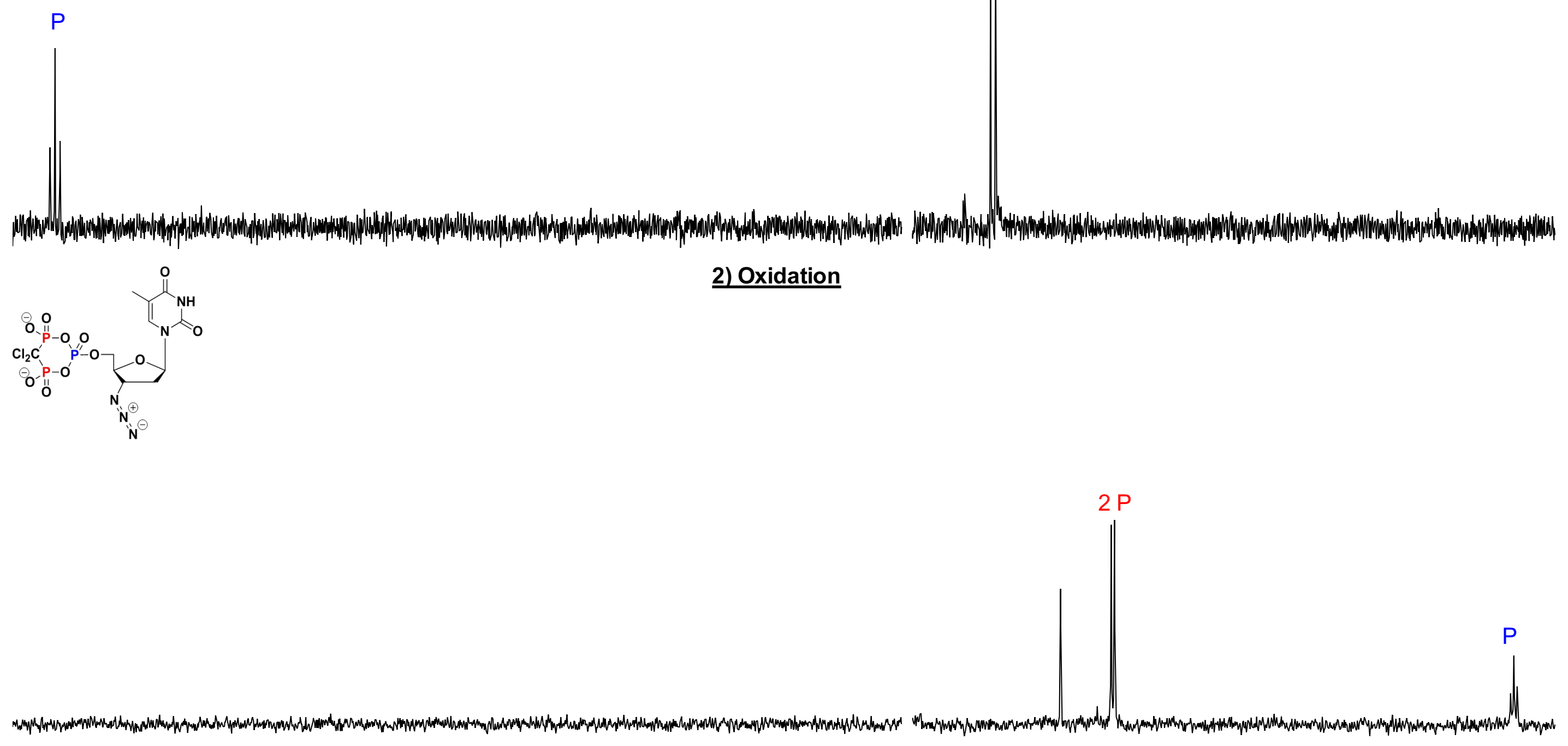

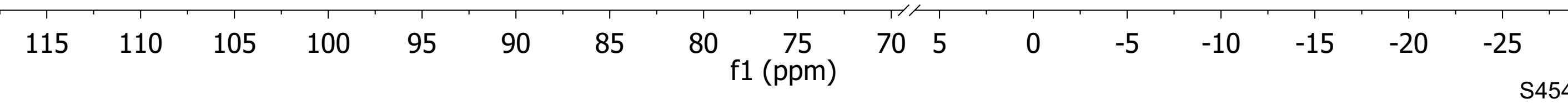




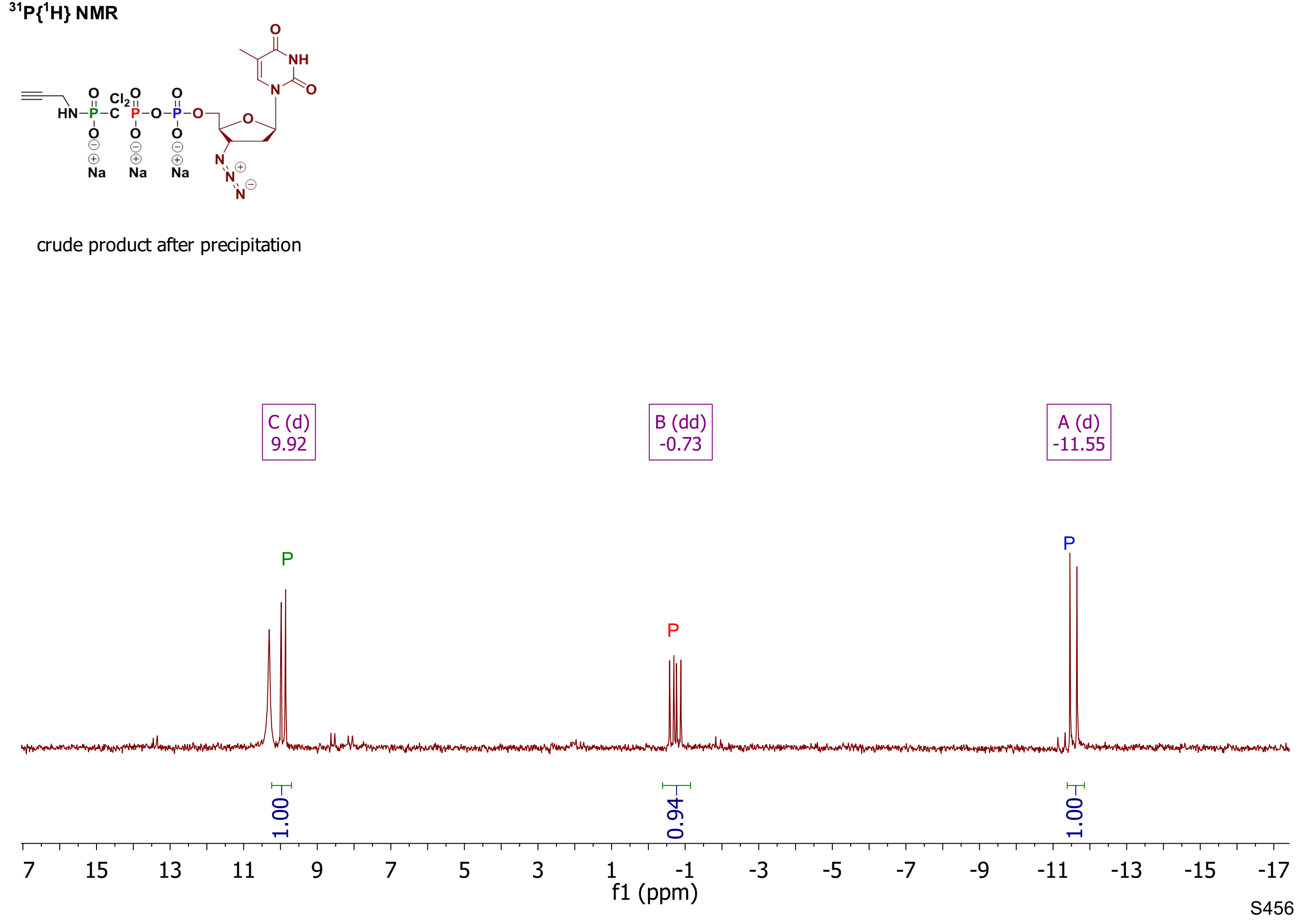




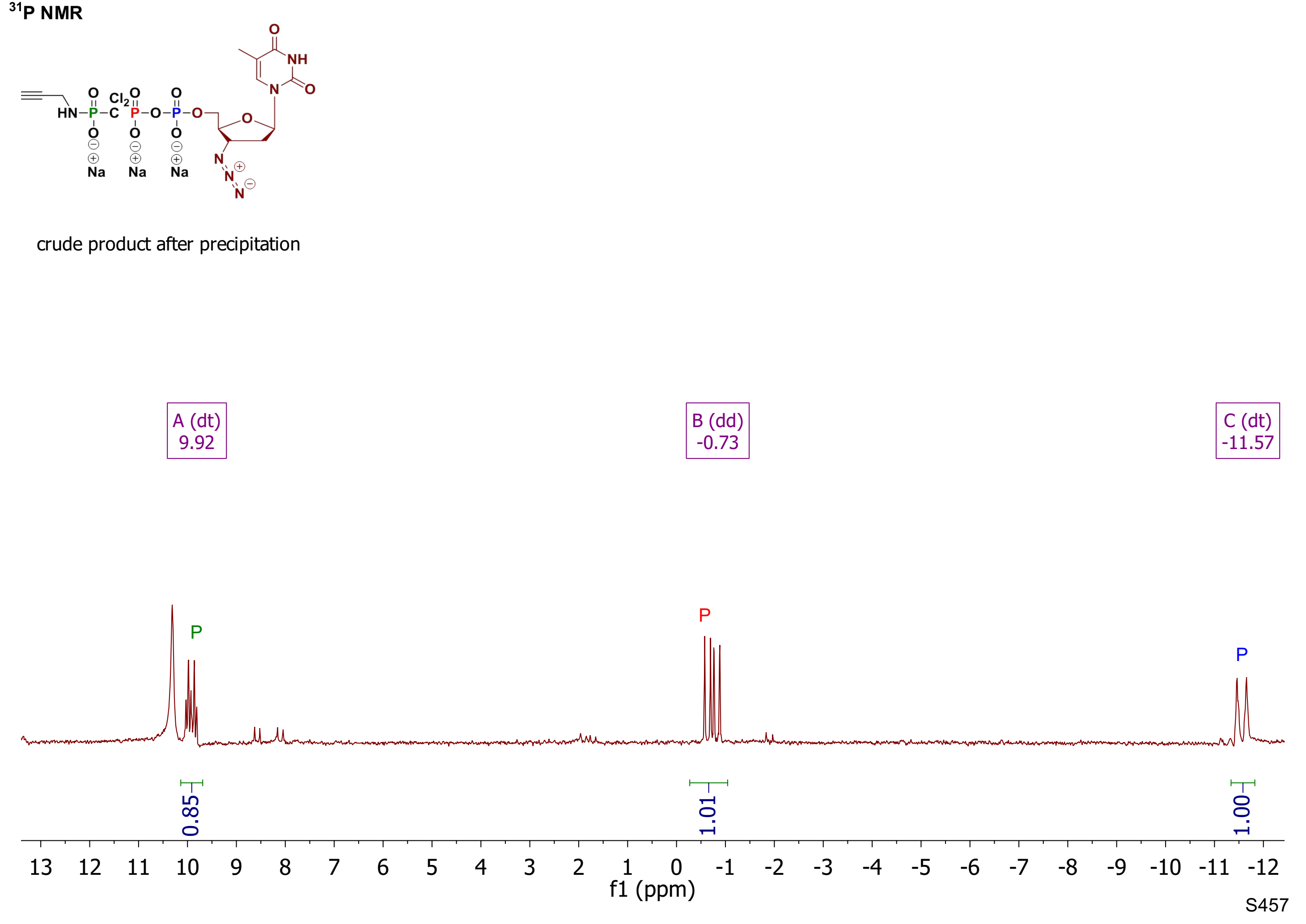




\section{Synthesis of triphosphates based}

on c-Py $\mathbf{N H}_{\mathrm{NH}} \mathrm{PA}\left(\mathbf{A}_{5}\right)$

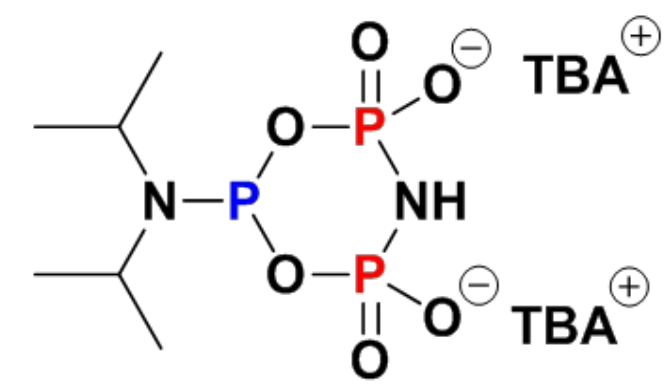




$$
{ }^{31} \mathrm{P}\left\{{ }^{1} \mathrm{H}\right\} \mathrm{NMR}
$$

The $\mathrm{CPy}_{\mathrm{NH}} \mathrm{PA}$ was not stable inside the nmr tube

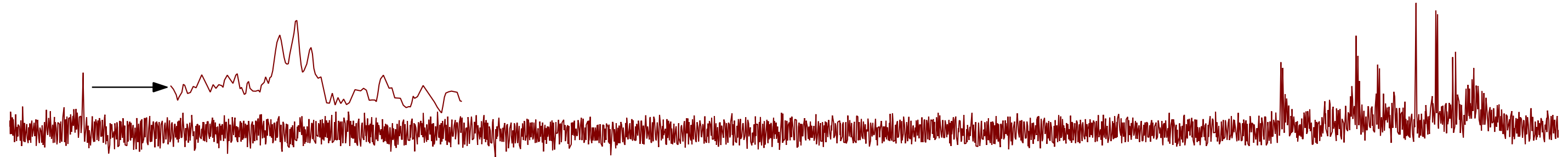

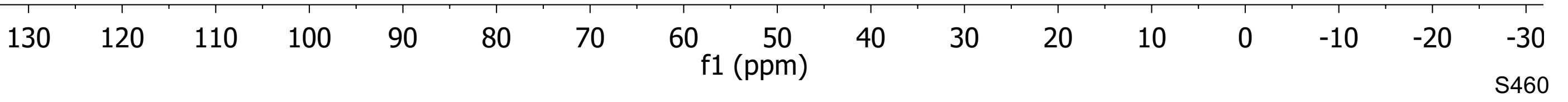




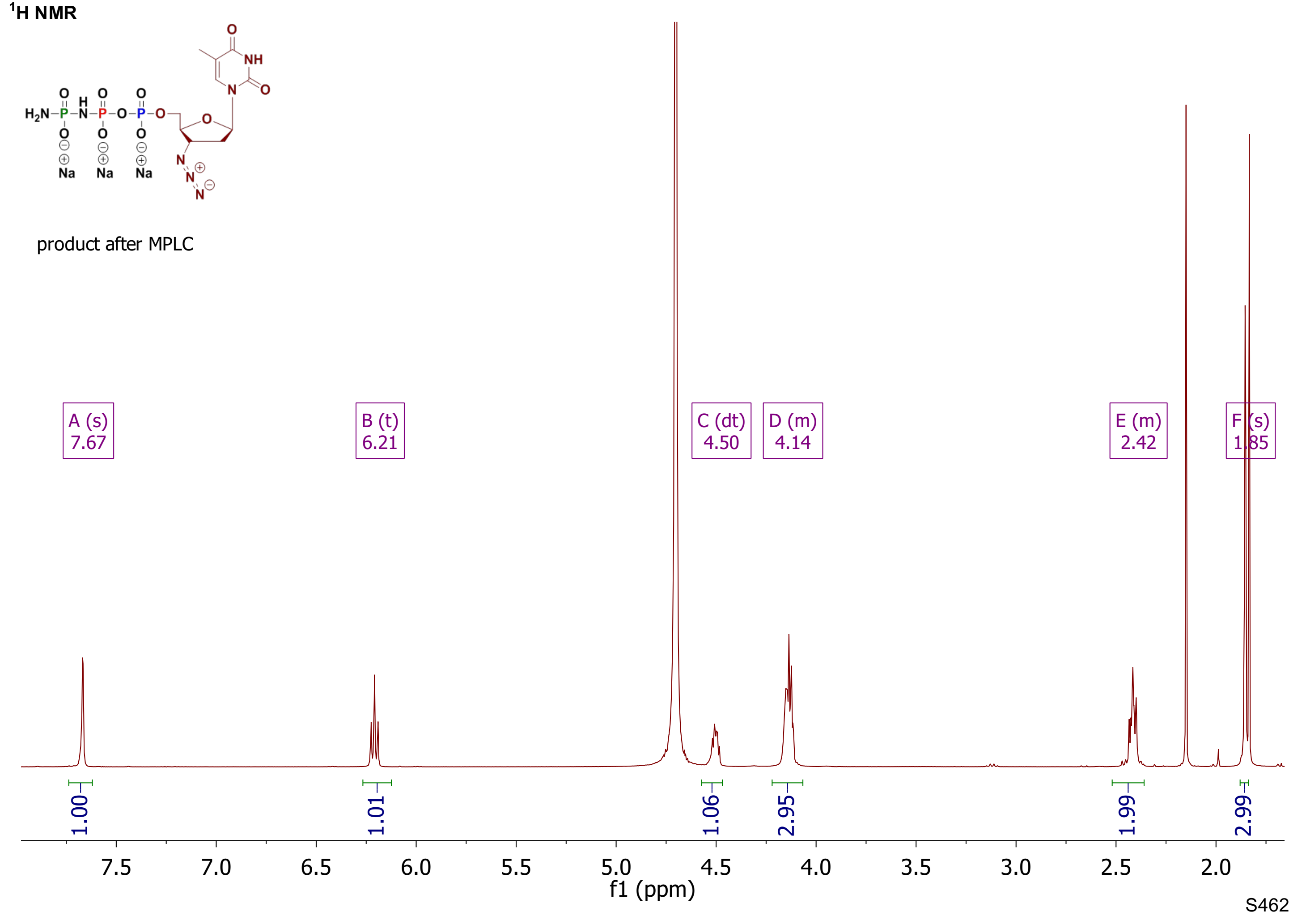




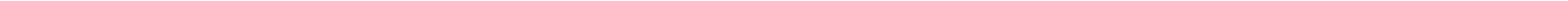




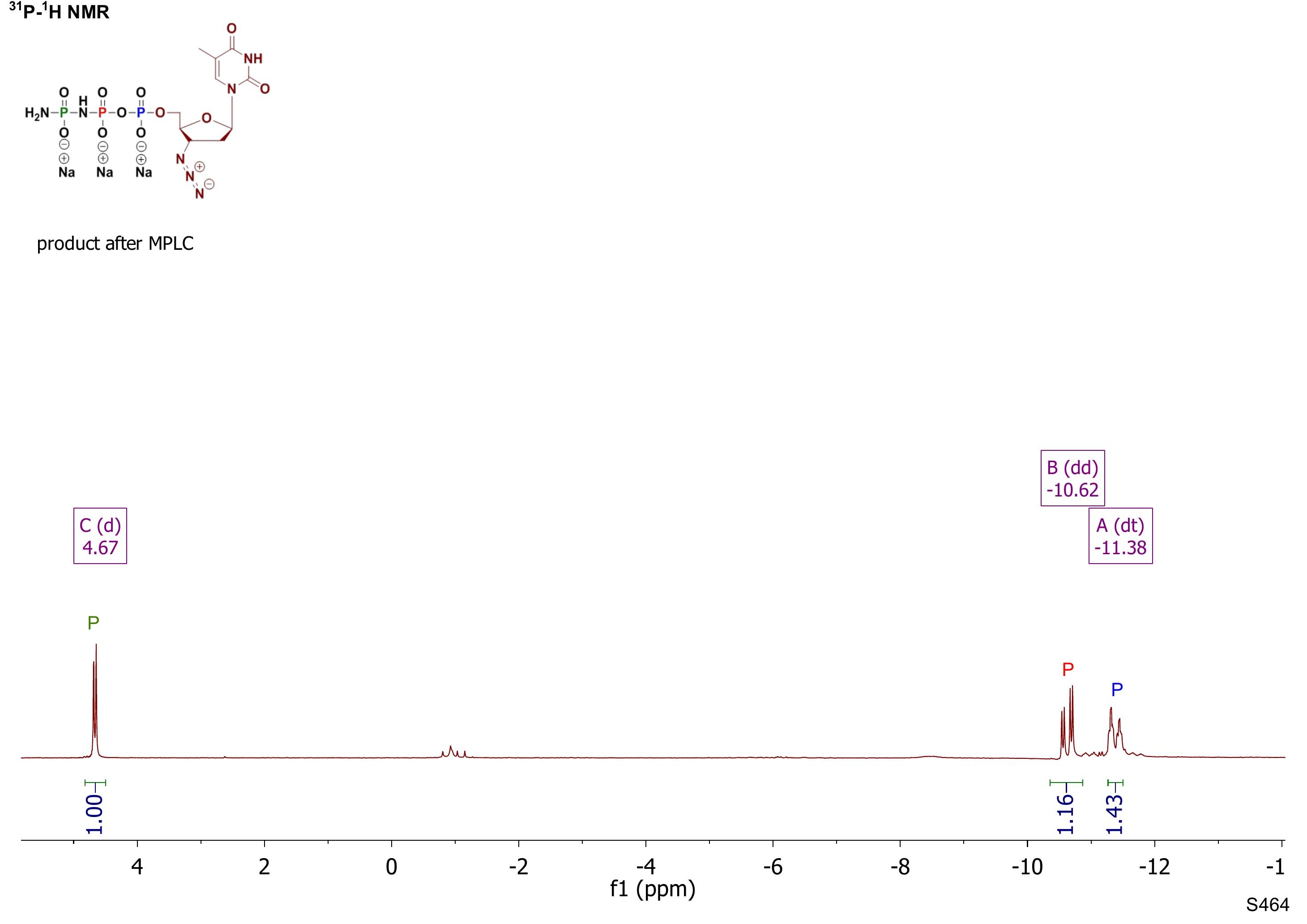




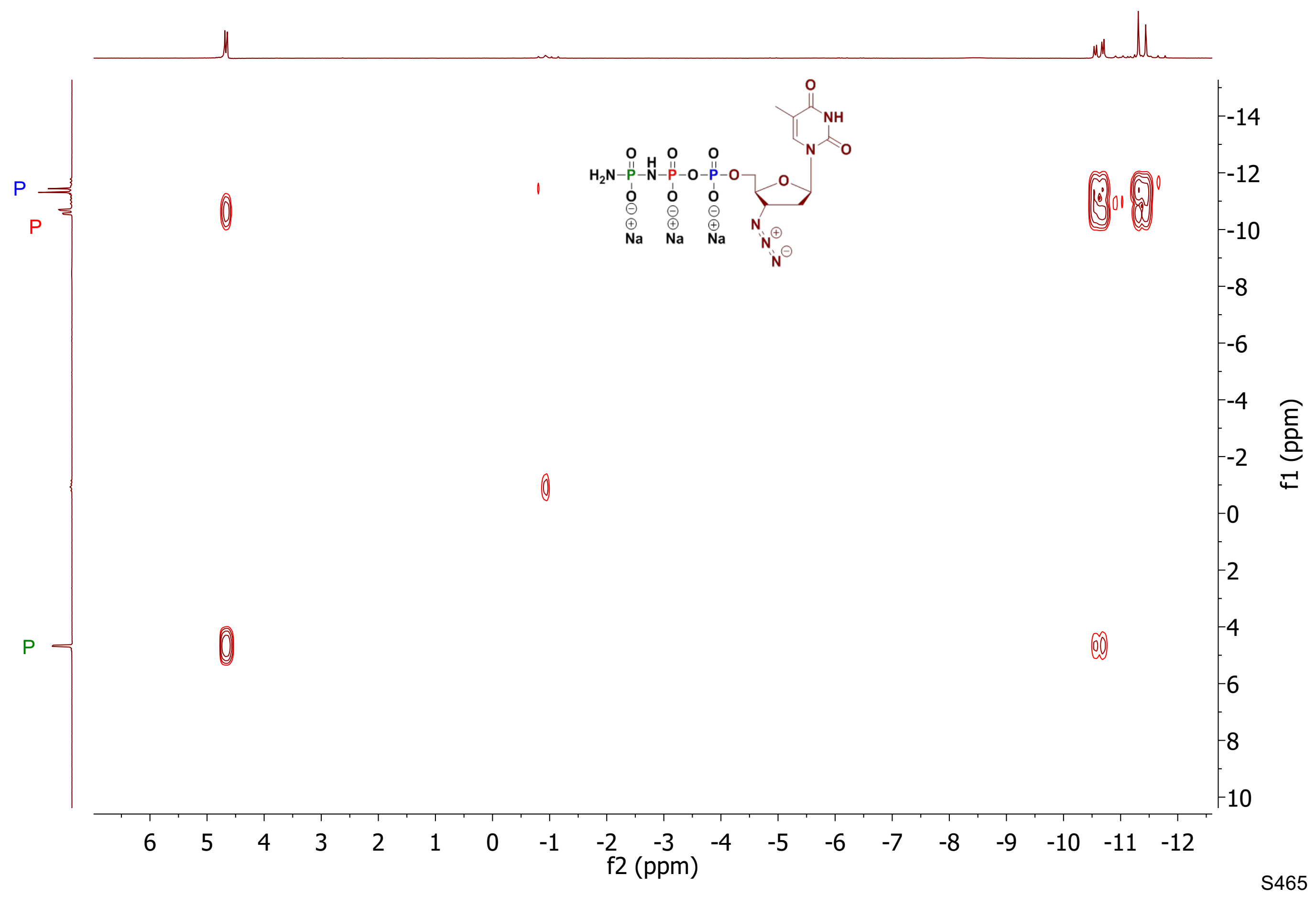




\section{${ }^{31} \mathrm{P}\left\{{ }^{1} \mathrm{H}\right\}$ NMR}

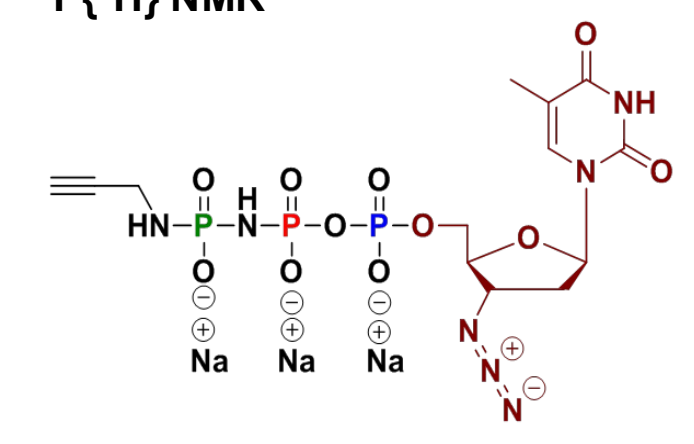

product after MPLC

C (d)

4.05
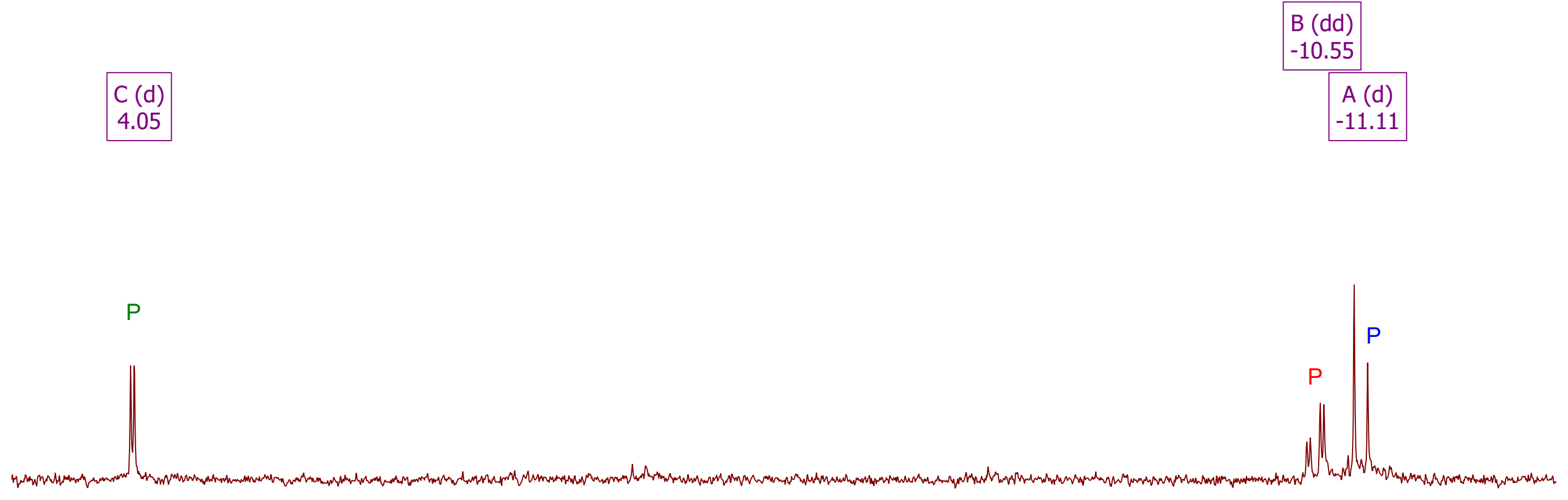

\begin{tabular}{|c|c|c|c|c|c|c|c|c|c|c|c|c|c|c|c|c|c|c|}
\hline & ?1 & & & & & & & & & & & & & & & $\begin{array}{c}1 \\
-1 \\
-1 \\
-1\end{array}$ & & \\
\hline 5 & 4 & 3 & 2 & 1 & 0 & -1 & -2 & -3 & 1 (ppm) & -5 & -6 & -7 & -8 & -9 & -10 & -11 & -12 & -13 \\
\hline
\end{tabular}




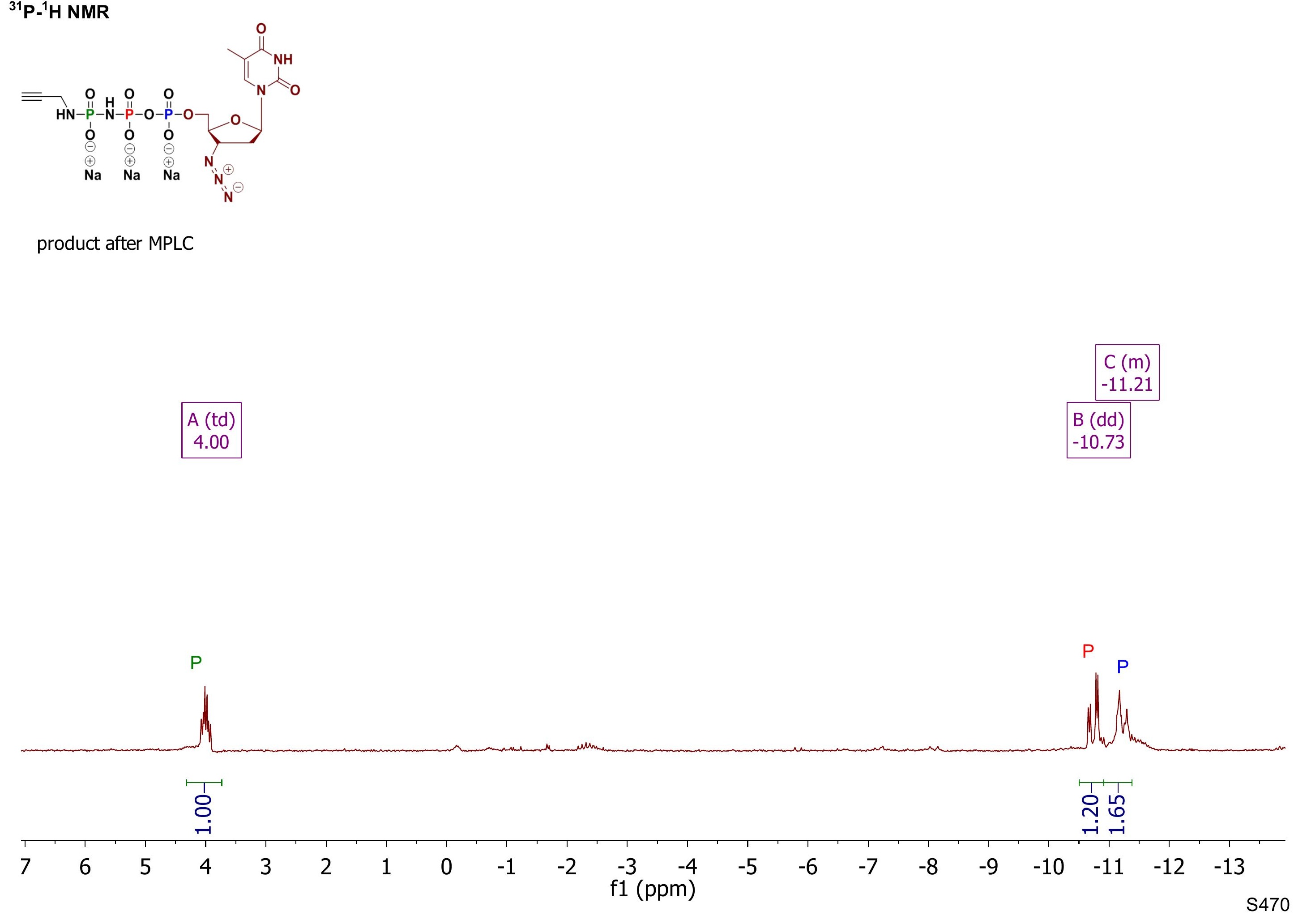




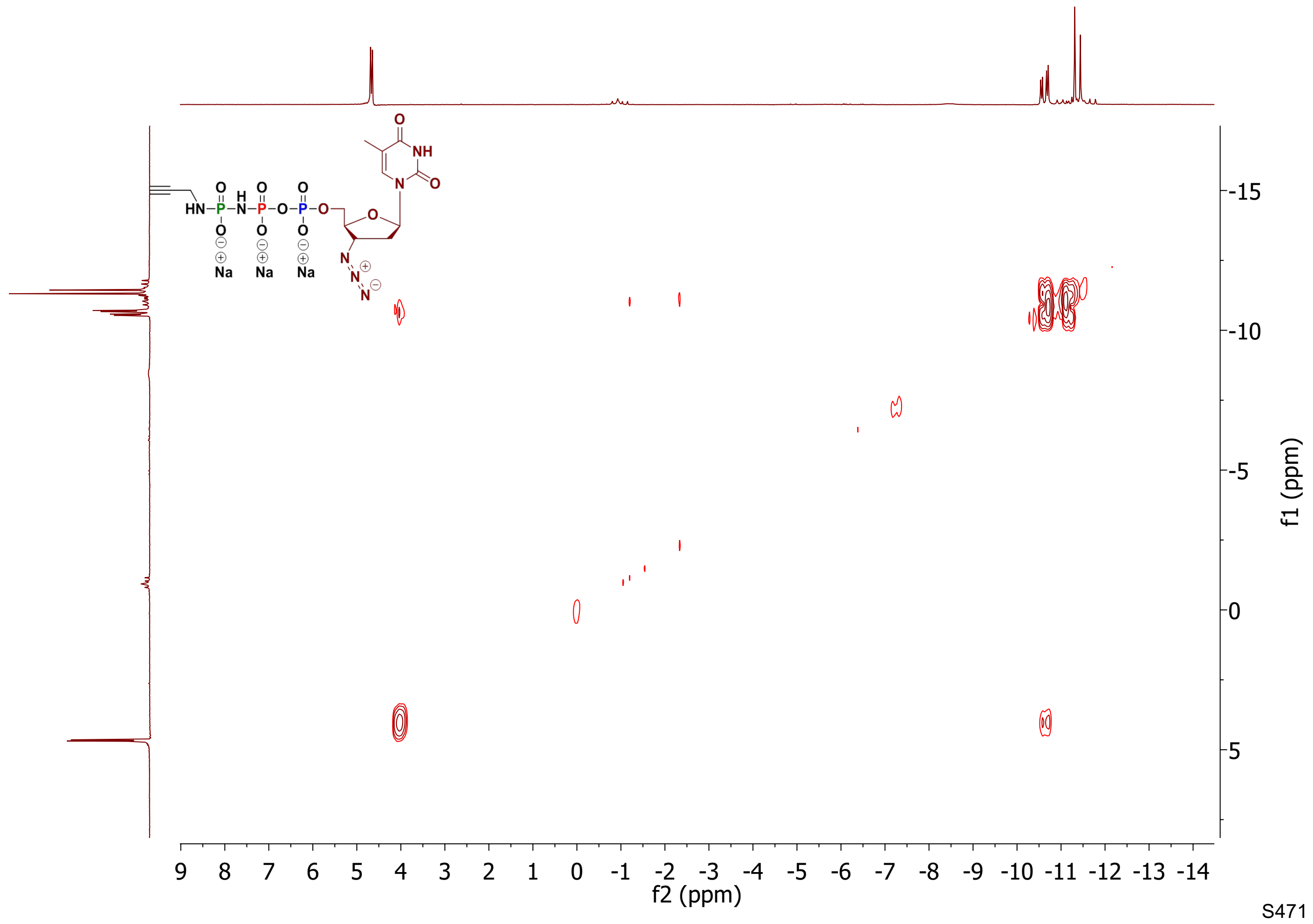




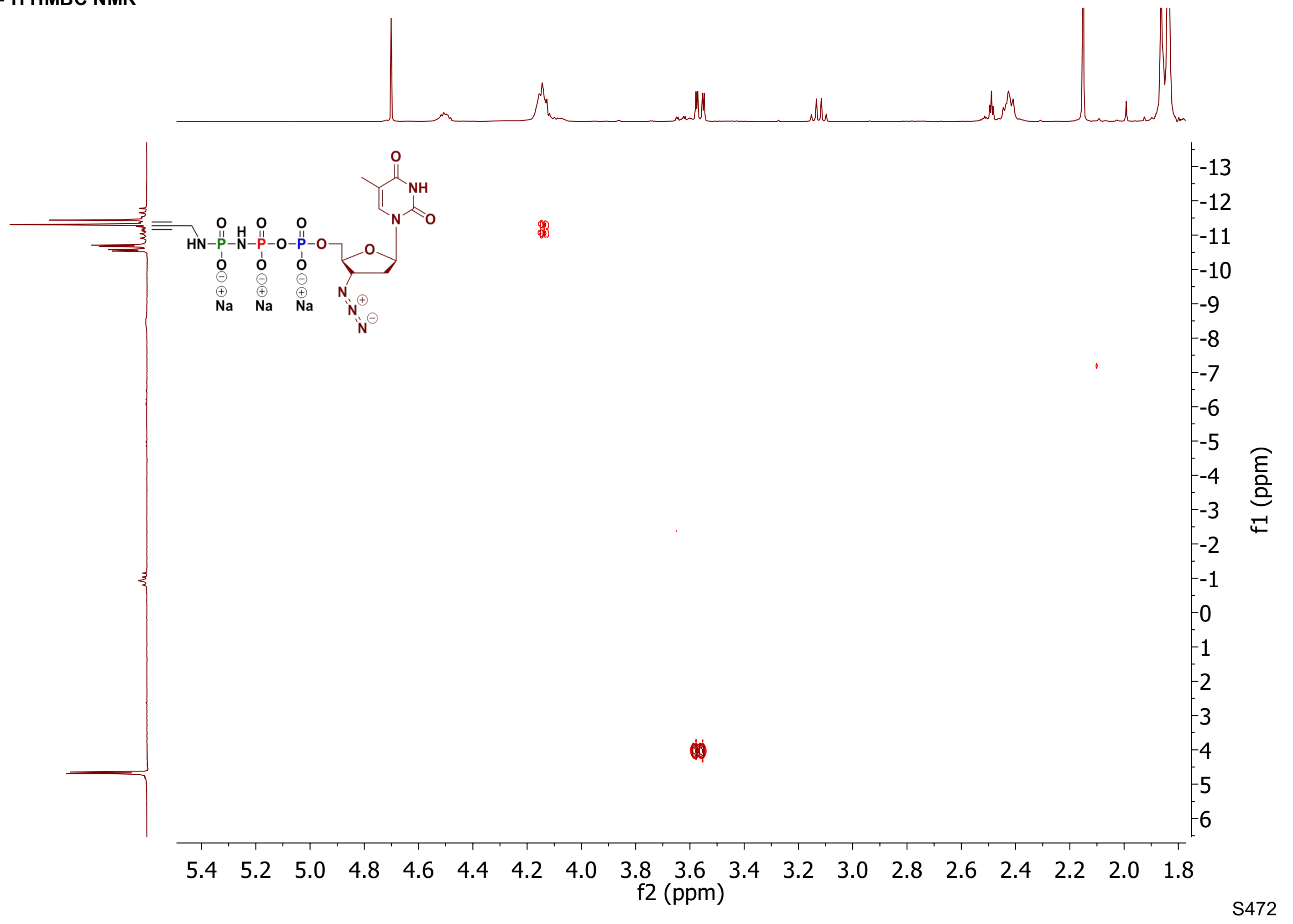




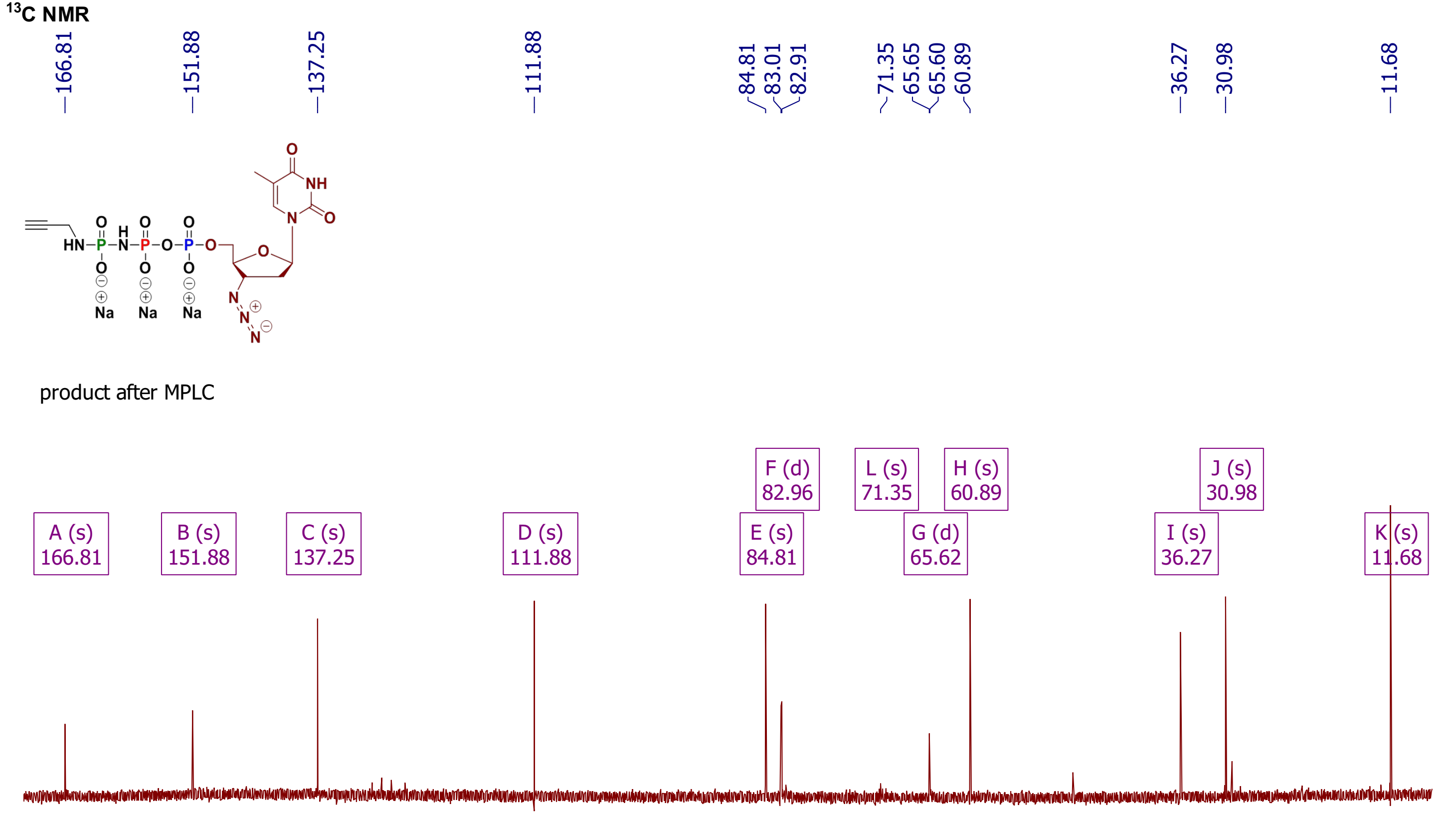

